for

\title{
Radical Aryl Migration from Boron to Carbon
}

\author{
Dinghai Wang, Christian Mück-Lichtenfeld, Constantin G. Daniliuc, Armido \\ Studer*
}

Corresponding author. e-mail: studer@uni-muenster.de

Organisch-Chemisches Institut, Westfälische Wilhelms-Universität, Corrensstraße 40, 48149 Münster, Germany

\section{Content}

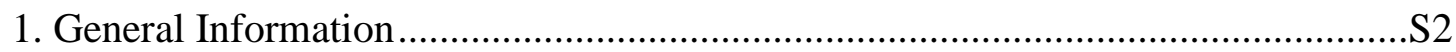

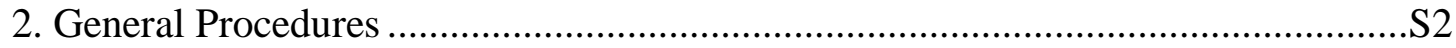

2.1 General procedures for radical aryl migration reactions of boronate complexes..................S2

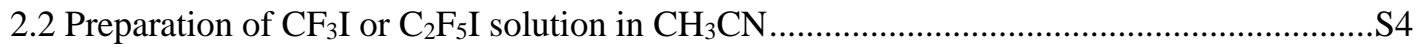

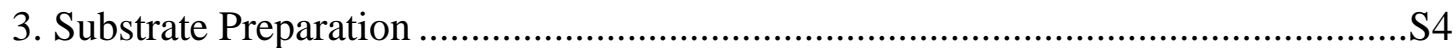

4. Characterization of Products .......................................................................S12

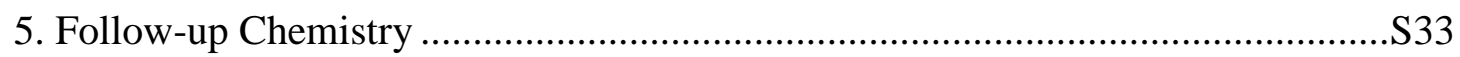

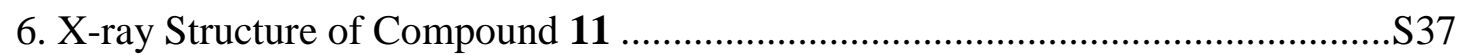

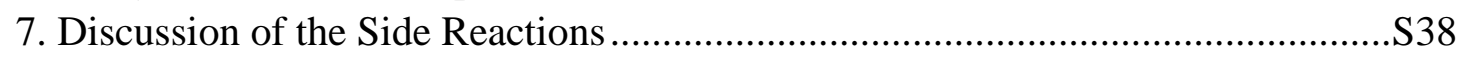

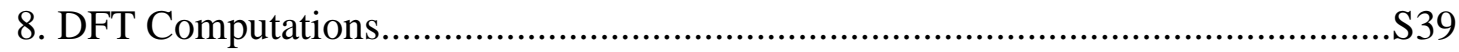

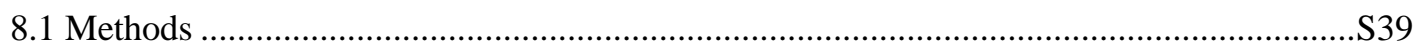

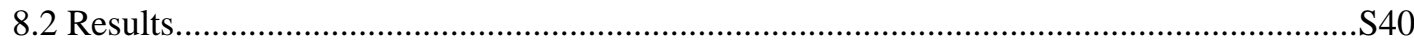

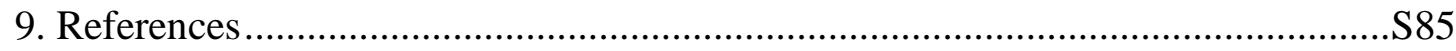

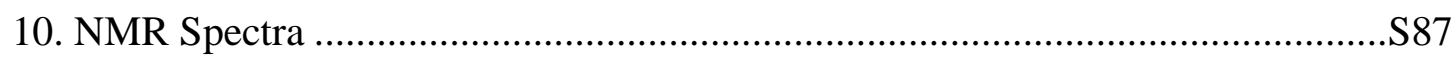




\section{General Information}

All reactions involving air or moisture sensitive reagents were carried out in flame-dried glassware under argon atmosphere using standard Schlenk techniques. Solvents used in reactions were either freshly distilled or obtained in extra-dry grade from commercial sources. Diethyl ether $\left(\mathrm{Et}_{2} \mathrm{O}\right)$ was refluxed over K and freshly distilled from K-Na-alloy (4:1) afterwards. Tetrahydrofuran (THF) was refluxed over $\mathrm{Na}$ and distilled from $\mathrm{K}$ afterwards. Acetonitrile (MeCN, 99.9\%, Extra Dry over Molecular Sieves) was purchased from Acros Organics. Solvents for extraction and for flash chromatography were distilled. Otherwise noted, commercially available chemicals were purchased from ABCR, Acros Organics, Alfa Aesar, Fluka, Sigma Aldrich and TCI and were used as received. Trifluoromethyl iodide was purchased from Sigma Aldrich and dissolved in $\mathrm{CH}_{3} \mathrm{CN}$. Flash chromatography (FC) was performed on Merck silica gel 60 (40-63 $\mu \mathrm{m})$. Merck silica gel 60 F254 plates were used for thin layer chromatography (TLC) using UV light $(254 / 366 \mathrm{~nm})$ or oxidation with $\mathrm{KMnO}_{4}\left(1.5 \mathrm{~g}\right.$ in $200 \mathrm{~mL} \mathrm{H}_{2} \mathrm{O}, 5 \mathrm{~g} \mathrm{NaHCO}$ ) for detection. Melting points (MP) were determined with a Stuart SMP10 and are uncorrected. Infrared spectra (IR) were measured on a Digilab 3100 FT-IR Excalibur Series spectrometer and the position of the absorption bands is given in wave numbers $v\left(\mathrm{~cm}^{-1}\right) .{ }^{1} \mathbf{H}$ NMR $(300 \mathrm{MHz}, 500 \mathrm{MHz}$ and $600 \mathrm{MHz}),{ }^{13} \mathbf{C}$ NMR (75 MHz, 126 MHz and $151 \mathrm{MHz}),{ }^{\mathbf{1}} \mathbf{F}$ NMR (282 MHz, $470 \mathrm{MHz}$ and $\left.564 \mathrm{MHz}\right)$ and ${ }^{11} \mathbf{B}$ NMR (96 MHz, 160 MHz and $192 \mathrm{MHz}$ ) spectra were measured on a Bruker DPX 300, Agilent DD2 500 or an Agilent $D D 2600$ spectrometer. The multiplicity of all signals were described as s (singlet), d (doublet), $\mathrm{t}$ (triplet), q (quartet) and $\mathrm{m}$ (multiplet). Chemical shifts ( $\delta$ in ppm) were referenced on the residual peak of $\mathrm{CDCl}_{3}\left({ }^{1} \mathrm{HNMR}: \delta=7.26 ;{ }^{13} \mathrm{C} \mathrm{NMR}: \delta=77.0\right)$ or on an external standard $\left(\mathrm{CFCl}_{3}:{ }^{19} \mathrm{~F} \mathrm{NMR}\right.$ : $\delta=0.0$; tetramethylsilane: ${ }^{1} \mathrm{H}$ NMR: $\left.\delta=0\right)$. HRMS ESI $(\mathrm{m} / \mathrm{z})$ measurements were performed on a Bruker MicroTof and HRMS EI (m/z) on a Waters-Micromass QuattroMicro GC-MS.

\section{General Procedures}

\subsection{General procedures for radical aryl migration reactions of boronate complexes}

\subsubsection{Gerneral procedure 1 for the radical induced aryl migration reaction of} boronate with commercially available phenyl lithium (GP1)

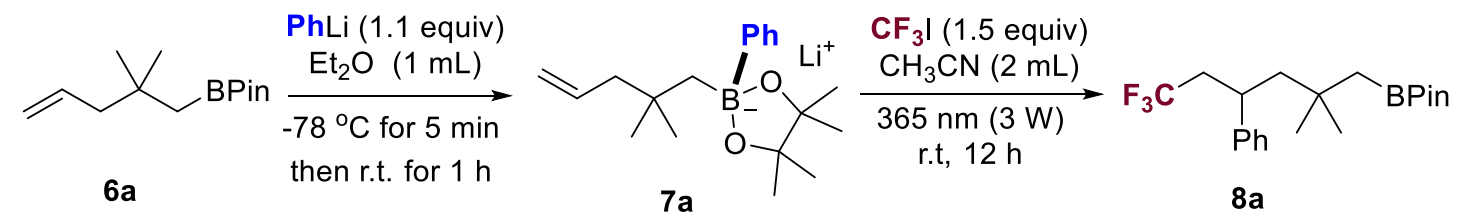

2,2-Dimethylpent-4-en-1-ylboronic acid pinacol ester 6a (44.8 mg, $0.20 \mathrm{mmol}, 1.0$ equiv) was dissolved in anhydrous diethyl ether $\left(\mathrm{Et}_{2} \mathrm{O}, 1.0 \mathrm{~mL}\right)$. Phenyl lithium $(1.9 \mathrm{M}$ in dibutyl ether, 0.12 $\mathrm{mL}, 0.22 \mathrm{mmol}, 1.1$ equiv) was added dropwise at $-78^{\circ} \mathrm{C}$ under Ar flow. The mixture was stirred at $-78{ }^{\circ} \mathrm{C}$ for 5 minutes then at room temperature for 1 hour. The solvent was carefully removed in vacuo and the resulting mixture was further dried for 5 min under high vacuum. After addition of 
acetonitrile $(2 \mathrm{~mL})$, the mixture was stirred until all solid was dissolved. Then $\mathrm{CF}_{3} \mathrm{I}(1.0 \mathrm{M}$ in $\mathrm{CH}_{3} \mathrm{CN}, 0.3 \mathrm{~mL}, 0.3 \mathrm{mmol}, 1.5$ equiv) was added to the reaction mixture and the reaction mixture was irradiated with a $3 \mathrm{~W}$ LED $(365 \mathrm{~nm})$ and stirred at room temperature for $12 \mathrm{~h}$. The reaction mixture was filtered through a pad of silica gel and rinsed with $40 \mathrm{mLEt}_{2} \mathrm{O}$. The organic solvent was removed under reduced pressure. Flash column chromatography eluting with pentane and dichloromethane (10:4) afforded the desired product 8a as a colorless sticky oil (52.4 $\mathrm{mg}, 71 \%$ yield). $\mathrm{Rf}=0.4$ (pentane/DCM = 2:1). ${ }^{1} \mathrm{H}$ NMR $(300 \mathrm{MHz}$, Chloroform- $d$ ) $\delta 7.24-7.06(\mathrm{~m}, 5 \mathrm{H})$, $3.07-2.94(\mathrm{~m}, 1 \mathrm{H}), 2.43-2.16(\mathrm{~m}, 2 \mathrm{H}), 1.86-1.60(\mathrm{~m}, 2 \mathrm{H}), 1.14(\mathrm{~s}, 12 \mathrm{H}), 0.75(\mathrm{~s}, 3 \mathrm{H}), 0.74(\mathrm{~s}$, 3H), 0.69 (s, 1H), $0.73-0.61(\mathrm{~m}, 2 \mathrm{H}) .{ }^{13} \mathrm{C}$ NMR $(151 \mathrm{MHz}$, Chloroform- $d$ ) $\delta$ 145.4, 128.4, 127.7, 126.5, 126.4, 82.8, 50.1, 42.9, 36.6, 33.1, 30.2, 29.7, 24.8, 24.8, carbon attached to boron not observed. ${ }^{19} \mathrm{~F}$ NMR (282 MHz, Chloroform- $d$ ) $\delta$-63.6. ${ }^{11} \mathrm{~B}$ NMR (96 MHz, Chloroform- $d$ ) $\delta$ 33.0. HRMS (ESI) $m / z=393.2187$ calcd. for $\mathrm{C}_{20} \mathrm{H}_{30} \mathrm{O}_{2} \mathrm{BF}_{3} \mathrm{Na}^{+}[\mathrm{M}+\mathrm{Na}]^{+}$, found: 393.2184 . FTIR (neat): $v\left(\mathrm{~cm}^{-1}\right)$ 2978, 2958, 2934, 2903, 1357, 1320, 1270, 1248, 1216, 1130, 1073, 969, 886, 847, 763, $700,665,605$.

\subsubsection{General procedure 2 for the radical induced aryl migration reactions of} boronate complexes with aryl lithium formed by lithium/halide exchange reaction (GP2)

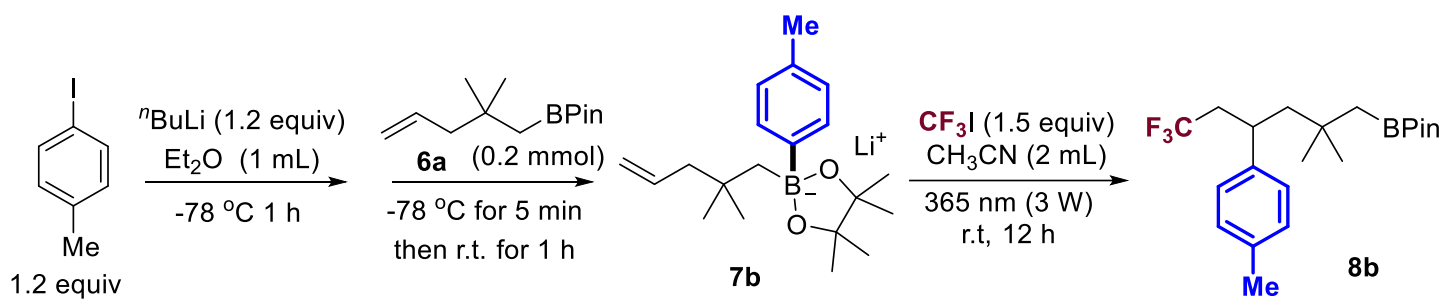

4-Methylphenyl iodide (52.3 mg, $0.24 \mathrm{mmol}, 1.2$ equiv) was dissolved in anhydrous diethyl ether ( $\mathrm{Et}_{2} \mathrm{O}, 1.0 \mathrm{~mL}$ ). $n$-Butyl lithium (1.6 M in hexane, $0.15 \mathrm{~mL}, 0.24 \mathrm{mmol}, 1.2$ equiv) was added dropwise at $-78{ }^{\circ} \mathrm{C}$ under Ar flow. The mixture was stirred at $-78{ }^{\circ} \mathrm{C}$ for 1 hour. 2,2-Dimethylpent4-en-1-ylboronic acid pinacol ester $6 \mathbf{a}(44.8 \mathrm{mg}, 0.20 \mathrm{mmol}, 1.0$ equiv) was added to the prepared aryl lithium solution by microsyringe at $-78{ }^{\circ} \mathrm{C}$. The reaction mixture was stirred at $-78{ }^{\circ} \mathrm{C}$ for 5 minutes then room temperature for 1 hour. The solvent was carefully removed in vacuo and the resulting mixture was further dried for $5 \mathrm{~min}$ under high vacuum. After addition of acetonitrile (2 $\mathrm{mL}$ ), the mixture was stirred until all solid was dissolved. Then $\mathrm{CF}_{3} \mathrm{I}\left(1.0 \mathrm{M}\right.$ in $\mathrm{CH}_{3} \mathrm{CN}, 0.3 \mathrm{~mL}, 0.3$ mmol, 1.5 equiv) was added to the reaction mixture and the reaction mixture was irradiated with a $3 \mathrm{~W}$ LED $(365 \mathrm{~nm})$ and stirred at room temperature for $12 \mathrm{~h}$. The reaction mixture was filtered through a pad of silica gel and rinsed with $40 \mathrm{~mL} \mathrm{Et}_{2} \mathrm{O}$. The organic solvent was removed under reduced pressure. Flash column chromatography eluting with pentane and dichloromethane (10:4) afforded the desired product $\mathbf{8 b}$ as a colorless sticky oil (52.7 $\mathrm{mg}, 69 \%$ yield). $\mathrm{Rf}=0.4$ (pentane/DCM = 2:1). ${ }^{1} \mathrm{H}$ NMR (300 MHz, Chloroform- $d$ ) $\delta 7.06-6.93(\mathrm{~m}, 4 \mathrm{H}), 3.03-2.90(\mathrm{~m}$, $1 \mathrm{H}), 2.38-2.17(\mathrm{~m}, 5 \mathrm{H}), 1.82-1.58(\mathrm{~m}, 2 \mathrm{H}), 1.14(\mathrm{~s}, 12 \mathrm{H}), 0.75(\mathrm{~s}, 3 \mathrm{H}), 0.74(\mathrm{~s}, 3 \mathrm{H}), 0.72-0.59$ 
(m, 2H). ${ }^{13} \mathrm{C}$ NMR (151 MHz, Chloroform- $d$ ) $\delta$ 142.4, 135.8, 129.1, 127.5, 126.6, 82.8, 50.2, 43.0, $36.1,33.1,30.3,29.7,24.8,24.8,21.0$, carbon attached to boron not observed. ${ }^{19} \mathrm{~F}$ NMR $(282 \mathrm{MHz}$, Chloroform- $d$ ) $\delta-63.5 .{ }^{11} \mathrm{~B}$ NMR (96 MHz, Chloroform- $d$ ) $\delta$ 32.9. HRMS (ESI) $m / z=407.2344$ calcd. for $\mathrm{C}_{21} \mathrm{H}_{32} \mathrm{O}_{2} \mathrm{BF}_{3} \mathrm{Na}^{+}[\mathrm{M}+\mathrm{Na}]^{+}$, found: 407.2342. FTIR (neat): $v\left(\mathrm{~cm}^{-1}\right)$ 2978, 2955, 2931, 2913, 2869, 1515, 1469, 1359, 1320, 1249, 1271, 1249, 1214, 1131, 1097, 970, 847, 814, 724, 606.

\subsection{Preparation of $\mathrm{CF}_{3} I$ or $\mathrm{C}_{2} \mathrm{~F}_{5} \mathrm{I}$ solution in $\mathrm{CH}_{3} \mathrm{CN}$}

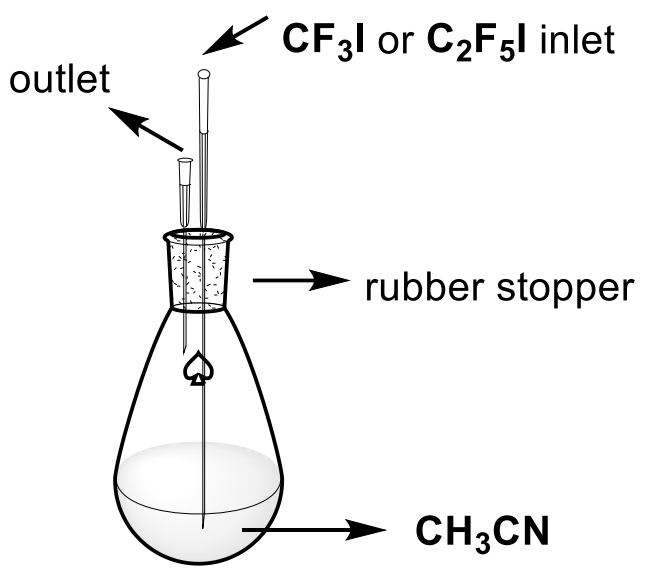

A $10 \mathrm{~mL}$ flask was charged with $5 \mathrm{~mL}$ anhydrous $\mathrm{CH}_{3} \mathrm{CN}$ under argon flow. The flask was sealed with a rubber stopper and the mass of the flask was weighed to be $\mathrm{m} 1$. After bubbling $\mathrm{CF}_{3} \mathrm{I}$ or $\mathrm{C}_{2} \mathrm{~F}_{5} \mathrm{I}$ gas into the $\mathrm{CH}_{3} \mathrm{CN}$ in the flask, the mass of the vial was weighed to be $\mathrm{m} 2$ and the volume of the solution was determined to be $\mathrm{V}$ by a $10 \mathrm{~mL}$ syringe. The concentration of the prepared $\mathrm{CF}_{3} \mathrm{I}$ solution was calculated to be $\frac{\mathrm{m} 2-\mathrm{m} 1}{\mathrm{~V} * \mathrm{M}}$. ( $\mathrm{M}$ is the molecular weight of $\mathrm{CF}_{3} \mathrm{I}$ or $\mathrm{C}_{2} \mathrm{~F}_{5} \mathrm{I}$.)

\section{Substrate Preparation}

3.1 General procedure 3 for the preparation of alkylboronic acid pinacol esters from alkyl bromides (GP3)

$$
\mathrm{R}-\mathrm{Br} \stackrel{\mathrm{Mg}(1.0 \text { equiv })}{\underset{\mathrm{THF}}{\longrightarrow}} \mathrm{R}-\mathrm{MgBr}
$$
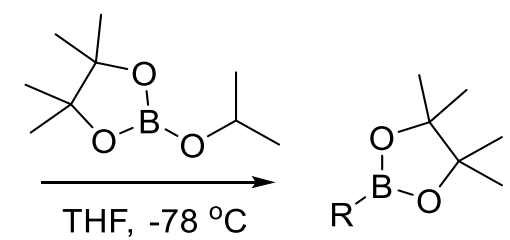

Magnesium chippings (2.0 equiv) were added to a dried flask under argon atmosphere and suspended with dry THF. A small amount of a solution of alkyl bromide (1.0 equiv) in dry THF was added to the magnesium and the activation of the Grignard formation was accelerated by addition of an iodine crystal and heating. Afterwards, the rest of the solution of alkyl bromide in THF was added dropwise. The reaction mixture was heated and stirred for another 1 hour at $60{ }^{\circ} \mathrm{C}$. The reaction mixture was cooled to $-78^{\circ} \mathrm{C}, 2$-isopropoxy-4,4,5,5-tetramethyl-1,3,2-dioxaborolane (1.0 equiv) was added in one portion. The reaction mixture was stirred overnight, quenched with saturated $\mathrm{NH}_{4} \mathrm{Cl}$ aqueous solution, and extracted with $\mathrm{Et}_{2} \mathrm{O}$. The combined organic layer was dried 
over $\mathrm{MgSO}_{4}$ and filtered. The filtrate was concentrated under reduced pressure and purified by flash column chromatography to afford the pure alkylboronic ester.

3.2 General procedure 4 for the preparation of alkylboronic acid pinacol esters from alkyl carboxylic acid esters (GP4)<smiles>COC(=O)C(C)C</smiles>

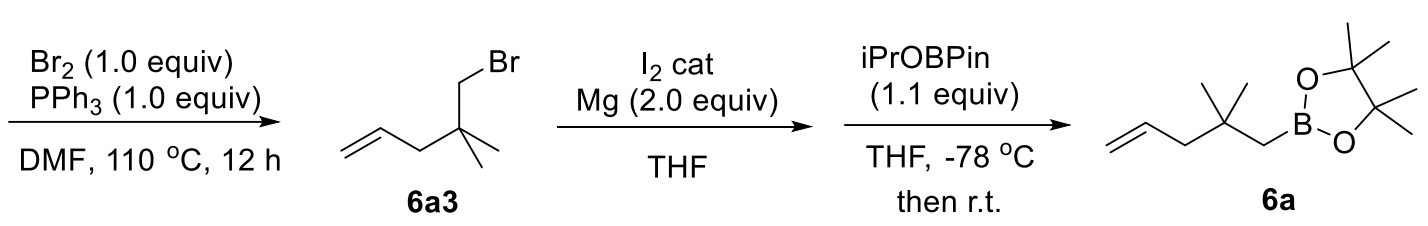

Step 1: To a stirred solution of diisopropylamine $(15.4 \mathrm{ml}, 12.1 \mathrm{~g}, 110 \mathrm{mmol}, 1.1 \mathrm{equiv})$ in THF $(100 \mathrm{ml})$ was added $n$-butyllithium $\left(68 \mathrm{ml}, 1.6 \mathrm{M}\right.$ in hexane, 1.1 equiv) dropwise at $-78{ }^{\circ} \mathrm{C}$. The mixture was stirred at room temperature for $30 \mathrm{~min}$, cooled again to $-78^{\circ} \mathrm{C}$, and treated with methyl isobutyrate ( $11.4 \mathrm{ml}, 10.2 \mathrm{~g}, 100 \mathrm{mmol}, 1.0$ equiv) slowly over $10 \mathrm{~min}$. The reaction mixture was stirred for 1.5 hours at $-78{ }^{\circ} \mathrm{C}$ and 2 hours at room temperature. The mixture was cooled again to $78{ }^{\circ} \mathrm{C}$ and allyl bromide $(13.1 \mathrm{ml}, 150 \mathrm{mmol}, 1.5$ equiv) was added dropwise. The mixture was stirred for 1 hour at room temperature then for 2 hours at $60{ }^{\circ} \mathrm{C}$. The reaction was quenched with water $(50 \mathrm{~mL})$ and extracted with diethyl ether $(100 \mathrm{~mL})$. The organic phase was separated and washed with water $(100 \mathrm{~mL})$, hydrochloric acid aqueous solution $(100 \mathrm{~mL}, 1.0 \mathrm{M})$ and brine $(100$ $\mathrm{mL}$ ), then dried over $\mathrm{MgSO}_{4}$ and filtered. The filtrate was concentrated under reduced pressure to afford the crude 6a1. 6a1 was used for the next step reaction without any further purification.

Step 2: 6a1 was dissolved in THF (150 mL). $\mathrm{LiAlH}_{4}(7.6 \mathrm{~g}, 200 \mathrm{mmol}, 2.0$ equiv) was added to the reaction flask at $0{ }^{\circ} \mathrm{C}$ portion wise. Then the cooling bath was removed and the reaction mixture was stirred for 2 hours. The reaction was carefully quenched as follows: addition of $7.6 \mathrm{ml}$ of water at $0{ }^{\circ} \mathrm{C}$ and stirring for $10 \mathrm{~min}$; addition of aqueous $\mathrm{NaOH}(15.2 \mathrm{ml}, 10 \% \mathrm{w} / \mathrm{w})$ and stirring for 10 $\mathrm{min}$; addition of $23 \mathrm{ml}$ of water and stirring for $10 \mathrm{~min}$. After warming to room temperature, the white solid was filtered off, and the filtrate was carefully concentrated. The residue was purified by column chromatography to afford $\mathbf{6 a 2}$ as a colorless liquid $(11.1 \mathrm{~g}, 87 \%$ yield for two steps). $\mathrm{Rf}=$ $0.4\left(\mathrm{PE} / \mathrm{Et}_{2} \mathrm{O}=2: 1\right) .{ }^{1} \mathrm{H}$ NMR $(300 \mathrm{MHz}$, Chloroform- $d$ ) $\delta 5.95-5.74(\mathrm{~m}, 1 \mathrm{H}), 5.12-4.94(\mathrm{~m}, 2 \mathrm{H})$, $3.32(\mathrm{~s}, 2 \mathrm{H}), 2.06-1.97(\mathrm{~m}, 2 \mathrm{H}), 1.60(\mathrm{~s}, 1 \mathrm{H}), 0.88(\mathrm{~s}, 6 \mathrm{H})$.

Step 3: Triphenylphosphine (7.86 g, $30 \mathrm{mmol}, 1.0$ equiv) was dissolved in $30 \mathrm{ml}$ DMF. Bromine $(1.55 \mathrm{~mL}, 4.8 \mathrm{~g}, 30 \mathrm{mmol}, 1.0$ equiv) was added to the triphenylphosphine solution dropwisely. Then $6 \mathbf{2} 2$ ( $3.82 \mathrm{~g}, 30 \mathrm{mmol}, 1.0$ equiv) was added in one portion. The reaction mixture was stirred at $110^{\circ} \mathrm{C}$ for 12 hours then quenched with water. The resulting mixture was extracted with $100 \mathrm{~mL}$ diethyl ether. The organic phase was washed with water $(100 \mathrm{~mL} \times 3)$, then dried over $\mathrm{MgSO}_{4}$ and filtered. The filtrate was evaporated under reduced pressure to remove the solvent and further purified by column chromatography with pentane as eluent to afford the alkylbromide $\mathbf{6 a} 3$ as colorless liquid (2.6 g, 50\% yield). $\mathrm{Rf}=0.7(\mathrm{PE}) .{ }^{1} \mathrm{H} \mathrm{NMR}(300 \mathrm{MHz}$, Chloroform- $d) \delta 5.86-5.68$ 
(m, $1 \mathrm{H}), 5.14-5.02(\mathrm{~m}, 2 \mathrm{H}), 3.28(\mathrm{~s}, 2 \mathrm{H}), 2.16-2.05(\mathrm{~m}, 2 \mathrm{H}), 1.02(\mathrm{~s}, 6 \mathrm{H})$.

Step 4: According to GP3, $\mathbf{6 a 3}$ ( $1.33 \mathrm{~g}, 7.5 \mathrm{mmol}, 1.0$ equiv) afforded $\mathbf{6 a}$ as a colorless liquid ( 0.77 $\mathrm{g}, 46 \%$ yield $) . \mathrm{Rf}=0.6\left(\mathrm{PE} / \mathrm{Et}_{2} \mathrm{O}=20: 1\right) .{ }^{1} \mathrm{H}$ NMR $(300 \mathrm{MHz}$, Chloroform- $d) \delta 5.92-5.74(\mathrm{~m}$, 1H), $5.05-4.90(\mathrm{~m}, 2 \mathrm{H}), 2.04-1.98(\mathrm{~m}, 2 \mathrm{H}), 1.24(\mathrm{~s}, 12 \mathrm{H}), 0.96(\mathrm{~s}, 6 \mathrm{H}), 0.78(\mathrm{~s}, 2 \mathrm{H}) .{ }^{13} \mathrm{C} \mathrm{NMR}$ (76 MHz, Chloroform- $d$ ) $\delta 136.3,116.6,82.8,49.0,32.5,29.2,24.9$, carbon attached to boron not observed. ${ }^{11} \mathrm{~B}$ NMR (96 MHz, Chloroform- $d$ ) $\delta 33.2$. HRMS (ESI) $\mathrm{m} / z=247.1842$ calcd. for $\mathrm{C}_{13} \mathrm{H}_{25} \mathrm{O}_{2} \mathrm{BNa}^{+}[\mathrm{M}+\mathrm{Na}]^{+}$, found: 247.1841. FTIR (neat): $v\left(\mathrm{~cm}^{-1}\right)$ 3076, 2979, 2955, 2360, 2341, 1469, 1355, 1324, 1312, 1274, 1245, 1215, 1142, 1106, 997, 970, 911, 874, 847.

\subsection{General procedure 5 for the preparation of alkylboronic acid pinacol esters from alkyl carboxylic acids (GP5)}
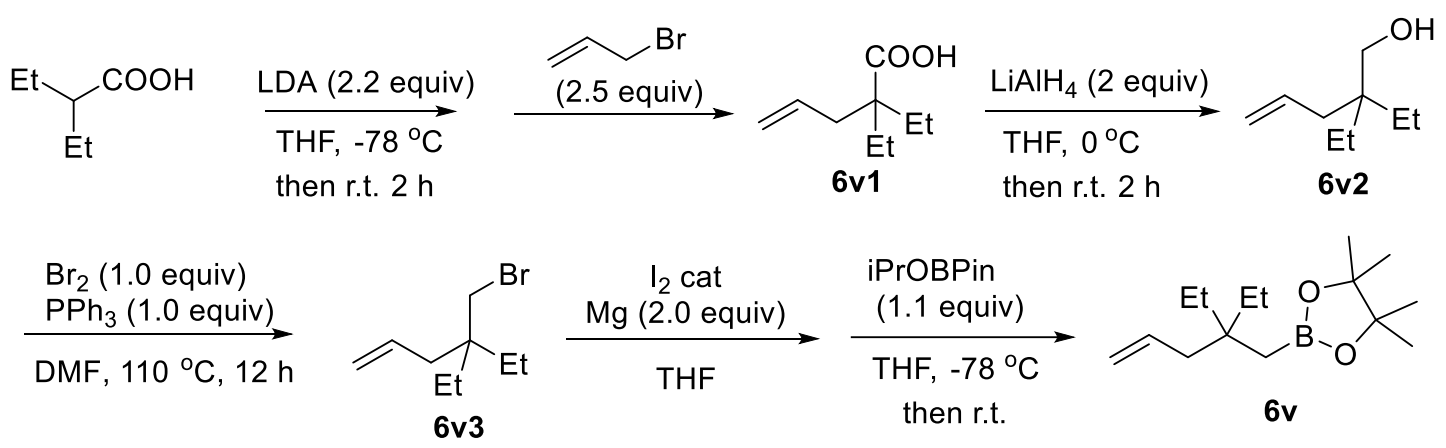

To a stirred solution of diisopropylamine $(6.2 \mathrm{ml}, 4.44 \mathrm{~g}, 110 \mathrm{mmol}, 2.2$ equiv) in THF ( $20 \mathrm{ml})$ was added $n$-butyllithium $\left(27.5 \mathrm{ml}, 1.6 \mathrm{M}\right.$ in hexane, $44 \mathrm{mmol}, 2.2$ equiv) dropwise at $-78{ }^{\circ} \mathrm{C}$. The mixture was stirred at room temperature for $30 \mathrm{~min}$, cooled again to $-78^{\circ} \mathrm{C}$, and treated with 2ethylbutanoic acid ( $2.32 \mathrm{~g}, 20 \mathrm{mmol}, 1.0$ equiv) slowly. The reaction mixture was stirred for 1 hour at room temperature and then 2 hours at $60{ }^{\circ} \mathrm{C}$. The mixture was cooled again to $-78{ }^{\circ} \mathrm{C}$ and allyl bromide ( $3.45 \mathrm{ml}, 50 \mathrm{mmol}, 2.5$ equiv) was added dropwise. The mixture was allowed to warm up to room temperature and stirred overnight. The reaction was quenched with water $(50 \mathrm{~mL})$ and washed with diethyl ether $(50 \mathrm{~mL} \times 3)$. The aqueous phase was separated and acidified with hydrochloric acid (40 mL, 1.0 M aqueous), then extracted with diethyl ether $(50 \mathrm{~mL} \times 3)$. The combined organic phase was dried over $\mathrm{MgSO}_{4}$ and filtered. The filtrate was concentrated under reduced pressure to afford the crude $6 \mathbf{v} \mathbf{1}$. $6 \mathbf{v} \mathbf{1}$ was used for the next step reaction without any further purification.

Step 2: 6v1 was dissolved in THF (100 mL). $\mathrm{LiAlH}_{4}(1.52 \mathrm{~g}, 40 \mathrm{mmol}, 2.0$ equiv) was added to the reaction flask at $0{ }^{\circ} \mathrm{C}$ portion wise. Then the cooling bath was removed and the reaction mixture was stirred for 2 hours. The reaction was carefully quenched as follows: addition of $1.5 \mathrm{ml}$ of water at $0{ }^{\circ} \mathrm{C}$ and stirring for $10 \mathrm{~min}$; addition of aqueous $\mathrm{NaOH}(3.0 \mathrm{ml}, 10 \% \mathrm{w} / \mathrm{w})$ and stirring for 10 $\mathrm{min}$; addition of $4.5 \mathrm{ml}$ of water and stirring for $10 \mathrm{~min}$. After warming to room temperature, the white solid was filtered off, and the filtrate was carefully concentrated. The residue was purified by column chromatography to afford $\mathbf{6 v 2}$ as a colorless liquid $(2.03 \mathrm{~g}, 71 \%$ yield for two steps). $\mathrm{Rf}=$ $0.4\left(\mathrm{PE} / \mathrm{Et}_{2} \mathrm{O}=2: 1\right) .{ }^{1} \mathrm{H}$ NMR $(300 \mathrm{MHz}$, Chloroform- $d) \delta 5.93-5.75(\mathrm{~m}, 1 \mathrm{H}), 5.14-5.00(\mathrm{~m}, 2 \mathrm{H})$, $3.38(\mathrm{~s}, 2 \mathrm{H}), 2.06-1.96(\mathrm{~m}, 2 \mathrm{H}), 1.34(\mathrm{~s}, 1 \mathrm{H}), 1.33-1.16(\mathrm{~m}, 4 \mathrm{H}), 0.82(\mathrm{t}, J=7.5 \mathrm{~Hz}, 6 \mathrm{H})$. 
Step 3: Triphenylphosphine (3.75 g, $14.3 \mathrm{mmol}, 1.0$ equiv) was dissolved in $30 \mathrm{ml}$ DMF. Bromine $(0.74 \mathrm{~mL}, 2.29 \mathrm{~g}, 14.3 \mathrm{mmol}, 1.0$ equiv) was added to the triphenylphosphine solution dropwise. Then $6 \mathrm{v} 2$ (2.03 g, $14.3 \mathrm{mmol}, 1.0$ equiv) was added in one portion. The reaction mixture was stirred at $110^{\circ} \mathrm{C}$ for 12 hours then quenched with water. The resulting mixture was extracted with $100 \mathrm{~mL}$ diethyl ether. The organic phase was washed with water $(100 \mathrm{~mL} \times 3)$, then dried over $\mathrm{MgSO}_{4}$ and filtered. The filtrate was evaporated under reduced pressure to remove the solvent and further purified by column chromatography with pentane as eluent to afford the alkylbromide $\mathbf{6 v} 3$ as colorless liquid ( $2.0 \mathrm{~g}, 69 \%$ yield). $\mathrm{Rf}=05$ (PE). ${ }^{1} \mathrm{H}$ NMR $(300 \mathrm{MHz}$, Chloroform- $d$ ) $\delta 5.83-5.62$ $(\mathrm{m}, 1 \mathrm{H}), 5.17-5.03(\mathrm{~m}, 2 \mathrm{H}), 3.27(\mathrm{~s}, 2 \mathrm{H}), 2.11-2.01(\mathrm{~m}, 2 \mathrm{H}), 1.38-1.26(\mathrm{~m}, 4 \mathrm{H}), 0.82(\mathrm{t}, J=7.5$ $\mathrm{Hz}, 6 \mathrm{H})$.

Step 4: According to $\mathbf{G P 3}, \mathbf{6 v 3}$ ( $2.0 \mathrm{~g}, 9.8 \mathrm{mmol}, 1.0$ equiv) afforded $\mathbf{6 v}$ as a colorless liquid $(0.7 \mathrm{~g}$, $28 \%$ yield $) . \mathrm{Rf}=0.5\left(\mathrm{PE}_{\mathrm{Et}} \mathrm{O}=20: 1\right) .{ }^{1} \mathrm{H}$ NMR $(300 \mathrm{MHz}$, Chloroform- $d) \delta 5.83-5.65(\mathrm{~m}, 1 \mathrm{H})$, $5.01-4.87$ (m, 2H), $2.03-1.92(\mathrm{~m}, 2 \mathrm{H}), 1.24(\mathrm{q}, J=7.5 \mathrm{~Hz}, 4 \mathrm{H}), 1.16(\mathrm{~s}, 12 \mathrm{H}), 0.71(\mathrm{t}, J=7.5$ $\mathrm{Hz}, 6 \mathrm{H}), 0.67$ (s, 2H). ${ }^{13} \mathrm{C}$ NMR (76 MHz, Chloroform- $d$ ) $\delta$ 136.1, 116.4, 82.3, 42.6, 37.7, 30.6, 24.8, 8.0, carbon attached to boron not observed. ${ }^{11} \mathrm{~B}$ NMR ( $96 \mathrm{MHz}$, Chloroform- $d$ ) $\delta 33.3$. HRMS (ESI) $m / z=275.2156$ calcd. for $\mathrm{C}_{15} \mathrm{H}_{29} \mathrm{O}_{2} \mathrm{BNa}^{+}[\mathrm{M}+\mathrm{Na}]^{+}$, found: 275.2154 . FTIR (neat): $v\left(\mathrm{~cm}^{-1}\right)$ 2979, 2956, 2930, 2872, 1638, 1466, 1457, 1369, 1353, 1315, 1269, 1213, 1141, 1109, 994, 968, $910,888,859,845$.

2-(2,2-Dipropylpent-4-en-1-yl)-4,4,5,5-tetramethyl-1,3,2-dioxaborolane (6w)

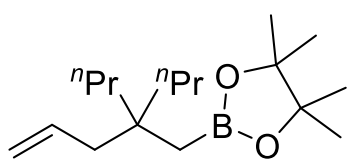

According to GP5, 2-propylpentanoic acid (2.88 g, $20 \mathrm{mmol}$ ) afforded $\mathbf{6 w}$ as a colorless liquid (0.43 $\mathrm{g}, 7 \%$ overall yield $) . \mathrm{Rf}=0.7\left(\mathrm{PE} / \mathrm{Et}_{2} \mathrm{O}=20: 1\right) .{ }^{1} \mathrm{H}$ NMR $(300 \mathrm{MHz}$, Chloroform- $d) \delta 5.90-5.72$ (m, 1H), $5.05-4.92(\mathrm{~m}, 2 \mathrm{H}), 2.10-2.00(\mathrm{~m}, 2 \mathrm{H}), 1.28-1.17(\mathrm{~m}, 20 \mathrm{H}), 0.92-0.79(\mathrm{~m}, 6 \mathrm{H}), 0.75$ (s, 2H). ${ }^{13} \mathrm{C}$ NMR (76 MHz, Chloroform- $d$ ) $\delta$ 136.1, 116.4, 82.6, 43.5, 41.6, 37.7, 24.8, 16.7, 14.9, carbon attached to boron not observed. ${ }^{11} \mathrm{~B}$ NMR $(96 \mathrm{MHz}$, Chloroform- $d$ ) $\delta 33.2$. HRMS (ESI) $m / z=303.2469$ calcd. for $\mathrm{C}_{17} \mathrm{H}_{33} \mathrm{O}_{2} \mathrm{BNa}^{+}[\mathrm{M}+\mathrm{Na}]^{+}$, found: 303.2466. FTIR (neat): $v$ (cm $\left.{ }^{-1}\right) 2979$, 2956, 2930, 2872, 1638, 1466, 1369, 1353, 1315, 1269, 1213, 1141, 1109, 994, 968, 910, 888, 859, $845,745,579$.

2-((1-Allylcyclopentyl)methyl)-4,4,5,5-tetramethyl-1,3,2-dioxaborolane (6x)

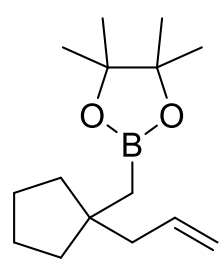

According to GP5, cyclopentanecarboxylic acid (2.28 g, $20 \mathrm{mmol})$ afforded $\mathbf{6 x}$ as a colorless liquid ( $0.54 \mathrm{~g}, 11 \%$ overall yield). $\mathrm{Rf}=0.5\left(\mathrm{PE}_{\mathrm{Et}} \mathrm{O}=20: 1\right) .{ }^{1} \mathrm{H}$ NMR $(300 \mathrm{MHz}$, Chloroform- $d$ ) $\delta 5.93$ $-5.76(\mathrm{~m}, 1 \mathrm{H}), 5.06-4.95(\mathrm{~m}, 2 \mathrm{H}), 2.15-2.07(\mathrm{~m}, 2 \mathrm{H}), 1.69-1.34(\mathrm{~m}, 8 \mathrm{H}), 1.23(\mathrm{~s}, 12 \mathrm{H}), 0.88$ (s, 2H). ${ }^{13} \mathrm{C}$ NMR $\left(76 \mathrm{MHz}, \mathrm{CDCl}_{3}\right) \delta 136.9,116.4,82.7,45.4,43.6,39.2,24.9,24.5$, carbon attached to boron not observed. ${ }^{11} \mathrm{~B}$ NMR $(96 \mathrm{MHz}$, Chloroform- $d$ ) $\delta 33.3$. HRMS (ESI) $\mathrm{m} / \mathrm{z}=$ 
273.1999 calcd. for $\mathrm{C}_{15} \mathrm{H}_{27} \mathrm{O}_{2} \mathrm{BNa}^{+}[\mathrm{M}+\mathrm{Na}]^{+}$, found: 73.1998. FTIR (neat): $v\left(\mathrm{~cm}^{-1}\right)$ 2977, 2952, 2869, 2362, 2339, 1356, 1311, 1269, 1214, 1144, 1109, 994, 968, 909, 876, 847, 668.

2-((1-Allylcyclohexyl)methyl)-4,4,5,5-tetramethyl-1,3,2-dioxaborolane (6y)

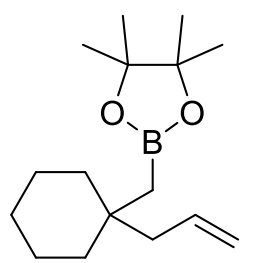

According to GP5, cyclohexanecarboxylic acid (2.56 g, $20 \mathrm{mmol})$ afforded $\mathbf{6 y}$ as a colorless liquid

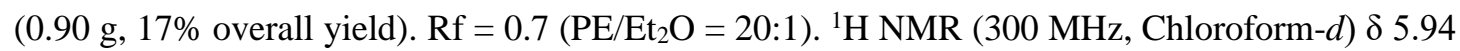
$-5.73(\mathrm{~m}, 1 \mathrm{H}), 5.09-4.93(\mathrm{~m}, 2 \mathrm{H}), 2.17-2.07(\mathrm{~m}, 2 \mathrm{H}), 1.50-1.29(\mathrm{~m}, 10 \mathrm{H}), 1.23(\mathrm{~s}, 12 \mathrm{H}), 0.82$ (s, 2H). ${ }^{13} \mathrm{C}$ NMR (76 MHz, Chloroform- $d$ ) $\delta$ 135.9, 116.6, 82.6, 44.1, 37.6, 35.1, 26.3, 24.9, 21.9, carbon attached to boron not observed. ${ }^{11} \mathrm{~B}$ NMR $(96 \mathrm{MHz}$, Chloroform- $d$ ) $\delta 33.2$. HRMS (ESI) $m / z=287.2156$ calcd. for $\mathrm{C}_{16} \mathrm{H}_{29} \mathrm{O}_{2} \mathrm{BNa}^{+}[\mathrm{M}+\mathrm{Na}]^{+}$, found: 287.2153. FTIR (neat): $v\left(\mathrm{~cm}^{-1}\right) 2977$, 2923, 2859, 1638, 1454, 1362, 1320, 1273, 1214, 1144, 1109, 999, 968, 909, 871, 847, 735.

2-((4-Allyltetrahydro-2H-pyran-4-yl)methyl)-4,4,5,5-tetramethyl-1,3,2-dioxaborolane (6z)

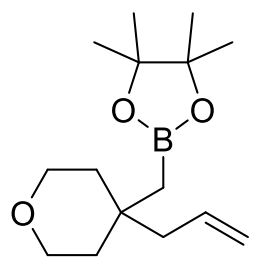

According to GP5, tetrahydro-2H-pyran-4-carboxylic acid (2.60 g, $20 \mathrm{mmol})$ afforded $\mathbf{6 z}$ as a

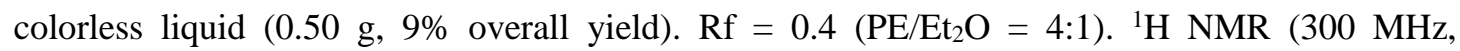
Chloroform- $d$ ) $\delta 5.95-5.71(\mathrm{~m}, 1 \mathrm{H}), 5.12-4.95(\mathrm{~m}, 2 \mathrm{H}), 3.78-3.56(\mathrm{~m}, 4 \mathrm{H}), 2.27-2.13(\mathrm{~m}, 2 \mathrm{H})$, $1.57-1.38(\mathrm{~m}, 4 \mathrm{H}), 1.23(\mathrm{~s}, 12 \mathrm{H}), 0.93(\mathrm{~s}, 2 \mathrm{H}) .{ }^{13} \mathrm{C} \mathrm{NMR}\left(76 \mathrm{MHz}, \mathrm{CDCl}_{3}\right) \delta 134.7,117.5,82.8$, $63.8,44.3,37.5,32.8,24.9$, carbon attached to boron not observed. ${ }^{11} \mathrm{~B} \mathrm{NMR}\left(96 \mathrm{MHz}, \mathrm{CDCl}_{3}\right) \delta$ 33.1. HRMS (ESI) $\mathrm{m} / \mathrm{z}=289.1948$ calcd. for $\mathrm{C}_{15} \mathrm{H}_{27} \mathrm{O}_{3} \mathrm{BNa}^{+}[\mathrm{M}+\mathrm{Na}]^{+}$, found: 289.1947. FTIR (neat): v $\left(\mathrm{cm}^{-1}\right)$ 2976, 2926, 2850, 1639, 1362, 1316, 1213, 1166, 1143, 1105, 1029, 1018, 1002, 968, 911, 848, 592 .

2-((1-Allylcycloheptyl)methyl)-4,4,5,5-tetramethyl-1,3,2-dioxaborolane (6aa)<smiles>C=CCC1(CC2(CC=C)CC(C)(C)C(C)(C)O2)CCCCCC1</smiles>

According to GP5, cycloheptanecarboxylic acid $(2.84 \mathrm{~g}, 20 \mathrm{mmol})$ afforded $\mathbf{6 a a}$ as a colorless liquid ( $0.8 \mathrm{~g}, 14 \%$ overall yield). $\mathrm{Rf}=0.5\left(\mathrm{PE} / \mathrm{Et}_{2} \mathrm{O}=20: 1\right) .{ }^{1} \mathrm{H}$ NMR $(300 \mathrm{MHz}$, Chloroform- $d) \delta 5.90-$ $5.67(\mathrm{~m}, 1 \mathrm{H}), 5.02-4.85(\mathrm{~m}, 2 \mathrm{H}), 2.10-1.95(\mathrm{~m}, 2 \mathrm{H}), 1.48-1.28(\mathrm{~m}, 12 \mathrm{H}), 1.17(\mathrm{~s}, 12 \mathrm{H}), 0.73$ (s, 2H). ${ }^{13} \mathrm{C} \mathrm{NMR}\left(76 \mathrm{MHz}, \mathrm{CDCl}_{3}\right) \delta 136.5,116.6,82.6,46.5,40.9,38.4,30.5,24.9,23.0$, carbon attached to boron not observed. ${ }^{11} \mathrm{~B}$ NMR $\left(96 \mathrm{MHz}, \mathrm{CDCl}_{3}\right) \delta 33.3$. HRMS (ESI) $\mathrm{m} / z=301.2313$ 
calcd. for $\mathrm{C}_{17} \mathrm{H}_{31} \mathrm{O}_{2} \mathrm{BNa}^{+}[\mathrm{M}+\mathrm{Na}]^{+}$, found: 301.2312. FTIR (neat): 2978, 2921, 2852, 1638, 1461 , 1444, 1366, 1349, 1313, 1270, 1214, 1145, 1107, 998, 969, 908, 846, 734, 680.

4,4,5,5-Tetramethyl-2-(2-methyl-2-phenylpent-4-en-1-yl)-1,3,2-dioxaborolane (6ab)

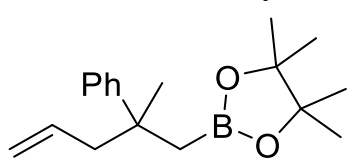

According to GP5, 2-phenylpropionic acid (2.19 g, $14.6 \mathrm{mmol})$ afforded 6ab as a colorless liquid (0.2 g, 5\% overall yield). $\mathrm{Rf}=0.6\left(\mathrm{PE} / \mathrm{Et}_{2} \mathrm{O}=10: 1\right) .{ }^{1} \mathrm{H}$ NMR $(300 \mathrm{MHz}$, Chloroform- $d) \delta 7.42-$ $7.33(\mathrm{~m}, 2 \mathrm{H}), 7.33-7.25(\mathrm{~m}, 2 \mathrm{H}), 7.21-7.11(\mathrm{~m}, 1 \mathrm{H}), 5.64-5.47(\mathrm{~m}, 1 \mathrm{H}), 5.04-4.89(\mathrm{~m}, 2 \mathrm{H})$, $2.59-2.37(\mathrm{~m}, 2 \mathrm{H}), 1.45(\mathrm{~s}, 3 \mathrm{H}), 1.31(\mathrm{~d}, J=56.5 \mathrm{~Hz}, 1 \mathrm{H}), 1.26(\mathrm{~d}, J=56.5 \mathrm{~Hz}, 1 \mathrm{H}), 1.09$ (d, $J=$ $8.0 \mathrm{~Hz}, 12 \mathrm{H}) .{ }^{13} \mathrm{C}$ NMR $(76 \mathrm{MHz}$, Chloroform- $d$ ) $\delta 149.2,135.8,127.7,126.1,125.3,116.9,82.7$, 49.7, 39.2, 26.8, 24.7, 24.6, carbon attached to boron not observed. ${ }^{11} \mathrm{~B}$ NMR $(96 \mathrm{MHz}$, Chloroform- $d$ ) $\delta 32.8$. HRMS (ESI) $\mathrm{m} / z=309.2000$ calcd. for $\mathrm{C}_{18} \mathrm{H}_{27} \mathrm{O}_{2} \mathrm{BNa}^{+}[\mathrm{M}+\mathrm{Na}]^{+}$, found: 309.1998. FTIR (neat): 3021, 2978, 2892, 1639, 1602, 1497, 1445, 1389, 1355, 1325, 1216, 1143 , 997, 970, 912, 846, 764, 697, 668, 632, 576.

${ }^{1} \mathrm{H}$ NMR (300 MHz, Chloroform- $d$ ) $\delta 7.42-7.33(\mathrm{~m}, 1 \mathrm{H}), 7.33-7.25(\mathrm{~m}, 1 \mathrm{H}), 7.21-7.11(\mathrm{~m}, 1 \mathrm{H})$.

4,4,5,5-Tetramethyl-2-(2-phenylpent-4-en-1-yl)-1,3,2-dioxaborolane (6ac)<smiles>C=CCC(CB1OC(C)(C)C(C)(C)O1)c1ccccc1</smiles>

According to GP5, 2-phenylpropionic acid (3.0 g, $14.6 \mathrm{mmol})$ afforded 6 ac as a colorless liquid (0.7 g, 13\% overall yield). $\mathrm{Rf}=0.4\left(\mathrm{PE}_{\mathrm{Et}} \mathrm{O}=20: 1\right) .{ }^{1} \mathrm{H}$ NMR $(300 \mathrm{MHz}$, Chloroform- $d) \delta 7.24-$ $7.02(\mathrm{~m}, 5 \mathrm{H}), 5.69-5.53(\mathrm{~m}, 1 \mathrm{H}), 4.97-4.77(\mathrm{~m}, 2 \mathrm{H}), 2.95-2.80(\mathrm{~m}, 1 \mathrm{H}), 2.38-2.18(\mathrm{~m}, 2 \mathrm{H})$, $1.21-0.98(\mathrm{~m}, 2 \mathrm{H}), 1.03(\mathrm{~s}, 6 \mathrm{H}), 1.02(\mathrm{~s}, 6 \mathrm{H}) .{ }^{13} \mathrm{C} \mathrm{NMR}\left(76 \mathrm{MHz}, \mathrm{CDCl}_{3}\right) \delta$ 146.8, 137.2, 128.0, $127.4,125.8,116.0,82.9,43.7,41.4,24.7,24.6$, carbon attached to boron not observed. ${ }^{11} \mathrm{~B}$ NMR $\left(96 \mathrm{MHz}, \mathrm{CDCl}_{3}\right) \delta$ 33.4. HRMS (ESI) $\mathrm{m} / z=295.1843$ calcd. for $\mathrm{C}_{17} \mathrm{H}_{25} \mathrm{O}_{2} \mathrm{BNa}^{+}[\mathrm{M}+\mathrm{Na}]^{+}$, found: 295.1841. FTIR (neat): $v\left(\mathrm{~cm}^{-1}\right)$ 3029, 2977, 2926, 1640, 1494, 1366, 1318, 1271, 1214, 1144, 968, 911, 846, 757, 699, 668, 579 .

2-(2-Isopropylpent-4-en-1-yl)-4,4,5,5-tetramethyl-1,3,2-dioxaborolane (6ad)<smiles>C=CCC(CB1OC(C)(C)C(C)(C)O1)C(C)C</smiles>

According to GP5, 4-methylbutanoic acid (2.04 g, $20 \mathrm{mmol})$ afforded $\mathbf{6 a d}$ as a colorless liquid (0.5 $\mathrm{g}, 10 \%$ overall yield $) . \mathrm{Rf}=0.5\left(\mathrm{PE} / \mathrm{Et}_{2} \mathrm{O}=20: 1\right) .{ }^{1} \mathrm{H}$ NMR $(300 \mathrm{MHz}$, Chloroform- $d) \delta 5.92-5.69$ (m, 1H), $5.07-4.89(\mathrm{~m}, 2 \mathrm{H}), 2.19-1.86(\mathrm{~m}, 2 \mathrm{H}), 1.72-1.52(\mathrm{~m}, 2 \mathrm{H}), 1.24(\mathrm{~s}, 12 \mathrm{H}), 0.89-0.79$ $(\mathrm{m}, 6 \mathrm{H}), 0.79-0.59(\mathrm{~m}, 2 \mathrm{H}) .{ }^{13} \mathrm{C} \mathrm{NMR}\left(76 \mathrm{MHz}, \mathrm{CDCl}_{3}\right) \delta 138.4,115.5,82.8,39.9,38.3,31.4$, 24.9, 24.8, 19.8, 18.4, carbon attached to boron not observed. ${ }^{11} \mathrm{~B} \mathrm{NMR}\left(96 \mathrm{MHz}, \mathrm{CDCl}_{3}\right) \delta 33.9$. HRMS (ESI) $m / z=261.1999$ calcd. for $\mathrm{C}_{14} \mathrm{H}_{27} \mathrm{O}_{2} \mathrm{BNa}^{+}[\mathrm{M}+\mathrm{Na}]^{+}$, found: 261.1996. FTIR (neat): 3075, 2978, 2931, 2873, 1640, 1466, 1371, 1346, 1317, 1273, 1215, 1146, 993, 969, 909, 848, 569. 


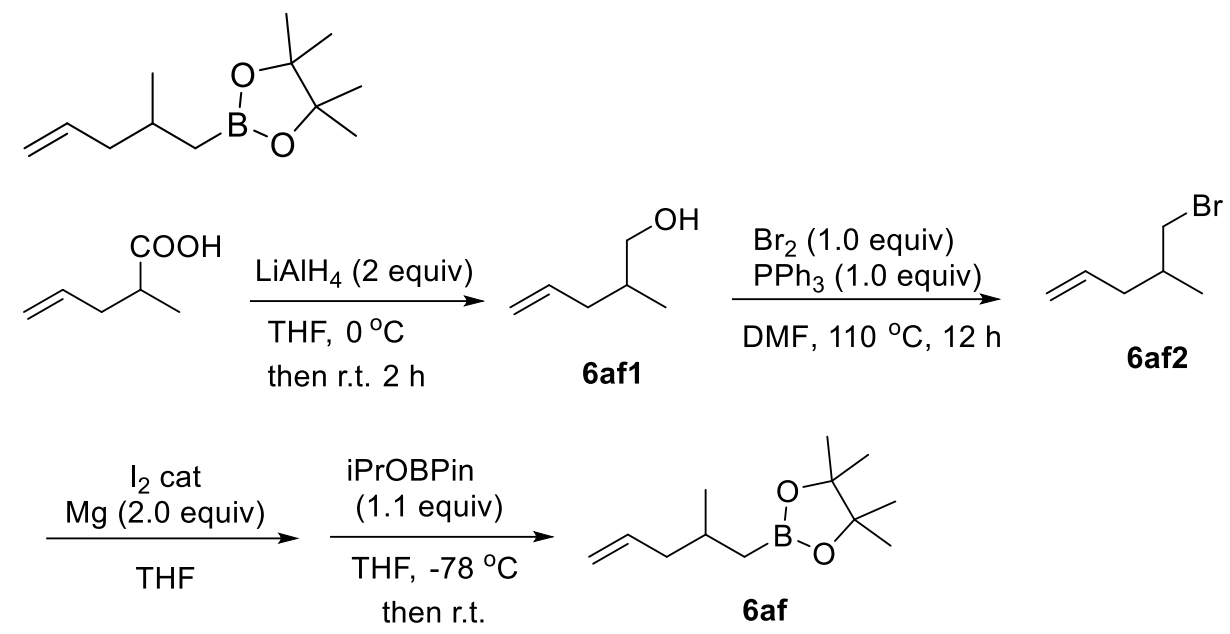

2-Methylpent-4-enoic acid (2.28 g, $20 \mathrm{mmol}, 1.0$ equiv) was dissolved in THF (50 mL). $\mathrm{LiAlH}_{4}$ $\left(1.52 \mathrm{~g}, 40 \mathrm{mmol}, 2.0\right.$ equiv) was added to the reaction flask at $0{ }^{\circ} \mathrm{C}$ portion wise. Then the cooling bath was removed and the reaction mixture was stirred for 2 hours. The reaction was carefully quenched as follows: addition of $1.5 \mathrm{ml}$ of water at $0{ }^{\circ} \mathrm{C}$ and stirring for $10 \mathrm{~min}$; addition of aqueous $\mathrm{NaOH}(3.0 \mathrm{ml}, 10 \% \mathrm{w} / \mathrm{w})$ and stirring for $10 \mathrm{~min}$; addition of $4.5 \mathrm{ml}$ of water and stirring for 10 min. After warming to room temperature, the white solid was filtered off, and the filtrate was carefully concentrated. The residue was purified by column chromatography to afford $\mathbf{6 a f 1}$ as a colorless liquid (1.51 g, 76\% yield for two steps). $\mathrm{Rf}=0.4\left(\mathrm{PE}_{\mathrm{Et}} \mathrm{O}=2: 1\right)$.

Triphenylphosphine ( $3.93 \mathrm{~g}, 15.0 \mathrm{mmol}, 1.0$ equiv) was dissolved in $30 \mathrm{ml}$ DMF. Bromine (0.77 $\mathrm{mL}, 2.4 \mathrm{~g}, 15.0 \mathrm{mmol}, 1.0$ equiv) was added to the triphenylphosphine solution dropwisely. Then 6af1 $(1.50 \mathrm{~g}, 15.0 \mathrm{mmol}, 1.0$ equiv) was added in one portion. The reaction mixture was stirred at $110{ }^{\circ} \mathrm{C}$ for 12 hours then quenched with water. The resulting mixture was extracted with $100 \mathrm{~mL}$ diethyl ether. The organic phase was washed with water $(100 \mathrm{~mL} \times 3)$, then dried over $\mathrm{MgSO}_{4}$ and filtered. The filtrate was evaporated under reduced pressure to remove the solvent and further purified by column chromatography with pentane as eluent to afford the alkylbromide $\mathbf{6 a f} 2$ as colorless liquid $(2.0 \mathrm{~g}, 81 \%$ yield $) . \mathrm{Rf}=0.7(\mathrm{PE})$.

According to GP3, 6af2 (2.0 g, $12.2 \mathrm{mmol}, 1.0$ equiv) afforded 6af as a colorless liquid (1.0 g, 39\%

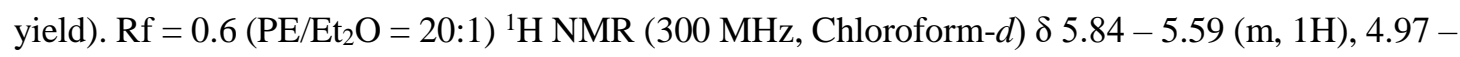
$4.84(\mathrm{~m}, 2 \mathrm{H}), 2.02-1.82(\mathrm{~m}, 2 \mathrm{H}), 1.80-1.64(\mathrm{~m}, 1 \mathrm{H}), 1.18(\mathrm{~s}, 12 \mathrm{H}), 0.86(\mathrm{~s}, 3 \mathrm{H}), 0.84(\mathrm{~s}, 3 \mathrm{H})$, $0.79(\mathrm{dd}, J=15.5,5.9 \mathrm{~Hz}, 1 \mathrm{H}), 0.58(\mathrm{dd}, J=15.4,8.4 \mathrm{~Hz}, 1 \mathrm{H}) .{ }^{13} \mathrm{C} \mathrm{NMR}\left(76 \mathrm{MHz}, \mathrm{CDCl}_{3}\right) \delta 137.8$, 115.6, 82.8, 43.9, 29.5, 24.9, 24.8, 22.1, carbon attached to boron not observed. ${ }^{11} \mathrm{~B} \mathrm{NMR}(96 \mathrm{MHz}$, $\left.\mathrm{CDCl}_{3}\right) \delta$ 33.8. HRMS (EI) $\mathrm{m} / \mathrm{z}=195.1553$ calcd. for $\mathrm{C}_{11} \mathrm{H}_{20} \mathrm{O}_{2} \mathrm{~B}\left[\mathrm{M}-\mathrm{CH}_{3}\right]^{+}$, found: 195.1552 . FTIR (neat): 2978, 2958, 2927, 2874, 1457, 1371, 1315, 1272, 1212, 1146, 994, 971, 910, 847.

2-(2,2-Dimethylhex-4-en-1-yl)-4,4,5,5-tetramethyl-1,3,2-dioxaborolane (6ag)

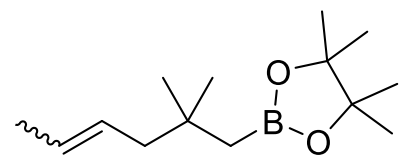

According to GP4, methyl isobutyrate $(2.04 \mathrm{~g}, 20 \mathrm{mmol})$ and 1-bromobut-2-ene (E/Z mixture, 4.05 $\mathrm{g}, 30 \mathrm{mmol})$ afforded $\mathbf{6 a g}$ as a colorless liquid $(1.0 \mathrm{~g}, 21 \%$ overall yield, $\mathrm{E} / \mathrm{Z}=3: 1)$. $\mathrm{Rf}=0.5$ $\left(\mathrm{PE} / \mathrm{Et}_{2} \mathrm{O}=20: 1\right) .{ }^{1} \mathrm{H}$ NMR $(300 \mathrm{MHz}$, Chloroform- $d) \delta 5.55-5.24(\mathrm{~m}, 2 \mathrm{H}), 1.97-1.90(\mathrm{~m}, 0.50 \mathrm{H})$, 
$1.88-1.82(\mathrm{~m}, 1.50 \mathrm{H}), 1.62-1.56(\mathrm{~m}, 2.25 \mathrm{H}), 1.54-1.51(\mathrm{~m}, 0.75 \mathrm{H}), 1.22-1.11(\mathrm{~m}, 12 \mathrm{H}), 0.89$ (s, 1.50H), $0.86(\mathrm{~s}, 4.50 \mathrm{H}), 0.74(\mathrm{~s}, 0.50 \mathrm{H}), 0.69(\mathrm{~s}, 1.50 \mathrm{H}),{ }^{13} \mathrm{C} \mathrm{NMR}\left(76 \mathrm{MHz}, \mathrm{CDCl}_{3}\right) \delta 128.6$ (major), 127.8 (minor), 126.9 (major), 125.2 (minor), 82.7 (minor), 82.7 (major), 47.8 (major), 41.3 (minor), 33.2 (minor), 32.6 (major), 29.2 (major), 29.1 (minor), 24.9 (major), 18.0 (major), 12.9 (minor), carbon attached to boron not observed. ${ }^{11} \mathrm{~B}$ NMR $\left(96 \mathrm{MHz}, \mathrm{CDCl}_{3}\right) \delta 33.4 . \mathbf{H R M S}$ (ESI) $m / z=261.1999$ calcd. for $\mathrm{C}_{14} \mathrm{H}_{27} \mathrm{O}_{2} \mathrm{BNa}^{+}[\mathrm{M}+\mathrm{Na}]^{+}$, found: 261.1997. FTIR (neat): 2979, 2960, $2875,1468,1356,1321,1272,1248,1143,968,873,847,586$.

4,4,5,5-Tetramethyl-2-((1-methylcyclopent-3-en-1-yl)methyl)-1,3,2-dioxaborolane (6ah)<smiles>CC1(CB2OC(C)(C)C(C)(C)O2)CC=CC1</smiles>

According to GP5, cyclopent-3-ene-1-carboxylic acid (2.24 g, $20 \mathrm{mmol})$ and $\mathrm{MeI}(2.5 \mathrm{~mL}, 5.68 \mathrm{~g}$, 2.0 equiv) afforded 6ah as a colorless liquid $\left(0.44 \mathrm{~g}, 10 \%\right.$ overall yield). $\mathrm{Rf}=0.5\left(\mathrm{PE}^{2} \mathrm{Et}_{2} \mathrm{O}=19: 1\right)$. ${ }^{1} \mathrm{H}$ NMR $(300 \mathrm{MHz}$, Chloroform- $d$ ) $\delta 5.65-5.51(\mathrm{~m}, 2 \mathrm{H}), 2.36-2.02(\mathrm{~m}, 4 \mathrm{H}), 1.23(\mathrm{~s}, 12 \mathrm{H}), 1.12$ (s, 3H), 0.98 (s, 2H). ${ }^{13} \mathrm{C}$ NMR (76 MHz, Chloroform- $d$ ) $\delta 129.5,82.7,48.2,39.7,30.0,24.9$, carbon attached to boron not observed. ${ }^{11} \mathrm{~B}$ NMR (96 MHz, Chloroform- $d$ ) $\delta 33.3$. HRMS (ESI) $\mathrm{m} / z=$ 245.1686 calcd. for $\mathrm{C}_{13} \mathrm{H}_{23} \mathrm{O}_{2} \mathrm{BNa}^{+}[\mathrm{M}+\mathrm{Na}]^{+}$, found: 245.1684. FTIR (neat): 3051, 2980, 2952, 2909, 2841, 1438, 1420, 1357, 1340, 1323, 1306, 1263, 1216, 1145, 971, 879, 846, 673.

(3aS,4S,6S,7aR)-2-(2,2-Dimethylpent-4-en-1-yl)-3a,5,5-trimethylhexahydro-4,6methanobenzo[d][1,3,2]dioxaborole (6ai)

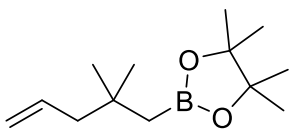

6c

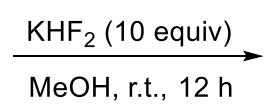

$\mathrm{MeOH}$, r.t., $12 \mathrm{~h}$

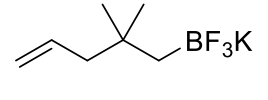

6ai-1

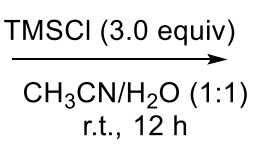

r.t., $12 \mathrm{~h}$

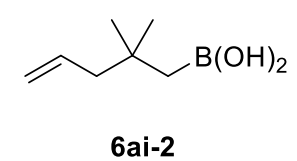

6ai-2
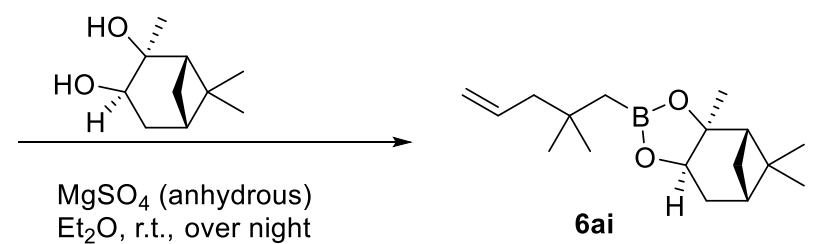

To a solution of $\mathbf{6 c}$ ( $224 \mathrm{mg}, 1.0 \mathrm{mmol}, 1.0$ equiv) in methanol $(2 \mathrm{~mL})$ was added $\mathrm{KHF}_{2}(0.78 \mathrm{~g}, 10$ mmol, 10 equiv). The resulting mixture was stirred for $12 \mathrm{~h}$, and then concentrated to dryness. The residue, a white solid, was extracted with hot acetone $(5 \mathrm{ml})$ two times, and the combined filtered extracts were concentrated under reduced pressure. Ether $(10 \mathrm{~mL})$ was added and the resultant precipitate was collected and dried to afford the corresponding potassium trifluoroborate 6ai-1 as a white solid. The prepared potassium trifluoroborate was dissolved in acetonitrile $(1 \mathrm{~mL})$ and water $(1 \mathrm{~mL})$ before addition of trimethylsilylchloride $(0.38 \mathrm{~mL}, 3 \mathrm{mmol}, 3$ equiv.). The resultant mixture was stirred for $12 \mathrm{~h}$. Saturated sodium bicarbonate solution $(2 \mathrm{~mL})$ and water $(10 \mathrm{~mL})$ were added to the residue, before extraction with ethyl acetate $(10 \mathrm{~mL})$ for two times. The combined extracts were washed with near saturated brine, dried $\left(\mathrm{Na}_{2} \mathrm{SO}_{4}\right)$, and the solvent was removed to provide the 
corresponding alkylboronic acid 6ai-2 as a white solid. 6ai-2 and (1S,2S,3R,5S)-(+)-pinanediol (170 mg, $1.0 \mathrm{mmol}, 1.0$ equiv) was dissolved in anhydrous diethyl ether $(2 \mathrm{~mL})$. Excess amount of anhydrous $\mathrm{MgSO}_{4}$ was added to the reaction mixture and the resulting mixture was stirred overnight. The reaction mixture was filtered through a pad of Celite and rinsed with $50 \mathrm{mLEt}_{2} \mathrm{O}$. Solvent was evaporated under reduced pressure for the filtrate. The residue was purified by column chromatography to afford 6ai as a colorless liquid (70 mg, 25\% yield). $\mathrm{Rf}=0.5$ ( $\mathrm{PE}_{\mathrm{Et}} \mathrm{O}=20: 1$ ) ${ }^{1} \mathrm{H}$ NMR (300 MHz, Chloroform- $d$ ) $\delta 5.92-5.75(\mathrm{~m}, 1 \mathrm{H}), 5.04-4.94(\mathrm{~m}, 2 \mathrm{H}), 4.24(\mathrm{dd}, J=8.8$, $2.1 \mathrm{~Hz}, 1 \mathrm{H}), 2.40-2.14(\mathrm{~m}, 2 \mathrm{H}), 2.07-1.99(\mathrm{~m}, 3 \mathrm{H}), 1.95-1.77(\mathrm{~m}, 2 \mathrm{H}), 1.36(\mathrm{~s}, 3 \mathrm{H}), 1.28(\mathrm{~s}$, $3 \mathrm{H}), 1.14(\mathrm{~d}, J=10.8 \mathrm{~Hz}, 1 \mathrm{H}), 0.97$ (s, 6H), 0.83 (s, 3H), 0.83 (s, 2H). ${ }^{13} \mathrm{C} \mathrm{NMR}$ (76 MHz, $\mathrm{CDCl}_{3}$ ) $\delta$ 136.2, 116.6, 85.1, 77.4, 51.2, 48.9, 39.5, 38.1, 35.7, 32.4, 29.3, 29.3, 28.8, 27.1, 26.6, 24.0, carbon attached to boron not observed. ${ }^{11} \mathrm{~B}$ NMR $\left(96 \mathrm{MHz}, \mathrm{CDCl}_{3}\right) \delta 32.7$. HRMS (ESI) $\mathrm{m} / z=299.2156$ calcd. for $\mathrm{C}_{17} \mathrm{H}_{29} \mathrm{O}_{2} \mathrm{BNa}^{+}[\mathrm{M}+\mathrm{Na}]^{+}$, found: 299.2156. FTIR (neat): 2922, 2919, 2870, 1639, 1471 , 1451, 1364, 1356, 1279, 1236, 1123, 1078, 1055, 1031, 992, 911, 842, 756, 628.

4,4,5,5-Tetramethyl-2-(pent-4-en-1-yl)-1,3,2-dioxaborolane (6ak)

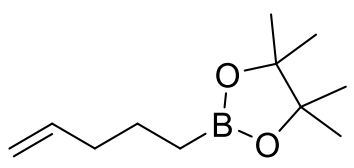

According to GP3, 5-bromopent-1-ene (2.23 g, $15 \mathrm{mmol}$ ) gave 6ak as a colorless liquid (1.0 g, 34\% yield $) . \mathrm{Rf}=0.5\left(\mathrm{PE} / \mathrm{Et}_{2} \mathrm{O}=20: 1\right) .{ }^{1} \mathrm{H}$ NMR $(300 \mathrm{MHz}$, Chloroform- $d) \delta 5.90-5.66(\mathrm{~m}, 1 \mathrm{H}), 5.05$ $-4.86(\mathrm{~m}, 2 \mathrm{H}), 2.10-1.99(\mathrm{~m}, 2 \mathrm{H}), 1.57-1.43(\mathrm{~m}, 2 \mathrm{H}), 1.24(\mathrm{~s}, 12 \mathrm{H}), 0.79(\mathrm{t}, J=7.8 \mathrm{~Hz}, 2 \mathrm{H})$. ${ }^{11} \mathrm{~B}$ NMR $\left(96 \mathrm{MHz}\right.$, Chloroform- $d$ ) $\delta 33.6$. The NMR spectra are identical to the reported data. ${ }^{\mathrm{S} 1}$

2-(But-3-en-1-yl)-4,4,5,5-tetramethyl-1,3,2-dioxaborolane (6al)

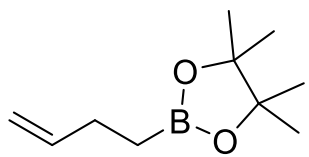

According to GP3, 4-bromobut-1-ene (1.35 g, $10 \mathrm{mmol}$ ) gave 6al as a colorless liquid ( $0.9 \mathrm{~g}, 55 \%$

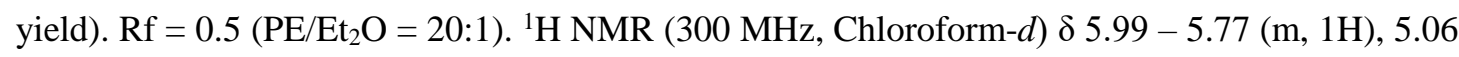
$-4.83(\mathrm{~m}, 2 \mathrm{H}), 2.26-2.08(\mathrm{~m}, 2 \mathrm{H}), 1.24(\mathrm{~s}, 12 \mathrm{H}), 0.89(\mathrm{t}, J=7.8 \mathrm{~Hz}, 2 \mathrm{H}) .{ }^{13} \mathrm{C}$ NMR $(76 \mathrm{MHz}$, Chloroform- $d$ ) $\delta 140.7,113.1,83.0,28.0,24.8$, carbon attached to boron not observed. ${ }^{11} \mathrm{~B}$ NMR $\left(96 \mathrm{MHz}\right.$, Chloroform- $d$ ) $\delta 33.7$. The NMR spectra are identical to the reported data. ${ }^{\mathrm{S} 2}$

\section{Characterization of Products}

2-(4-(4-(Tert-butyl)phenyl)-6,6,6-trifluoro-2,2-dimethylhexyl)-4,4,5,5-tetramethyl-1,3,2dioxaborolane $(\mathbf{8 c})$<smiles>CC(C)(CB1OC(C)(C)C(C)(C)O1)CC(CC(F)(F)F)c1ccc(C(C)(C)C)cc1</smiles>

Following GP2, 4-tert-butylphenyl iodide (95\% purity, $65.7 \mathrm{mg}, 0.24 \mathrm{mmol}, 1.2$ equiv), $n$-butyl lithium (1.6 M, $0.15 \mathrm{~mL}, 0.24 \mathrm{mmol}, 1.2$ equiv), 2,2-dimethylpent-4-en-1-ylboronic acid pinacol 
ester 6a (44.8 mg, $0.20 \mathrm{mmol}, 1.0$ equiv) and trifluoromethyl iodide (1.0 $\mathrm{M}$ in $\mathrm{CH}_{3} \mathrm{CN}, 0.3 \mathrm{~mL}, 0.3$ mmol, 1.5 equiv) afforded the desired product $8 \mathbf{c}$ as a colorless sticky oil ( $73.6 \mathrm{mg}, 86 \%$ yield). Rf $=0.5($ pentane/DCM $=2: 1) .{ }^{1} \mathrm{H}$ NMR $(300 \mathrm{MHz}$, Chloroform- $d$ ) $\delta 7.23-7.14(\mathrm{~m}, 2 \mathrm{H}), 7.07-6.98$ $(\mathrm{m}, 2 \mathrm{H}), 3.04-2.87(\mathrm{~m}, 1 \mathrm{H}), 2.42-2.14(\mathrm{~m}, 2 \mathrm{H}), 1.84-1.59(\mathrm{~m}, 2 \mathrm{H}), 1.21(\mathrm{~s}, 9 \mathrm{H}), 1.13(\mathrm{~s}, 12 \mathrm{H})$, $0.76(\mathrm{~s}, 3 \mathrm{H}), 0.74(\mathrm{~s}, 3 \mathrm{H}), 0.70(\mathrm{~d}, J=15 \mathrm{~Hz}, 1 \mathrm{H}), 0.64(\mathrm{~d}, J=15 \mathrm{~Hz}, 1 \mathrm{H}),{ }^{13} \mathrm{C}\left\{{ }^{1} \mathrm{H},{ }^{19} \mathrm{~F}\right\}$ NMR $(126$ MHz, Chloroform- $d$ ) $\delta$ 149.1, 142.4, 127.2, 126.6, 125.2, 82.7, 50.1, 42.9, 35.9, 34.3, 33.1, 31.4, 30.2, 29.6, 24.9, 24.8, 24.8, carbon attached to boron not observed. ${ }^{19} \mathrm{~F}\left\{{ }^{1} \mathrm{H}\right\}$ NMR $(282 \mathrm{MHz}$, Chloroform- $d$ ) $\delta$-63.6. ${ }^{11} \mathrm{~B}$ NMR (96 MHz, Chloroform- $d$ ) $\delta$ 33.1. HRMS (ESI) $\mathrm{m} / z=449.2814$ calcd. for $\mathrm{C}_{24} \mathrm{H}_{38} \mathrm{O}_{2} \mathrm{BF}_{3} \mathrm{Na}^{+}[\mathrm{M}+\mathrm{Na}]^{+}$, found: 449.2807. FTIR (neat): $v\left(\mathrm{~cm}^{-1}\right)$ 2961, 2908, 2870, 1516, 1469, 1361, 1320, 1270, 1248, 1214, 1131, 1097, 1046, 1020, 970, 909, 847, 828, 733, 607.

2-(4-([1,1'-Biphenyl]-4-yl)-6,6,6-trifluoro-2,2-dimethylhexyl)-4,4,5,5-tetramethyl-1,3,2dioxaborolane $(\mathbf{8 d})$<smiles>CC(C)(CB1OC(C)(C)C(C)(C)O1)CC(CC(F)(F)F)c1ccc(-c2ccccc2)cc1</smiles>

Following GP2, 4-iodobiphenyl (61.6 mg, $0.22 \mathrm{mmol}, 1.1$ equiv), $n$-butyl lithium (1.6 M, $0.14 \mathrm{~mL}$, $0.22 \mathrm{mmol}, 1.1$ equiv), 2,2-dimethylpent-4-en-1-ylboronic acid pinacol ester $6 \mathbf{6}$ (44.8 mg, 0.20 mmol, 1.0 equiv) and trifluoromethyl iodide (1.0 $\mathrm{M}$ in $\mathrm{CH}_{3} \mathrm{CN}, 0.3 \mathrm{~mL}, 0.3 \mathrm{mmol}, 1.5$ equiv) afforded the desired product 8d as a colorless sticky oil $(63.2 \mathrm{mg}, 71 \%$ yield). $\mathrm{Rf}=0.5$ (pentane/DCM = 5:3). ${ }^{1} \mathrm{H}$ NMR $(300 \mathrm{MHz}$, Chloroform- $d$ ) $\delta 7.54-7.47(\mathrm{~m}, 2 \mathrm{H}), 7.46-7.40(\mathrm{~m}$, 2H), $7.38-7.29(\mathrm{~m}, 2 \mathrm{H}), 7.27-7.15(\mathrm{~m}, 3 \mathrm{H}), 3.12-2.96(\mathrm{~m}, 1 \mathrm{H}), 2.44-2.20(\mathrm{~m}, 2 \mathrm{H}), 1.91-1.60$ $(\mathrm{m}, 2 \mathrm{H}), 1.14(\mathrm{~s}, 12 \mathrm{H}), 0.82-0.61(\mathrm{~m}, 8 \mathrm{H}) .{ }^{13} \mathrm{C}\left\{{ }^{1} \mathrm{H},{ }^{19} \mathrm{~F}\right\}$ NMR (151 MHz, Chloroform- $d$ ) $\delta$ 144.6, 140.8, 139.2, 128.7, 128.1, 127.1, 127.1, 126.9, 126.5, 82.8, 50.2, 42.9, 36.2, 33.2, 30.3, 29.8, 24.8, 24.8, carbon attached to boron not observed. ${ }^{19} \mathrm{~F}\left\{{ }^{1} \mathrm{H}\right\} \mathrm{NMR}\left(282 \mathrm{MHz}, \mathrm{CDCl}_{3}\right) \delta$-63.5. ${ }^{11} \mathrm{~B} \mathrm{NMR}$ $\left(96 \mathrm{MHz}, \mathrm{CDCl}_{3}\right) \delta 32.9$. HRMS (ESI) $\mathrm{m} / z=469.2501$ calcd. for $\mathrm{C}_{26} \mathrm{H}_{34} \mathrm{O}_{2} \mathrm{BF}_{3} \mathrm{Na}^{+}[\mathrm{M}+\mathrm{Na}]^{+}$, found: 469.2497. FTIR (neat): $v\left(\mathrm{~cm}^{-1}\right)$ 2979, 2932, 2896, 1487, 1369, 1358, 1320, 1272, 1247, 1130, 1095, 1008, 969, 909, 847, 835, 766, 732, 697, 657, 605.

4,4,5,5-Tetramethyl-2-(6,6,6-trifluoro-4-(4-methoxyphenyl)-2,2-dimethylhexyl)-1,3,2dioxaborolane $(\mathbf{8 e})$<smiles>COc1ccc(C(CC(F)(F)F)CC(C)(C)CB2OC(C)(C)C(C)(C)O2)cc1</smiles>

Following GP2, 4-iodoanisole ( $51.5 \mathrm{mg}, 0.22 \mathrm{mmol}, 1.1$ equiv), $n$-butyl lithium (1.6 M, $0.14 \mathrm{~mL}$, $0.22 \mathrm{mmol}, 1.1$ equiv), 2,2-dimethylpent-4-en-1-ylboronic acid pinacol ester 6a (44.8 $\mathrm{mg}, 0.20$ mmol, 1.0 equiv) and trifluoromethyl iodide (1.0 $\mathrm{M}$ in $\mathrm{CH}_{3} \mathrm{CN}, 0.3 \mathrm{~mL}, 0.3 \mathrm{mmol}, 1.5$ equiv) afforded the desired product $8 \mathrm{e}$ as a colorless sticky oil ( $49.4 \mathrm{mg}, 62 \%$ yield). $\mathrm{Rf}=0.3$ (pentane/ $\mathrm{Et}_{2} \mathrm{O}$ $=10: 1) .{ }^{1} \mathrm{H}$ NMR $(300 \mathrm{MHz}$, Chloroform- $d) \delta 7.07-7.00(\mathrm{~m}, 2 \mathrm{H}), 6.78-6.70(\mathrm{~m}, 2 \mathrm{H}), 3.70(\mathrm{~s}$, $3 \mathrm{H}), 3.04-2.89(\mathrm{~m}, 1 \mathrm{H}), 2.41-2.09(\mathrm{~m}, 2 \mathrm{H}), 1.82-1.58(\mathrm{~m}, 2 \mathrm{H}), 1.15(\mathrm{~s}, 12 \mathrm{H}), 0.79-0.57(\mathrm{~m}$, 
$8 \mathrm{H}) .{ }^{13} \mathrm{C}\left\{{ }^{1} \mathrm{H},{ }^{19} \mathrm{~F}\right\}$ NMR $(126 \mathrm{MHz}$, Chloroform- $d$ ) $\delta 158.0,137.4,128.5,126.5,113.8,82.8,55.1$, 50.2, 43.1, 35.7, 33.1, 30.3, 29.7, 24.8, 24.8, carbon attached to boron not observed. ${ }^{19} \mathrm{~F}\left\{{ }^{1} \mathrm{H}\right\} \mathrm{NMR}$ $\left(282 \mathrm{MHz}\right.$, Chloroform- $d$ ) $\delta-63.5 .{ }^{11} \mathrm{~B}$ NMR $(96 \mathrm{MHz}$, Chloroform- $d$ ) $\delta 32.8$. HRMS (ESI) $\mathrm{m} / z=$ 423.2293 calcd. for $\mathrm{C}_{21} \mathrm{H}_{32} \mathrm{O}_{2} \mathrm{BF}_{3} \mathrm{Na}^{+}[\mathrm{M}+\mathrm{Na}]^{+}$, found: 423.2289. FTIR (neat): $v$ ( $\left.\mathrm{cm}^{-1}\right) 2979,2938$, 2890, 1613, 1513, 1467, 1443, 1358, 1320, 1303, 1246, 1179, 1129, 1095, 1039, 970, 847, 827, 809, 604.

2-(4-(4-(Benzyloxy)phenyl)-6,6,6-trifluoro-2,2-dimethylhexyl)-4,4,5,5-tetramethyl-1,3,2dioxaborolane (8f)<smiles>CC(C)(CB1OC(C)(C)C(C)(C)O1)CC(CC(F)(F)F)c1ccc(OCc2ccccc2)cc1</smiles>

Following GP2, 1-(benzyloxy)-4-iodobenzene (74.4 mg, $0.24 \mathrm{mmol}, 1.2$ equiv), $n$-butyl lithium (1.6 M, $0.15 \mathrm{~mL}, 0.24 \mathrm{mmol}, 1.2$ equiv), 2,2-dimethylpent-4-en-1-ylboronic acid pinacol ester 6a (44.8 mg, $0.20 \mathrm{mmol}, 1.0$ equiv) and trifluoromethyl iodide $\left(1.0 \mathrm{M}^{\text {in }} \mathrm{CH}_{3} \mathrm{CN}, 0.3 \mathrm{~mL}, 0.3 \mathrm{mmol}\right.$, 1.5 equiv) afforded the desired product $\mathbf{8 f}$ as a colorless sticky oil $(57.2 \mathrm{mg}, 60 \%$ yield). $\mathrm{Rf}=0.6$ (pentane/Et $2 \mathrm{O}=5: 1) .{ }^{1} \mathrm{H}$ NMR $(300 \mathrm{MHz}$, Chloroform- $d$ ) $\delta 7.41-7.19(\mathrm{~m}, 5 \mathrm{H}), 7.10-6.98(\mathrm{~m}$, $2 \mathrm{H}), 6.87-6.76(\mathrm{~m}, 2 \mathrm{H}), 4.94(\mathrm{~s}, 2 \mathrm{H}), 3.04-2.89(\mathrm{~m}, 1 \mathrm{H}), 2.38-2.10(\mathrm{~m}, 2 \mathrm{H}), 1.84-1.57(\mathrm{~m}$, 2H), $1.14(\mathrm{~s}, 12 \mathrm{H}), 0.78-0.58(\mathrm{~m}, 8 \mathrm{H}) \cdot{ }^{13} \mathrm{C}\left\{{ }^{1} \mathrm{H},{ }^{19} \mathrm{~F}\right\}$ NMR (151 MHz, Chloroform- $d$ ) $\delta 157.3$, 137.7, 137.1, 128.6, 128.5, 127.9, 127.5, 126.5, 114.8, 82.8, 70.0, 50.2, 43.0, 35.7, 33.1, 30.3, 29.7, 24.8, 24.8, carbon attached to boron not observed. ${ }^{19} \mathrm{~F}\left\{{ }^{1} \mathrm{H}\right\} \mathrm{NMR}\left(282 \mathrm{MHz}, \mathrm{CDCl}_{3}\right) \delta-63.5 .{ }^{11} \mathrm{~B}$ NMR (96 MHz, $\mathrm{CDCl}_{3}$ ) $\delta 32.7$. HRMS (ESI) $m / z=499.2607$ calcd. for $\mathrm{C}_{27} \mathrm{H}_{36} \mathrm{O}_{2} \mathrm{BF}_{3} \mathrm{Na}^{+}[\mathrm{M}+\mathrm{Na}]^{+}$, found: 499.2604. FTIR (neat): $v\left(\mathrm{~cm}^{-1}\right)$ 2978, 2931, 2903, 2872, 1611, 1586, 1511, 1469, 1455, 1358, 1320, 1270, 1129, 1095, 1026, 969, 847, 827, 736, 696, 607.

Trimethyl(4-(1,1,1-trifluoro-5,5-dimethyl-6-(4,4,5,5-tetramethyl-1,3,2-dioxaborolan-2-yl)hexan-3yl)phenyl)silane (8g)<smiles>CSc1ccc(C(CC(F)(F)F)CC(C)(C)CB2OC(C)(C)C(C)(C)O2)cc1</smiles>

Following GP2, (4-iodophenyl)trimethylsilane ( $66.2 \mathrm{mg}, 0.24 \mathrm{mmol}, 1.2$ equiv), $n$-butyl lithium (1.6 M, $0.15 \mathrm{~mL}, 0.24 \mathrm{mmol}, 1.2$ equiv), 2,2-dimethylpent-4-en-1-ylboronic acid pinacol ester $6 \mathbf{a}$

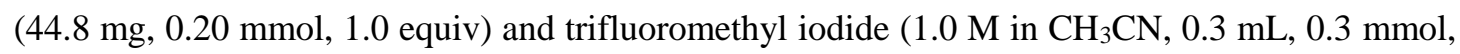
1.5 equiv) afforded the desired product $8 \mathrm{~g}$ as a colorless sticky oil $(67.9 \mathrm{mg}, 77 \%$ yield). $\mathrm{Rf}=0.5$ (pentane/DCM = 2:1). ${ }^{1} \mathrm{H}$ NMR $(300 \mathrm{MHz}$, Chloroform- $d$ ) $\delta 7.37-7.30(\mathrm{~m}, 2 \mathrm{H}), 7.15-7.06(\mathrm{~m}$, 2H), $3.06-2.89(\mathrm{~m}, 1 \mathrm{H}), 2.43-2.14(\mathrm{~m}, 2 \mathrm{H}), 1.87-1.59(\mathrm{~m}, 2 \mathrm{H}), 1.13(\mathrm{~s}, 12 \mathrm{H}), 0.81-0.58(\mathrm{~m}$, $8 \mathrm{H}), 0.16$ (s, 9H). ${ }^{13} \mathrm{C}\left\{{ }^{1} \mathrm{H},{ }^{19} \mathrm{~F}\right\}$ NMR (126 MHz, Chloroform- $d$ ) $\delta 146.1,138.0,133.5,127.1,126.6$, 82.8, 50.0, 42.8, 36.4, 33.1, 30.2, 29.7, 24.9, 24.8, -1.1, carbon attached to boron not observed. ${ }^{19} \mathrm{~F}\left\{{ }^{1} \mathrm{H}\right\}$ NMR (282 MHz, Chloroform- $d$ ) $\delta$-63.6. ${ }^{11} \mathrm{~B}$ NMR (96 MHz, Chloroform- $d$ ) $\delta 32.9$. HRMS (ESI) $m / z=465.2583$ calcd. for $\mathrm{C}_{23} \mathrm{H}_{38} \mathrm{O}_{2} \mathrm{BF}_{3} \mathrm{Na}^{+}[\mathrm{M}+\mathrm{Na}]^{+}$, found: 465.2580 . FTIR (neat): 
$v\left(\mathrm{~cm}^{-1}\right) 2979,2956,1359,1320,1273,1248,1135,1107,1094,969,908,838,817,728$.

4,4,5,5-Tetramethyl-2-(6,6,6-trifluoro-4-(4-iodophenyl)-2,2-dimethylhexyl)-1,3,2-dioxaborolane $(\mathbf{8 h})$<smiles>CC(C)(C)CC(C)(C)CC(CC(F)(F)F)c1ccc(I)cc1</smiles>

Following GP2, 1,4-diiodobenzene (72.6 mg, $0.22 \mathrm{mmol}, 1.1$ equiv), $n$-butyl lithium (1.6 M, 0.14 $\mathrm{mL}, 0.22 \mathrm{mmol}, 1.1$ equiv), 2,2-dimethylpent-4-en-1-ylboronic acid pinacol ester $\mathbf{6 a}$ ( $44.8 \mathrm{mg}, 0.20$ mmol, 1.0 equiv) and trifluoromethyl iodide (1.0 $\mathrm{M}$ in $\mathrm{CH}_{3} \mathrm{CN}, 0.3 \mathrm{~mL}, 0.3 \mathrm{mmol}, 1.5$ equiv) afforded the desired product $\mathbf{8 h}$ as a colorless sticky oil $\left(79.8 \mathrm{mg}\right.$, containing $7 \% \mathbf{8 a}$ by ${ }^{19} \mathrm{~F}$ NMR, $76 \%$ yield). $\mathrm{Rf}=0.5$ (pentane/DCM $=2: 1) .{ }^{1} \mathrm{H}$ NMR $(300 \mathrm{MHz}$, Chloroform- $d$ ) $\delta 7.57-7.46(\mathrm{~m}$, $2 \mathrm{H}), 6.94-6.86(\mathrm{~m}, 2 \mathrm{H}), 3.04-2.88(\mathrm{~m}, 1 \mathrm{H}), 2.41-2.11(\mathrm{~m}, 2 \mathrm{H}), 1.81-1.58(\mathrm{~m}, 2 \mathrm{H}), 1.14(\mathrm{~s}$, 12H), $0.82-0.57(\mathrm{~m}, 8 \mathrm{H}) .{ }^{13} \mathrm{C}\left\{{ }^{1} \mathrm{H},{ }^{19} \mathrm{~F}\right\}$ NMR $\left(126 \mathrm{MHz}, \mathrm{cdcl}_{3}\right) \delta 145.1,137.5,129.8,126.3,91.4$, $82.8,50.0,42.8,36.2,33.1,30.3,29.8,24.8,24.8$, carbon attached to boron not observed. ${ }^{19} \mathrm{~F}\left\{{ }^{1} \mathrm{H}\right\}$ $\operatorname{NMR}\left(282 \mathrm{MHz}, \mathrm{CDCl}_{3}\right) \delta-63.5 .{ }^{11} \mathrm{~B} \mathrm{NMR}\left(96 \mathrm{MHz}, \mathrm{CDCl}_{3}\right) \delta 32.9$. HRMS (ESI) $\mathrm{m} / z=519.1153$ calcd. for $\mathrm{C}_{20} \mathrm{H}_{29} \mathrm{O}_{2} \mathrm{BF}_{3} \mathrm{INa}^{+}$[M+Na] ${ }^{+}$, found: 519.1150. FTIR (neat): $v\left(\mathrm{~cm}^{-1}\right)$ 2980, 2900, 2875, 1486, 1358, 1321, 1270, 1249, 1132, 1093, 1006, 969, 907, 847, 817, 730, 649, 602.

4,4,5,5-Tetramethyl-2-(6,6,6-trifluoro-4-(4-fluorophenyl)-2,2-dimethylhexyl)-1,3,2-dioxaborolane (8i)<smiles>CC(C)(CB1OC(C)(C)C(C)(C)O1)CC(CC(F)(F)F)c1ccc(F)cc1</smiles>

Following GP2, 1-fluoro-4-iodobenzene ( $48.8 \mathrm{mg}, 0.22 \mathrm{mmol}, 1.1$ equiv), $n$-butyl lithium (1.6 M, $0.14 \mathrm{~mL}, 0.22 \mathrm{mmol}, 1.1$ equiv), 2,2-dimethylpent-4-en-1-ylboronic acid pinacol ester $6 \mathbf{a}$ ( $44.8 \mathrm{mg}$, $0.20 \mathrm{mmol}, 1.0$ equiv) and trifluoromethyl iodide (1.0 $\mathrm{M}$ in $\mathrm{CH}_{3} \mathrm{CN}, 0.3 \mathrm{~mL}, 0.3 \mathrm{mmol}, 1.5$ equiv) afforded the desired product $\mathbf{8 i}$ as a colorless sticky oil ( $46.7 \mathrm{mg}$, containing $5 \% \mathbf{8 h}, 57 \%$ yield). $\mathrm{Rf}$ $=0.4$ (pentane/DCM = 2:1). ${ }^{1} \mathrm{H}$ NMR (300 MHz, Chloroform- $d$ ) $\delta 7.14-7.04(\mathrm{~m}, 2 \mathrm{H}), 6.94-6.84$ (m, 2H), $3.08-2.91(\mathrm{~m}, 1 \mathrm{H}), 2.40-2.12(\mathrm{~m}, 2 \mathrm{H}), 1.82-1.57(\mathrm{~m}, 2 \mathrm{H}), 1.14(\mathrm{~s}, 12 \mathrm{H}), 0.78-0.57$ $(\mathrm{m}, 8 \mathrm{H}) .{ }^{13} \mathrm{C}\left\{{ }^{1} \mathrm{H},{ }^{19} \mathrm{~F}\right\}$ NMR $(126 \mathrm{MHz}$, Chloroform- $d$ ) $\delta 161.5,141.0,129.1,126.4,115.2,82.8$, $50.3,43.0,35.9,33.1,30.2,29.8,24.8$, carbon attached to boron not observed. ${ }^{19} \mathrm{~F}\left\{{ }^{1} \mathrm{H}\right\} \operatorname{NMR}(282$ MHz, Chloroform- $d$ ) $\delta-63.5,-116.8 .{ }^{11} \mathrm{~B}$ NMR (96 MHz, Chloroform- $d$ ) $\delta 32.8$. HRMS (ESI) $\mathrm{m} / \mathrm{z}$ $=411.2093$ calcd. for $\mathrm{C}_{20} \mathrm{H}_{29} \mathrm{O}_{2} \mathrm{BF}_{4} \mathrm{Na}^{+}[\mathrm{M}+\mathrm{Na}]^{+}$, found: 411.2093. FTIR (neat): $v\left(\mathrm{~cm}^{-1}\right) 2979$, 2938, 2871, 1605, 1511, 1470, 1438, 1359, 1321, 1271, 1249, 1225, 1139, 1091, 970, 847, 832, 735, 603.

4,4,5,5-Tetramethyl-2-(6,6,6-trifluoro-2,2-dimethyl-4-(m-tolyl)hexyl)-1,3,2-dioxaborolane (8j) 
<smiles>Cc1cccc(C(CCC(F)(F)F)CC(C)(C)CB2OC(C)(C)C(C)(C)O2)c1</smiles>

Following GP2, 1-methyl-3-iodobenzene (48.0 mg, $0.22 \mathrm{mmol}, 1.1$ equiv), $n$-butyl lithium (1.6 M, $0.14 \mathrm{~mL}, 0.22 \mathrm{mmol}, 1.1$ equiv), 2,2-dimethylpent-4-en-1-ylboronic acid pinacol ester $6 \mathbf{a}$ (44.8 $\mathrm{mg}$, $0.20 \mathrm{mmol}, 1.0$ equiv) and trifluoromethyl iodide (1.0 $\mathrm{M}$ in $\mathrm{CH}_{3} \mathrm{CN}, 0.3 \mathrm{~mL}, 0.3 \mathrm{mmol}, 1.5$ equiv) afforded the desired product $\mathbf{8 j}$ as a colorless sticky oil (45.8 $\mathrm{mg}, 60 \%$ yield). $\mathrm{Rf}=0.5$ (pentane/DCM = 5:3). ${ }^{1} \mathrm{H}$ NMR $(300 \mathrm{MHz}$, Chloroform- $d) \delta 7.12-7.02(\mathrm{~m}, 1 \mathrm{H}), 6.96-6.88(\mathrm{~m}$, $3 \mathrm{H}), 3.03-2.87(\mathrm{~m}, 1 \mathrm{H}), 2.41-2.16(\mathrm{~m}, 5 \mathrm{H}), 1.84-1.58(\mathrm{~m}, 2 \mathrm{H}), 1.15(\mathrm{~s}, 12 \mathrm{H}), 0.81-0.58(\mathrm{~m}$, $8 \mathrm{H}) .{ }^{13} \mathrm{C}\left\{{ }^{1} \mathrm{H},{ }^{19} \mathrm{~F}\right\}$ NMR $(126 \mathrm{MHz}$, Chloroform- $d) \delta 145.5,137.9,128.4,128.3,127.1,126.6,124.7$, 82.8, 50.1, 42.9, 36.4, 33.1, 30.2, 29.7, 24.8, 21.4, carbon attached to boron not observed. ${ }^{19} \mathrm{~F}\left\{{ }^{1} \mathrm{H}\right\}$ NMR (282 MHz, Chloroform- $d$ ) $\delta$-63.6. ${ }^{11} \mathrm{~B}$ NMR (96 MHz, Chloroform- $d$ ) $\delta 33.0$. HRMS (ESI) $m / z=407.2344$ calcd. for $\mathrm{C}_{21} \mathrm{H}_{32} \mathrm{O}_{2} \mathrm{BF}_{3} \mathrm{Na}^{+}[\mathrm{M}+\mathrm{Na}]^{+}$, found: 407.2341. FTIR (neat): $v\left(\mathrm{~cm}^{-1}\right) 2977$, 2957, 2911, 1609, 1469, 1358, 1319, 1250, 1215, 1129, 1106, 1090, 1044, 969, 883, 847, 784, 734, $707,664$.

2-(4-([1,1'-Biphenyl]-3-yl)-6,6,6-trifluoro-2,2-dimethylhexyl)-4,4,5,5-tetramethyl-1,3,2dioxaborolane $(\mathbf{8 k})$<smiles>CC(C)(CB1OC(C)(C)C(C)(C)O1)CC(CC(F)(F)F)c1cccc(-c2ccccc2)c1</smiles>

Following GP2, 3-iodobiphenyl (67.2 mg, $0.24 \mathrm{mmol}, 1.2$ equiv), $n$-butyl lithium (1.6 M, $0.15 \mathrm{~mL}$, $0.24 \mathrm{mmol}, 1.2$ equiv), 2,2-dimethylpent-4-en-1-ylboronic acid pinacol ester $6 \mathbf{a}$ (44.8 mg, 0.20 mmol, 1.0 equiv) and trifluoromethyl iodide (1.0 $\mathrm{M}$ in $\mathrm{CH}_{3} \mathrm{CN}, 0.3 \mathrm{~mL}, 0.3 \mathrm{mmol}, 1.5$ equiv) afforded the desired product $\mathbf{8 k}$ as a colorless sticky oil $(51.1 \mathrm{mg}, 57 \%$ yield $)$. $\mathrm{Rf}=0.3$ (pentane/DCM = 2:1). ${ }^{1} \mathrm{H}$ NMR $(300 \mathrm{MHz}$, Chloroform- $d) \delta 7.55-7.46(\mathrm{~m}, 2 \mathrm{H}), 7.41-7.21(\mathrm{~m}$, $6 \mathrm{H}), 7.12(\mathrm{dt}, J=7.5,1.6 \mathrm{~Hz}, 1 \mathrm{H}), 3.14-3.00(\mathrm{~m}, 1 \mathrm{H}), 2.45-2.23(\mathrm{~m}, 2 \mathrm{H}), 1.93-1.62(\mathrm{~m}, 2 \mathrm{H})$, $1.12(\mathrm{~s}, 12 \mathrm{H}), 0.83-0.61(\mathrm{~m}, 8 \mathrm{H}) .{ }^{13} \mathrm{C}\left\{{ }^{1} \mathrm{H},{ }^{19} \mathrm{~F}\right\} \mathrm{NMR}(126 \mathrm{MHz}$, Chloroform-d) $\delta 146.0,141.4$, 141.3, 128.8, 128.9, 127.2, 127.2, 126.7, 126.6, 126.5, 125.3, 82.8, 50.1, 43.0, 36.7, 33.2, 30.3, 29.8, 24.8, 24.8, carbon attached to boron not observed. ${ }^{19} \mathrm{~F}\left\{{ }^{1} \mathrm{H}\right\}$ NMR $(282 \mathrm{MHz}$, Chloroform- $d$ ) $\delta-$ 63.5. ${ }^{11} \mathrm{~B}$ NMR (96 MHz, Chloroform- $d$ ) $\delta$ 32.6. HRMS (ESI) $\mathrm{m} / \mathrm{z}=469.2501$ calcd. for $\mathrm{C}_{26} \mathrm{H}_{34} \mathrm{O}_{2} \mathrm{BF}_{3} \mathrm{Na}^{+}[\mathrm{M}+\mathrm{Na}]^{+}$, found: 469.2498. FTIR (neat): $v\left(\mathrm{~cm}^{-1}\right)$

4,4,5,5-Tetramethyl-2-(6,6,6-trifluoro-4-(3-methoxyphenyl)-2,2-dimethylhexyl)-1,3,2dioxaborolane $(\mathbf{8 1})$<smiles>COc1cccc(C(CC(F)(F)F)CC(C)(C)CB2OC(C)(C)C(C)(C)O2)c1</smiles>

Following GP2, 3-iodoanisole (56.2 mg, $0.24 \mathrm{mmol}, 1.2$ equiv), $n$-butyl lithium (1.6 M, $0.15 \mathrm{~mL}$, $0.24 \mathrm{mmol}, 1.2$ equiv), 2,2-dimethylpent-4-en-1-ylboronic acid pinacol ester $6 \mathbf{a}$ (44.8 mg, 0.20 mmol, 1.0 equiv) and trifluoromethyl iodide (1.0 $\mathrm{M}$ in $\mathrm{CH}_{3} \mathrm{CN}, 0.3 \mathrm{~mL}, 0.3 \mathrm{mmol}, 1.5$ equiv) 
afforded the desired product $8 \mathrm{I}$ as a colorless sticky oil $(52.2 \mathrm{mg}, 65 \%$ yield). $\mathrm{Rf}=0.7$ (pentane/Et $2 \mathrm{O}$ = 5:1). ${ }^{1} \mathrm{H}$ NMR $(300 \mathrm{MHz}$, Chloroform- $d$ ) $\delta 7.19(\mathrm{t}, J=7.8 \mathrm{~Hz}, 1 \mathrm{H}), 6.85-6.76(\mathrm{~m}, 1 \mathrm{H}), 6.79-$ $6.67(\mathrm{~m}, 2 \mathrm{H}), 3.79(\mathrm{~s}, 3 \mathrm{H}), 3.14-2.97(\mathrm{~m}, 1 \mathrm{H}), 2.50-2.23(\mathrm{~m}, 2 \mathrm{H}), 1.94-1.65(\mathrm{~m}, 2 \mathrm{H}), 1.22(\mathrm{~s}$, 12H), $0.88-0.62(\mathrm{~m}, 8 \mathrm{H}) .{ }^{13} \mathrm{C}\left\{{ }^{1} \mathrm{H},{ }^{19} \mathrm{~F}\right\}$ NMR (151 MHz, Chloroform- $d$ ) $\delta$ 159.6, 147.2, 129.4, 126.5, 120.2, 113.8, 111.3, 82.8, 55.1, 50.0, 42.9, 36.6, 33.1, 30.2, 29.6, 24.8, 24.8, carbon attached to boron not observed. ${ }^{19} \mathrm{~F}\left\{{ }^{1} \mathrm{H}\right\}$ NMR $\left(282 \mathrm{MHz}\right.$, Chloroform- $d$ ) $\delta$-63.6. ${ }^{11} \mathrm{~B}$ NMR $(96 \mathrm{MHz}$, Chloroform- $d$ ) $\delta 32.8$. HRMS (ESI) $m / z=423.2293$ calcd. for $\mathrm{C}_{21} \mathrm{H}_{32} \mathrm{O}_{2} \mathrm{BF}_{3} \mathrm{Na}^{+}[\mathrm{M}+\mathrm{Na}]^{+}$, found: 423.2287. FTIR (neat): $v\left(\mathrm{~cm}^{-1}\right)$ 2978, 2944, 1601, 1587, 1489, 1468, 1438, 1358, 1318, 1249, 1127 , 1105, 1051, 969, 875, 847, 778, 703, 666 .

4,4,5,5-Tetramethyl-2-(6,6,6-trifluoro-4-(3-iodophenyl)-2,2-dimethylhexyl)-1,3,2-dioxaborolane $(\mathbf{8 m})$<smiles>CC(C)(CB1OC(C)(C)C(C)(C)O1)CC(CC(F)(F)F)c1cccc(I)c1</smiles>

Following GP2, 1,3-diiodobenzene (79.2 mg, $0.24 \mathrm{mmol}, 1.2$ equiv), $n$-butyl lithium (1.6 M, 0.15 $\mathrm{mL}, 0.24 \mathrm{mmol}, 1.2$ equiv), 2,2-dimethylpent-4-en-1-ylboronic acid pinacol ester $\mathbf{6 a}$ ( $44.8 \mathrm{mg}, 0.20$ mmol, 1.0 equiv) and trifluoromethyl iodide (1.0 $\mathrm{M}$ in $\mathrm{CH}_{3} \mathrm{CN}, 0.3 \mathrm{~mL}, 0.3 \mathrm{mmol}, 1.5$ equiv) afforded the desired product $\mathbf{8 m}$ as a colorless sticky oil (71.9 $\mathrm{mg}$, containing $4 \mathrm{~mol} \% \mathbf{8 a}, 69 \%$ yield). $\mathrm{Rf}=0.3$ (pentane/DCM $=5: 3) .{ }^{1} \mathrm{H}$ NMR $(300 \mathrm{MHz}$, Chloroform- $d$ ) $\delta 7.52-7.41(\mathrm{~m}, 2 \mathrm{H})$, $7.15-7.07$ (m, 1H), $6.93(\mathrm{t}, J=7.7 \mathrm{~Hz}, 1 \mathrm{H}), 3.01-2.85(\mathrm{~m}, 1 \mathrm{H}), 2.41-2.15(\mathrm{~m}, 2 \mathrm{H}), 1.83-1.58$ (m, 2H), $1.15(\mathrm{~s}, 12), 0.80-0.62(\mathrm{~m}, 8 \mathrm{H}) .{ }^{13} \mathrm{C}\left\{{ }^{1} \mathrm{H},{ }^{19} \mathrm{~F}\right\} \mathrm{NMR}\left(126 \mathrm{MHz}, \mathrm{cdcl}_{3}\right) \delta 148.0,136.6$, 135.6, 130.2, 127.2, 126.3, 94.4, 82.9, 49.9, 42.8, 36.2, 33.1, 30.2, 29.8, 24.9, 24.8, carbon attached to boron not observed. ${ }^{19} \mathrm{~F}\left\{{ }^{1} \mathrm{H}\right\} \mathrm{NMR}\left(282 \mathrm{MHz}, \mathrm{CDCl}_{3}\right) \delta$-63.6. ${ }^{11} \mathrm{~B} \mathrm{NMR}\left(96 \mathrm{MHz}, \mathrm{CDCl}_{3}\right) \delta$ 33.0. HRMS (ESI) $\mathrm{m} / z=519.1150$ calcd. for $\mathrm{C}_{20} \mathrm{H}_{29} \mathrm{O}_{2} \mathrm{BF}_{3} \mathrm{INa}^{+}[\mathrm{M}+\mathrm{Na}]^{+}$, found: 519.1151. FTIR (neat): v $\left(\mathrm{cm}^{-1}\right)$ 2978, 2956, 2929, 2896, 1591, 1565, 1471, 1425, 1357, 1320, 1249, 1135, 1106, 1087, 995, 968, 907, 847, 782, 730, 700, 650 .

4,4,5,5-Tetramethyl-2-(6,6,6-trifluoro-2,2-dimethyl-4-(o-tolyl)hexyl)-1,3,2-dioxaborolane (8n)<smiles>Cc1ccccc1C(CC(F)(F)F)CC(C)(C)CB1OC(C)(C)C(C)(C)O1</smiles>

Following GP2, 2-iodotoluene ( $52.3 \mathrm{mg}, 0.24 \mathrm{mmol}, 1.2$ equiv), $n$-butyl lithium (1.6 M, $0.15 \mathrm{~mL}$, $0.24 \mathrm{mmol}, 1.2$ equiv), 2,2-dimethylpent-4-en-1-ylboronic acid pinacol ester $6 \mathbf{6}(44.8 \mathrm{mg}, 0.20$ mmol, 1.0 equiv) and trifluoromethyl iodide (1.0 $\mathrm{M}$ in $\mathrm{CH}_{3} \mathrm{CN}, 0.3 \mathrm{~mL}, 0.3 \mathrm{mmol}, 1.5$ equiv) afforded the desired product $8 \mathbf{n}$ as a colorless sticky oil $(23.4 \mathrm{mg}, 30 \%$ yield). $\mathrm{Rf}=0.4$ (pentane/DCM = 5:3). ${ }^{1} \mathrm{H}$ NMR (300 MHz, Chloroform- $d$ ) $\delta 7.16-6.92(\mathrm{~m}, 4 \mathrm{H}), 3.38-3.22(\mathrm{~m}$, 1H), $2.45-2.05(\mathrm{~m}, 5 \mathrm{H}), 1.91-1.64(\mathrm{~m}, 2 \mathrm{H}), 1.15(\mathrm{~s}, 12 \mathrm{H}), 0.86-0.57(\mathrm{~m}, 8 \mathrm{H}) .{ }^{13} \mathrm{C}\left\{{ }^{1} \mathrm{H},{ }^{19} \mathrm{~F}\right\}$ NMR (126 MHz, Chloroform- $d$ ) $\delta 144.1,135.0,130.5,126.7,126.3,126.2,125.9,82.8,50.3,42.8$, $33.1,30.3,30.0,29.7,24.8,24.8,19.7$, carbon attached to boron not observed. ${ }^{19} \mathrm{~F}\left\{{ }^{1} \mathrm{H}\right\} \mathrm{NMR}(282$ MHz, Chloroform- $d$ ) $\delta$-64.0. ${ }^{11} \mathrm{~B}$ NMR (96 MHz, Chloroform- $d$ ) $\delta 32.8$. HRMS (ESI) $\mathrm{m} / z=$ 407.2344 calcd. for $\mathrm{C}_{21} \mathrm{H}_{32} \mathrm{O}_{2} \mathrm{BF}_{3} \mathrm{Na}^{+}[\mathrm{M}+\mathrm{Na}]^{+}$, found: 407.2340. FTIR (neat): $v$ (cm ${ }^{-1}$ ) 2978, 2934 , 
2-(4-(3,5-Dimethylphenyl)-6,6,6-trifluoro-2,2-dimethylhexyl)-4,4,5,5-tetramethyl-1,3,2dioxaborolane (8o)<smiles>Cc1cc(C)cc(C(CC(F)(F)F)CC(C)(C)CB2OC(C)(C)C(C)(C)O2)c1</smiles>

Following GP2, 3,5-dimethyliodobenzene ( $55.7 \mathrm{mg}, 0.24 \mathrm{mmol}, 1.2$ equiv), $n$-butyl lithium (1.6 M, $0.15 \mathrm{~mL}, 0.24 \mathrm{mmol}, 1.2$ equiv), 2,2-dimethylpent-4-en-1-ylboronic acid pinacol ester $6 \mathbf{a}$ ( $44.8 \mathrm{mg}$, $0.20 \mathrm{mmol}, 1.0$ equiv) and trifluoromethyl iodide (1.0 $\mathrm{M}$ in $\mathrm{CH}_{3} \mathrm{CN}, 0.3 \mathrm{~mL}, 0.3 \mathrm{mmol}, 1.5$ equiv) afforded the desired product 80 as a colorless sticky oil $(44.8 \mathrm{mg}, 56 \%$ yield). $\mathrm{Rf}=0.4$ (pentane/DCM = 5:3). ${ }^{1} \mathrm{H}$ NMR $(300 \mathrm{MHz}$, Chloroform- $d$ ) $\delta 6.83-6.74(\mathrm{~m}, 3 \mathrm{H}), 3.06-2.90(\mathrm{~m}$, $1 \mathrm{H}), 2.46-2.19(\mathrm{~m}, 8 \mathrm{H}), 1.92-1.66(\mathrm{~m}, 2 \mathrm{H}), 1.22(\mathrm{~s}, 12 \mathrm{H}), 0.89-0.66(\mathrm{~m}, 8 \mathrm{H}) .{ }^{13} \mathrm{C}\left\{{ }^{1} \mathrm{H},{ }^{19} \mathrm{~F}\right\}$ NMR (126 MHz, Chloroform- $d$ ) $\delta$ 145.6, 137.7, 127.9, 126.6, 125.4, 82.8, 50.1, 42.9, 36.3, 33.2, 30.1, 29.6, 24.8, 21.3, carbon attached to boron not observed. ${ }^{19} \mathrm{~F}\left\{{ }^{1} \mathrm{H}\right\}$ NMR $(282 \mathrm{MHz}$, Chloroform- $d$ ) $\delta$-63.6. ${ }^{11} \mathrm{~B}$ NMR $(96 \mathrm{MHz}$, Chloroform- $d$ ) $\delta$ 32.7. HRMS (ESI) $\mathrm{m} / z=421.2500$ calcd. for $\mathrm{C}_{22} \mathrm{H}_{34} \mathrm{O}_{2} \mathrm{BF}_{3} \mathrm{Na}^{+}[\mathrm{M}+\mathrm{Na}]^{+}$, found: 421.2496. FTIR (neat): $v\left(\mathrm{~cm}^{-1}\right)$ 2977, 2924, 1604, $1469,1357,1320,1271,1248,1131,1102,970,846,711$.

4,4,5,5-Tetramethyl-2-(6,6,6-trifluoro-2,2-dimethyl-4-(naphthalen-2-yl)hexyl)-1,3,2dioxaborolane $\mathbf{( 8 p )}$<smiles>CC(C)(C)CC(C)(C)CC(CC(F)(F)F)c1ccc2ccccc2c1</smiles>

Following GP2, 2-iodonaphthalene ( $45.5 \mathrm{mg}, 0.22 \mathrm{mmol}, 1.1$ equiv), $n$-butyl lithium (1.6 M, 0.14 $\mathrm{mL}, 0.22 \mathrm{mmol}, 1.1$ equiv), 2,2-dimethylpent-4-en-1-ylboronic acid pinacol ester $\mathbf{6 a}$ ( $44.8 \mathrm{mg}, 0.20$ mmol, 1.0 equiv) and trifluoromethyl iodide (1.0 $\mathrm{M}$ in $\mathrm{CH}_{3} \mathrm{CN}, 0.3 \mathrm{~mL}, 0.3 \mathrm{mmol}, 1.5$ equiv) afforded the desired product $\mathbf{8 p}$ as a colorless sticky oil (37.4 $\mathrm{mg}, 45 \%$ yield). $\mathrm{Rf}=0.4$ (pentane/DCM = 5:3). ${ }^{1} \mathrm{H}$ NMR $(300 \mathrm{MHz}$, Chloroform- $d$ ) $\delta 7.74-7.66(\mathrm{~m}, 3 \mathrm{H}), 7.61-7.56(\mathrm{~m}$, $1 \mathrm{H}), 7.41-7.24(\mathrm{~m}, 3 \mathrm{H}), 3.33-3.08(\mathrm{~m}, 1 \mathrm{H}), 2.52-2.26(\mathrm{~m}, 2 \mathrm{H}), 2.00-1.66(\mathrm{~m}, 2 \mathrm{H}), 1.13(\mathrm{~s}$, $6 \mathrm{H}), 1.12(\mathrm{~s}, 6 \mathrm{H}), 0.82-0.62(\mathrm{~m}, 8 \mathrm{H}) .{ }^{13} \mathrm{C}\left\{{ }^{1} \mathrm{H},{ }^{19} \mathrm{~F}\right\}$ NMR (126 MHz, Chloroform- $d$ ) $\delta 142.8,133.5$, 132.3, 128.2, 127.6, 127.6, 126.5, 126.4, 125.9, 125.7, 125.4, 82.8, 50.0, 42.9, 36.7, 33.2, 30.3, 29.8, 24.8, carbon attached to boron not observed. ${ }^{19} \mathrm{~F}\left\{{ }^{1} \mathrm{H}\right\} \mathrm{NMR}\left(282 \mathrm{MHz}, \mathrm{CDCl}_{3}\right) \delta-63.5 .{ }^{11} \mathrm{~B} \mathrm{NMR}$ $\left(96 \mathrm{MHz}, \mathrm{CDCl}_{3}\right) \delta 33.0$. HRMS (ESI) $\mathrm{m} / z=443.2344$ calcd. for $\mathrm{C}_{24} \mathrm{H}_{32} \mathrm{O}_{2} \mathrm{BF}_{3} \mathrm{Na}^{+}[\mathrm{M}+\mathrm{Na}]^{+}$, found: 443.2340. FTIR (neat): $v\left(\mathrm{~cm}^{-1}\right)$ 2978, 2935, 1601, 1509, 1469, 1439, 1357, 1319, 1271, 1248, 1215 , 1133, 1099, 968, 909, 847, 816, 731, 649.

2-(4-(Benzo[d][1,3]dioxol-5-yl)-6,6,6-trifluoro-2,2-dimethylhexyl)-4,4,5,5-tetramethyl-1,3,2dioxaborolane $\mathbf{( 8 q})$ 
<smiles>CC(C)(CB1OC(C)(C)C(C)(C)O1)CC(CC(F)(F)F)c1ccc2c(c1)OCO2</smiles>

Following GP2, 5-iodobenzo[d][1,3]dioxole (54.6 mg, $0.22 \mathrm{mmol}, 1.1$ equiv), $n$-butyl lithium (1.6 M, $0.14 \mathrm{~mL}, 0.22 \mathrm{mmol}, 1.1$ equiv), 2,2-dimethylpent-4-en-1-ylboronic acid pinacol ester $6 \mathbf{a}$ (44.8 $\mathrm{mg}, 0.20 \mathrm{mmol}, 1.0$ equiv) and trifluoromethyl iodide (1.0 $\mathrm{M}$ in $\mathrm{CH}_{3} \mathrm{CN}, 0.3 \mathrm{~mL}, 0.3 \mathrm{mmol}, 1.5$ equiv) afforded the desired product $\mathbf{8 q}$ as a colorless sticky oil $(47.3 \mathrm{mg}, 57 \%$ yield). $\mathrm{Rf}=0.3$ (pentane/Et $2 \mathrm{O}=20: 3) .{ }^{1} \mathrm{H}$ NMR (300 MHz, Chloroform- $d$ ) $\delta 6.69-6.54(\mathrm{~m}, 3 \mathrm{H}), 5.85(\mathrm{~s}, 2 \mathrm{H}), 3.01$ $-2.84(\mathrm{~m}, 1 \mathrm{H}), 2.35-2.11(\mathrm{~m}, 2 \mathrm{H}), 1.82-1.50(\mathrm{~m}, 2 \mathrm{H}), 1.15(\mathrm{~s}, 12 \mathrm{H}), 0.76(\mathrm{~s}, 6 \mathrm{H}), 0.73-0.59$ (m, 2H). ${ }^{13} \mathrm{C}\left\{{ }^{1} \mathrm{H},{ }^{19} \mathrm{~F}\right\}$ NMR $\left(126 \mathrm{MHz}, \mathrm{cdcl}_{3}\right) \delta 147.6,146.0,139.2,126.5,120.9,108.1,107.7$, $100.8,82.8,50.1,43.0,36.3,33.0,30.2,29.8,24.8,24.8$, carbon attached to boron not observed. ${ }^{19} \mathrm{~F}\left\{{ }^{1} \mathrm{H}\right\}$ NMR (282 MHz, $\left.\mathrm{CDCl}_{3}\right) \delta-63.5 .{ }^{11} \mathrm{~B}$ NMR $\left(96 \mathrm{MHz}, \mathrm{CDCl}_{3}\right) \delta 32.9$. HRMS (ESI) $\mathrm{m} / \mathrm{z}=$ 437.2085 calcd. for $\mathrm{C}_{21} \mathrm{H}_{30} \mathrm{O}_{4} \mathrm{BF}_{3} \mathrm{Na}^{+}[\mathrm{M}+\mathrm{Na}]^{+}$, found: 437.2081. FTIR (neat): $v$ ( $\mathrm{cm}^{-1}$ ) 2979, 1505 , 1489, 1441, 1358, 1320, 1239, 1140, 1116, 1098, 1040, 969, 937, 908, 847, 811, 729, 641.

2-(4-(Dibenzo[b,d]furan-2-yl)-6,6,7,7,8,8,9,9,9-nonafluoro-2,2-dimethylnonyl)-4,4,5,5tetramethyl-1,3,2-dioxaborolane (8r)<smiles>CC(C)(CB1OC(C)(C)C(C)(C)O1)CC(CC(F)(F)C(F)(F)C(F)(F)C(F)(F)F)c1ccc2oc3ccccc3c2c1</smiles>

Following GP2, 2-bromodibenzo[b,d]furan ( $59.3 \mathrm{mg}, 0.24 \mathrm{mmol}, 1.2$ equiv), $n$-butyl lithium (1.6 M, $0.15 \mathrm{~mL}, 0.24 \mathrm{mmol}, 1.1$ equiv), 2,2-dimethylpent-4-en-1-ylboronic acid pinacol ester $\mathbf{6 a}$ (44.8 $\mathrm{mg}, 0.20 \mathrm{mmol}, 1.0$ equiv) and nonafluorobutyl bromide $(89.7 \mathrm{mg}, 0.3 \mathrm{mmol}, 1.5$ equiv) afforded the desired product $8 \mathbf{r}$ as a colorless sticky oil ( $79.8 \mathrm{mg}, 65 \%$ yield). $\mathrm{Rf}=0.4$ (pentane/DCM $=5: 3$ ). ${ }^{1} \mathrm{H}$ NMR $(300 \mathrm{MHz}$, Chloroform- $d$ ) $\delta 7.90-7.82(\mathrm{~m}, 1 \mathrm{H}), 7.73(\mathrm{~d}, J=1.9 \mathrm{~Hz}, 1 \mathrm{H}), 7.51-7.31(\mathrm{~m}$, $3 \mathrm{H}), 7.29-7.21(\mathrm{~m}, 2 \mathrm{H}), 3.45-3.26(\mathrm{~m}, 1 \mathrm{H}), 2.53-2.18(\mathrm{~m}, 2 \mathrm{H}), 2.01-1.71(\mathrm{~m}, 2 \mathrm{H}), 1.12(\mathrm{~s}$, $6 \mathrm{H}), 1.11(\mathrm{~s}, 6 \mathrm{H}), 0.83-0.62(\mathrm{~m}, 8 \mathrm{H}) .{ }^{13} \mathrm{C}\left\{{ }^{1} \mathrm{H},{ }^{19} \mathrm{~F}\right\}$ NMR $(126 \mathrm{MHz}$, Chloroform- $d$ ) $\delta 156.6,155.0$, 140.5, 127.1, 126.8, 124.4, 124.2, 122.6, 120.6, 119.5, 118.3, 117.4, 111.7, 111.6, 110.3, 108.8, $82.8,51.1,39.8,35.2,33.3,30.4,30.0,24.8,24.8$, carbon attached to boron not observed. ${ }^{19} \mathrm{~F}\left\{{ }^{1} \mathrm{H}\right\}$ NMR (470 MHz, Chloroform- $d$ ) $\delta-81.1$ - -81.2 (m, 3F), -111.7 - -114.3 (m, 2F), -124.5 - -124.7 (m, 2F), $-125.8--126.0$ (m, 2F). ${ }^{11} \mathrm{~B}$ NMR (96 MHz, Chloroform- $d$ ) $\delta 32.6$. HRMS (ESI) $\mathrm{m} / \mathrm{z}=$ 633.2198 calcd. for $\mathrm{C}_{29} \mathrm{H}_{32} \mathrm{O}_{3} \mathrm{BF}_{9} \mathrm{Na}^{+}[\mathrm{M}+\mathrm{Na}]^{+}$, found: 633.2200. FTIR (neat): $v\left(\mathrm{~cm}^{-1}\right)$ 2980, 2920, 2871, 1481, 1451, 1354, 1320, 1234, 1217, 1197, 1166, 1133, 1023, 969, 907, $883,845,815,730,648$.

2-(4-(Benzo[b]thiophen-6-yl)-6,6,6-trifluoro-2,2-dimethylhexyl)-4,4,5,5-tetramethyl-1,3,2dioxaborolane (8s) 
<smiles>CC(C)(CB1OC(C)(C)C(C)(C)O1)CC(CC(F)(F)F)c1ccc2ccsc2c1</smiles>

Following GP2, 6-bromobenzo[b]thiophene (51.1 mg, $0.24 \mathrm{mmol}, 1.2$ equiv), $n$-butyl lithium (1.6 M, $0.15 \mathrm{~mL}, 0.24 \mathrm{mmol}, 1.1$ equiv), 2,2-dimethylpent-4-en-1-ylboronic acid pinacol ester $6 \mathbf{a}$ (44.8 $\mathrm{mg}, 0.20 \mathrm{mmol}, 1.0$ equiv) and trifluoromethyl iodide (1.0 $\mathrm{M}$ in $\mathrm{CH}_{3} \mathrm{CN}, 0.3 \mathrm{~mL}, 0.3 \mathrm{mmol}, 1.5$ equiv) afforded the desired product $\mathbf{8 s}$ as a colorless sticky oil (48.3 $\mathrm{mg}, 57 \%$ yield). $\mathrm{Rf}=0.4$ (pentane/DCM = 5:3). ${ }^{1} \mathrm{H}$ NMR $(300 \mathrm{MHz}$, Chloroform- $d$ ) $\delta 7.70-7.62(\mathrm{~m}, 2 \mathrm{H}), 7.29(\mathrm{~d}, J=5.4$ $\mathrm{Hz}, 1 \mathrm{H}), 7.22-7.11(\mathrm{~m}, 2 \mathrm{H}), 3.24-3.05(\mathrm{~m}, 1 \mathrm{H}), 2.47-2.22(\mathrm{~m}, 2 \mathrm{H}), 1.96-1.64(\mathrm{~m}, 2 \mathrm{H}), 1.13$ (s, 6H), 1.12 (s, 6H), $0.78-0.61(\mathrm{~m}, 8 \mathrm{H}) .{ }^{13} \mathrm{C}\left\{{ }^{1} \mathrm{H},{ }^{19} \mathrm{~F}\right\}$ NMR (126 MHz, Chloroform- $d$ ) $\delta 141.8$, 140.0, 138.2, 126.5, 125.8, 124.2, 123.6, 121.4, 82.8, 50.2, 43.1, 36.7, 33.2, 30.3, 29.8, 24.8, carbon attached to boron not observed. ${ }^{19} \mathrm{~F}\left\{{ }^{1} \mathrm{H}\right\}$ NMR $\left(282 \mathrm{MHz}\right.$, Chloroform- $d$ ) $\delta$-63.5. ${ }^{11} \mathrm{~B}$ NMR $(96$ MHz, Chloroform- $d$ ) $\delta 32.8$. HRMS (ESI) $m / z=449.1908$ calcd. for $\mathrm{C}_{22} \mathrm{H}_{30} \mathrm{O}_{2} \mathrm{SBF}_{3} \mathrm{Na}^{+}[\mathrm{M}+\mathrm{Na}]^{+}$, found: 449.1904. FTIR (neat): $v\left(\mathrm{~cm}^{-1}\right)$ 2976, 2955, 2933, 2907, 2871, 1468, 1357, 1320, 1271 , $1247,1138,1131,1101,1082,969,883,847,818,759,698,630$.

2-(4-(Dibenzo[b,d]thiophen-4-yl)-6,6,6-trifluoro-2,2-dimethylhexyl)-4,4,5,5-tetramethyl-1,3,2dioxaborolane $(\mathbf{8 t})$<smiles>CC(C)(CB1OC(C)(C)C(C)(C)O1)CC(CCC(F)(F)F)c1cccc2c1sc1ccccc12</smiles>

Following GP2, 4-iododibenzo[b,d]thiophene ( $74.4 \mathrm{mg}, 0.24 \mathrm{mmol}, 1.2$ equiv), $n$-butyl lithium (1.6 M, $0.15 \mathrm{~mL}, 0.24 \mathrm{mmol}, 1.1$ equiv), 2,2-dimethylpent-4-en-1-ylboronic acid pinacol ester $\mathbf{6 a}$ (44.8 $\mathrm{mg}, 0.20 \mathrm{mmol}, 1.0$ equiv) and trifluoromethyl iodide $\left(1.0 \mathrm{M}\right.$ in $\mathrm{CH}_{3} \mathrm{CN}, 0.3 \mathrm{~mL}, 0.3 \mathrm{mmol}, 1.5$ equiv) afforded the desired product $8 \mathrm{t}$ as a light yellow sticky oil (49.9 $\mathrm{mg}, 52 \%$ yield). $\mathrm{Rf}=0.4$ (pentane/DCM = 5:3). ${ }^{1} \mathrm{H}$ NMR (300 MHz, Chloroform- $d$ ) $\delta 8.11-8.01(\mathrm{~m}, 1 \mathrm{H}), 7.93(\mathrm{dd}, J=7.6$, $1.3 \mathrm{~Hz}, 1 \mathrm{H}), 7.83-7.72(\mathrm{~m}, 1 \mathrm{H}), 7.41-7.24(\mathrm{~m}, 4 \mathrm{H}), 3.44-3.21(\mathrm{~m}, 1 \mathrm{H}), 2.72-2.29(\mathrm{~m}, 2 \mathrm{H})$, $2.26-1.70(\mathrm{~m}, 2 \mathrm{H}), 1.12(\mathrm{~s}, 12 \mathrm{H}), 0.84-0.63(\mathrm{~m}, 8 \mathrm{H}) .{ }^{13} \mathrm{C}\left\{{ }^{1} \mathrm{H},{ }^{19} \mathrm{~F}\right\}$ NMR $(126 \mathrm{MHz}$, Chloroformd) $\delta 139.0,126.7,126.4,124.9,124.4,122.7,121.6,119.8,82.8,33.1,29.9,29.4,24.8,24.8$, carbon attached to boron not observed. ${ }^{19} \mathrm{~F}\left\{{ }^{1} \mathrm{H}\right\}$ NMR $\left(282 \mathrm{MHz}\right.$, Chloroform- $d$ ) $\delta$-63.9. ${ }^{11} \mathrm{~B}$ NMR $(96$ MHz, Chloroform- $d$ ) $\delta 32.6$. HRMS (ESI) $m / z=499.2065$ calcd. for $\mathrm{C}_{26} \mathrm{H}_{32} \mathrm{O}_{2} \mathrm{SBF}_{3} \mathrm{Na}^{+}[\mathrm{M}+\mathrm{Na}]^{+}$, found: 499.2064. FTIR (neat): $v\left(\mathrm{~cm}^{-1}\right)$ 2979, 2932, 2870, 1444, 1358, 1320, 1269, 1248, 1134 , 1091, 1046, 968, 908, 847, 798, 751, 731, 648, 627.

3-(1,1,1-Trifluoro-5,5-dimethyl-6-(4,4,5,5-tetramethyl-1,3,2-dioxaborolan-2-yl)hexan-3yl)quinolone (8u) 
<smiles>CC(C)(CB1OC(C)(C)C(C)(C)O1)CC(CC(F)(F)F)c1cnc2ccccc2c1</smiles>

Following GP2, 3-bromoquinoline (49.9 mg, $0.24 \mathrm{mmol}, 1.2$ equiv), $n$-butyl lithium (1.6 M, 0.15 $\mathrm{mL}, 0.24 \mathrm{mmol}, 1.1$ equiv), 2,2-dimethylpent-4-en-1-ylboronic acid pinacol ester $\mathbf{6 a}$ ( $44.8 \mathrm{mg}, 0.20$ mmol, 1.0 equiv) and trifluoromethyl iodide $\left(1.0 \mathrm{M}\right.$ in $\mathrm{CH}_{3} \mathrm{CN}, 0.3 \mathrm{~mL}, 0.3 \mathrm{mmol}, 1.5$ equiv) afforded the desired product $8 \mathbf{~ u}$ as a light yellow sticky oil $(32.7 \mathrm{mg}, 39 \%$ yield). $\mathrm{Rf}=0.3$ (pentane/Et $2 \mathrm{O}=1: 1) .{ }^{1} \mathrm{H}$ NMR $(300 \mathrm{MHz}$, Chloroform- $d$ ) $\delta 8.76(\mathrm{~d}, J=2.3 \mathrm{~Hz}, 1 \mathrm{H}), 8.03(\mathrm{dd}, J=$ $8.5,1.1 \mathrm{~Hz}, 1 \mathrm{H}), 7.91(\mathrm{~d}, J=2.2 \mathrm{~Hz}, 1 \mathrm{H}), 7.77-7.68(\mathrm{~m}, 1 \mathrm{H}), 7.66-7.57(\mathrm{~m}, 1 \mathrm{H}), 7.52-7.42(\mathrm{~m}$, $1 \mathrm{H}), 3.32-3.16(\mathrm{~m}, 1 \mathrm{H}), 2.57-2.30(\mathrm{~m}, 2 \mathrm{H}), 2.02-1.73(\mathrm{~m}, 2 \mathrm{H}), 1.12(\mathrm{~s}, 6 \mathrm{H}), 1.11(\mathrm{~s}, 6 \mathrm{H}), 0.83$ $-0.66(\mathrm{~m}, 8 \mathrm{H}) .{ }^{13} \mathrm{C}\left\{{ }^{1} \mathrm{H},{ }^{19} \mathrm{~F}\right\}$ NMR $(126 \mathrm{MHz}$, Chloroform- $d$ ) $\delta$ 150.7, 146.8, 138.1, 134.4, 129.1, 129.0, 128.0, 127.5, 126.8, 126.2, 82.9, 50.0, 42.7, 34.4, 33.3, 30.4, 30.0, 24.8, 24.8, carbon attached to boron not observed. ${ }^{19} \mathrm{~F}\left\{{ }^{1} \mathrm{H}\right\}$ NMR $\left(282 \mathrm{MHz}\right.$, Chloroform- $d$ ) $\delta$-63.4. ${ }^{11} \mathrm{~B}$ NMR (96 MHz, Chloroform- $d$ ) $\delta 32.9$. HRMS (ESI) $m / z=422.2477$ calcd. for $\mathrm{C}_{23} \mathrm{H}_{31} \mathrm{NO}_{2} \mathrm{BF}_{3} \mathrm{Na}^{+}[\mathrm{M}+\mathrm{Na}]^{+}$, found: 422.2472. FTIR (neat): $v\left(\mathrm{~cm}^{-1}\right)$ 2977, 2873, 1496, 1469, 1358, 1321, 1269, 1248, 1215, $1139,1100,969,847,788,752,649$.

2-(4-(4-(Tert-butyl)phenyl)-2,2-diethyl-6,6,6-trifluorohexyl)-4,4,5,5-tetramethyl-1,3,2dioxaborolane $(\mathbf{8 v})$<smiles>CCC(CC)(CB1OC(C)(C)C(C)(C)O1)CC(CC(F)(F)F)c1ccc(C(C)(C)C)cc1</smiles>

Following GP2, 4-tert-butylphenyl iodide (95\% purity, $65.7 \mathrm{mg}, 0.24 \mathrm{mmol}, 1.2$ equiv), $n$-butyl lithium (1.6 M, $0.15 \mathrm{~mL}, 0.24 \mathrm{mmol}, 1.2$ equiv), 2,2-diethylpent-4-en-1-ylboronic acid pinacol ester 6v $\left(50.4 \mathrm{mg}, 0.20 \mathrm{mmol}, 1.0\right.$ equiv) and trifluoromethyl iodide $\left(1.0 \mathrm{M}\right.$ in $\mathrm{CH}_{3} \mathrm{CN}, 0.3 \mathrm{~mL}, 0.3 \mathrm{mmol}$, 1.5 equiv) afforded the desired product $8 \mathrm{v}$ as a colorless sticky oil ( $77.1 \mathrm{mg}, 85 \%$ yield). $\mathrm{Rf}=0.3$ (pentane/DCM = 4:1). ${ }^{1} \mathrm{H}$ NMR $(300 \mathrm{MHz}$, Chloroform- $d$ ) $\delta 7.21-7.14(\mathrm{~m}, 2 \mathrm{H}), 7.09-7.01(\mathrm{~m}$, $2 \mathrm{H}), 3.02-2.86(\mathrm{~m}, 1 \mathrm{H}), 2.46-2.17(\mathrm{~m}, 2 \mathrm{H}), 1.85-1.50(\mathrm{~m}, 2 \mathrm{H}), 1.21(\mathrm{~s}, 9 \mathrm{H}), 1.16-1.00(\mathrm{~m}$, 16H), $0.65-0.47(\mathrm{~m}, 8 \mathrm{H}) .{ }^{13} \mathrm{C}\left\{{ }^{1} \mathrm{H},{ }^{19} \mathrm{~F}\right\}$ NMR (126 MHz, Chloroform- $d$ ) $\delta 149.0,142.4,127.3$, 126.7, 125.1, 82.6, 44.1, 42.9, 38.3, 35.3, 34.3, 31.4, 30.7, 30.6, 24.8, 24.7, 7.8, 7.8, carbon attached to boron not observed. ${ }^{19} \mathrm{~F}\left\{{ }^{1} \mathrm{H}\right\}$ NMR $\left(282 \mathrm{MHz}\right.$, Chloroform- $d$ ) $\delta-63.5 .{ }^{11} \mathrm{~B}$ NMR $(96 \mathrm{MHz}$, Chloroform- $d$ ) $\delta$ 32.9. HRMS (ESI) $m / z=477.3127$ calcd. for $\mathrm{C}_{26} \mathrm{H}_{42} \mathrm{O}_{2} \mathrm{BF}_{3} \mathrm{Na}^{+}[\mathrm{M}+\mathrm{Na}]^{+}$, found: 477.3124. FTIR (neat): $v\left(\mathrm{~cm}^{-1}\right)$ 2976, 2955, 2936, 2907, 1468, 1357, 1320, 1271, 1247, 1138, 1131, 1101, 1082, 1048 969, 883, 847, 818, 759, 698, 630.

2-(4-(4-(Tert-butyl)phenyl)-6,6,6-trifluoro-2,2-dipropylhexyl)-4,4,5,5-tetramethyl-1,3,2dioxaborolane $(\mathbf{8 w})$ 


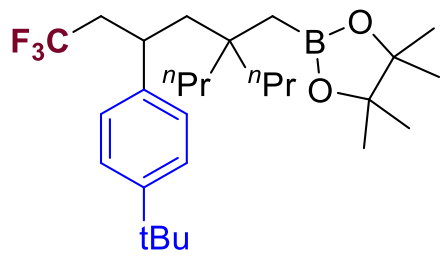

Following GP2, 4-tert-butylphenyl iodide (95\% purity, $65.7 \mathrm{mg}, 0.24 \mathrm{mmol}, 1.2$ equiv), $n$-butyl lithium (1.6 M, $0.15 \mathrm{~mL}, 0.24 \mathrm{mmol}, 1.2$ equiv), 2,2-dipropylpent-4-en-1-ylboronic acid pinacol ester $6 \mathbf{w}$ (56.0 mg, $0.20 \mathrm{mmol}, 1.0$ equiv) and trifluoromethyl iodide (1.0 $\mathrm{M}_{\text {in }} \mathrm{CH}_{3} \mathrm{CN}, 0.3 \mathrm{~mL}, 0.3$ mmol, 1.5 equiv) afforded the desired product $8 \mathbf{w}$ as a colorless sticky oil $(71.7 \mathrm{mg}, 74 \%$ yield). $\mathrm{Rf}$ $=0.5$ (pentane/DCM = 5:3). ${ }^{1} \mathrm{H}$ NMR (300 MHz, Chloroform- $d$ ) $\delta 7.22-7.14(\mathrm{~m}, 2 \mathrm{H}), 7.09-7.02$ (m, 2H), $3.04-2.87(\mathrm{~m}, 1 \mathrm{H}), 2.42-2.16(\mathrm{~m}, 2 \mathrm{H}), 1.86-1.51(\mathrm{~m}, 2 \mathrm{H}), 1.21(\mathrm{~s}, 8 \mathrm{H}), 1.14(\mathrm{~s}, 5 \mathrm{H})$, $1.13(\mathrm{~s}, 6 \mathrm{H}), 1.08-0.81(\mathrm{~m}, 5 \mathrm{H}), 0.67-0.48(\mathrm{~m}, 6 \mathrm{H}) .{ }^{13} \mathrm{C}\left\{{ }^{1} \mathrm{H},{ }^{19} \mathrm{~F}\right\}$ NMR $(126 \mathrm{MHz}$, Chloroformd) $\delta 149.1,142.1,127.3,126.7,125.1,82.6,44.7,43.0,41.9,41.5,38.4,35.4,34.3,31.4,24.9,24.7$, 16.6, 16.6, 14.6, 14.5, carbon attached to boron not observed. ${ }^{19} \mathrm{~F}\left\{{ }^{1} \mathrm{H}\right\}$ NMR $(282 \mathrm{MHz}$, Chloroform- $d$ ) $\delta-63.5 .{ }^{11} \mathrm{~B}$ NMR $(96 \mathrm{MHz}$, Chloroform- $d$ ) $\delta 33.1$. HRMS (ESI) $\mathrm{m} / z=505.3440$ calcd. for $\mathrm{C}_{28} \mathrm{H}_{46} \mathrm{O}_{2} \mathrm{BF}_{3} \mathrm{Na}^{+}[\mathrm{M}+\mathrm{Na}]^{+}$, found: 505.3439. FTIR (neat): v ( $\left.\mathrm{cm}^{-1}\right) 2959,2933,2873$, $1466,1371,1324,1269,1251,1131,1095,968,848,827,736,611$.

2-((1-(2-(4-(Tert-butyl)phenyl)-4,4,4-trifluorobutyl)cyclopentyl)methyl)-4,4,5,5-tetramethyl1,3,2-dioxaborolane $(\mathbf{8 x})$<smiles>CC(C)(C)c1ccc(C(CC(F)(F)F)CC2(CB3OC(C)(C)C(C)(C)O3)CCCC2)cc1</smiles>

Following GP2, 4-tert-butylphenyl iodide (95\% purity, $65.7 \mathrm{mg}, 0.24 \mathrm{mmol}, 1.2$ equiv), $n$-butyl lithium (1.6 M, $0.15 \mathrm{~mL}, 0.24 \mathrm{mmol}, 1.2$ equiv), 2-((1-allylcyclopentyl)methyl)-4,4,5,5tetramethyl-1,3,2-dioxaborolane $\mathbf{6 x}(50.0 \mathrm{mg}, 0.20 \mathrm{mmol}, 1.0$ equiv) and trifluoromethyl iodide (1.0 $\mathrm{M}$ in $\mathrm{CH}_{3} \mathrm{CN}, 0.3 \mathrm{~mL}, 0.3 \mathrm{mmol}, 1.5$ equiv) afforded the desired product $\mathbf{8 x}$ as a colorless sticky oil ( $68.5 \mathrm{mg}, 76 \%$ yield). $\mathrm{Rf}=0.6$ (pentane/DCM $=5: 3) .{ }^{1} \mathrm{H}$ NMR $(300 \mathrm{MHz}$, Chloroform- $d$ ) $\delta 7.23-$ $7.14(\mathrm{~m}, 2 \mathrm{H}), 7.08-7.00(\mathrm{~m}, 2 \mathrm{H}), 3.08-2.94(\mathrm{~m}, 1 \mathrm{H}), 2.40-2.20(\mathrm{~m}, 2 \mathrm{H}), 1.90-1.74(\mathrm{~m}, 2 \mathrm{H})$, $1.52-1.29(\mathrm{~m}, 6 \mathrm{H}), 1.22(\mathrm{~s}, 9 \mathrm{H}), 1.13(\mathrm{~s}, 6 \mathrm{H}), 1.13(\mathrm{~s}, 6 \mathrm{H}), 1.01-0.90(\mathrm{~m}, 2 \mathrm{H}), 0.85-0.64(\mathrm{~m}$, $2 \mathrm{H}) .{ }^{13} \mathrm{C}\left\{{ }^{1} \mathrm{H},{ }^{19} \mathrm{~F}\right\}$ NMR (126 MHz, Chloroform- $d$ ) $\delta 149.1,142.0,127.3,126.7,125.1,82.7,47.3$, 44.3, 42.5, 40.5, 40.1, 36.6, 34.3, 31.4, 24.9, 24.8, 23.9, 23.4, carbon attached to boron not observed. ${ }^{19} \mathrm{~F}\left\{{ }^{1} \mathrm{H}\right\}$ NMR (282 MHz, Chloroform- $d$ ) $\delta$-63.5. ${ }^{11} \mathrm{~B}$ NMR (96 MHz, Chloroform- $d$ ) $\delta 32.7$. HRMS (ESI) $m / z=475.2970$ calcd. for $\mathrm{C}_{26} \mathrm{H}_{40} \mathrm{O}_{2} \mathrm{BF}_{3} \mathrm{Na}^{+}[\mathrm{M}+\mathrm{Na}]^{+}$, found: 475.2968. FTIR (neat): $v\left(\mathrm{~cm}^{-1}\right)$ 2957, 2920, 2876, 1513, 1362, 1316, 1268, 1248, 1133, 969, 848, 829, 735, 581.

2-((1-(2-(4-(tert-butyl)phenyl)-4,4,4-trifluorobutyl)cyclohexyl)methyl)-4,4,5,5-tetramethyl-1,3,2dioxaborolane $(\mathbf{8 y})$ 
<smiles>CC(C)(C)c1ccc(C(CC(F)(F)F)CC2(CB3OC(C)(C)C(C)(C)O3)CCCCC2)cc1</smiles>

Following GP2, 4-tert-butylphenyl iodide (95\% purity, $65.7 \mathrm{mg}, 0.24 \mathrm{mmol}, 1.2$ equiv), $n$-butyl lithium (1.6 M, $0.15 \mathrm{~mL}, 0.24 \mathrm{mmol}, 1.2$ equiv), 2-((1-allylcyclohexyl)methyl)-4,4,5,5-tetramethyl1,3,2-dioxaborolane 6y (52.8 mg, $0.20 \mathrm{mmol}, 1.0$ equiv) and trifluoromethyl iodide $(1.0 \mathrm{M}$ in $\mathrm{CH}_{3} \mathrm{CN}, 0.3 \mathrm{~mL}, 0.3 \mathrm{mmol}, 1.5$ equiv) afforded the desired product $8 \mathbf{y}$ as a colorless sticky oil (73.8 $\mathrm{mg}, 79 \%$ yield). $\mathrm{Rf}=0.6$ (pentane/DCM $=5: 3) .{ }^{1} \mathrm{H}$ NMR $(300 \mathrm{MHz}$, Chloroform- $d$ ) $\delta 7.21-7.12$ (m, 2H), $7.09-7.02(\mathrm{~m}, 2 \mathrm{H}), 3.09-2.93(\mathrm{~m}, 1 \mathrm{H}), 2.46-2.16(\mathrm{~m}, 2 \mathrm{H}), 1.98-1.58(\mathrm{~m}, 2 \mathrm{H}), 1.40$ $-0.90(\mathrm{~m}, 10 \mathrm{H}) 1.21(\mathrm{~s}, 9 \mathrm{H}), 1.12(\mathrm{~s}, 12 \mathrm{H}), 0.83-0.63(\mathrm{~m}, 2 \mathrm{H}) .{ }^{13} \mathrm{C}\left\{{ }^{1} \mathrm{H},{ }^{19} \mathrm{~F}\right\}$ NMR $(126 \mathrm{MHz}$, Chloroform- $d$ ) $\delta$ 149.0, 142.4, 127.3, 126.7, 125.1, 82.7, 42.9, 38.8, 38.0, 35.7, 35.1, 34.3, 31.4, 26.2, 24.9, 24.8, 21.9, 21.7, carbon attached to boron not observed. ${ }^{19} \mathrm{~F}\left\{{ }^{1} \mathrm{H}\right\}$ NMR $(282 \mathrm{MHz}$, Chloroform- $d$ ) $\delta-63.5 .{ }^{11} \mathrm{~B}$ NMR (96 MHz, Chloroform- $d$ ) $\delta 32.9$. HRMS (ESI) $m / z=489.3127$ calcd. for $\mathrm{C}_{27} \mathrm{H}_{42} \mathrm{O}_{2} \mathrm{BF}_{3} \mathrm{Na}^{+}[\mathrm{M}+\mathrm{Na}]^{+}$, found: 489.3124. FTIR (neat): v $\left(\mathrm{cm}^{-1}\right)$ 2969, 2928, 2852 , 1364, 1321, 1268, 1247, 1126, 1098, 967, 907, 849, 731, 649, 605.

2-((4-(2-(4-(Tert-butyl)phenyl)-4,4,4-trifluorobutyl)tetrahydro-2H-pyran-4-yl)methyl)-4,4,5,5tetramethyl-1,3,2-dioxaborolane ( $\mathbf{8 z})$<smiles>CC(C)(C)c1ccc(C(CC(F)(F)F)CC2(CB3OC(C)(C)C(C)(C)O3)CCOCC2)cc1</smiles>

Following GP2, 4-tert-butylphenyl iodide (95\% purity, $65.7 \mathrm{mg}, 0.24 \mathrm{mmol}, 1.2$ equiv), $n$-butyl lithium (1.6 M, $0.15 \mathrm{~mL}, 0.24 \mathrm{mmol}, 1.2$ equiv), 2-((4-allyltetrahydro-2H-pyran-4-yl)methyl)4,4,5,5-tetramethyl-1,3,2-dioxaborolane $\mathbf{6 z}(53.2 \mathrm{mg}, 0.20 \mathrm{mmol}, 1.0$ equiv) and trifluoromethyl iodide (1.0 M in $\mathrm{CH}_{3} \mathrm{CN}, 0.3 \mathrm{~mL}, 0.3 \mathrm{mmol}, 1.5$ equiv) afforded the desired product $8 \mathrm{z}$ as a colorless sticky oil (69.8 mg, 75\% yield). $\mathrm{Rf}=0.4$ (pentane/ $\left.\mathrm{Et}_{2} \mathrm{O}=3: 1\right) .{ }^{1} \mathrm{H}$ NMR (300 MHz, Chloroform- $d$ ) $\delta 7.23-7.15(\mathrm{~m}, 2 \mathrm{H}), 7.09-7.02(\mathrm{~m}, 2 \mathrm{H}), 3.64-3.44(\mathrm{~m}, 2 \mathrm{H}), 3.41-3.24(\mathrm{~m}, 2 \mathrm{H}), 3.12-2.96$ (m, 1H), $2.41-2.19(\mathrm{~m}, 2 \mathrm{H}), 2.11-1.65(\mathrm{~m}, 2 \mathrm{H}), 1.46-1.30(\mathrm{~m}, 2 \mathrm{H}), 1.21(\mathrm{~s}, 9 \mathrm{H}), 1.13(\mathrm{~s}, 6 \mathrm{H})$, $1.13(\mathrm{~s}, 6 \mathrm{H}), 1.10-0.94(\mathrm{~m}, 2 \mathrm{H}), 0.92-0.82(\mathrm{~m}, 2 \mathrm{H}) .{ }^{13} \mathrm{C}\left\{{ }^{1} \mathrm{H},{ }^{19} \mathrm{~F}\right\}$ NMR (151 MHz, Chloroformd) $\delta 149.4,141.7,127.2,126.5,125.3,82.9,63.7,63.6,46.5,42.9,38.6,38.0,35.2,34.3,33.4,31.3$, 24.8, 24.8, carbon attached to boron not observed. ${ }^{19} \mathrm{~F}\left\{{ }^{1} \mathrm{H}\right\}$ NMR (282 MHz, Chloroform- $d$ ) $\delta$ 63.4. ${ }^{11} \mathrm{~B}$ NMR (96 MHz, Chloroform- $d$ ) $\delta$ 32.7. HRMS (ESI) $\mathrm{m} / \mathrm{z}=491.2920$ calcd. for $\mathrm{C}_{26} \mathrm{H}_{40} \mathrm{O}_{3} \mathrm{BF}_{3} \mathrm{Na}^{+}[\mathrm{M}+\mathrm{Na}]^{+}$, found: 491.2915. FTIR (neat): $v\left(\mathrm{~cm}^{-1}\right)$ 2962, 2941, 2916, 2868, 2862 , 
2856, 1516, 1471, 1447, 1364, 1325, 1267, 1248, 1214, 1127, 1108, 1018, 968, 910, 848, 827, 732, 598.

2-((1-(2-(4-(Tert-butyl)phenyl)-4,4,4-trifluorobutyl)cycloheptyl)methyl)-4,4,5,5-tetramethyl1,3,2-dioxaborolane (8aa)<smiles>CC(C)(C)c1ccc(C(CC(F)(F)F)CC2(CB3OC(C)(C)C(C)(C)O3)CCCCCC2)cc1</smiles>

Following GP2, 4-tert-butylphenyl iodide (95\% purity, $65.7 \mathrm{mg}, 0.24 \mathrm{mmol}, 1.2$ equiv), $n$-butyl lithium (1.6 M, $0.15 \mathrm{~mL}, 0.24 \mathrm{mmol}, 1.2$ equiv), 2-((1-allylcycloheptyl)methyl)-4,4,5,5tetramethyl-1,3,2-dioxaborolane 6aa $(55.6 \mathrm{mg}, 0.20 \mathrm{mmol}, 1.0$ equiv) and trifluoromethyl iodide (1.0 $\mathrm{M}$ in $\mathrm{CH}_{3} \mathrm{CN}, 0.3 \mathrm{~mL}, 0.3 \mathrm{mmol}, 1.5$ equiv) afforded the desired product $8 \mathbf{a a}$ as a colorless sticky oil (75.5 mg, 79\% yield). $\mathrm{Rf}=0.3$ (pentane/DCM $=4: 1) .{ }^{1} \mathrm{H}$ NMR $(300 \mathrm{MHz}$, Chloroformd) $\delta 7.21-7.12(\mathrm{~m}, 2 \mathrm{H}), 7.09-7.01(\mathrm{~m}, 2 \mathrm{H}), 3.09-2.90(\mathrm{~m}, 1 \mathrm{H}), 2.47-2.16(\mathrm{~m}, 2 \mathrm{H}), 1.99-1.58$ $(\mathrm{m}, 2 \mathrm{H}), 1.53-1.05(\mathrm{~m}, 12 \mathrm{H}), 1.21(\mathrm{~s}, 9 \mathrm{H}), 1.12(\mathrm{~s}, 6 \mathrm{H}), 1.11(\mathrm{~s}, 6 \mathrm{H}), 0.76-0.50(\mathrm{~m}, 2 \mathrm{H}) .{ }^{13} \mathrm{C}\left\{{ }^{1} \mathrm{H}\right.$, ${ }^{19} \mathrm{~F}$ \} NMR $\left(126 \mathrm{MHz}, \mathrm{cdcl}_{3}\right) \delta 148.9,142.5,127.3,126.7,125.1,82.6,47.7,43.0,41.6,41.1,38.8$, 35.5, 34.3, 31.4, 30.6, 30.4, 24.9, 24.7, 22.7, carbon attached to boron not observed. ${ }^{19} \mathrm{~F}\left\{{ }^{1} \mathrm{H}\right\} \mathrm{NMR}$ $\left(282 \mathrm{MHz}, \mathrm{CDCl}_{3}\right) \delta-63.4 .{ }^{11} \mathrm{~B} \mathrm{NMR}\left(96 \mathrm{MHz}, \mathrm{CDCl}_{3}\right) \delta 32.8$. HRMS (ESI) $\mathrm{m} / z=503.3284 \mathrm{calcd}$. for $\mathrm{C}_{28} \mathrm{H}_{44} \mathrm{O}_{2} \mathrm{BF}_{3} \mathrm{Na}^{+}[\mathrm{M}+\mathrm{Na}]^{+}$, found: 503.3281. FTIR (neat): $v\left(\mathrm{~cm}^{-1}\right)$ 2969, 2919, 2855, 1516, 1463, 1445, 1367, 1319, 1268, 1247, 1200, 1127, 1097, 969, 847, 827, 607.

2-(4-(4-(Tert-butyl)phenyl)-6,6,6-trifluoro-2-methyl-2-phenylhexyl)-4,4,5,5-tetramethyl-1,3,2dioxaborolane (8ab)<smiles>CC(C)(C)c1ccc(C(CC(F)(F)F)CC(C)(C)c2ccc(C(C)(C)C)cc2)cc1</smiles>

Following GP2, 4-tert-butylphenyl iodide (95\% purity, $65.7 \mathrm{mg}, 0.24 \mathrm{mmol}, 1.2$ equiv), $n$-butyl lithium (1.6 M, $0.15 \mathrm{~mL}, 0.24 \mathrm{mmol}, 1.2$ equiv), 4,4,5,5-tetramethyl-2-(2-methyl-2-phenylpent-4en-1-yl)-1,3,2-dioxaborolane 6ab (57.2 $\mathrm{mg}, 0.20 \mathrm{mmol}, 1.0$ equiv) and trifluoromethyl iodide (1.0 $\mathrm{M}$ in $\mathrm{CH}_{3} \mathrm{CN}, 0.3 \mathrm{~mL}, 0.3 \mathrm{mmol}, 1.5$ equiv) afforded the desired product $8 \mathbf{a b}$ as a colorless sticky oil (57.2 mg, 59\% yield, d.r. $=5.7: 1$ by $\left.{ }^{19} \mathrm{~F} \mathrm{NMR}\right) . \mathrm{Rf}=0.3$ (pentane/DCM $\left.=10: 7\right) .{ }^{1} \mathrm{H}$ NMR $(300$ MHz, Chloroform- $d$ ) $\delta 7.22-6.99(\mathrm{~m}, 7 \mathrm{H}), 6.87-6.79(\mathrm{~m}, 1.68 \mathrm{H}), 6.78-6.68(\mathrm{~m}, 0.32 \mathrm{H}), 2.81-$ $2.62(\mathrm{~m}, 1 \mathrm{H}), 2.25-1.86(\mathrm{~m}, 4 \mathrm{H}), 1.25-1.12(\mathrm{~m}, 13 \mathrm{H}), 1.09-0.89(\mathrm{~m}, 13 \mathrm{H}) .{ }^{13} \mathrm{C}\left\{{ }^{1} \mathrm{H},{ }^{19} \mathrm{~F}\right\}$ NMR $\left(151 \mathrm{MHz}, \mathrm{cdcl}_{3}\right) \delta 148.9,148.7$ (minor), 148.6 (minor), 148.4, 141.7, 141.7 (minor), 127.8, 127.7 (minor), 127.0, 127.0 (minor), 126.5 (minor), 126.4, 126.2, 126.1 (minor), 125.5, 125.3 (minor), 125.1, 125.0 (minor), 82.7, 51.9, 51.4 (minor), 41.8 (minor), 41.6, 39.7, 39.6 (minor), 36.2, 36.1 (minor), 34.3, 34.2 (minor), 31.4, 31.3 (minor), 27.5 (minor), 26.6, 24.7 (minor), 24.8, 24.5, 24.5 
(minor), carbon attached to boron not observed. ${ }^{19} \mathrm{~F}\left\{{ }^{1} \mathrm{H}\right\}$ NMR $\left(282 \mathrm{MHz}, \mathrm{CDCl}_{3}\right) \delta$-63.6 (major), -63.6 (minor). ${ }^{11} \mathrm{~B}$ NMR $\left(96 \mathrm{MHz}, \mathrm{CDCl}_{3}\right.$ ) $\delta$ 32.6. HRMS (ESI) $\mathrm{m} / \mathrm{z}=511.2971$ calcd. for $\mathrm{C}_{29} \mathrm{H}_{40} \mathrm{O}_{2} \mathrm{BF}_{3} \mathrm{Na}^{+}[\mathrm{M}+\mathrm{Na}]^{+}$, found: 511.2968. FTIR (neat): $v\left(\mathrm{~cm}^{-1}\right)$ 2966, 2908, 1513, 1467, 1446, 1359, 1325, 1266, 1245, 1215, 1129, 1096, 969, 910, 847, 829, 764, 734, 698, 581.

2-(4-(4-(Tert-butyl)phenyl)-6,6,7,7,8,8,9,9,9-nonafluoro-2-phenylnonyl)-4,4,5,5-tetramethyl1,3,2-dioxaborolane (8ac)<smiles>CC(C)(C)c1ccc(C(C[C@H](CB2OC(C)(C)C(C)(C)O2)c2ccccc2)CC(F)(F)C(F)(F)C(F)(F)C(F)(F)F)cc1</smiles>

Following GP2, 4-tert-butylphenyl iodide (95\% purity, $65.7 \mathrm{mg}, 0.24 \mathrm{mmol}, 1.2$ equiv), $n$-butyl lithium (1.6 M, $0.15 \mathrm{~mL}, 0.24 \mathrm{mmol}, 1.2$ equiv), 4,4,5,5-tetramethyl-2-(2-phenylpent-4-en-1-yl)1,3,2-dioxaborolane 6ac (54.4 mg, $0.20 \mathrm{mmol}, 1.0$ equiv) and nonafluorobutyl bromide ( $89.7 \mathrm{mg}$, $0.3 \mathrm{mmol}, 1.5$ equiv) afforded the desired product $8 \mathbf{a c}$ as a colorless sticky oil ( $65.8 \mathrm{mg}, 53 \%$ yield, d.r. $=32: 1$ by crude GC and GC/MS analysis). $\mathrm{Rf}=0.5$ (pentane/DCM $=5: 3) .{ }^{1} \mathrm{H} \mathrm{NMR}(300 \mathrm{MHz}$, Chloroform- $d$ ) $\delta 7.26-7.15(\mathrm{~m}, 4 \mathrm{H}), 7.14-7.06(\mathrm{~m}, 1 \mathrm{H}), 7.04-6.98(\mathrm{~m}, 2 \mathrm{H}), 6.96-6.89(\mathrm{~m}, 2 \mathrm{H})$, $2.70-2.56(\mathrm{~m}, 1 \mathrm{H}), 2.52-2.38(\mathrm{~m}, 1 \mathrm{H}), 2.26-1.95(\mathrm{~m}, 4 \mathrm{H}), 1.26(\mathrm{~s}, 9 \mathrm{H}), 1.07-0.92(\mathrm{~m}, 14 \mathrm{H})$. ${ }^{13} \mathrm{C}\left\{{ }^{1} \mathrm{H},{ }^{19} \mathrm{~F}\right\} \mathrm{NMR}\left(126 \mathrm{MHz}, \mathrm{cdcl}_{3}\right) \delta 149.4,145.9,140.3,128.2,127.6,127.2,126.1,125.4,118.2$, 117.4, 110.3, 108.7, 82.9, 45.5, 38.7, 37.9, 35.7, 34.4, 31.4, 24.7, 24.6, carbon attached to boron not observed. ${ }^{19} \mathrm{~F}\left\{{ }^{1} \mathrm{H}\right\}$ NMR (470 MHz, Chloroform- $d$ ) $\delta-81.1--81.2(\mathrm{~m}, 3 \mathrm{~F}),-111.8--114.0(\mathrm{~m}$, 2F), $-124.3--124.8(\mathrm{~m}, 2 \mathrm{~F}),-125.7--126.2(\mathrm{~m}, 2 \mathrm{~F}) .{ }^{11} \mathrm{~B} \mathrm{NMR}\left(96 \mathrm{MHz}, \mathrm{CDCl}_{3}\right) \delta 32.0$. HRMS (ESI) $\mathrm{m} / \mathrm{z}=647.2719$ calcd. for $\mathrm{C}_{31} \mathrm{H}_{38} \mathrm{O}_{2} \mathrm{BF}_{9} \mathrm{Na}^{+}[\mathrm{M}+\mathrm{Na}]^{+}$, found: 647.2717. FTIR (neat): $v$ (cm 1) $3027,2969,2870,1371,1323,1371,1323,1233,1218,1166,1145,1133,1115,1019,967,909$, $880,848,832,758,733,700,649,566$.

2-(4-([1,1'-biphenyl]-4-yl)-6,6,6-trifluoro-2-isopropylhexyl)-4,4,5,5-tetramethyl-1,3,2dioxaborolane (8ad)<smiles>CC(C)[C@H](CB1OC(C)(C)C(C)(C)O1)CC(CC(F)(F)F)c1ccc(-c2ccccc2)cc1</smiles>

Following GP2, 4-iodobiphenyl (67.2 mg, $0.24 \mathrm{mmol}, 1.2$ equiv), $n$-butyl lithium (1.6 M, $0.15 \mathrm{~mL}$, 0.24 mmol, 1.2 equiv), 2-(2-isopropylpent-4-en-1-yl)-4,4,5,5-tetramethyl-1,3,2-dioxaborolane 6ad

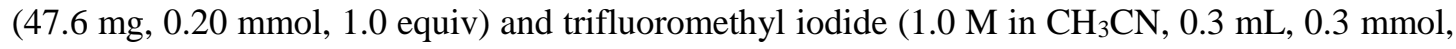
1.5 equiv) afforded the desired product $8 \mathrm{ad}$ as a colorless sticky oil ( $46.5 \mathrm{mg}, 50 \%$ yield, $d . r .=17: 1$ by ${ }^{19} \mathrm{~F}$ NMR). $\mathrm{R}_{\mathrm{f}}=0.4$ (pentane/DCM $\left.=10: 7\right) .{ }^{1} \mathrm{H}$ NMR $(300 \mathrm{MHz}$, Chloroform- $d$ ) $\delta 7.54-7.40$ (m, 4H), $7.39-7.30(\mathrm{~m}, 2 \mathrm{H}), 7.28-7.13(\mathrm{~m}, 3 \mathrm{H}), 3.08-2.89(\mathrm{~m}, 1 \mathrm{H}), 2.47-2.19(\mathrm{~m}, 2 \mathrm{H}), 1.80$ $-1.47(\mathrm{~m}, 3 \mathrm{H}), 1.39-1.26(\mathrm{~m}, 1 \mathrm{H}), 1.15(\mathrm{~s}, 6 \mathrm{H}), 1.14(\mathrm{~s}, 6 \mathrm{H}), 0.72(\mathrm{~d}, J=6.8 \mathrm{~Hz}, 6 \mathrm{H}), 0.71(\mathrm{~d}, J=$ $6.8 \mathrm{~Hz}, 6 \mathrm{H}), 0.65(\mathrm{dd}, J=11.1,4.5 \mathrm{~Hz}, 1 \mathrm{H}), 0.47(\mathrm{dd}, J=15.6,9.2 \mathrm{~Hz}, 1 \mathrm{H}) .{ }^{13} \mathrm{C}\left\{{ }^{1} \mathrm{H},{ }^{19} \mathrm{~F}\right\}$ NMR $(151 \mathrm{MHz}$, Chloroform- $d$ ) $\delta 142.8,140.9,139.4,128.7,127.9,127.1,127.1,127.0,126.7,82.9$, 
40.8, 40.7, 36.8, 36.5, 29.4, 24.9, 24.8, 20.5, 16.4, carbon attached to boron not observed. ${ }^{19} \mathrm{~F}\left\{{ }^{1} \mathrm{H}\right\}$ NMR (282 MHz, $\left.\mathrm{CDCl}_{3}\right) \delta-63.3$ (major), -63.4 (minor). ${ }^{11} \mathrm{~B} \mathrm{NMR}\left(96 \mathrm{MHz}, \mathrm{CDCl}_{3}\right) \delta 33.7$. HRMS (ESI) $m / z=483.2669$ calcd. for $\mathrm{C}_{27} \mathrm{H}_{36} \mathrm{O}_{2} \mathrm{BF}_{3} \mathrm{Na}^{+}[\mathrm{M}+\mathrm{Na}]^{+}$, found: 483.2652 .

2-(4-(4-(Tert-butyl)phenyl)-6,6,7,7,8,8,9,9,9-nonafluoro-2-isopropylnonyl)-4,4,5,5-tetramethyl1,3,2-dioxaborolane (8ae)

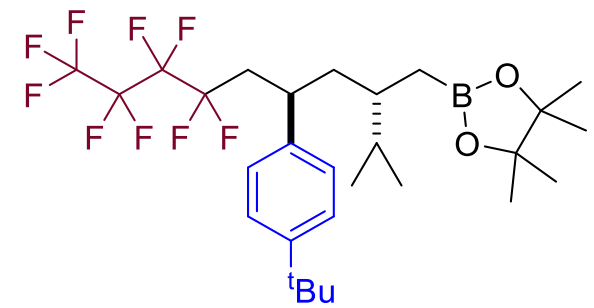

Following GP2, 4-tert-butylphenyl iodide (95\% purity, $65.7 \mathrm{mg}, 0.24 \mathrm{mmol}, 1.2$ equiv), $n$-butyl lithium (1.6 M, $0.15 \mathrm{~mL}, 0.24 \mathrm{mmol}, 1.2$ equiv), 2-(2-isopropylpent-4-en-1-yl)-4,4,5,5-tetramethyl1,3,2-dioxaborolane 6ad (47.6 mg, $0.20 \mathrm{mmol}, 1.0$ equiv) and nonafluorobutyl bromide $(89.7 \mathrm{mg}$, $0.3 \mathrm{mmol}, 1.5$ equiv) afforded the desired product 8 ae as a colorless sticky oil $(89.1 \mathrm{mg}, 76 \%$ yield, d.r. $>20: 1$ by crude GC and GC/MS analysis). $\mathrm{Rf}=0.6$ (pentane/DCM $=10: 7) .{ }^{1} \mathrm{H} \mathrm{NMR}(300 \mathrm{MHz}$, Chloroform- $d$ ) $\delta 7.25-7.19(\mathrm{~m}, 2 \mathrm{H}), 7.08-7.00(\mathrm{~m}, 2 \mathrm{H}), 3.14-2.98(\mathrm{~m}, 1 \mathrm{H}), 2.40-2.10(\mathrm{~m}, 2 \mathrm{H})$, $1.83-1.46(\mathrm{~m}, 2 \mathrm{H}), 1.36-1.25(\mathrm{~m}, 1 \mathrm{H}), 1.15(\mathrm{~s}, 6 \mathrm{H}), 1.13(\mathrm{~s}, 6 \mathrm{H}), 0.72(\mathrm{~s}, 3 \mathrm{H}), 0.70(\mathrm{~s}, 3 \mathrm{H}), 0.67$ $-0.36(\mathrm{~m}, 2 \mathrm{H}) .{ }^{13} \mathrm{C}\left\{{ }^{1} \mathrm{H},{ }^{19} \mathrm{~F}\right\}$ NMR $(126 \mathrm{MHz}$, Chloroform- $d$ ) $\delta$ 149.1, 141.1, 127.0, 125.3, 118.4, 117.4, 110.4, 108.8, 82.8, 41.3, 37.3, 36.6, 35.2, 34.4, 31.4, 29.4, 24.9, 24.7, 20.6, 16.3, carbon attached to boron not observed. ${ }^{19} \mathrm{~F}\left\{{ }^{1} \mathrm{H}\right\}$ NMR $(470 \mathrm{MHz}$, Chloroform- $d$ ) $\delta-81.1--81.2(\mathrm{~m}, 3 \mathrm{~F})$, $-112.7--112.9(\mathrm{~m}, 2 \mathrm{~F}),-124.3--124.5$ (m, 2F), -125.8 - -126.0 (m, 2F). ${ }^{11} \mathrm{~B}$ NMR (96 MHz, Chloroform- $d$ ) $\delta$ 33.7. HRMS (ESI) $m / z=613.2875$ calcd. for $\mathrm{C}_{28} \mathrm{H}_{40} \mathrm{O}_{2} \mathrm{BF}_{9} \mathrm{Na}^{+}[\mathrm{M}+\mathrm{Na}]^{+}$, found: 613.2873. FTIR (neat): $v\left(\mathrm{~cm}^{-1}\right)$ 2964, 2876, 1512, 1466, 1371, 1319, 1271, 1233, 1217, 1166, 1144 , $1133,1018,966,909,879,848,831,734,649,582$.

2-(4-(4-(Tert-butyl)phenyl)-6,6,7,7,8,8,9,9,9-nonafluoro-2-methylnonyl)-4,4,5,5-tetramethyl1,3,2-dioxaborolane (8af)<smiles>C[C@H](CB1OC(C)(C)C(C)(C)O1)CC(CC(F)(F)C(F)(F)C(F)(F)C(F)(F)F)c1ccc(C(C)(C)C)cc1</smiles>

Following GP2, 4-tert-butylphenyl iodide (95\% purity, $65.7 \mathrm{mg}, 0.24 \mathrm{mmol}, 1.2$ equiv), $n$-butyl lithium (1.6 M, $0.15 \mathrm{~mL}, 0.24 \mathrm{mmol}, 1.2$ equiv), 4,4,5,5-tetramethyl-2-(2-methylpent-4-en-1-yl)1,3,2-dioxaborolane 6af ( $42.0 \mathrm{mg}, 0.20 \mathrm{mmol}, 1.0$ equiv) and nonafluorobutyl bromide $(89.7 \mathrm{mg}$, $0.3 \mathrm{mmol}, 1.5$ equiv) afforded the desired product 8 af as a colorless sticky oil ( $66.3 \mathrm{mg}, 59 \%$ yield, the minor diastereoisomer was not detected by GC and GC/MS). $\mathrm{Rf}=0.6$ (pentane/DCM $=10: 6$ ). ${ }^{1} \mathrm{H}$ NMR $(300 \mathrm{MHz}$, Chloroform- $d$ ) $\delta 7.26-7.19(\mathrm{~m}, 2 \mathrm{H}), 7.07-6.99(\mathrm{~m}, 2 \mathrm{H}), 3.12-2.98(\mathrm{~m}, 1 \mathrm{H})$, $2.42-2.07(\mathrm{~m}, 2 \mathrm{H}), 1.80-1.64(\mathrm{~m}, 1 \mathrm{H}), 1.55-1.31(\mathrm{~m}, 2 \mathrm{H}), 1.23(\mathrm{~s}, 9 \mathrm{H}), 1.14(\mathrm{~s}, 6 \mathrm{H}), 1.13(\mathrm{~s}$, $6 \mathrm{H}), 0.85(\mathrm{~d}, J=6.3 \mathrm{~Hz}, 3 \mathrm{H}), 0.72-0.53(\mathrm{~m}, 2 \mathrm{H}) .{ }^{13} \mathrm{C}\left\{{ }^{1} \mathrm{H},{ }^{19} \mathrm{~F}\right\}$ NMR $(126 \mathrm{MHz}$, Chloroform- $d$ ) $\delta$ 149.3, 140.7, 127.0, 125.4, 118.4, 117.4, 110.4, 108.8, 82.9, 46.3, 37.9, 35.6, 34.4, 31.4, 26.5, 24.8, 
24.8, 21.4, carbon attached to boron not observed. ${ }^{19} \mathrm{~F}\left\{{ }^{1} \mathrm{H}\right\}$ NMR $(470 \mathrm{MHz}$, Chloroform- $d) \delta$ 81.1 - -81.2 (m, 3F), -112.0 - -113.6 (m, 2F), -124.3--124.8 (m, 2F), -125.7--126.1 (m, 2F). ${ }^{11} \mathrm{~B}$ NMR (96 MHz, Chloroform- $d$ ) $\delta 33.3$. HRMS (ESI) $m / z=585.2562$ calcd. for $\mathrm{C}_{26} \mathrm{H}_{36} \mathrm{O}_{2} \mathrm{BF}_{9} \mathrm{Na}^{+}$ [M+Na $]^{+}$, found: 585.2556. FTIR (neat): $v\left(\mathrm{~cm}^{-1}\right)$ 2968, 2871, 1512, 1463, 1407, 1372, 1320, 1233 , 1218, 1166, 1134, 1019, 970, 909, 881, 847, 830, 734, 582.

2-(4-(4-(Tert-butyl)phenyl)-6,6,7,7,8,8,9,9,9-nonafluoro-2,2,5-trimethylnonyl)-4,4,5,5tetramethyl-1,3,2-dioxaborolane (8ag)<smiles>CC(CC(C)(C)CB1OC(C)(C)C(C)(C)O1)C(c1ccc(Br)cc1)C(F)(F)C(F)(F)C(F)(F)C(F)(F)F</smiles>

Following GP2, 4-tert-butylphenyl iodide (95\% purity, $65.7 \mathrm{mg}, 0.24 \mathrm{mmol}, 1.2$ equiv), $n$-butyl lithium (1.6 M, $0.15 \mathrm{~mL}, 0.24 \mathrm{mmol}, 1.2$ equiv), 2-(2,2-dimethylhex-4-en-1-yl)-4,4,5,5-tetramethyl1,3,2-dioxaborolane 6ag ( $\mathrm{E} / \mathrm{Z}=3: 1,47.6 \mathrm{mg}, 0.20 \mathrm{mmol}, 1.0$ equiv) and nonafluorobutyl bromide (89.7 mg, $0.3 \mathrm{mmol}, 1.5$ equiv) afforded the desired product 8 ag as a colorless sticky oil (59.8 $\mathrm{mg}$, $50 \%$ yield, the minor diastereoisomer was not detected by GC and GC/MS). $\mathrm{Rf}=0.7$ (pentane/DCM = 10:6). ${ }^{1} \mathrm{H}$ NMR (300 MHz, Chloroform- $d$ ) $\delta 7.24-7.18(\mathrm{~m}, 2 \mathrm{H}), 7.14-7.08(\mathrm{~m}, 2 \mathrm{H}), 3.37-3.26$ (m, 1H), $2.40-2.13(\mathrm{~m}, 1 \mathrm{H}), 1.96(\mathrm{dd}, 1 \mathrm{H}), 1.64(\mathrm{~d}, J=14.2 \mathrm{~Hz}, 1 \mathrm{H}), 1.23(\mathrm{~s}, 9 \mathrm{H}), 1.16(\mathrm{~s}, 6 \mathrm{H})$, $1.15(\mathrm{~s}, 6 \mathrm{H}), 1.03(\mathrm{dd}, J=7.3,1.5 \mathrm{~Hz}, 3 \mathrm{H}), 0.78(\mathrm{~s}, 3 \mathrm{H}), 0.72-0.57(\mathrm{~m}, 5 \mathrm{H}) .{ }^{13} \mathrm{C}\left\{{ }^{1} \mathrm{H},{ }^{19} \mathrm{~F}\right\}$ NMR (151 MHz, Chloroform- $d$ ) $\delta 149.2,141.3,128.1,125.2,119.6,117.5,111.4,109.0,82.7,43.0,40.6$, 38.6, 34.3, 33.0, 31.4, 30.1, 29.3, 24.9, 24.8, 7.4, carbon attached to boron not observed. ${ }^{19} \mathrm{~F}\left\{{ }^{1} \mathrm{H}\right\}$ NMR (564 MHz, Chloroform- $d$ ) $\delta-80.9--81.0$ (m, 3F), -112.6 - -113.3 (m, 1F), -116.2 - -116.8 (m, 1F), -121.0--122.2 (m, 2F), -125.1 - -125.8 (m, 1F), -126.2 - -126.8 (m, 1F). ${ }^{11} \mathrm{~B}$ NMR (96 $\mathrm{MHz}, \mathrm{CDCl}_{3}$ ) $\delta$ 32.7. HRMS (ESI) $\mathrm{m} / \mathrm{z}=613.2875$ calcd. for $\mathrm{C}_{28} \mathrm{H}_{40} \mathrm{O}_{2} \mathrm{BF}_{9} \mathrm{Na}^{+}[\mathrm{M}+\mathrm{Na}]^{+}$, found: 613.2869. FTIR (neat): $v\left(\mathrm{~cm}^{-1}\right)$ 2966, 2873, 1469, 1354, 1323, 1235, 1217, 1167, 1134, 1017, 969, $880,845,722,601$.

2-((3-(4-(Tert-butyl)phenyl)-1-methyl-4-(perfluorobutyl)cyclopentyl)methyl)-4,4,5,5-tetramethyl1,3,2-dioxaborolane (8ah)<smiles>CC(C)(C)c1ccc([C@H]2C[C@](C)(CB3OC(C)(C)C(C)(C)O3)C[C@H]2C(F)(F)C(F)(F)C(F)(F)C(F)(F)F)cc1</smiles>

Following GP2, 4-tert-butylphenyl iodide (95\% purity, $65.7 \mathrm{mg}, 0.24 \mathrm{mmol}, 1.2$ equiv), $n$-butyl lithium (1.6 M, $0.15 \mathrm{~mL}, 0.24 \mathrm{mmol}, 1.2$ equiv), 4,4,5,5-tetramethyl-2-((1-methylcyclopent-3-en1-yl)methyl)-1,3,2-dioxaborolane 6ah (44.4 mg, $0.20 \mathrm{mmol}, 1.0$ equiv) and nonafluorobutyl bromide ( $89.7 \mathrm{mg}, 0.3 \mathrm{mmol}, 1.5$ equiv) afforded the desired product 8ah as a colorless sticky oil ( $20.6 \mathrm{mg}, 18 \%$ yield, $d . r .=45: 1$ by crude GC and GC/MS analysis). $\mathrm{Rf}=0.6$ (pentane/DCM $=$ 
10:6). ${ }^{1} \mathrm{H}$ NMR (300 MHz, Chloroform- $d$ ) $\delta 7.26-7.19(\mathrm{~m}, 2 \mathrm{H}), 7.14-7.07$ (m, 2H), $3.43-3.29$ (m, 1H), $3.17-2.90(\mathrm{~m}, 1 \mathrm{H}), 2.04-1.80(\mathrm{~m}, 2 \mathrm{H}), 1.80-1.63(\mathrm{~m}, 2 \mathrm{H}), 1.23(\mathrm{~s}, 9 \mathrm{H}), 1.19(\mathrm{~s}, 6 \mathrm{H})$, $1.18(\mathrm{~s}, 6 \mathrm{H}), 1.10(\mathrm{~s}, 3 \mathrm{H}), 0.96(\mathrm{~s}, 2 \mathrm{H}) .{ }^{13} \mathrm{C}\left\{{ }^{1} \mathrm{H},{ }^{19} \mathrm{~F}\right\} \mathrm{NMR}(126 \mathrm{MHz}$, Chloroform- $d$ ) $\delta 149.0,140.3$, 126.8, 125.2, 119.2, 117.4, 110.9, 108.8, 83.0, 51.6, 47.4, 45.1, 41.1, 39.2, 34.4, 31.4, 30.2, 24.9, 24.9, carbon attached to boron not observed. ${ }^{19} \mathrm{~F}\left\{{ }^{1} \mathrm{H}\right\}$ NMR $(470 \mathrm{MHz}$, Chloroform- $d) \delta$-81.0 - 81.1 (m, 3F), -110.7 - -111.6 (m, 1F), -121.1 - -121.8 (m, 1F), -122.1 - -122.3 (m, 2F), -125.0 - $125.8(\mathrm{~m}, 1 \mathrm{~F}),-126.4--127.2(\mathrm{~m}, 1 \mathrm{~F}) .{ }^{11} \mathrm{~B}$ NMR (96 MHz, Chloroform- $d$ ) $\delta 33.0$. HRMS (ESI) $m / z=597.2562$ calcd. for $\mathrm{C}_{27} \mathrm{H}_{36} \mathrm{O}_{2} \mathrm{BF}_{9} \mathrm{Na}^{+}[\mathrm{M}+\mathrm{Na}]^{+}$, found: 597.2560. FTIR (neat): $v\left(\mathrm{~cm}^{-1}\right) 2965$, $2874,1365,1320,1235,1165,1134,970,877,719$.

(3aS,4S,6S)-2-(4-(4-(Tert-butyl)phenyl)-6,6,6-trifluoro-2,2-dimethylhexyl)-3a,5,5trimethylhexahydro-4,6-methanobenzo[d][1,3,2]dioxaborole (8ai)<smiles>CC(C)(CB1OC2CC3CC(C2(C)C)[C@]3(C)O1)CC(CC(F)(F)F)c1ccc(C(C)(C)C)cc1</smiles>

Following GP2, 4-tert-butylphenyl iodide (95\% purity, $65.7 \mathrm{mg}, 0.24 \mathrm{mmol}, 1.25$ equiv), $n$-butyl lithium (1.6 M, $0.15 \mathrm{~mL}, 0.24 \mathrm{mmol}, 1.25$ equiv), (3aS,4S,6S)-2-(2,2-dimethylpent-4-en-1-yl)3a,5,5-trimethylhexahydro-4,6-methanobenzo[d][1,3,2]dioxaborole 6ai $(52.9 \mathrm{mg}, 0.192 \mathrm{mmol}, 1.0$ equiv) and trifluoromethyl iodide (1.0 $\mathrm{M}$ in $\mathrm{CH}_{3} \mathrm{CN}, 0.3 \mathrm{~mL}, 0.3 \mathrm{mmol}, 1.5$ equiv) afforded the desired product 8ai as a colorless sticky oil (58.3 mg, $64 \%$ yield, d.r. $=71: 29$ by GC analysis). $\mathrm{Rf}$ $=0.4$ (pentane/DCM = 3:1). ${ }^{1} \mathrm{H}$ NMR (300 MHz, Chloroform- $d$ ) $\delta 7.24-7.16(\mathrm{~m}, 2 \mathrm{H}), 7.09-6.97$ (m, 2H), $4.25-4.06(\mathrm{~m}, 1 \mathrm{H}), 3.06-2.90(\mathrm{~m}, 1 \mathrm{H}), 2.37-2.18(\mathrm{~m}, 3 \mathrm{H}), 2.17-2.04(\mathrm{~m}, 1 \mathrm{H}), 2.00$ - $1.90(\mathrm{~m}, 1 \mathrm{H}), 1.86-1.60(\mathrm{~m}, 4 \mathrm{H}), 1.27(\mathrm{~s}, 3 \mathrm{H}), 1.22(\mathrm{~s}, 9 \mathrm{H}), 1.20(\mathrm{~s}, 3 \mathrm{H}), 1.05-0.97(\mathrm{~m}, 1 \mathrm{H})$, $0.80-0.74(\mathrm{~m}, 9 \mathrm{H}), 0.74-0.64(\mathrm{~m}, 2 \mathrm{H}) .{ }^{13} \mathrm{C}\left\{{ }^{1} \mathrm{H},{ }^{19} \mathrm{~F}\right\} \mathrm{NMR}\left(126 \mathrm{MHz}, \mathrm{cdcl}_{3}\right) \delta 149.1,142.4,142.4$ (minor), 127.2, 127.2 (minor), 126.6, 125.2, 85.2, 85.2 (minor), 77.4 (minor), 77.4, 51.2, 50.1 (minor), 50.1, 42.9, 39.6, 38.1, 36.0 (minor), 36.0, 35.6 (minor), 35.6 (minor), 34.3, 33.0, 33.0 (minor), 31.4, 30.2 (minor), 30.2, 29.7, 29.6 (minor), 28.8 (minor), 28.7, 27.1, 26.6 (minor), 26.6, 24.0, carbon attached to boron not observed. ${ }^{19} \mathrm{~F}\left\{{ }^{1} \mathrm{H}\right\} \mathrm{NMR}\left(282 \mathrm{MHz}, \mathrm{CDCl}_{3}\right) \delta-63.6$ (minor), 63.6 (major). ${ }^{11} \mathrm{~B}$ NMR $\left(96 \mathrm{MHz}, \mathrm{CDCl}_{3}\right) \delta 32.0$. HRMS (ESI) $\mathrm{m} / z=501.3127$ calcd. for $\mathrm{C}_{28} \mathrm{H}_{42} \mathrm{O}_{2} \mathrm{BF}_{3} \mathrm{Na}^{+}[\mathrm{M}+\mathrm{Na}]^{+}$, found: 501.3123. FTIR (neat): $v\left(\mathrm{~cm}^{-1}\right)$ 2958, 2873, 1473, 1367, 1347, 1269, 1248, 1131, 1078, 1030, 992, 907, 828, 731, 649, 598.

2-(4-(4-(Tert-butyl)phenyl)-6,6,7,7,8,8,9,9,10,10,11,11,11-tridecafluoroundecyl)-4,4,5,5tetramethyl-1,3,2-dioxaborolane (8ak)<smiles>CC(C)(C)c1ccc(C(CCCB2OC(C)(C)C(C)(C)O2)CC(F)(F)C(F)(F)C(F)(F)C(F)(F)C(F)(F)C(F)(F)F)cc1</smiles>

Following GP2, three reactions were set up with 4-tert-butylphenyl iodide (95\% purity, $65.7 \mathrm{mg} \times 3$, 0.24 mmol $\times 3,1.2$ equiv), $n$-butyl lithium (1.6 M, $0.15 \mathrm{~mL} \times 3,0.24 \mathrm{mmol} \times 3,1.2$ equiv), 4,4,5,5- 
tetramethyl-2-(pent-4-en-1-yl)-1,3,2-dioxaborolane 6ak (39.2 $\mathrm{mg} \times 3,0.20 \mathrm{mmol} \times 3,1.0$ equiv) and pentafluoropropyl iodide $(88.8 \mathrm{mg} \times 3,0.3 \mathrm{mmol} \times 3,1.5$ equiv). Silica gel chromatography afforded the desired product 8ak as a colorless sticky oil ( $11.9 \mathrm{mg}, 3 \%$ yield). $\mathrm{Rf}=0.6$ (pentane/DCM $=5: 3$ ). ${ }^{1} \mathrm{H}$ NMR $(300 \mathrm{MHz}$, Chloroform- $d$ ) $\delta 7.27-7.20(\mathrm{~m}, 2 \mathrm{H}), 7.06-6.99(\mathrm{~m}, 2 \mathrm{H}), 3.02-2.86(\mathrm{~m}, 1 \mathrm{H})$, $2.39-2.18(\mathrm{~m}, 2 \mathrm{H}), 1.77-1.55(\mathrm{~m}, 2 \mathrm{H}), 1.24(\mathrm{~s}, 9 \mathrm{H}), 1.20-1.18(\mathrm{~m}, 2 \mathrm{H}), 1.15(\mathrm{~s}, 12 \mathrm{H}), 0.66(\mathrm{t}$, $J=7.6 \mathrm{~Hz}, 2 \mathrm{H}) .{ }^{13} \mathrm{C}\left\{{ }^{1} \mathrm{H},{ }^{19} \mathrm{~F}\right\}$ NMR $(151 \mathrm{MHz}$, Chloroform- $d$ ) $\delta 149.3,140.9,126.9,125.4,118.5$, 117.2, 111.1, 99.4, 82.9, 39.5, 37.7, 37.2, 34.4, 31.4, 24.8, 24.8, 21.5, carbon attached to boron not observed. ${ }^{19} \mathrm{~F}$ NMR $(564 \mathrm{MHz}$, Chloroform- $d$ ) $\delta-80.78--80.88(\mathrm{~m}),-111.0--114.6(\mathrm{~m}, 3 \mathrm{~F}),-$ 121.5 - -122.1 (m, 2F), -122.7 - -123.1 (m, 2F), -123.4--123.8 (m, 2F), -125.9--126.3 (m, 2F). ${ }^{11} \mathrm{~B}$ NMR (192 MHz, Chloroform- $d$ ) $\delta$ 33.6. HRMS (ESI) $\mathrm{m} / \mathrm{z}=671.2341$ calcd. for $\mathrm{C}_{27} \mathrm{H}_{34} \mathrm{O}_{2} \mathrm{BF}_{13} \mathrm{Na}^{+}[\mathrm{M}+\mathrm{Na}]^{+}$, found: 671.2340. FTIR (neat): $v\left(\mathrm{~cm}^{-1}\right)$

2-(3-(4-(Tert-butyl)phenyl)-5,5,6,6,7,7,8,8,8-nonafluorooctyl)-4,4,5,5-tetramethyl-1,3,2dioxaborolane (8al)

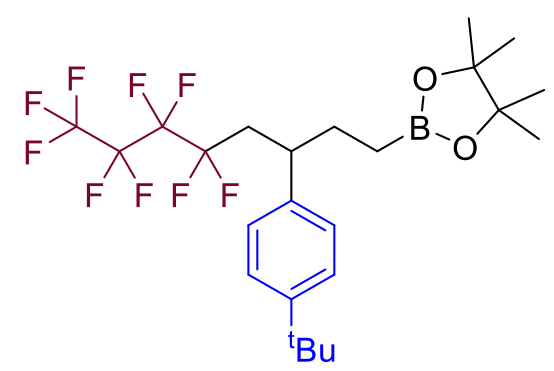

Following GP2, 4-tert-butylphenyl iodide (95\% purity, $65.7 \mathrm{mg}, 0.24 \mathrm{mmol}, 1.2$ equiv), $n$-butyl lithium (1.6 M, $0.15 \mathrm{~mL}, 0.24 \mathrm{mmol}, 1.2$ equiv), 2-(but-3-en-1-yl)-4,4,5,5-tetramethyl-1,3,2dioxaborolane 6al $(39.2 \mathrm{mg}, 0.20 \mathrm{mmol}, 1.0$ equiv) and pentafluoropropyl iodide ( $88.8 \mathrm{mg}, 0.3$ mmol, 1.5 equiv) afforded the desired product 8 al as a colorless sticky oil (13.4 mg, 13\% yield). Rf $=0.6($ pentane $/ \mathrm{DCM}=5: 3) .{ }^{1} \mathrm{H}$ NMR $(300 \mathrm{MHz}$, Chloroform- $d$ ) $\delta 7.25-7.20(\mathrm{~m}, 2 \mathrm{H}), 7.04-6.97$ (m, 2H), $2.97-2.81(\mathrm{~m}, 1 \mathrm{H}), 2.44-2.19(\mathrm{~m}, 2 \mathrm{H}), 1.89-1.74(\mathrm{~m}, 1 \mathrm{H}), 1.71-1.55(\mathrm{~m}, 1 \mathrm{H}), 1.23$ (s, 9H), $1.14(\mathrm{~s}, 12 \mathrm{H}), 0.54(\mathrm{dd}, J=9.0,7.3 \mathrm{~Hz}, 2 \mathrm{H}) .{ }^{13} \mathrm{C}\left\{{ }^{1} \mathrm{H},{ }^{19} \mathrm{~F}\right\} \mathrm{NMR}\left(151 \mathrm{MHz}, \mathrm{cdcl}_{3}\right) \delta 149.3$, 140.5, 127.0, 125.3, 118.3, 117.4, 110.4, 108.8, 83.0, 39.9, 36.6, 34.4, 31.6, 31.4, 24.8, 24.8, carbon attached to boron not observed. ${ }^{19} \mathrm{~F}$ NMR $(564 \mathrm{MHz}$, Chloroform- $d$ ) $\delta-81.1--81.2(\mathrm{~m}, 3 \mathrm{~F}),-112.9$ - -113.2 (m, 2F), -124.4 - -124.6 (m, 2F), -125.9 - -126.0 (m, 2F). ${ }^{11} \mathrm{~B}$ NMR (96 MHz, $\left.\mathrm{CDCl}_{3}\right) \delta$ 33.2. HRMS (ESI) $m / z=557.2248$ calcd. for $\mathrm{C}_{24} \mathrm{H}_{32} \mathrm{O}_{2} \mathrm{BF}_{9} \mathrm{Na}^{+}[\mathrm{M}+\mathrm{Na}]^{+}$, found: 557.2248. FTIR (neat): $v\left(\mathrm{~cm}^{-1}\right)$ 2969, 2872, 1511, 1408, 1372, 1321, 1233, 1217, 1166, 1144, 1132, 1018, 969, 929 , $879,847,830,734,721,581,574$.

4,4,5,5-Tetramethyl-2-(6,6,7,7,8,8,9,9,10,10,11,11,11-tridecafluoro-2,2-dimethyl-4phenylundecyl)-1,3,2-dioxaborolane (8an)

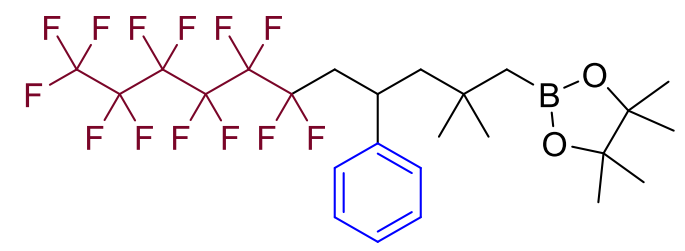

Following GP1, phenyl lithium (1.9 M in dibutyl ether, $0.11 \mathrm{~mL}, 022 \mathrm{mmol}, 1.1$ equiv), 2,2dimethylpent-4-en-1-ylboronic acid pinacol ester 6a (44.8 mg, $0.20 \mathrm{mmol}, 1.0$ equiv) and 
perfluorohexyl bromide (119.7 $\mathrm{mg}, 0.3 \mathrm{mmol}, 1.5$ equiv) afforded the desired product 8an as a colorless sticky oil $(84.7 \mathrm{mg}, 68 \%$ yield). $\mathrm{Rf}=0.3$ (pentane/DCM $=3: 1) .{ }^{1} \mathrm{H}$ NMR $(300 \mathrm{MHz}$, Chloroform- $d$ ) $\delta 7.25-7.07(\mathrm{~m}, 5 \mathrm{H}), 3.25-3.06(\mathrm{~m}, 1 \mathrm{H}), 2.47-2.12(\mathrm{~m}, 2 \mathrm{H}), 1.87-1.66(\mathrm{~m}, 2 \mathrm{H})$, $1.14(\mathrm{~s}, 12 \mathrm{H}), 0.78-0.58(\mathrm{~m}, 8 \mathrm{H}) .{ }^{13} \mathrm{C}\left\{{ }^{1} \mathrm{H},{ }^{19} \mathrm{~F}\right\}$ NMR (126 MHz, Chloroform- $d$ ) $\delta 145.9,128.5$, 127.7, 126.4, 118.4, 117.2, 111.1, 110.8, 110.2, 108.5, 82.8, 50.6, 39.4, 35.2, 33.2, 30.3, 29.8, 24.8, 24.8, carbon attached to boron not observed. ${ }^{19} \mathrm{~F}\left\{{ }^{1} \mathrm{H}\right\}$ NMR $(470 \mathrm{MHz}$, Chloroform- $d) \delta-80.9--$ 81.0 (m, 3F), -111.7 - -114.2 (m, 2F), -121.7 - -122.1 (m, 2F), -122.6 - -123.2 (m, 2F), -123.5 - $124.0(\mathrm{~m}, 2 \mathrm{~F}),-126.2--126.3$ (m, 2F). ${ }^{11} \mathrm{~B}$ NMR (96 MHz, Chloroform- $d$ ) $\delta 32.8$. HRMS (ESI) $m / z=597.2562$ calcd. for $\mathrm{C}_{27} \mathrm{H}_{36} \mathrm{O}_{2} \mathrm{BF}_{9} \mathrm{Na}^{+}[\mathrm{M}+\mathrm{Na}]^{+}$, found: 597.2560. FTIR (neat): $v$ ( $\mathrm{cm}^{-1}$ ) 2969, 2923, 2855, 1516, 1463, 1367, 1319, 1268, 1247, 1127, 1097, 969, 847, 827, 607, 559.

2-(4-(4-(Tert-butyl)phenyl)-6,6,7,7,7-pentafluoro-2,2-dimethylheptyl)-4,4,5,5-tetramethyl-1,3,2dioxaborolane (8ao)<smiles>CC(C)(C)CC(CC(F)(F)C(F)(F)F)c1ccc(C(C)(C)C)cc1</smiles>

Following GP2, 4-tert-butylphenyl iodide (95\% purity, $65.7 \mathrm{mg}, 0.24 \mathrm{mmol}, 1.2$ equiv), $n$-butyl lithium (1.6 M, $0.15 \mathrm{~mL}, 0.24 \mathrm{mmol}, 1.2$ equiv), 2,2-dimethylpent-4-en-1-ylboronic acid pinacol ester $6 \mathbf{a}$ (44.8 mg, $0.20 \mathrm{mmol}, 1.0$ equiv) and pentafluoroethyl iodide (1.0 $\mathrm{M}$ in $\mathrm{CH} 3 \mathrm{CN}, 0.3 \mathrm{~mL}$, $0.3 \mathrm{mmol}, 1.5$ equiv) afforded the desired product $8 \mathbf{a o}$ as a colorless sticky oil (70.4 $\mathrm{mg}, 74 \%$ yield). $\mathrm{Rf}=0.5$ (pentane/DCM = 5:3). ${ }^{1} \mathrm{H}$ NMR $(300 \mathrm{MHz}$, Chloroform- $d$ ) $\delta 7.23-7.16(\mathrm{~m}, 2 \mathrm{H}), 7.09-$ $7.01(\mathrm{~m}, 2 \mathrm{H}), 3.17-3.03(\mathrm{~m}, 1 \mathrm{H}), 2.39-2.05(\mathrm{~m}, 2 \mathrm{H}), 1.81(\mathrm{dd}, J=14.0,8.6 \mathrm{~Hz}, 1 \mathrm{H}), 1.68(\mathrm{dd}, J$ $=14.0,3.7 \mathrm{~Hz}, 1 \mathrm{H}), 1.22(\mathrm{~s}, 9 \mathrm{H}), 1.14(\mathrm{~s}, 12 \mathrm{H}), 0.76(\mathrm{~s}, 3 \mathrm{H}), 0.74(\mathrm{~s}, 3 \mathrm{H}), 0.71-0.59(\mathrm{~m}, 2 \mathrm{H})$. ${ }^{13} \mathrm{C}\left\{{ }^{1} \mathrm{H},{ }^{19} \mathrm{~F}\right\}$ NMR $(151 \mathrm{MHz}$, Chloroform- $d$ ) $\delta 149.1,142.8,127.2,125.3,119.1,115.7,82.8,50.5$, $39.2,34.6,34.3,33.2,31.4,30.3,29.7,24.8,24.8$, carbon attached to boron not observed. ${ }^{19} \mathrm{~F}$ NMR $(564 \mathrm{MHz}$, Chloroform- $d$ ) $\delta-86.0$ (s, 3F), $-116.4(\mathrm{~d}, J=265.0 \mathrm{~Hz}, 1 \mathrm{~F}),-117.6$ (d, $J=265.0 \mathrm{~Hz}, 1 \mathrm{~F})$. ${ }^{11} \mathrm{~B}$ NMR $\left(96 \mathrm{MHz}, \mathrm{CDCl}_{3}\right) \delta 32.9$. HRMS (ESI) $\mathrm{m} / z=499.2782$ calcd. for $\mathrm{C}_{25} \mathrm{H}_{38} \mathrm{O}_{2} \mathrm{BF}_{5} \mathrm{Na}^{+}$ [M+Na $]^{+}$, found: 499.2777. FTIR (neat): $v\left(\mathrm{~cm}^{-1}\right)$ 2963, 2875, 1512, 1469, 1362, 1320, 1194, 1141, 1112, 1094, 1058, 1018, 971, 847, 830, 721, 610.

2-(4-(4-(Tert-butyl)phenyl)-6,6,7,7,8,8,8-heptafluoro-2,2-dimethyloctyl)-4,4,5,5-tetramethyl1,3,2-dioxaborolane (8ap)<smiles>CC(C)(C)CC(CC(F)(F)C(F)(F)C(F)(F)F)c1ccc(Br)cc1</smiles>

Following GP2, 4-tert-butylphenyl iodide (95\% purity, $65.7 \mathrm{mg}, 0.24 \mathrm{mmol}, 1.2$ equiv), $n$-butyl lithium (1.6 M, $0.15 \mathrm{~mL}, 0.24 \mathrm{mmol}, 1.2$ equiv), 2,2-dimethylpent-4-en-1-ylboronic acid pinacol ester $6 \mathbf{a}$ (44.8 mg, $0.20 \mathrm{mmol}, 1.0$ equiv) and heptafluoropropyl iodide ( $88.8 \mathrm{mg}, 0.3 \mathrm{mmol}, 1.5$ 
equiv) afforded the desired product $8 \mathrm{ap}$ as a colorless sticky oil (67.6 $\mathrm{mg}, 64 \%$ yield). $\mathrm{Rf}=0.6$ (pentane/DCM = 2:1). ${ }^{1} \mathrm{H}$ NMR (300 MHz, Chloroform- $d$ ) $\delta 7.23-7.16(\mathrm{~m}, 2 \mathrm{H}), 7.09-7.02(\mathrm{~m}$, $2 \mathrm{H}), 3.20-3.03(\mathrm{~m}, 1 \mathrm{H}), 2.45-2.07(\mathrm{~m}, 2 \mathrm{H}), 1.87-1.63(\mathrm{~m}, 2 \mathrm{H}), 1.22(\mathrm{~s}, 9 \mathrm{H}), 1.14(\mathrm{~s}, 12 \mathrm{H}), 0.76$ (s, 3H), $0.74(\mathrm{~s}, 3 \mathrm{H}), 0.72-0.59(\mathrm{~m}, 2 \mathrm{H}) .{ }^{13} \mathrm{C}\left\{{ }^{1} \mathrm{H},{ }^{19} \mathrm{~F}\right\}$ NMR $\left(126 \mathrm{MHz}, \mathrm{cdcl}_{3}\right) \delta 149.1,142.8$, 127.1, 125.3, 117.9, 117.7, 108.7, 82.8, 50.6, 39.0, 34.5, 34.3, 33.2, 31.4, 30.3, 29.7, 24.8, 24.8, carbon attached to boron not observed. ${ }^{19} \mathrm{~F}\left\{{ }^{1} \mathrm{H}\right\}$ NMR $(470 \mathrm{MHz}$, Chloroform- $d) \delta-80.4(\mathrm{t}, J=9.8$ $\mathrm{Hz}, 3 \mathrm{~F}),-112.8--115.0$ (m, 2F), -127.9 (dd, $J=13.3,4.6 \mathrm{~Hz}, 2 \mathrm{~F}) .{ }^{11} \mathrm{~B} \mathrm{NMR}\left(96 \mathrm{MHz}, \mathrm{CDCl}_{3}\right) \delta$ 33.0. HRMS (ESI) $m / z=549.2750$ calcd. for $\mathrm{C}_{26} \mathrm{H}_{38} \mathrm{O}_{2} \mathrm{BF}_{7} \mathrm{Na}^{+}[\mathrm{M}+\mathrm{Na}]^{+}$, found: 549.2748. FTIR (neat): $v\left(\mathrm{~cm}^{-1}\right)$ 2964, 2916, 2870, 1353, 1320, 1221, 1168, 1140, 1115, 968, 952, 910, 847, 830, $728,586$.

2-(4-(4-(Tert-butyl)phenyl)-6,6,7,7,8,8,9,9,10,10,11,11,12,12,13,13,13-heptadecafluoro-2,2dimethyltridecyl)-4,4,5,5-tetramethyl-1,3,2-dioxaborolane (8aq)

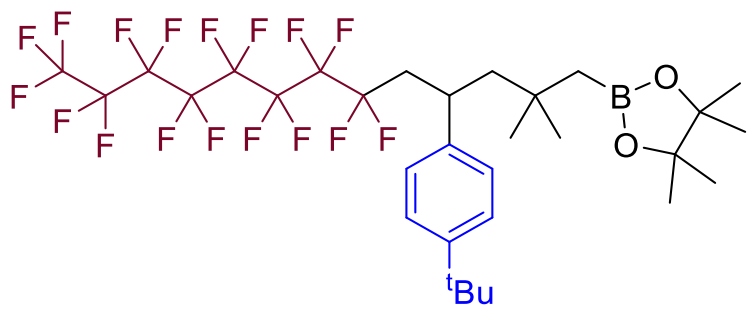

Following GP2, 4-tert-butylphenyl iodide (95\% purity, $65.7 \mathrm{mg}, 0.24 \mathrm{mmol}, 1.2$ equiv), $n$-butyl lithium (1.6 M, $0.15 \mathrm{~mL}, 0.24 \mathrm{mmol}, 1.2$ equiv), 2,2-dimethylpent-4-en-1-ylboronic acid pinacol ester 6a (44.8 mg, $0.20 \mathrm{mmol}, 1.0$ equiv) and perfluorooctyl bromide (149.7 mg, $0.3 \mathrm{mmol}, 1.5$ equiv) afforded the desired product $\mathbf{8 a q}$ as a colorless sticky oil (116.8 $\mathrm{mg}, 75 \%$ yield). $\mathrm{Rf}=0.6$ (pentane/DCM = 5:3). ${ }^{1} \mathrm{H}$ NMR $(300 \mathrm{MHz}$, Chloroform- $d$ ) $\delta 7.23-7.16(\mathrm{~m}, 2 \mathrm{H}), 7.10-7.01(\mathrm{~m}$, $2 \mathrm{H}), 3.20-3.06(\mathrm{~m}, 1 \mathrm{H}), 2.45-2.09(\mathrm{~m}, 2 \mathrm{H}), 1.87-1.62(\mathrm{~m}, 2 \mathrm{H}), 1.21(\mathrm{~s}, 9 \mathrm{H}), 1.13(\mathrm{~s}, 12 \mathrm{H}), 0.76$ (s, 3H), 0.74 (s, 3H), $0.73-0.59(\mathrm{~m}, 2 \mathrm{H}) .{ }^{13} \mathrm{C}\left\{{ }^{1} \mathrm{H},{ }^{19} \mathrm{~F}\right\}$ NMR (126 MHz, Chloroform- $d$ ) $\delta 149.1$, 142.9, 127.2, 125.3, 118.5, 117.2, 111.2, 111.0, 110.8, 110.8, 110.3, 108.4, 82.8, 50.6, 39.4, 34.6, $34.3,33.2,31.4,30.3,29.7,24.8,24.8$, carbon attached to boron not observed. ${ }^{19} \mathrm{~F}\left\{{ }^{1} \mathrm{H}\right\} \mathrm{NMR}(470$ MHz, Chloroform- $d$ ) $\delta$-80.9 - -81.0 (m, 3F), -111.9 - -113.9 (m, 2F), -121.5 - -121.8 (m, 2F), 121.9 - -122.2 (m, 4F), -122.7 - -123.0 (m, 2F), -123.6--123.9 (m, 2F), -126.1 - -126.4 (m, 2F). ${ }^{11} \mathrm{~B}$ NMR (96 MHz, Chloroform- $d$ ) $\delta$ 33.0. HRMS (ESI) $\mathrm{m} / \mathrm{z}=799.2591$ calcd. for $\mathrm{C}_{31} \mathrm{H}_{38} \mathrm{O}_{2} \mathrm{BF}_{17} \mathrm{Na}^{+}[\mathrm{M}+\mathrm{Na}]^{+}$, found: 799.2592. FTIR (neat): $v\left(\mathrm{~cm}^{-1}\right)$ 2963, 2883, 1512, 1470, 1362, 1322, 1240, 1206, 1144, 1019, 969, 908, 880, 847, 829, 732, 705, 650, 559.

Ethyl 4-(4-(tert-butyl)phenyl)-6,6-dimethyl-7-(4,4,5,5-tetramethyl-1,3,2-dioxaborolan-2yl)heptanoate (8ar)<smiles>CCOC(=O)CCC(CC(C)(C)CB1OC(C)(C)C(C)(C)O1)c1ccc(Br)cc1</smiles>

Following GP2, 4-tert-butylphenyl iodide (95\% purity, $65.7 \mathrm{mg}, 0.24 \mathrm{mmol}, 1.2$ equiv), $n$-butyl lithium (1.6 M, $0.15 \mathrm{~mL}, 0.24 \mathrm{mmol}, 1.2$ equiv), 2,2-dimethylpent-4-en-1-ylboronic acid pinacol 
ester 6a (44.8 mg, $0.20 \mathrm{mmol}, 1.0$ equiv) and ethyl bromoacetate (50.1 mg, $0.3 \mathrm{mmol}, 1.5$ equiv) afforded the desired product 8ar as a colorless sticky oil (16.5 mg, 19\% yield). Rf $=0.4$ (pentane/Et $2 \mathrm{O}=5: 1) .{ }^{1} \mathrm{H}$ NMR $(300 \mathrm{MHz}$, Chloroform- $d$ ) $\delta 7.19-7.14(\mathrm{~m}, 2 \mathrm{H}), 7.02-6.95(\mathrm{~m}$, $2 \mathrm{H}), 4.03-3.93(\mathrm{~m}, 2 \mathrm{H}), 2.60-2.46(\mathrm{~m}, 1 \mathrm{H}), 2.12-1.48(\mathrm{~m}, 6 \mathrm{H}), 1.22(\mathrm{~s}, 9 \mathrm{H}), 1.17-1.11(\mathrm{~m}$, 15H), 0.77 (s, 3H), 0.76 (s, 3H), $0.73-0.61(\mathrm{~m}, 2 \mathrm{H}) .{ }^{13} \mathrm{C} \mathrm{NMR}(126 \mathrm{MHz}$, Chloroform- $d$ ) $\delta$ 173.9, 148.5, 143.4, 127.5, 125.0, 82.7, 60.1, 50.8, 41.4, 34.6, 34.3, 33.1, 32.7, 31.4, 30.2, 29.8, 24.9, 24.8, 14.2, carbon attached to boron not observed. ${ }^{11} \mathrm{~B}$ NMR ( $96 \mathrm{MHz}$, Chloroform- $d$ ) $\delta$ 32.4. HRMS (ESI) $\mathrm{m} / z=467.3308$ calcd. for $\mathrm{C}_{27} \mathrm{H}_{45} \mathrm{O}_{4} \mathrm{BNa}^{+}[\mathrm{M}+\mathrm{Na}]^{+}$, found: 467.3312. FTIR (neat): $v\left(\mathrm{~cm}^{-1}\right)$ 2956, 2903, 2869, 1735, 1511, 1467, 1357, 1318, 1271, 1249, 1213, 1142, 1111, 1038, 971, 847, $830,588$.

Ethyl 4-(4-(tert-butyl)phenyl)-2,2-difluoro-6,6-dimethyl-7-(4,4,5,5-tetramethyl-1,3,2dioxaborolan-2-yl)heptanoate (8as)<smiles>CCOC(=O)C(F)(F)CC(CC(C)(C)CB1OC(C)(C)C(C)(C)O1)c1ccc(C(C)(C)C)cc1</smiles>

Following GP2, 4-tert-butylphenyl iodide ( $95 \%$ purity, $65.7 \mathrm{mg}, 0.24 \mathrm{mmol}, 1.2$ equiv), $n$-butyl lithium (1.6 M, $0.15 \mathrm{~mL}, 0.24 \mathrm{mmol}, 1.2$ equiv), 2,2-dimethylpent-4-en-1-ylboronic acid pinacol ester 6a (44.8 mg, $0.20 \mathrm{mmol}, 1.0$ equiv) and ethyl bromodifluoroacetate $(60.9 \mathrm{mg}, 0.3 \mathrm{mmol}, 1.5$ equiv) afforded the desired product 8 as as a colorless sticky oil (13.0 mg, 14\% yield). $\mathrm{Rf}=0.5$ (pentane/Et $2 \mathrm{O}=5: 1) .{ }^{1} \mathrm{H}$ NMR (300 MHz, Chloroform- $d$ ) $\delta 7.19-7.14(\mathrm{~m}, 2 \mathrm{H}), 7.06-6.98(\mathrm{~m}$, $2 \mathrm{H}), 3.81-3.55(\mathrm{~m}, 2 \mathrm{H}), 2.98-2.84(\mathrm{~m}, 1 \mathrm{H}), 2.55-2.18(\mathrm{~m}, 2 \mathrm{H}), 1.79-1.54(\mathrm{~m}, 2 \mathrm{H}), 1.20(\mathrm{~s}$, 9H), $1.14(\mathrm{~s}, 12 \mathrm{H}), 1.06(\mathrm{t}, J=7.2 \mathrm{~Hz}, 3 \mathrm{H}), 0.76(\mathrm{~s}, 3 \mathrm{H}), 0.75(\mathrm{~s}, 3 \mathrm{H}), 0.72-0.58(\mathrm{~m}, 2 \mathrm{H}) .{ }^{13} \mathrm{C}\left\{{ }^{1} \mathrm{H}\right.$, ${ }^{19} \mathrm{~F}$ \} NMR (126 MHz, Chloroform- $d$ ) $\delta$ 163.9, 149.0, 141.7, 127.9, 125.0, 115.8, 82.7, 62.3, 51.3, 43.5, 35.7, 34.3, 33.2, 31.4, 30.1, 29.7, 24.9, 24.8, 13.7, carbon attached to boron not observed. ${ }^{19} \mathrm{~F}\left\{{ }^{1} \mathrm{H}\right\}$ NMR $(470 \mathrm{MHz}$, Chloroform- $d$ ) $\delta-98.5(\mathrm{~d}, J=260.6 \mathrm{~Hz}, 1 \mathrm{~F}),-107.8(\mathrm{~d}, J=260.6 \mathrm{~Hz}, 1 \mathrm{~F})$. ${ }^{11} \mathrm{~B}$ NMR (96 MHz, Chloroform- $d$ ) $\delta$ 32.4. HRMS (ESI) $\mathrm{m} / z=503.3120$ calcd. for $\mathrm{C}_{27} \mathrm{H}_{43} \mathrm{O}_{4} \mathrm{BF}_{2} \mathrm{Na}^{+}$ [M+Na] $]^{+}$, found: 503.3114. FTIR (neat): $v\left(\mathrm{~cm}^{-1}\right)$ 2967, 2901, 1771, 1469, 1362, 1322, 1271, 1218, 1142, 1112, 1087, 1024, 970, 847, 830.

6-(4-(Tert-butyl)phenyl)-2,2,8,8-tetramethyl-9-(4,4,5,5-tetramethyl-1,3,2-dioxaborolan-2yl)nonan-3-one (8at)<smiles>CC(C)(C)CC(=O)CCC(CC(C)(C)CB1OC(C)(C)C(C)(C)O1)c1ccc(C(C)(C)C)cc1</smiles>

Following GP2, 4-tert-butylphenyl iodide (95\% purity, $65.7 \mathrm{mg}, 0.24 \mathrm{mmol}, 1.2$ equiv), $n$-butyl lithium (1.6 M, $0.15 \mathrm{~mL}, 0.24 \mathrm{mmol}, 1.2$ equiv), 2,2-dimethylpent-4-en-1-ylboronic acid pinacol ester $6 \mathbf{a}$ (44.8 mg, $0.20 \mathrm{mmol}, 1.0$ equiv) and 1-bromo-3,3-dimethylbutan-2-one (53.7 mg, $0.3 \mathrm{mmol}$, 
1.5 equiv) afforded the desired product 8at as a colorless sticky oil ( $20.9 \mathrm{mg}, 23 \%$ yield). $\mathrm{Rf}=0.5$ (pentane/Et $2 \mathrm{O}=5: 1) .{ }^{1} \mathrm{H}$ NMR $(300 \mathrm{MHz}$, Chloroform- $d$ ) $\delta 7.19-7.13(\mathrm{~m}, 2 \mathrm{H}), 7.00-6.94(\mathrm{~m}$, $2 \mathrm{H}), 2.57-2.46(\mathrm{~m}, 1 \mathrm{H}), 2.31-2.01(\mathrm{~m}, 2 \mathrm{H}), 1.88-1.47(\mathrm{~m}, 4 \mathrm{H}), 1.21(\mathrm{~s}, 9 \mathrm{H}), 1.13(\mathrm{~s}, 12 \mathrm{H}), 0.94$ (s, 9H), 0.78 (s, 3H), 0.76 (s, 3H), $0.73-0.60(\mathrm{~m}, 2 \mathrm{H}) .{ }^{13} \mathrm{C}$ NMR (126 MHz, Chloroform- $d$ ) $\delta 216.1$, 148.4, 143.9, 127.5, 125.0, 82.6, 51.3, 44.0, 41.2, 34.6, 34.3, 33.5, 33.1, 31.4, 30.2, 29.8, 26.4, 24.9, 24.8, 24.8, carbon attached to boron not observed. ${ }^{11} \mathrm{~B}$ NMR $(96 \mathrm{MHz}$, Chloroform- $d$ ) $\delta 32.4$. HRMS (ESI) $m / z=479.3672$ calcd. for $\mathrm{C}_{29} \mathrm{H}_{43} \mathrm{O}_{3} \mathrm{BNa}^{+}[\mathrm{M}+\mathrm{Na}]^{+}$, found: 479.3666. FTIR (neat): $v$ $\left(\mathrm{cm}^{-1}\right)$ 2961, 2906, 2871, 1706, 1476, 1466, 1361, 1320, 1270, 1214, 1143, 1111, 1084, 971, 847, $831,571$.

Diethyl (1,1-difluoro-5,5-dimethyl-3-phenyl-6-(4,4,5,5-tetramethyl-1,3,2-dioxaborolan-2yl)hexyl)phosphonate (8au)<smiles>CCOP(=O)(OCC)C(F)(F)CC(CC(C)(C)CB1OC(C)(C)C(C)(C)O1)c1ccccc1</smiles>

Following GP1, phenyl lithium (1.9 M in dibutyl ether, $0.13 \mathrm{~mL}, 0.24 \mathrm{mmol}, 1.2$ equiv), 2,2dimethylpent-4-en-1-ylboronic acid pinacol ester $\mathbf{6 a}(44.8 \mathrm{mg}, 0.20 \mathrm{mmol}, 1.0$ equiv) and diethyl (bromodifluoromethyl)phosphonate ( $80.1 \mathrm{mg}, 0.3 \mathrm{mmol}, 1.5$ equiv) afforded the desired product 8au as a colorless sticky oil $(42.1 \mathrm{mg}, 43 \%$ yield). $\mathrm{Rf}=0.3$ (pentane/Et $2 \mathrm{O}=1: 3) .{ }^{1} \mathrm{H}$ NMR (300 MHz, Chloroform- $d$ ) $\delta 7.22-7.03(\mathrm{~m}, 5 \mathrm{H}), 4.21-4.04(\mathrm{~m}, 4 \mathrm{H}), 3.28-3.13(\mathrm{~m}, 1 \mathrm{H}), 2.45-2.15$ (m, 2H), $1.89-1.63(\mathrm{~m}, 2 \mathrm{H}), 1.31-1.22(\mathrm{~m}, 6 \mathrm{H}), 1.14(\mathrm{~s}, 12 \mathrm{H}), 0.78(\mathrm{~s}, 3 \mathrm{H}), 0.73(\mathrm{~s}, 3 \mathrm{H}), 0.70-$ $0.59(\mathrm{~m}, 2 \mathrm{H}) .{ }^{13} \mathrm{C}\left\{{ }^{1} \mathrm{H},{ }^{19} \mathrm{~F}\right\}$ NMR (126 MHz, Chloroform- $d$ ) $\delta 146.8,128.2,127.7,125.9,120.7(\mathrm{~d}$, $J=215.1 \mathrm{~Hz}), 82.7,64.3(\mathrm{~d}, J=3.5 \mathrm{~Hz}), 64.3(\mathrm{~d}, J=3.5 \mathrm{~Hz}), 51.0,42.4(\mathrm{~d}, J=13.6 \mathrm{~Hz}), 35.2(\mathrm{~d}$, $J=6.9 \mathrm{~Hz}), 33.2,30.0(\mathrm{~d}, J=2.4 \mathrm{~Hz}), 29.6,24.8,16.3,16.3$, carbon attached to boron not observed. ${ }^{19} \mathrm{~F}\left\{{ }^{1} \mathrm{H}\right\}$ NMR (470 MHz, Chloroform- $d$ ) $\delta-110.5(\mathrm{dd}, J=296.6,108.7 \mathrm{~Hz}, 1 \mathrm{~F}),-111.9$ (dd, $J=$ 296.6, 109.2 Hz, 1F). ${ }^{31} \mathrm{P}$ NMR (122 MHz, Chloroform- $d$ ) $\delta 7.2(\mathrm{t}, J=108.9 \mathrm{~Hz}) .{ }^{11} \mathrm{~B}$ NMR $(96$ $\mathrm{MHz}, \mathrm{CDCl}_{3}$ ) $\delta 32.6$. HRMS (ESI) $\mathrm{m} / z=511.2567$ calcd. for $\mathrm{C}_{24} \mathrm{H}_{40} \mathrm{O}_{5} \mathrm{PBF}_{2} \mathrm{Na}^{+}[\mathrm{M}+\mathrm{Na}]^{+}$, found: 511.2567. FTIR (neat): $v\left(\mathrm{~cm}^{-1}\right)$ 2979, 2932, 2914, 1358, 1321, 1272, 1164, 1141, 1018, 971, 846, $796,763,701$.

\section{Follow-up Chemistry}

4-([1,1'-biphenyl]-4-yl)-6,6,6-trifluoro-2-isopropylhexyl 6-bromo-2-naphthoate (11)

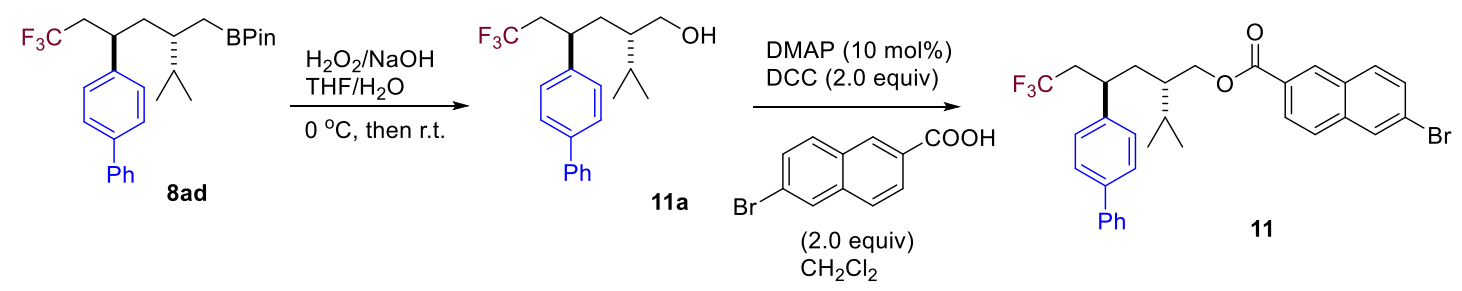

To a solution of $8 \mathbf{a d}(88.4 \mathrm{mg}, 0.1922 \mathrm{mmol})$ in tetrahydrofuran $(1 \mathrm{~mL})$ at $0{ }^{\circ} \mathrm{C}$ was added aqueous sodium hydroxide solution $(1 \mathrm{~mL}, 3 \mathrm{mmol}, 3 \mathrm{M})$. Aqueous hydrogen peroxide solution $(0.5 \mathrm{~mL}$, 
$30 \% \mathrm{w} / \mathrm{w}$ ) was added dropwise. The mixture was stirred at room temperature overnight. Upon the completion of the reaction as determined by TLC, the mixture was cooled to $0{ }^{\circ} \mathrm{C}$ and saturated aqueous sodium thiosulfate solution $(2 \mathrm{~mL})$ was added dropwise. The aqueous layer was extracted with diethyl ether $(15 \mathrm{~mL} \times 3)$. The combined organic layers were dried over magnesium sulfate, filtered and concentrated. The product was purified by flash column chromatography on silica gel with $n$-pentane/diethyl ether (10:1 to 2:1) as eluent to give the corresponding product 11a (56.7 mg, $84 \%$ yield) as a colorless sticky oil. $\mathrm{Rf}=0.4$ (pentane/ $\left.\mathrm{Et}_{2} \mathrm{O}=10: 7\right) .{ }^{1} \mathrm{H}$ NMR $(300 \mathrm{MHz}$, Chloroform- $d$ ) $\delta 7.54-7.44(\mathrm{~m}, 4 \mathrm{H}), 7.40-7.31(\mathrm{~m}, 2 \mathrm{H}), 7.30-7.21(\mathrm{~m}, 1 \mathrm{H}), 7.21-7.13(\mathrm{~m}, 2 \mathrm{H})$, $3.49-3.39(\mathrm{~m}, 1 \mathrm{H}), 3.35-3.22(\mathrm{~m}, 1 \mathrm{H}), 2.98(\mathrm{p}, J=7.3 \mathrm{~Hz}, 1 \mathrm{H}), 2.49-2.27(\mathrm{~m}, 2 \mathrm{H}), 1.88-1.73$ $(\mathrm{m}, 1 \mathrm{H}), 1.71-1.63(\mathrm{~m}, 2 \mathrm{H}), 1.24-1.09(\mathrm{~m}, 1 \mathrm{H}), 0.92(\mathrm{~s}, 1 \mathrm{H}), 0.83(\mathrm{~d}, J=7.0 \mathrm{~Hz}, 3 \mathrm{H}), 0.80(\mathrm{~d}, J$ $=7.0 \mathrm{~Hz}, 3 \mathrm{H}) .{ }^{13} \mathrm{C}\left\{{ }^{1} \mathrm{H},{ }^{19} \mathrm{~F}\right\}$ NMR $(151 \mathrm{MHz}$, Chloroform- $d) \delta 142.5,140.7,139.7,128.7,127.8$, 127.3, 127.2, 127.0, 126.6, 63.6, 43.7, 40.9, 37.8, 35.3, 27.6, 19.8, 18.5. ${ }^{19} \mathrm{~F}\left\{{ }^{1} \mathrm{H}\right\}$ NMR $(282 \mathrm{MHz}$, Chloroform- $d$ ) $\delta-63.5$.

To a solution of 11a $(40.8 \mathrm{mg}, 0.1166 \mathrm{mmol})$ in dichloromethane $(1 \mathrm{~mL})$ at $0{ }^{\circ} \mathrm{C}$ was added $4-$ dimethylaminopyridine (DMAP, $1.5 \mathrm{mg}, 0.012 \mathrm{mmol}, 0.1$ equiv), N,N'-Dicyclohexylcarbodiimide (DCC, $47.7 \mathrm{mg}, 0.23 \mathrm{mmol}, 2.0$ equiv), 6-bromo-2-naphthoic acid (57.7 mg, $0.23 \mathrm{mmol}, 2.0$ equiv). The reaction mixture was stirred for $24 \mathrm{~h}$. Then solvent was removed under reduced pressure. The residue was subjected to flash column chromatography on silica gel (pentane/DCM $=1: 1$ ) to afford 11 (46.4 mg, 68\% yield) as colorless white powder. ${ }^{1} \mathrm{H}$ NMR (300 MHz, Chloroform- $d$ ) $\delta 8.34$ (d, $J=1.6 \mathrm{~Hz}, 1 \mathrm{H}), 7.97-7.86(\mathrm{~m}, 2 \mathrm{H}), 7.70-7.60(\mathrm{~m}, 2 \mathrm{H}), 7.51-7.21(\mathrm{~m}, 8 \mathrm{H}), 7.19-7.11(\mathrm{~m}, 2 \mathrm{H})$, $4.19(\mathrm{dd}, J=11.1,4.8 \mathrm{~Hz}, 1 \mathrm{H}), 4.07(\mathrm{dd}, J=11.1,7.2 \mathrm{~Hz}, 1 \mathrm{H}), 3.14-2.93(\mathrm{~m}, 1 \mathrm{H}), 2.48-2.29(\mathrm{~m}$, $2 \mathrm{H}), 2.00-1.68(\mathrm{~m}, 3 \mathrm{H}), 1.62-1.49(\mathrm{~m}, 1 \mathrm{H}), 0.92(\mathrm{~d}, J=6.9 \mathrm{~Hz}, 3 \mathrm{H}), 0.92(\mathrm{~d}, J=6.9 \mathrm{~Hz}, 3 \mathrm{H})$. ${ }^{13} \mathrm{C}\left\{{ }^{1} \mathrm{H},{ }^{19} \mathrm{~F}\right\}$ NMR (151 MHz, Chloroform- $d$ ) $\delta 166.3,142.2,140.5,139.7,136.4,130.9,130.8$, $130.8,130.2,129.9,128.7,128.0,127.7,127.4,127.2,127.2,126.9,126.6,126.3,122.6,66.1,40.9$, 40.5, 37.6, 36.1, 28.2, 20.4, 18.2. ${ }^{19} \mathrm{~F}\left\{{ }^{1} \mathrm{H}\right\}$ NMR $\left(282 \mathrm{MHz}, \mathrm{CDCl}_{3}\right) \delta-63.5$. HRMS (ESI) $\mathrm{m} / \mathrm{z}=$ 605.1274 calcd. for $\mathrm{C}_{32} \mathrm{H}_{30} \mathrm{O}_{2} \mathrm{BrF}_{3} \mathrm{Na}^{+}$[M+Na] ${ }^{+}$, found: 605.1276. FTIR (neat): v ( $\mathrm{cm}^{-1}$ ) 2962, 2363 , 2341, 1715, 1653, 1628, 1487, 1462, 1387, 1332, 1262, 1220, 1176, 1131, 1097, 1064, 1008, 908, $882,806,766,732,697$.

2-(5-(4-(Tert-butyl)phenyl)-3,3-diethyl-7,7,7-trifluoroheptyl)-4,4,5,5-tetramethyl-1,3,2dioxaborolane (12)
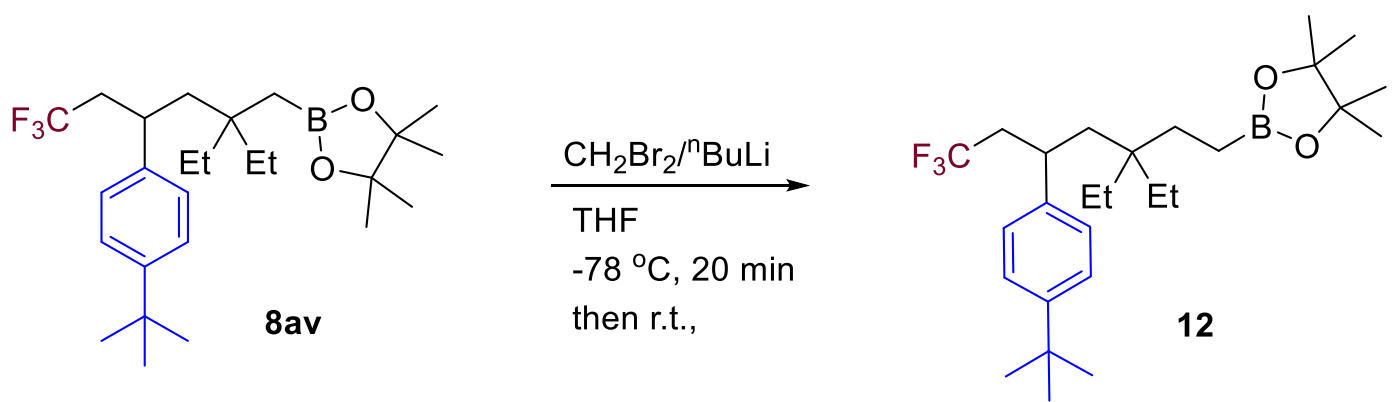

12 was prepared according to the reported procedure. ${ }^{\mathrm{S3}} \mathbf{8 a v}(60.4 \mathrm{mg}, 0.133 \mathrm{mmol})$ was dissolved in tetrahydrofuran $(1 \mathrm{~mL})$, and dibromomethane $(23.2 \mu \mathrm{L}, 0.333 \mathrm{mmol}, 2.5$ equiv) was added. The mixture was cooled to $-78^{\circ} \mathrm{C}, n$-butyl lithium $(0.18 \mathrm{~mL}, 0.29 \mathrm{mmol}, 1.6 \mathrm{M}$ in hexane) was added dropwise. The reaction mixture was stirred at $-78{ }^{\circ} \mathrm{C}$ for $20 \mathrm{~min}$, and then warmed to room 
temperature for another 2 hours. The mixture was quenched with saturated aqueous ammonium chloride solution, extracted with diethyl ether $(15 \mathrm{~mL} \times 3)$, dried over magnesium sulfate, filtered and concentrated. The product was purified by flash column chromatography on silica gel with $n$ pentane/dichloromethane (3:1) as eluent to give the corresponding product $\mathbf{1 2}$ as a colorless sticky oil $(33.0 \mathrm{mg}, 53 \%$ yield). $\mathrm{Rf}=0.7$ (pentane/dichloromethane $=5: 3) .{ }^{1} \mathrm{H}$ NMR $(300 \mathrm{MHz}$, Chloroform- $d$ ) $\delta 7.22-7.15(\mathrm{~m}, 2 \mathrm{H}), 7.08-7.00(\mathrm{~m}, 2 \mathrm{H}), 2.96-2.81(\mathrm{~m}, 1 \mathrm{H}), 2.37-2.18(\mathrm{~m}, 2 \mathrm{H})$, $1.63-1.52(\mathrm{~m}, 1 \mathrm{H}), 1.48-1.34(\mathrm{~m}, 1 \mathrm{H}), 1.22(\mathrm{~s}, 9 \mathrm{H}), 1.19-1.08(\mathrm{~m}, 14 \mathrm{H}), 1.07-0.92(\mathrm{~m}, 4 \mathrm{H})$, $0.58-0.47(\mathrm{~m}, 6 \mathrm{H}), 0.46-0.37(\mathrm{~m}, 2 \mathrm{H}) .{ }^{13} \mathrm{C}\left\{{ }^{1} \mathrm{H},{ }^{19} \mathrm{~F}\right\}$ NMR $(151 \mathrm{MHz}$, Chloroform- $d$ ) $\delta 149.1$, 142.4, 127.1, 126.6, 125.1, 82.8, 42.8, 41.4, 39.0, 34.9, 34.3, 31.4, 28.9, 27.5, 27.5, 24.8, 7.4, 7.3, carbon attached to boron not observed. ${ }^{19} \mathrm{~F}\left\{{ }^{1} \mathrm{H}\right\}$ NMR (282 MHz, Chloroform- $d$ ) $\delta-63.6 .{ }^{11} \mathrm{~B}$ NMR $\left(96 \mathrm{MHz}, \mathrm{CDCl}_{3}\right) \delta 33.7$. HRMS (ESI) $\mathrm{m} / z=491.3284$ calcd. for $\mathrm{C}_{27} \mathrm{H}_{44} \mathrm{O}_{2} \mathrm{BF}_{3} \mathrm{Na}^{+}[\mathrm{M}+\mathrm{Na}]^{+}$, found: 491.3276. FTIR (neat): $v\left(\mathrm{~cm}^{-1}\right)$ 2966, 2939, 2873, 1516, 1464, 1372, 1336, 1321, 1267, 1249, 1216 , 1146, 1132, 1096, 1019, 968, 888, 849, 677, 602.

2-((1-(2-(4-(Tert-butyl)phenyl)-4,4,4-trifluorobutyl)cyclopentyl)methyl)thiophene (13)
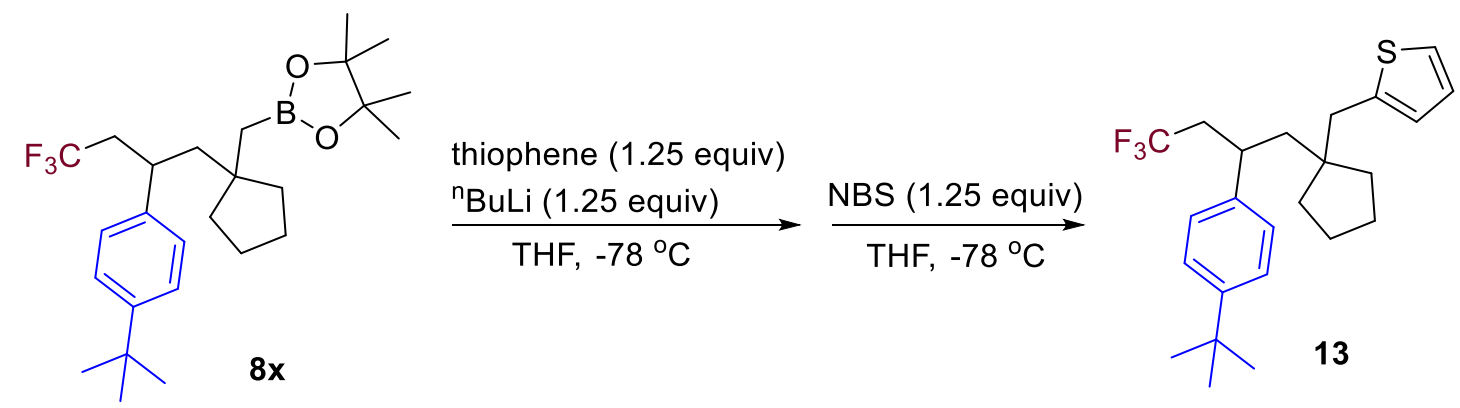

13 was prepared according to the reported procedure. ${ }^{\mathrm{S} 4}$ Thiophene $(15 \mu \mathrm{L}, 15.8 \mathrm{mg}, 0.188 \mathrm{mmol})$ in tetrahydrofuran $(1 \mathrm{~mL})$ was cooled to $-78^{\circ} \mathrm{C}$ and $n$-butyl lithium $(0.12 \mathrm{~mL}, 0.188 \mathrm{mmol}, 1.6 \mathrm{M}$ in hexane) was added dropwise. Then the mixture was allowed to warm up to $0{ }^{\circ} \mathrm{C}$ and stirred for 30 minutes. After cooling to $-78^{\circ} \mathrm{C}$ again, a solution of $8 \mathbf{x}(67.8 \mathrm{mg}, 0.150 \mathrm{mmol})$ in tetrahydrofuran $(0.5 \mathrm{~mL})$ was added dropwise. The reaction mixture was allowed to stir at $-78{ }^{\circ} \mathrm{C}$ for 1 hour. $\mathrm{N}$ bromosuccinimide $(33.5 \mathrm{mg}, 0.188 \mathrm{mmol})$ in tetrahydrofuran $(0.5 \mathrm{~mL})$ was added dropwise and the mixture was stirred at $-78{ }^{\circ} \mathrm{C}$ for 1 hour. Saturated aqueous sodium thiosulfate solution $(2 \mathrm{~mL})$ was added. The reaction mixture was allowed to warm to room temperature and diluted with water and diethyl ether. The aqueous layer was extracted with diethyl ether $(15 \mathrm{~mL} \times 3)$. The combined organic layers were dried over magnesium sulfate, filtered and concentrated. The product was purified by flash column chromatography on silica gel with $n$-pentane as eluent to give the corresponding product 13 (41.7 mg, 68\% yield) as a colorless sticky oil. $\mathrm{Rf}=0.4$ (pentane/dichloromethane $=20: 1)$. ${ }^{1} \mathrm{H}$ NMR (300 MHz, Chloroform- $d$ ) $\delta 7.27-7.19(\mathrm{~m}, 2 \mathrm{H}), 7.09-6.99(\mathrm{~m}, 3 \mathrm{H}), 6.82(\mathrm{dd}, J=5.2$, $3.4 \mathrm{~Hz}, 1 \mathrm{H}), 6.59-6.51(\mathrm{~m}, 1 \mathrm{H}), 3.17-3.01(\mathrm{~m}, 1 \mathrm{H}), 2.76(\mathrm{~d}, J=14.9 \mathrm{~Hz}, 1 \mathrm{H}), 2.60(\mathrm{~d}, J=14.9$ $\mathrm{Hz}, 1 \mathrm{H}), 2.38-2.17(\mathrm{~m}, 2 \mathrm{H}), 1.84-1.62(\mathrm{~m}, 2 \mathrm{H}), 1.55-1.26(\mathrm{~m}, 6 \mathrm{H}), 1.23(\mathrm{~s}, 9 \mathrm{H}), 1.15-0.92$ (m, 2H). ${ }^{13} \mathrm{C}\left\{{ }^{1} \mathrm{H},{ }^{19} \mathrm{~F}\right\}$ NMR (151 MHz, Chloroform- $d$ ) $\delta 149.6,141.4,141.4,127.3,126.6,126.5$, $126.4,125.3,123.5,47.0,44.5,42.6,37.7,37.5,37.3,36.3,34.4,31.4,23.9,23.4 .{ }^{19} \mathrm{~F}\left\{{ }^{1} \mathrm{H}\right\} \mathrm{NMR}$ $\left(282 \mathrm{MHz}, \mathrm{CDCl}_{3}\right) \delta-63.5$. HRMS (ESI) $\mathrm{m} / z=431.1991$ calcd. for $\mathrm{C}_{24} \mathrm{H}_{31} \mathrm{SF}_{3} \mathrm{Na}^{+}[\mathrm{M}+\mathrm{Na}]^{+}$, found: 431.1990. FTIR (neat): $v\left(\mathrm{~cm}^{-1}\right)$ 2955, 2872, 1513, 1457, 1437, 1419, 1364, 1293, 1266, 1246, 1128, 1096, 1041, 1018, 997, 851, 828, 692, 664. 
N-benzyl-1-(1-(2-(4-(tert-butyl)phenyl)-4,4,4-trifluorobutyl)cyclohexyl)methanamine (14)
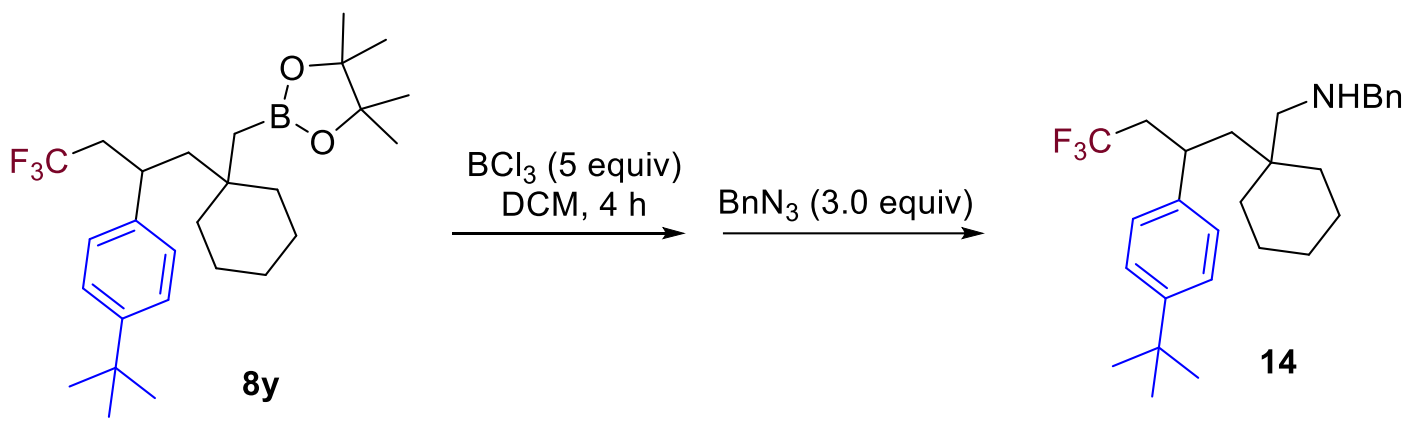

$\mathbf{1 4}$ was prepared according to the reported procedure.$^{\mathrm{S5}} \mathbf{8 y}$ (46.6 $\mathrm{mg}, 0.100 \mathrm{mmol}$ ) was added into a flame-dried round bottom flask. The reaction vessel was evacuated and backfilled with Argon for three times. Dichloromethane $(0.40 \mathrm{~mL})$ was added followed by dropwise addition of boron trichloride ( $0.5 \mathrm{~mL}, 0.5 \mathrm{mmol}, 1 \mathrm{M}$ in dichloromethane). The reaction mixture was stirred under an argon atmosphere at room temperature for 4 hours. The volatiles were removed under vacuum and the reaction vessel was evacuated and backfilled with Argon for three times again. The resulting mixture was dissolved in dichloromethane $(1.2 \mathrm{~mL})$. Benzyl azide $(38 \mu \mathrm{L}, 0.30 \mathrm{mmol})$ was added dropwise at $0{ }^{\circ} \mathrm{C}$. After warming to room temperature, the reaction mixture was allowed to stir for 12 hours. The reaction was quenched with aqueous sodium hydroxide solution $(5.0 \mathrm{~mL}, 3 \mathrm{M})$ and the aqueous layer was extracted with dichloromethane ( $3 \times 15 \mathrm{~mL})$. The combined organic layers were dried over magnesium sulfate, filtered and concentrated. The product was purified by flash column chromatography on silica gel with $n$-pentane/ethyl acetate/triethylamine (300:100:1) as eluent to give the corresponding product $\mathbf{1 4}(25.3 \mathrm{mg}, 57 \%$ yield $)$ as a yellow sticky oil. $\mathrm{Rf}=0.5$ ( $n$-pentane/ethyl acetate/trimethylamine $=300: 100: 1) .{ }^{1} \mathrm{H}$ NMR $(300 \mathrm{MHz}$, Chloroform- $d) \delta 7.27-$ $7.10(\mathrm{~m}, 7 \mathrm{H}), 7.07-7.00(\mathrm{~m}, 2 \mathrm{H}), 3.41-3.19(\mathrm{~m}, 2 \mathrm{H}), 3.01-2.89(\mathrm{~m}, 1 \mathrm{H}), 2.47-2.12(\mathrm{~m}, 3 \mathrm{H})$, $2.01(\mathrm{~d}, J=12.0 \mathrm{~Hz}, 1 \mathrm{H}), 1.85(\mathrm{dd}, J=14.4,9.3 \mathrm{~Hz}, 1 \mathrm{H}), 1.63(\mathrm{dd}, J=14.4,3.3 \mathrm{~Hz}, 1 \mathrm{H}), 1.39-$ $1.07(\mathrm{~m}, 19 \mathrm{H}) .{ }^{13} \mathrm{C}\left\{{ }^{1} \mathrm{H},{ }^{19} \mathrm{~F}\right\}$ NMR $\left(126 \mathrm{MHz}, \mathrm{cdcl}_{3}\right) \delta 149.4,142.2,128.2,128.0,127.1,126.7$, 126.6, 125.7, 125.3, 54.3, 42.8, 37.0, 35.0, 34.7, 34.7, 34.4, 31.4, 31.3, 26.3, 21.5, 21.4. ${ }^{19} \mathrm{~F}\left\{{ }^{1} \mathrm{H}\right\}$ NMR $\left(282 \mathrm{MHz}, \mathrm{CDCl}_{3}\right) \delta-63.5$. HRMS (ESI) $\mathrm{m} / z=446.3029$ calcd. for $\mathrm{C}_{28} \mathrm{H}_{38} \mathrm{NF}_{3} \mathrm{H}^{+}[\mathrm{M}+\mathrm{H}]^{+}$, found: 446.3031. FTIR (neat): $v\left(\mathrm{~cm}^{-1}\right)$ 2928, 2863, 2813, 1513, 1495, 1453, 1415, 1364, 1267 , $1249,1128,1098,851,827,735,698,636,593$.

2-(6-(4-(Tert-butyl)phenyl)-8,8,8-trifluoro-2,4,4-trimethyloctan-2-yl)-4,4,5,5-tetramethyl-1,3,2dioxaborolane (15)
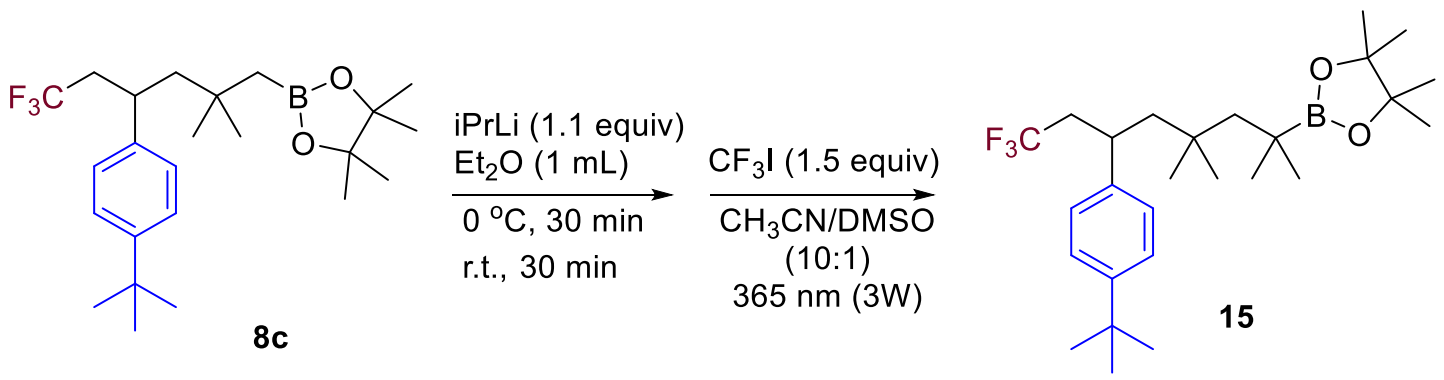

15 was prepared according to the reported procedure ${ }^{\mathrm{S} 6} \mathbf{8 c}(65.0 \mathrm{mg}, 0.153 \mathrm{mmol}, 1.0$ equiv) was dissolved in diethyl ether $(1.0 \mathrm{~mL})$. Isopropyl lithium solution $(0.24 \mathrm{~mL}, 0.7 \mathrm{M}$ solution in pentane, 
$0.17 \mathrm{mmol}, 1.1$ equiv) was added dropwise over $5 \mathrm{~min}$ at $0{ }^{\circ} \mathrm{C}$. The mixture was stirred at $0{ }^{\circ} \mathrm{C}$ for $30 \mathrm{~min}$ then room temperature for another $30 \mathrm{~min}$. The solvent was carefully removed in vacuo and further dried for $5 \mathrm{~min}$ under high vacuum. After subsequent addition of acetonitrile $(1.8 \mathrm{~mL})$, DMSO $(0.2 \mathrm{~mL})$ under argon, the mixture was stirred until all solid was dissolved. Then $\mathrm{CF}_{3} \mathrm{I}(1.0$ $\mathrm{M}$ in $\mathrm{CH}_{3} \mathrm{CN}, 0.23 \mathrm{~mL}, 0.23 \mathrm{mmol}, 1.5$ equiv) was added to the reaction mixture and the reaction mixture was immediately irradiated by a $3 \mathrm{~W} \operatorname{LED}(365 \mathrm{~nm})$ and stirred for $1 \mathrm{~h}$. The reaction mixture was diluted with $20 \mathrm{~mL} \mathrm{CH}_{2} \mathrm{Cl}_{2}$ and quenched with saturated $\mathrm{NH}_{4} \mathrm{Cl}$ aqueous solution. The organic phase was separated and dried over $\mathrm{MgSO}_{4}$ and concentrated under reduced pressure. Flash column chromatography eluting with pentane and $\mathrm{CH}_{2} \mathrm{Cl}_{2}$ (3:1 to 5:3) afforded $\mathbf{1 5}$ as colorless sticky oil (35.7 $\mathrm{mg}, 50 \%$ yield) and $\mathbf{8 c}$ as colorless sticky oil (24.9 $\mathrm{mg}, 38 \%$ recovered yield).

15: $\mathrm{Rf}=0.7$ (pentane/dichloromethane $=5: 3),{ }^{1} \mathrm{H}$ NMR $(300 \mathrm{MHz}$, Chloroform- $d) \delta 7.23-7.16(\mathrm{~m}$, $2 \mathrm{H}), 7.08-6.99(\mathrm{~m}, 2 \mathrm{H}), 3.10-2.90(\mathrm{~m}, 1 \mathrm{H}), 2.38-2.18(\mathrm{~m}, 2 \mathrm{H}), 1.69(\mathrm{dd}, J=14.0,8.0 \mathrm{~Hz}, 1 \mathrm{H})$, $1.56(\mathrm{dd}, J=13.9,4.0 \mathrm{~Hz}, 1 \mathrm{H}), 1.23$ (s, 3H), $1.22(\mathrm{~s}, 9 \mathrm{H}), 1.13$ (s, 12H), 0.85 (s, 3H), $0.82(\mathrm{~s}, 3 \mathrm{H})$, $0.78(\mathrm{~s}, 3 \mathrm{H}), 0.69(\mathrm{~s}, 3 \mathrm{H}) .{ }^{13} \mathrm{C}\left\{{ }^{1} \mathrm{H},{ }^{19} \mathrm{~F}\right\}$ NMR $(151 \mathrm{MHz}$, Chloroform- $d$ ) $\delta 149.1,142.6,127.2,126.6$, 125.2, 82.9, 53.4, 50.8, 42.9, 35.6, 35.1, 34.3, 31.4, 28.4, 28.2, 27.4, 27.4, 24.7, 24.7, carbon attached to boron not observed. ${ }^{19} \mathrm{~F}\left\{{ }^{1} \mathrm{H}\right\}$ NMR $\left(282 \mathrm{MHz}\right.$, Chloroform- $d$ ) $\delta-63.6 .{ }^{11} \mathrm{~B}$ NMR $(96$ $\left.\mathrm{MHz}, \mathrm{CDCl}_{3}\right) \delta 34.4$. HRMS (ESI) $\mathrm{m} / z=491.3284$ calcd. for $\mathrm{C}_{27} \mathrm{H}_{44} \mathrm{O}_{2} \mathrm{BF}_{3} \mathrm{Na}^{+}[\mathrm{M}+\mathrm{Na}]^{+}$, found: 491.3279. FTIR (neat): $v\left(\mathrm{~cm}^{-1}\right)$ 2959, 2870, 1514, 1479, 1446, 1389, 1371, 1306, 1266, 1248, 1209 , 1165, 1130, 1097, 1046, 1024, 967, 934, 867, 847, 829, 715, 692, 669, 635, 611, 580.

\section{X-ray Structure of 11}

X-Ray diffraction: Data sets for compound 11 were collected with a Bruker D8 Venture CMOS diffractometer. Programs used: data collection: APEX3 V2016.1-0 (Bruker AXS Inc., 2016); cell refinement: SAINT V8.37A (Bruker AXS Inc., 2015); data reduction: SAINT V8.37A (Bruker AXS Inc., 2015); absorption correction, SADABS V2014/7 (Bruker AXS Inc., 2014); structure solution SHELXT-2015 (Sheldrick, G. M. Acta Cryst., 2015, A71, 3-8); structure refinement SHELXL-2015 (Sheldrick, G. M. Acta Cryst., 2015, $C 71$ (1), 3-8) and graphics, XP (Version 5.1, Bruker AXS Inc., Madison, Wisconsin, USA, 1998). $R$-values are given for observed reflections, and $w \mathrm{R}^{2}$ values are given for all reflections.

$\mathrm{X}$-ray crystal structure analysis of 11 (stu10027): A colorless prism-like specimen of $\mathrm{C}_{32} \mathrm{H}_{30} \mathrm{BrF}_{3} \mathrm{O}_{2}$, approximate dimensions $0.076 \mathrm{~mm} \times 0.077 \mathrm{~mm} \times 0.179 \mathrm{~mm}$, was used for the $\mathrm{X}$ ray crystallographic analysis. The X-ray intensity data were measured on a Bruker D8 Venture Bruker D8 Venture Photon III Diffractometer system equipped with a micro focus tube $\mathrm{CuK} \alpha$ $(\mathrm{CuK} \alpha, \lambda=1.54178 \AA$ ) and a MX mirror monochromator. A total of 1602 frames were collected. The total exposure time was 22.34 hours. The frames were integrated with the Bruker SAINT software package using a wide-frame algorithm. The integration of the data using a monoclinic unit cell yielded a total of 48861 reflections to a maximum $\theta$ angle of $66.59^{\circ}$ ( $0.84 \AA$ resolution), of which 4825 were independent (average redundancy 10.127 , completeness $=99.8 \%, \mathrm{R}_{\text {int }}=6.99 \%$, $\left.\mathrm{R}_{\mathrm{sig}}=3.07 \%\right)$ and $4176(86.55 \%)$ were greater than $2 \sigma\left(\mathrm{F}^{2}\right)$. The final cell constants of $\underline{a}=10.9035(2)$ $\AA, \underline{b}=8.5349(2) \AA, \underline{c}=29.4427(5) \AA, \beta=92.1110(10)^{\circ}$, volume $=2738.09(9) \AA^{3}$, are based upon the refinement of the XYZ-centroids of 9942 reflections above $20 \sigma(\mathrm{I})$ with $8.757^{\circ}<2 \theta<133.2^{\circ}$. 
Data were corrected for absorption effects using the Multi-Scan method (SADABS). The ratio of minimum to maximum apparent transmission was 0.902 . The calculated minimum and maximum transmission coefficients (based on crystal size) are 0.6680 and 0.8350 . The structure was solved and refined using the Bruker SHELXTL Software Package, using the space group $P 2_{1} / n$, with $\mathrm{Z}=$ 4 for the formula unit, $\mathrm{C}_{32} \mathrm{H}_{30} \mathrm{BrF}_{3} \mathrm{O}_{2}$. The final anisotropic full-matrix least-squares refinement on $\mathrm{F}^{2}$ with 345 variables converged at $\mathrm{R} 1=3.54 \%$, for the observed data and $\mathrm{wR} 2=7.35 \%$ for all data. The goodness-of-fit was 1.097 . The largest peak in the final difference electron density synthesis was $0.259 \mathrm{e}^{-} / \AA^{3}$ and the largest hole was $-0.256 \mathrm{e}^{-} / \AA^{3}$ with an RMS deviation of $0.057 \mathrm{e} / \AA^{3}$. On the basis of the final model, the calculated density was $1.415 \mathrm{~g} / \mathrm{cm}^{3}$ and $\mathrm{F}(000), 1200 \mathrm{e} \cdot \mathrm{CCDC}$ number: 2078808.

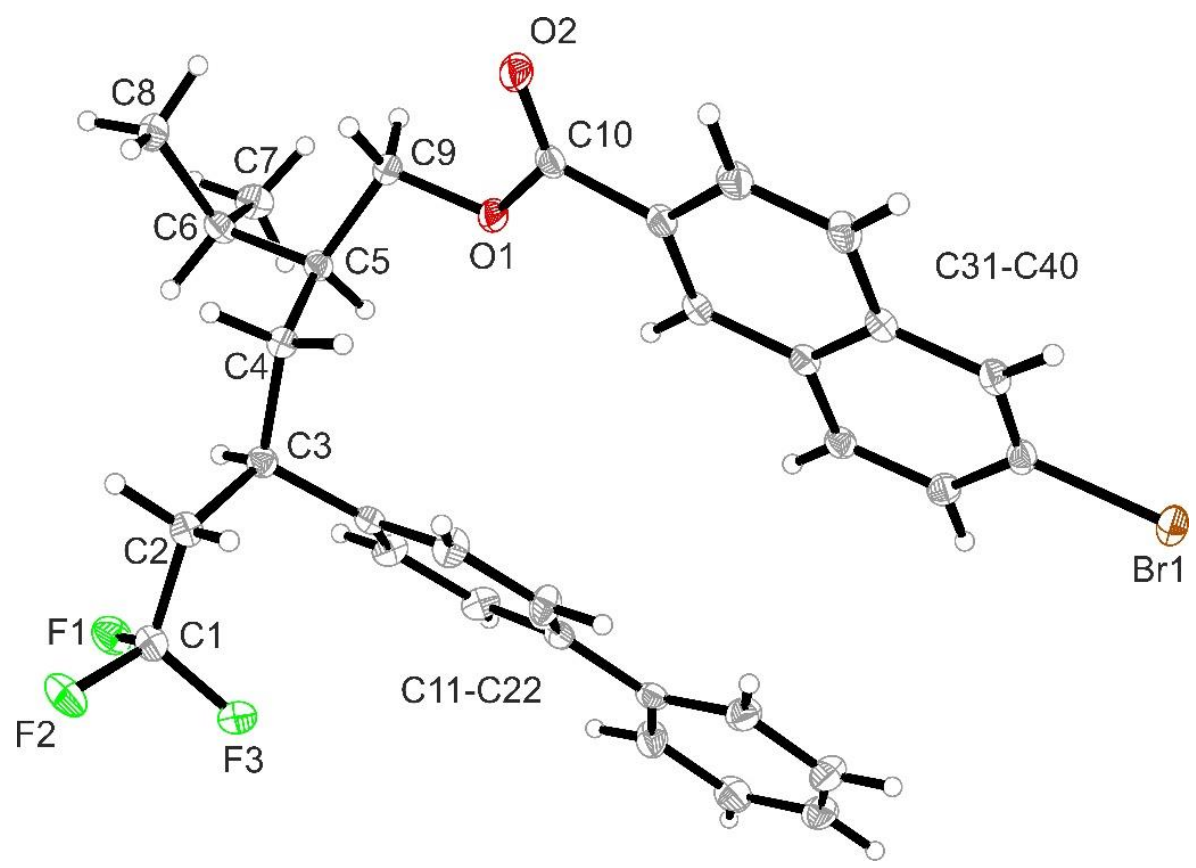

Figure S1: Crystal structure of compound 11. (Thermal ellipsoids are shown at 30\% probability.)

\section{Reference:}

1. APEX3 (2016), SAINT (2015) and SADABS (2015), Bruker AXS Inc., Madison, Wisconsin, USA.

2. Sheldrick, G. M., SHELXT - Integrated space-group and crystal-structure determination, Acta Cryst., 2015, A71, 3-8.

3. Sheldrick, G.M., Crystal structure refinement with SHELXL, Acta Cryst., 2015, C71 (1), 3-8.

4. XP-Interactive molecular graphics, Version 5.1, Bruker AXS Inc., Madison, Wisconsin, USA, 1998.

\section{Discussion of the Side Reactions}

For the reactions of boronate complexes generated from halogenated aryl lithium reagents, halogen/iodine exchange product $\mathbf{8 h}$ and proton dehalogenation product $\mathbf{8 a}$ were detected by GC analysis (Scheme $\mathrm{S} 1$ ). $\mathrm{CF}_{3}$ radical addition to the pending olefin resulted in the distal radical anion 
intermediate I, from which radical 1,5-aryl migration gave II. According to the DFT optimized structure of II (see the DFT part below), an interaction existed between the boron radial anion center and the aryl ring. Intramolecular single electron transfer from boron to the aryl ring and Ar-X bond dissociation would deliver aryl radical III. III could abstract hydrogen atom from the solvent to give the proton dehalogenation product 8a. Or iodine atom transfer from $\mathrm{CF}_{3}$ I to III would give the halogen/iodine exchange product $\mathbf{8 h}$. When $\mathrm{X}=\mathrm{Br}$ or $\mathrm{Cl}$, Ar-X bond dissociation was facile thus $\mathbf{8 h}$ was generated with much higher yields (68\% and 63\% GC yield) compared to the direct aryl migration products $(4 \%, 11 \%)$. As Ar-F dissociation was not favored thus the direct aryl migration product $8 \mathbf{i}$ was generated predominantly (57\%), while the fluorine/iodine exchange product $8 \mathbf{h}$ was formed with much lower yield (5\%).

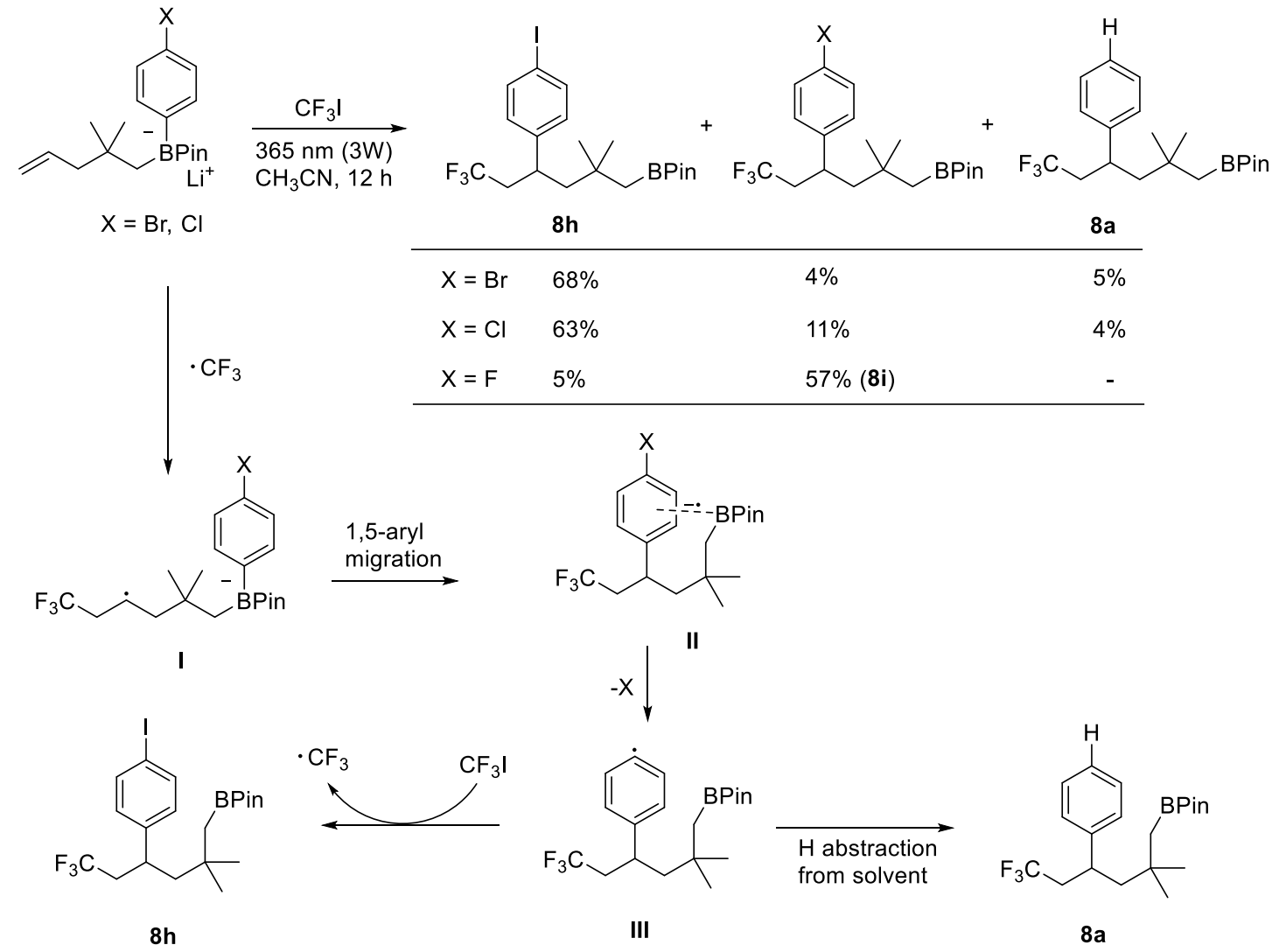

Scheme S1. Possible pathway for the side product generation

\section{DFT Computations}

\subsection{Methods}

All structures were optimized without geometry constraints using the PBE0 hybrid functional ${ }^{\mathrm{S} 7, \mathrm{~S} 8}$ and an atom-pairwise dispersion correction (D3). ${ }^{\mathrm{S}, \mathrm{S} 10}$ A flexible triple zeta basis set (def2-TZVP) ${ }^{\mathrm{S} 11}$ was used in all calculations. The nature of the optimized stationary points was proven by the presence of either 0 (minimum) or 1 (transition structure) imaginary vibrational frequency. For the determination of the free enthalpy contributions at $298 \mathrm{~K}\left(\mathrm{G}^{\mathrm{RRHO}}\right)$, a rotor approximation was applied for vibrational modes with wave numbers below $100 \mathrm{~cm}^{-1}{ }^{S 12}$ Electronic energies were 
recalculated with the hybrid meta-GGA functional PW6B95(-D3) ${ }^{\mathrm{S} 13}$ using the structures optimized with PBE0-D3. Solvation free energies $\left(\mathrm{G}_{\text {solv }}\right)$ for $\mathrm{T}=298.15 \mathrm{~K}$ in acetonitrile were obtained with COSMO-RS ${ }^{\mathrm{S} 14, \mathrm{~S} 15}$ using the COSMOtherm program package. ${ }^{\mathrm{S} 16}$ The final value for the relative free enthalpy in solution $(\Delta \mathrm{G} 298)$ was obtained using the sum of the differences in PW6B95-D3 electronic energies, $\mathrm{G}^{\mathrm{RRHO}}(298 \mathrm{~K})$, and $\mathrm{G}_{\text {solv }}$ as

$$
\Delta \mathrm{G}_{298}=\Delta \mathrm{E}(\mathrm{PW} 6 \mathrm{~B} 95-\mathrm{D} 3)+\Delta \mathrm{G}^{\text {rrho }}+\Delta \mathrm{G}_{\text {solv }}
$$

All quantum chemical calculations were performed with the TURBOMOLE 7.4 program. ${ }^{S 17}$ For the determination of the prevailing conformers of boronic esters 8a and $\mathbf{8} \mathbf{a v}_{\mathbf{s y n}} / \mathbf{a n t}$, we performed a conformational search with the semiempirical GFN2-xTB method, ${ }^{\mathrm{S} 18, \mathrm{~S} 19}$ and re-optimized the ten most stable conformers with the abovementioned procedure. Only the most stable conformer is reported here for brevity.

\subsection{Results}

Calculated energies of all species, and relative free enthalpies in solution for the reaction of boronate anions 9a, 9av, 9ad, and 9aw are reported in Table S1. Optimized structures are depicted in Figure $\mathrm{S} 1-\mathrm{S} 5$. The $\mathrm{Li}^{+}$cation is expected to have little impact on the rearrangement, as the highly polar solvent $\left(\mathrm{CH}_{3} \mathrm{CN}\right)$ would promote the formation of separated ion pairs. Therefore only the (distonic) radical boronate anions which are generated by addition of $\mathrm{CF}_{3} \cdot$ radical to the double bond of 7 were used as reference point of the energy calculations for 9a and 9av.

Table S1 Energies, free energy contributions $G^{\text {rho }}$, solvation free energies $G_{\text {solv }}$ (COSMO-RS, $\mathrm{CH}_{3} \mathrm{CN}$ ) and resulting relative free enthalpies $\Delta \mathrm{G}_{298}$ for reactants and products in the aryl migration reaction of radical anions $9 \mathbf{a}, 9 \mathbf{9 a v}, 9 \mathbf{9 d}$ and 9 aw. ${ }^{[a]}$

\begin{tabular}{|c|c|c|c|c|c|}
\hline & E(PBE0-D3) & $\mathrm{G}^{\text {rrho }}(298 \mathrm{~K})$ & $\mathrm{E}$ (PW6B95-D3) & $\mathrm{G}_{\text {solv }}$ & $\Delta \mathbf{G}_{298}$ \\
\hline & {$\left[\mathrm{E}_{\mathrm{h}}\right]$} & {$[\mathrm{kcal} / \mathrm{mol}]$} & {$\left[\mathrm{E}_{\mathrm{h}}\right]$} & {$[\mathrm{kcal} / \mathrm{mol}]$} & {$[\mathrm{kcal} / \mathrm{mol}]$} \\
\hline $\mathrm{CF}_{3} \mathrm{I}$ & -635.180684 & -9.441 & -636.034506 & 1.95 & \\
\hline $\mathrm{CF}_{3}$ & -337.393943 & -7.931 & -338.075589 & 4.34 & \\
\hline $\mathrm{I}^{-}$ & -297.809353 & -10.573 & -297.981232 & -62.53 & \\
\hline $9 a$ & -1254.259603 & 260.171 & -1257.189773 & -46.20 & 0.00 \\
\hline 9a-TS & -1254.247634 & 262.210 & -1257.176152 & -45.89 & 10.91 \\
\hline 10a(cycl,ipso) & -1254.279304 & 263.506 & -1257.204821 & -45.97 & -5.87 \\
\hline 10a(cycl,ortho & -1254.278529 & 263.850 & -1257.205592 & -46.86 & -6.90 \\
\hline $10 a$ & -1254.244571 & 260.954 & -1257.175402 & -51.80 & 4.21 \\
\hline $8 \mathbf{a}$ & -1254.294949 & 265.878 & -1257.225824 & -7.89 & $-61.81^{[\mathrm{c}]}$ \\
\hline 9av & -1293.538552 & 277.234 & -1296.565731 & -45.75 & 0.00 \\
\hline
\end{tabular}




\begin{tabular}{cccccc} 
9av-TS $_{\text {syn }}$ & -1293.526260 & 279.817 & -1296.553702 & -45.90 & 9.98 \\
9av-TS $_{\text {anti }}$ & -1293.525759 & 278.882 & -1296.551862 & -46.59 & 9.51 \\
$\mathbf{1 0 a v}_{\text {syn }}(\mathbf{c y c l})$ & -1293.553414 & 281.322 & -1296.577852 & -46.37 & -4.14 \\
$\mathbf{1 0 a v}_{\text {anti }}$ (cycl) & -1293.558774 & 280.351 & -1296.581861 & -46.99 & -8.24 \\
$\mathbf{1 0 a v}_{\text {syn }}$ & -1293.530659 & 277.042 & -1296.558415 & -51.31 & -1.17 \\
$\mathbf{1 0 a v}_{\text {anti }}$ & -1293.535515 & 277.167 & -1296.562975 & -50.93 & -3.52 \\
$\mathbf{8 a v}_{\text {syn }}$ & -1293.576323 & 283.435 & -1296.605608 & -7.94 & $-64.22^{[\mathrm{c}]}$ \\
$\mathbf{8 a v}_{\text {anti }}$ & -1293.576023 & 282.364 & -1296.604453 & -8.57 & $-65.20^{[\mathrm{c}]}$ \\
\hline & & & & & $\Delta$ \\
\hline 9ad-TS $_{\text {syn }}$ & -1524.407016 & 327.605 & -1528.014375 & -47.72 & 0.00 \\
9ad-TS $_{\text {anti }}$ & -1524.406458 & 326.767 & -1528.012493 & -48.56 & -0.50 \\
\hline 9aw-TS $_{\text {syn }}$ & -1450.654379 & 347.032 & -1454.073072 & -46.06 & 0.00 \\
9aw-TS $_{\text {anti }}$ & -1450.653818 & 346.208 & -1454.071206 & -46.79 & -0.39 \\
\hline
\end{tabular}

[a] all DFT energies were obtained with the def2-TZVP basis set and gridsize m5 (Turbomole)

[b] relative free enthalpies of the two diastereomeric transition structures.

[c] free enthalpy of the process $9 \mathbf{a}(\mathbf{v})+\mathrm{CF}_{3} \mathrm{I} \rightarrow \mathrm{CF}_{3}+\mathrm{I}^{-}+\mathbf{8 a}(\mathbf{v})$ 
The aryl migration of radical anion $9 a\left(\mathrm{R}_{\mathrm{f}}=\mathrm{CF}_{3}, \mathrm{Ar}=\mathrm{Ph}, \mathrm{R}^{1}=\mathrm{R}^{2}=\mathrm{CH}_{3}\right)$, shown in Scheme $\mathrm{S} 2$, proceeds via a chair like transition state with the (trifluormethyl)methyl group in a equatorial position (Figure S2). The barrier is comparably low (11 kcal/mol), allowing for fast reaction at room temperature or lower temperatures. The resulting boronate radical anion may be stabilized by addition to the aryl ring with formation of a pentadienyl anion $(\mathbf{1 0 a}(\mathbf{c y c l}))$, in which case the ortho position is the preferred site for addition. Nevertheless, electron transfer to $\mathrm{CF}_{3} \mathrm{I}$, with subsequent dissociation of the resulting anion into iodide and the radical chain carrier $\mathrm{CF}_{3}$, is extremely exothermic (almost $-62 \mathrm{kcal} / \mathrm{mol}$ ) and results in the formation of boronic ester $\mathbf{8 a}$.
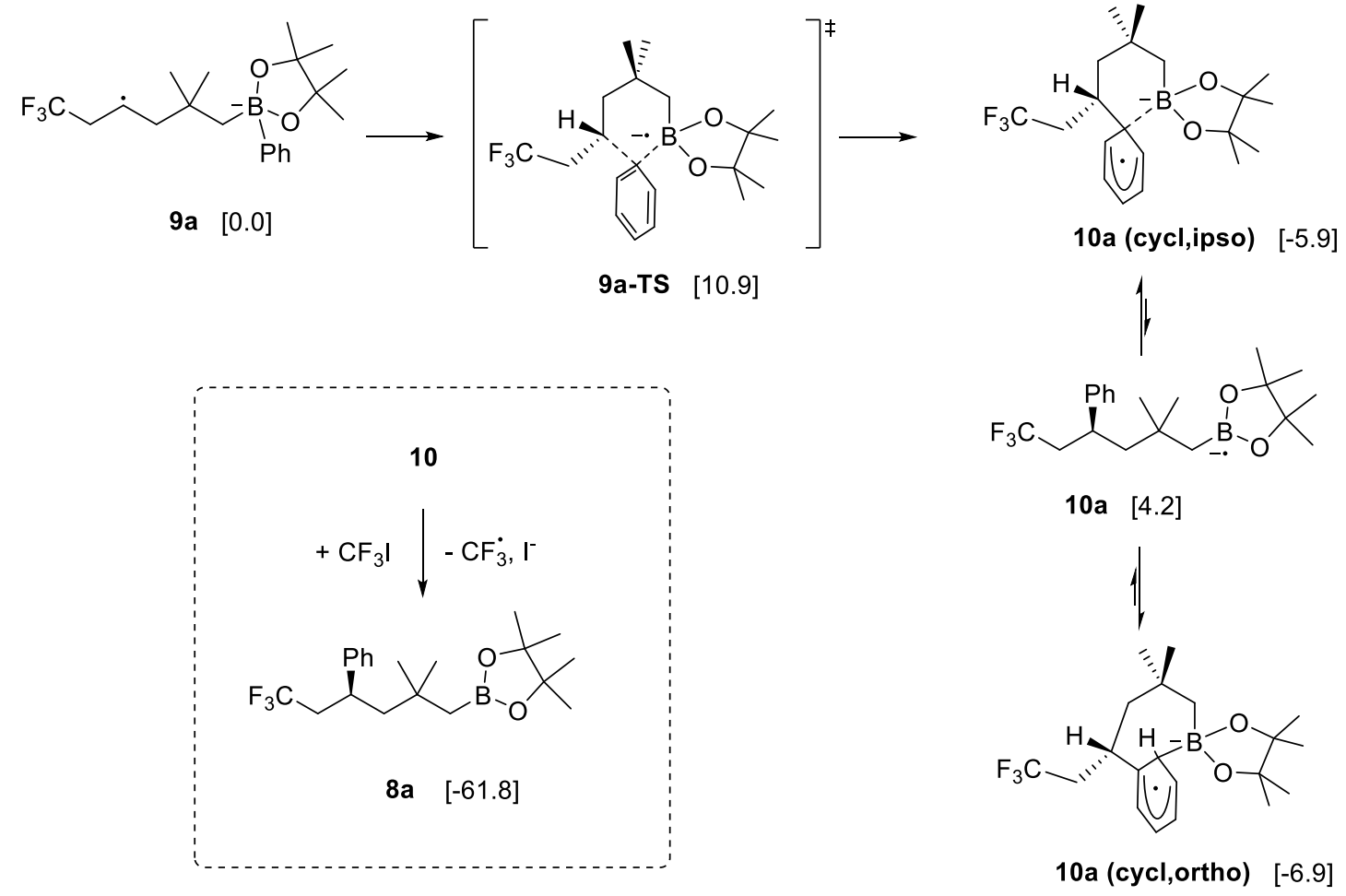

Scheme S2

In the reaction of radical anion $9 \mathbf{a v}\left(\mathrm{R}_{\mathrm{f}}=\mathrm{CF}_{3}, \mathrm{Ar}=\mathrm{Ph}, \mathrm{R}^{1}=i \mathrm{Pr}, \mathrm{R}^{2}=\mathrm{H}\right)$, we have identified the two diastereoisomeric transition structures with lowest energy for the formation of syn- and anti-8av, respectively (Scheme S3). In both, the $\mathrm{CF}_{3} \mathrm{CH}_{2}$ group attains an equatorial position while the position of the isopropyl group differs: the axial position in 9av-TS $\mathbf{S S}_{\text {syn }}$ leads to a barrier which is higher by $0.5 \mathrm{kcal} / \mathrm{mol}$ for the syn pathway (Figure S3). This is qualitatively correct, as anti boronic esters are observed as the predominant products $(\mathbf{8 a d}, \mathbf{8 a e})$, although the product ratio would imply a larger difference of activation barriers ( $>1 \mathrm{kcal} / \mathrm{mol})$. The aryl stabilized boronate radical anions 10av transfer the excess electron to $\mathrm{CF}_{3} \mathrm{I}$, and products $\mathbf{8} \mathbf{a v}_{\text {syn }}$ and $\mathbf{8} \mathbf{a v}$ anti are formed with similar free enthalpies of reaction as in the formation of dimethyl compound $\mathbf{8 a}$. The anti product is slightly more stable than $\mathbf{8} \mathbf{a v}_{\text {syn }}$. 


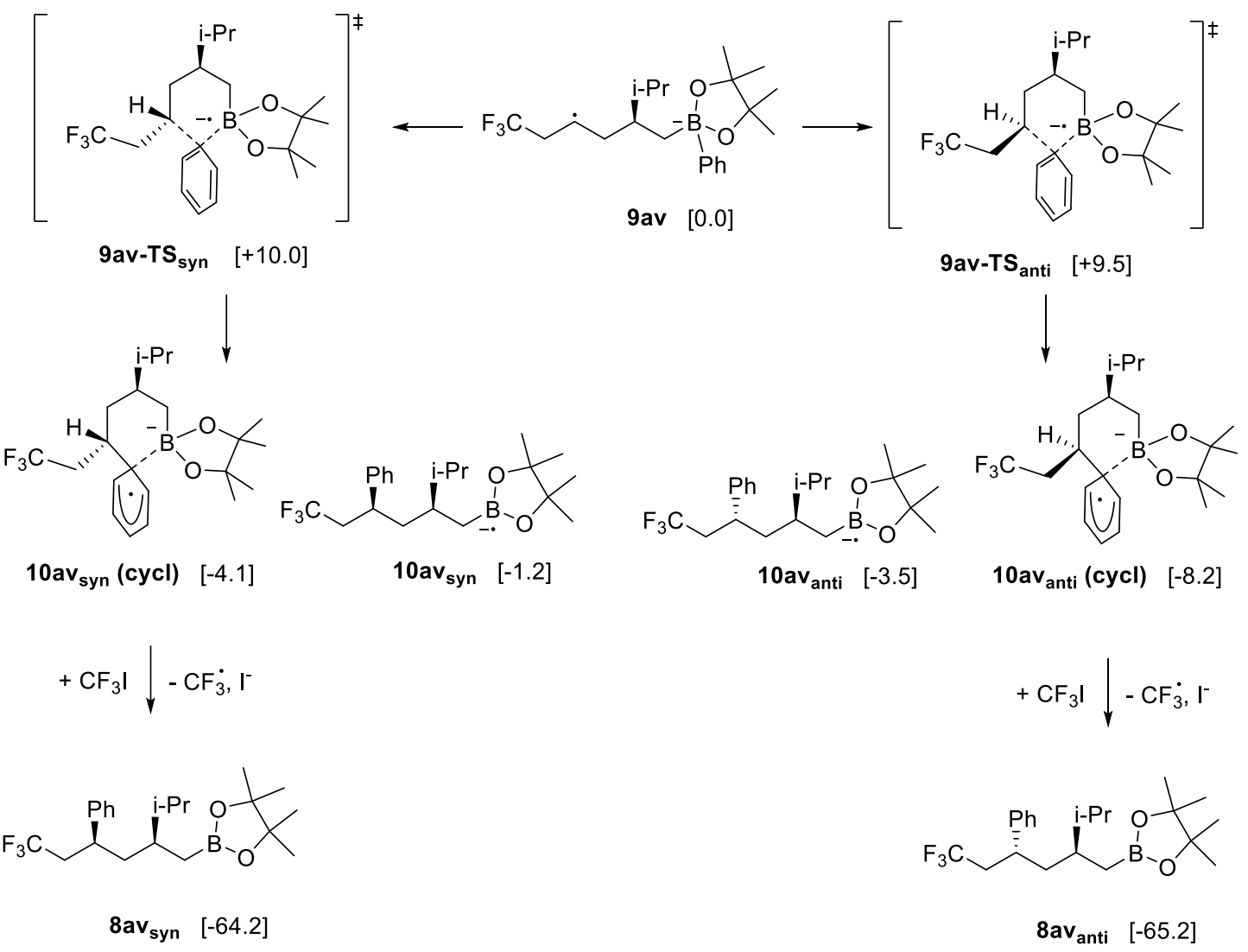

Scheme S3

Finally, we have compared the diastereoisomeric transition states of reactions with a migrating aryl group which is substituted in the para position ( $\mathrm{Ar}=4-t \mathrm{Bu}-\mathrm{Ph}$ and biphenyl). As depicted in Scheme S4, the anti transition state is favoured for both 10ad-TS and 10aw-TS (for the molecular structures, see Figures S4 and S5). Obviously, the calculated difference of activation barriers is not changed significantly by the para substituent. We see a higher selectivity in the experiments when $\mathrm{CF}_{3}$ is replaced with the larger $\mathrm{C}_{4} \mathrm{~F}_{9}$ group, which suggests that the size of the fluoroalkyl group has a larger effect on the selectivity than the aryl substitutent.

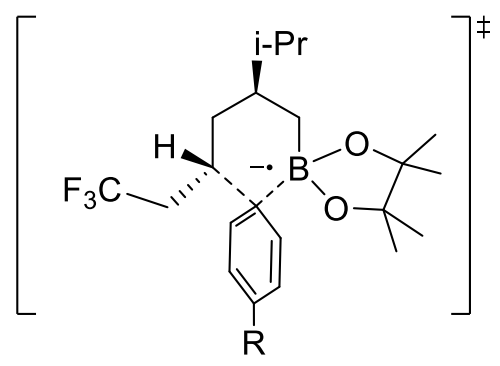

$$
\begin{array}{ll}
R=P h: \text { 9ad-TS } & {[0.0]} \\
R=t B u: 9 a w-T S_{\text {syn }} & {[0.0]}
\end{array}
$$<smiles>[R]c1ccc([As]2(OC(C)(C)C)(OC(C)(C)C)CC([3H])C[C@H](CC)C2)cc1</smiles>

$\mathrm{R}=\mathrm{Ph}:$ 9ad-TS anti $[-0.5]$

$\mathrm{R}=\mathrm{tBu}: 9 \mathrm{aw}-\mathrm{TS}_{\mathrm{anti}} \quad[-0.4]$

Scheme S4 
Figure S2 Optimized molecular structures in the aryl migration reaction of 9a

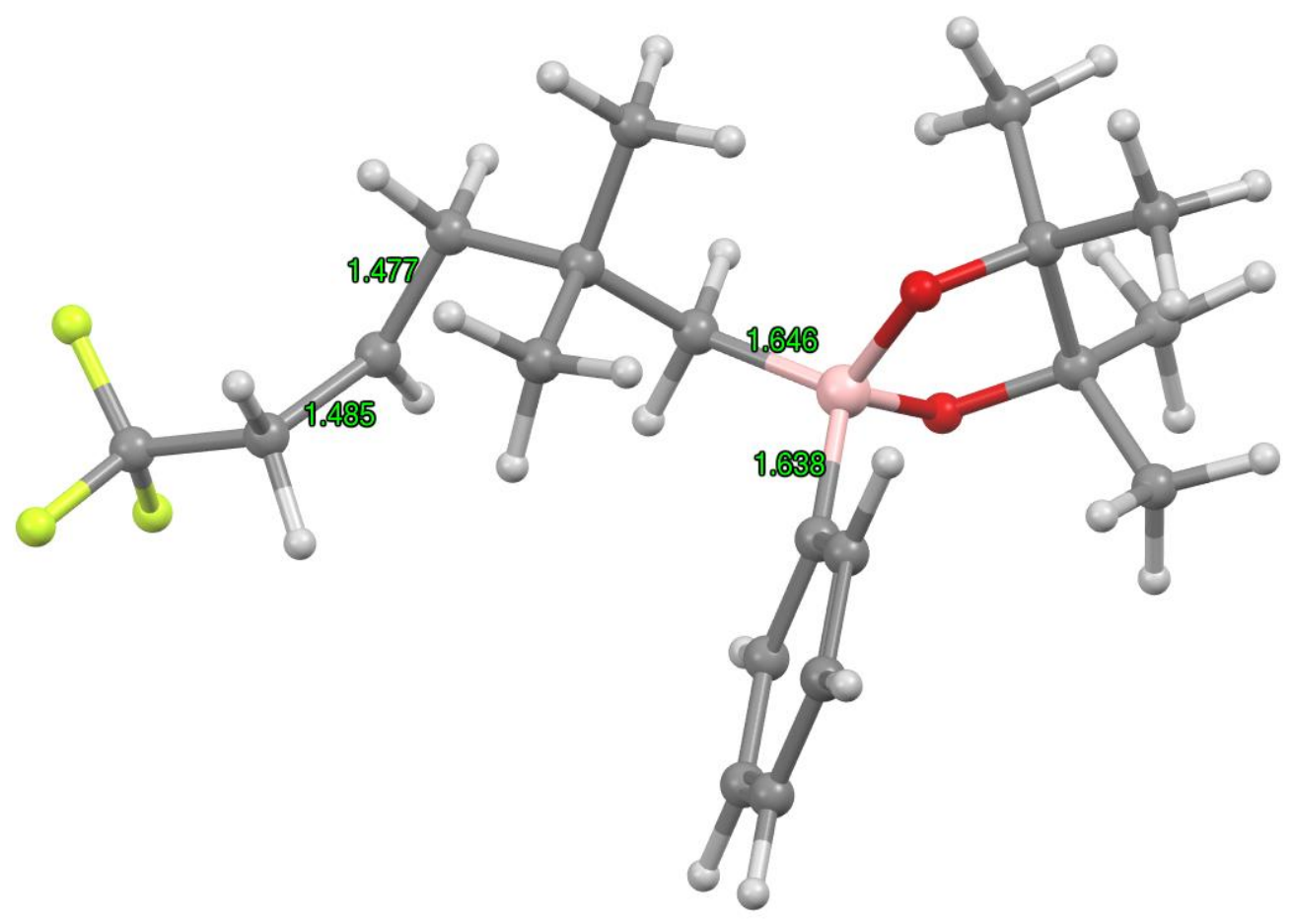

9a

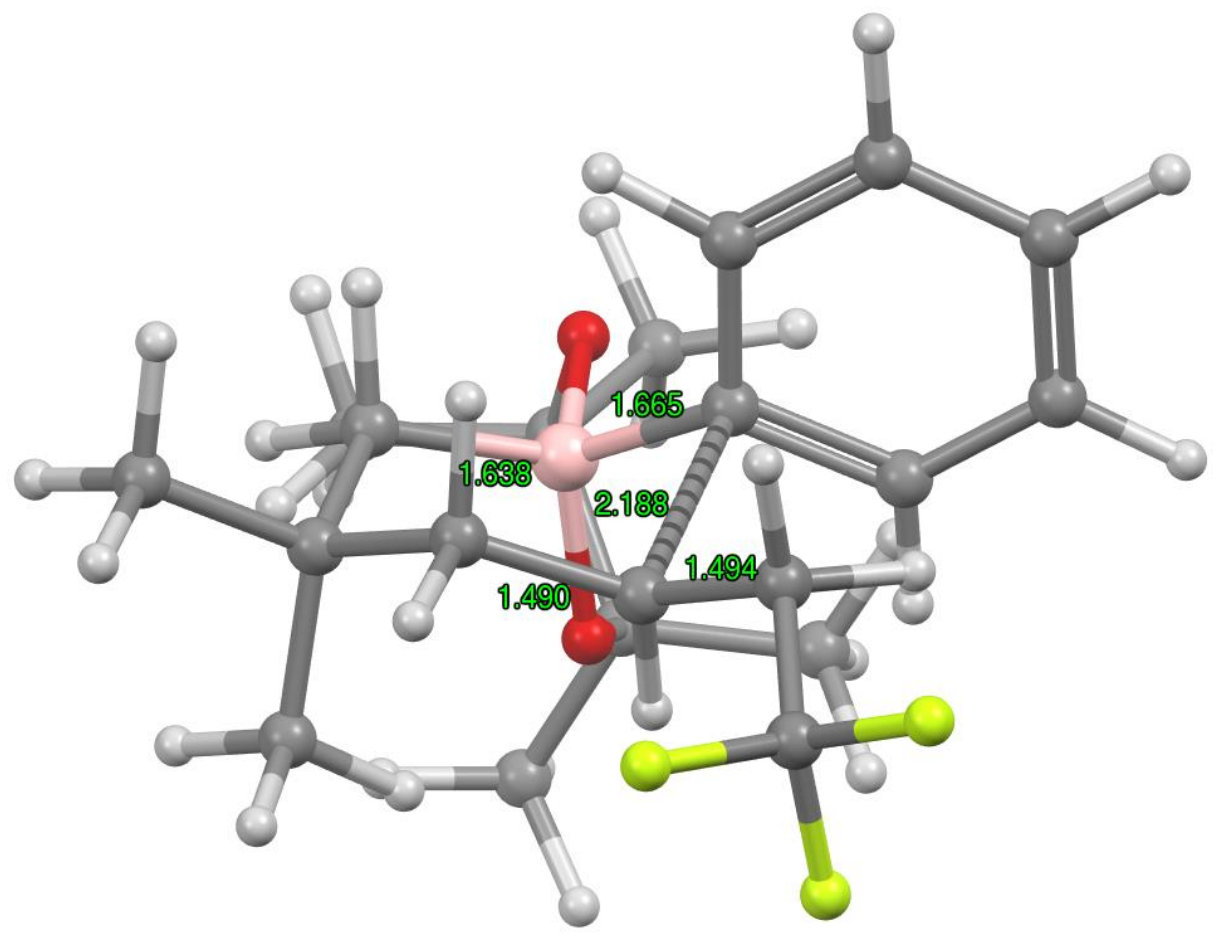

9a-TS 
Figure S2 (continued) Optimized molecular structures in the aryl migration reaction of $9 \mathbf{a}$

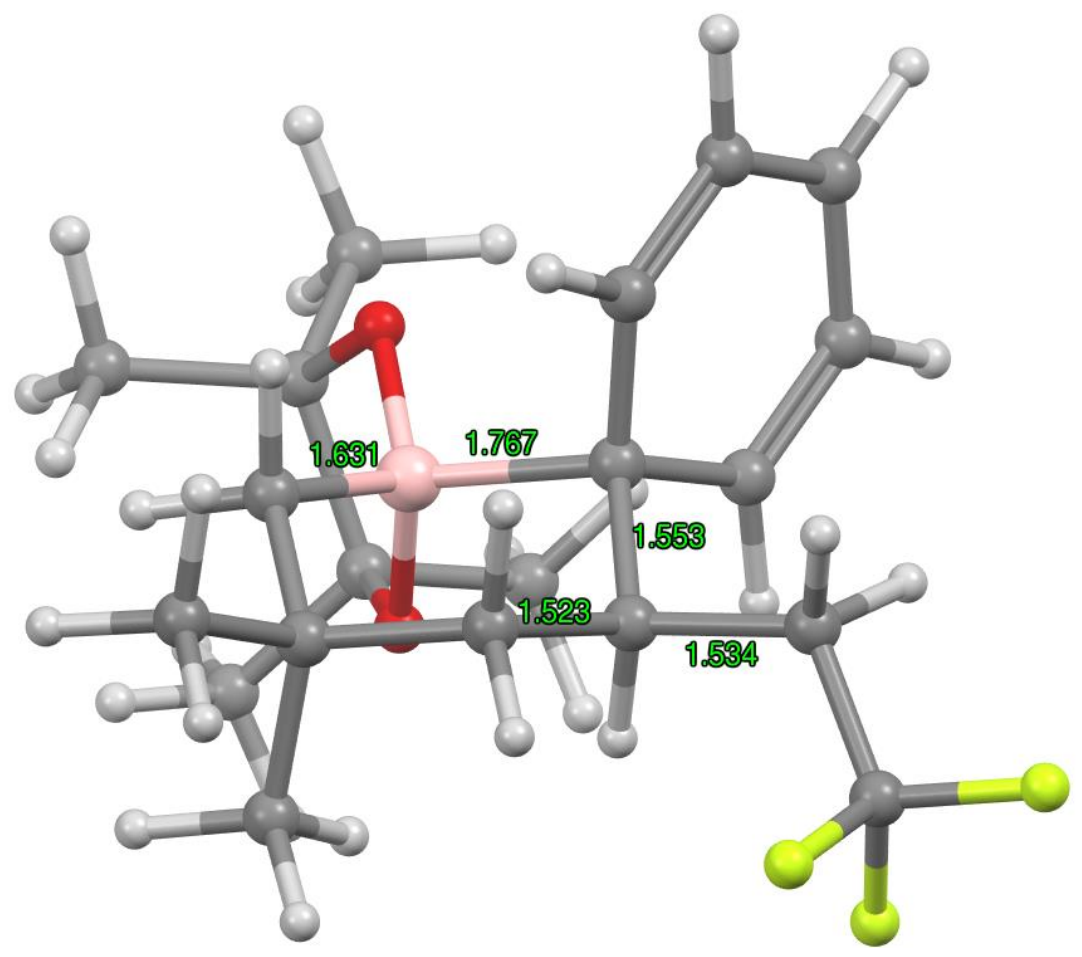

10a (cycl)

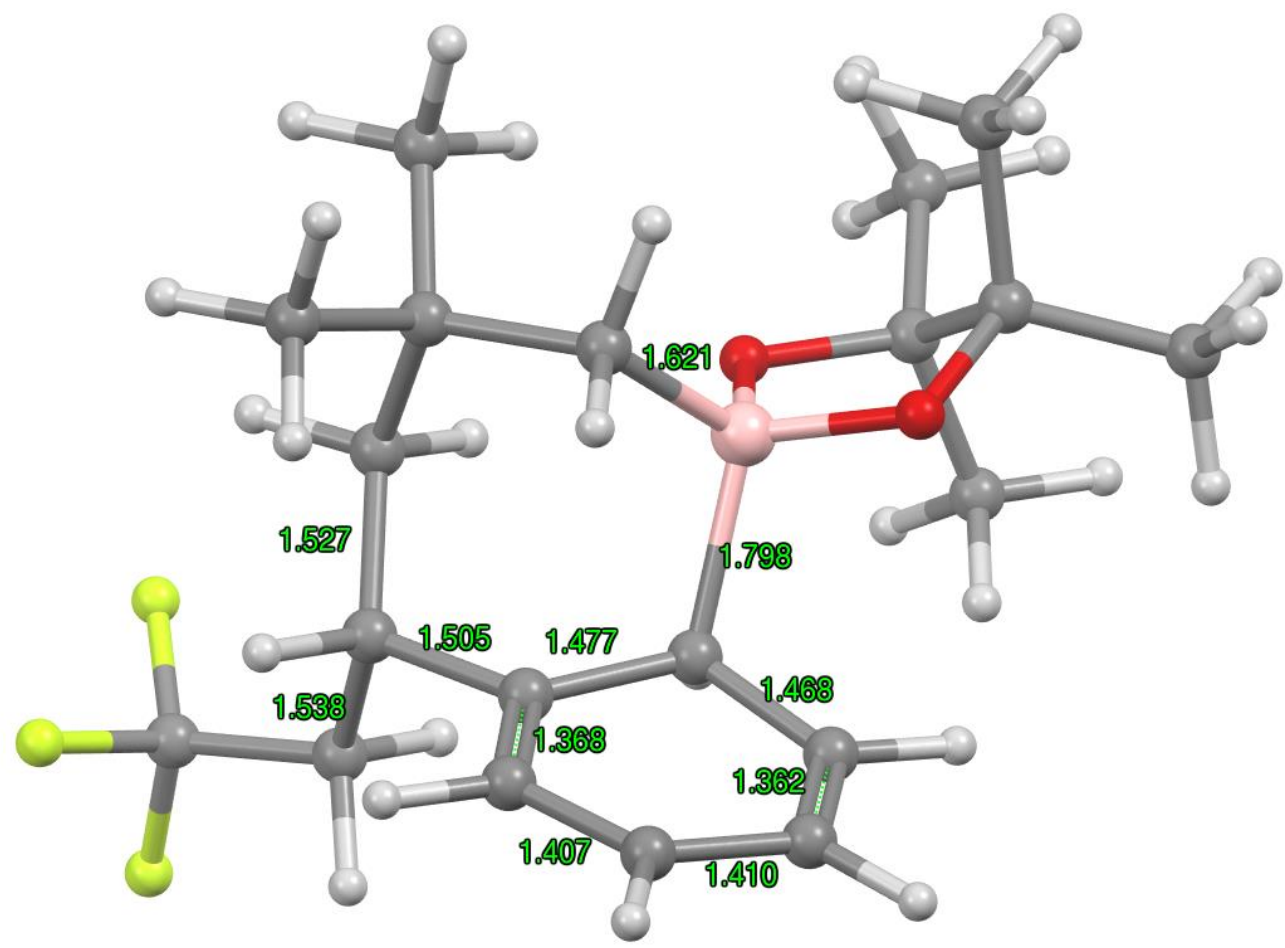

10a (cycl, ortho) 
Figure S2 (continued) Optimized molecular structures in the aryl migration reaction of $\mathbf{9 a}$

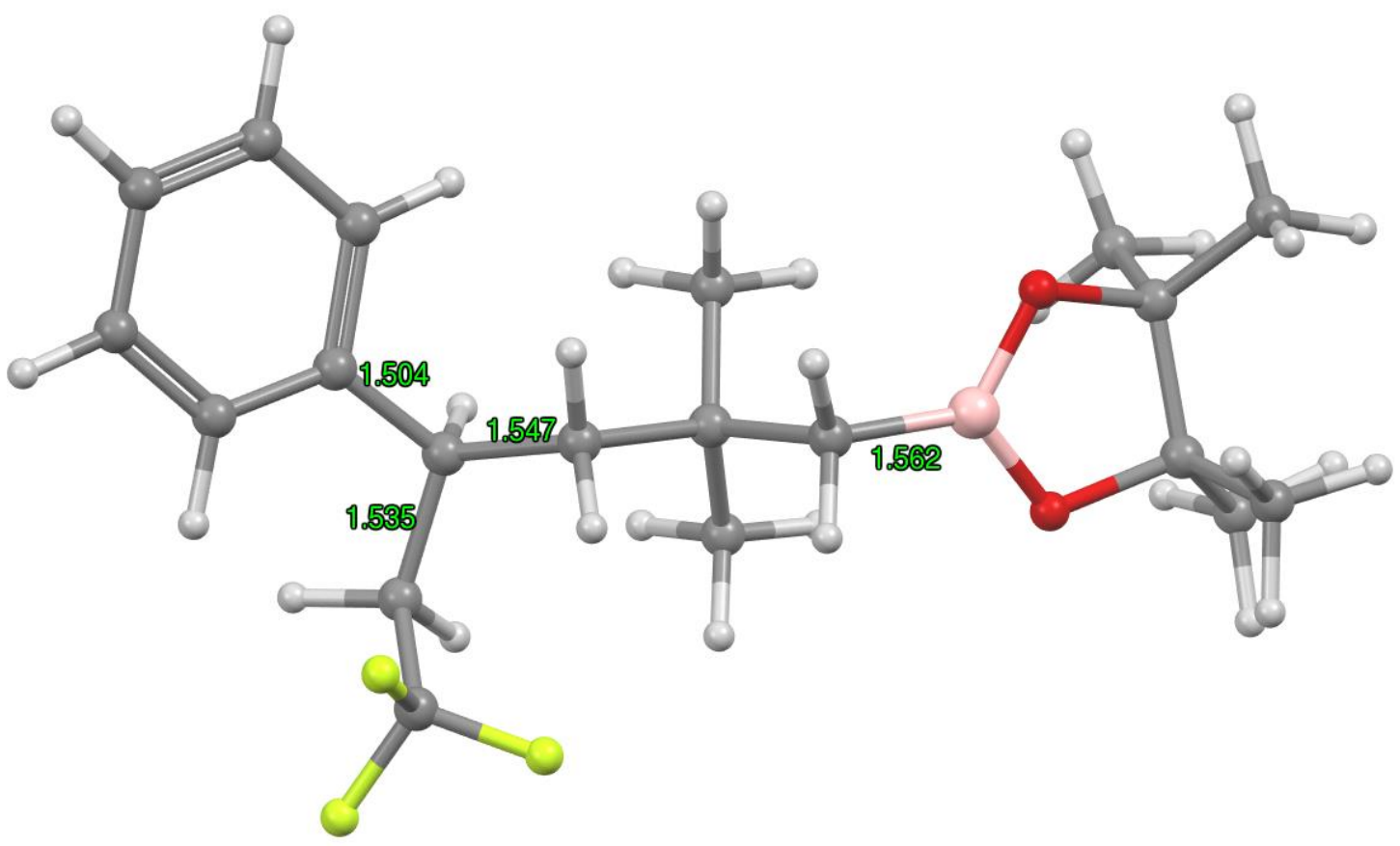

$10 a$

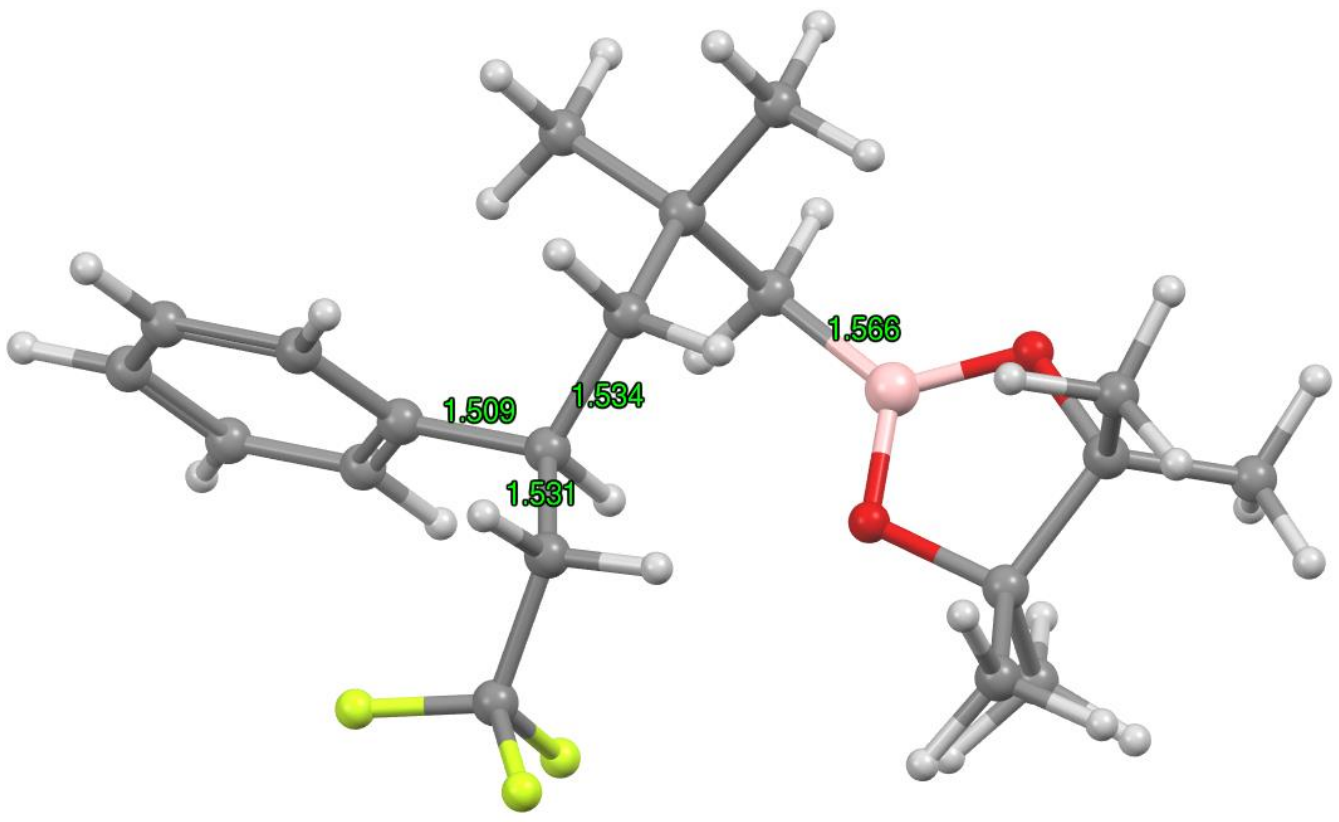


Figure S3 Optimized molecular structures in the aryl migration reaction of 9av

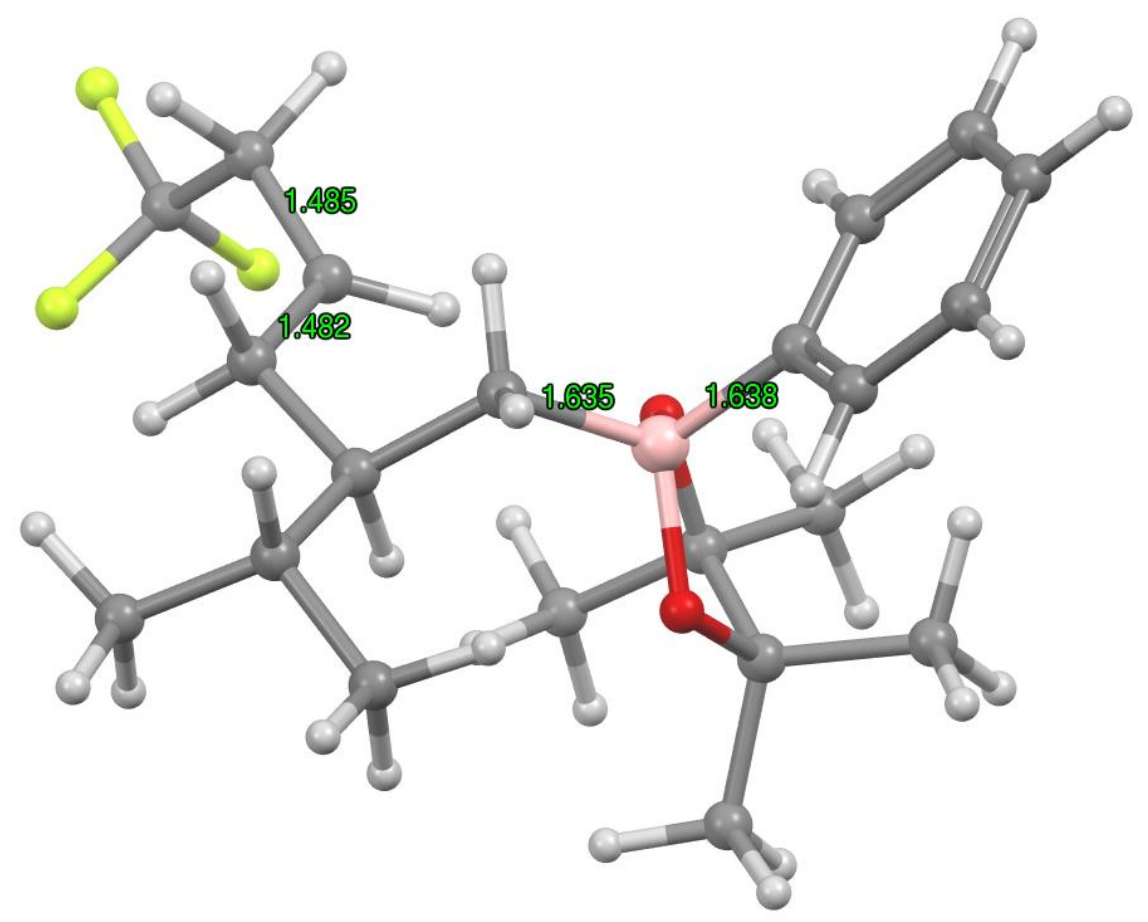

9av

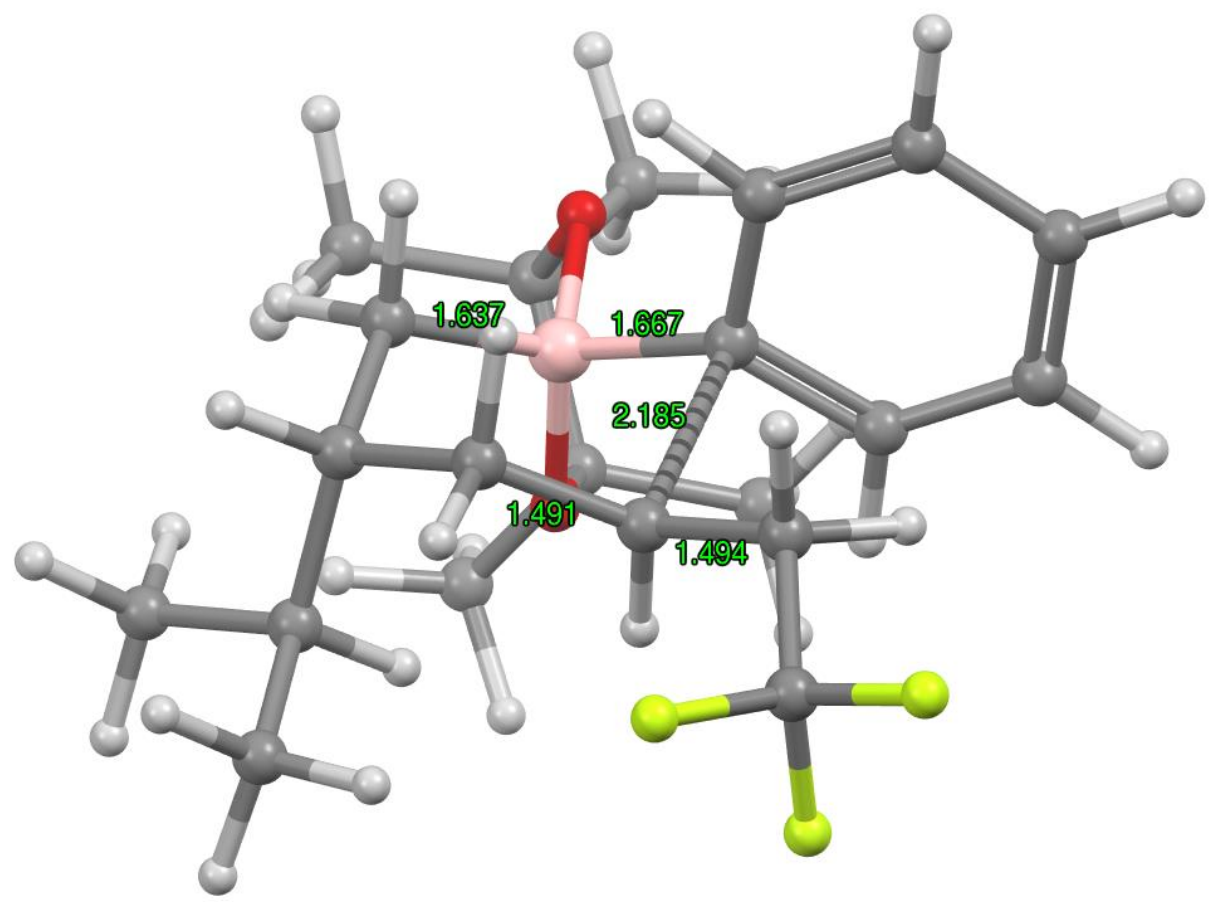

9av-TS syn 
Figure S3 (continued) Optimized molecular structures in the aryl migration reaction of 9av

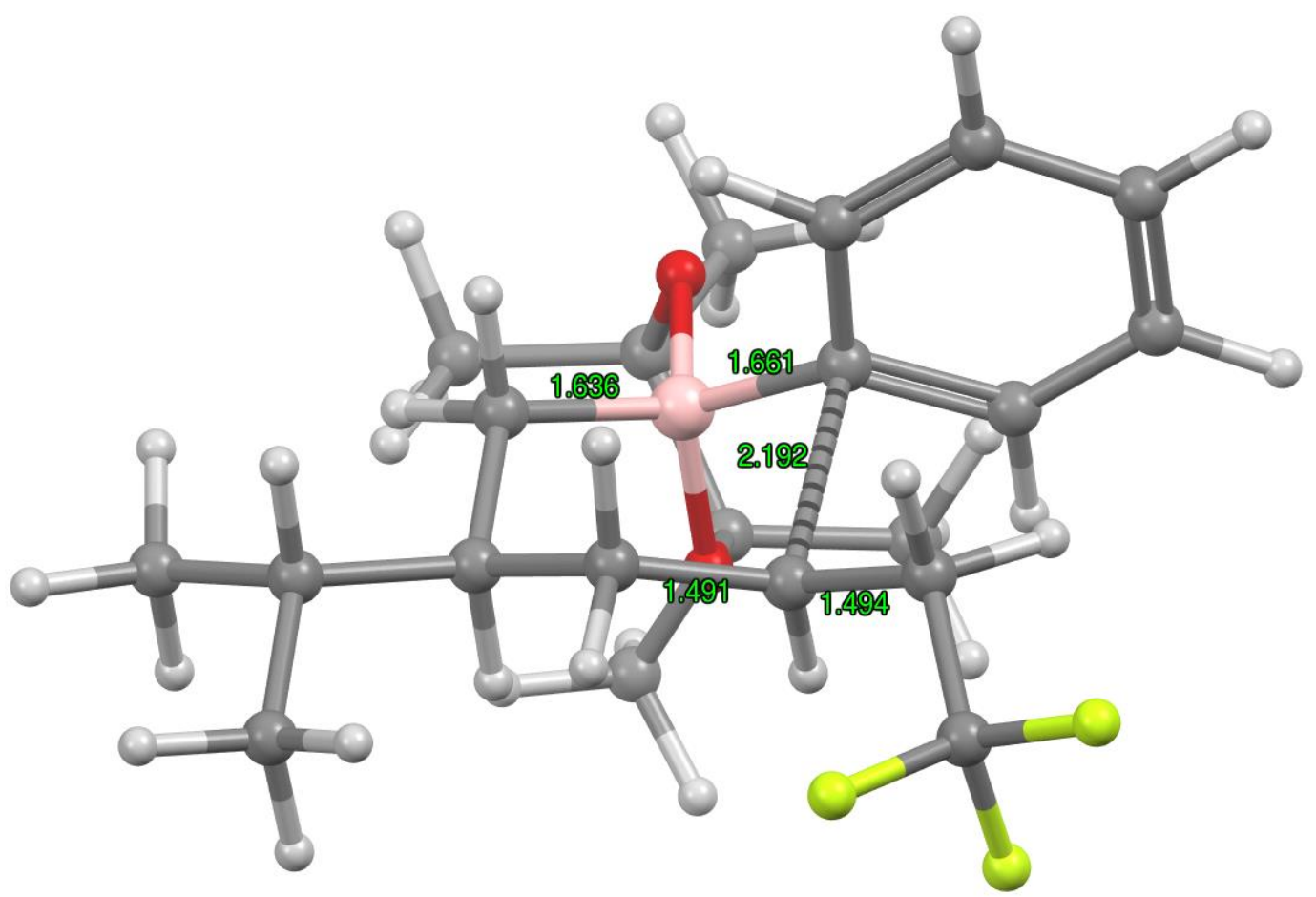

9av-TS $_{\text {anti }}$

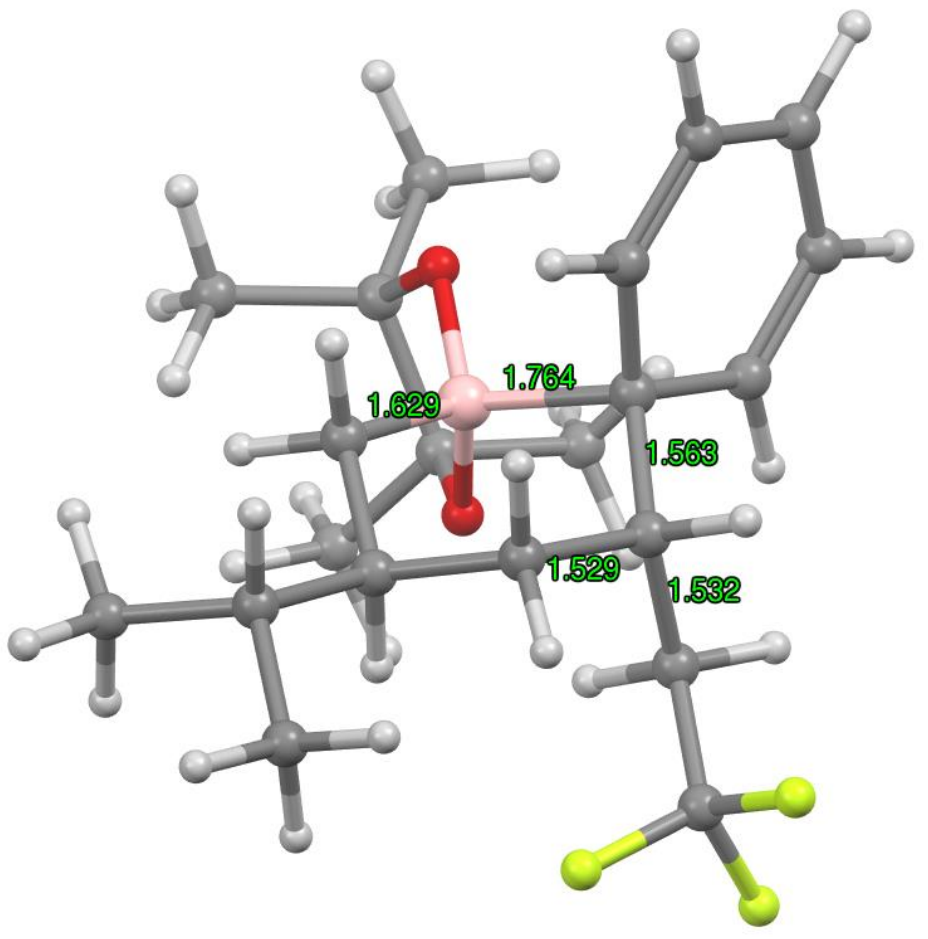

$10 \mathrm{av}_{\text {syn }}(\mathrm{cycl})$ 
Figure S3 (continued) Optimized molecular structures in the aryl migration reaction of 9av

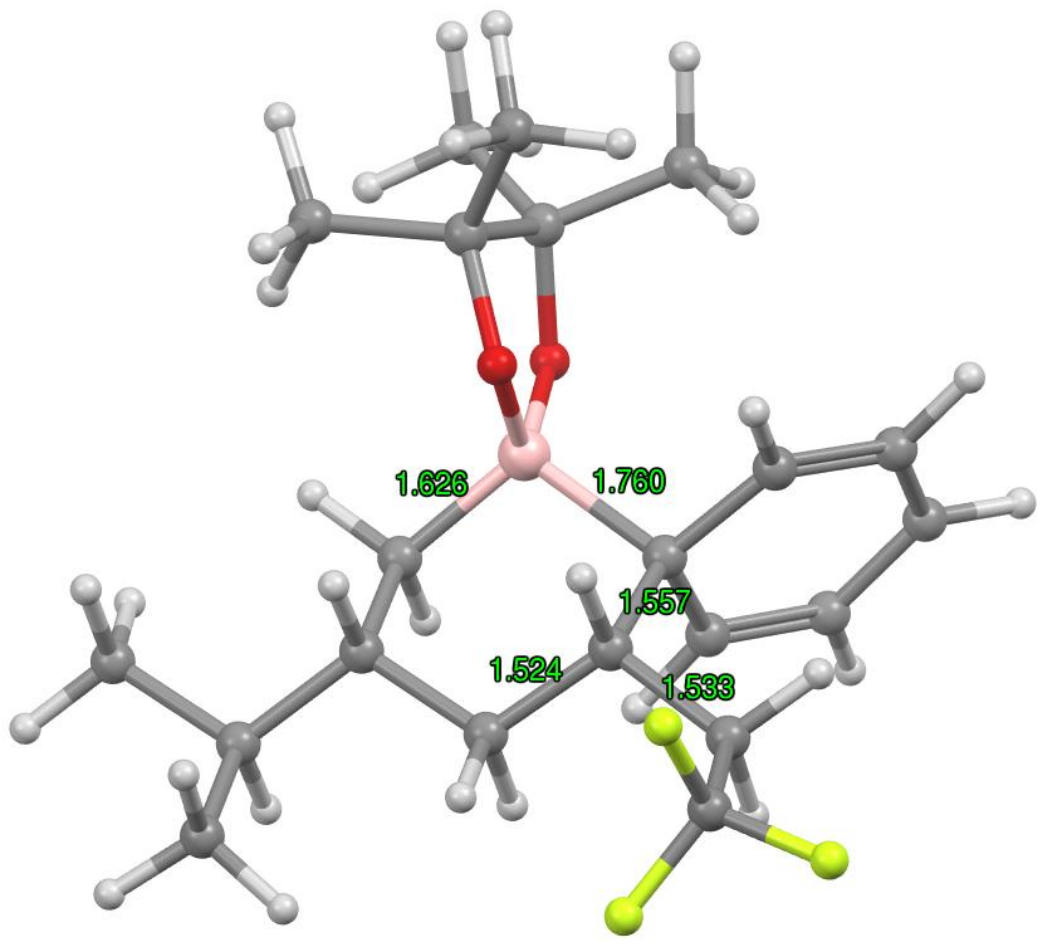

$10 \mathbf{a v}_{\text {anti }}$ (cycl)

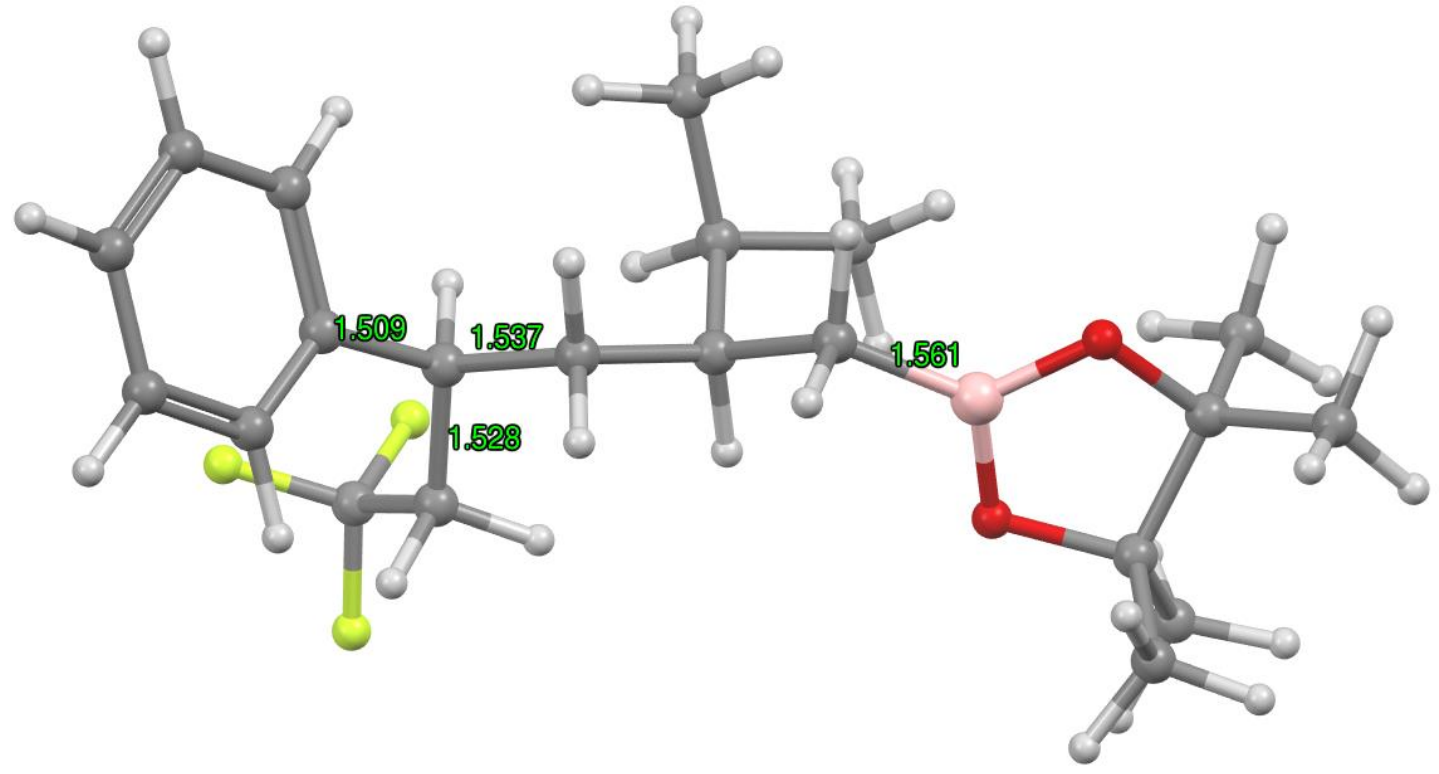

$10 \mathbf{a v}_{\text {syn }}$ 
Figure S3 (continued) Optimized molecular structures in the aryl migration reaction of 9av

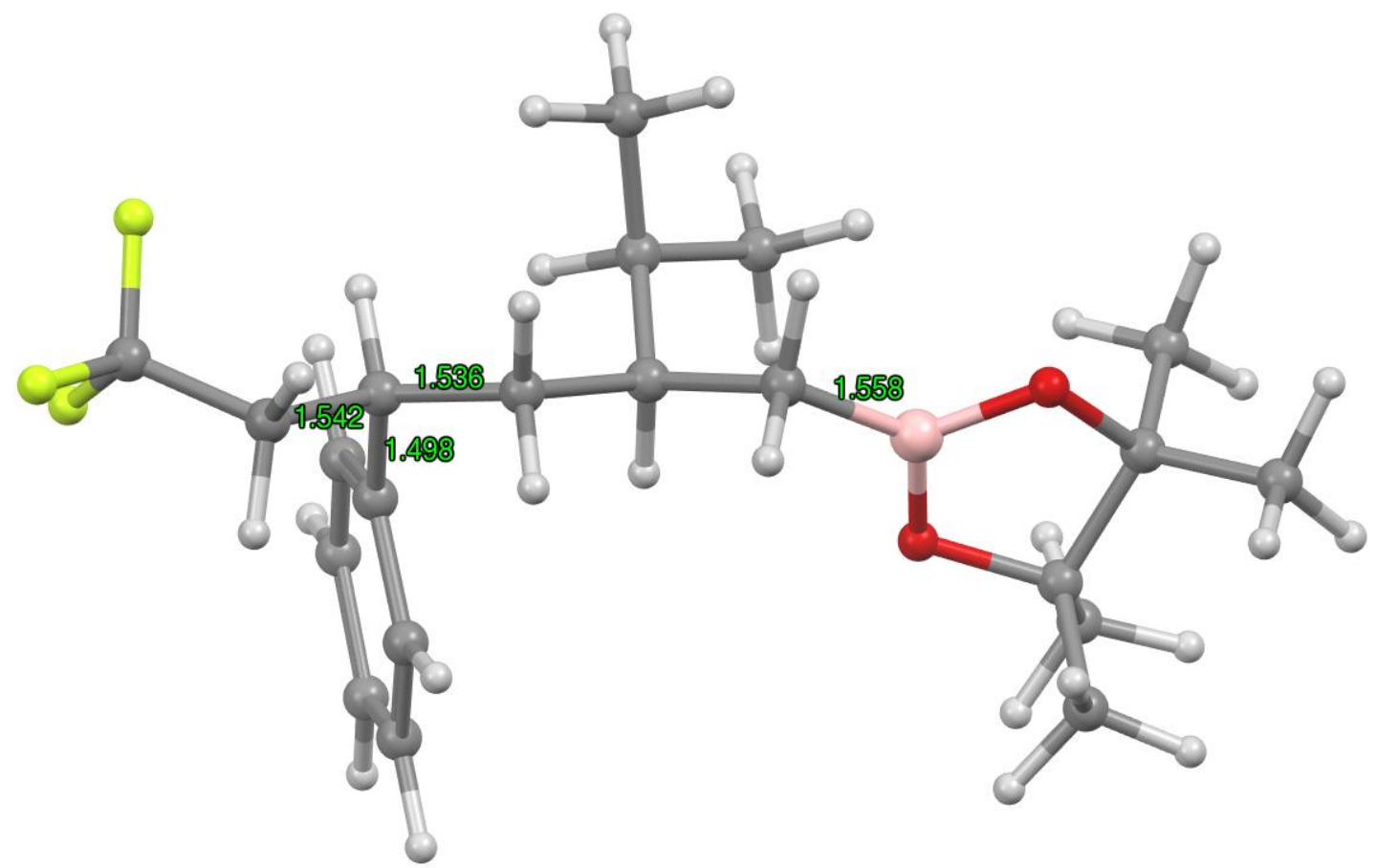

$10 \mathbf{a v}_{\text {anti }}$

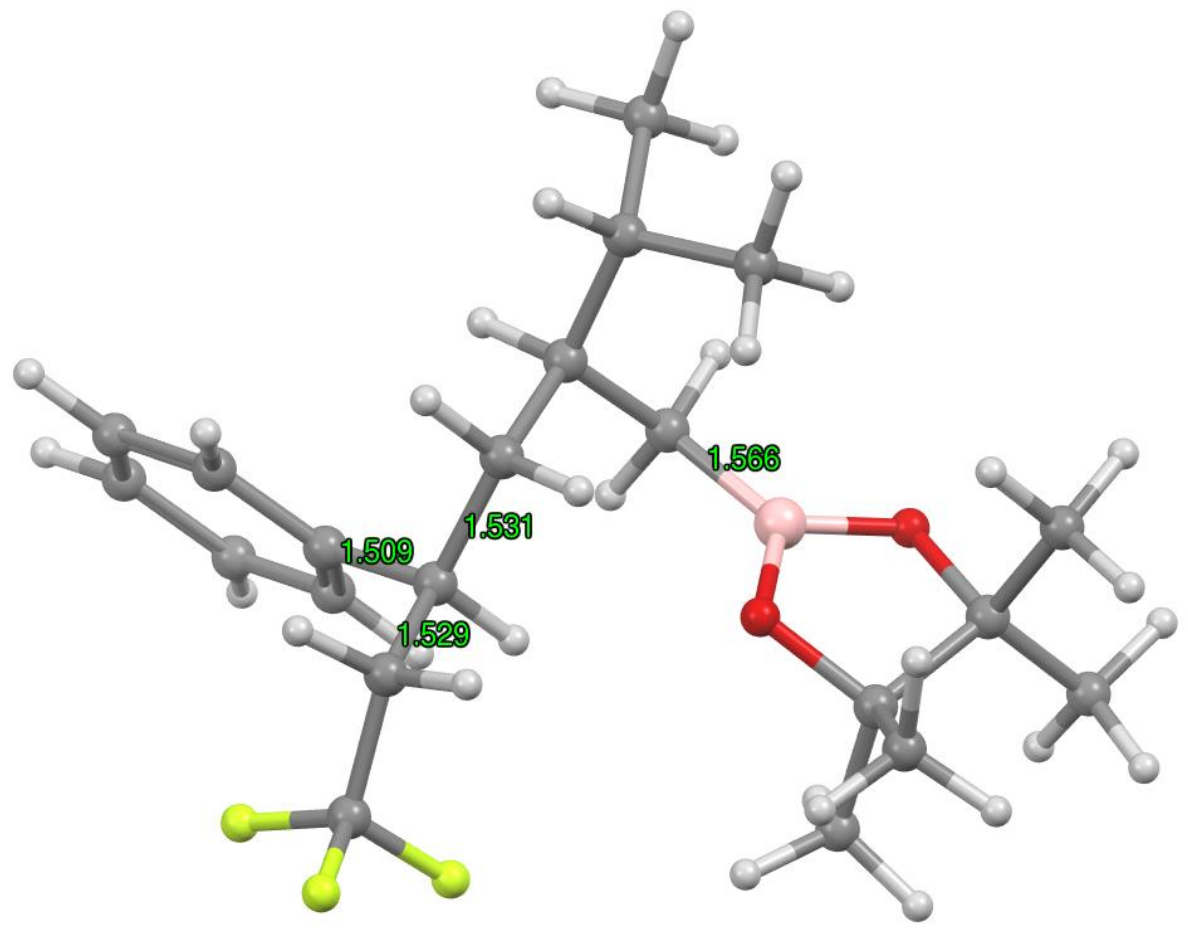

$8 \mathbf{a v}_{\text {syn }}$ 


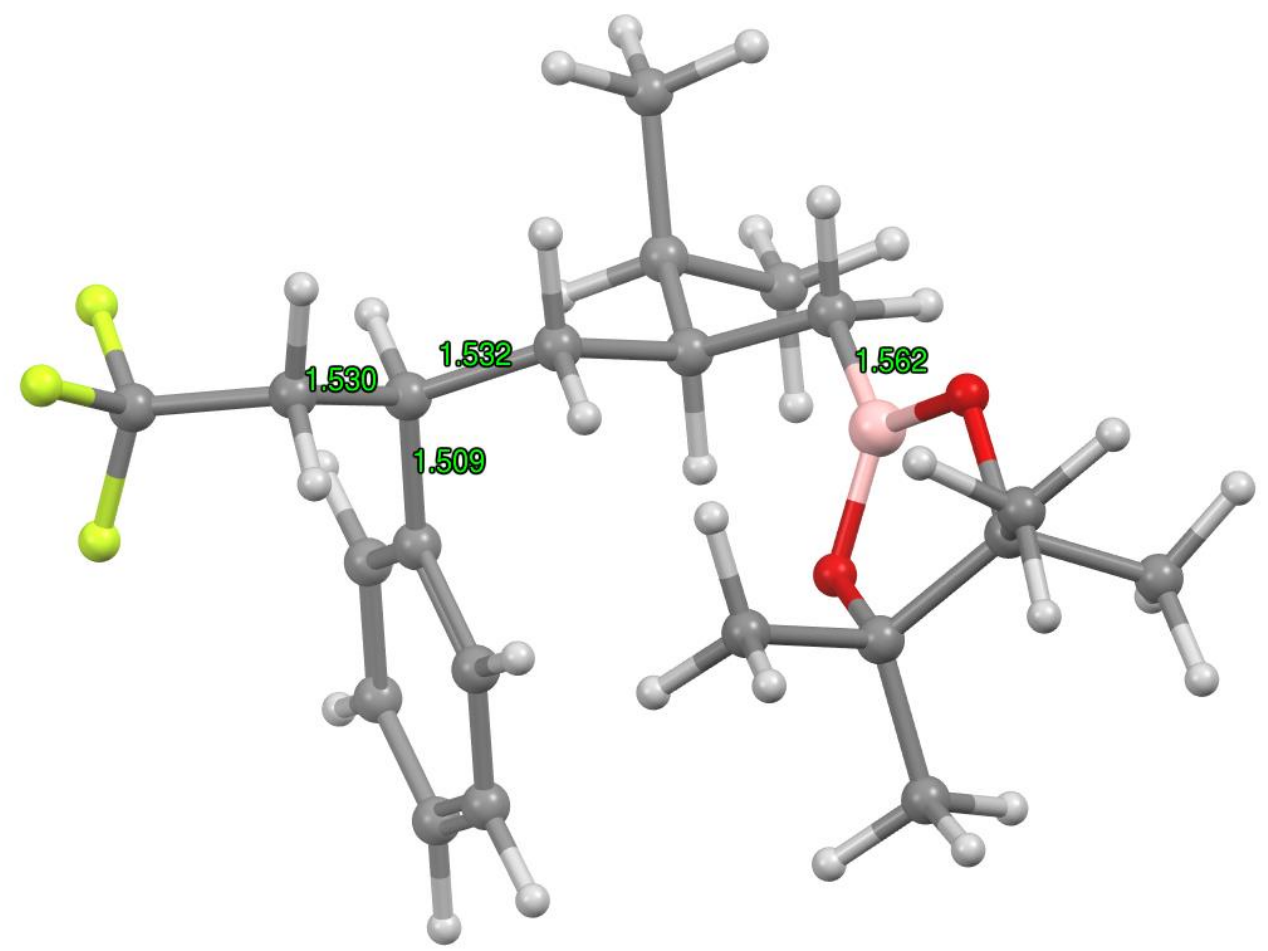

8avanti 
Figure S4 Optimized transition structures of the aryl migration reaction of 9ad

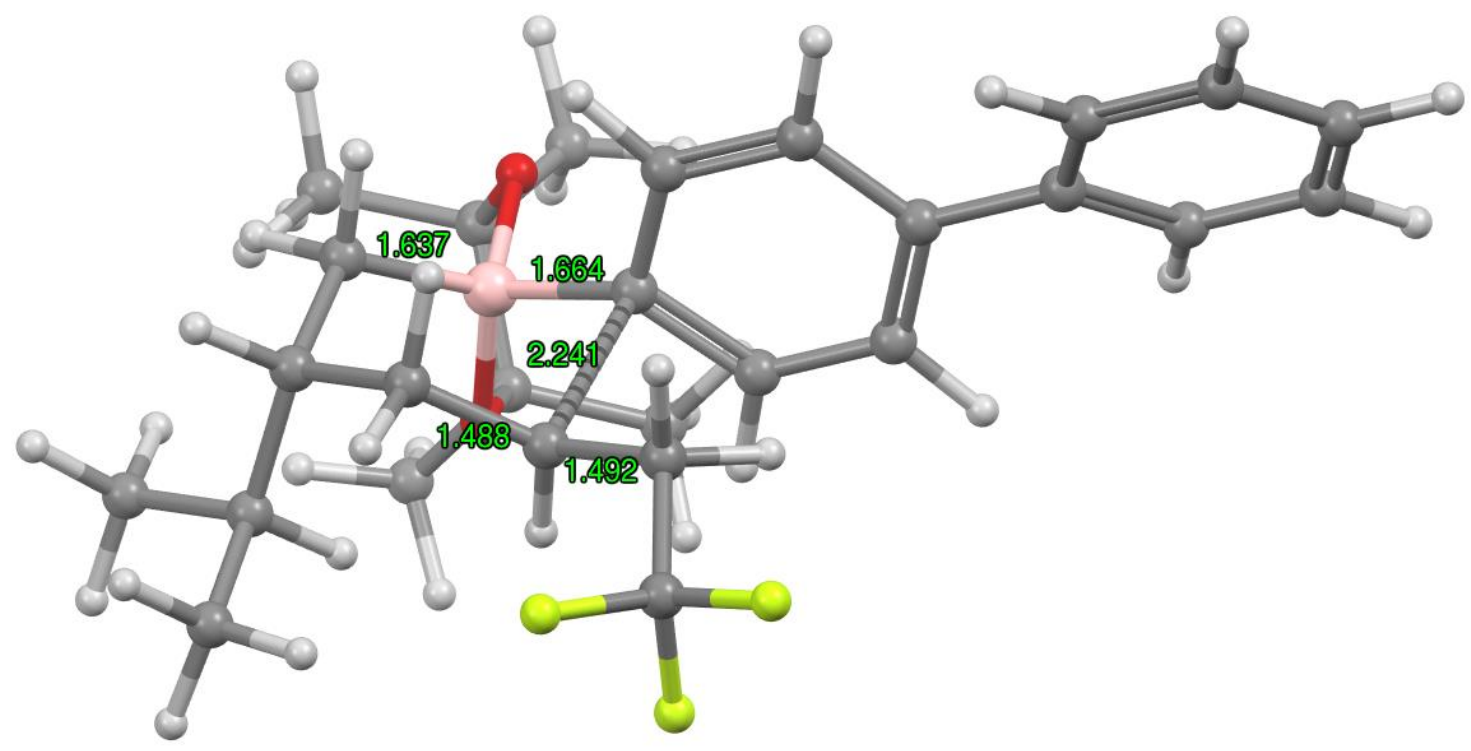

9ad-TS $_{\text {syn }}$

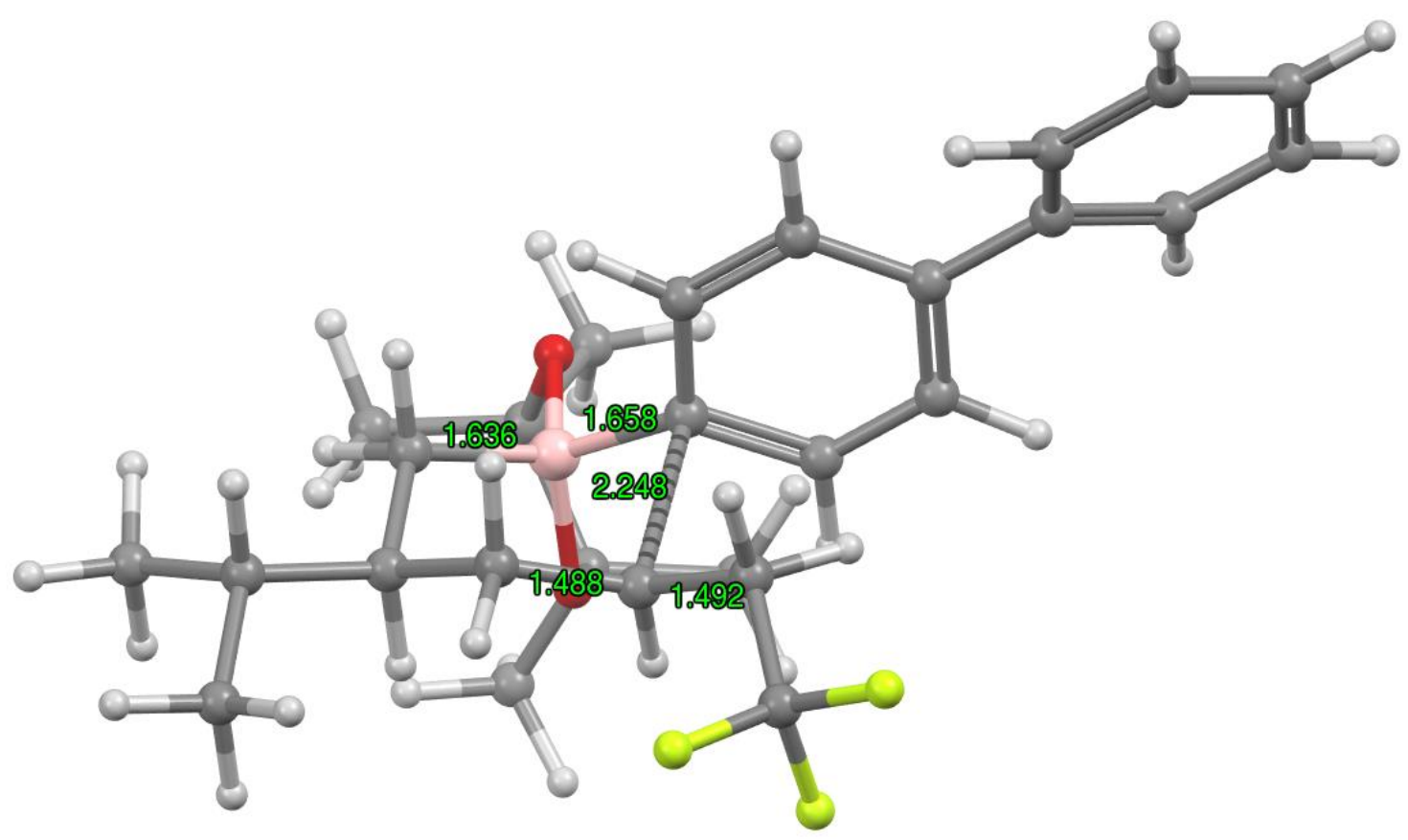

9ad-TS 
Figure S5 Optimized transition structures of the aryl migration reaction of 9aw

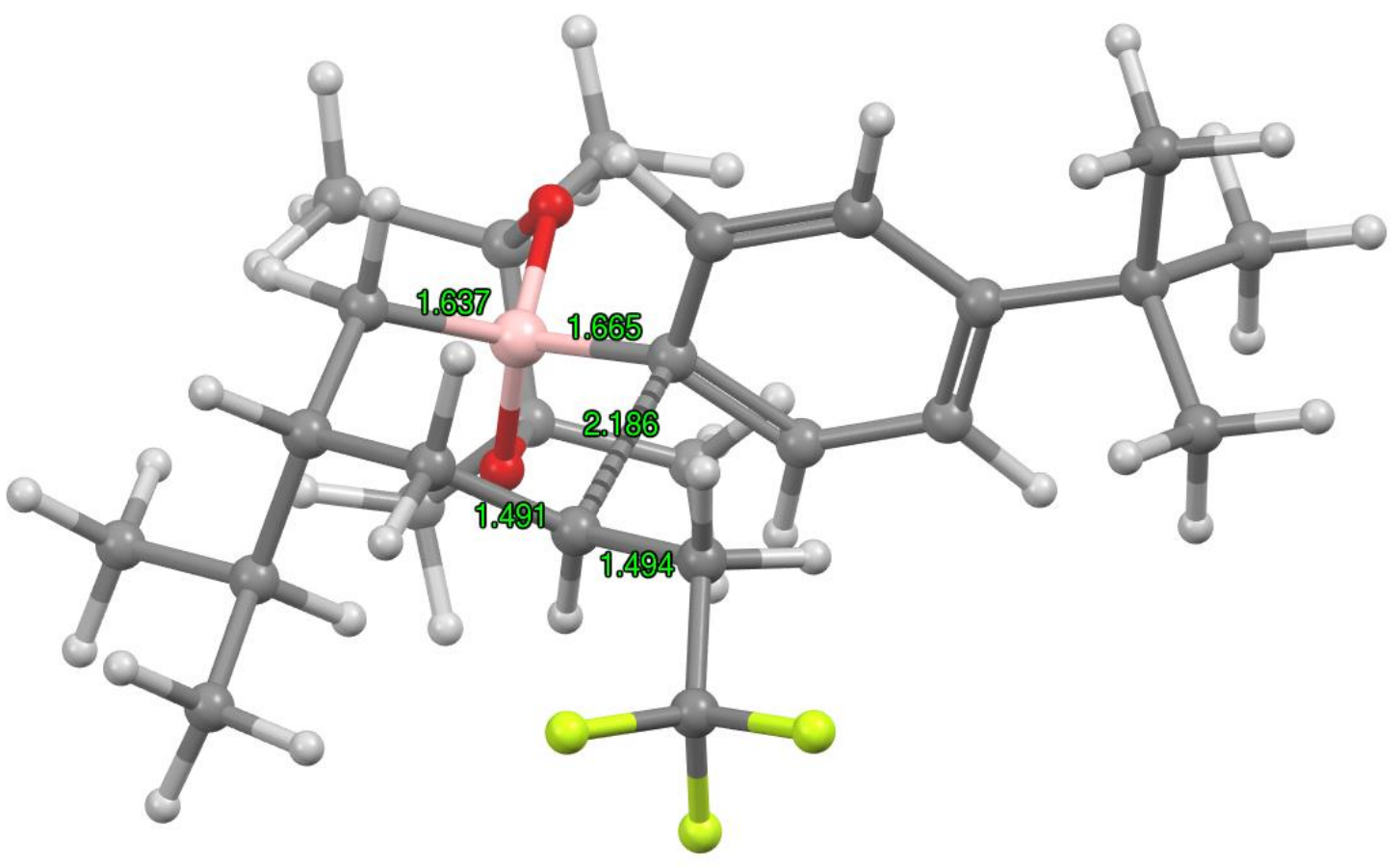

9aw-TS

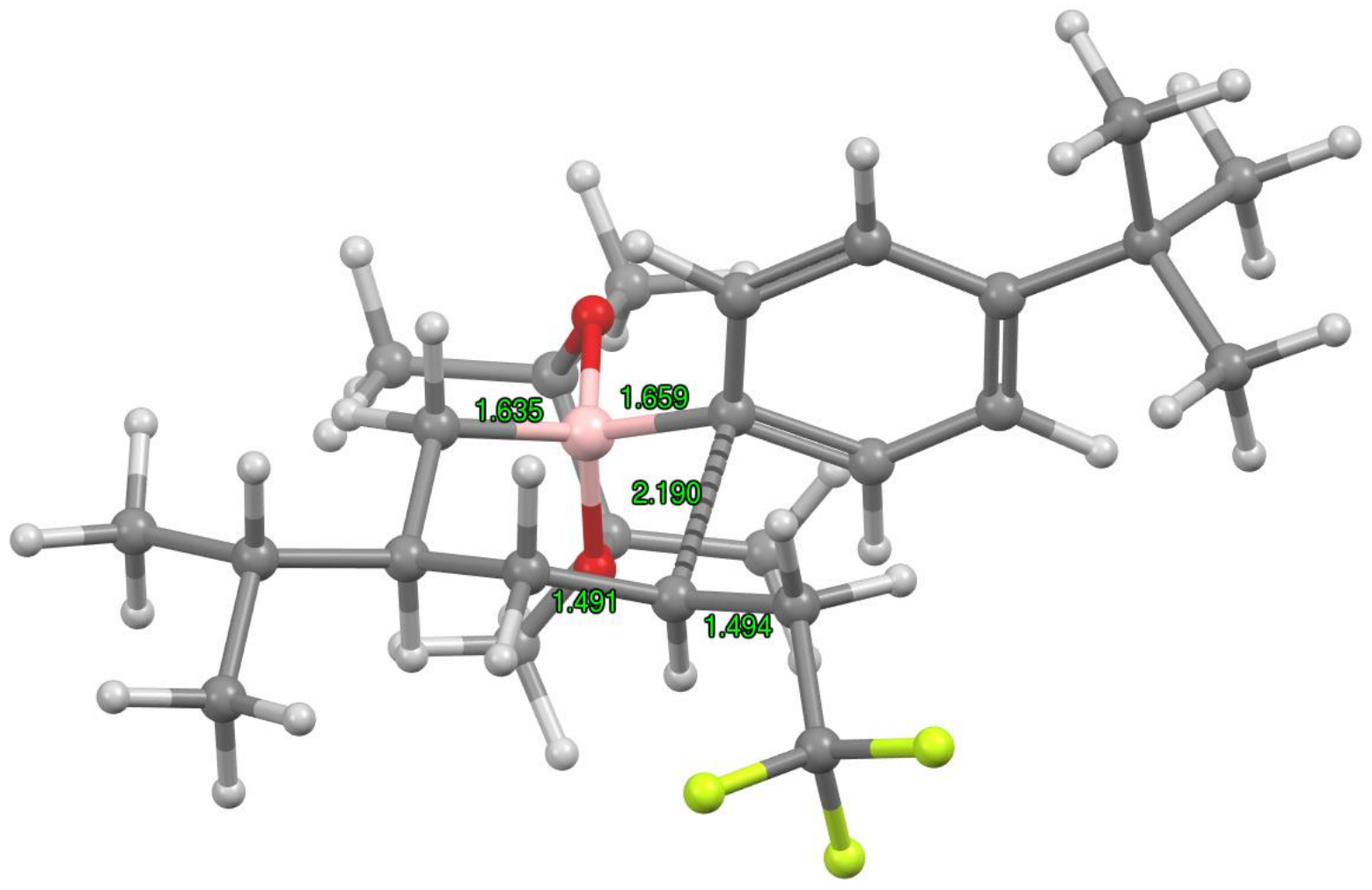

9aw-TS anti 


\section{Cartesian coordinates $(\AA)$ of all DFT-optimized species}

\begin{tabular}{|c|c|c|c|}
\hline \multicolumn{4}{|c|}{$9 a$} \\
\hline & est Freq. = & \multirow{2}{*}{\multicolumn{2}{|c|}{$8.80 \mathrm{~cm}^{\wedge}-1$}} \\
\hline 56 & & & \\
\hline \multicolumn{4}{|c|}{$9 \mathrm{a}(004 \mathrm{c} 1 / \mathrm{opt})$} \\
\hline $\mathrm{C}$ & -5.9427925 & -1.4457521 & -0.3193245 \\
\hline $\mathrm{C}$ & -4.4400744 & -1.5388160 & -0.3853549 \\
\hline $\mathrm{F}$ & -6.5273926 & -2.2577001 & -1.2233469 \\
\hline $\mathrm{F}$ & -6.3932146 & -0.2063623 & -0.5718660 \\
\hline $\mathrm{F}$ & -6.4299358 & -1.7993458 & 0.8803586 \\
\hline $\mathrm{H}$ & -4.1722328 & -1.3246469 & -1.4291284 \\
\hline $\mathrm{H}$ & -4.1804318 & -2.5829387 & -0.1971771 \\
\hline $\mathrm{C}$ & -3.7440965 & -0.6336927 & 0.5641469 \\
\hline $\mathrm{H}$ & -3.8822313 & 0.4318142 & 0.4231949 \\
\hline $\mathrm{C}$ & -1.2608665 & -1.1618773 & 1.0138466 \\
\hline $\mathrm{C}$ & -1.1793920 & -2.1069895 & -0.1805598 \\
\hline $\mathrm{C}$ & -0.4161349 & -1.7355568 & 2.1476668 \\
\hline $\mathrm{C}$ & -0.7875091 & 0.2530997 & 0.6631655 \\
\hline $\mathrm{H}$ & -0.7333866 & -2.7549052 & 2.3992041 \\
\hline $\mathrm{H}$ & -0.5077534 & -1.1188141 & 3.0475630 \\
\hline $\mathrm{H}$ & 0.6353606 & -1.7423711 & 1.8581591 \\
\hline $\mathrm{H}$ & -1.6315358 & -3.0779823 & 0.0577751 \\
\hline $\mathrm{H}$ & -0.1405683 & -2.2768105 & -0.4628631 \\
\hline $\mathrm{H}$ & -1.6795079 & -1.6936820 & -1.0597018 \\
\hline $\mathrm{C}$ & 3.1509847 & 2.2009969 & -0.8383132 \\
\hline $\mathrm{C}$ & 2.2954686 & 2.1583997 & 0.4304669 \\
\hline $\mathrm{C}$ & 2.4590306 & 3.4741097 & 1.1760663 \\
\hline $\mathrm{O}$ & 0.9481886 & 1.9886265 & 0.0971862 \\
\hline B & 0.6786687 & 0.5234251 & -0.0343692 \\
\hline $\mathrm{O}$ & 1.8444624 & -0.1074785 & 0.6562105 \\
\hline $\mathrm{C}$ & 2.5982601 & 0.8881476 & 1.2889863 \\
\hline $\mathrm{C}$ & 2.1133214 & 1.0568520 & 2.7309648 \\
\hline $\mathrm{C}$ & 4.0608099 & 0.4714178 & 1.3053966 \\
\hline $\mathrm{H}$ & -0.8212414 & 0.8506944 & 1.5859098 \\
\hline $\mathrm{H}$ & -1.5342775 & 0.7095619 & -0.0018264 \\
\hline $\mathrm{H}$ & 2.2452457 & 4.3040718 & 0.4971708 \\
\hline $\mathrm{H}$ & 3.4795348 & 3.5965155 & 1.5536605 \\
\hline $\mathrm{H}$ & 1.7620979 & 3.5353109 & 2.0121965 \\
\hline $\mathrm{H}$ & 4.4018312 & 0.2206241 & 0.3007919 \\
\hline $\mathrm{H}$ & 4.1806463 & -0.4148764 & 1.9348528 \\
\hline $\mathrm{H}$ & 4.6989287 & 1.2645841 & 1.7085119 \\
\hline $\mathrm{H}$ & 2.7427455 & 2.9683525 & -1.5004546 \\
\hline
\end{tabular}




$\begin{array}{lrrr}\mathrm{H} & 3.1081281 & 1.2485992 & -1.3670994 \\ \mathrm{H} & 4.1965340 & 2.4443872 & -0.6244229 \\ \mathrm{H} & 1.0831587 & 1.4153403 & 2.7432714 \\ \mathrm{H} & 2.7373571 & 1.7512273 & 3.3020137 \\ \mathrm{H} & 2.1407037 & 0.0814601 & 3.2228276 \\ \mathrm{H} & 2.2254127 & -1.3898923 & -1.4107781 \\ \mathrm{C} & 1.5334694 & -0.9206244 & -2.1048574 \\ \mathrm{C} & 0.6806206 & 0.0665255 & -1.6075894 \\ \mathrm{C} & -0.1822778 & 0.6622798 & -2.5312822 \\ \mathrm{C} & -0.2044490 & 0.2935181 & -3.8695734 \\ \mathrm{H} & -0.8870622 & 0.7836979 & -4.5585508 \\ \mathrm{C} & 0.6479828 & -0.7012801 & -4.3320646 \\ \mathrm{H} & 0.6341546 & -0.9964717 & -5.3766506 \\ \mathrm{C} & 1.5222523 & -1.3063383 & -3.4394085 \\ \mathrm{H} & 2.1995427 & -2.0815011 & -3.7880711 \\ \mathrm{H} & -0.8500869 & 1.4487529 & -2.1889398 \\ \mathrm{C} & -2.7336794 & -1.1171716 & 1.5273357 \\ \mathrm{H} & -2.7376138 & -0.4698029 & 2.4120585 \\ \mathrm{H} & -3.0051576 & -2.1287124 & 1.8626149\end{array}$

\section{9a-TS}

$\mathrm{E}(\mathrm{PBE} 0-\mathrm{D} 3 / \mathrm{def} 2-\mathrm{TZVP})=-1254.247634160$ (conv)

Lowest Freq. $=-376.17 \mathrm{~cm}^{\wedge}-1$

56

9a-TS (010TSc4/opt)

$\begin{array}{llll}\text { C } & -2.7866118 & 3.6170882 & -0.5627237\end{array}$

$\begin{array}{llll}\text { C } & -2.6552528 & 2.1244575 & -0.4529706\end{array}$

F $\quad-3.9365144 \quad 3.9673520 \quad-1.1834965$

F $\quad-1.7921595 \quad 4.1747123 \quad-1.2741627$

$\begin{array}{llll}\text { F } & -2.8036951 & 4.2484783 & 0.6268840\end{array}$

$\begin{array}{llll}\mathrm{H} & -2.7450237 & 1.7427099 & -1.4747164\end{array}$

$\begin{array}{llll}\mathrm{H} & -3.5278428 & 1.7695678 & 0.1029449\end{array}$

$\begin{array}{llll}\text { C } & -1.3904091 & 1.6425506 & 0.1798397\end{array}$

$\begin{array}{llll}\mathrm{H} & -0.4763195 & 1.9547379 & -0.3151563\end{array}$

$\begin{array}{llll}\text { C } & -0.0874740 & 1.0542721 & 2.3062050\end{array}$

$\begin{array}{llll}\text { C } & 0.1935399 & -0.3667376 & 1.8146449\end{array}$

$\begin{array}{llll}\text { C } & 1.9558934 & -0.8745058 & -2.6053755\end{array}$

$\begin{array}{llll}\text { C } & 2.2496414 & -0.7838131 & -1.1068303\end{array}$

$\begin{array}{llll}\text { C } & 3.6603655 & -0.2342807 & -0.9358147\end{array}$

$\begin{array}{llll}\text { O } & 1.3462361 & 0.0923997 & -0.4872495\end{array}$

$\begin{array}{llll}\text { B } & 0.2639088 & -0.6659620 & 0.2052617\end{array}$

$\begin{array}{llll}\text { O } & 0.6420634 & -2.0872346 & -0.0406280\end{array}$

$\begin{array}{llll}\text { C } & 1.9946107 & -2.1514904 & -0.3847949\end{array}$

$\begin{array}{llll}\text { C } & 2.8645607 & -2.3105140 & 0.8679451\end{array}$ 


$\begin{array}{lrrr}\mathrm{C} & 2.2165584 & -3.3768585 & -1.2615863 \\ \mathrm{H} & -0.5721731 & -1.0304518 & 2.2432510 \\ \mathrm{H} & 1.1318573 & -0.6977289 & 2.2759061 \\ \mathrm{H} & 3.7488112 & 0.7077417 & -1.4839577 \\ \mathrm{H} & 4.4125099 & -0.9274812 & -1.3262254 \\ \mathrm{H} & 3.8763332 & -0.0325186 & 0.1131981 \\ \mathrm{H} & 1.5214297 & -3.3856604 & -2.1005922 \\ \mathrm{H} & 2.0385143 & -4.2779108 & -0.6685148 \\ \mathrm{H} & 3.2410725 & -3.4178447 & -1.6455858 \\ \mathrm{H} & 1.9300583 & 0.1381668 & -3.0167981 \\ \mathrm{H} & 0.9890033 & -1.3431619 & -2.7870382 \\ \mathrm{H} & 2.7259900 & -1.4416913 & -3.1370692 \\ \mathrm{H} & 2.8325662 & -1.4191162 & 1.4926282 \\ \mathrm{H} & 3.9089104 & -2.5186264 & 0.6148787 \\ \mathrm{H} & 2.4735785 & -3.1476378 & 1.4511145 \\ \mathrm{H} & -2.3694064 & -1.3263070 & 1.0673305 \\ \mathrm{C} & -2.3731826 & -1.0439776 & 0.0193804 \\ \mathrm{C} & -1.2130083 & -0.4217064 & -0.5227626 \\ \mathrm{C} & -1.2777418 & -0.1785364 & -1.9234795 \\ \mathrm{C} & -2.3950363 & -0.4517615 & -2.6825690 \\ \mathrm{H} & -2.3898594 & -0.2348531 & -3.7475896 \\ \mathrm{C} & -3.5319112 & -1.0142370 & -2.0958200 \\ \mathrm{H} & -4.4127264 & -1.2319275 & -2.6899458 \\ \mathrm{C} & -3.5015037 & -1.3062117 & -0.7328921 \\ \mathrm{H} & -4.3642584 & -1.7721543 & -0.2641815 \\ \mathrm{H} & -0.4117772 & 0.2720032 & -2.3939075 \\ \mathrm{C} & -1.3701489 & 1.5898545 & 1.6682807 \\ \mathrm{H} & -1.5624645 & 2.5982052 & 2.0720357 \\ \mathrm{H} & -2.2184135 & 0.9763066 & 2.0054649 \\ \mathrm{C} & 1.0871815 & 1.9782069 & 1.9859308 \\ \mathrm{H} & 1.3396561 & 1.9496036 & 0.9265643 \\ \mathrm{H} & 1.9766228 & 1.6526260 & 2.5353775 \\ \mathrm{H} & 0.8660864 & 3.0105235 & 2.2819192 \\ & -0.2926587 & 1.0562582 & 3.8208592 \\ -1.1438754 & 0.4260787 & 4.0977526 \\ & -0.4796844 & 2.0678062 & 4.2021590 \\ \mathrm{H} & 0.6611920 & 4.3266776\end{array}$

\section{0a(cycl,ipso)}

$\mathrm{E}(\mathrm{PBE}-\mathrm{D} 3 / \mathrm{def} 2-\mathrm{TZVP})=-1254.279303968$ (conv)

Lowest Freq. $=10.38 \mathrm{~cm}^{\wedge}-1$

56

10a(cycl ipso) (006c3/opt)

$\begin{array}{llll}\text { C } & -3.9426478 & 1.2288045 & 2.0150126\end{array}$ 


\begin{tabular}{|c|c|c|c|}
\hline & & & \\
\hline & .2797704 & & \\
\hline & -3.4881160 & & \\
\hline & & & \\
\hline & & & \\
\hline & 46 & 90 & 1318 \\
\hline & & & \\
\hline & & & \\
\hline & & & \\
\hline & & & \\
\hline & & & \\
\hline & & & \\
\hline & 23 & & 267 \\
\hline & & & \\
\hline & & & \\
\hline & & 052 & \\
\hline & 402 & -1.6 & \\
\hline & 0.1830477 & -2.6 & \\
\hline & & & \\
\hline & 1.807 & $1.26 ?$ & \\
\hline & 3.0209630 & 006 & \\
\hline & & & \\
\hline & & & \\
\hline & 1.06 & -0.9 & \\
\hline & 2.15 & $-0.1\}$ & \\
\hline & & & \\
\hline & & -0.3 & \\
\hline & 3368 & -2.1 & \\
\hline & 9935130 & & \\
\hline & & & \\
\hline & 3.7299006 & 2.2125868 & \\
\hline & 3.5351364 & 1.6040852 & 7233 \\
\hline & 2987893 & -0.0848099 & \\
\hline & & & \\
\hline & 3.0285032 & 0.3324131 & -3.7149574 \\
\hline & 0.65854 & 9794461 & -1.8 \\
\hline & & & \\
\hline & 1.6916978 & 2.3763491 & -3.1670666 \\
\hline & 3.4402923 & -0.6173518 & -0.0901343 \\
\hline & 4.33314 & -0.22241 & \\
\hline & 3.5141541 & -1.7897669 & -1.4066367 \\
\hline & -1.4820144 & & \\
\hline & -1.6507394 & -1.7978218 & -0.4828882 \\
\hline & -1.2597819 & -0.3963915 & -0.2504183 \\
\hline
\end{tabular}




$\begin{array}{lrrr}\mathrm{C} & -1.6748428 & 0.4800249 & -1.3444683 \\ \mathrm{C} & -2.1523749 & 0.0196934 & -2.5338369 \\ \mathrm{H} & -2.3791857 & 0.7273946 & -3.3276425 \\ \mathrm{C} & -2.3667150 & -1.3563674 & -2.7510628 \\ \mathrm{H} & -2.7423472 & -1.7188407 & -3.7004559 \\ \mathrm{C} & -2.1271439 & -2.2397624 & -1.6824160 \\ \mathrm{H} & -2.3286206 & -3.2996669 & -1.8138973 \\ \mathrm{H} & -1.5323660 & 1.5462642 & -1.1992306 \\ \mathrm{C} & -1.2754547 & -0.6106972 & 2.3310832 \\ \mathrm{H} & -1.7304023 & -0.2005807 & 3.2385767 \\ \mathrm{H} & -1.6463525 & -1.6406846 & 2.2505533\end{array}$

\section{0a(cycl,ortho)}

$\mathrm{E}(\mathrm{PBE} 0-\mathrm{D} 3 /$ def2-TZVP $)=-1254.278528771$ (conv)

Lowest Freq. $=18.46 \mathrm{~cm}^{\wedge}-1$

56

$10 \mathrm{a}$ (cycl ortho) (007c1/opt)

$\begin{array}{lrrr}\text { C } & -1.3921274 & -1.5356029 & 3.7401965 \\ \text { C } & -1.6646314 & -1.3277819 & 2.2787802 \\ \text { F } & -2.5482035 & -1.6171305 & 4.4386477 \\ \text { F } & -0.6860987 & -0.5489388 & 4.3148805 \\ \text { F } & -0.7257181 & -2.6785069 & 3.9883915 \\ \text { H } & -2.0987403 & -0.3304563 & 2.1715827 \\ \mathrm{H} & -2.4389284 & -2.0515226 & 2.0136430 \\ \mathrm{C} & -0.4760383 & -1.5010011 & 1.3182538 \\ \mathrm{H} & -0.0432573 & -2.4863291 & 1.5235930 \\ \mathrm{C} & 1.7603448 & -0.3998343 & 0.5470704 \\ \mathrm{C} & 2.5366154 & -1.7133526 & 0.6187583 \\ \mathrm{C} & 2.6778422 & 0.7361073 & 1.0016974 \\ \mathrm{C} & 1.3122897 & -0.1214314 & -0.9028584 \\ \mathrm{H} & 3.0012913 & 0.5968671 & 2.0400448 \\ \mathrm{H} & 2.1518707 & 1.6898600 & 0.9230690 \\ \mathrm{H} & 3.5710096 & 0.7815582 & 0.3701821 \\ \mathrm{H} & 2.8847568 & -1.9224599 & 1.6379462 \\ \mathrm{H} & 3.4117171 & -1.6707885 & -0.0372658 \\ \mathrm{H} & 1.9195067 & -2.5522855 & 0.2867807 \\ \mathrm{C} & -2.0102294 & 3.1898001 & -0.8906278 \\ \mathrm{C} & -0.4867426 & 3.0830535 & -0.9775602 \\ \mathrm{C} & 0.1281278 & 4.3499467 & -0.4052256 \\ \mathrm{O} & -0.0447209 & 1.9823415 & -0.2303397 \\ \mathrm{~B} & -0.0179529 & 0.7830030 & -1.1019356 \\ \mathrm{O} & -0.1836268 & 1.3308103 & -2.4576116 \\ \mathrm{C} & -0.0109317 & 2.7199046 & -2.4172924 \\ \mathrm{C} & 1.4678334 & 3.0567350 & -2.6218105\end{array}$




$\begin{array}{lrrr}\mathrm{C} & -0.8237924 & 3.3565304 & -3.5329231 \\ \mathrm{H} & 1.1396727 & -1.0704105 & -1.4227194 \\ \mathrm{H} & 2.1529704 & 0.3511399 & -1.4296165 \\ \mathrm{H} & -0.2664146 & 4.5254758 & 0.5991511 \\ \mathrm{H} & -0.1096455 & 5.2222549 & -1.0222712 \\ \mathrm{H} & 1.2114437 & 4.2563950 & -0.3294515 \\ \mathrm{H} & -1.8631911 & 3.0304173 & -3.4914241 \\ \mathrm{H} & -0.4146124 & 3.0517948 & -4.4995874 \\ \mathrm{H} & -0.7921709 & 4.4494226 & -3.4771620 \\ \mathrm{H} & -2.2960387 & 3.2116930 & 0.1639048 \\ \mathrm{H} & -2.4870781 & 2.3255892 & -1.3539487 \\ \mathrm{H} & -2.3882076 & 4.0972334 & -1.3707792 \\ \mathrm{H} & 2.0652563 & 2.6682572 & -1.7959217 \\ \mathrm{H} & 1.6411000 & 4.1337120 & -2.7087578 \\ \mathrm{H} & 1.8050490 & 2.5735798 & -3.5417221 \\ \mathrm{H} & -0.7293850 & -3.6272975 & -0.2398352 \\ \mathrm{C} & -1.1121043 & -2.7365399 & -0.7351804 \\ \mathrm{C} & -1.0191325 & -1.5365783 & -0.0852589 \\ \mathrm{C} & -1.4251408 & -0.2768452 & -0.7399976 \\ \mathrm{C} & -2.1987633 & -0.5000477 & -1.9676744 \\ \mathrm{H} & -2.6408608 & 0.3650743 & -2.4488125 \\ \mathrm{C} & -2.2764751 & -1.7111095 & -2.5870063 \\ \mathrm{H} & -2.7942213 & -1.7933695 & -3.5387845 \\ \mathrm{C} & -1.7001306 & -2.8594797 & -2.0071972 \\ \mathrm{H} & -1.7543093 & -3.8226528 & -2.5006855 \\ \mathrm{H} & -1.9412637 & 0.4006547 & -0.0447476 \\ \mathrm{C} & 0.5879363 & -0.4278167 & 1.5406421 \\ \mathrm{H} & 0.1104381 & 0.5555485 & 1.5191479 \\ \mathrm{H} & 1.0039179 & -0.5526211 & 2.5468883\end{array}$

\section{$10 \mathbf{a}$}

$\mathrm{E}(\mathrm{PBE}-\mathrm{D} 3 / \mathrm{def} 2-\mathrm{TZVP})=-1254.244570795$ (conv)

\begin{tabular}{|c|c|c|c|}
\hline \multirow{2}{*}{\multicolumn{2}{|c|}{$\begin{array}{l}\text { Lowest Freq. = } \\
56 \\
10 \mathrm{a}(029 \mathrm{c} 1 / \mathrm{opt})\end{array}$}} & \multicolumn{2}{|l|}{$7.73 \mathrm{~cm}^{\wedge}-1$} \\
\hline & & & \\
\hline & -2.7638562 & 3.2636972 & 1.4145815 \\
\hline & -2.6326433 & 1.9797617 & 2.1910638 \\
\hline & -3.1231596 & 4.2744303 & 2.2427287 \\
\hline & -3.6644448 & 3.2440920 & 0.4293978 \\
\hline & -1.5989224 & 3.6604632 & 0.8545410 \\
\hline & -3.4601108 & 1.9665719 & 2.9030489 \\
\hline & -1.7165279 & 2.1083109 & 2.7727331 \\
\hline & -2.6124354 & 0.6354045 & 1.4505301 \\
\hline & -2.0950030 & -0.0444717 & \\
\hline
\end{tabular}




\begin{tabular}{|c|c|c|c|}
\hline & & & \\
\hline & & .2915514 & \\
\hline & & & \\
\hline & & & \\
\hline & & & \\
\hline & & & 834 \\
\hline & & & \\
\hline & & & \\
\hline & & & \\
\hline & -0.6573738 & -1.8 & \\
\hline & & & \\
\hline & & & \\
\hline & 3067 & -2.12 & \\
\hline & & & \\
\hline & & & \\
\hline & 2.92 & & \\
\hline & 4.1073230 & 39059 & \\
\hline & 4.47 & & \\
\hline & & & \\
\hline & 0.2867 & & \\
\hline & 345 & & \\
\hline & & -3.1 & \\
\hline & & & \\
\hline & & & \\
\hline & 6827 & & \\
\hline & & & \\
\hline & & & \\
\hline & 6741 & -3.1227511 & \\
\hline & & & \\
\hline & & & \\
\hline & & & \\
\hline & 5.4061909 & & \\
\hline & & & \\
\hline & & & \\
\hline & -4.0045721 & -1.3599967 & 0.8576293 \\
\hline & -3.97 & 82 & \\
\hline & -5.1778569 & & \\
\hline & -6.4115141 & 0.0464875 & 1.2366867 \\
\hline & -7.3321 & 5997027 & 6542 \\
\hline & & & \\
\hline & -7.3752623 & & \\
\hline & -5.2393027 & & \\
\hline & -5.2554161 & -3.0350099 & 0.3638957 \\
\hline & -5.1885579 & 7237844 & 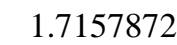 \\
\hline
\end{tabular}




$\begin{array}{lrrr}\mathrm{C} & -1.7708055 & 0.6224063 & 0.1529477 \\ \mathrm{H} & -2.2598261 & -0.0768269 & -0.5318308 \\ \mathrm{H} & -1.8169455 & 1.5988972 & -0.3343335\end{array}$

\section{8a}

$\mathrm{E}(\mathrm{PBE} 0-\mathrm{D} 3 / \mathrm{def} 2-\mathrm{TZVP})=-1254.294948529$ (conv)

Lowest Freq. $=7.65 \mathrm{~cm}^{\wedge}-1$

56

$8 \mathrm{a}(028 \mathrm{c} 8 / \mathrm{opt})$

$\begin{array}{llll}\text { C } & -1.9042778 & 2.8543513 & -0.8607034\end{array}$

F $\quad-1.5500611 \quad 3.8293536 \quad-1.7186297$

$\begin{array}{llll}\text { F } & -1.3358386 & 3.1521554 & 0.3188415\end{array}$

F $\quad-3.2276066 \quad 2.9515143 \quad-0.6940422$

$\begin{array}{llll}\text { C } & -1.4619271 & 1.5126748 & -1.3810294\end{array}$

$\begin{array}{llll}\text { C } & -1.5820778 & 0.3498273 & -0.3923317\end{array}$

$\begin{array}{llll}\text { C } & -3.0093265 & 0.0807223 & 0.0156655\end{array}$

$\begin{array}{llll}\text { C } & -3.4179402 & 0.3109319 & 1.3240214\end{array}$

$\begin{array}{llll}\text { C } & -4.7223630 & 0.0576005 & 1.7210212\end{array}$

$\begin{array}{llll}\text { C } & -5.6443849 & -0.4269005 & 0.8066524\end{array}$

$\begin{array}{llll}\text { C } & -5.2522233 & -0.6488706 & -0.5059810\end{array}$

$\begin{array}{llll}\text { C } & -3.9473254 & -0.3957747 & -0.8951591\end{array}$

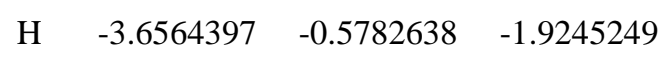

$\mathrm{H} \quad-5.9676072 \quad-1.0221452 \quad-1.2299453$

$\mathrm{H} \quad-6.6644773 \quad-0.6273220 \quad 1.1126103$

$\begin{array}{llll}\mathrm{H} & -5.0185803 & 0.2406750 & 2.7475516\end{array}$

$\begin{array}{llll}\mathrm{H} & -2.7000782 & 0.6949500 & 2.0415554\end{array}$

$\begin{array}{llll}\text { C } & -0.8753408 & -0.8665448 & -1.0040930\end{array}$

$\begin{array}{llll}\text { C } & -0.4663101 & -2.0183947 & -0.0707698\end{array}$

$\begin{array}{llll}\text { C } & -1.6741401 & -2.8103335 & 0.4242765\end{array}$

$\begin{array}{llll}\mathrm{H} & -2.2704650 & -3.1807979 & -0.4142610\end{array}$

$\begin{array}{llll}\mathrm{H} & -2.3283157 & -2.2098590 & 1.0562496\end{array}$

$\begin{array}{llll}\mathrm{H} & -1.3399494 & -3.6729710 & 1.0074177\end{array}$

$\begin{array}{llll}\text { C } & 0.3339320 & -1.5022621 & 1.1460599\end{array}$

$\begin{array}{llll}\mathrm{H} & 0.6913150 & -2.3710946 & 1.7077838\end{array}$

$\begin{array}{llll}\mathrm{H} & -0.3442804 & -0.9605903 & 1.8139291\end{array}$

$\begin{array}{llll}\text { B } & 1.5521950 & -0.5824571 & 0.7947767\end{array}$

$\begin{array}{llll}\text { O } & 2.8549958 & -1.0014352 & 0.7692159\end{array}$

$\begin{array}{llll}\text { C } & 3.6407451 & 0.0512405 & 0.1748711\end{array}$

$\begin{array}{llll}\text { C } & 4.9977998 & 0.0897051 & 0.8413088\end{array}$

$\begin{array}{llll}\mathrm{H} & 4.9097659 & 0.1862928 & 1.9224807\end{array}$

$\begin{array}{llll}\mathrm{H} & 5.5375610 & -0.8336822 & 0.6247935\end{array}$

$\begin{array}{llll}\mathrm{H} & 5.5894760 & 0.9259310 & 0.4614059\end{array}$

$\begin{array}{llll}\text { C } & 3.7890128 & -0.2899506 & -1.2993122\end{array}$

$\begin{array}{llll}\mathrm{H} & 4.2373045 & -1.2807029 & -1.3877472\end{array}$ 


$\begin{array}{lrrr}\text { H } & 2.8187222 & -0.3108568 & -1.7999251 \\ \text { H } & 4.4297835 & 0.4273400 & -1.8150108 \\ \text { C } & 2.7523344 & 1.3109988 & 0.4206357 \\ \text { C } & 2.7995813 & 2.3532912 & -0.6747745 \\ \text { H } & 2.4975016 & 1.9401310 & -1.6365559 \\ \text { H } & 2.1217392 & 3.1721180 & -0.4271861 \\ \text { H } & 3.8067604 & 2.7645176 & -0.7741242 \\ \text { C } & 2.9987409 & 1.9597842 & 1.7728509 \\ \text { H } & 2.2132822 & 2.6942184 & 1.9573502 \\ \text { H } & 3.9632473 & 2.4695182 & 1.8035275 \\ \text { H } & 2.9693879 & 1.2206110 & 2.5756737 \\ \text { O } & 1.4292250 & 0.7439436 & 0.4649590 \\ \text { C } & 0.4183547 & -2.9601708 & -0.8872842 \\ \text { H } & -0.1208126 & -3.3377571 & -1.7607637 \\ \text { H } & 1.3241294 & -2.4594713 & -1.2395149 \\ \text { H } & 0.7278228 & -3.8184354 & -0.2857305 \\ \text { H } & 0.0375949 & -0.4999297 & -1.4856168 \\ \text { H } & -1.4931068 & -1.2794872 & -1.8098030 \\ \text { H } & -1.0286312 & 0.6496421 & 0.4986933 \\ \text { H } & -2.0270161 & 1.3204510 & -2.2967156 \\ \text { H } & -0.4114074 & 1.6319701 & -1.6546436\end{array}$

\section{9av}

$\mathrm{E}(\mathrm{PBE} 0-\mathrm{D} 3 / \mathrm{def} 2-\mathrm{TZVP})=-1293.538552165$ (conv)

\begin{tabular}{|c|c|c|c|}
\hline \multirow{2}{*}{\multicolumn{2}{|c|}{$\begin{array}{l}\text { Lowest Freq. = } \\
59 \\
9 \mathrm{av}(013 \mathrm{c} 1 / \mathrm{opt})\end{array}$}} & \multicolumn{2}{|l|}{$9.31 \mathrm{~cm}^{\wedge}-1$} \\
\hline & & & \\
\hline & 3.7134314 & 0.8641318 & 2.7098303 \\
\hline & 2.8417203 & -0.2807922 & 3.1687694 \\
\hline & 5.0138492 & 0.6407898 & 3.0058565 \\
\hline & 3.3883915 & 2.0282760 & 3.2942453 \\
\hline & 3.6538056 & 1.0604379 & 1.3864900 \\
\hline & 3.0291869 & -0.3831383 & 4.2441136 \\
\hline & 3.2432428 & -1.1712397 & 2.6740237 \\
\hline & 1.3953482 & -0.1075207 & 2.8814559 \\
\hline & 0.9949108 & -0.4348342 & 1.9269460 \\
\hline & -0.9832178 & 0.4661283 & 3.4535514 \\
\hline & -1.9111331 & 1.0971321 & 4.5036012 \\
\hline & -1.4145005 & -0.9611111 & 3.0824998 \\
\hline & -1.9633150 & 0.3978456 & 5.3513116 \\
\hline & -3.2803708 & -1.1562761 & -1.1652882 \\
\hline & -2.5858548 & -0.0757468 & -0.3310388 \\
\hline & -3.1867811 & 1.2713379 & -0.7046807 \\
\hline & -2.8079925 & -0.3014125 & 1.0322762 \\
\hline
\end{tabular}




\begin{tabular}{|c|c|c|c|}
\hline & & & \\
\hline & .6287327 & .0000869 & \\
\hline & & & \\
\hline & & & \\
\hline & 31 & & \\
\hline & 68 & 765 & 53035 \\
\hline & & & \\
\hline & & & \\
\hline & -2.9 & & \\
\hline & -2.81 & & \\
\hline & & & \\
\hline & & & \\
\hline & -0.8707376 & 402 & 425 \\
\hline & -4.3 & & \\
\hline & -2.86 & & \\
\hline & -3.20 & 39 & \\
\hline & -0.7 & & \\
\hline & -0.5 & & \\
\hline & & & \\
\hline & -0.39 & & \\
\hline & -1.38 & & \\
\hline & -2.24 & & \\
\hline & -3.5 & & \\
\hline & -3.92 & & \\
\hline & -4.9 & & \\
\hline & & & \\
\hline & & & \\
\hline & -1.77 & -5.1 & \\
\hline & -1.07 & & \\
\hline & & & \\
\hline & & & \\
\hline & 0.5904460 & -0.0309211 & 3491 \\
\hline & 0.8089050 & & \\
\hline & -1.0690324 & & \\
\hline & -1.4181782 & 2.4404379 & 5.0306127 \\
\hline & -1.2411847 & 3.1359789 & 4.2027577 \\
\hline & -0.48 & & 7403 \\
\hline & -2.1669883 & 2.8948189 & 5.6872618 \\
\hline & -3.3149352 & & \\
\hline & -3.6935491 & & 3.4934121 \\
\hline & -3.3069914 & 2.0175495 & 3.1382561 \\
\hline & & 1.6060276 & 4.7118866 \\
\hline
\end{tabular}

\section{9av-TS-syn}




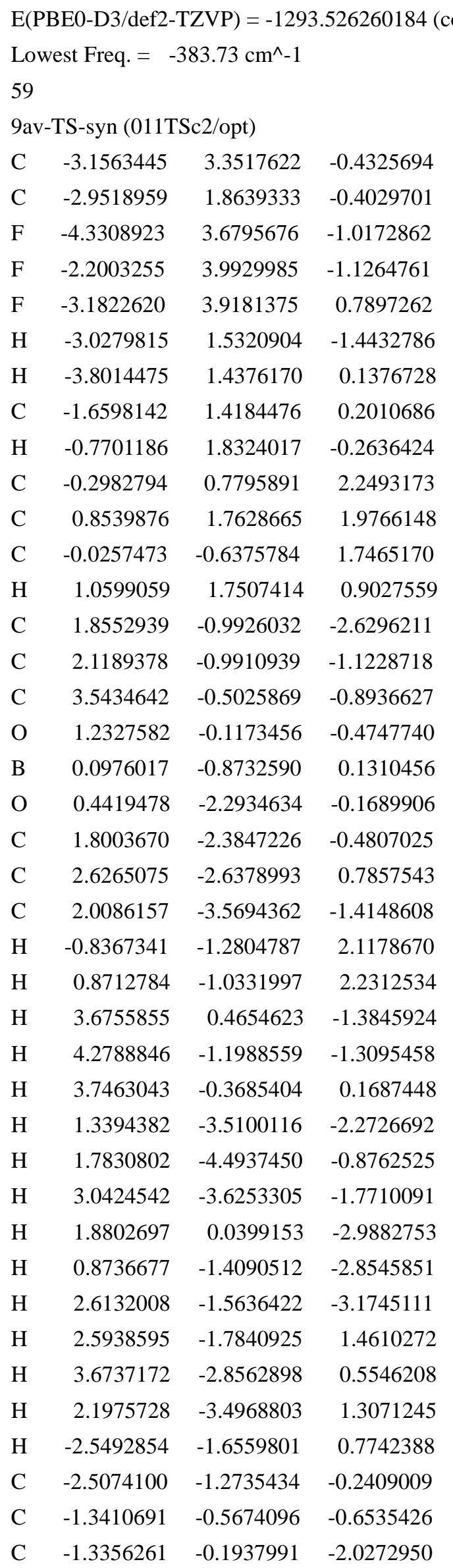




$\begin{array}{lrrr}\mathrm{C} & -2.3989141 & -0.4260762 & -2.8725403 \\ \mathrm{H} & -2.3427572 & -0.1080290 & -3.9103088 \\ \mathrm{C} & -3.5452222 & -1.0785429 & -2.4092223 \\ \mathrm{H} & -4.3828706 & -1.2655154 & -3.0721827 \\ \mathrm{C} & -3.5809269 & -1.4981134 & -1.0797229 \\ \mathrm{H} & -4.4507228 & -2.0341373 & -0.7092488 \\ \mathrm{H} & -0.4583153 & 0.3205353 & -2.4022898 \\ \mathrm{C} & -1.6312827 & 1.2613034 & 1.6834737 \\ \mathrm{H} & -1.9244238 & 2.2085560 & 2.1591079 \\ \mathrm{H} & -2.4130044 & 0.5444809 & 1.9711494 \\ \mathrm{H} & -0.4195854 & 0.7606572 & 3.3466846 \\ \mathrm{C} & 0.5110120 & 3.1860148 & 2.3995036 \\ \mathrm{H} & 0.2170787 & 3.2206058 & 3.4557304 \\ \mathrm{H} & -0.3075181 & 3.6075715 & 1.8126795 \\ \mathrm{H} & 1.3763496 & 3.8447009 & 2.2763099 \\ \mathrm{C} & 2.1316994 & 1.3178434 & 2.6742098 \\ \mathrm{H} & 2.4756881 & 0.3557109 & 2.2957322 \\ \mathrm{H} & 1.9754813 & 1.2234632 & 3.7557398 \\ \mathrm{H} & 2.9347669 & 2.0442793 & 2.5147314\end{array}$

\section{9av-TS-anti}

$\mathrm{E}(\mathrm{PBE} 0-\mathrm{D} 3 / \mathrm{def} 2-\mathrm{TZVP})=-1293.525759261$ (conv)

Lowest Freq. $=-369.72 \mathrm{~cm}^{\wedge}-1$

59

9av-TS-anti (012TSc3/opt)

C $\quad-2.8244774 \quad 3.4992146$

$-0.8987794$

$\begin{array}{llll}\text { C } & -2.6928264 & 2.0057258 & -0.8009619\end{array}$

$\begin{array}{llll}\text { F } & -3.9757431 & 3.8546847 & -1.5137310\end{array}$

F $\quad-1.8317852 \quad 4.0637976 \quad-1.6069332$

$\begin{array}{llll}\text { F } & -2.8395960 & 4.1208918 & 0.2967710\end{array}$

$\begin{array}{llll}\mathrm{H} & -2.7919622 & 1.6314233 & -1.8245876\end{array}$

$\begin{array}{llll}\mathrm{H} & -3.5613715 & 1.6482165 & -0.2403062\end{array}$

$\begin{array}{llll}\text { C } & -1.4238695 & 1.5150832 & -0.1836668\end{array}$

$\begin{array}{llll}\mathrm{H} & -0.5078770 & 1.8141287 & -0.6856373\end{array}$

$\begin{array}{llll}\text { C } & -0.0776247 & 0.9141722 & 1.8829310\end{array}$

$\begin{array}{llll}\text { C } & -0.0062072 & 1.1339263 & 3.4063508\end{array}$

$\begin{array}{llll}\text { C } & 0.0683321 & -0.5407338 & 1.4603377\end{array}$

$\begin{array}{llll}\mathrm{H} & -0.8786923 & 0.6313069 & 3.8502527\end{array}$

$\begin{array}{llll}\text { C } & 2.0129027 & -0.8135351 & -2.8769698\end{array}$

$\begin{array}{llll}\text { C } & 2.2273182 & -0.8037507 & -1.3620349\end{array}$

$\begin{array}{llll}\text { C } & 3.6156739 & -0.2389639 & -1.0881418\end{array}$

$\begin{array}{llll}\text { O } & 1.2750014 & 0.0187537 & -0.7434355\end{array}$

$\begin{array}{llll}\text { B } & 0.1772481 & -0.8004576 & -0.1508024\end{array}$

$\begin{array}{llll}\text { O } & 0.5977090 & -2.1978890 & -0.4526096\end{array}$ 


$\begin{array}{lrrr}\mathrm{C} & 1.9664330 & -2.2167744 & -0.7332613 \\ \mathrm{C} & 2.7819030 & -2.4360087 & 0.5474448 \\ \mathrm{C} & 2.2550934 & -3.3846893 & -1.6676461 \\ \mathrm{H} & -0.7629176 & -1.1181931 & 1.8940365 \\ \mathrm{H} & 0.9707085 & -0.9628587 & 1.9055991 \\ \mathrm{H} & 3.7108255 & 0.7344247 & -1.5770922 \\ \mathrm{H} & 4.4016327 & -0.8943164 & -1.4774156 \\ \mathrm{H} & 3.7741336 & -0.0935404 & -0.0196734 \\ \mathrm{H} & 1.5991133 & -3.3582101 & -2.5372179 \\ \mathrm{H} & 2.0700465 & -4.3220694 & -1.1361489 \\ \mathrm{H} & 3.2966223 & -3.3835563 & -2.0051778 \\ \mathrm{H} & 1.9775852 & 0.2208752 & -3.2292425 \\ \mathrm{H} & 1.0712824 & -1.2969587 & -3.1358120 \\ \mathrm{H} & 2.8248508 & -1.3278666 & -3.4001433 \\ \mathrm{H} & 2.7163976 & -1.5809567 & 1.2187190 \\ \mathrm{H} & 3.8382655 & -2.6219257 & 0.3293090 \\ \mathrm{H} & 2.3747799 & -3.3072384 & 1.0661535 \\ \mathrm{H} & -2.4750870 & -1.4498267 & 0.6388341 \\ \mathrm{C} & -2.4539389 & -1.1561093 & -0.4057195 \\ \mathrm{C} & -1.2745461 & -0.5452501 & -0.9162785 \\ \mathrm{C} & -1.3086473 & -0.2797568 & -2.3138441 \\ \mathrm{C} & -2.4155370 & -0.5240443 & -3.0978630 \\ \mathrm{H} & -2.3871512 & -0.2901774 & -4.1589203 \\ \mathrm{H} & 0.0593623 & 2.7332996 & 4.8750966 \\ \mathrm{C} & -3.5716544 & -1.0782324 & -2.5417029 \\ \mathrm{H} & -4.4442449 & -1.2728764 & -3.1556262 \\ \mathrm{C} & -3.5717135 & -1.3903539 & -1.1831440 \\ \mathrm{H} & -4.4506371 & -1.8486133 & -0.7374262 \\ \mathrm{H} & -0.4291592 & 0.1670987 & -2.7620090 \\ \mathrm{C} & -1.3697895 & 1.4819093 & 1.3059534 \\ \mathrm{H} & -1.5342322 & 2.5042074 & 1.6721407 \\ \mathrm{H} & -2.2212809 & 0.8952131 & 1.6843672 \\ \mathrm{C} & 0.7594871 & 1.4750258 & 1.4384321 \\ \mathrm{H} & 2.2467268 & 0.5170929 & 4.0164567 \\ \mathrm{H} & 1.2681290 & -0.5661851 & 3.9003229 \\ \mathrm{H} & -1.0607179 & 2.6092337 & 3.7941126 \\ \mathrm{H} & 0.9200360 & 3.5336729 \\ \mathrm{H} & 3.0864310 & 3.5120594 \\ \mathrm{H} & & \\ \mathrm{H} & & \end{array}$

\section{0av-syn(cycl)}

$\mathrm{E}(\mathrm{PBE}-\mathrm{D} 3 /$ def2-TZVP) $=-1293.553414086$ (conv)

Lowest Freq. $=28.54 \mathrm{~cm}^{\wedge}-1$ 
10av-syn(cycl) (020c2/opt)

$\begin{array}{lll}\text { C } & -1.7684717 & 2.1449083\end{array}$

2.8388123

C $\quad-1.3472899 \quad 1.7989170$

1.4432564

F -1.3758318

3.3990271

3.1628376

F -1.2484065

1.3484423

3.7907916

F $\quad-3.1049839$

2.1197091

3.0149475

$\begin{array}{llll}\mathrm{H} & -0.2589859 & 1.7106350 & 1.4269685\end{array}$

$\mathrm{H} \quad-1.5995403$

2.6825545

0.8552941

C -2.0145850

0.5731141

0.8114819

$\mathrm{H} \quad-3.0906542 \quad 0.7913085$

0.7759278

$\begin{array}{lll}\text { C } & -0.4216638 & -1.2015391\end{array}$

1.7883603

$\begin{array}{lll}\text { C } & -0.3486542 & -2.4190008\end{array}$

2.7284413

C $\quad 0.1345869-1.4711606$

0.3964407

$\begin{array}{lll}\mathrm{H} & -0.9837827 & -3.2027853\end{array}$

2.2889261

C $\quad 1.6133048 \quad 2.4903713$

$-1.8196524$

C 1.9830697

1.1634499

$-1.1577636$

C $\quad 3.3550361$

1.3236335

$-0.5152243$

O 1.0511269

0.8546891

$-0.1518642$

B 0.1134685

$-0.1950515$

$-0.6156891$

$\begin{array}{lll}\text { O } & 0.5622609 & -0.5060467\end{array}$

$-1.9721295$

C 1.8720523

$-0.0493173$

$-2.1501019$

C 2.8581405

$-1.1719638$

$-1.8110399$

C 2.0612166

0.3206684

$-3.6143998$

$\mathrm{H} \quad-0.4136139 \quad-2.3115221$

$-0.0533815$

$\mathrm{H} \quad 1.1772178 \quad-1.7948051$

0.4690489

$\mathrm{H} \quad 3.3401666 \quad 2.1900616$

0.1514741

$\mathrm{H}$

$\mathrm{H}$

$\mathrm{H}$

$$
\text { H }
$$

.

H

H

H

$\mathrm{H}$

H

H

H

C

$$
\text { C }
$$

C

$\begin{array}{llll}\mathrm{C} & -2.0985019 & 1.7081526 & -2.7239365 \\ \mathrm{H} & -2.0250867 & 2.6512522 & -3.2603328\end{array}$ 


$\begin{array}{lrrr}\mathrm{C} & -2.7089168 & 0.6048008 & -3.3473915 \\ \mathrm{H} & -3.1030643 & 0.6774083 & -4.3539623 \\ \mathrm{C} & -2.8271113 & -0.5830208 & -2.6033667 \\ \mathrm{H} & -3.3322134 & -1.4394164 & -3.0423600 \\ \mathrm{H} & -1.1200260 & 2.5055615 & -1.0462397 \\ \mathrm{C} & -1.8524226 & -0.6890644 & 1.6589114 \\ \mathrm{H} & -2.2895307 & -0.4975368 & 2.6406225 \\ \mathrm{H} & -2.4636168 & -1.4845437 & 1.2176435 \\ \mathrm{H} & 0.1903853 & -0.4131130 & 2.2513187 \\ \mathrm{C} & -0.8677684 & -2.1181876 & 4.1310939 \\ \mathrm{H} & -0.7451825 & -2.9873050 & 4.7853227 \\ \mathrm{H} & -1.9242048 & -1.8468849 & 4.1383691 \\ \mathrm{H} & -0.3115822 & -1.2857207 & 4.5740650 \\ \mathrm{C} & 1.0668094 & -2.9723906 & 2.8353059 \\ \mathrm{H} & 1.4404882 & -3.3253341 & 1.8741389 \\ \mathrm{H} & 1.1063768 & -3.8067211 & 3.5428895 \\ \mathrm{H} & 1.7530111 & -2.1975206 & 3.1946320\end{array}$

\section{0av-anti(cycl)}

$\mathrm{E}(\mathrm{PBE}-\mathrm{D} 3 / \mathrm{def} 2-\mathrm{TZVP})=-1293.558774276$ (conv)

Lowest Freq. $=14.01 \mathrm{~cm}^{\wedge}-1$

59

10av-anti(cycl) (021c2/opt)

$\begin{array}{lrrr}\text { C } & -3.2334546 & -0.1222474 & 3.1869483 \\ \text { C } & -2.7073997 & 0.6597702 & 2.0218568 \\ \text { F } & -4.5189992 & 0.2076436 & 3.4541582 \\ \text { F } & -2.5642823 & 0.0839782 & 4.3395502 \\ \text { F } & -3.2251608 & -1.4502621 & 2.9777869 \\ \text { H } & -2.6382521 & 1.7053296 & 2.3367481 \\ \text { H } & -3.4845971 & 0.6143864 & 1.2538274 \\ \text { C } & -1.3828577 & 0.2023938 & 1.4012604 \\ \text { H } & -1.4582244 & -0.8695541 & 1.1849848 \\ \text { C } & 1.1197103 & -0.1113266 & 1.8451398 \\ \text { C } & 2.2359431 & 0.0782993 & 2.8876112 \\ \text { C } & 1.4196830 & 0.5215828 & 0.4962391 \\ \text { H } & 2.3484760 & 1.1610731 & 3.0471062 \\ \text { C } & -0.6105746 & -0.0476376 & -3.7745840 \\ \text { C } & 0.6377537 & -0.2115797 & -2.9088315 \\ \text { C } & 1.8573189 & -0.0382853 & -3.8064490 \\ \text { O } & 0.6621653 & 0.7841621 & -1.9218270 \\ \text { B } & 0.2738497 & 0.2375363 & -0.6218552 \\ \text { O } & 0.0322636 & -1.1967348 & -0.8729870 \\ \text { C } & 0.6343784 & -1.5523690 & -2.0879883 \\ \text { C } & 2.0474809 & -2.0749318 & -1.8132391\end{array}$




\begin{tabular}{lrrr}
$\mathrm{C}$ & -0.1715128 & -2.6743784 & -2.7254739 \\
$\mathrm{H}$ & 2.3618968 & 0.1387624 & 0.0921084 \\
$\mathrm{H}$ & 1.5679918 & 1.6037750 & 0.6235694 \\
$\mathrm{H}$ & 1.7768687 & 0.9150507 & -4.3349608 \\
$\mathrm{H}$ & 1.9252648 & -0.8373457 & -4.5518838 \\
$\mathrm{H}$ & 2.7769179 & -0.0210527 & -3.2216346 \\
$\mathrm{H}$ & -1.2186312 & -2.3908250 & -2.8295564 \\
$\mathrm{H}$ & -0.1219465 & -3.5608886 & -2.0875943 \\
$\mathrm{H}$ & 0.2216003 & -2.9398626 & -3.7117918 \\
$\mathrm{H}$ & -0.6354191 & 0.9797582 & -4.1449215 \\
$\mathrm{H}$ & -1.5152157 & -0.2037086 & -3.1906569 \\
$\mathrm{H}$ & -0.6096360 & -0.7305949 & -4.6300548 \\
$\mathrm{H}$ & 2.6786871 & -1.2947974 & -1.3884538 \\
$\mathrm{H}$ & 2.5296979 & -2.4616048 & -2.7159872 \\
$\mathrm{H}$ & 1.9756543 & -2.8845273 & -1.0828066 \\
$\mathrm{H}$ & -2.5364304 & -0.5494737 & -0.9527772 \\
$\mathrm{C}$ & -2.3297087 & 0.5141603 & -0.8742042 \\
$\mathrm{C}$ & -1.2237488 & 0.9027675 & 0.0196853 \\
$\mathrm{C}$ & -1.0914351 & 2.3689867 & 0.1124948 \\
$\mathrm{C}$ & -1.8026070 & 3.2366093 & -0.6631944 \\
$\mathrm{H}$ & -1.6091359 & 4.3029631 & -0.5801673 \\
$\mathrm{C}$ & -2.7837429 & 2.7834218 & -1.5654367 \\
$\mathrm{H}$ & -3.3443704 & 3.4816173 & -2.1754825 \\
$\mathrm{C}$ & -3.0366436 & 1.3993133 & -1.6312736 \\
$\mathrm{H}$ & -3.8039573 & 1.0298177 & -2.3071208 \\
$\mathrm{H}$ & -0.3493338 & 2.7721847 & 0.7930667 \\
$\mathrm{C}$ & -0.2168084 & 0.4097679 & 2.3604670 \\
$\mathrm{H}$ & -0.1201745 & 1.4804559 & 2.5911252 \\
$\mathrm{H}$ & -0.4646082 & -0.0783264 & 3.3040095 \\
$\mathrm{H}$ & 1.0098642 & -1.1957814 & 1.6853909 \\
$\mathrm{C}$ & 1.9094489 & -0.5657047 & 4.2315667 \\
$\mathrm{H}$ & 2.7564206 & -0.4821962 & 4.9200161 \\
& 1.6925779 & -1.6317864 & 4.1012985 \\
$\mathrm{H}$ & 1.0450011 & -0.1093738 & 4.7158867 \\
\hline & -5711321 & -0.4616266 & 2.3908649 \\
$\mathrm{H}$ & -3459185 & -0.3533283 & 3.1565712 \\
$\mathrm{H}$ & 0.0535952 & 1.4917729 \\
$\mathrm{H}$ & -1.5270502 & 2.1500825
\end{tabular}

\section{0av-syn}

$\mathrm{E}(\mathrm{PBE} 0-\mathrm{D} 3 /$ def2-TZVP $)=-1293.530659463$ (conv)

Lowest Freq. $=8.62 \mathrm{~cm}^{\wedge}-1$

59

10av-syn (015c2/opt) 


\begin{tabular}{|c|c|c|c|}
\hline & & & \\
\hline & -2.3955795 & & \\
\hline & -2.5491049 & 4.0245484 & 50011 \\
\hline & -1.1518181 & 2.7815518 & \\
\hline & 72072 & 1489 & 4951 \\
\hline & -1.60 & .0928 & 053 \\
\hline & -3.3466434 & & \\
\hline & -2.1986981 & 0.3184098 & 1246 \\
\hline & -1.4827990 & .3084578 & 8380 \\
\hline & 3087 & -0.1 & \\
\hline & 0.8358120 & & \\
\hline & 0.2727199 & 627 & 4142 \\
\hline & 0.4924691 & 0.6385237 & \\
\hline & 4.4391444 & -0.12 & \\
\hline & 3.2438802 & -0.4 & \\
\hline & 3.7045360 & -1.129 & 2767 \\
\hline & 3657 & -1.25 & \\
\hline & 1.3719523 & -0.52 & \\
\hline & 1.4198844 & 0.7752752 & -2.1733066 \\
\hline & 5911 & 0.834 & -3 \\
\hline & 3912 & 0.73 & \\
\hline & 3.0781112 & 2.1585667 & \\
\hline & -0.6095154 & -1.2430819 & -1.39 \\
\hline & 0.5482562 & -2.05 & \\
\hline & & & \\
\hline & 3096572 & -0.45 & \\
\hline & 2.8608133 & -1.4878186 & -4.8 \\
\hline & 3.5537920 & 2.3226768 & \\
\hline & 2.3685567 & 2.9707694 & \\
\hline & 3.8440824 & 2.2006822 & -4.0091926 \\
\hline & 4.8967532 & -1.0768235 & -1.8369598 \\
\hline & 4.1382356 & 0.3976241 & -1.2222844 \\
\hline & 5.1884939 & & \\
\hline & 0.9902395 & -0.2141094 & -4.6014533 \\
\hline & 2.1577613 & 0.8367773 & -5.4358280 \\
\hline & .7901023 & & \\
\hline & -2.2870171 & -1.5453429 & 3.9095477 \\
\hline & -3.2557471 & -1.4096999 & 3.4387028 \\
\hline & -3.4411145 & -0.3751472 & 2.4725049 \\
\hline & -4.6979749 & -0.1434822 & 1.9643311 \\
\hline & -5.8228584 & -0.8936990 & 2.3754525 \\
\hline & -6.8079691 & -0.6701802 & 1.9839794 \\
\hline & -5.6304196 & -1.9141172 & \\
\hline & 6.4749943 & -2.5111070 & $3.6 / 8044$ \\
\hline
\end{tabular}




$\begin{array}{lrrr}\mathrm{C} & -4.3806457 & -2.1573239 & 3.8589120 \\ \mathrm{H} & -4.2519812 & -2.9426005 & 4.6000221 \\ \mathrm{H} & -4.8432463 & 0.6244016 & 1.2075573 \\ \mathrm{C} & -1.5837926 & -0.4895197 & 0.8156875 \\ \mathrm{H} & -1.6335542 & -1.5445822 & 1.1026836 \\ \mathrm{H} & -2.2448125 & -0.3907458 & -0.0536579 \\ \mathrm{H} & -0.1548804 & 0.8793711 & -0.0191281 \\ \mathrm{C} & 2.2406967 & 0.2453207 & 1.1182948 \\ \mathrm{H} & 2.2373966 & 1.1683533 & 0.5320538 \\ \mathrm{H} & 2.6959967 & -0.5417190 & 0.5082289 \\ \mathrm{H} & 2.8910250 & 0.3952123 & 1.9849284 \\ \mathrm{C} & 0.8632627 & -1.4513151 & 2.3327306 \\ \mathrm{H} & -0.1211182 & -1.7204948 & 2.7200426 \\ \mathrm{H} & 1.5474320 & -1.3738480 & 3.1824423 \\ \mathrm{H} & 1.2145381 & -2.2769341 & 1.7067127\end{array}$

\section{0av-anti}

$\mathrm{E}(\mathrm{PBE} 0-\mathrm{D} 3 /$ def2-TZVP $)=-1293.535515229$ (conv)

Lowest Freq. $=15.00 \mathrm{~cm}^{\wedge}-1$

59

10av-anti (026c1/opt)

$\begin{array}{lrrr}\text { C } & -4.3697023 & -0.4815406 & 3.8689786 \\ \text { C } & -3.8599080 & -0.7260402 & 2.4828358 \\ \text { F } & -5.6249641 & -0.9763361 & 4.0192771 \\ \text { F } & -4.4253796 & 0.8019245 & 4.2195576 \\ \text { F } & -3.6195878 & -1.1073585 & 4.8046638 \\ \text { H } & -4.5410033 & -0.2297347 & 1.7853300 \\ \text { H } & -3.9453522 & -1.8075939 & 2.3308532 \\ \text { C } & -2.4229060 & -0.2327903 & 2.2195639 \\ \text { H } & -1.8507255 & -0.3589446 & 3.1460561 \\ \text { C } & -0.4307406 & -0.7227454 & 0.6549273 \\ \text { C } & 0.5803482 & -0.5599037 & 1.8014392 \\ \text { C } & 0.0487734 & -1.6774001 & -0.4484084 \\ \text { H } & 0.1594676 & 0.2154917 & 2.4493652 \\ \text { C } & 4.1223382 & -0.6557476 & -1.9990855 \\ \text { C } & 2.9021755 & -1.0059324 & -2.8370035 \\ \text { C } & 3.3319281 & -1.7349696 & -4.0928238 \\ \text { O } & 2.0810096 & -1.8815254 & -2.0483065 \\ \text { B } & 1.0934403 & -1.1199737 & -1.4611910 \\ \text { O } & 1.0854377 & 0.1626936 & -1.9449058 \\ \text { C } & 1.9591279 & 0.2168611 & -3.0781366 \\ \text { C } & 1.0885378 & 0.0608884 & -4.3166718 \\ \text { C } & 2.6520285 & 1.5627996 & -3.1001819 \\ \text { H } & -0.8250380 & -1.9342663 & -1.0666599\end{array}$




\begin{tabular}{lrrr}
$\mathrm{H}$ & 0.3929885 & -2.6304098 & -0.0320879 \\
$\mathrm{H}$ & 3.9779279 & -2.5736009 & -3.8235387 \\
$\mathrm{H}$ & 3.8950536 & -1.0685600 & -4.7529597 \\
$\mathrm{H}$ & 2.4745089 & -2.1272947 & -4.6394330 \\
$\mathrm{H}$ & 3.1643118 & 1.7624802 & -2.1595513 \\
$\mathrm{H}$ & 1.9092507 & 2.3480566 & -3.2521595 \\
$\mathrm{H}$ & 3.3781030 & 1.6118092 & -3.9174134 \\
$\mathrm{H}$ & 4.6203566 & -1.5810237 & -1.7020739 \\
$\mathrm{H}$ & 3.8370703 & -0.1179392 & -1.0934850 \\
$\mathrm{H}$ & 4.8316251 & -0.0442536 & -2.5622119 \\
$\mathrm{H}$ & 0.5844673 & -0.9083418 & -4.3232229 \\
$\mathrm{H}$ & 1.6720533 & 0.1611345 & -5.2354763 \\
$\mathrm{H}$ & 0.3229384 & 0.8385769 & -4.3012056 \\
$\mathrm{H}$ & -3.4446039 & 0.9348667 & -0.0865262 \\
$\mathrm{C}$ & -2.9829215 & 1.6450021 & 0.5916071 \\
$\mathrm{C}$ & -2.3741085 & 1.2120569 & 1.8280763 \\
$\mathrm{C}$ & -1.7527555 & 2.1565342 & 2.6234306 \\
$\mathrm{C}$ & -1.6798394 & 3.5076584 & 2.2832873 \\
$\mathrm{H}$ & -1.1849588 & 4.2152083 & 2.9383830 \\
$\mathrm{C}$ & -2.3080876 & 3.9360953 & 1.0664117 \\
$\mathrm{H}$ & -2.2868763 & 4.9813178 & 0.7740452 \\
$\mathrm{C}$ & -2.9295505 & 3.0060063 & 0.2619655 \\
$\mathrm{H}$ & -3.3943123 & 3.3383958 & -0.6648147 \\
$\mathrm{H}$ & -1.3054628 & 1.8232591 & 3.5608371 \\
$\mathrm{C}$ & -1.8097431 & -1.1475265 & 1.1484075 \\
$\mathrm{H}$ & -1.7804374 & -2.1820952 & 1.5171565 \\
$\mathrm{H}$ & -2.4786396 & -1.1566285 & 0.2794281 \\
$\mathrm{H}$ & -0.5603463 & 0.2735207 & 0.2168484 \\
$\mathrm{C}$ & 1.9219904 & -0.0405522 & 1.3076798 \\
$\mathrm{H}$ & 1.7936888 & 0.8511666 & 0.6901376 \\
$\mathrm{H}$ & 2.4502785 & -0.7979920 & 0.7165455 \\
$\mathrm{H}$ & 2.5717573 & 0.2178263 & 2.1492133 \\
& 0.7766461 & -1.8192831 & 2.6347723 \\
$\mathrm{H}$ & 1.1606292 & -2.1703571 & 3.0719467 \\
\hline
\end{tabular}

\section{8av-syn}

E $($ PBE0-D3/def2-TZVP) $=-1293.576322497$ (conv)

Lowest Freq. $=15.04 \mathrm{~cm}^{\wedge}-1$

59

8av-syn (019c0/opt)

$\begin{array}{llll}\text { C } & -2.6479511 & -2.9467233 & 0.7801809\end{array}$

F $\quad-1.7437595 \quad-3.3970478 \quad-0.1084501$ 


\begin{tabular}{|c|c|c|c|}
\hline & & & \\
\hline & -3.816 & $-2.8 c$ & \\
\hline & & & \\
\hline & & & \\
\hline & -3.01 & & \\
\hline & 10 & & \\
\hline & -3.0788022 & & \\
\hline & & & \\
\hline & & & \\
\hline & -5.28 & 979 & \\
\hline & & & \\
\hline & & & \\
\hline & -2.39 & & \\
\hline & & & \\
\hline & & & \\
\hline & -5.9 & & \\
\hline & -4.053 & 5677 & \\
\hline & -1.1 & & \\
\hline & & & \\
\hline & -1.7 & 729 & \\
\hline & -0.63 & 945 & \\
\hline & -1.52 & & \\
\hline & & & \\
\hline & & & \\
\hline & 2.60 & 77373 & \\
\hline & & & \\
\hline & & & \\
\hline & 384 & -0.7 & \\
\hline & & & \\
\hline & & & \\
\hline & 4.3378879 & -1.2296455 & \\
\hline & 78654 & -1.5805162 & \\
\hline & & & \\
\hline & & & \\
\hline & 2.4536322 & -1.6973106 & -0.1248 \\
\hline & 587996 & -2.7156883 & \\
\hline & & -3.4426768 & \\
\hline & & -2.2277546 & -2.0597507 \\
\hline & 1.1060818 & -3.2494384 & -0.7169681 \\
\hline & & -2.3958806 & \\
\hline & 2.1340313 & -3.0738523 & \\
\hline & & -2.9871545 & \\
\hline & & -1.6847365 & \\
\hline & 1.3315367 & -0.8516570 & 0.1943275 \\
\hline
\end{tabular}




$\begin{array}{lrrr}\mathrm{H} & -0.4232616 & 0.7685851 & -1.6023603 \\ \mathrm{H} & 0.6274172 & 2.1305283 & -1.4005604 \\ \mathrm{C} & 0.0489749 & 2.7980037 & 1.1723110 \\ \mathrm{C} & 1.4245768 & 2.3839406 & 1.6777384 \\ \mathrm{H} & 1.8114422 & 3.1198130 & 2.3869863 \\ \mathrm{H} & 1.4068087 & 1.4150925 & 2.1817349 \\ \mathrm{H} & 2.1424761 & 2.3253081 & 0.8539795 \\ \mathrm{C} & 0.1346836 & 4.1356477 & 0.4505821 \\ \mathrm{H} & -0.8488084 & 4.4636764 & 0.1048520 \\ \mathrm{H} & 0.7944868 & 4.0794011 & -0.4194290 \\ \mathrm{H} & 0.5352481 & 4.9091752 & 1.1106968 \\ \mathrm{H} & -0.5979609 & 2.9420581 & 2.0477457 \\ \mathrm{H} & -1.1851464 & -0.9551648 & -0.3041960\end{array}$

\section{8av-anti}

$\mathrm{E}(\mathrm{PBE}-\mathrm{D} 3 / \mathrm{def} 2-\mathrm{TZVP})=-1293.576023291$ (conv)

Lowest Freq. $=8.35 \mathrm{~cm}^{\wedge}-1$

59

8av-anti (027c1/opt)

C $\quad-4.2383525$

$1.1373799 \quad-2.1134432$

F $\quad-5.0293418 \quad 0.0510704 \quad-2.1324736$

F $\quad-4.6653689 \quad 1.9153424 \quad-1.1139140$

$\begin{array}{llll}\text { F } & -4.4708093 & 1.8075098 & -3.2553600\end{array}$

$\begin{array}{llll}\text { C } & -2.7825243 & 0.7708703 & -2.0027769\end{array}$

$\begin{array}{llll}\text { C } & -2.4240968 & -0.1550565 & -0.8386989\end{array}$

$\begin{array}{llll}\text { C } & -2.5884406 & 0.5082264 & 0.5071563\end{array}$

$\begin{array}{llll}\text { C } & -3.5874211 & 0.0964649 & 1.3804339\end{array}$

$\begin{array}{llll}\text { C } & -3.7393421 & 0.6921935 & 2.6239312\end{array}$

$\begin{array}{llll}\text { C } & -2.8868278 & 1.7130391 & 3.0130732\end{array}$

$\begin{array}{llll}\text { C } & -1.8828720 & 2.1307877 & 2.1506816\end{array}$

$\begin{array}{llll}\text { C } & -1.7365438 & 1.5326771 & 0.9104680\end{array}$

$\begin{array}{llll}\mathrm{H} & -0.9301841 & 1.8510743 & 0.2592316\end{array}$

$\begin{array}{llll}\mathrm{H} & -1.2055431 & 2.9229043 & 2.4492100\end{array}$

$\begin{array}{llll}\mathrm{H} & -3.0011697 & 2.1798402 & 3.9844155\end{array}$

$\begin{array}{llll}\mathrm{H} & -4.5266245 & 0.3569824 & 3.2893141\end{array}$

$\begin{array}{llll}\mathrm{H} & -4.2615419 & -0.6983803 & 1.0777668\end{array}$

$\begin{array}{llll}\text { C } & -0.9989825 & -0.6714343 & -1.0591724\end{array}$

$\begin{array}{llll}\text { C } & -0.4238333 & -1.5417919 & 0.0549554\end{array}$

$\begin{array}{llll}\text { C } & 1.0358755 & -1.8646234 & -0.2667542\end{array}$

$\begin{array}{llll}\mathrm{H} & 1.4415711 & -2.5699827 & 0.4696860\end{array}$

$\begin{array}{llll}\text { B } & 1.9901578 & -0.6274876 & -0.2619738\end{array}$

$\begin{array}{llll}\text { O } & 1.7394394 & 0.5286602 & 0.4319682\end{array}$

$\begin{array}{llll}\text { C } & 2.7335688 & 1.4938164 & 0.0405047\end{array}$

$\begin{array}{llll}\text { C } & 3.0769963 & 2.3570199 & 1.2340156\end{array}$ 


$\begin{array}{lrrr}\mathrm{H} & 3.3835257 & 1.7547551 & 2.0879192 \\ \mathrm{H} & 2.2017180 & 2.9397510 & 1.5267524 \\ \mathrm{H} & 3.8817356 & 3.0537679 & 0.9879741 \\ \mathrm{C} & 2.1173965 & 2.3423952 & -1.0608816 \\ \mathrm{H} & 1.2028976 & 2.8029120 & -0.6826486 \\ \mathrm{H} & 1.8609468 & 1.7344306 & -1.9308330 \\ \mathrm{H} & 2.7920803 & 3.1383413 & -1.3804019 \\ \mathrm{C} & 3.8937103 & 0.5841640 & -0.4721280 \\ \mathrm{C} & 4.6921138 & 1.1571881 & -1.6219271 \\ \mathrm{H} & 4.0627841 & 1.3500060 & -2.4897003 \\ \mathrm{H} & 5.4683214 & 0.4488169 & -1.9160502 \\ \mathrm{H} & 5.1786153 & 2.0898440 & -1.3265002 \\ \mathrm{C} & 4.8248669 & 0.1318556 & 0.6412674 \\ \mathrm{H} & 4.2630975 & -0.2764152 & 1.4836388 \\ \mathrm{H} & 5.4776578 & -0.6527849 & 0.2558793 \\ \mathrm{H} & 5.4470571 & 0.9525526 & 1.0025948 \\ \mathrm{O} & 3.1858409 & -0.5845146 & -0.9289170 \\ \mathrm{H} & 1.1249465 & -2.3774862 & -1.2309002 \\ \mathrm{C} & -1.2851466 & -2.7800219 & 0.3526868 \\ \mathrm{C} & -0.8261078 & -3.4906949 & 1.6180709 \\ \mathrm{H} & -0.7514174 & -2.7932230 & 2.4564511 \\ \mathrm{H} & -1.5310808 & -4.2776125 & 1.8973574 \\ \mathrm{H} & 0.1501236 & -3.9647061 & 1.4849298 \\ \mathrm{C} & -1.3735289 & -3.7565592 & -0.8114814 \\ \mathrm{H} & -2.0374429 & -4.5884526 & -0.5642244 \\ \mathrm{H} & -0.3940895 & -4.1803121 & -1.0491993 \\ \mathrm{H} & -1.7628199 & -3.2863407 & -1.7176584 \\ \mathrm{H} & -2.2972890 & -2.4112613 & 0.5558219 \\ \mathrm{H} & -0.4315872 & -0.9366170 & 0.9686546 \\ \mathrm{H} & -0.9715858 & -1.2186553 & -2.0083405 \\ \mathrm{H} & -0.3357672 & 0.1903192 & -1.1931579 \\ \mathrm{H} & -3.1113187 & -1.0052312 & -0.8754502 \\ & -2.5198020 & 0.2878628 & -2.9475454 \\ & -2.2182409 & 1.7048240 & -1.9442979\end{array}$

\section{9ad-TS-syn}

$\mathrm{E}(\mathrm{PBE} 0-\mathrm{D} 3 /$ def2-TZVP) $=-1524.407015797$ (conv)

Lowest Freq. $=-359.32 \mathrm{~cm}^{\wedge}-1$

69

9ad-TS-syn (022TSc1/opt)

$\begin{array}{lrrr}\text { C } & -2.3283226 & 3.5487160 & 0.2111570 \\ \text { C } & -2.1066104 & 2.0629929 & 0.2646923 \\ \text { F } & -3.5165760 & 3.8523373 & -0.3533273 \\ \text { F } & -1.3920029 & 4.1845973 & -0.5129672\end{array}$




\begin{tabular}{|c|c|c|c|}
\hline & & & \\
\hline & -2.1904479 & .7120639 & \\
\hline & -2.9440275 & 6372593 & \\
\hline & & & \\
\hline & & & \\
\hline & & & \\
\hline & & & \\
\hline & & & \\
\hline & & & \\
\hline & 1687 & -0.771 & \\
\hline & & & \\
\hline & & & \\
\hline & 910 & & \\
\hline & 61 & -0.6 & \\
\hline & & & \\
\hline & & & \\
\hline & 3.552 & -2.3 & \\
\hline & 69 & -3.3 & \\
\hline & & & \\
\hline & & & \\
\hline & 999 & & \\
\hline & 413 & -0.9 & \\
\hline & & & \\
\hline & & & \\
\hline & 2.728 & -4.26 & \\
\hline & & & \\
\hline & & & \\
\hline & 2224 & -1.1 & \\
\hline & 292 & -1.34 & \\
\hline & & & \\
\hline & & & \\
\hline & 1345548 & $-3.2 ?$ & \\
\hline & 247 & & \\
\hline & & & \\
\hline & -0.4297199 & -0.3732920 & -0.0178416 \\
\hline & -0.4353543 & 0.0342670 & \\
\hline & -1.4977836 & -0.1811822 & -2.22 \\
\hline & -1.4496789 & 0.1906200 & -3.2417604 \\
\hline & -2.6590859 & -0.8436105 & -1.7847023 \\
\hline & -2.6714708 & -1.2821007 & -0.4510228 \\
\hline & -3.5236182 & -1.8498524 & -0.0892682 \\
\hline & & & \\
\hline & -0.7588230 & & 2.3403621 \\
\hline & -1.0618520 & .4162847 & $2.8256 / 03$ \\
\hline
\end{tabular}




$\begin{array}{lrrr}\mathrm{H} & -1.5296237 & 0.7477197 & 2.6272419 \\ \mathrm{H} & 0.4724484 & 0.9896355 & 3.9912806 \\ \mathrm{C} & 1.3693626 & 3.4233045 & 3.0402319 \\ \mathrm{H} & 1.0890989 & 3.4525587 & 4.1002361 \\ \mathrm{H} & 0.5386269 & 3.8373086 & 2.4651704 \\ \mathrm{H} & 2.2254015 & 4.0918810 & 2.9070629 \\ \mathrm{C} & 3.0131123 & 1.5712577 & 3.2917586 \\ \mathrm{H} & 3.3665332 & 0.6178514 & 2.9003758 \\ \mathrm{H} & 2.8683789 & 1.4647039 & 4.3736482 \\ \mathrm{H} & 3.8043325 & 2.3102722 & 3.1316334 \\ \mathrm{C} & -3.7963852 & -1.0689282 & -2.6758995 \\ \mathrm{C} & -5.1046422 & -1.1572479 & -2.1817586 \\ \mathrm{C} & -3.6263322 & -1.2038185 & -4.0602790 \\ \mathrm{C} & -4.7037429 & -1.4139717 & -4.9029650 \\ \mathrm{C} & -6.1819288 & -1.3729818 & -3.0235483 \\ \mathrm{C} & -5.9923967 & -1.5020178 & -4.3928851 \\ \mathrm{H} & -2.6251735 & -1.1682999 & -4.4733512 \\ \mathrm{H} & -5.2753280 & -1.0266286 & -1.1196388 \\ \mathrm{H} & -4.5345309 & -1.5210137 & -5.9693580 \\ \mathrm{H} & -7.1823418 & -1.4282376 & -2.6071434 \\ \mathrm{H} & -6.8362978 & -1.6675537 & -5.0529734\end{array}$

\section{9ad-TS-anti}

$\mathrm{E}(\mathrm{PBE}-\mathrm{D} 3 / \mathrm{def} 2-\mathrm{TZVP})=-1524.406458318$ (conv)

Lowest Freq. $=-341.86 \mathrm{~cm}^{\wedge}-1$

69

9ad-TS-anti (023TSc1/opt)

C $\quad-1.9420513 \quad 3.7168460$

$-0.2882903$

$\begin{array}{llll}\text { C } & -1.8138670 & 2.2249061 & -0.1536599\end{array}$

F $\quad-3.1053544 \quad 4.0612415 \quad-0.8816831$

F $\quad-0.9633869 \quad 4.2546705 \quad-1.0348483$

$\begin{array}{llll}\text { F } & -1.9215270 & 4.3658602 & 0.8922130\end{array}$

$\begin{array}{llll}\mathrm{H} & -1.9244232 & 1.8248531 & -1.1665788\end{array}$

$\begin{array}{llll}\mathrm{H} & -2.6762732 & 1.8848172 & 0.4264555\end{array}$

$\begin{array}{llll}\text { C } & -0.5390980 & 1.7582213 & 0.4648127\end{array}$

$\begin{array}{llll}\mathrm{H} & 0.3766675 & 2.0399844 & -0.0461767\end{array}$

$\begin{array}{llll}\text { C } & 0.8175591 & 1.1475696 & 2.5218145\end{array}$

$\begin{array}{llll}\text { C } & 0.8913519 & 1.3796265 & 4.0435472\end{array}$

$\begin{array}{llll}\text { C } & 0.9693403 & -0.3080153 & 2.1035106\end{array}$

$\begin{array}{llll}\mathrm{H} & -0.0056989 & 0.9230105 & 4.4883005\end{array}$

$\begin{array}{llll}\text { C } & 2.9159740 & -0.5774604 & -2.2311058\end{array}$

$\begin{array}{llll}\text { C } & 3.1389035 & -0.5518755 & -0.7177967\end{array}$

$\begin{array}{llll}\text { C } & 4.5185173 & 0.0381306 & -0.4554292\end{array}$

$\begin{array}{llll}\text { O } & 2.1755133 & 0.2593145 & -0.0993282\end{array}$ 


\begin{tabular}{|c|c|c|c|}
\hline & & & \\
\hline & 23 & 1.9670000 & \\
\hline & 9043635 & .9634898 & \\
\hline & & & \\
\hline & & & \\
\hline & 93 & & \\
\hline & 8713094 & & \\
\hline & & & \\
\hline & & & \\
\hline & 0551 & & \\
\hline & & & \\
\hline & 205 & & \\
\hline & 184 & & \\
\hline & 203 & & \\
\hline & 349 & & \\
\hline & 160 & & \\
\hline & 3.639 & -1.2 & \\
\hline & 963 & & \\
\hline & & & \\
\hline & 50 & & \\
\hline & 40 & & \\
\hline & -0.35 & 38 & \\
\hline & -0.3 & & \\
\hline & -1.50 & & \\
\hline & -1.4815319 & -0.0160987 & \\
\hline & & & \\
\hline & & & \\
\hline & -3.5228960 & -1.6 & \\
\hline & 965 & & \\
\hline & -0.481 & & \\
\hline & -0.6606771 & & \\
\hline & -1.32 & & \\
\hline & & & \\
\hline & & & \\
\hline & 3.0284757 & 1.0637446 & 4.1851190 \\
\hline & 2.0795255 & -0.3691475 & 6118 \\
\hline & & & \\
\hline & 0.9016089 & 2.8613050 & \\
\hline & 0.0014565 & 3850092 & 4.0842882 \\
\hline & & & \\
\hline & 0.9864090 & 2.9959487 & 5.4925350 \\
\hline & -3.8640047 & -1.0908914 & -2.7411051 \\
\hline & -5.1532974 & -1.0572464 & \\
\hline & -3.7591105 & -1.3610295 & -4.1119555 \\
\hline
\end{tabular}




$\begin{array}{llll}\mathrm{C} & -4.8804829 & -1.5842015 & -4.8917179 \\ \mathrm{C} & -6.2748915 & -1.2855924 & -2.9719507 \\ \mathrm{C} & -6.1496780 & -1.5500485 & -4.3291626 \\ \mathrm{H} & -2.7753852 & -1.4217007 & -4.5623543 \\ \mathrm{H} & -5.2720524 & -0.8204913 & -1.1428227 \\ \mathrm{H} & -4.7618072 & -1.7980724 & -5.9488220 \\ \mathrm{H} & -7.2587136 & -1.2439527 & -2.5162632 \\ \mathrm{H} & -7.0280829 & -1.7257447 & -4.9397745\end{array}$

\section{9aw-TS-syn}

$\mathrm{E}(\mathrm{PBE} 0-\mathrm{D} 3 / \mathrm{def} 2-\mathrm{TZVP})=-1450.654378594$ (conv)

Lowest Freq. $=-385.46 \mathrm{~cm}^{\wedge}-1$

71

9ax-TS-syn (024TSc1/opt)

$\begin{array}{llll}\text { C } & -2.2765915 & 3.5798025 & 0.1209357\end{array}$

$\begin{array}{llll}\text { C } & -2.0610557 & 2.0955632 & 0.1967923\end{array}$

F $\quad-3.4597577 \quad 3.8782628 \quad-0.4633136$

F $\quad-1.3326948 \quad 4.2050354 \quad-0.6031904$

$\begin{array}{llll}\text { F } & -2.2958621 & 4.1857053 & 1.3239030\end{array}$

$\begin{array}{llll}\mathrm{H} & -2.1419661 & 1.7320605 & -0.8327009\end{array}$

$\begin{array}{llll}\mathrm{H} & -2.9042066 & 1.6810913 & 0.7563516\end{array}$

$\begin{array}{llll}\text { C } & -0.7627707 & 1.6742679 & 0.8043030\end{array}$

$\begin{array}{llll}\mathrm{H} & 0.1217455 & 2.0805762 & 0.3231439\end{array}$

$\begin{array}{llll}\text { C } & 0.6081075 & 1.0851464 & 2.8626589\end{array}$

$\begin{array}{llll}\text { C } & 1.7644814 & 2.0520290 & 2.5520653\end{array}$

$\begin{array}{llll}\text { C } & 0.8692532 & -0.3492446 & 2.4043752\end{array}$

$\begin{array}{llll}\mathrm{H} & 1.9615734 & 2.0073298 & 1.4773909\end{array}$

$\begin{array}{llll}\text { C } & 2.7276461 & -0.8607778 & -1.9643545\end{array}$

$\begin{array}{llll}\text { C } & 3.0024330 & -0.8153471 & -0.4602152\end{array}$

$\begin{array}{llll}\text { C } & 4.4341301 & -0.3371152 & -0.2560710\end{array}$

$\begin{array}{llll}\text { O } & 2.1310533 & 0.0881769 & 0.1667263\end{array}$

$\begin{array}{llll}\text { B } & 0.9881711 & -0.6358026 & 0.7972221\end{array}$

$\begin{array}{llll}\text { O } & 1.3156745 & -2.0686996 & 0.5395345\end{array}$

$\begin{array}{llll}\text { C } & 2.6720082 & -2.1843440 & 0.2267591\end{array}$

$\begin{array}{llll}\text { C } & 3.4997471 & -2.4049024 & 1.4981513\end{array}$

$\begin{array}{llll}\text { C } & 2.8648028 & -3.4002061 & -0.6698382\end{array}$

$\begin{array}{llll}\mathrm{H} & 0.0530155 & -0.9736577 & 2.7954988\end{array}$

$\begin{array}{llll}\mathrm{H} & 1.7635378 & -0.7364121 & 2.9010030\end{array}$

$\begin{array}{llll}\mathrm{H} & 4.5744840 & 0.6133199 & -0.7781423\end{array}$

$\mathrm{H} \quad 5.1582552 \quad-1.0550496 \quad-0.6545602$

$\begin{array}{llll}\mathrm{H} & 4.6459098 & -0.1722209 & 0.8002319\end{array}$

$\mathrm{H} \quad 2.1928994 \quad-3.3613520 \quad-1.5267319$

$\begin{array}{llll}\mathrm{H} & 2.6323966 & -4.3047099 & -0.1013588\end{array}$

H $\quad 3.8965790 \quad-3.4777125 \quad-1.0278936$ 


\begin{tabular}{|c|c|c|c|}
\hline & & & \\
\hline & .7395450 & .2724504 & 1431 \\
\hline & 4746016 & 71 & \\
\hline & & & \\
\hline & 8607 & & \\
\hline & 3.0637566 & & \\
\hline & -1.6702534 & -1.39 & \\
\hline & 7 & & \\
\hline & -0.4465370 & & \\
\hline & -0.44 & & 710 \\
\hline & -1.51 & -0.2 & \\
\hline & 195 & & \\
\hline & -2.68 & & \\
\hline & -2.70 & & \\
\hline & -3.56 & & \\
\hline & & & \\
\hline & -0.72 & 1.557 & 1796 \\
\hline & -1.0 & & \\
\hline & -1.50 & & \\
\hline & & & \\
\hline & 1.43 & & \\
\hline & 1.14 & & 3272 \\
\hline & & & \\
\hline & & & \\
\hline & 3.045 & & 5476 \\
\hline & 3.38 & & \\
\hline & & & \\
\hline & 3.8505448 & & \\
\hline & -3.8577612 & -1.0670388 & -2.6969407 \\
\hline & -5.0353999 & & 168 \\
\hline & -5.85 & & \\
\hline & -5.4141042 & -1.1708236 & -1.1779367 \\
\hline & -4.7580322 & -2.74 & 347 \\
\hline & -4.34151 & 0.2888048 & -3.22 \\
\hline & -5.1810321 & & \\
\hline & -3.5447788 & 081499 & \\
\hline & -4.6689899 & 87 & \\
\hline & -3.4131022 & -1.9346482 & -3.8794009 \\
\hline & -2.5843998 & & -4.4201920 \\
\hline & & & \\
\hline & -3.0774148 & -2.9141297 & -3.530402 \\
\hline
\end{tabular}

\section{9aw-TS-anti}

$\mathrm{E}(\mathrm{PBE} 0-\mathrm{D} 3 / \mathrm{def} 2-\mathrm{TZVP})=-1450.653818414$ (conv) 


$\begin{array}{lrrc}\text { Lowest Freq. }= & -372.66 \mathrm{~cm}^{\wedge}-1 & \\ \text { 71 } & & & \\ \text { 9ax-TS-anti }(025 \mathrm{TSc} 1 / \mathrm{opt}) & \\ \mathrm{C} & -1.9246808 & 3.7153142 & -0.3282188 \\ \mathrm{C} & -1.7902323 & 2.2261027 & -0.1856091 \\ \mathrm{~F} & -3.0889134 & 4.0497943 & -0.9314654 \\ \mathrm{~F} & -0.9471964 & 4.2565540 & -1.0743781 \\ \mathrm{~F} & -1.9163027 & 4.3750456 & 0.8464447 \\ \mathrm{H} & -1.8997409 & 1.8219835 & -1.1970229 \\ \mathrm{H} & -2.6524631 & 1.8854151 & 0.3948841 \\ \mathrm{C} & -0.5152879 & 1.7535637 & 0.4330989 \\ \mathrm{H} & 0.3970629 & 2.0464951 & -0.0790190 \\ \mathrm{C} & 0.8390217 & 1.1893411 & 2.5064225 \\ \mathrm{C} & 0.9136408 & 1.4487393 & 4.0236948 \\ \mathrm{C} & 0.9813757 & -0.2750859 & 2.1168161 \\ \mathrm{H} & 0.0181061 & 0.9973522 & 4.4768244 \\ \mathrm{C} & 2.9078358 & -0.6420470 & -2.2213844 \\ \mathrm{C} & 3.1298531 & -0.6025012 & -0.7079671 \\ \mathrm{C} & 4.5213053 & -0.0369484 & -0.4522458 \\ \mathrm{O} & 2.1832077 & 0.2353083 & -0.1015313 \\ \mathrm{~B} & 1.0847105 & -0.5684114 & 0.5111947 \\ \mathrm{O} & 1.4996171 & -1.9730982 & 0.2352836 \\ \mathrm{C} & 2.8673468 & -2.0017324 & -0.0498224 \\ \mathrm{C} & -0.3667896 & -0.3208367 & -0.2527049 \\ \mathrm{C} & -0.4103341 & -0.0835757 & -1.6547386 \\ \mathrm{H} & 3.6868088 & -2.1970450 & 1.2320910 \\ \mathrm{C} & 3.1494587 & -3.1891008 & -0.9613752 \\ \mathrm{H} & 0.1505185 & -0.8422756 & 2.5649010 \\ \mathrm{H} & 1.8849712 & -0.6877840 & 2.5684374 \\ \mathrm{H} & 4.6170985 & 0.9263205 & -0.9607203 \\ \mathrm{H} & 5.3032724 & -0.7023687 & -0.8323380 \\ \mathrm{H} & 4.6854624 & 0.1289662 & 0.6123606 \\ \mathrm{H} & 2.4911873 & -3.1780495 & -1.8295722 \\ \mathrm{H} & 2.9630069 & -4.1150384 & -0.4106799 \\ \mathrm{H} & 4.1899888 & -3.1981315 & -1.3018184 \\ \mathrm{H} & 2.8729132 & 0.3852272 & -2.5939951 \\ \mathrm{H} & 1.9639983 & -1.1287135 & -2.4658711 \\ \mathrm{H} & 3.7159275 & -1.1685164 & -2.7383768 \\ \mathrm{H} & 3.6244124 & -1.3289467 & 1.8866869 \\ \mathrm{H} & -2804228 & -3.0574355 & 1.7691323 \\ \mathrm{H} & -2.4235946\end{array}$




$\begin{array}{lrrr}\mathrm{H} & -1.4816245 & -0.1003266 & -3.4865721 \\ \mathrm{C} & -2.7049341 & -0.8528005 & -1.8735058 \\ \mathrm{C} & -2.6764166 & -1.1429880 & -0.5081879 \\ \mathrm{H} & -3.5456532 & -1.5826150 & -0.0309114 \\ \mathrm{H} & 0.4686938 & 0.3446398 & -2.1216822 \\ \mathrm{C} & -0.4557372 & 1.7446614 & 1.9227858 \\ \mathrm{H} & -0.6244542 & 2.7714998 & 2.2747143 \\ \mathrm{H} & -1.3035863 & 1.1598102 & 2.3120242 \\ \mathrm{H} & 1.6754850 & 1.7375683 & 2.0454140 \\ \mathrm{C} & 2.1352115 & 0.7989915 & 4.6621773 \\ \mathrm{H} & 3.0517372 & 1.1426523 & 4.1699348 \\ \mathrm{H} & 2.1077956 & -0.2877005 & 4.5858837 \\ \mathrm{H} & 2.2062690 & 1.0639605 & 5.7220198 \\ \mathrm{C} & 0.9200372 & 2.9363095 & 4.3652291 \\ \mathrm{H} & 0.0155839 & 3.4502017 & 4.0364241 \\ \mathrm{H} & 1.7734653 & 3.4301001 & 3.8878269 \\ \mathrm{H} & 1.0110222 & 3.0892504 & 5.4453397 \\ \mathrm{C} & -3.9237784 & -1.0758867 & -2.7557976 \\ \mathrm{C} & -5.1035559 & -1.6503640 & -1.9783803 \\ \mathrm{H} & -4.8602845 & -2.6213521 & -1.5401832 \\ \mathrm{H} & -5.9565244 & -1.7879322 & -2.6494592 \\ \mathrm{H} & -5.4143502 & -0.9817349 & -1.1719601 \\ \mathrm{C} & -3.5746837 & -2.0529799 & -3.8836060 \\ \mathrm{H} & -2.7485781 & -1.6786666 & -4.4910620 \\ \mathrm{H} & -4.4368944 & -2.2091652 & -4.5410549 \\ \mathrm{H} & -3.2726883 & -3.0193620 & -3.4727747 \\ \mathrm{C} & -4.3624338 & 0.2595696 & -3.3677136 \\ \mathrm{H} & -3.5640217 & 0.7021564 & -3.9663326 \\ \mathrm{H} & -4.6228172 & 0.9749193 & -2.5836153 \\ \mathrm{H} & -5.2359149 & 0.1216120 & -4.0142281 \\ & & & \\ \end{array}$

\section{CF3}

$\mathrm{E}(\mathrm{PBE} 0-\mathrm{D} 3 /$ def2-TZVP $)=-337.3939426721$ (conv)

Lowest Freq. $=513.14 \mathrm{~cm}^{\wedge}-1$

4

CF3 (017c1/opt)

$\begin{array}{llll}\text { C } & -0.0000000 & 0.0000000 & -0.2955589\end{array}$

$\begin{array}{llll}\text { F } & 0.6260960 & 1.0844301 & 0.0985196\end{array}$

F $\quad 0.6260960 \quad-1.0844301 \quad 0.0985196$

F $\quad-1.2521921 \quad 0.0000000 \quad 0.0985196$

\section{CF3I}

$\mathrm{E}(\mathrm{PBE}-\mathrm{D} 3 / \mathrm{def} 2-\mathrm{TZVP})=-635.1806836457$ (conv)

Lowest Freq. $=269.37 \mathrm{~cm}^{\wedge}-1$ 
5

CF3I (016c1/opt)

$\begin{array}{llll}\text { I } & -0.0000000 & 0.0000000 & -1.9929517\end{array}$

$\begin{array}{llll}\text { C } & -0.0000000 & 0.0000000 & 0.1545778\end{array}$

$\begin{array}{llll}\text { F } & 0.6203768 & 1.0745242 & 0.6148603\end{array}$

F $\quad 0.6203768 \quad-1.0745242 \quad 0.6148603$

$\begin{array}{llll}\text { F } & -1.2407537 & 0.0000000 & 0.6148603\end{array}$ 
Figure S6 Optimized molecular structures of $\mathrm{CF}_{3}$ radical and $\mathrm{CF}_{3} \mathrm{I}$
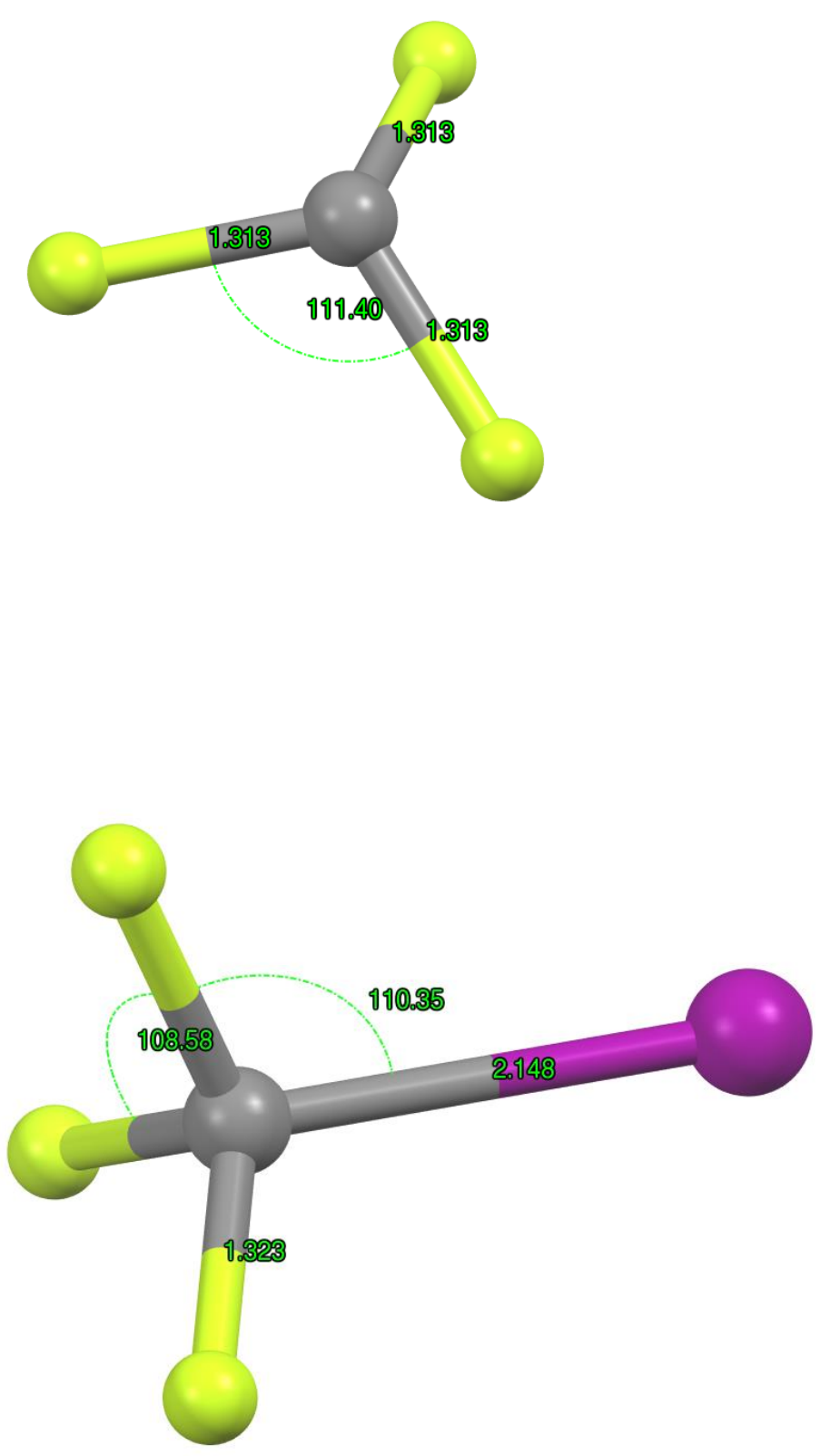


\section{References}

S1 Atack, T. C.; Cook, S. P. J. Am. Chem. Soc. 2016, 138, 6139.

S2 Wang, J.; Shang, M.; Lundberg, H.; Feu, K. S.; Hecker, S. J.; Qin, T.; Blackmond, D. G.; Baran, P. S. ACS Catal. 2018, 8, 9537.

S3 Sonawane, R. P.; Jheengut, V.; Rabalakos, C.; Larouche-Gauthier, R.; Scott, H. K.; Aggarwal, V. K. Angew. Chem. Int. Ed. 2011, 50, 3760.

S4 Bonet, A.; Odachowski, M.; Leonori, D.; Essafi, S.; Aggarwal, V. K. Nat. Chem. 2014, 6, 584 .

S5 Hupe, E.; Marek, I.; Knochel, P. Org. Lett. 2002, 4, 2861.

S6 Wang, D.; Mück-Lichtenfeld, C.; Studer, A. J. Am. Chem. Soc. 2019, 141, 14126.

S7. Perdew, J. P.; Ernzerhof, M.; Burke, K. Rationale for mixing exact exchange with density functional approximations. J. Chem. Phys. 105. 9982-9985 (1996).

S8. Adamo, C.; Barone, V. Toward reliable density functional methods without adjustable parameters: The PBE0 model. J. Chem. Phy. 110. 6158-6170 (1999)

S9. Grimme, S.; Antony, J.; Ehrlich, S.; Krieg, H. A consistent and accurate ab initio parametrization of density functional dispersion correction (DFT-D) for the 94 elements H-Pu. $J$. Chem. Phys. 132. 154104 (2010).

S10. Grimme, S.; Ehrlich., S.; Goerigk, L. Effect of the damping function in dispersion corrected density functional theory. J. Comput. Chem. 32. 1456-1465 (2011).

S11. Weigend, F; Ahlrichs, R. Balanced basis sets of split valence. triple zeta valence and quadruple zeta valence quality for $\mathrm{H}$ to Rn: Design and assessment of accuracy. Phys. Chem. Chem. Phys. 7. 3297-3305 (2005).

S12. Grimme, S. Supramolecular binding thermodynamics by dispersion-corrected density functional theory. Chem. Eur. J. 18. 9955-9964 (2012).

S13. Zhao, Y; Truhlar, D.G. Design of Density Functionals That Are Broadly Accurate for Thermochemistry, Thermochemical Kinetics, and Nonbonded Interactions. J. Phys. Chem. A 2005, 109, 5656-5667.

S14. Klamt, A. Conductor-like Screening Model for Real Solvents: A New Approach to the Quantitative Calculation of Solvation Phenomena. J. Phys. Chem. 1995, 99, 2224-2235.

S15. Klamt, A.; Jonas V.; Bürger, T.; Lohrenz, J. C. Refinement and Parametrization of COSMORS. J. Phys. Chem. A 1998, 102, 5074-5085 
S16. COSMOtherm, Release 19; @ 2019 COSMOlogic GmbH \& Co. KG, a Dassault Systèmes company.

S17. TURBOMOLE V7.4.1 (2020), a development of University of Karlsruhe and Forschungszentrum Karlsruhe GmbH, 1989-2007, TURBOMOLE GmbH, since 2007; available from http://www.turbomole.com.

S18. Pracht, P.; Bohle, F; Grimme, S. Automated exploration of the low-energy chemical space with fast quantum chemical methods. Phys. Chem. Chem. Phys. 2020, 22, 7169-7192.

S19. Bannwarth, C.; Ehlert, S; Grimme, S. GFN2-xTB - An Accurate and Broadly Parametrized Self-Consistent Tight-Binding Quantum Chemical Method with Multipole Electrostatics and Density-Dependent Dispersion Contributions. J. Chem. Theory Comput. 2019, 15, $1652-1671$. 


\section{NMR Spectra}
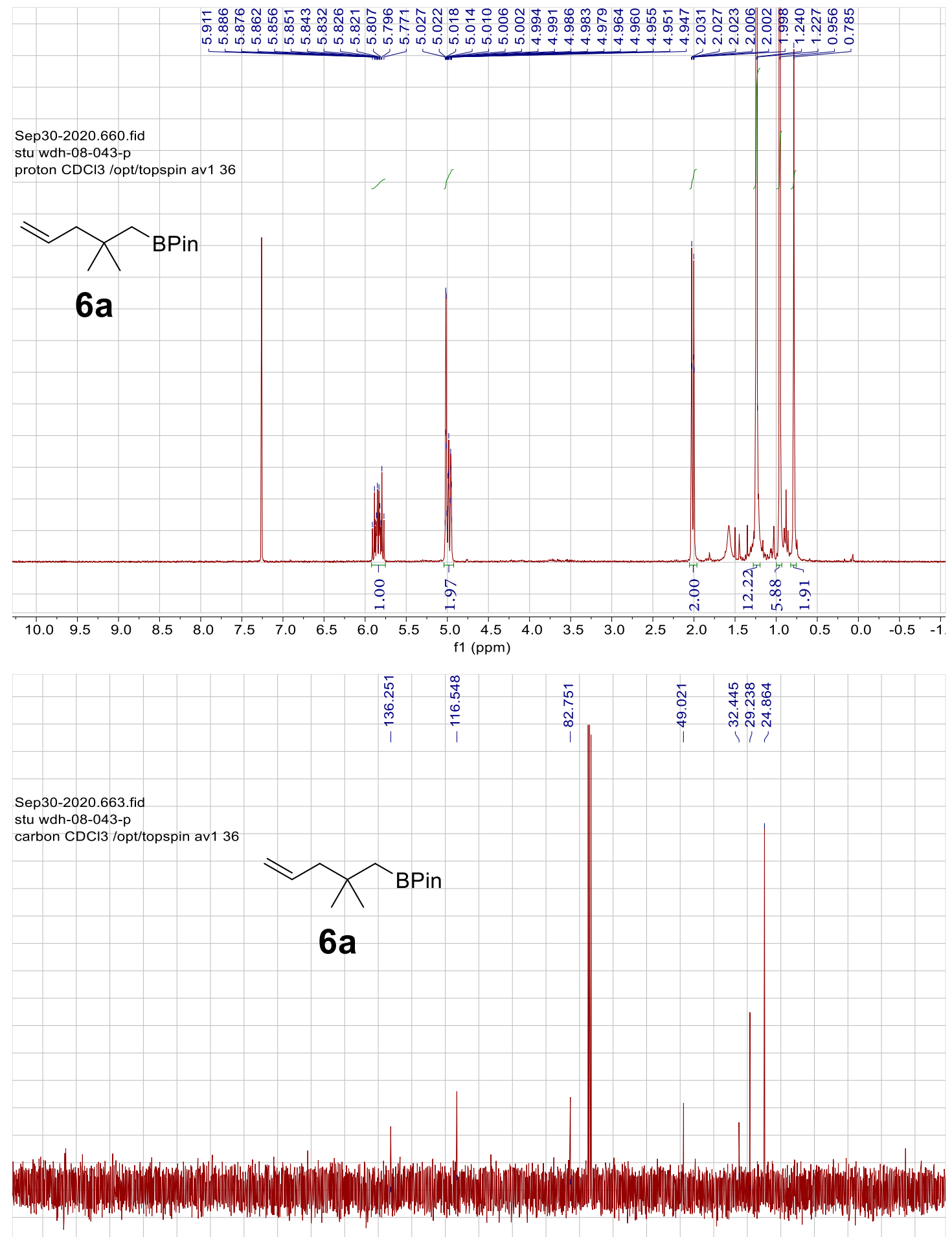

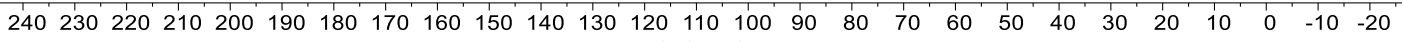
f1 (ppm) 

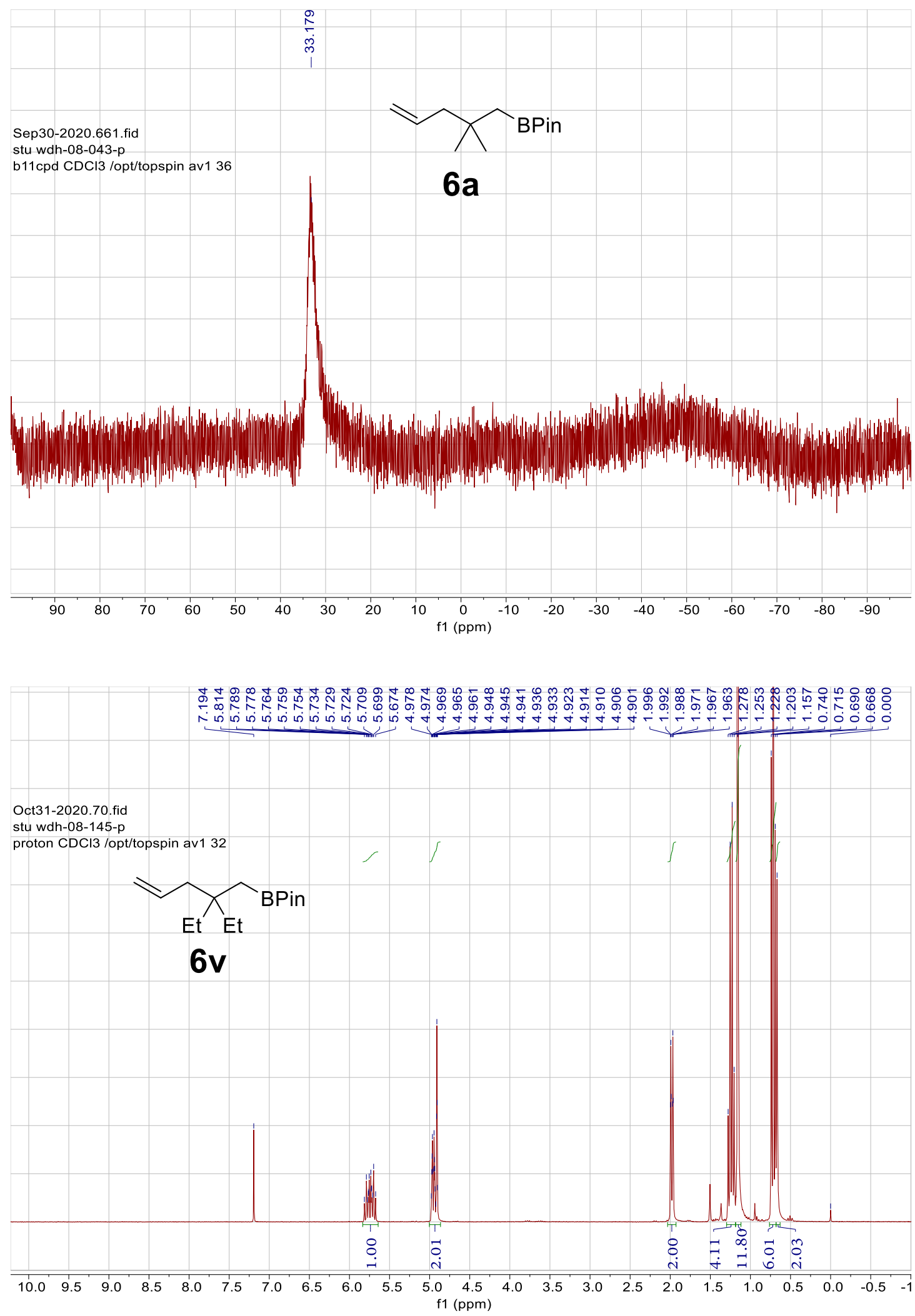

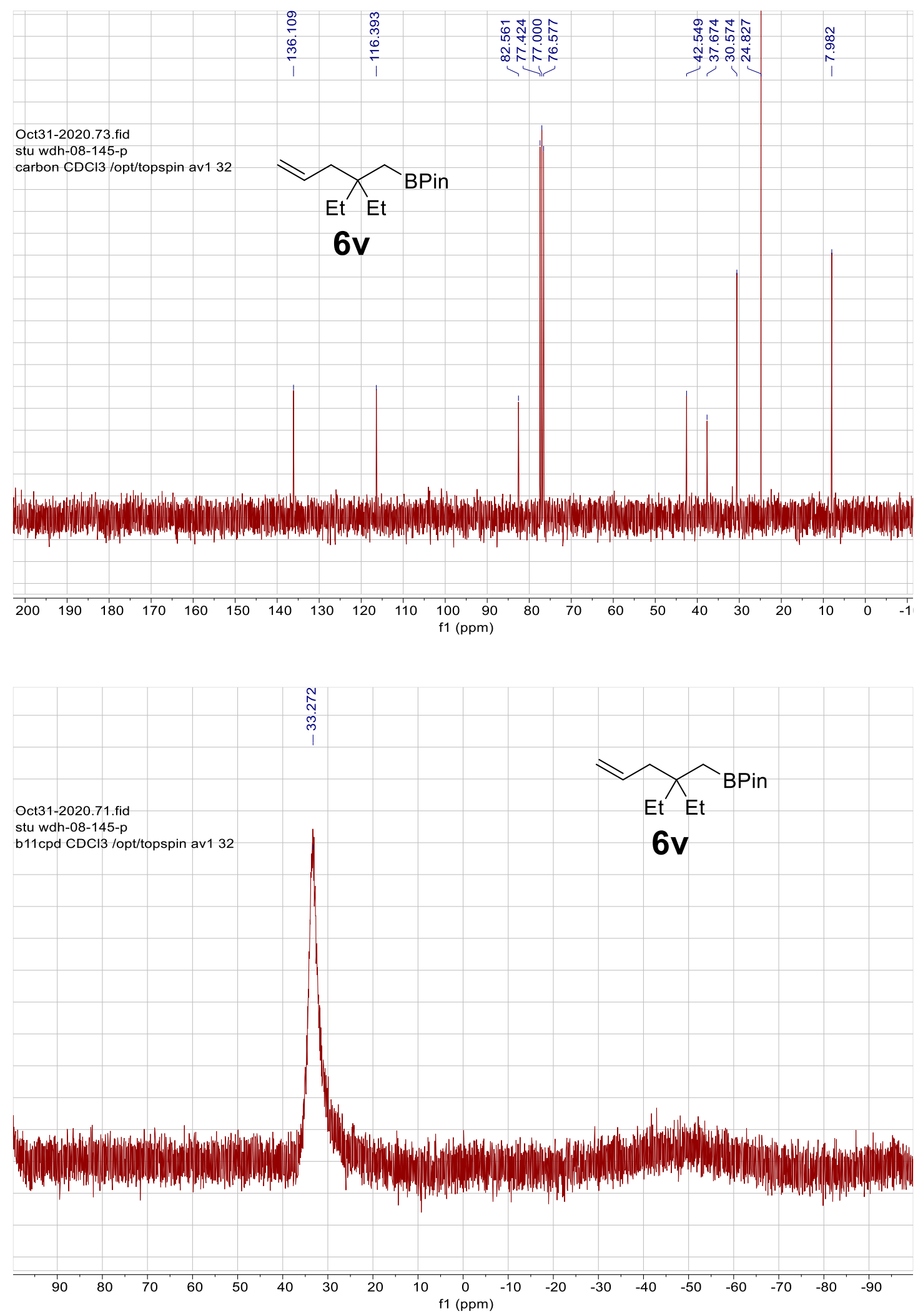

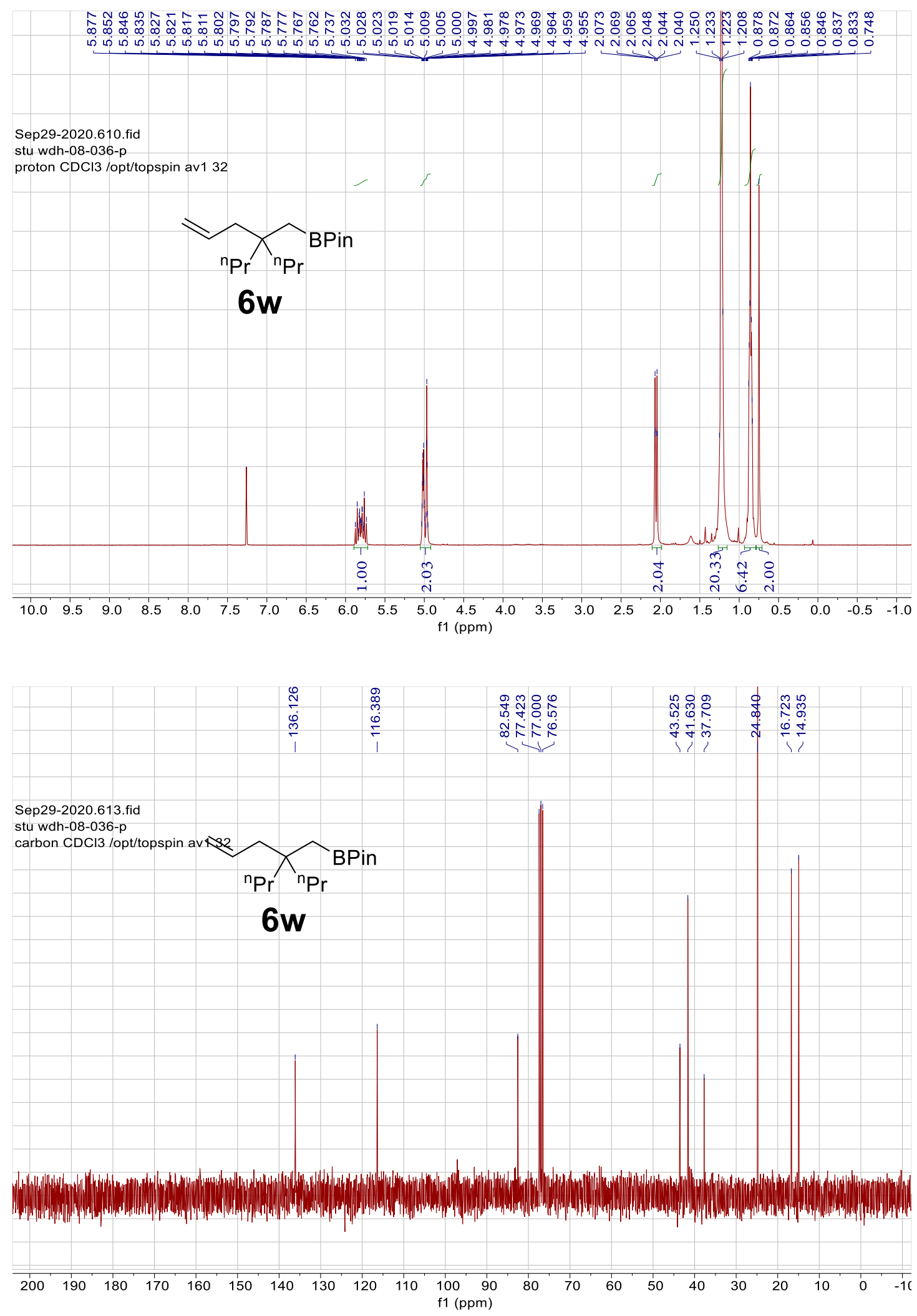

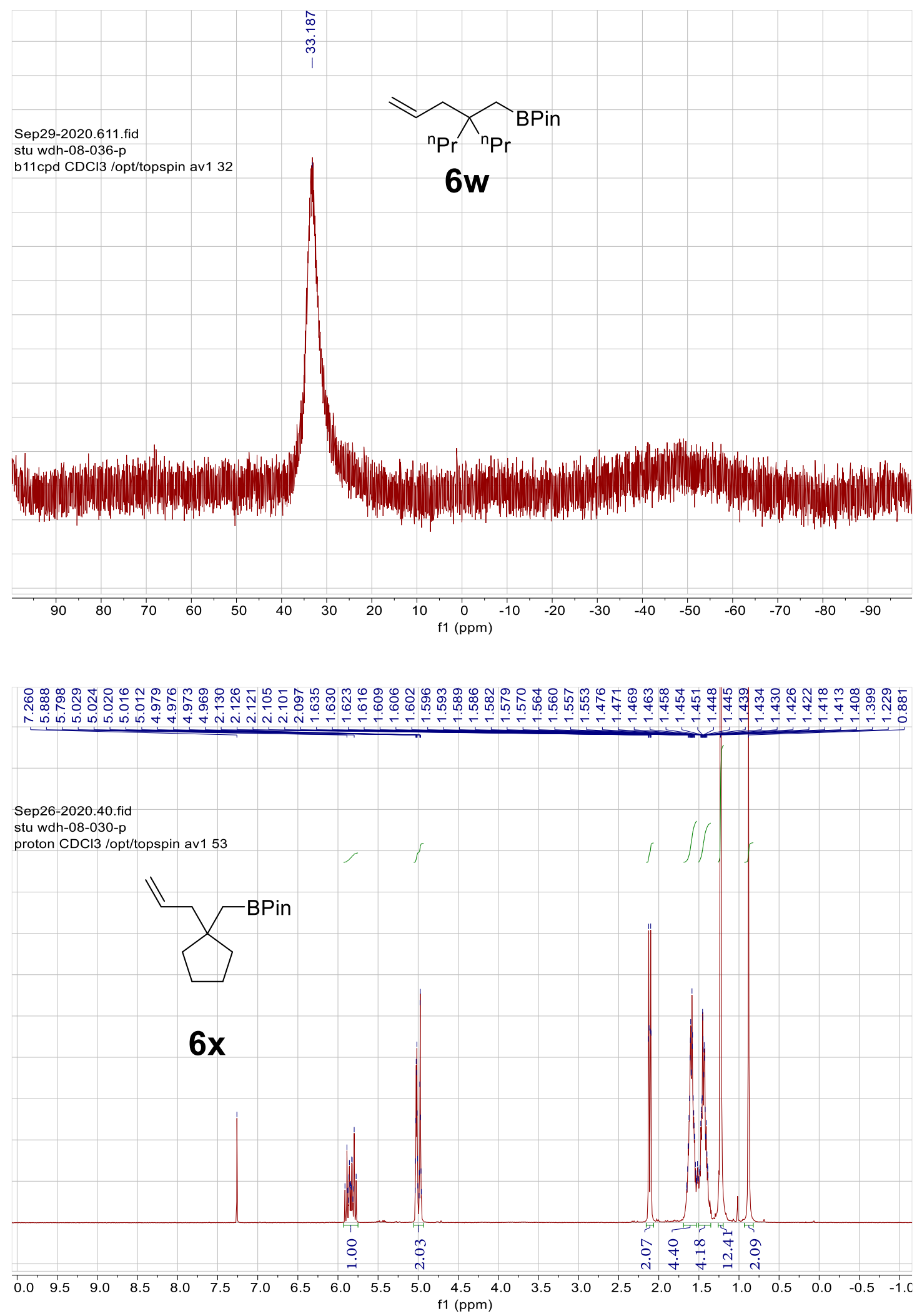

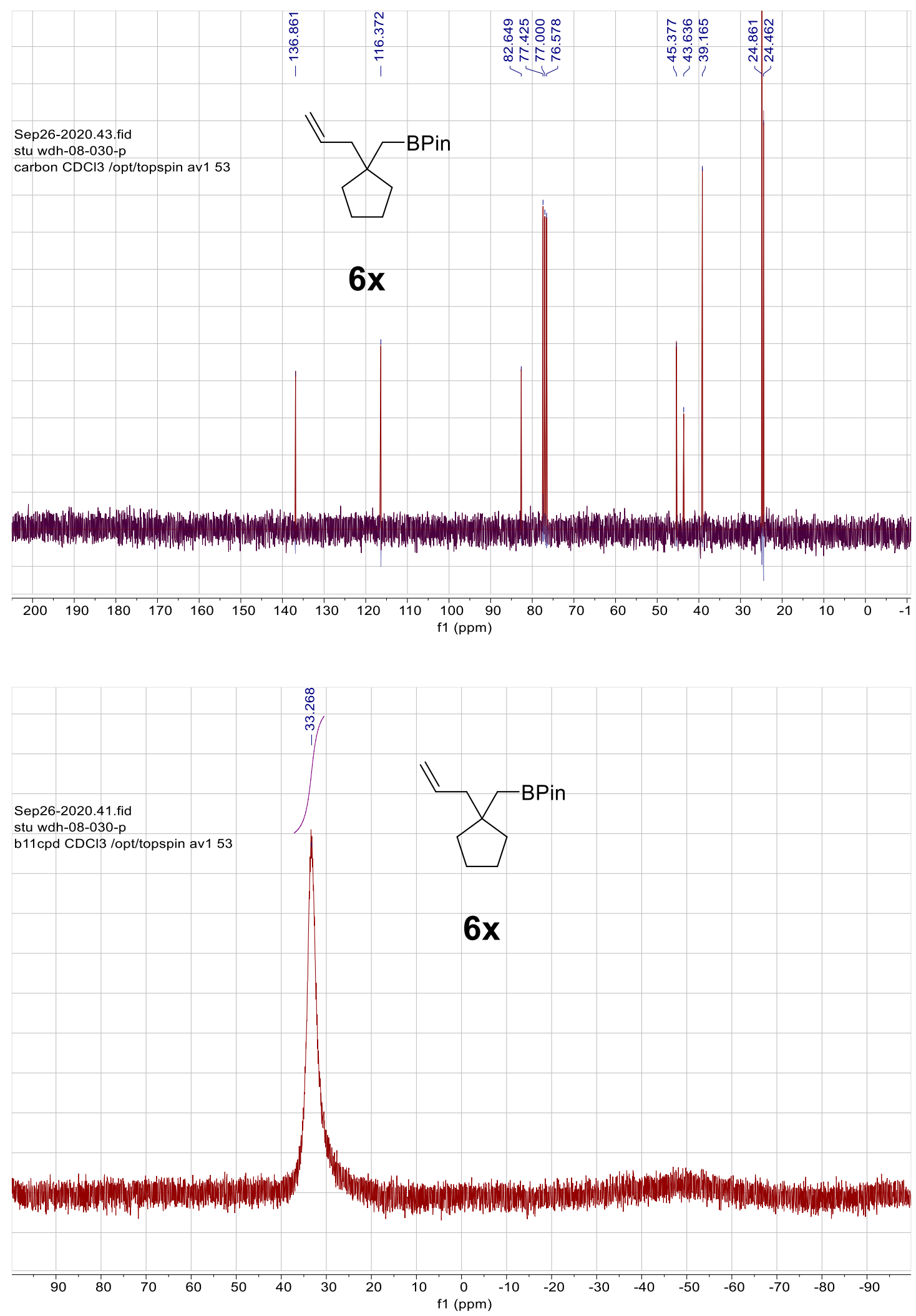
proton $\mathrm{CDCl} 3$ /opt/topspin av1 31
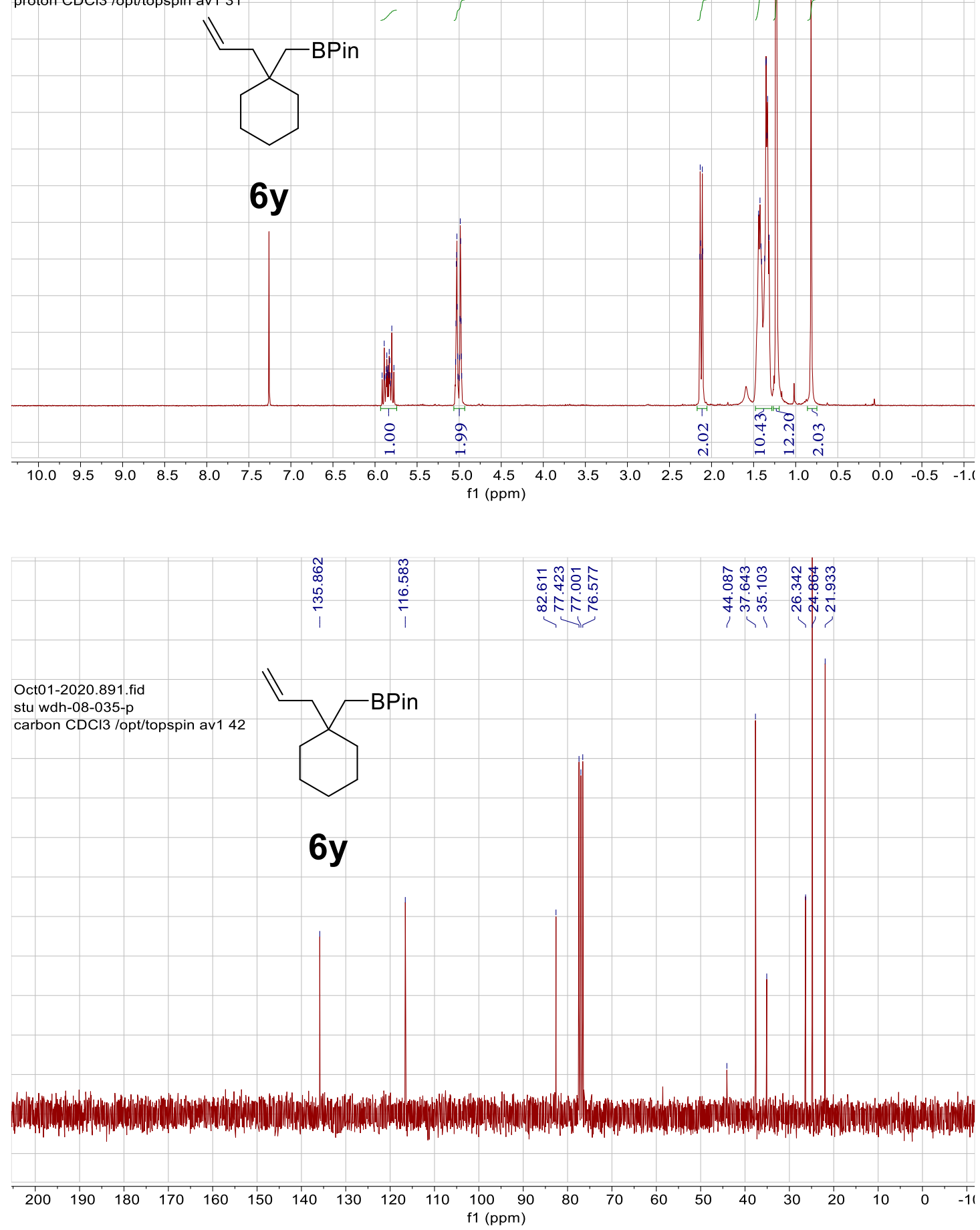

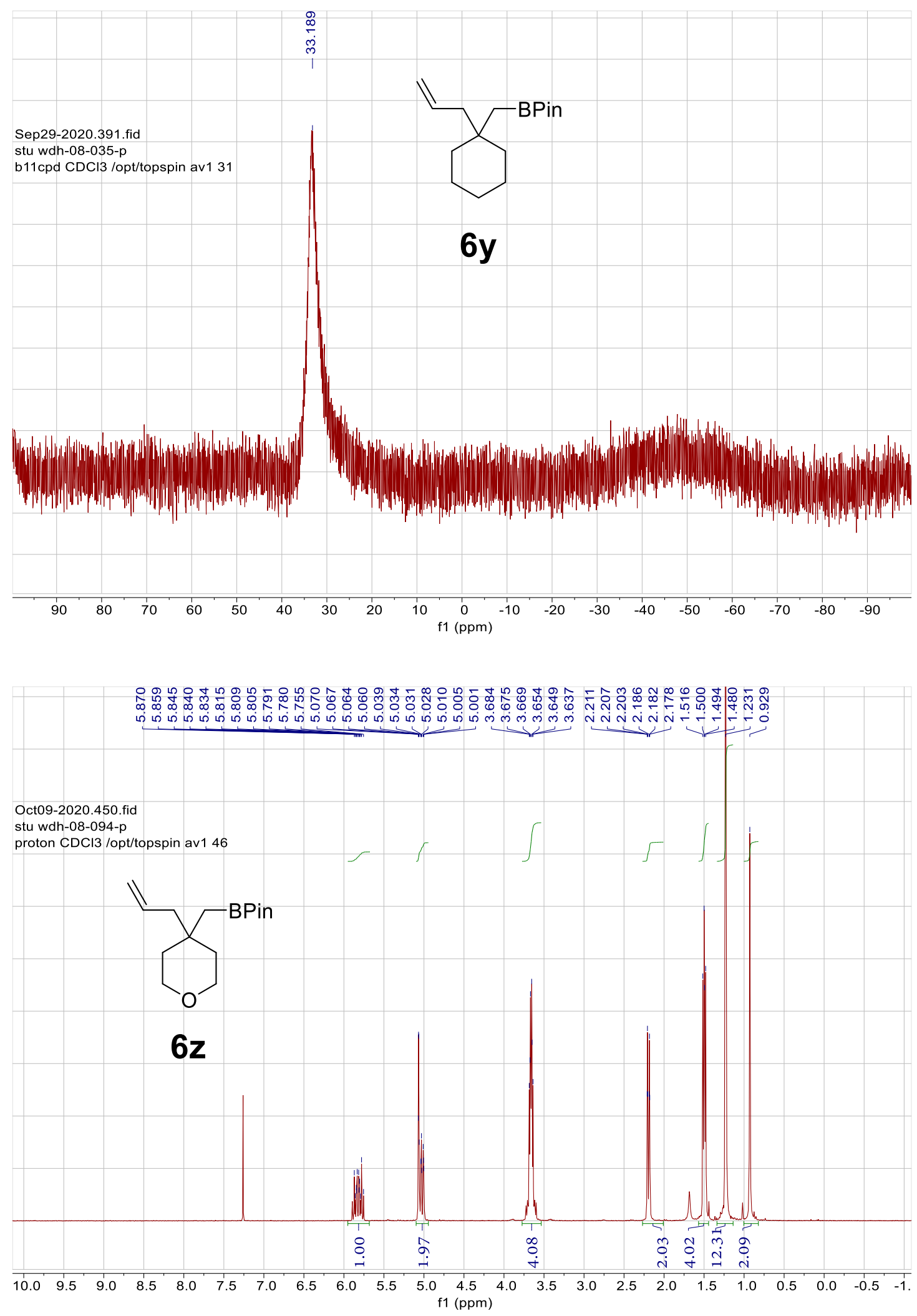


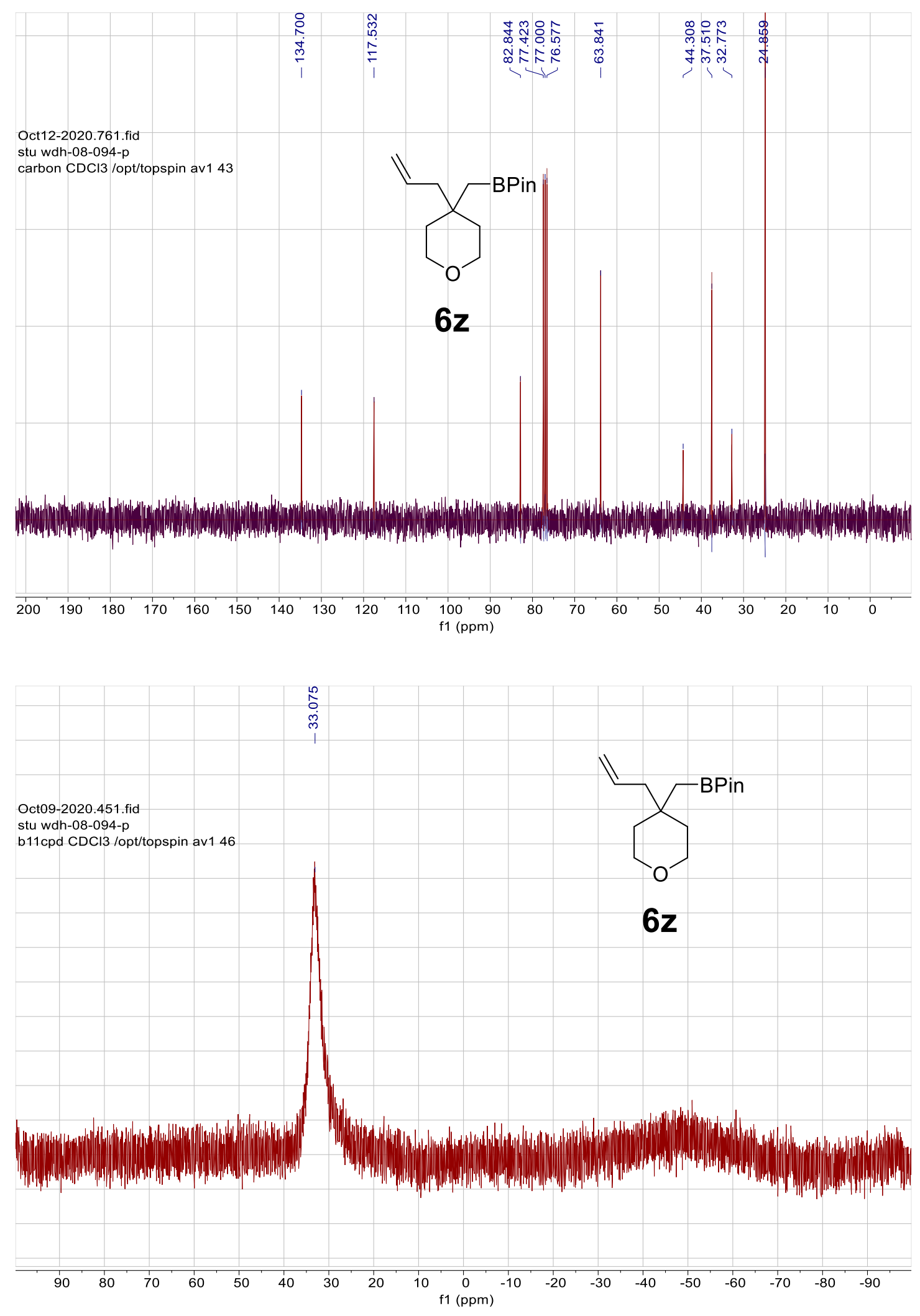



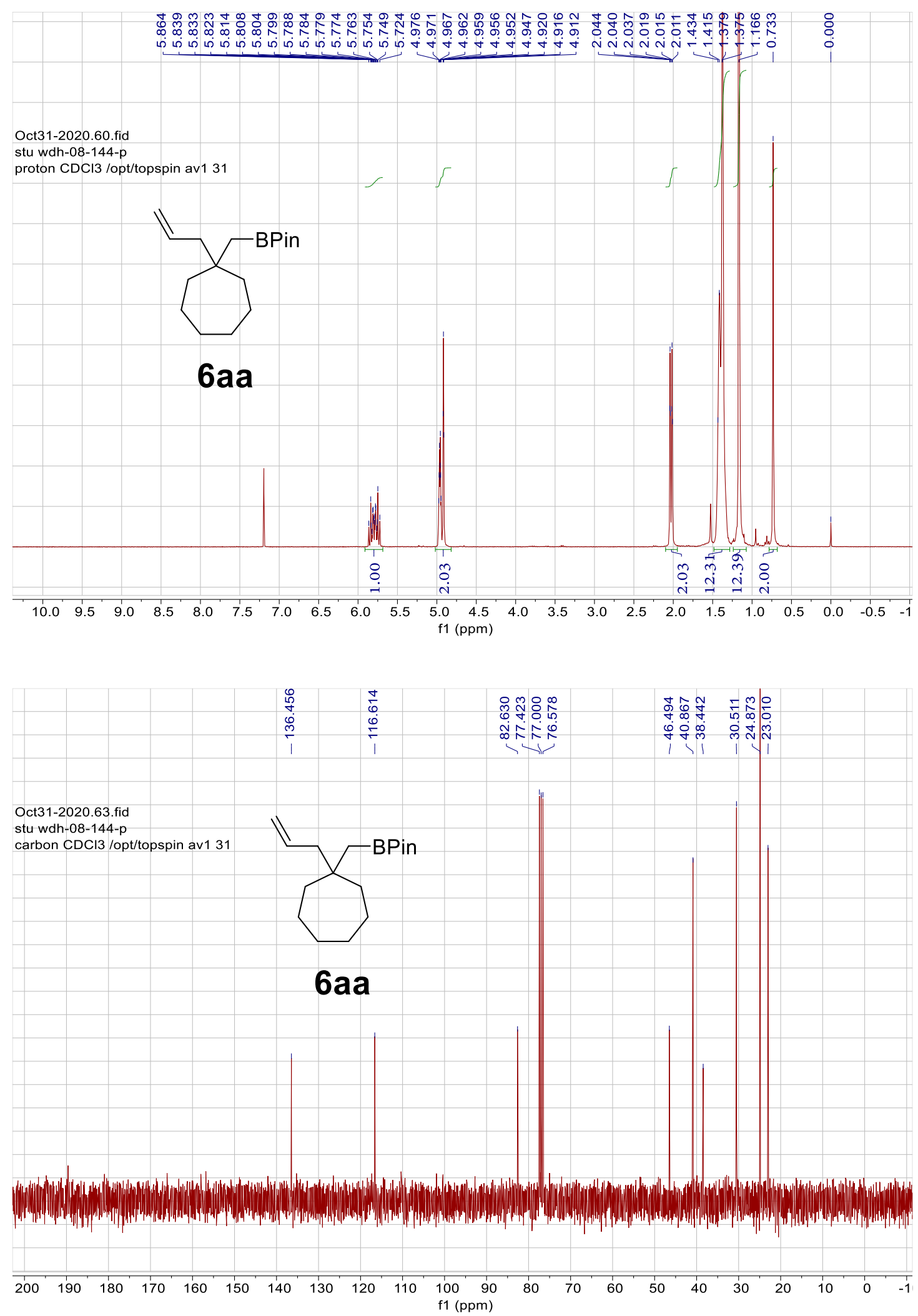


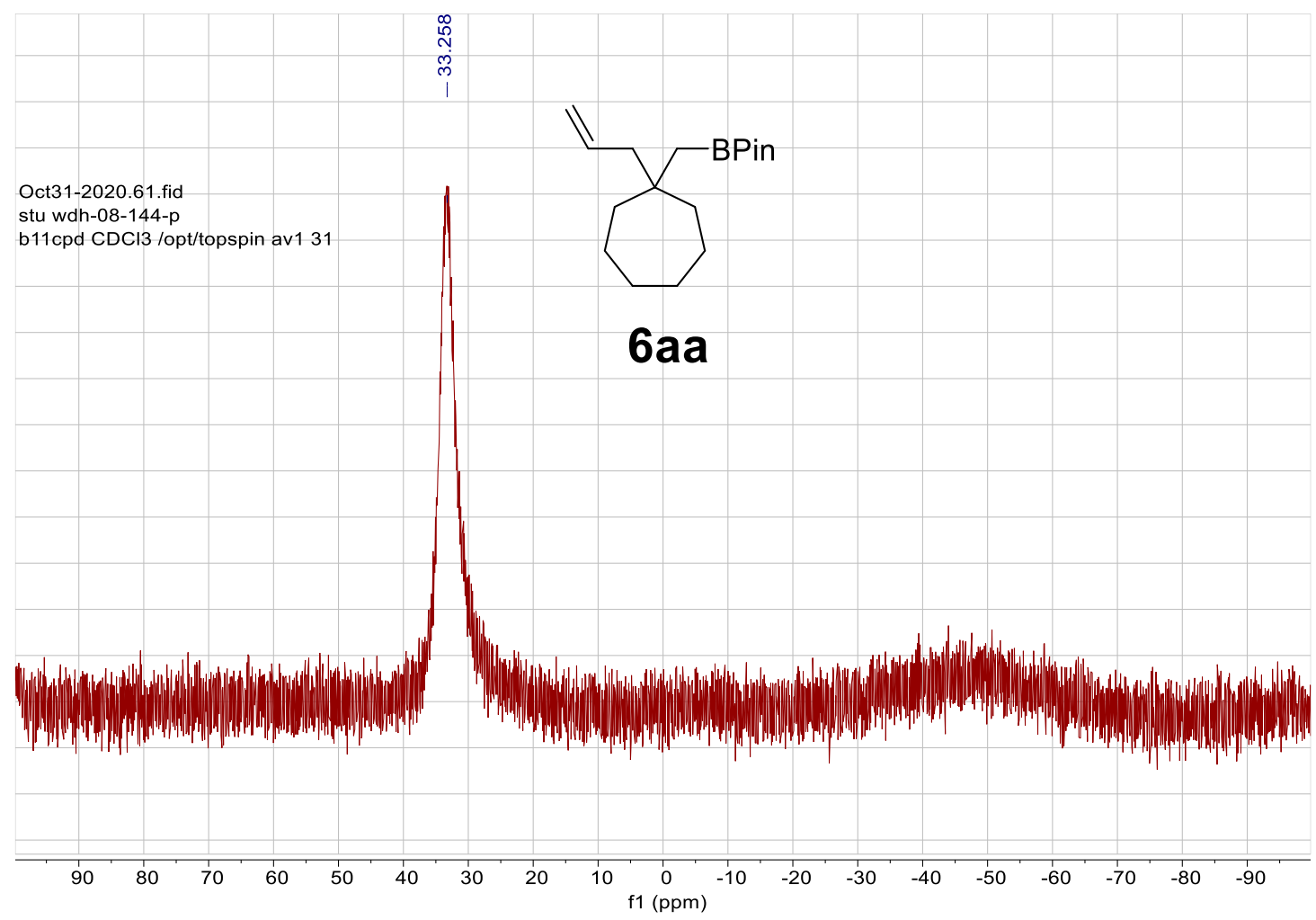

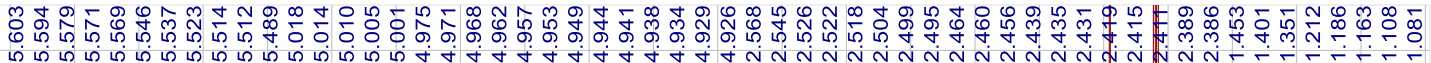

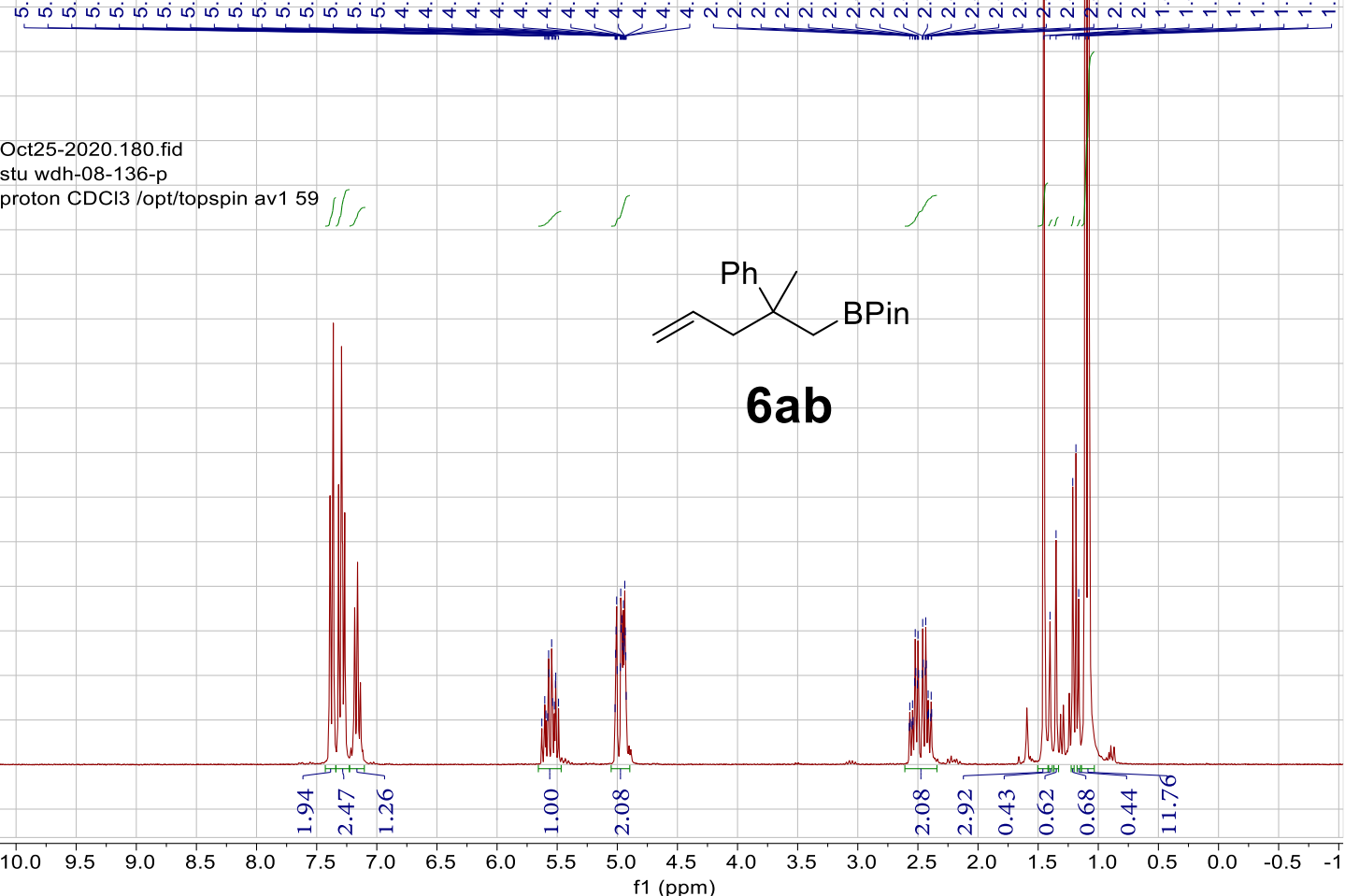




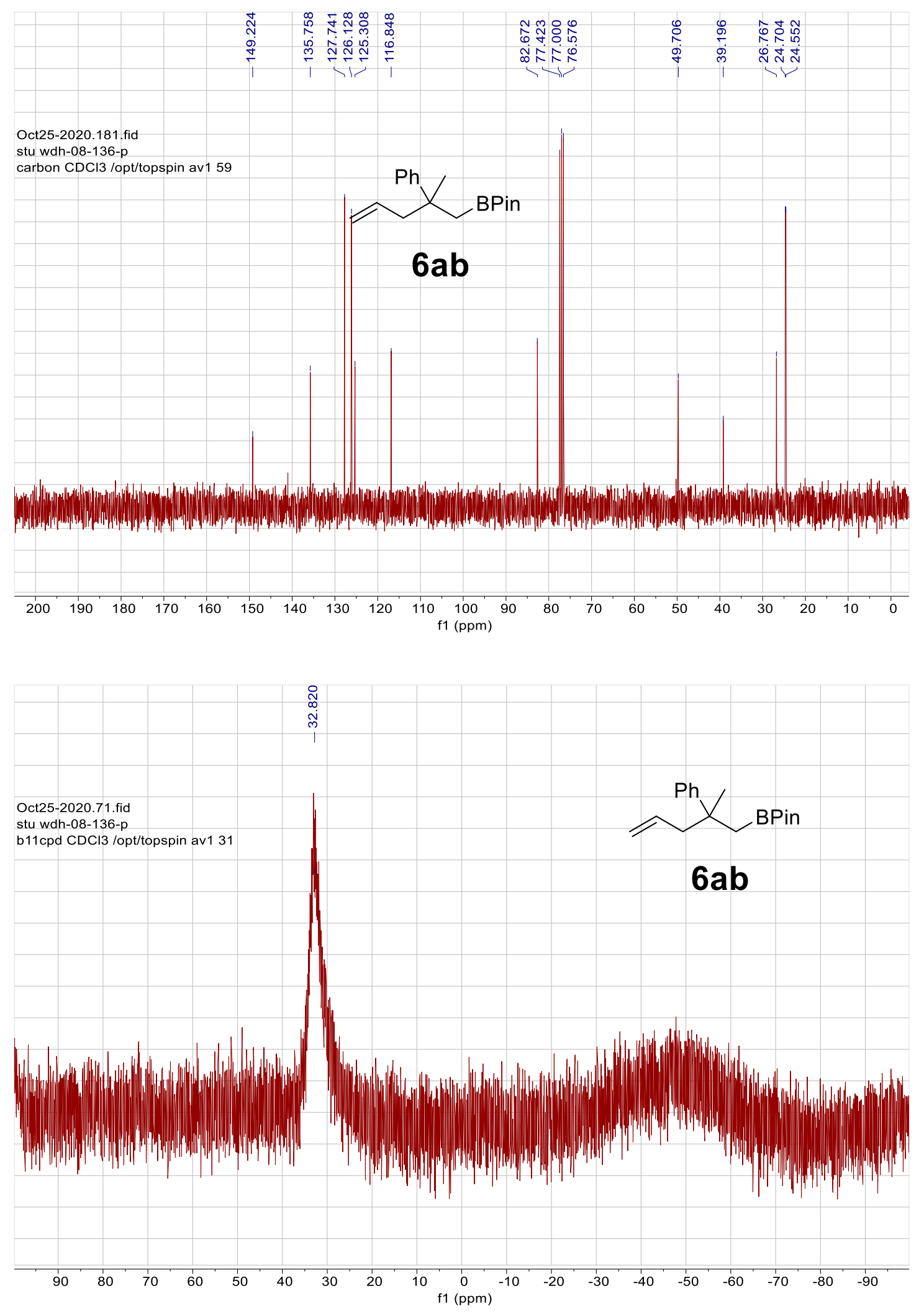



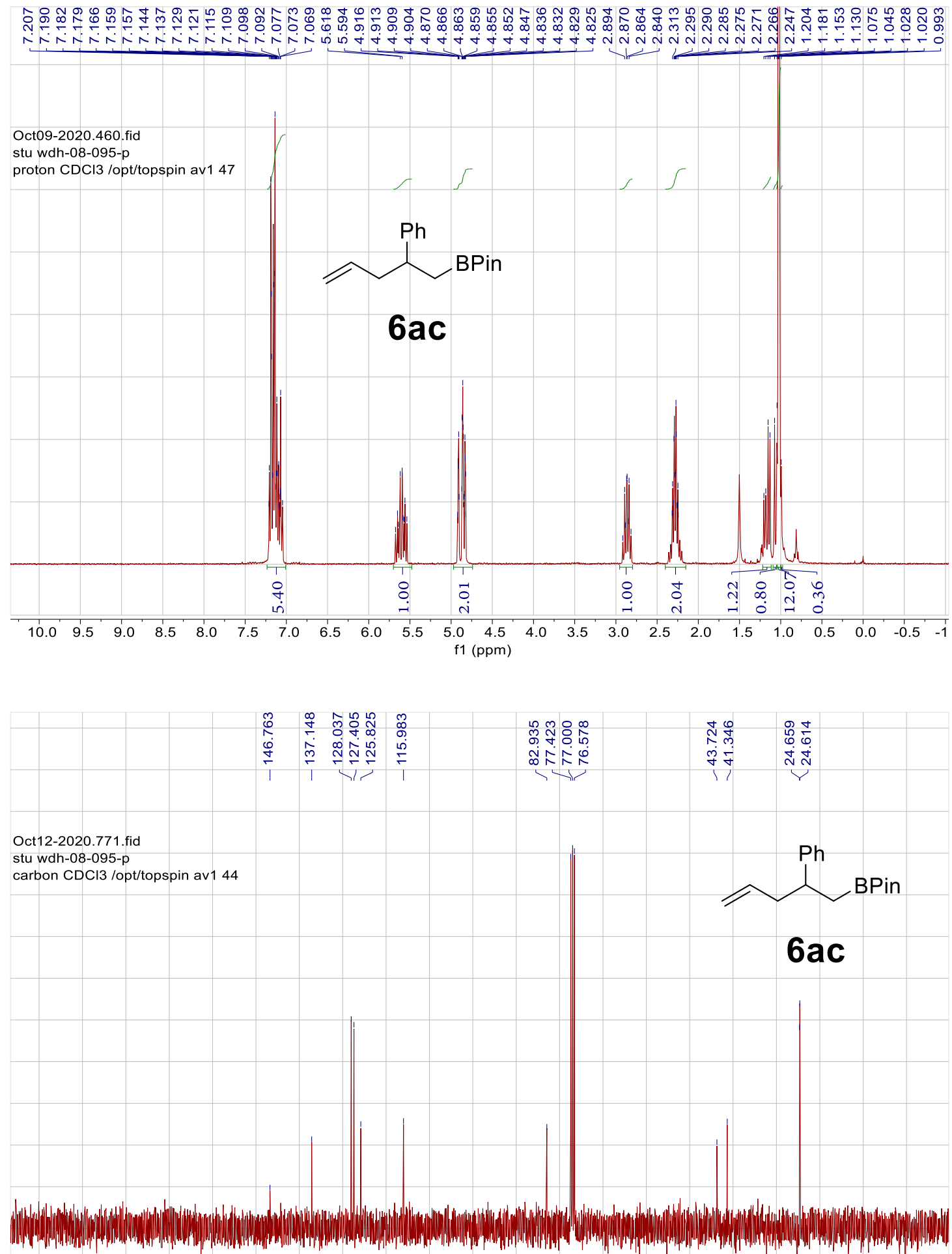

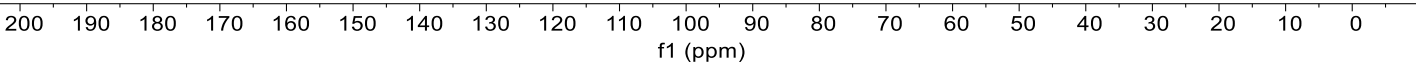



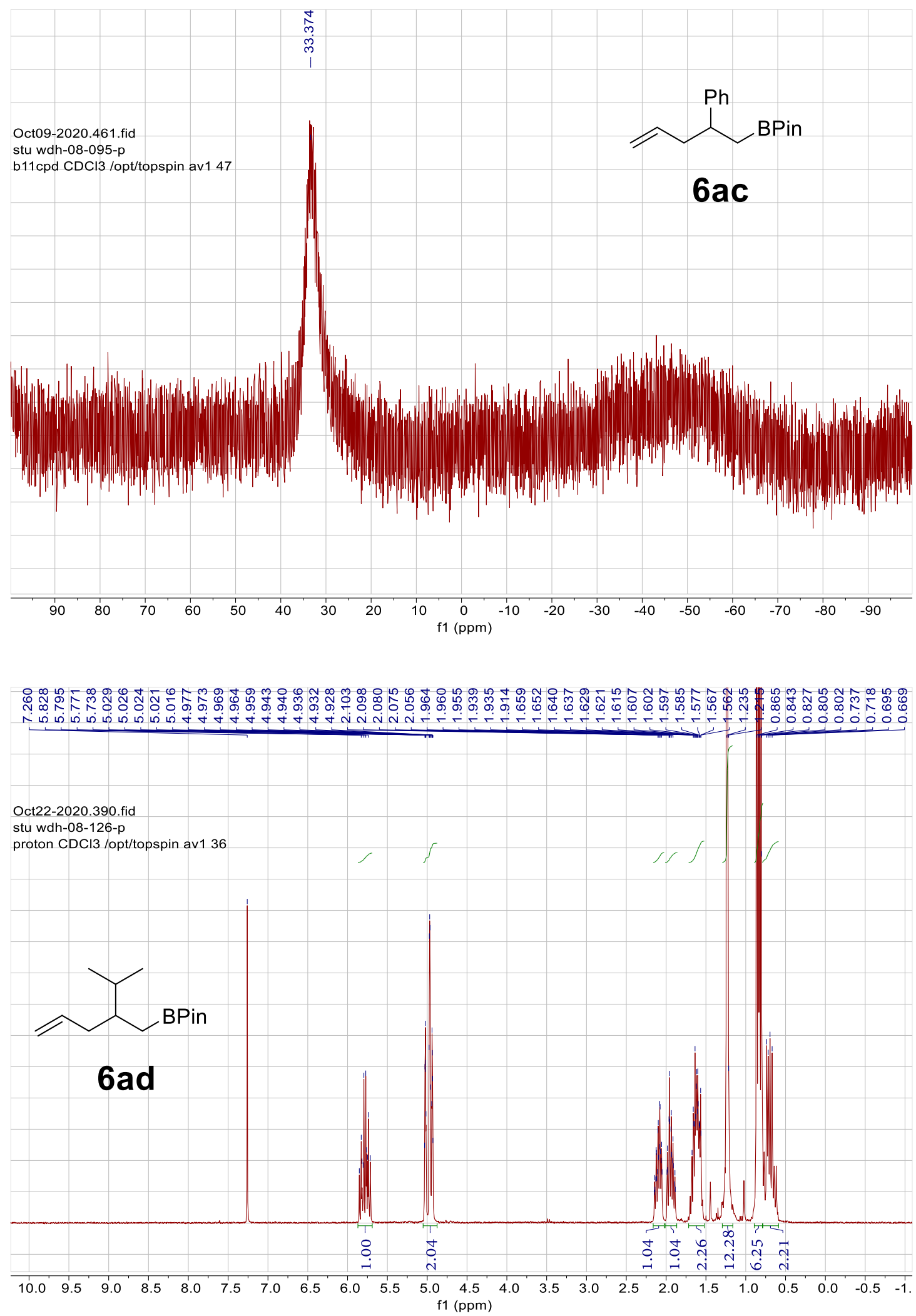

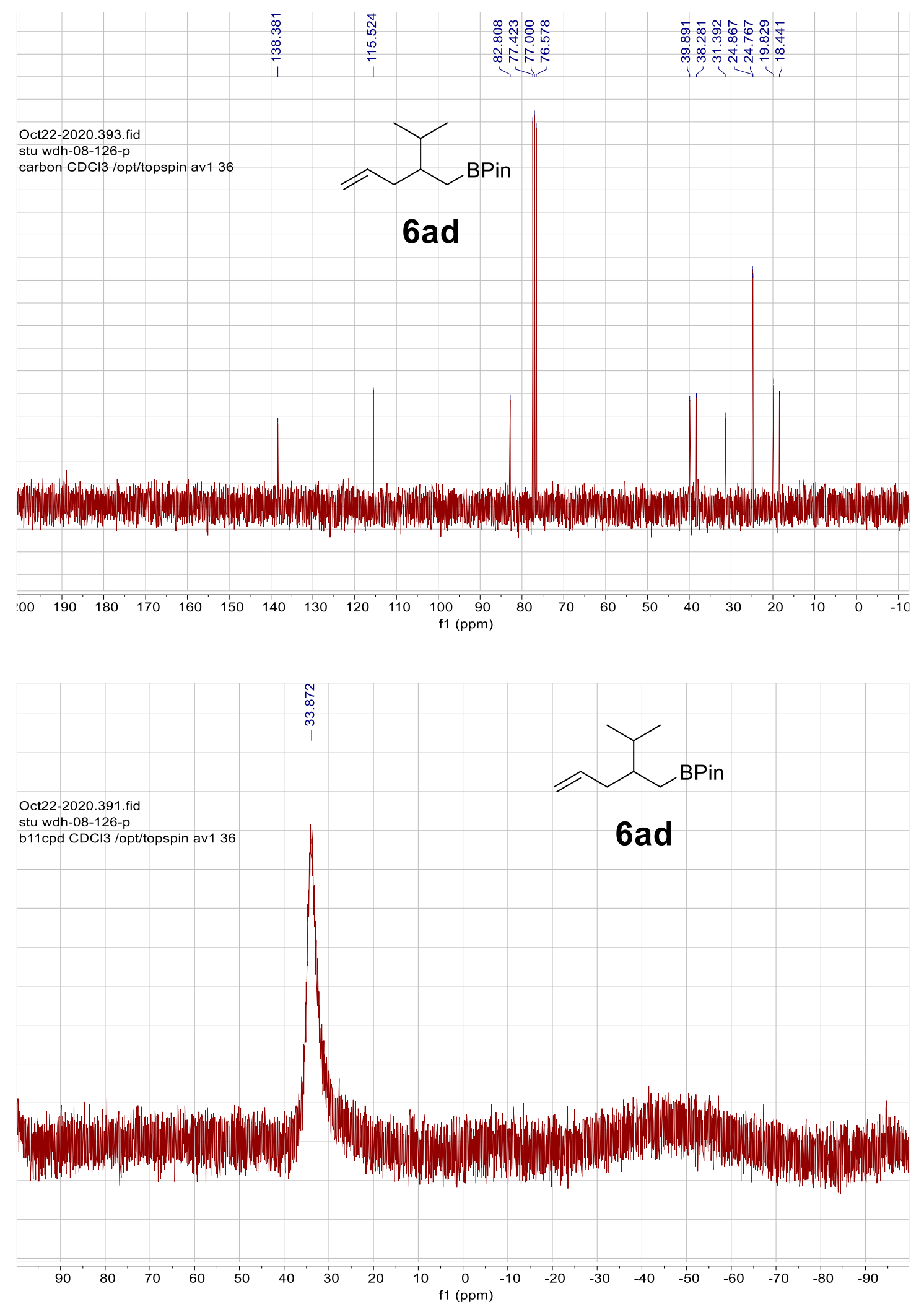

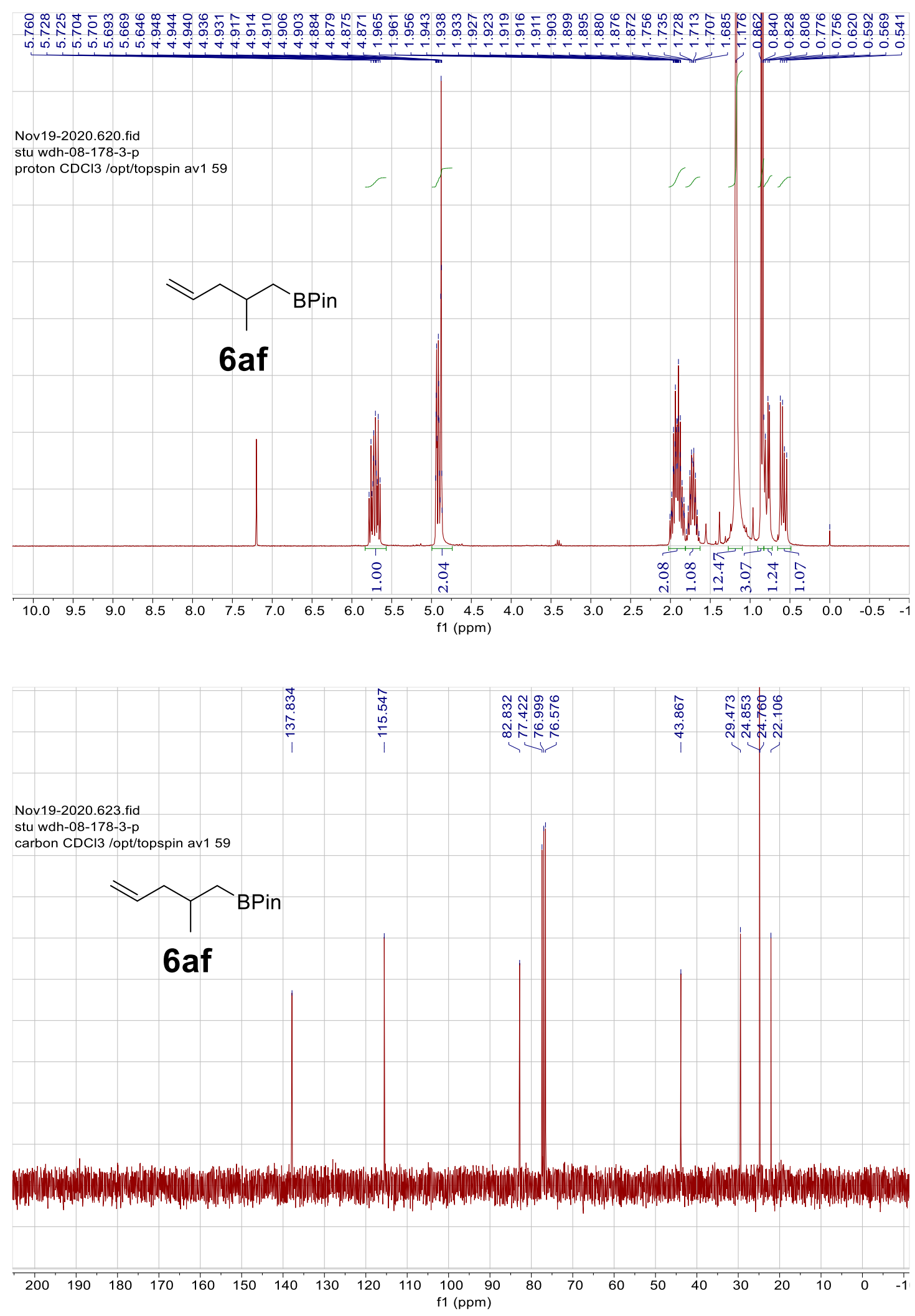

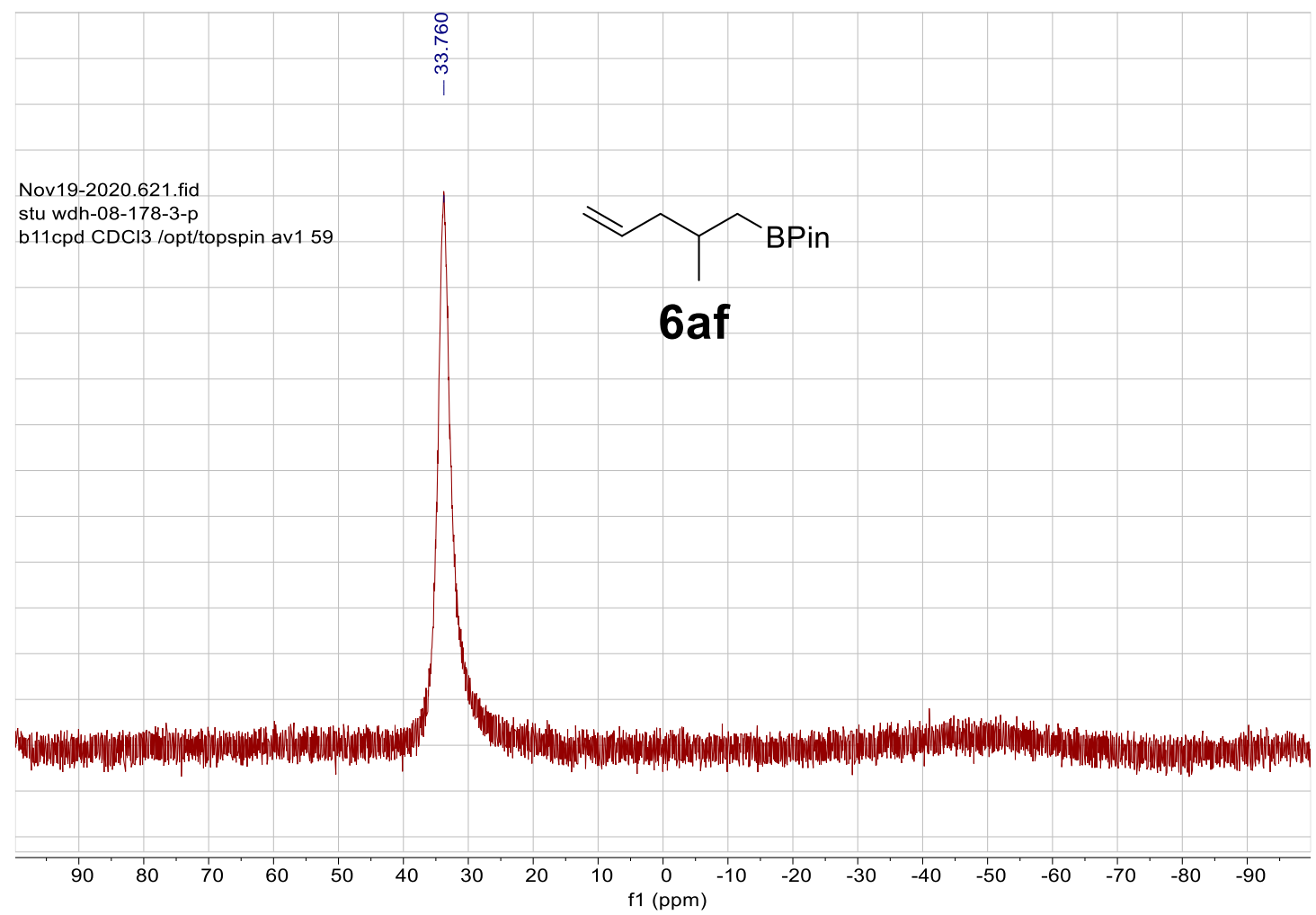

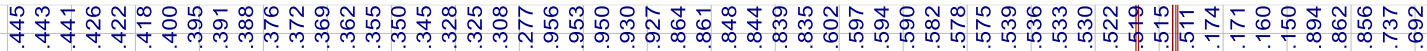

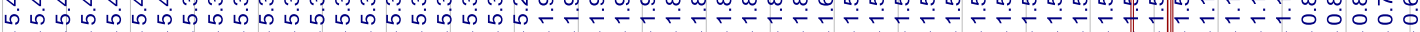

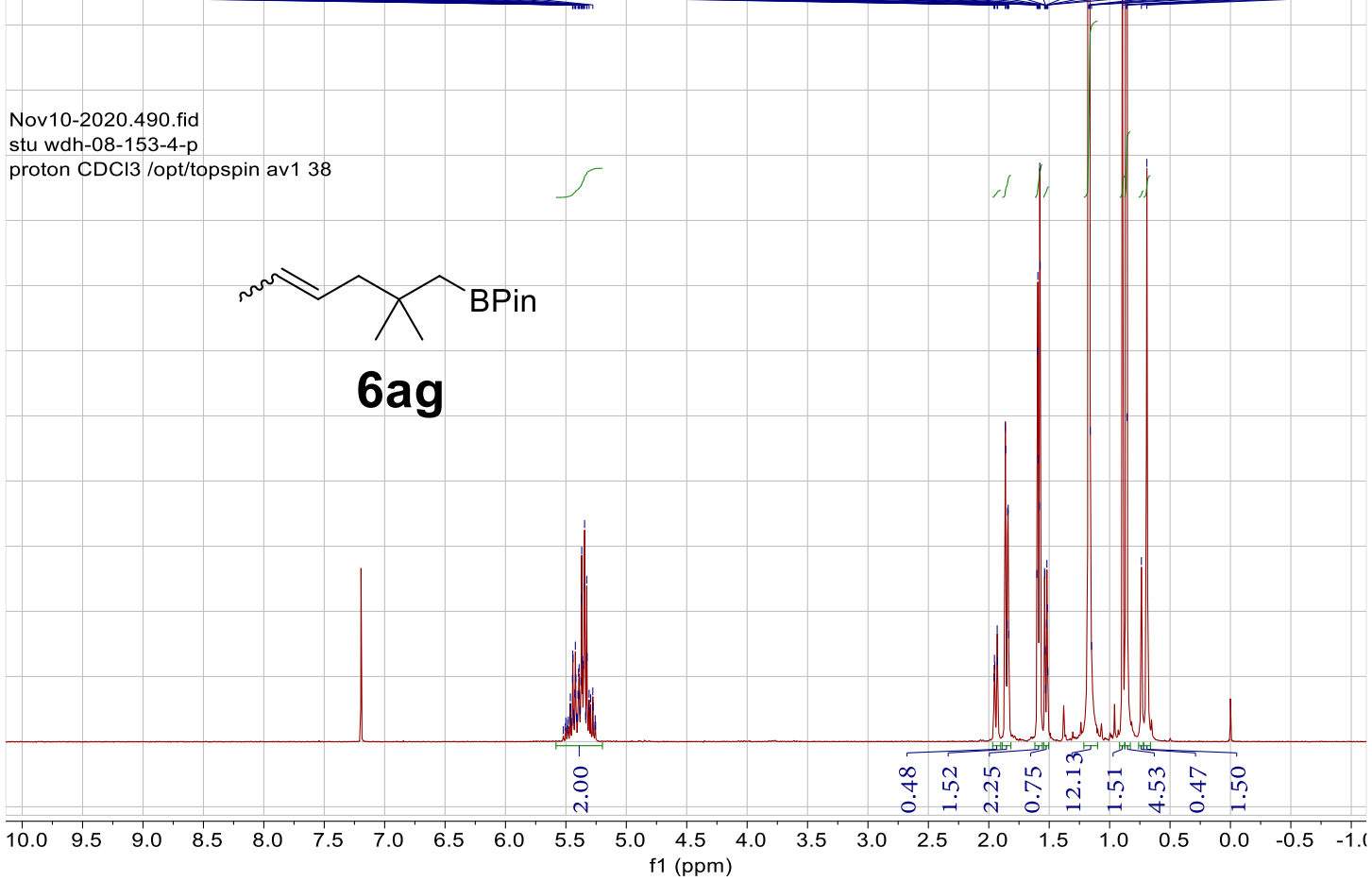




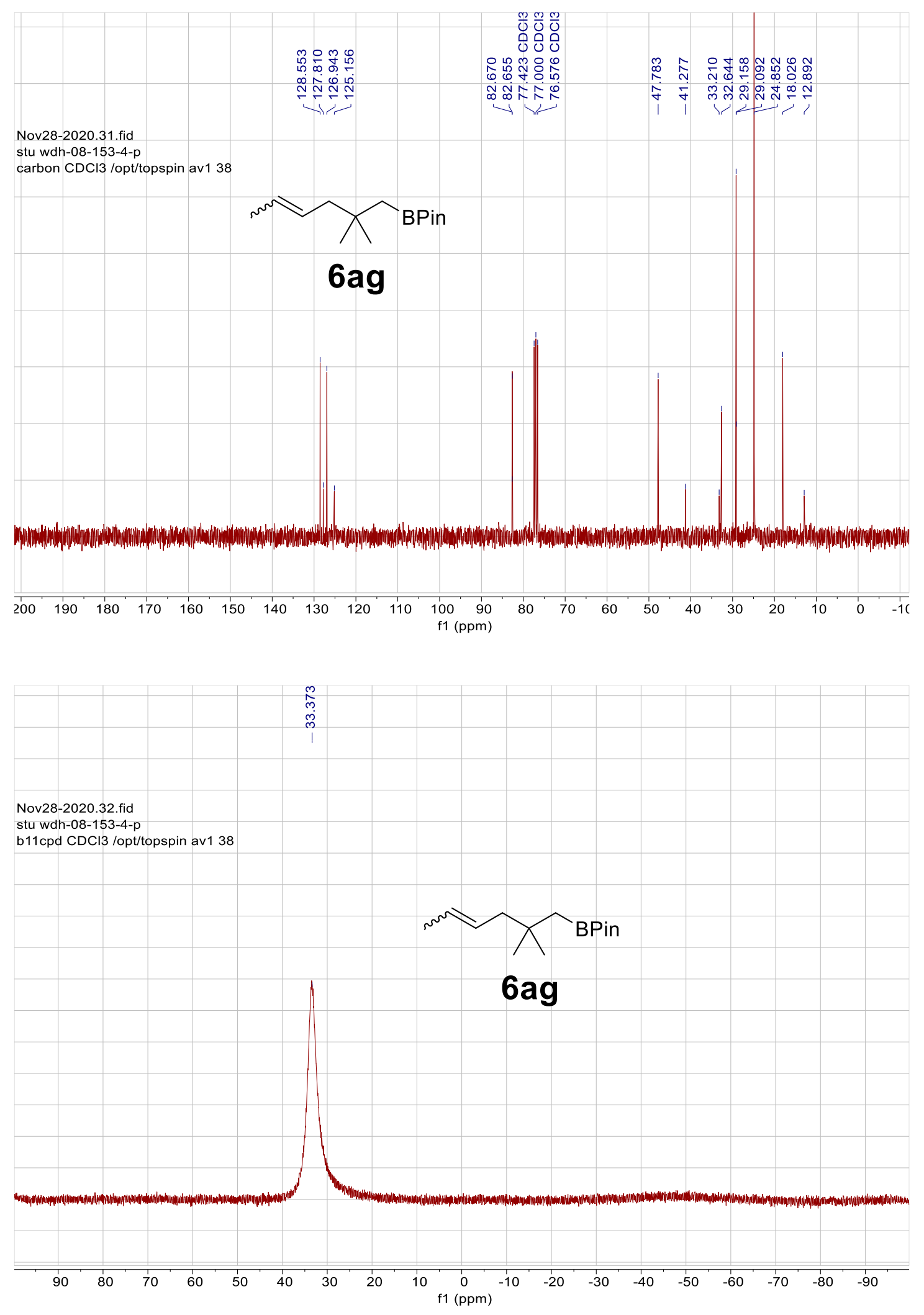



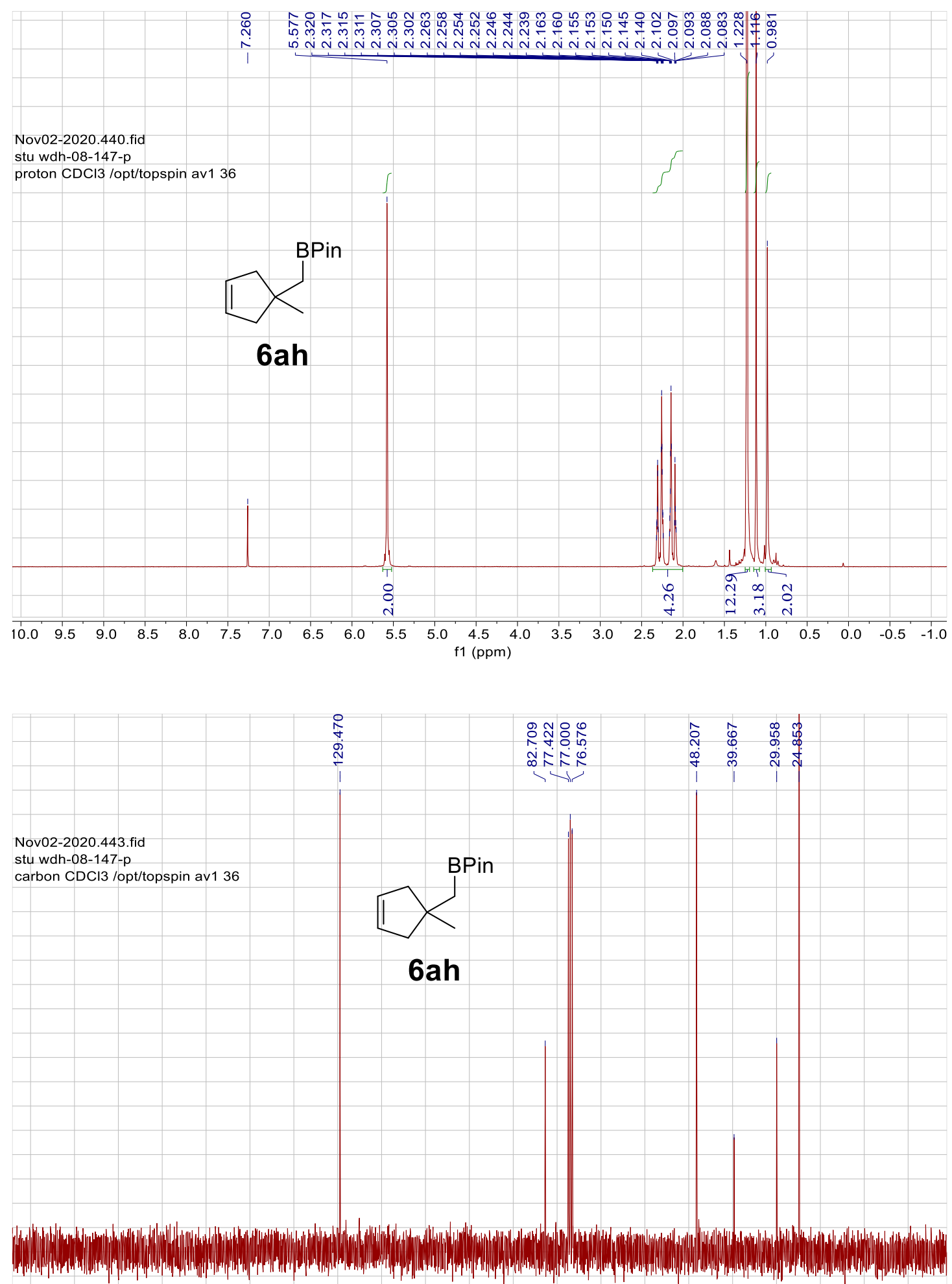

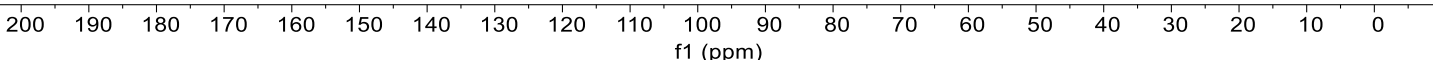



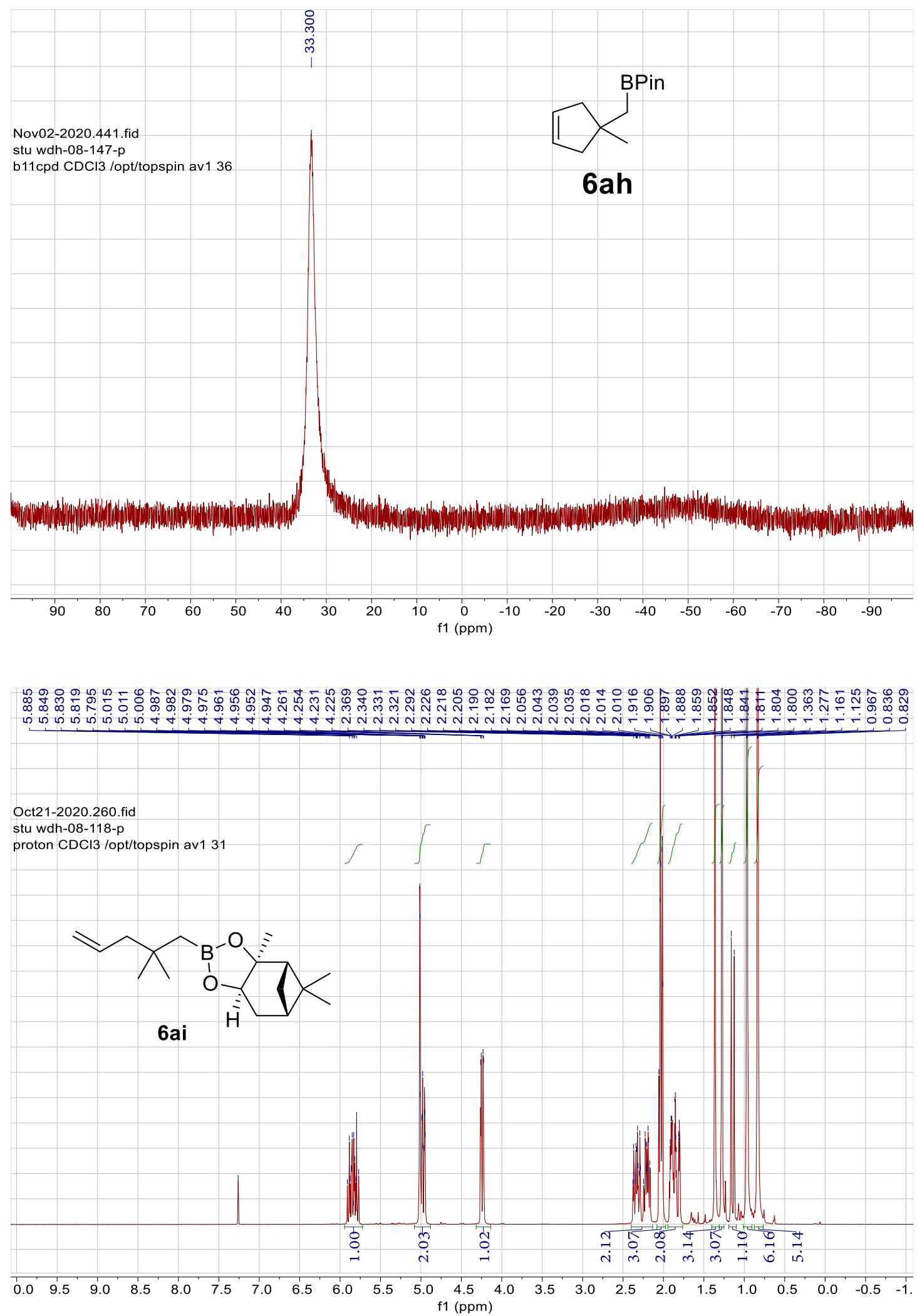

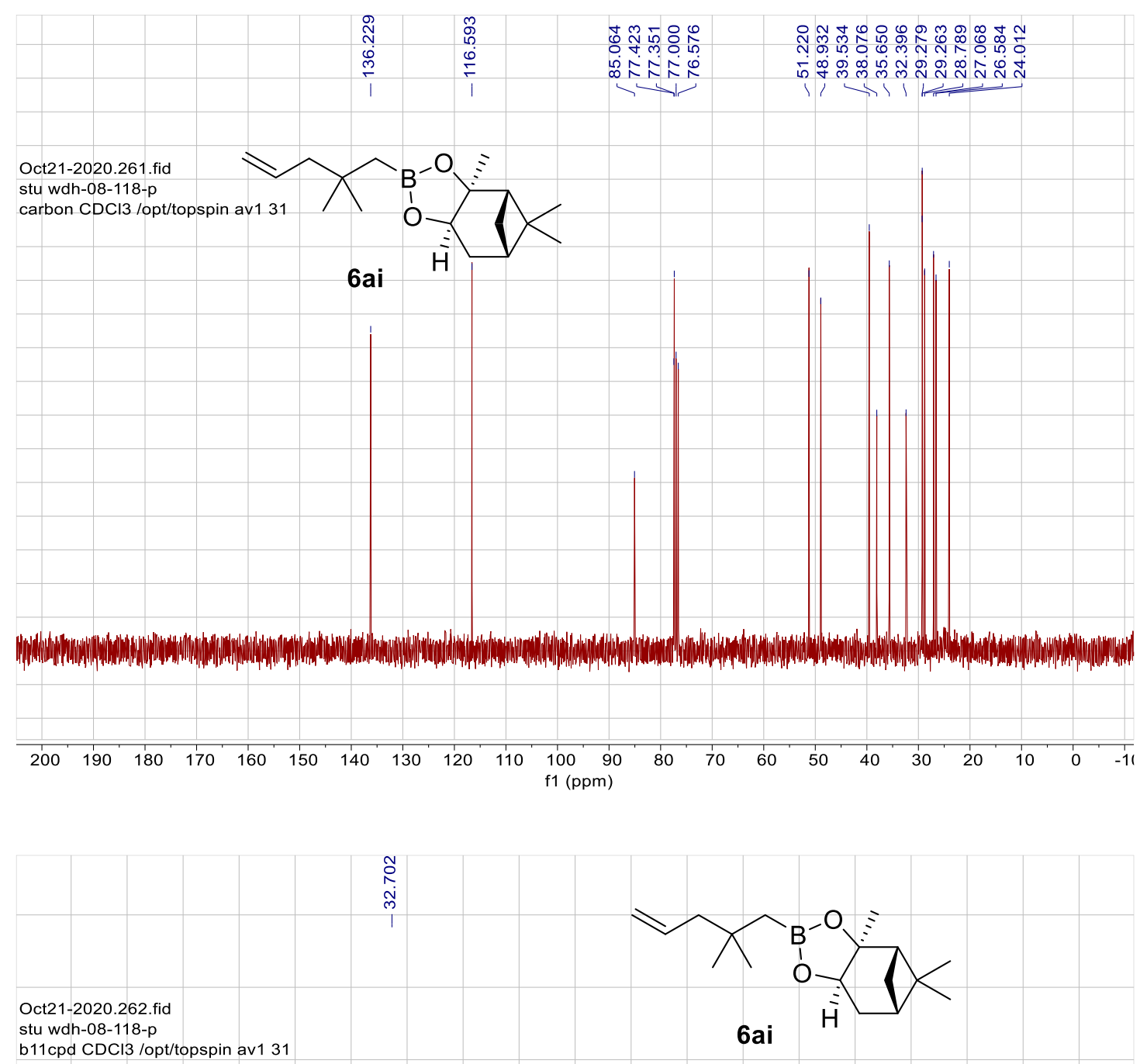

b11cpd $\mathrm{CDCl} 3$ /opt/topspin av1 31
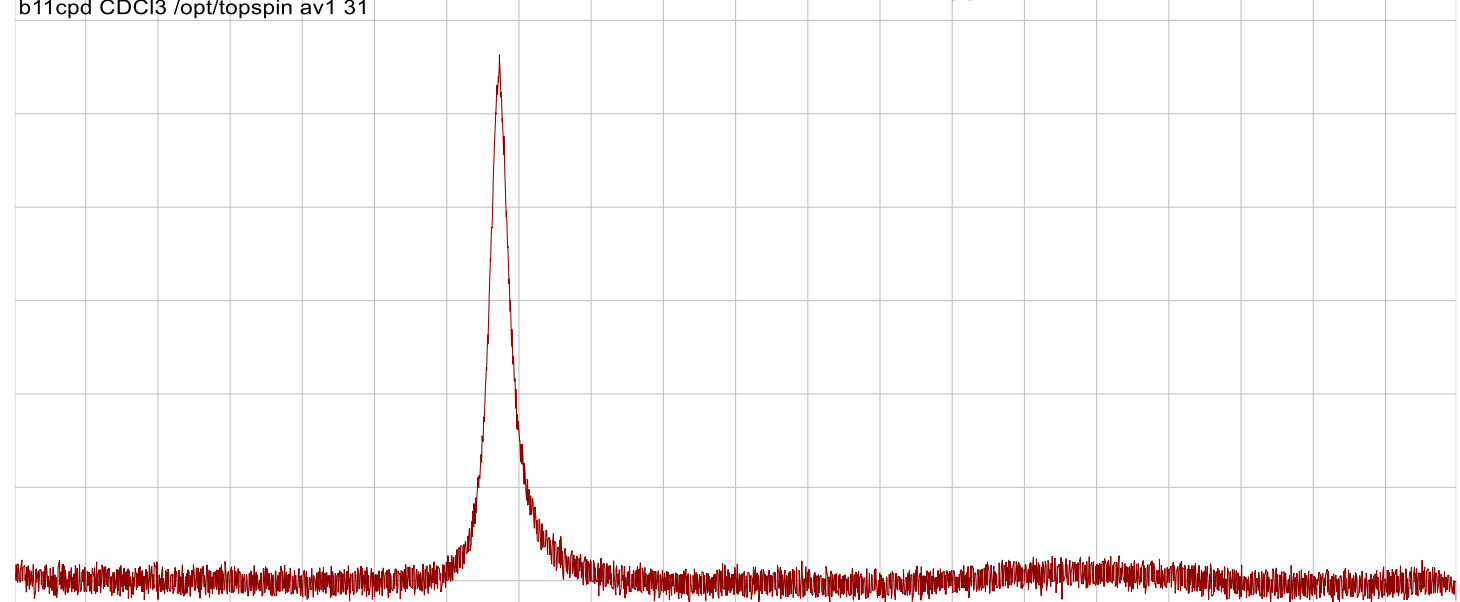

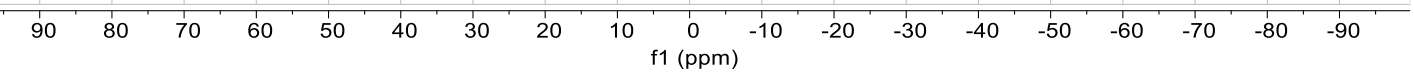




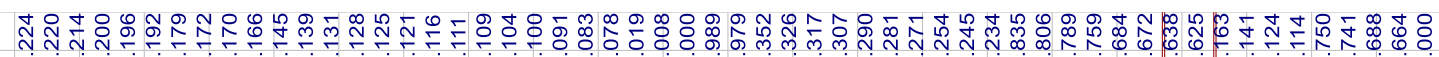
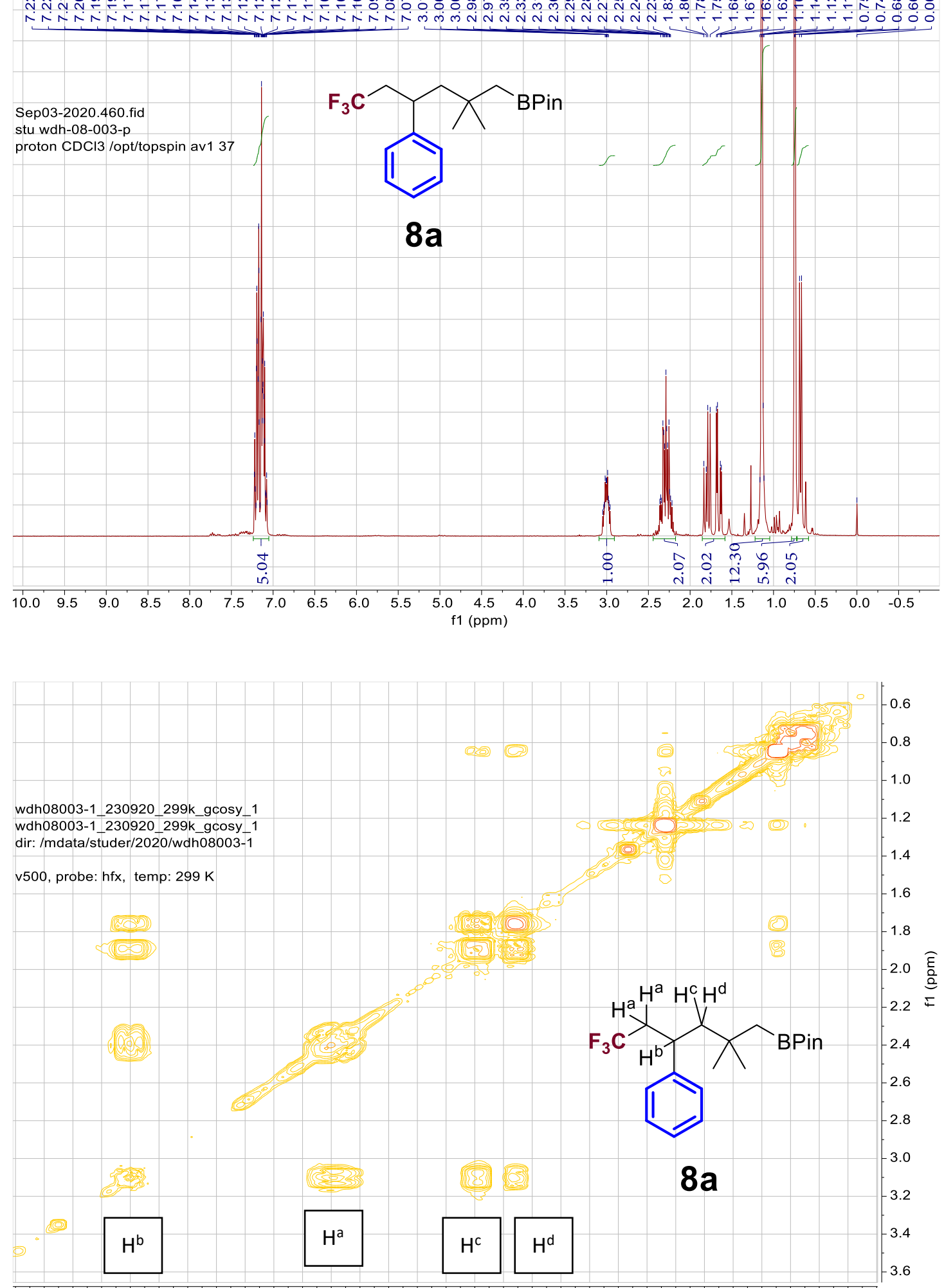

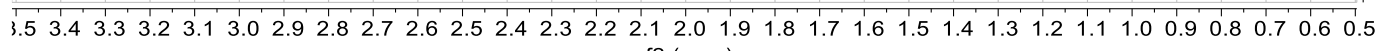
f2 (ppm) 

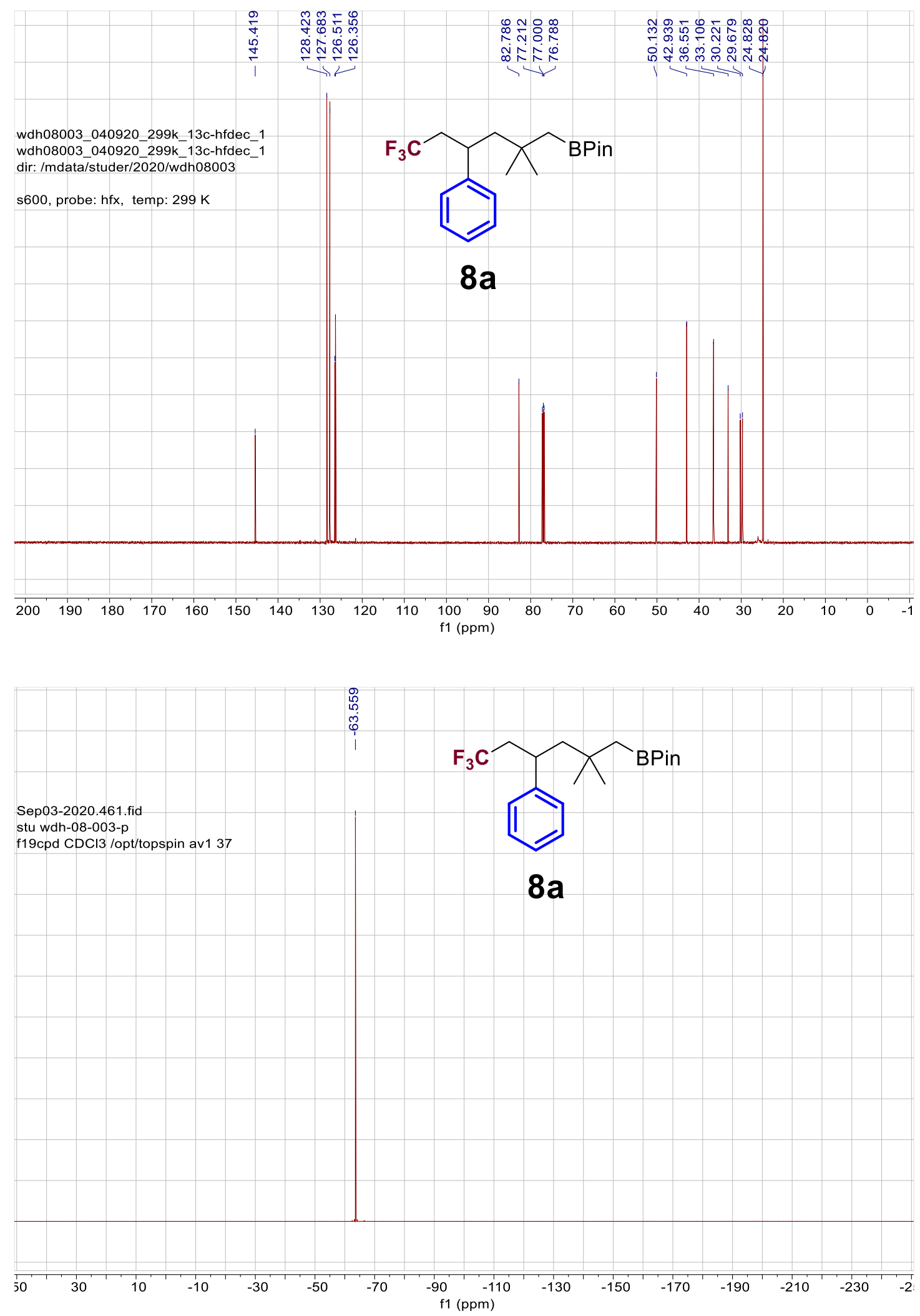

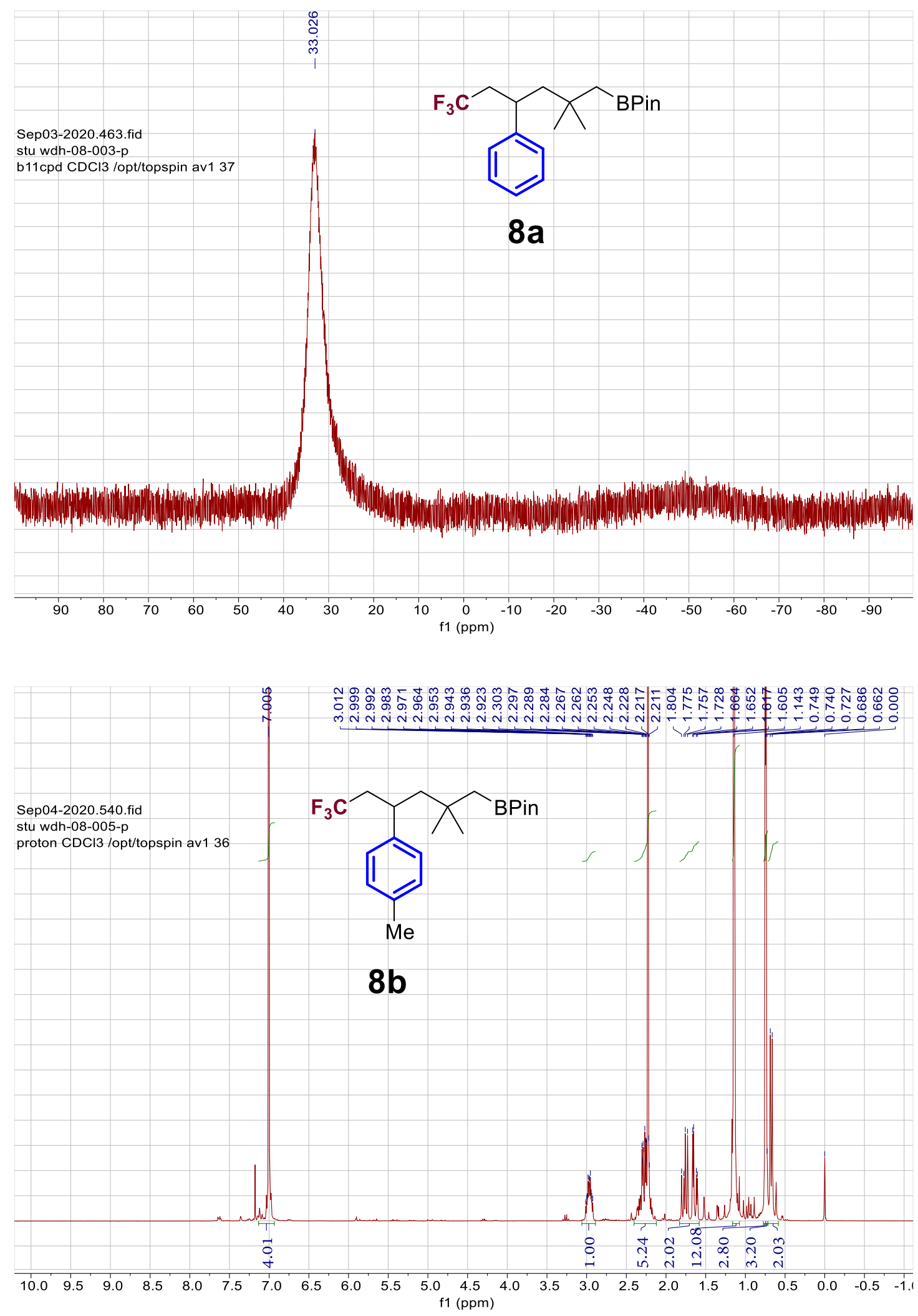

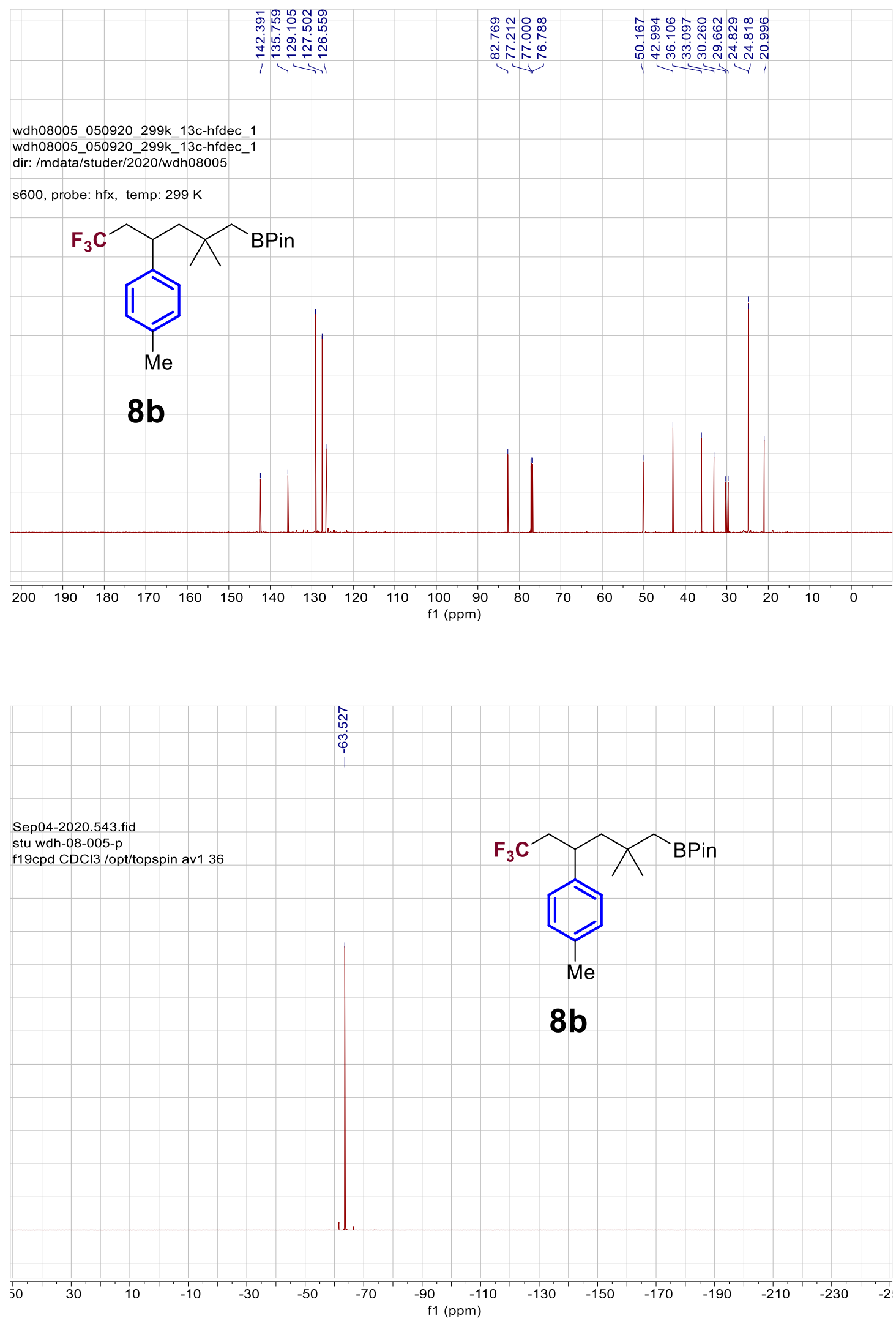

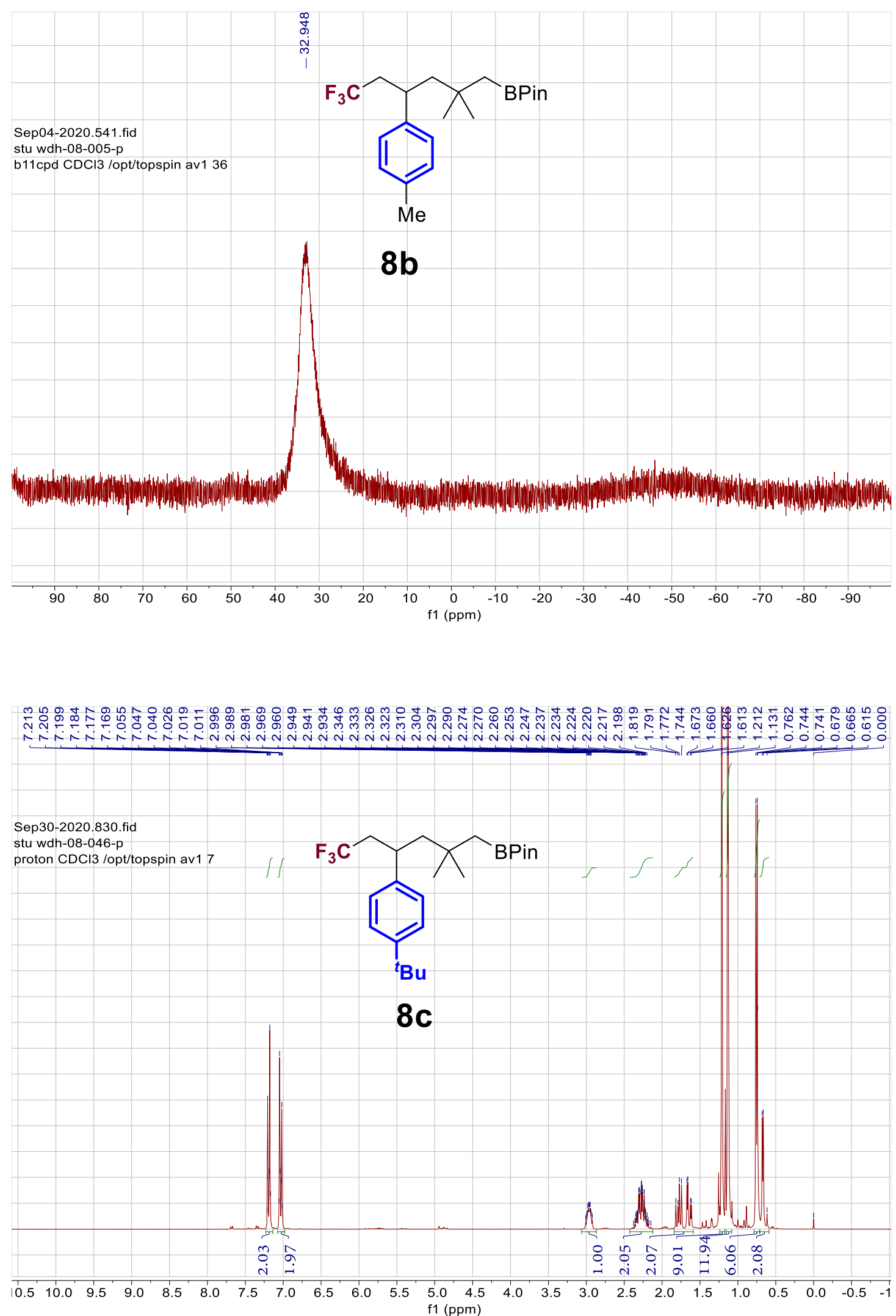

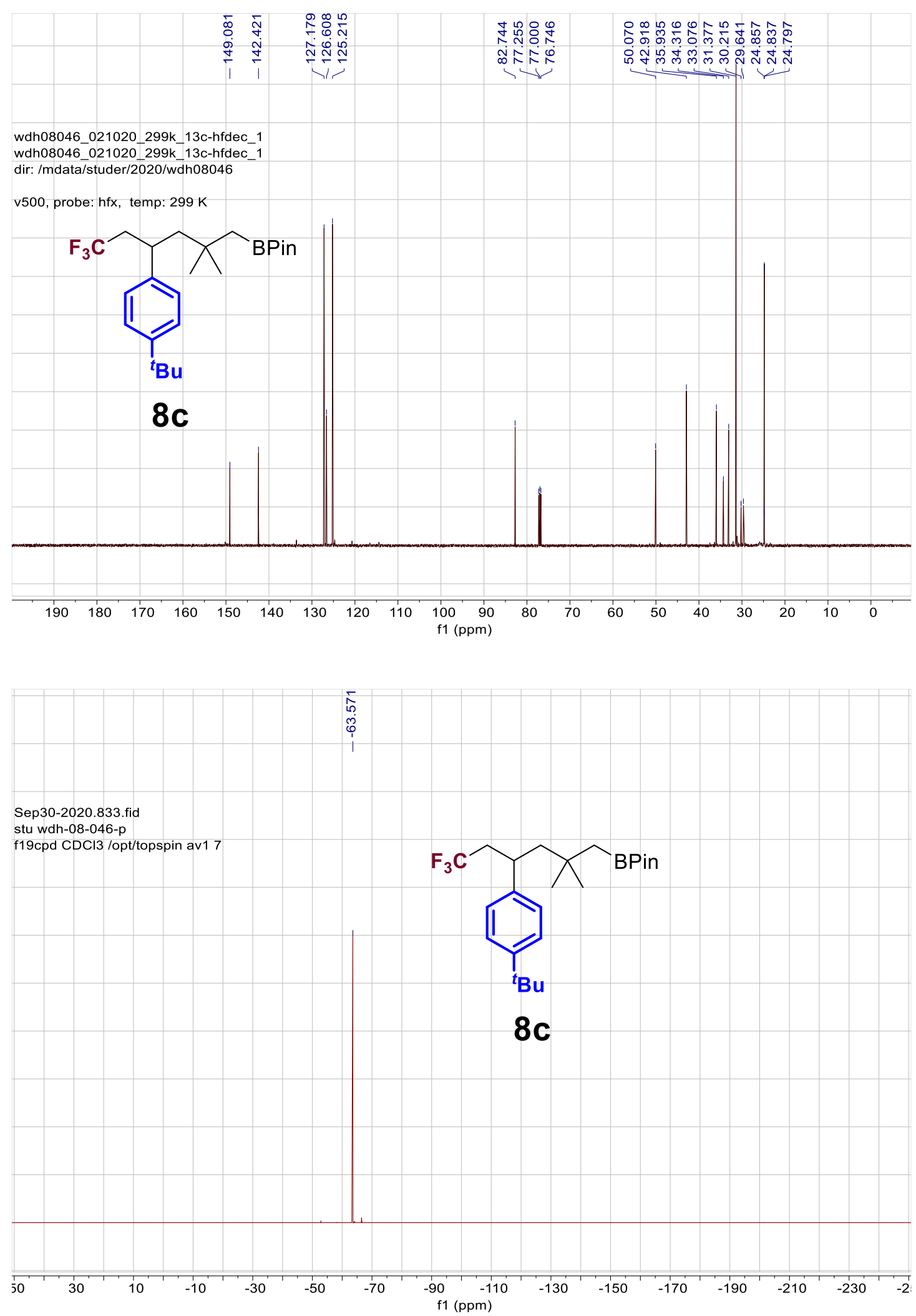

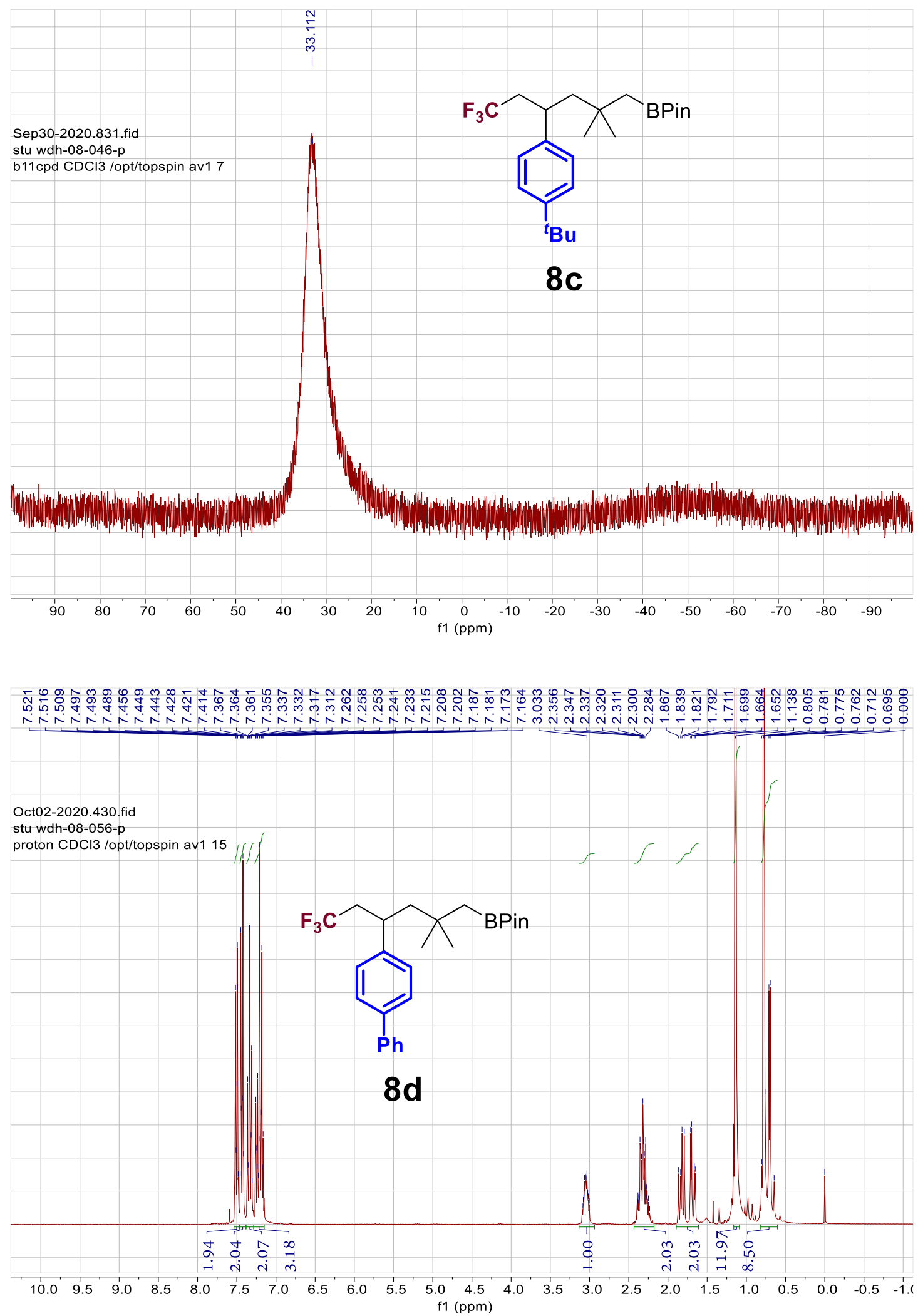

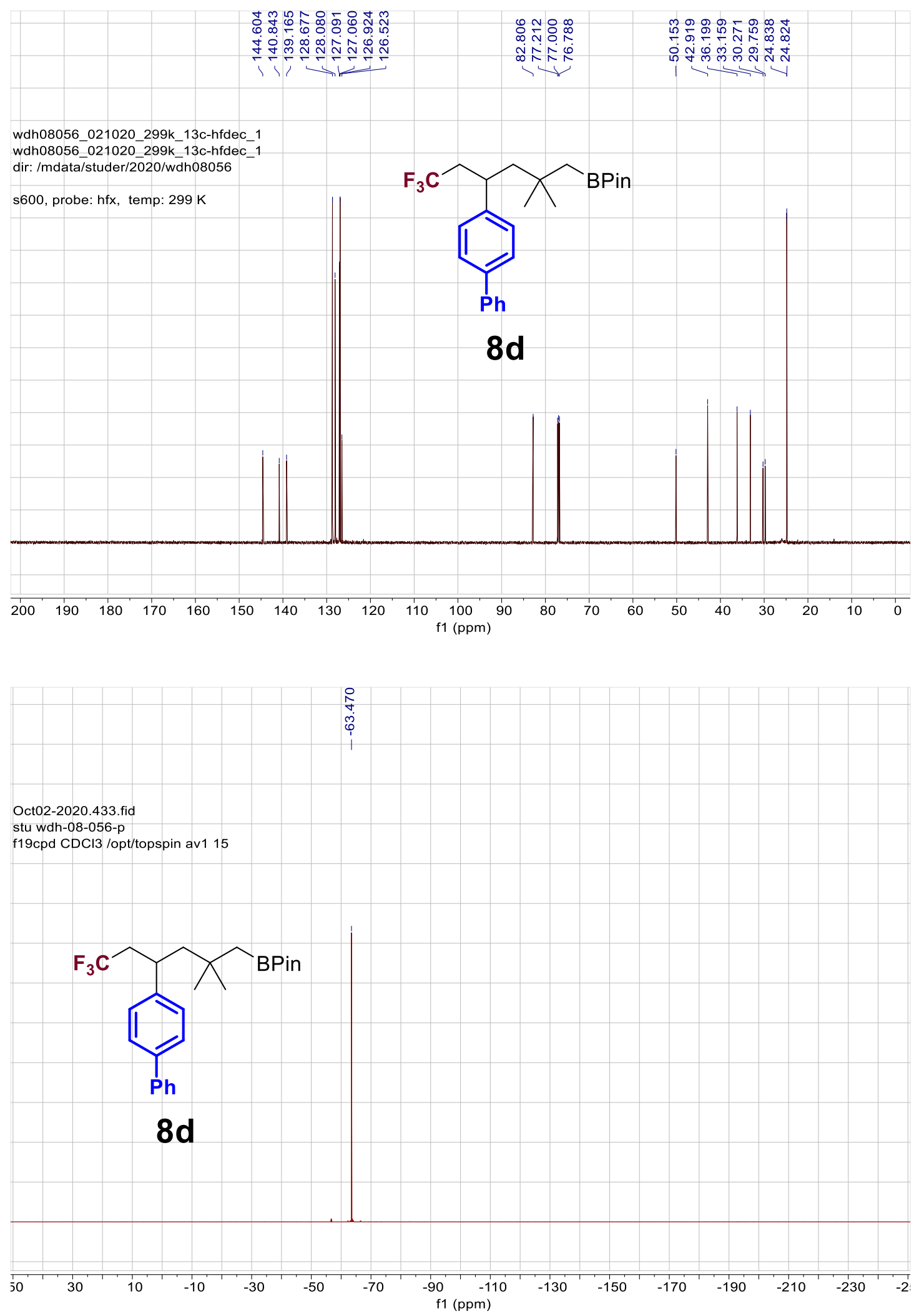


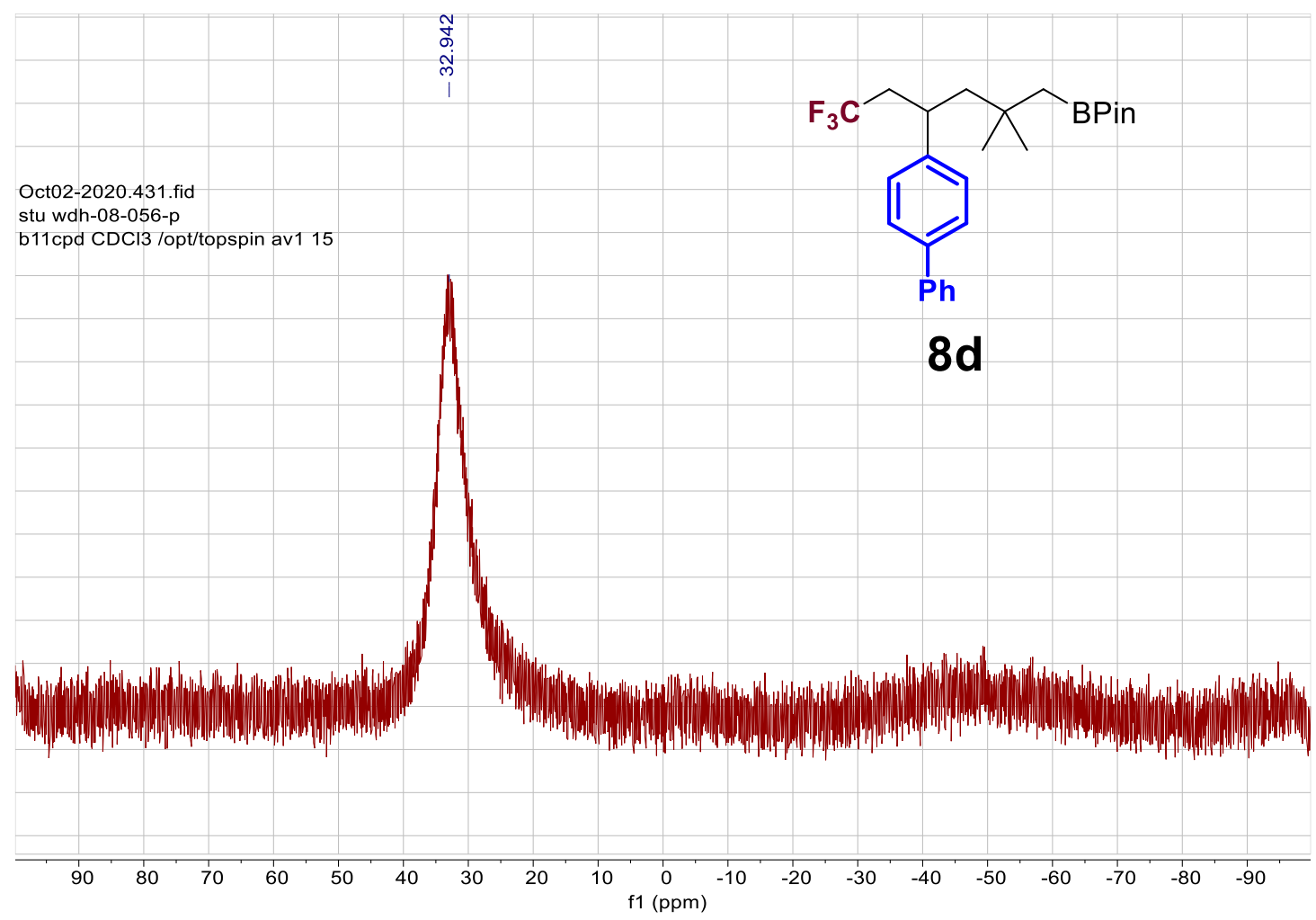

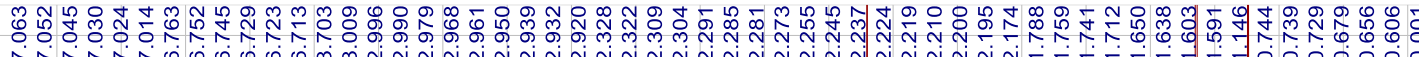

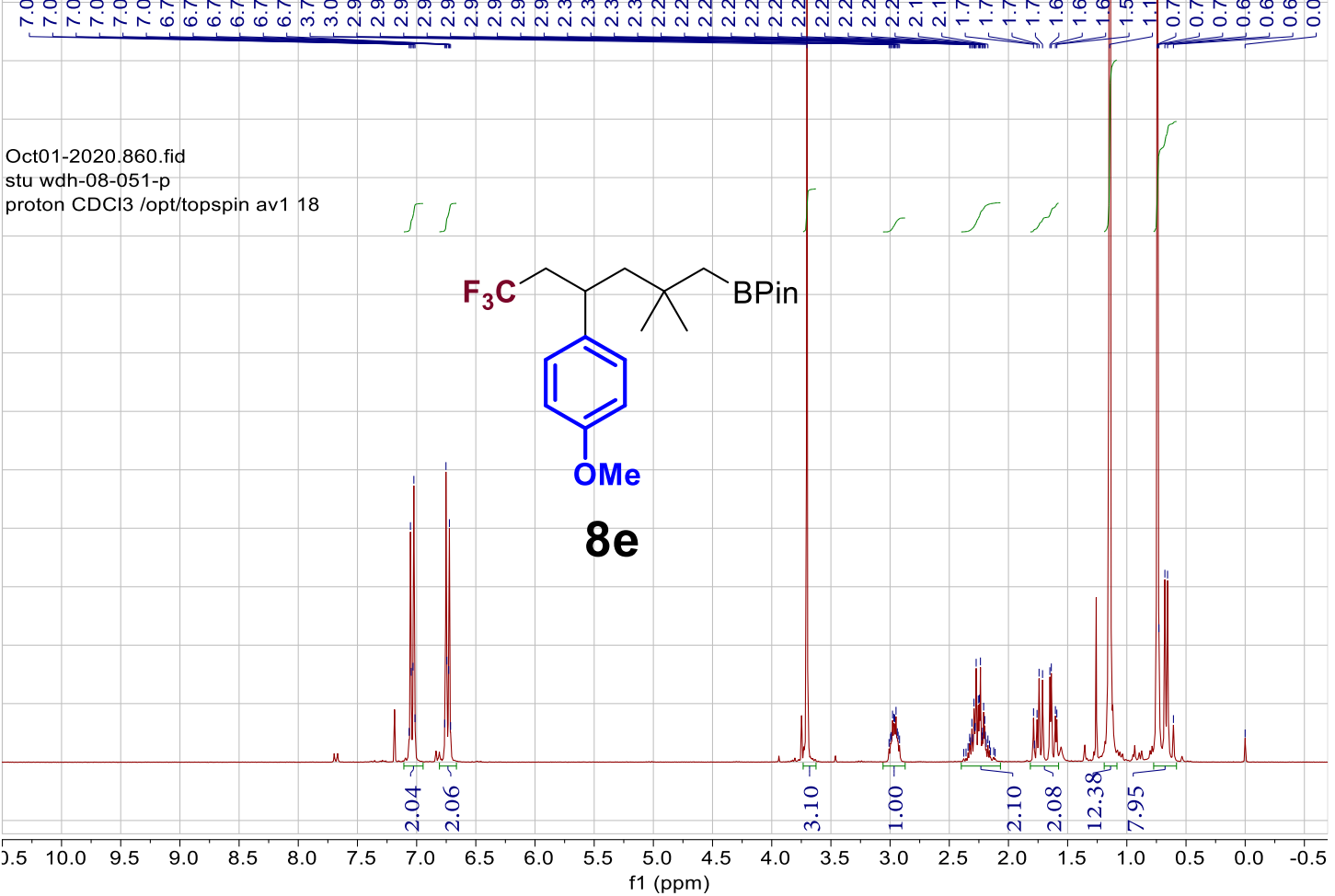



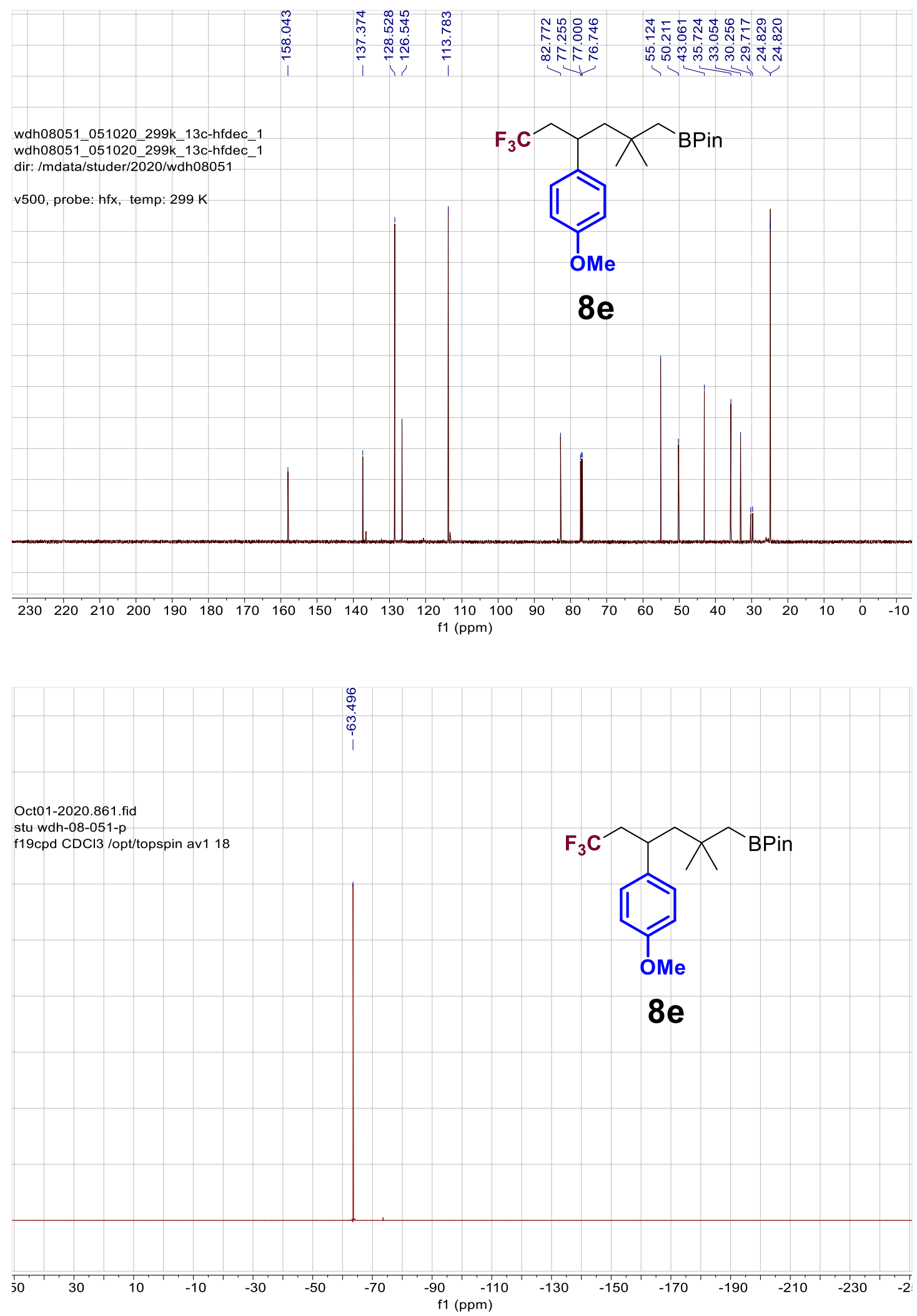

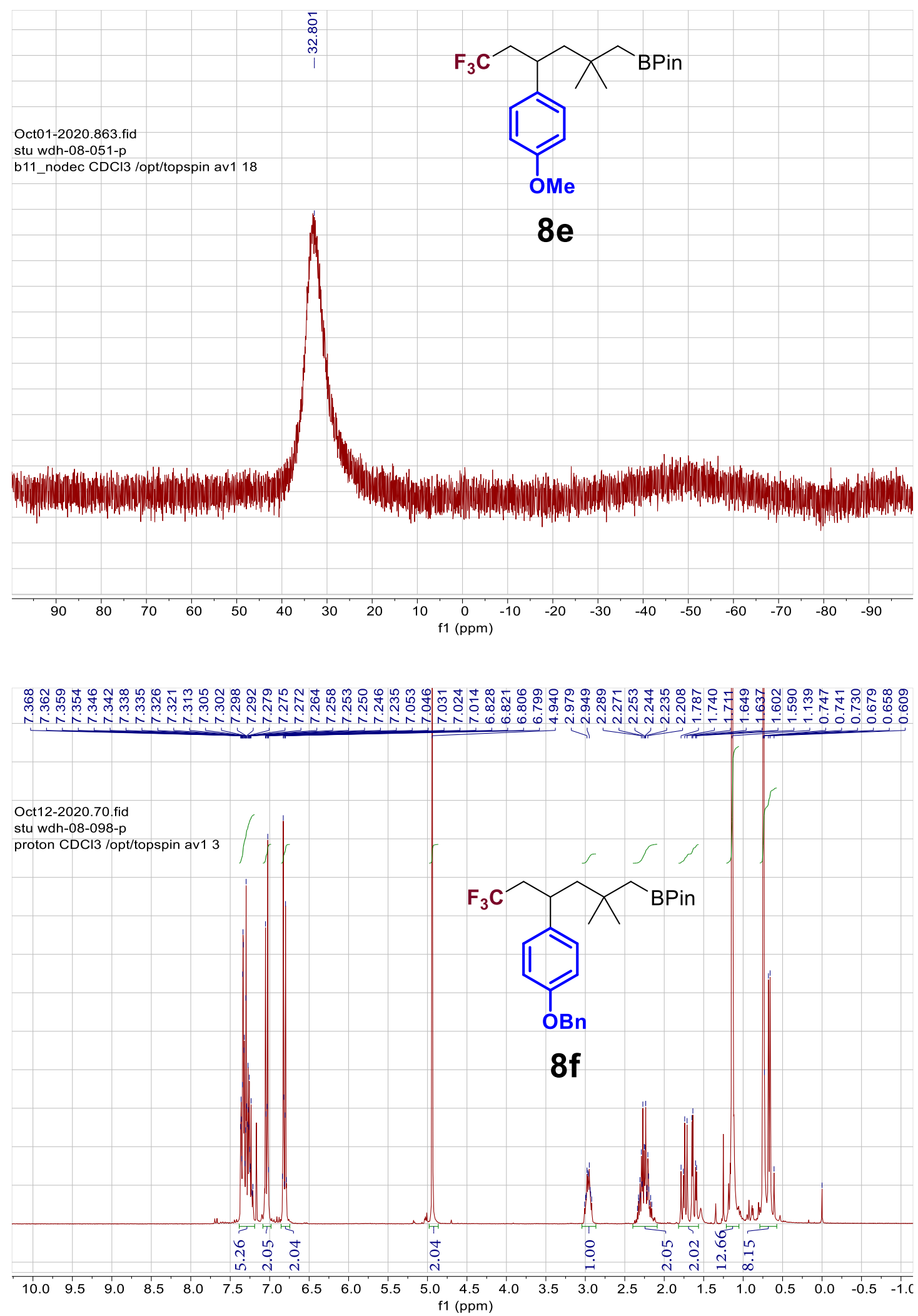

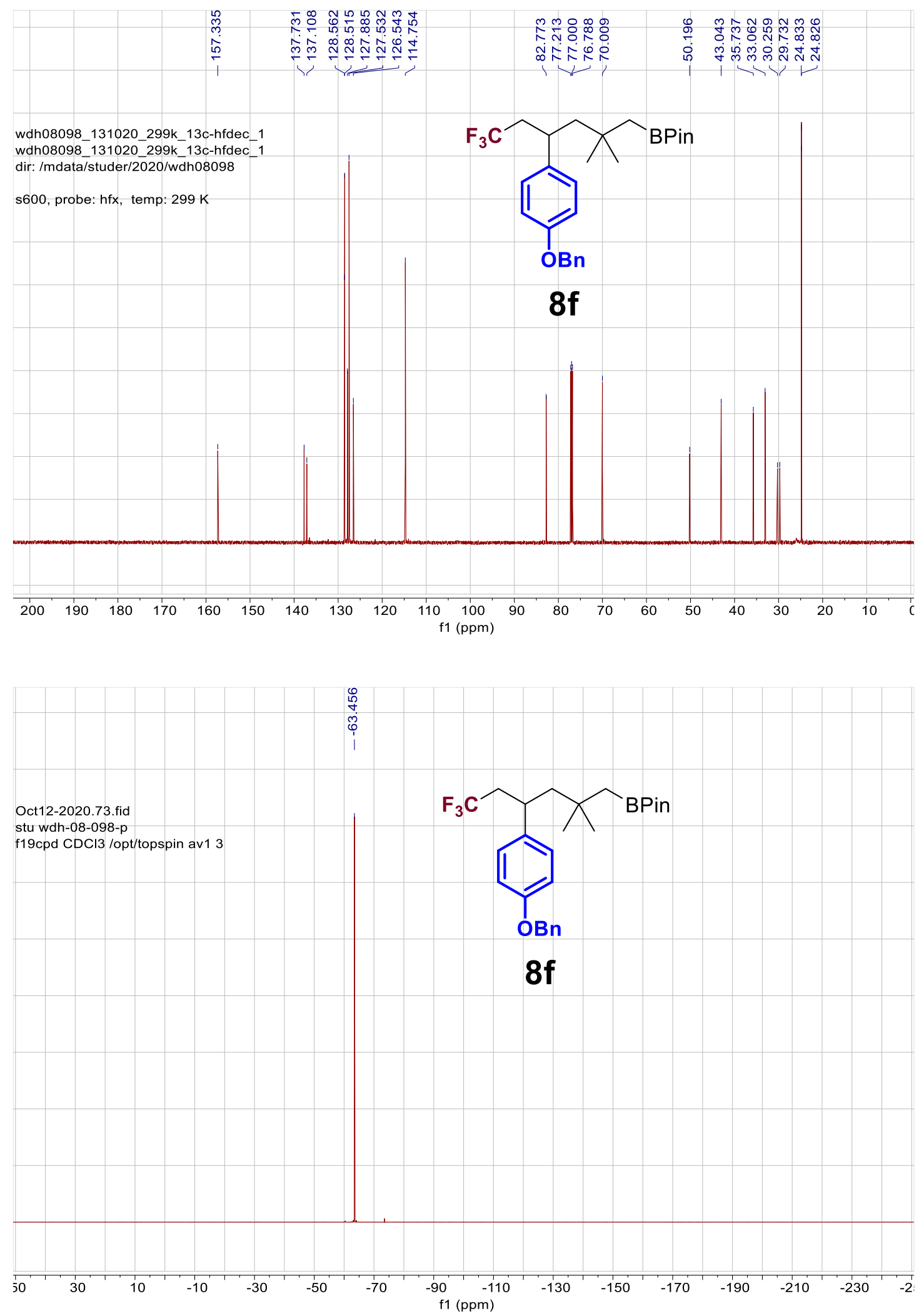

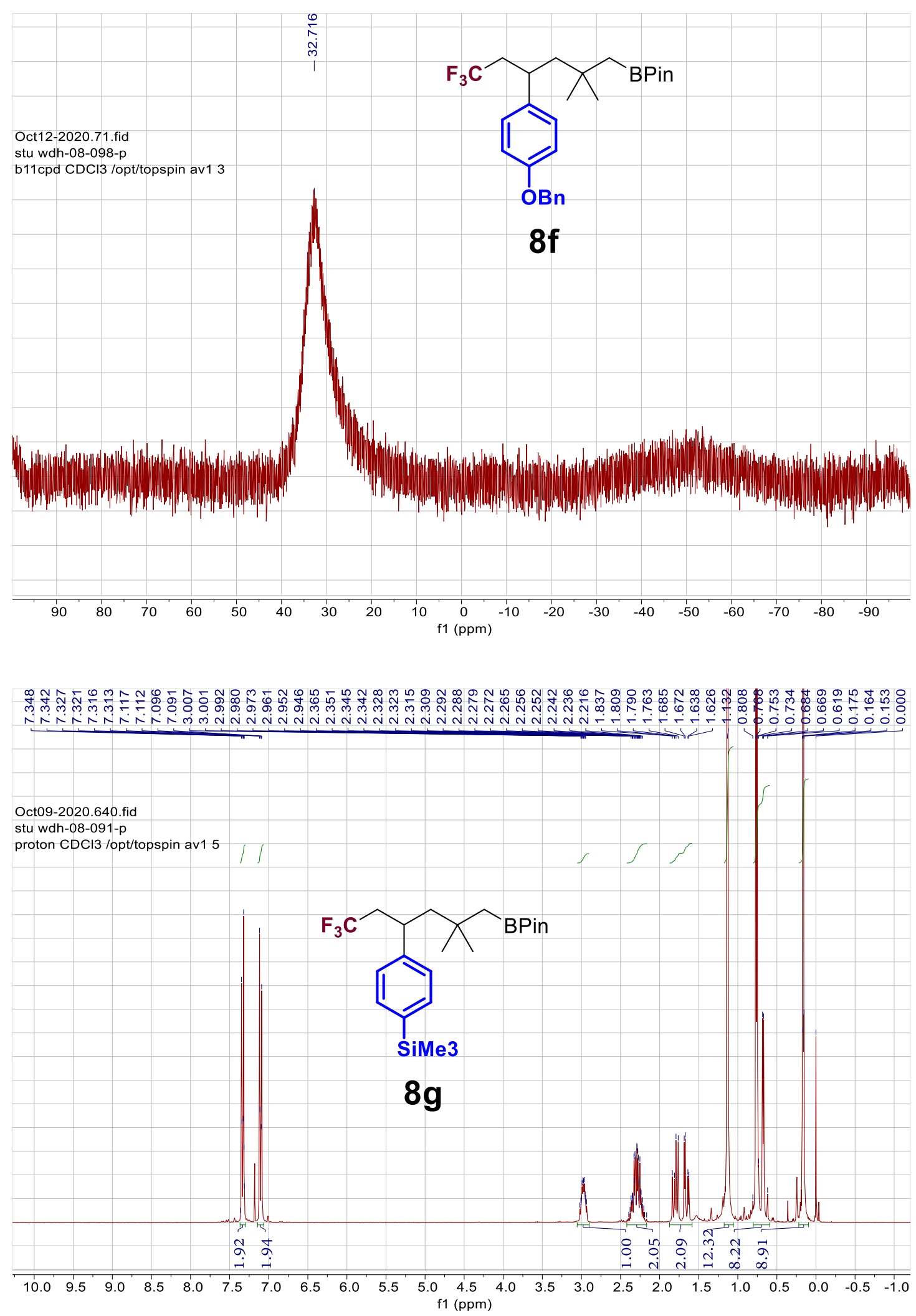

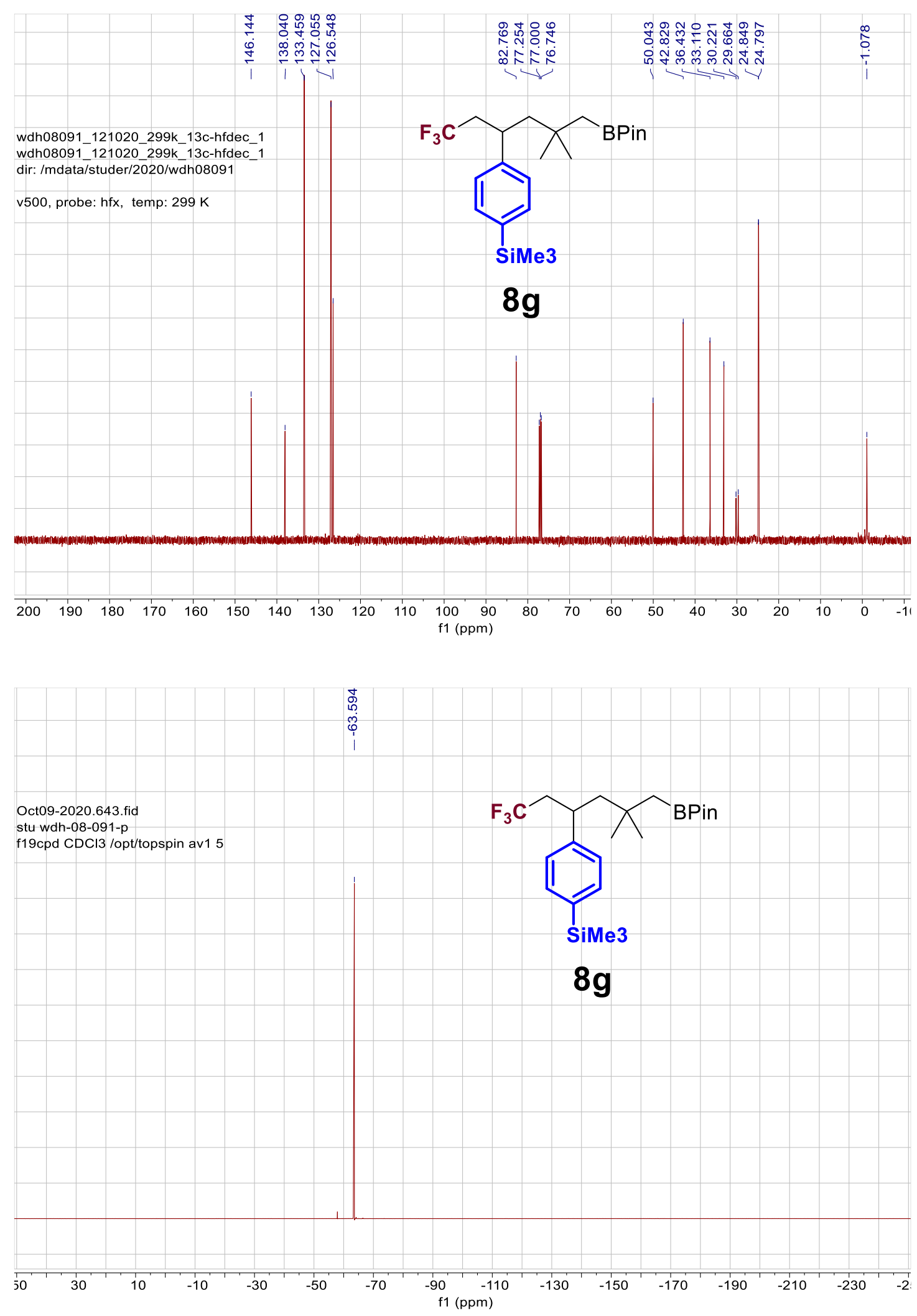

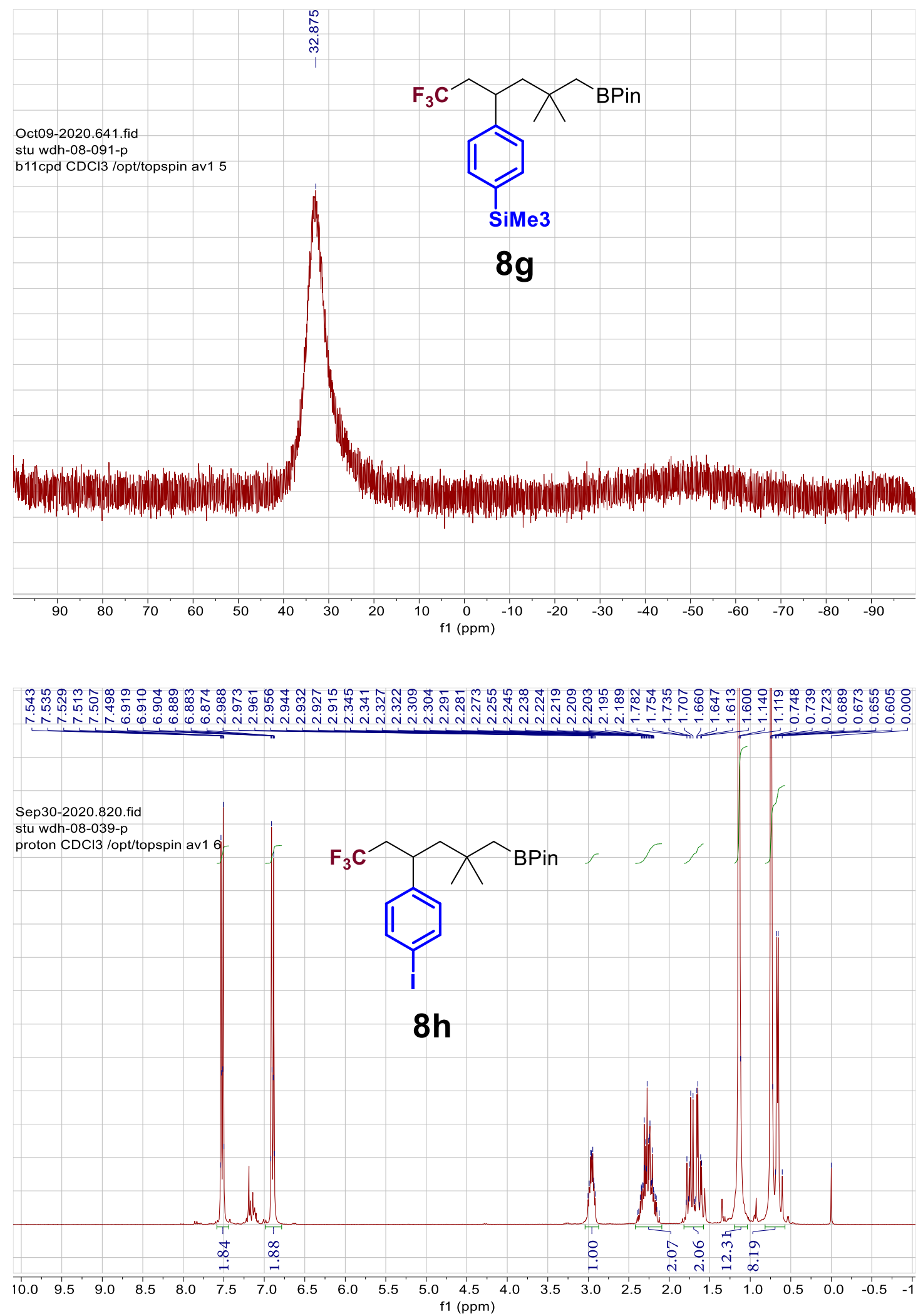

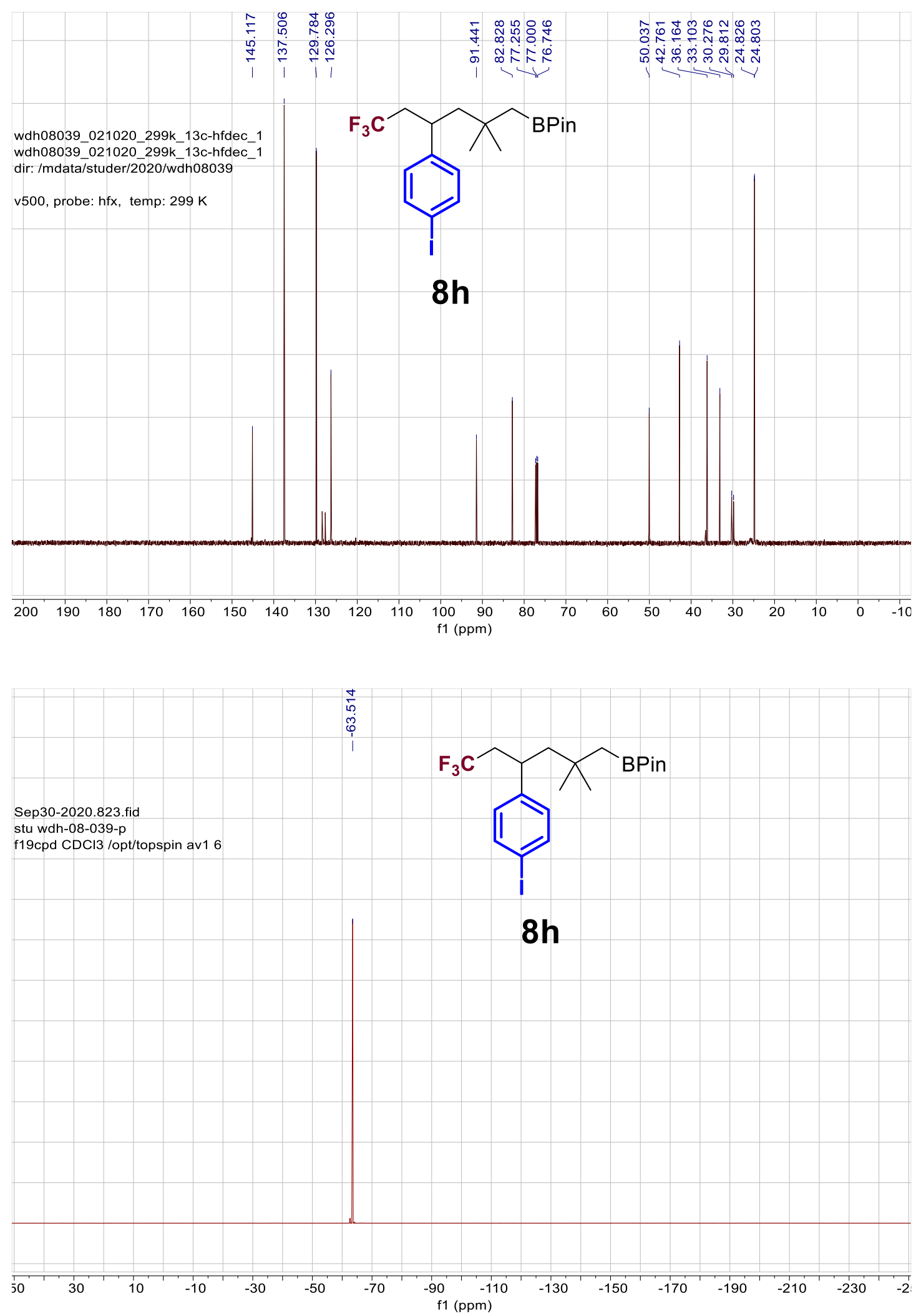


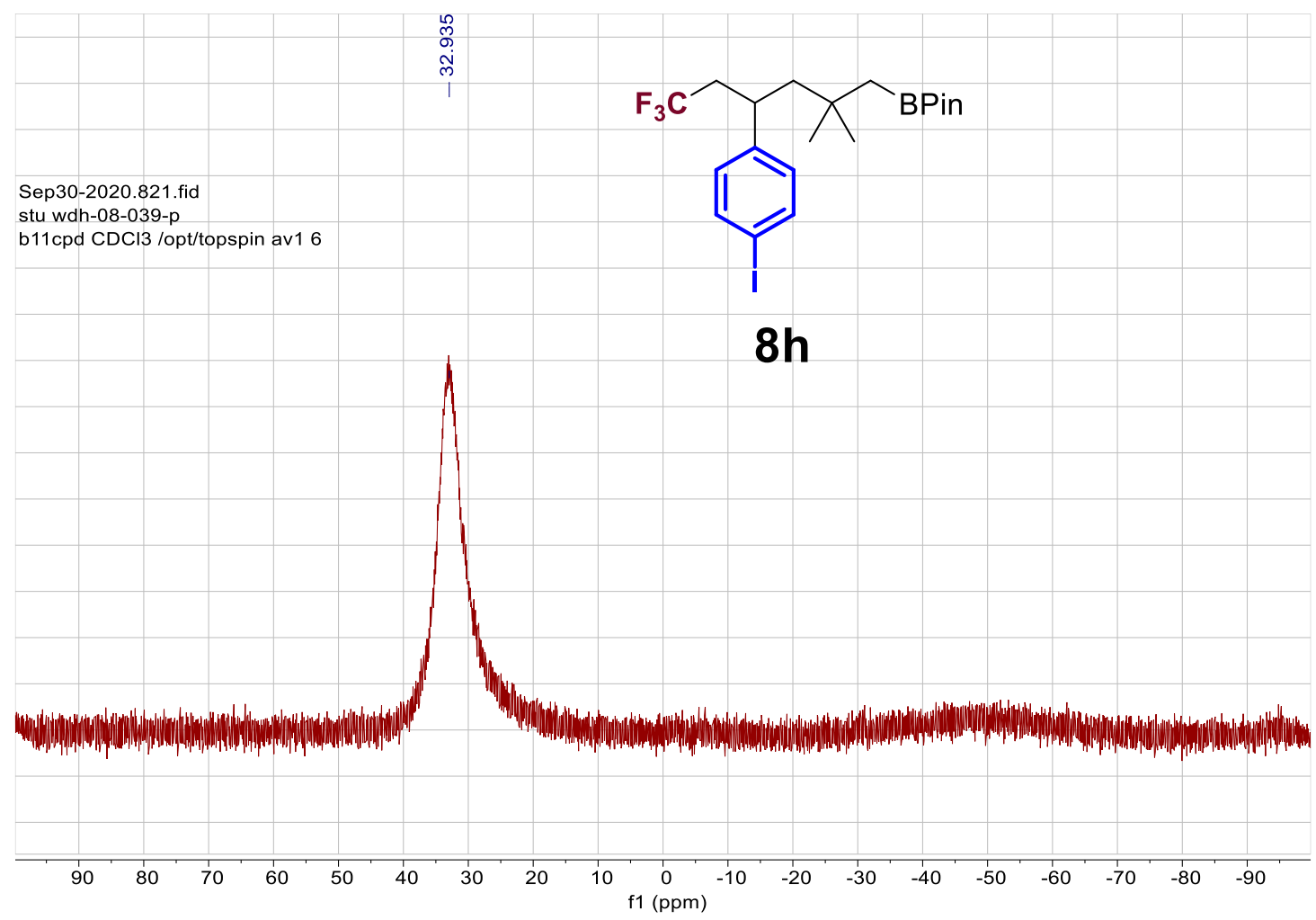

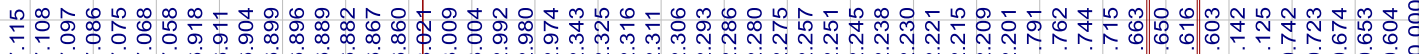

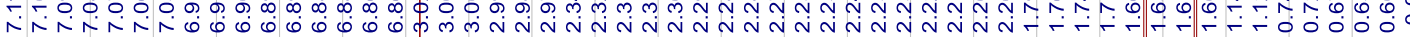

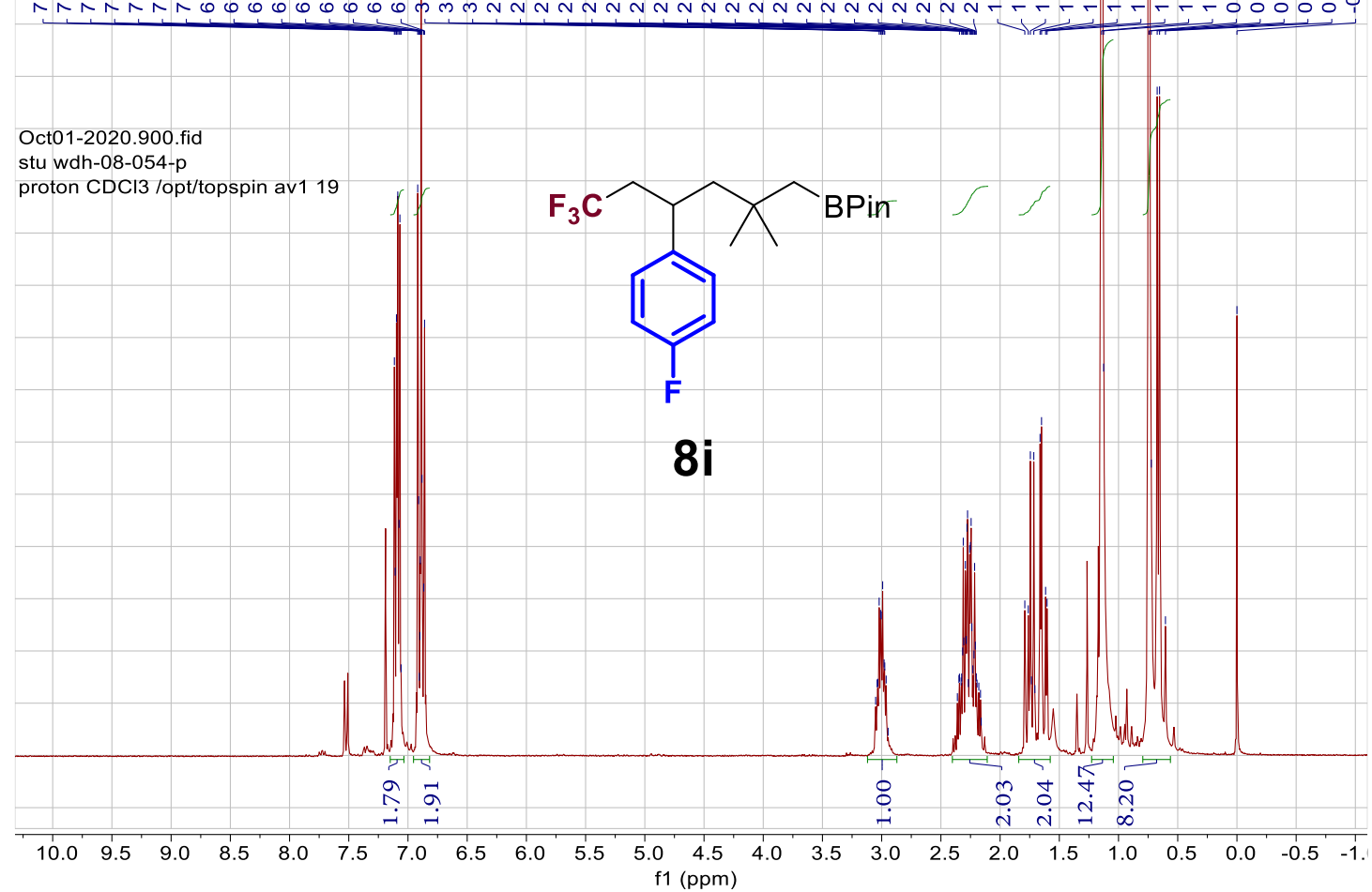



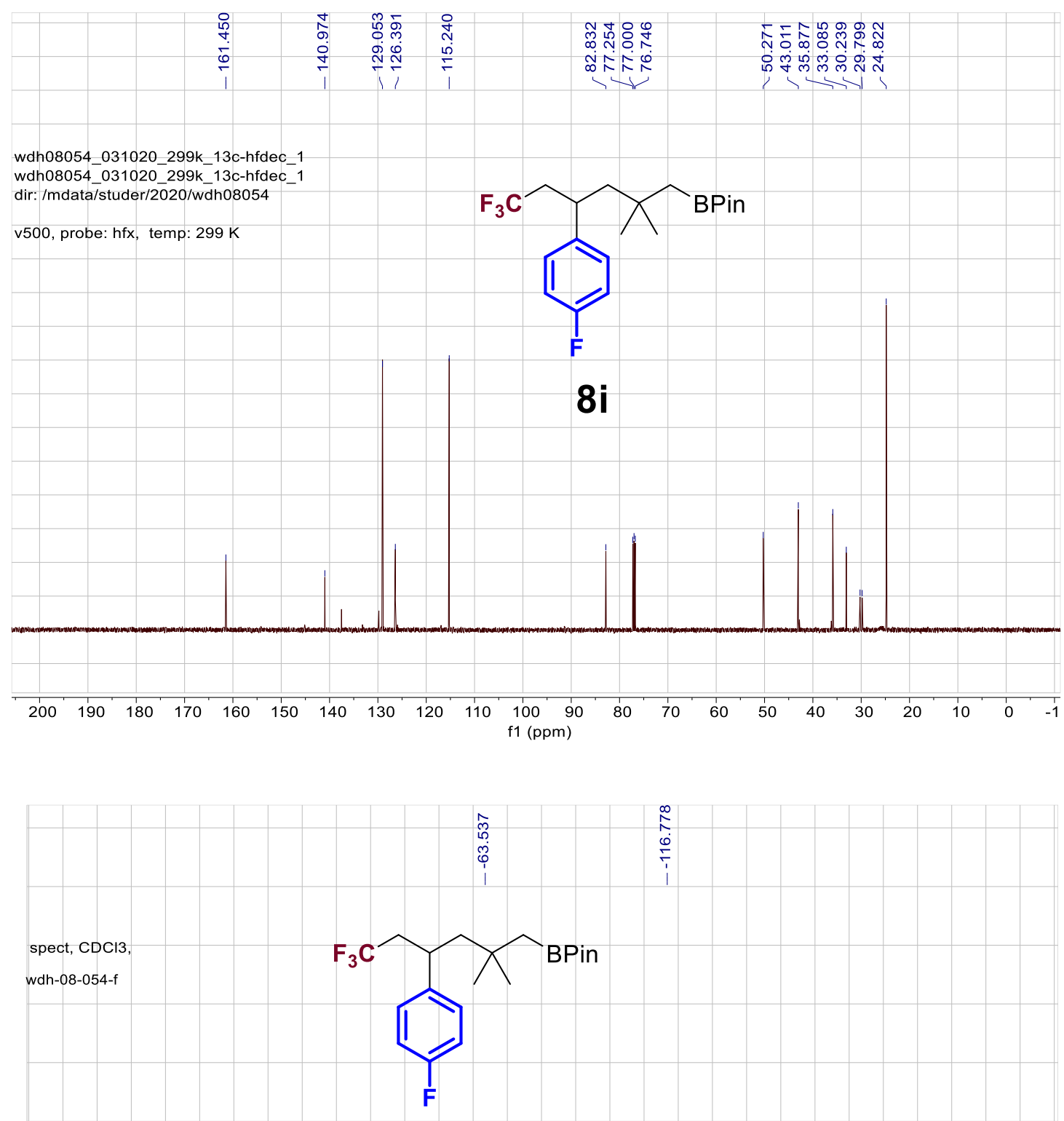

\section{$8 i$}

\begin{tabular}{llllllllllllllllll}
\hline & 50 & 30 & 10 & -10 & -30 & -50 & -70 & -90 & -110 & -130 & -150 & -170 & -190 & -210 & $-2 ;$ \\
\hline
\end{tabular}



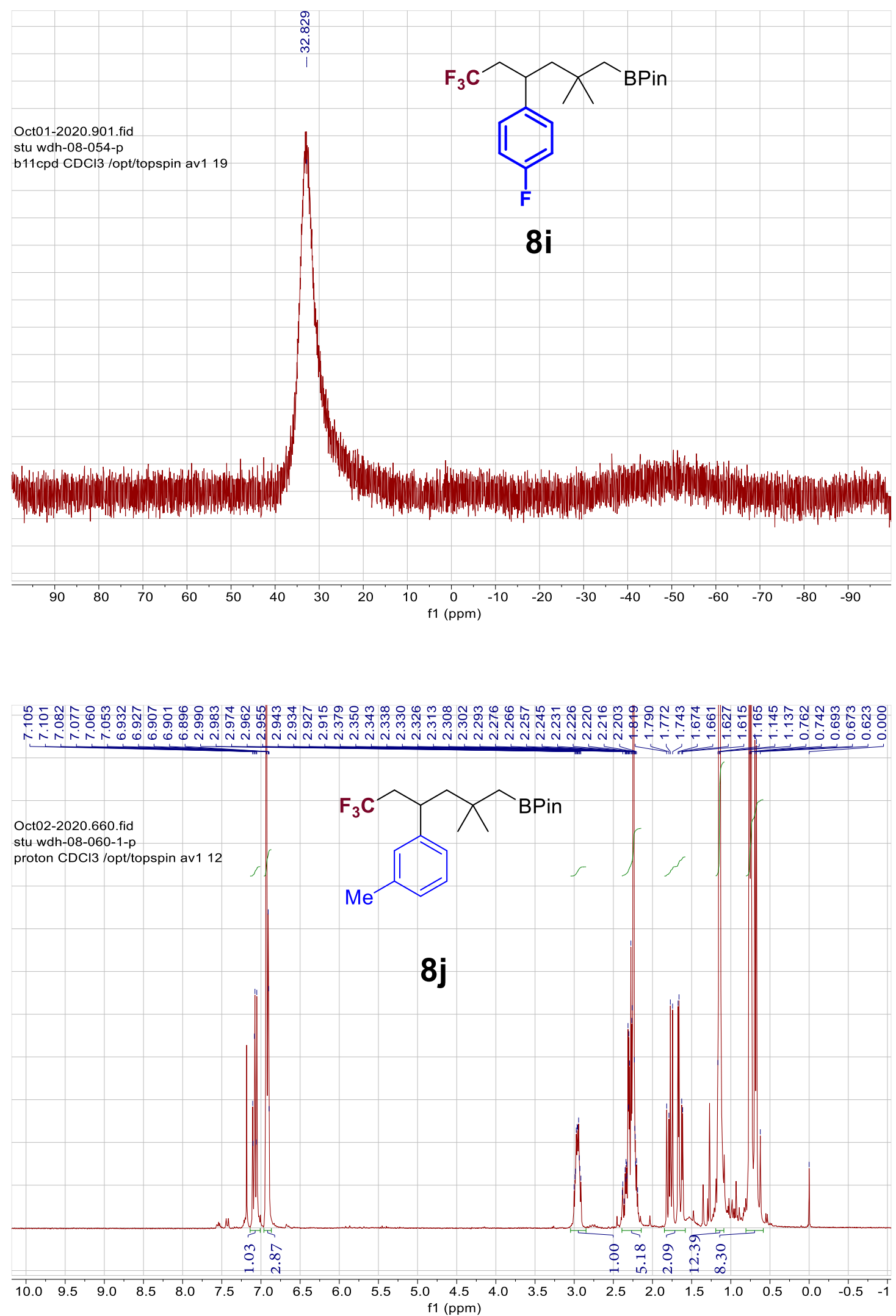

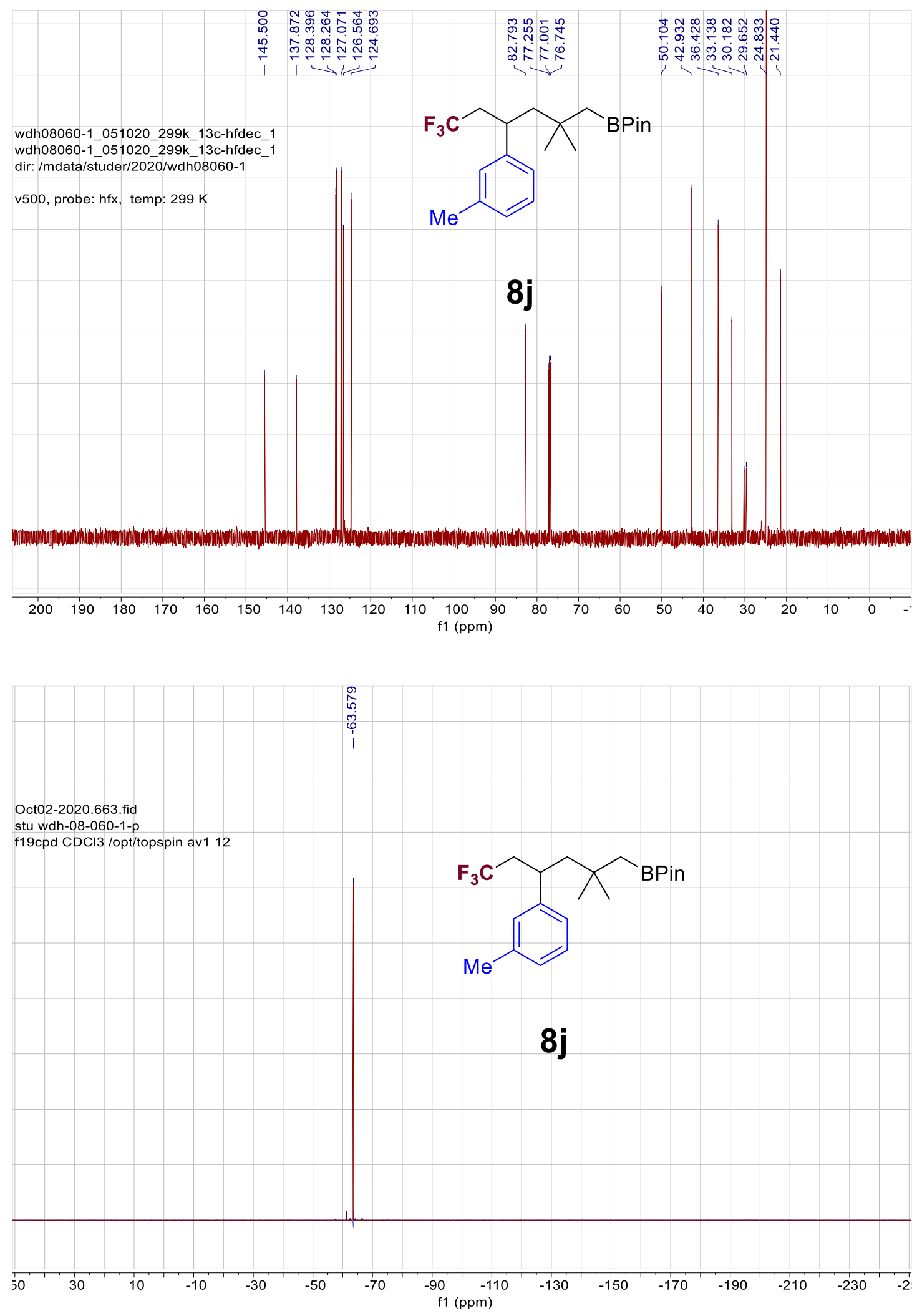

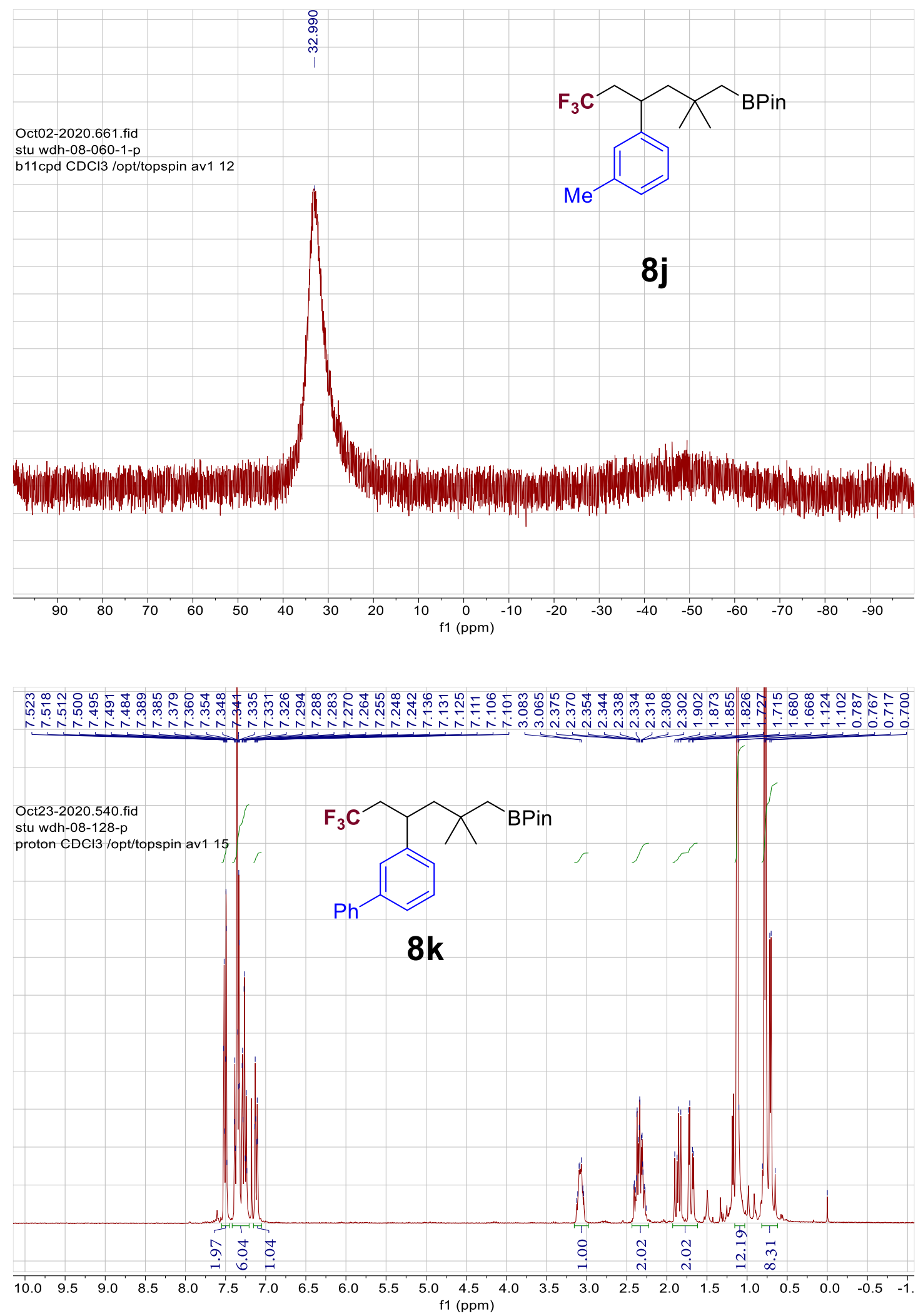

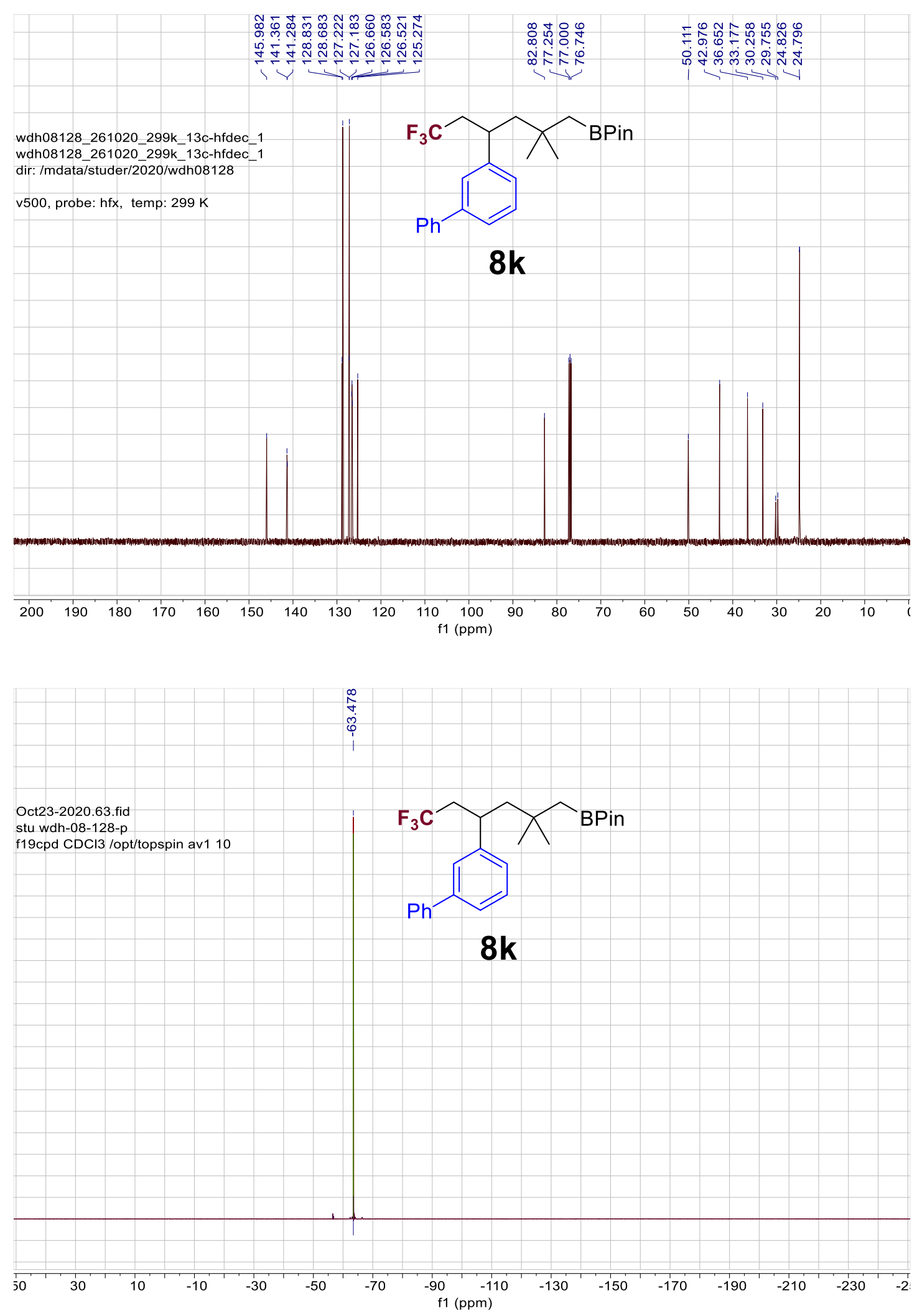

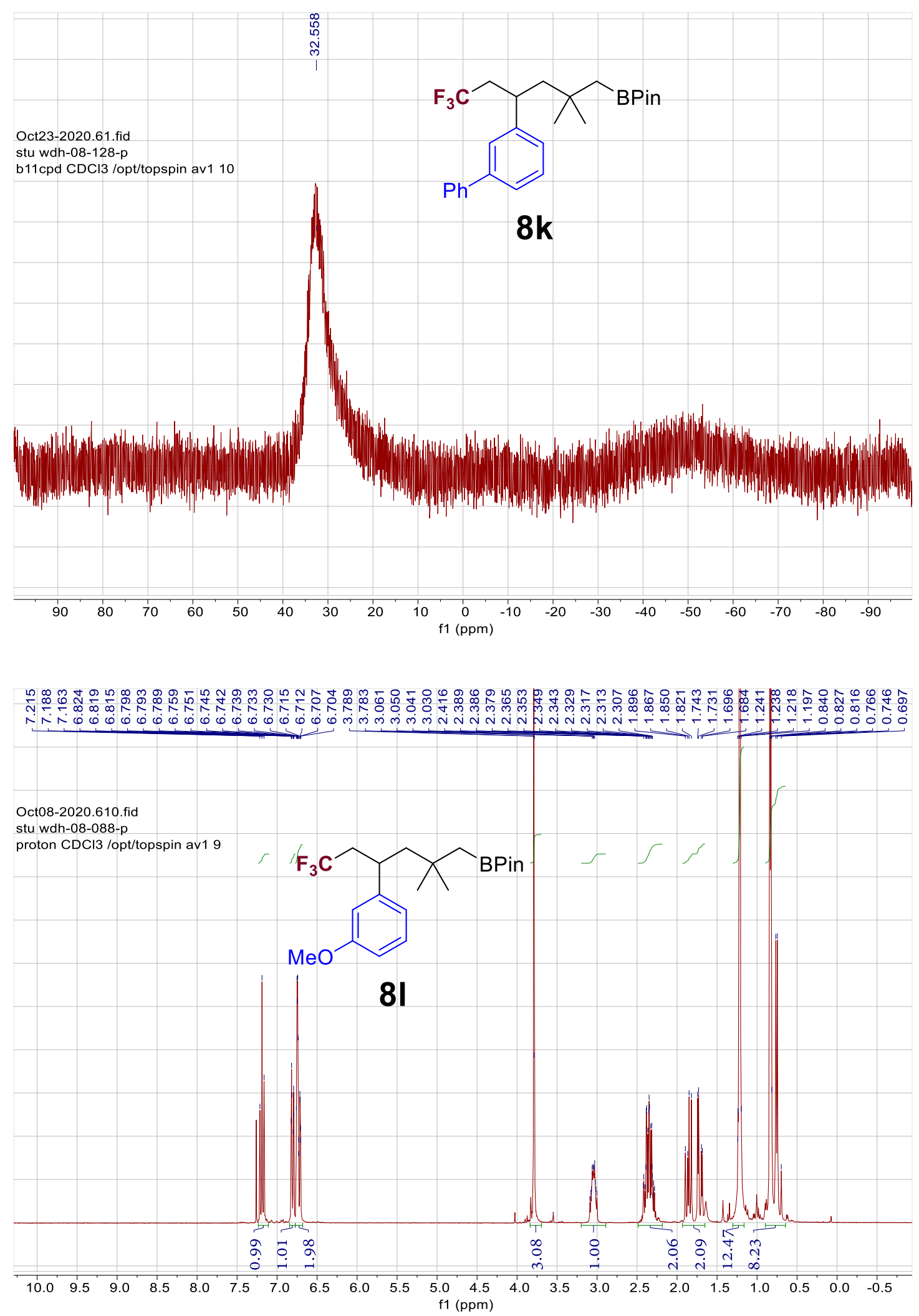

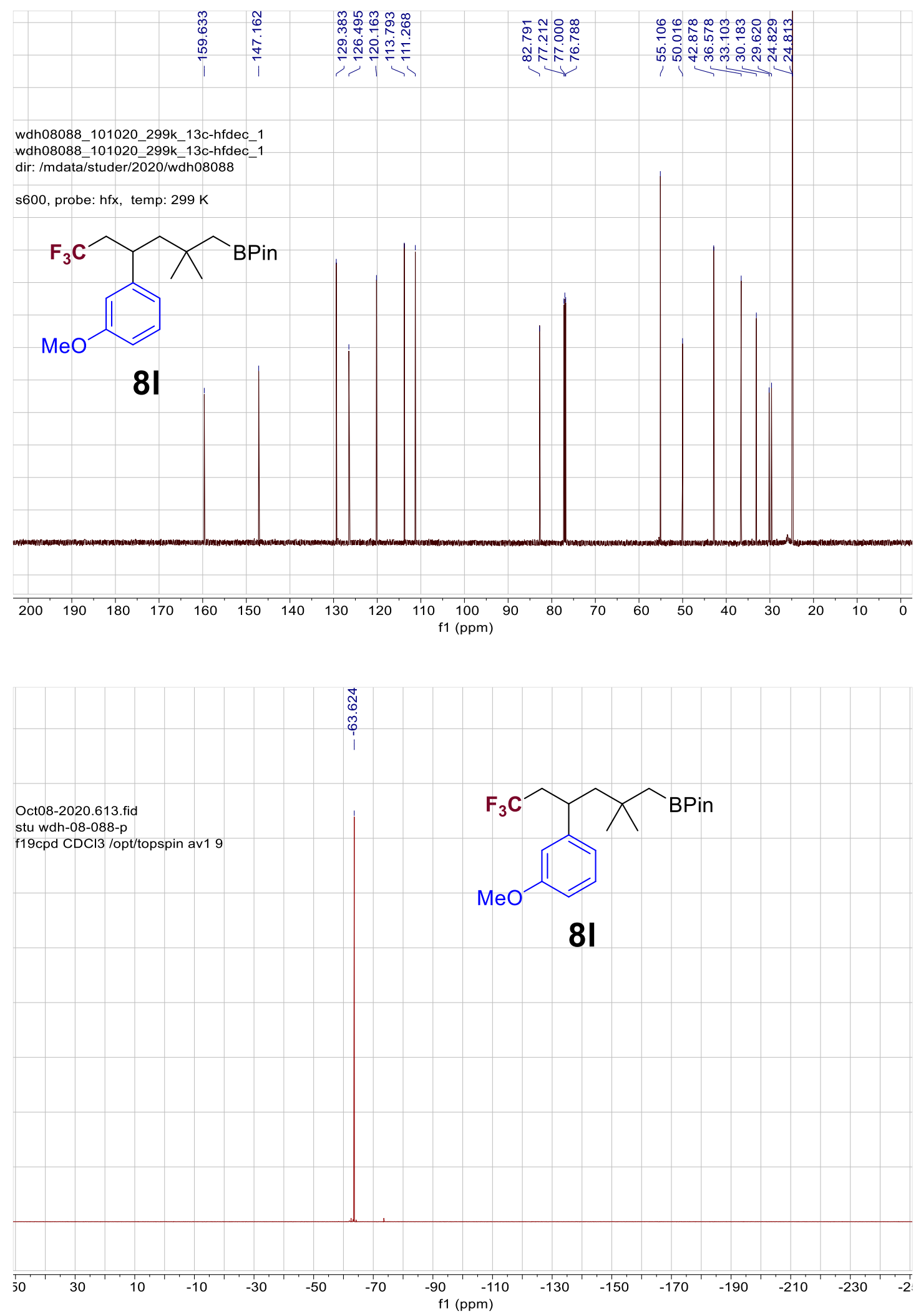

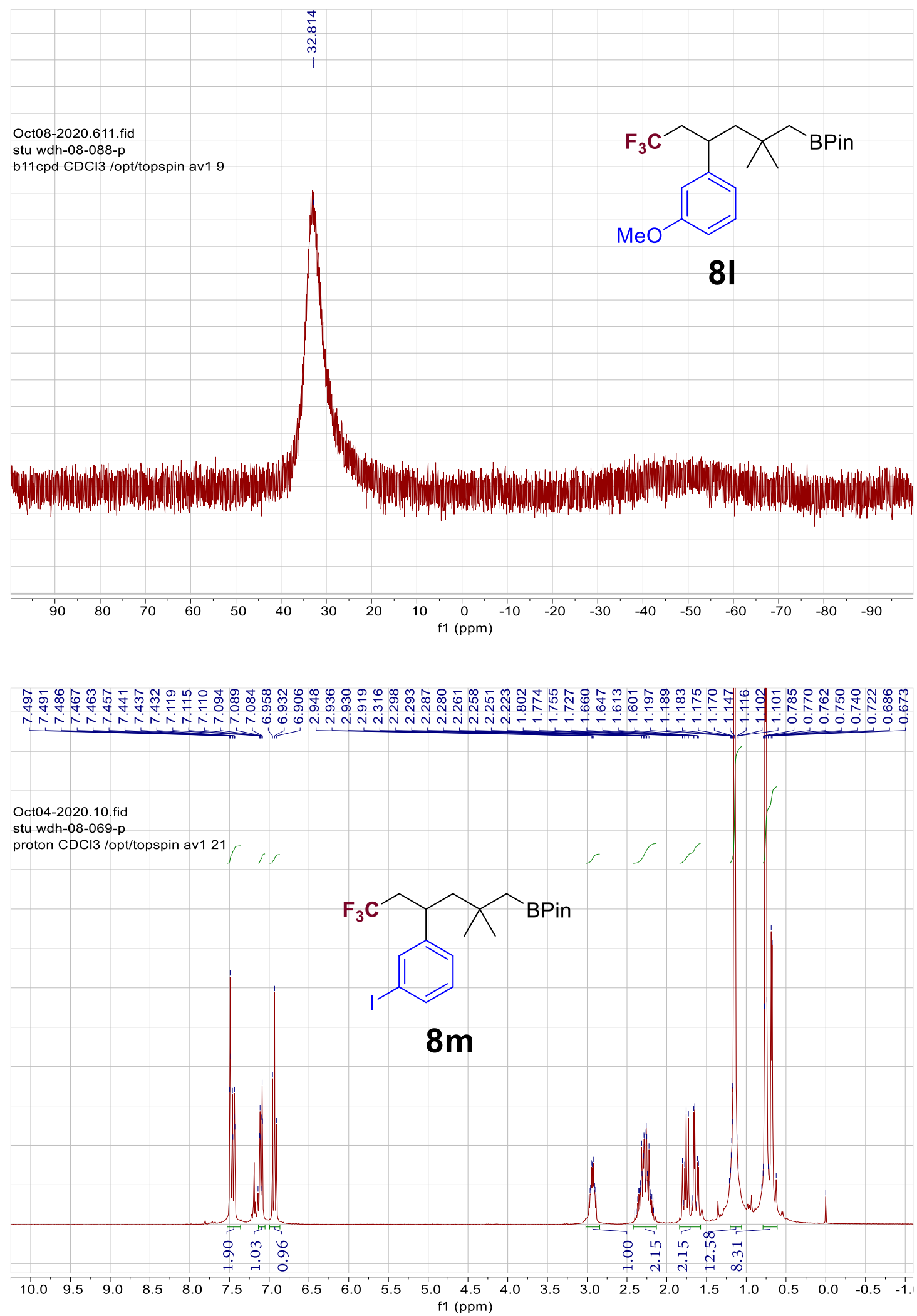

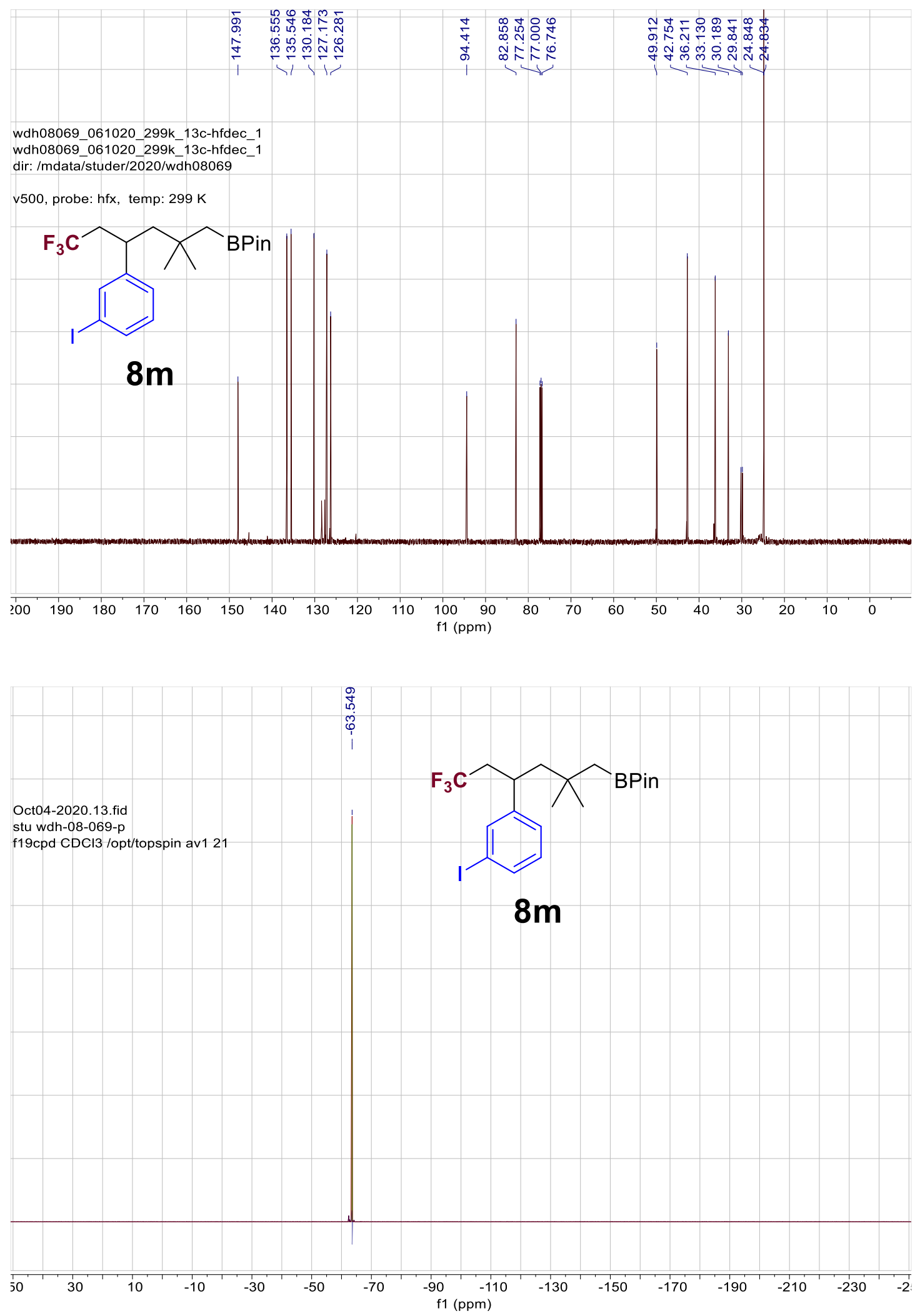

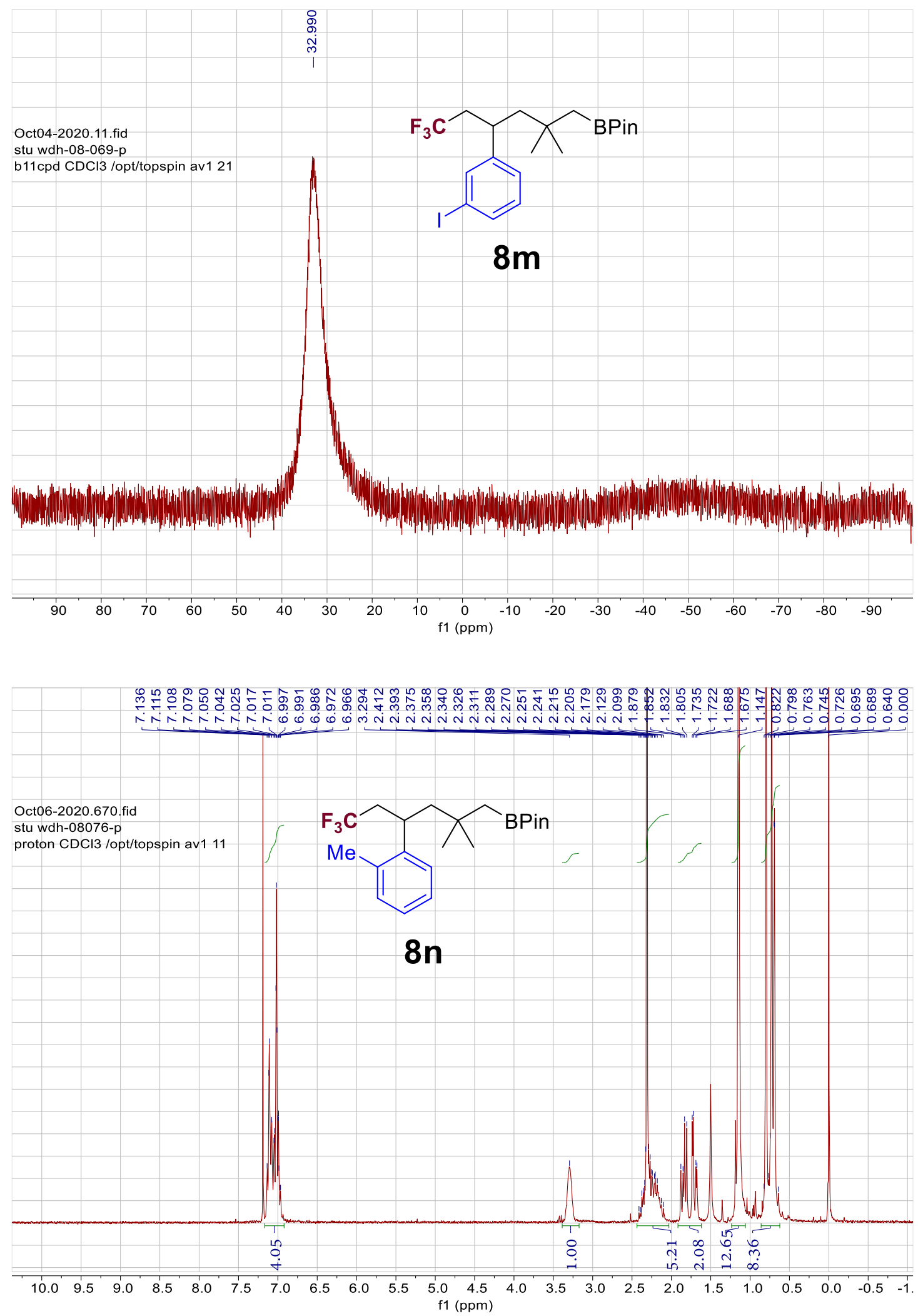

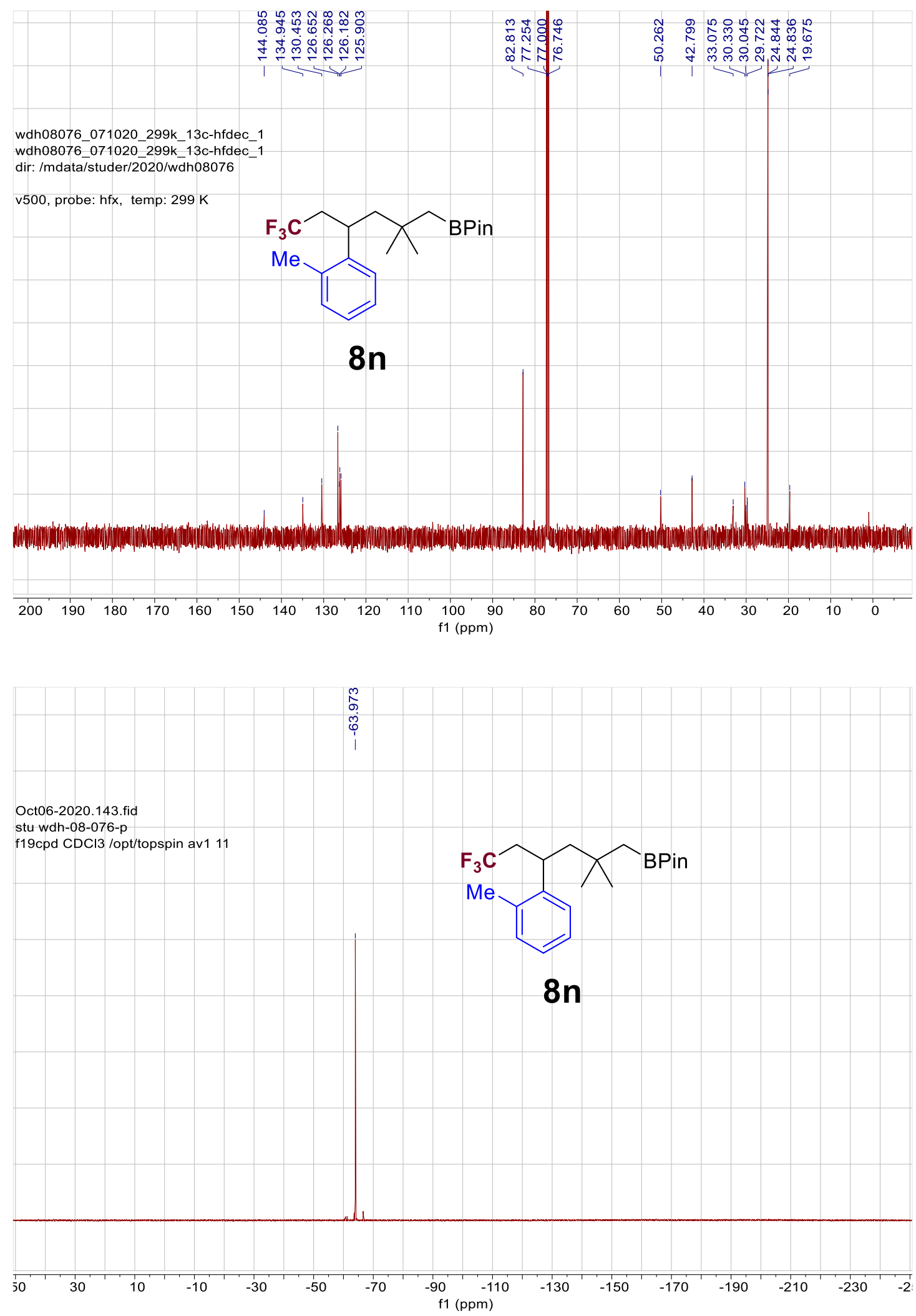

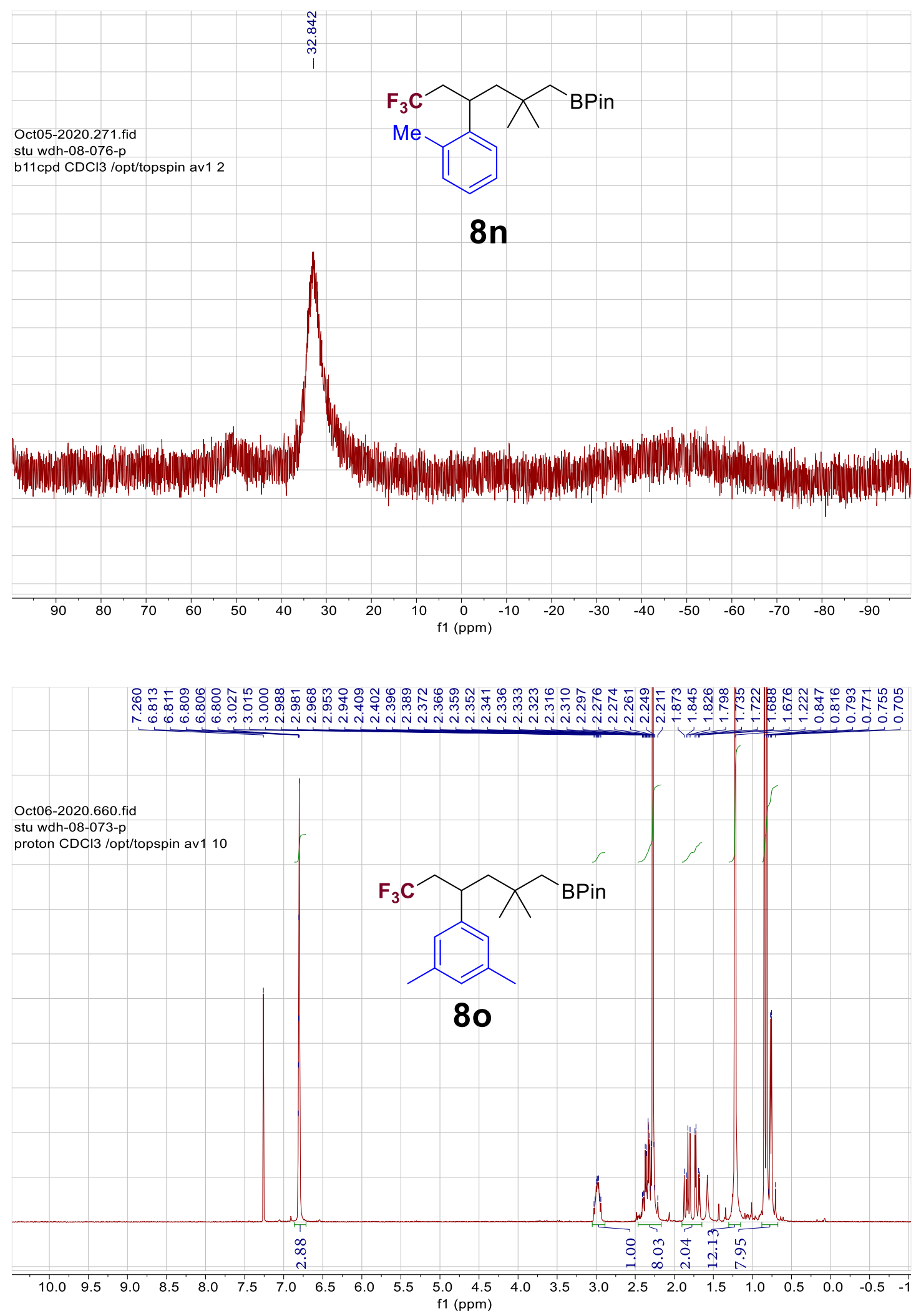

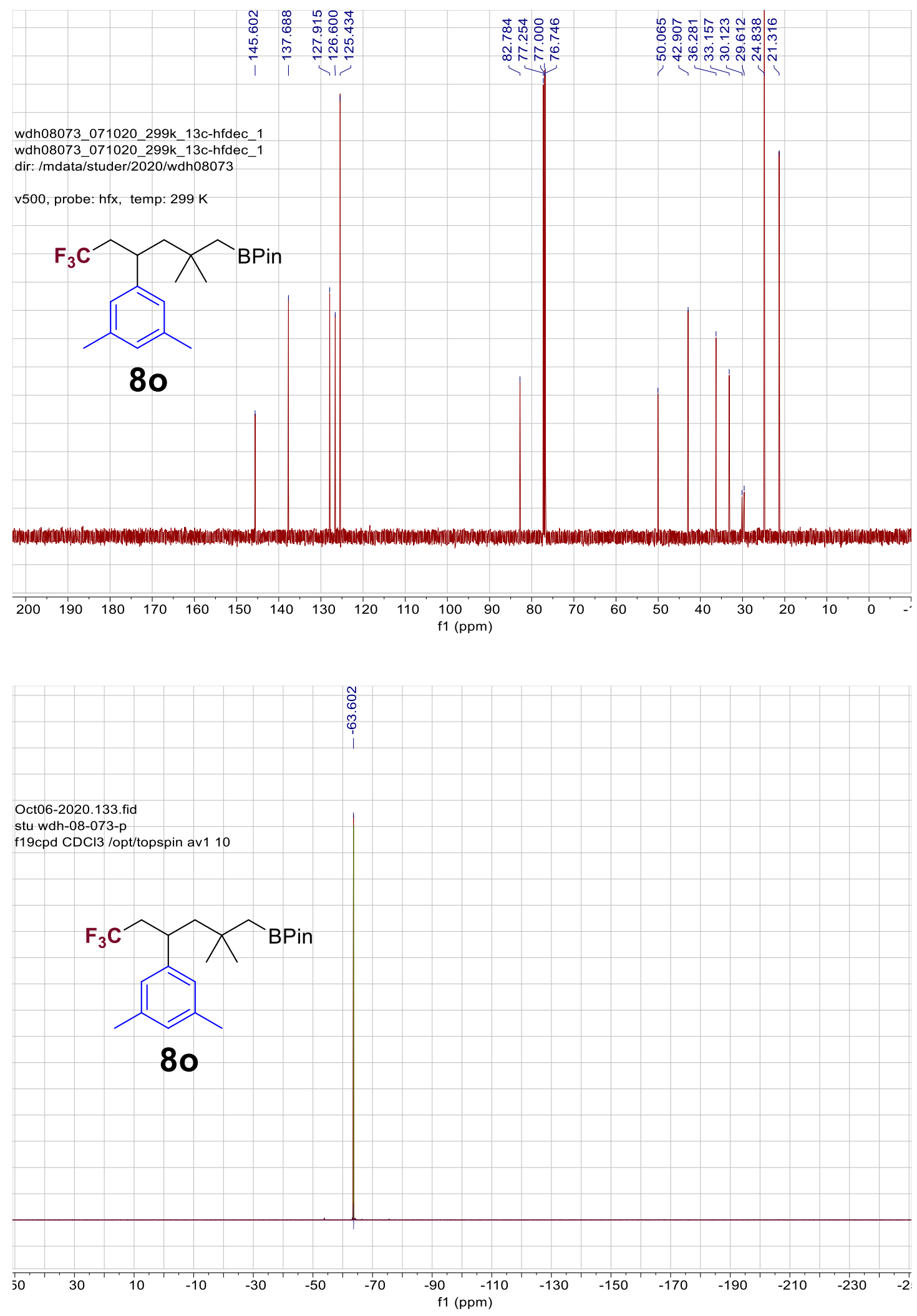

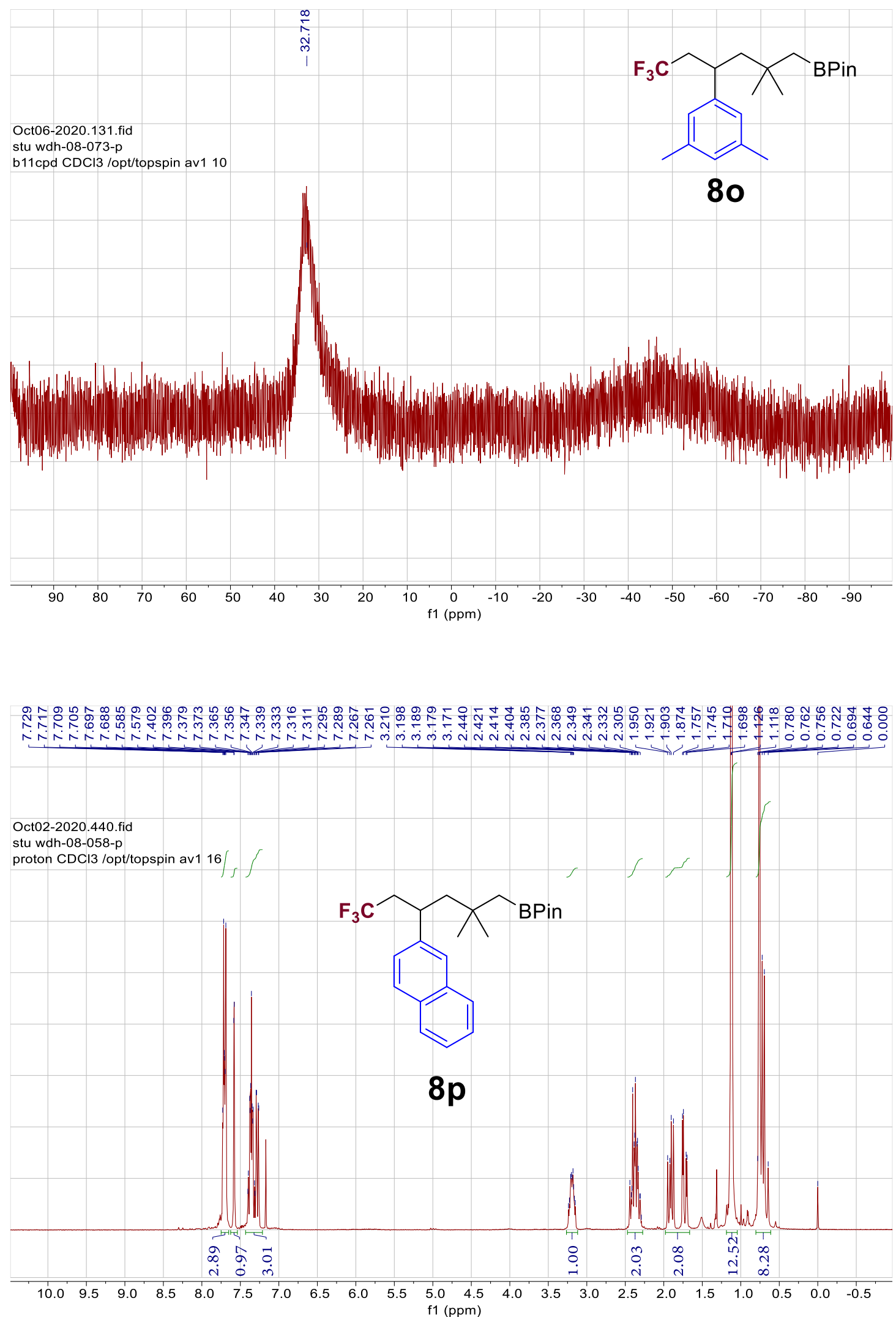

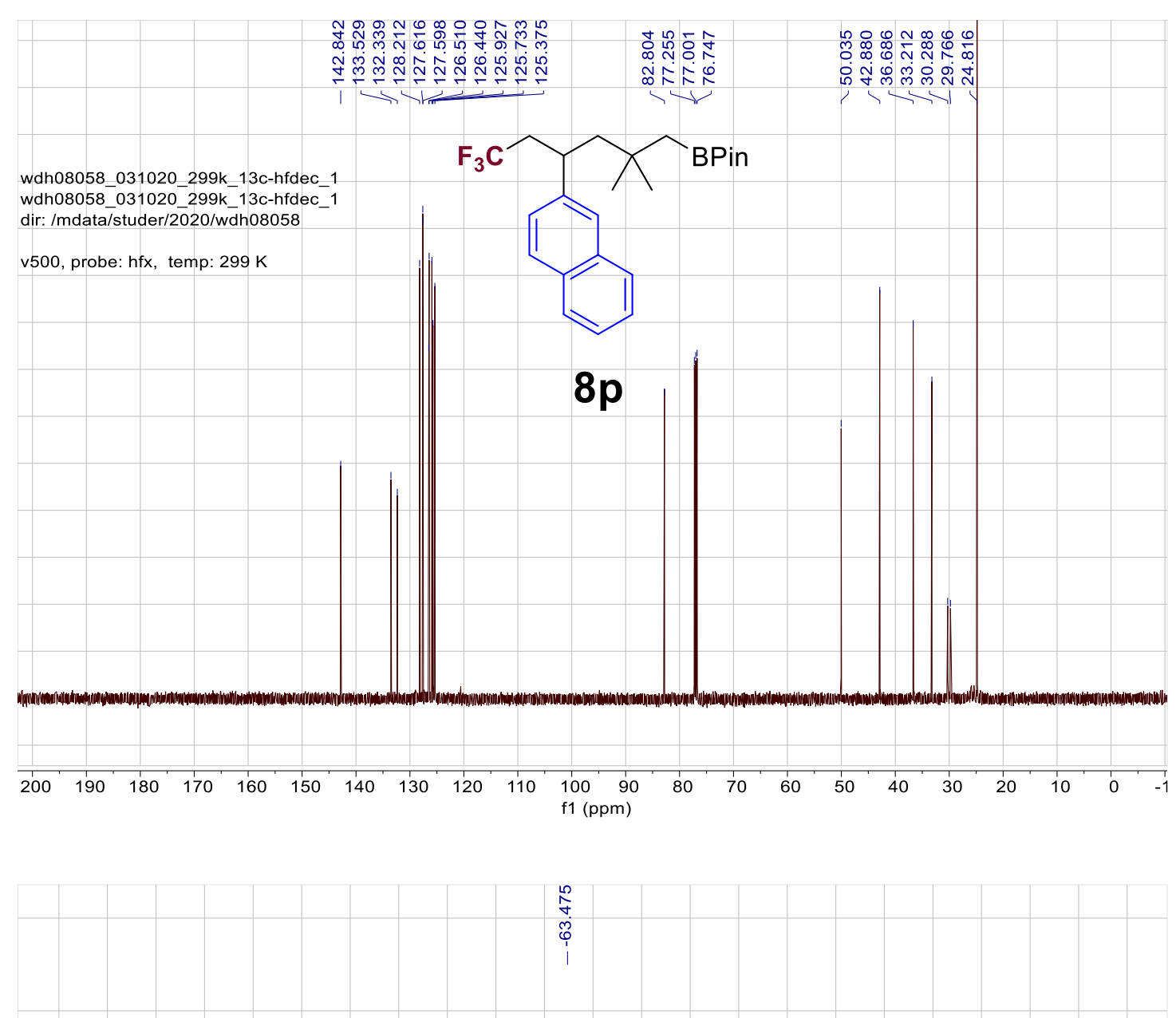

\section{Oct02-2020.443.fid}

stu wdh-08-058-p

f19cpd $\mathrm{CDCl} 3$ /opt/topspin av1 16

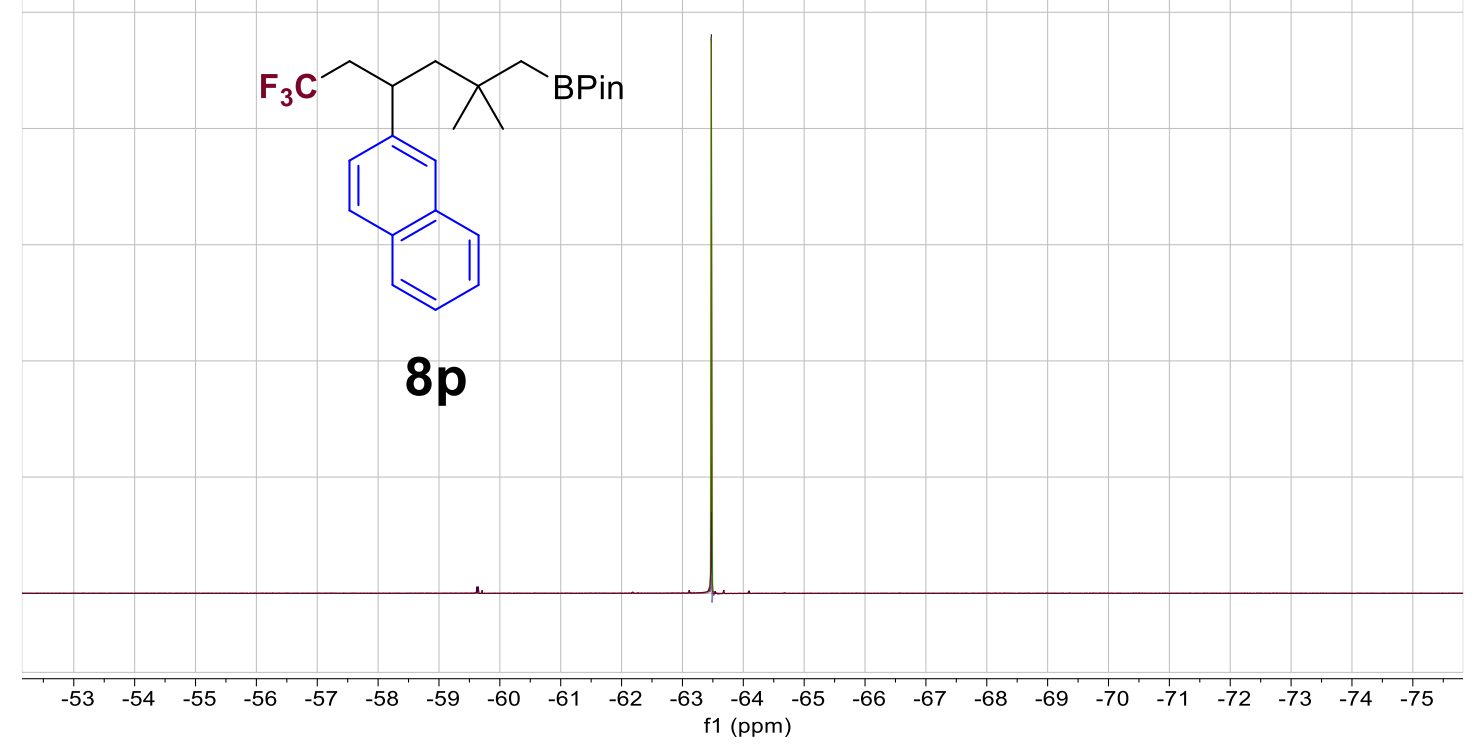




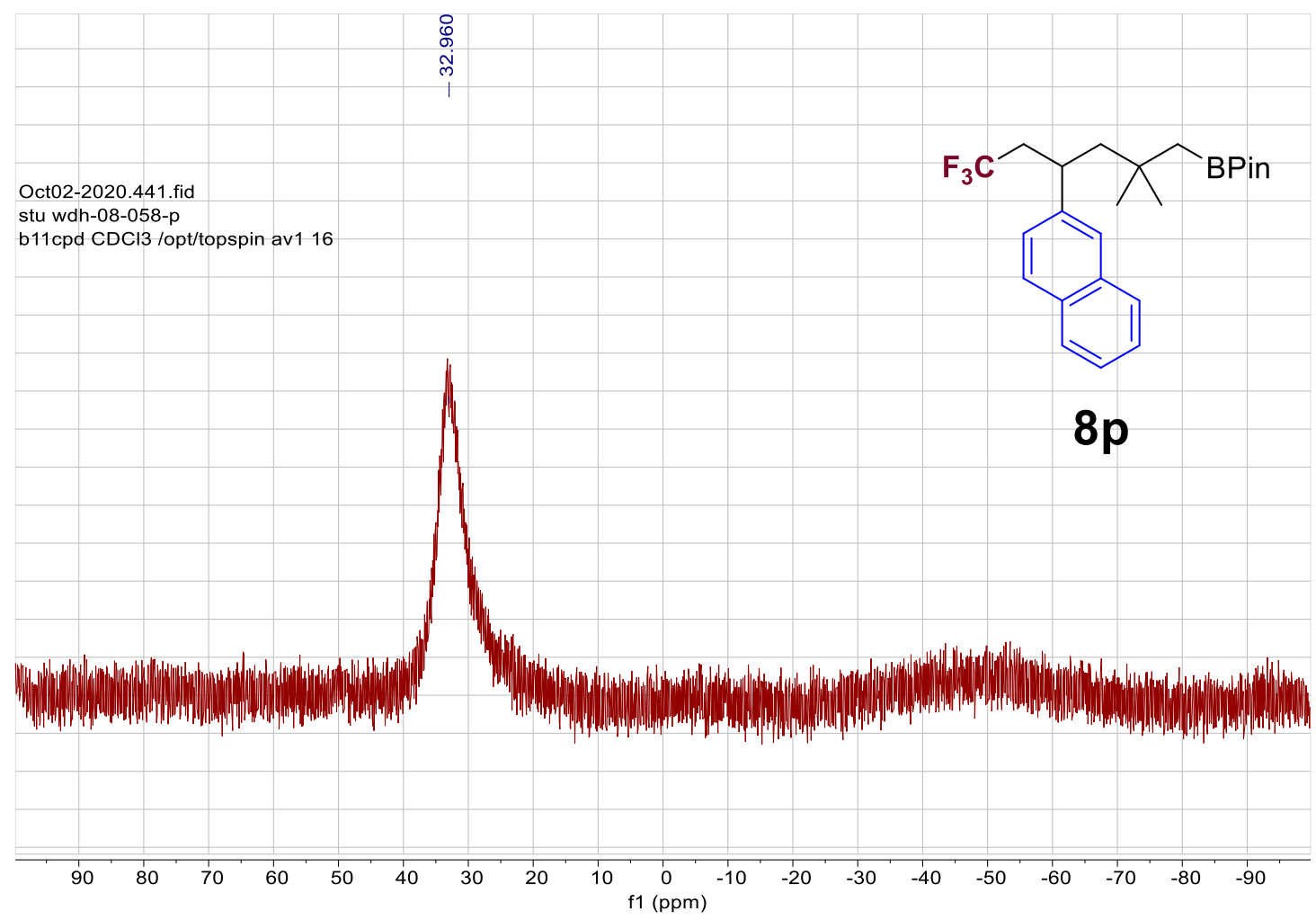

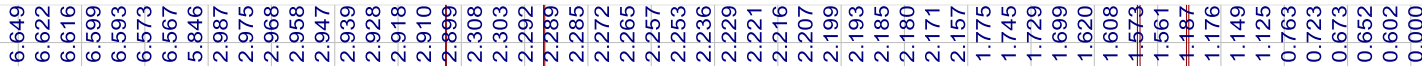

Oct02-2020.750.fid

stu wdh-08-059-p

proton $\mathrm{CDCl} 3$ /opt/topspin av1 20

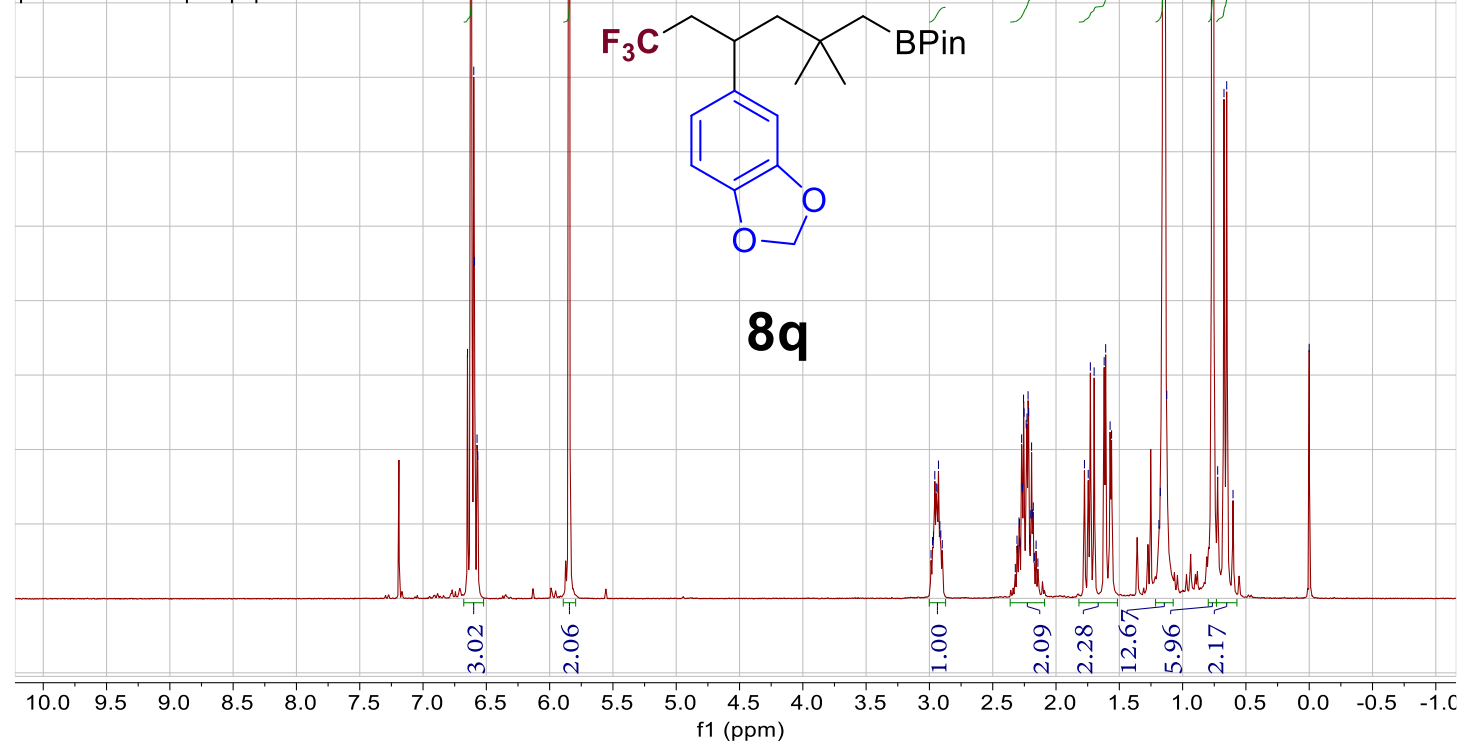



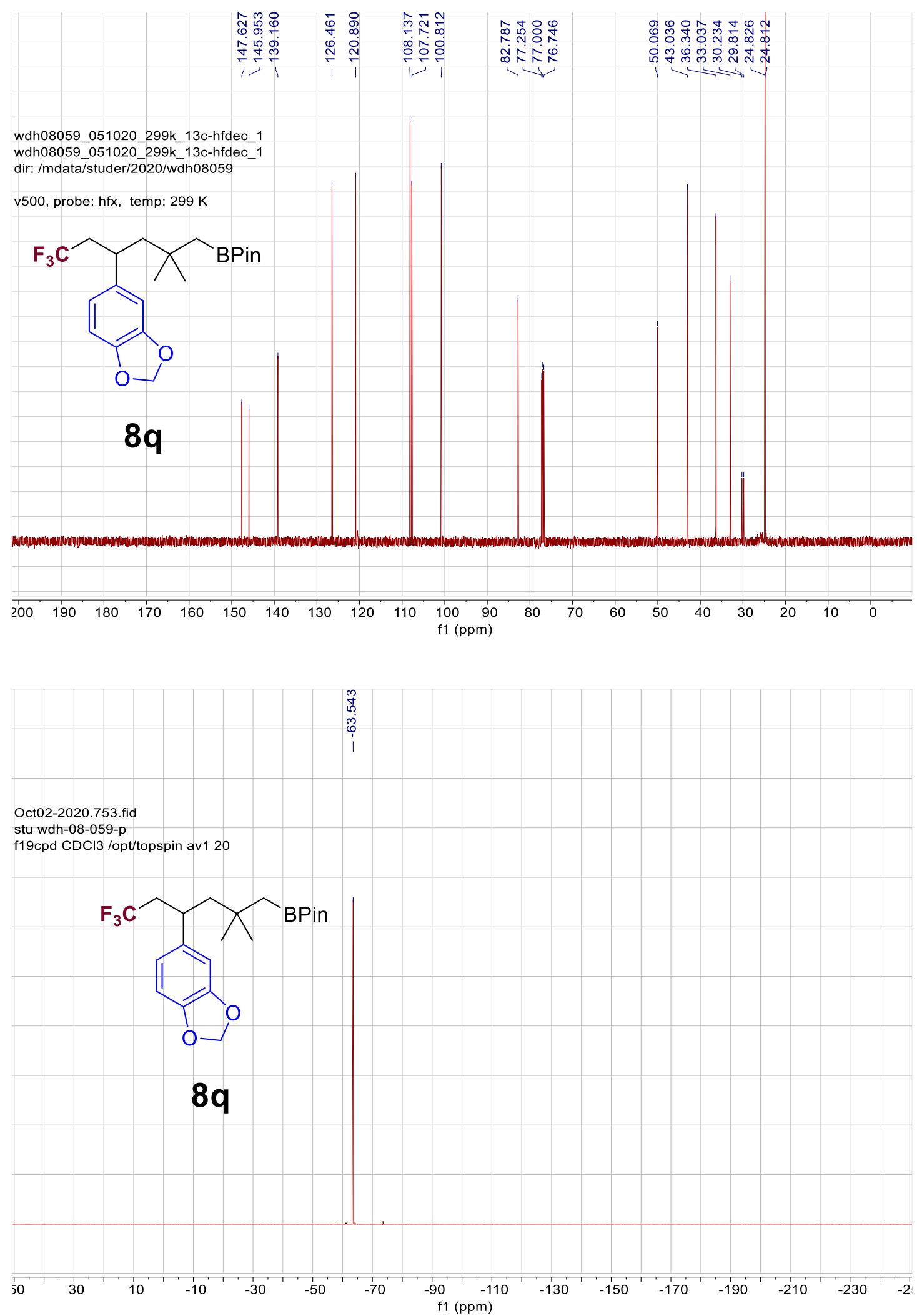

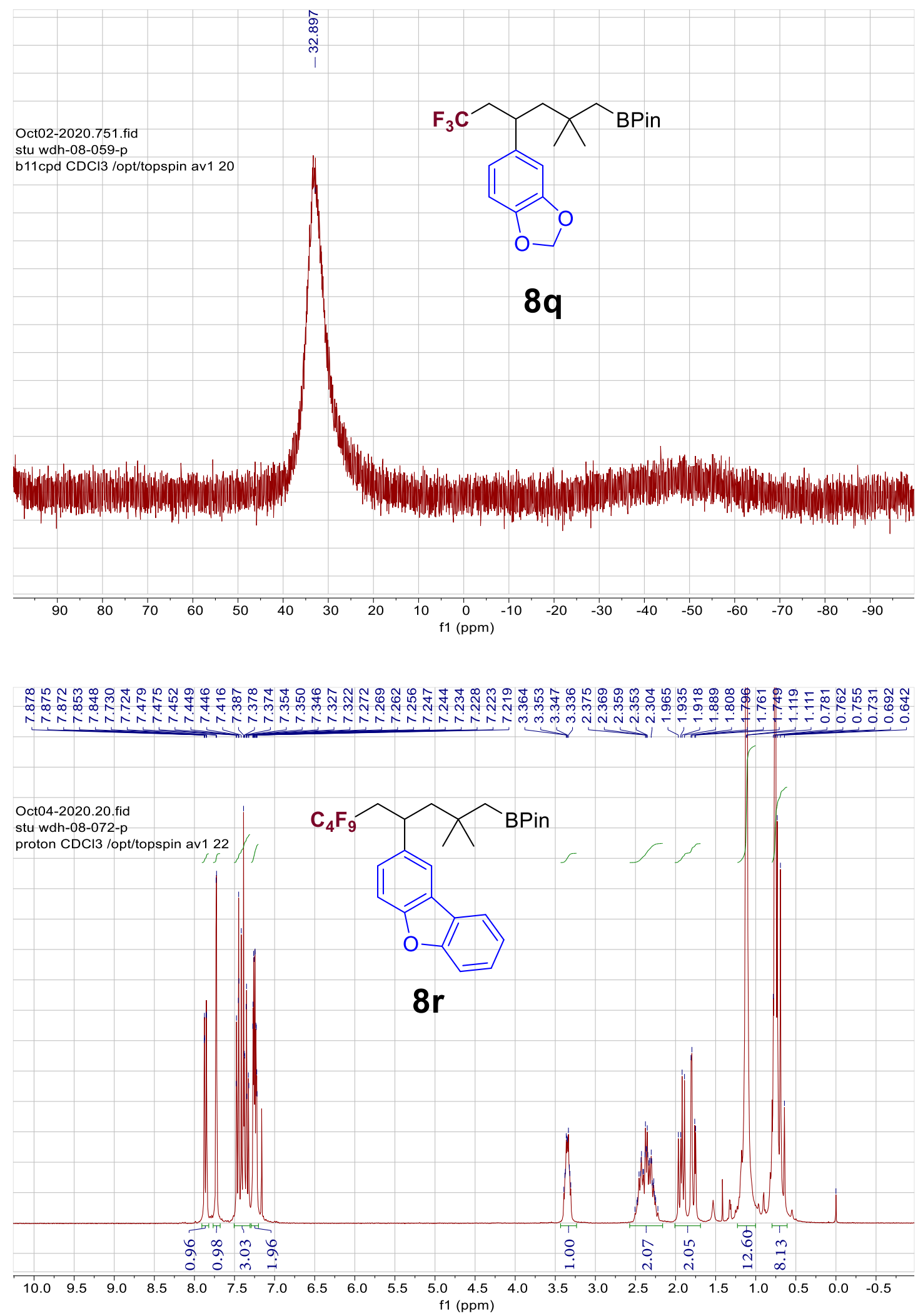

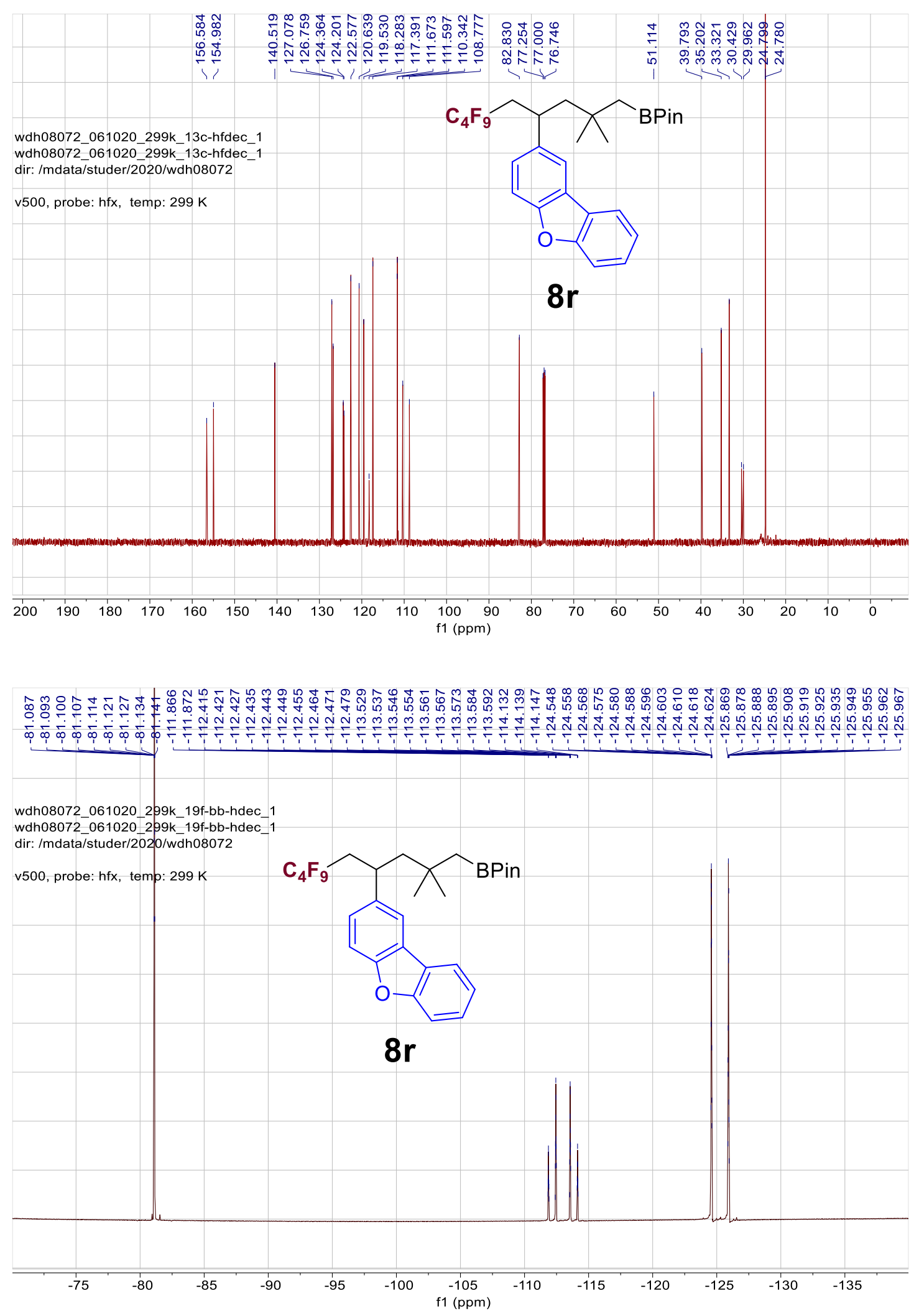

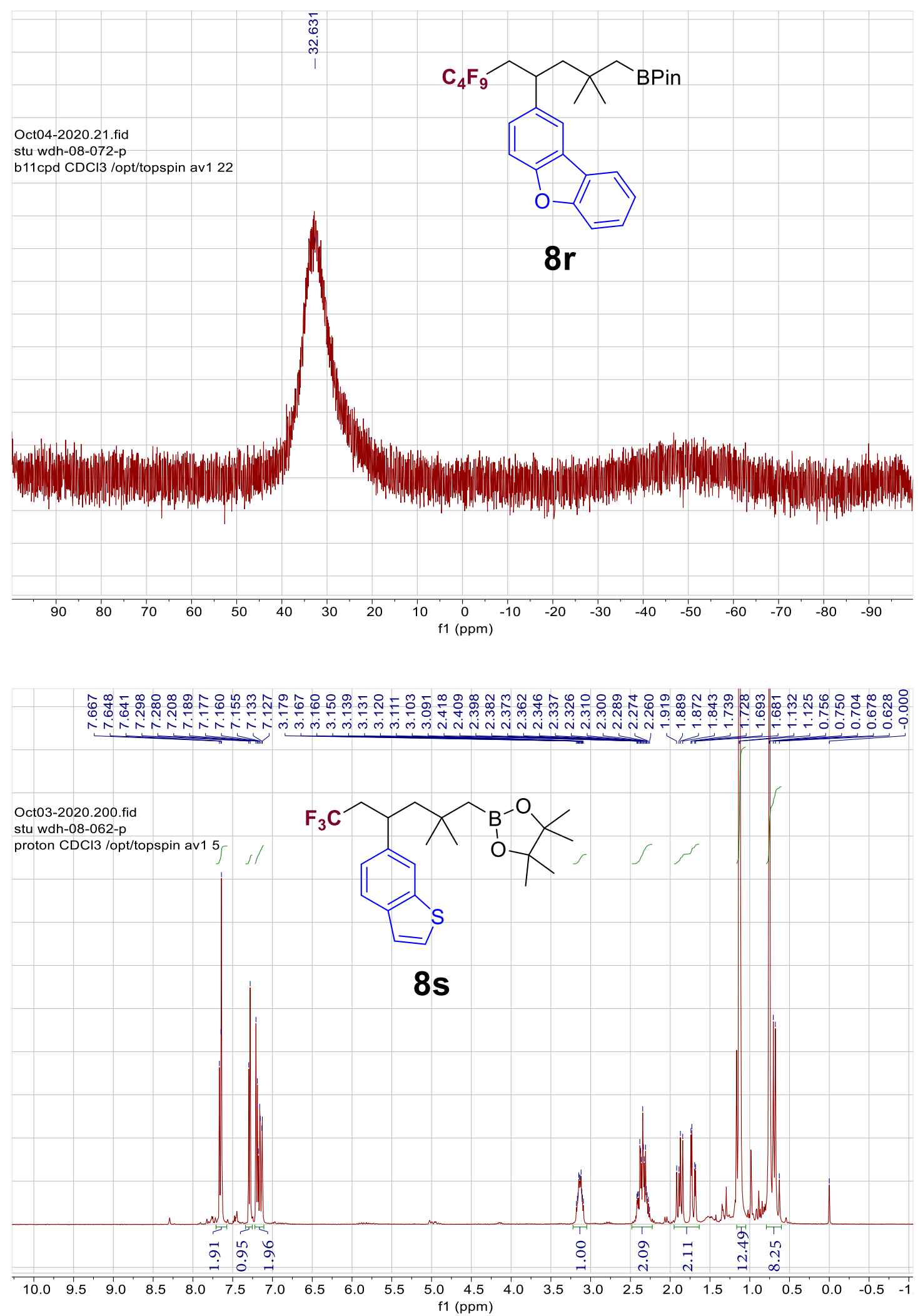

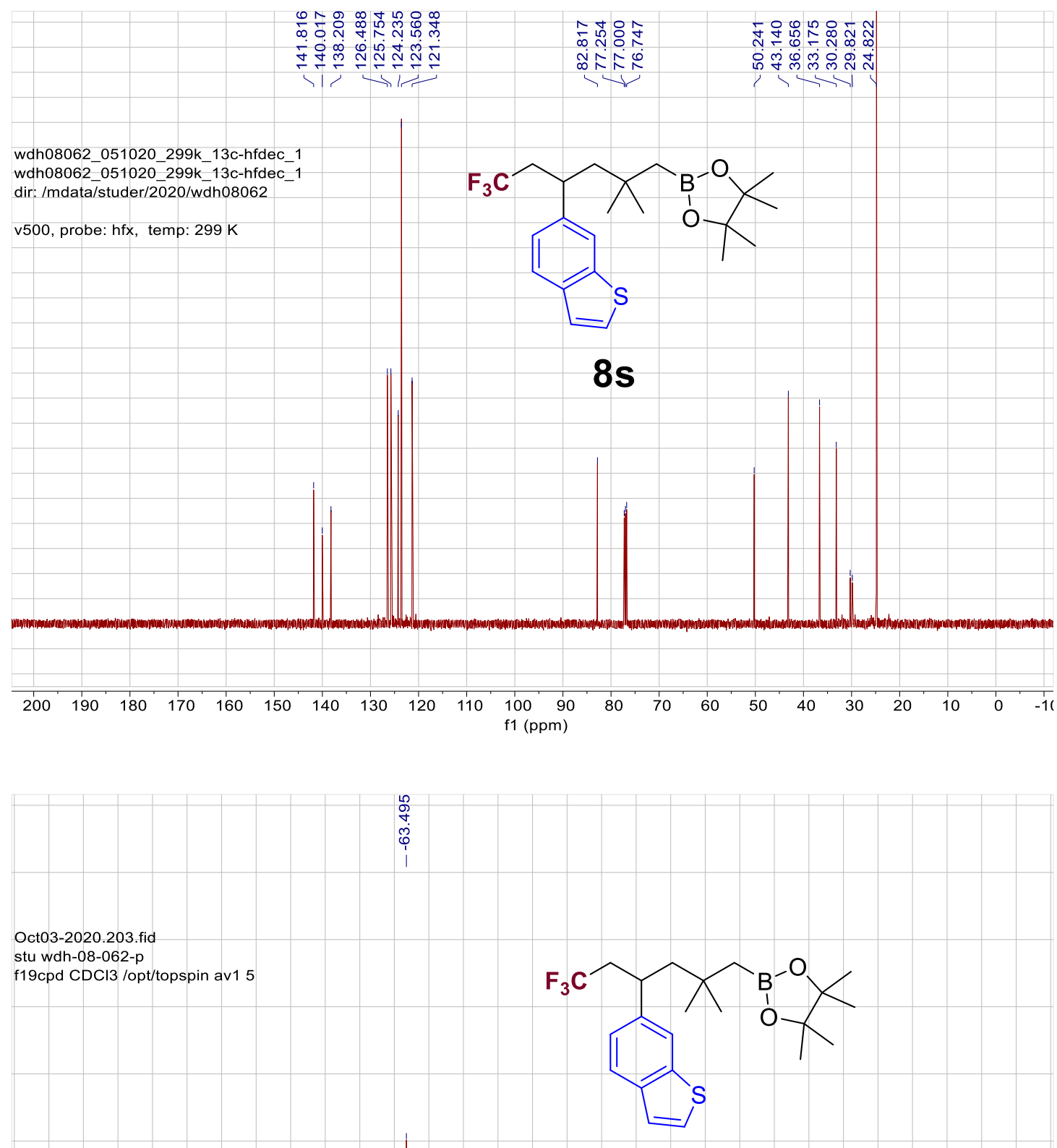

85

\begin{tabular}{llllllllllllllll}
50 & 30 & 10 & -10 & -30 & -50 & -70 & -90 & -110 & -130 & -150 & -170 & -190 & -210 & -230 & -2 \\
\hline
\end{tabular} 

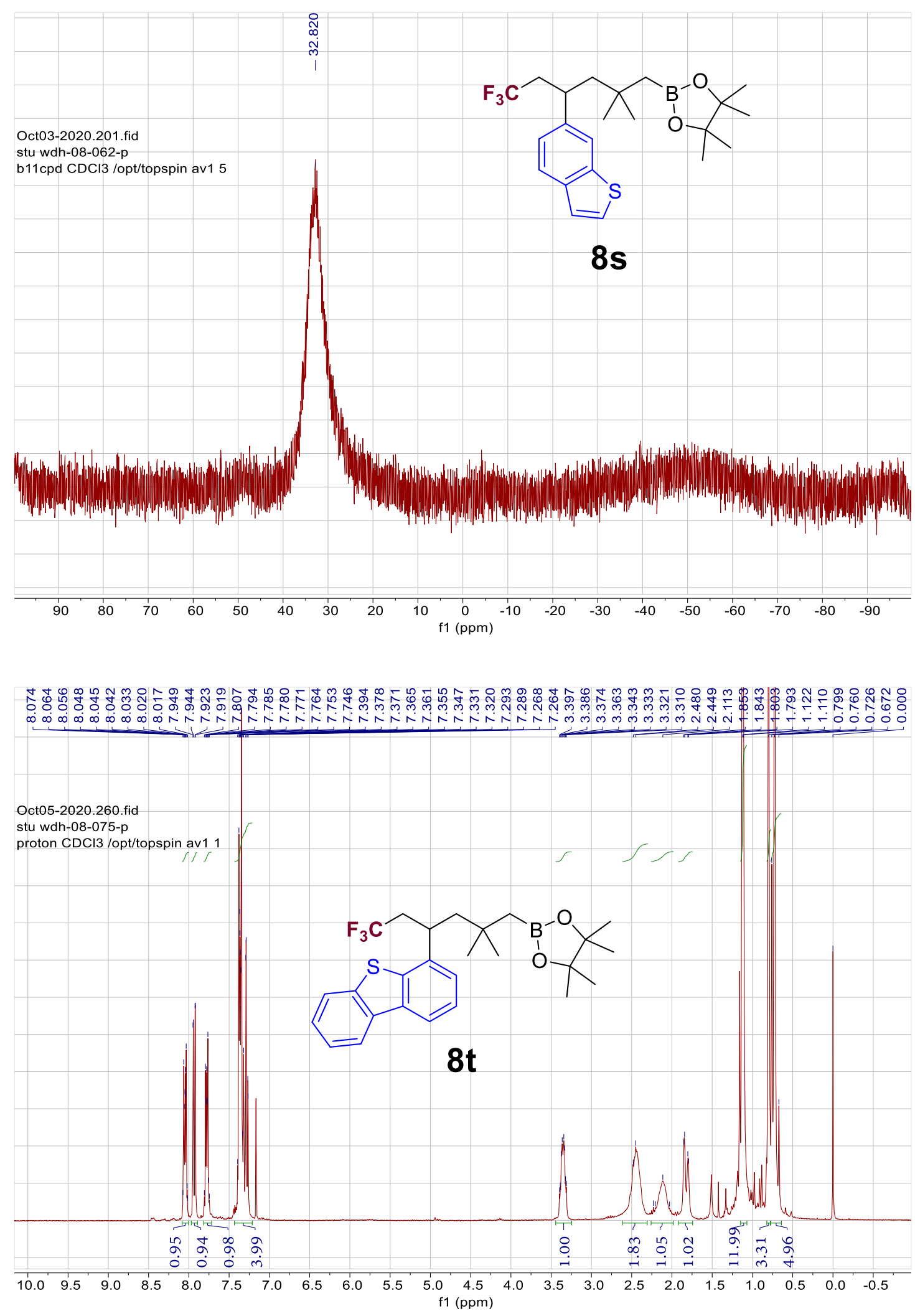

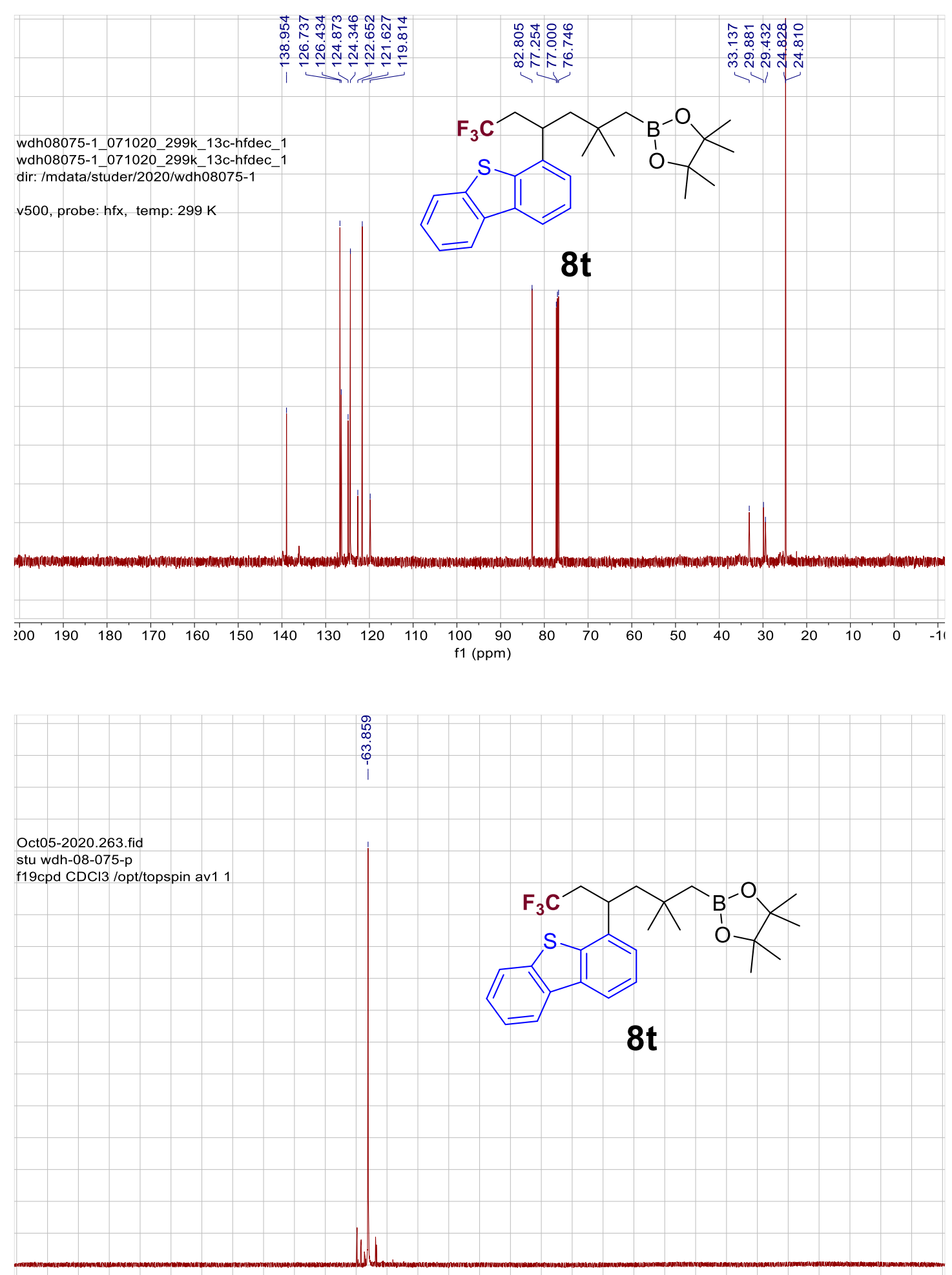

\begin{tabular}{cccccccccccccccc}
\hline 50 & 30 & 10 & -10 & -30 & -50 & -70 & -90 & -110 & -130 & -150 & -170 & -190 & -210 & -1 & -1 \\
$\mathrm{f} 1(\mathrm{ppm})$
\end{tabular} 

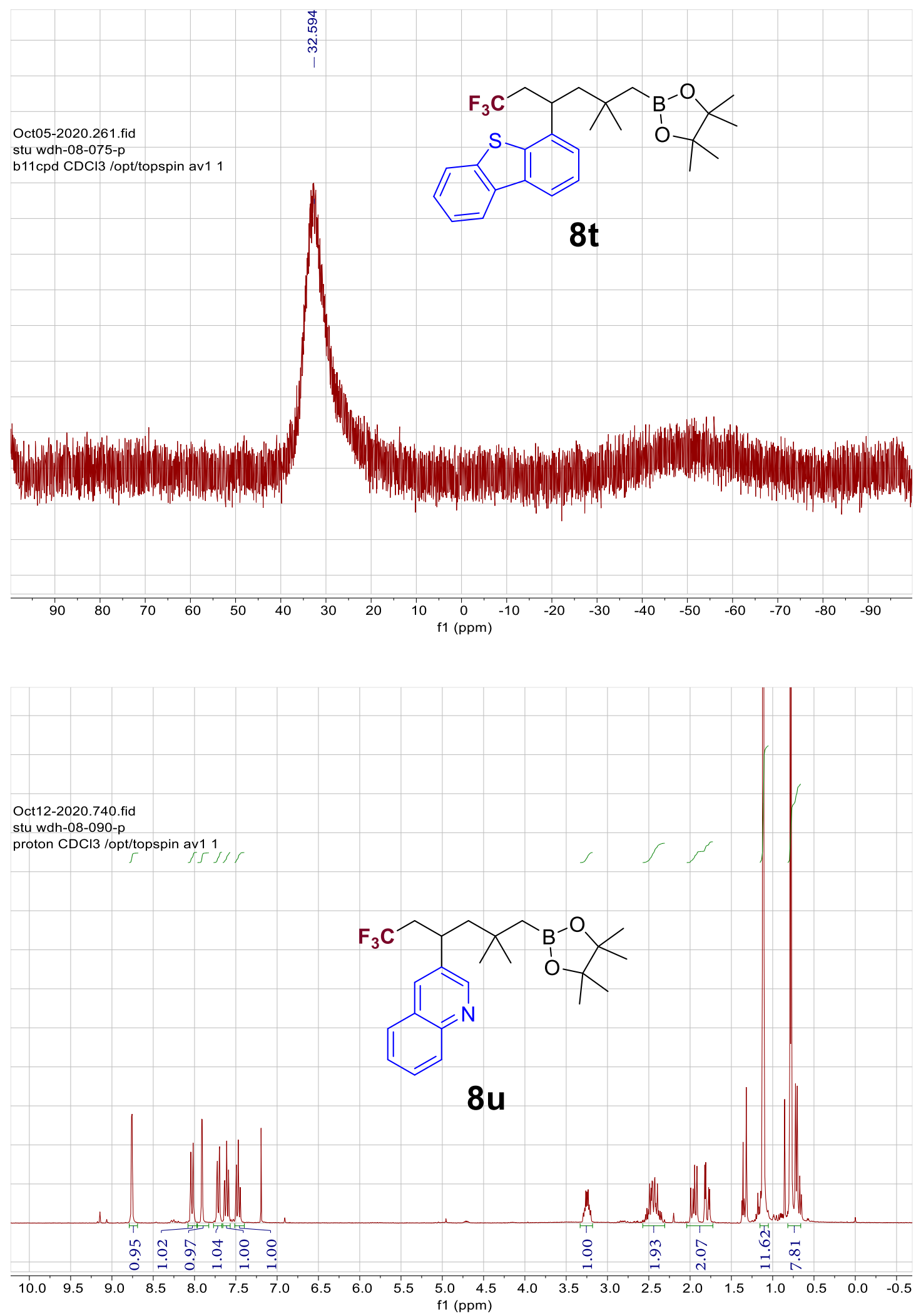

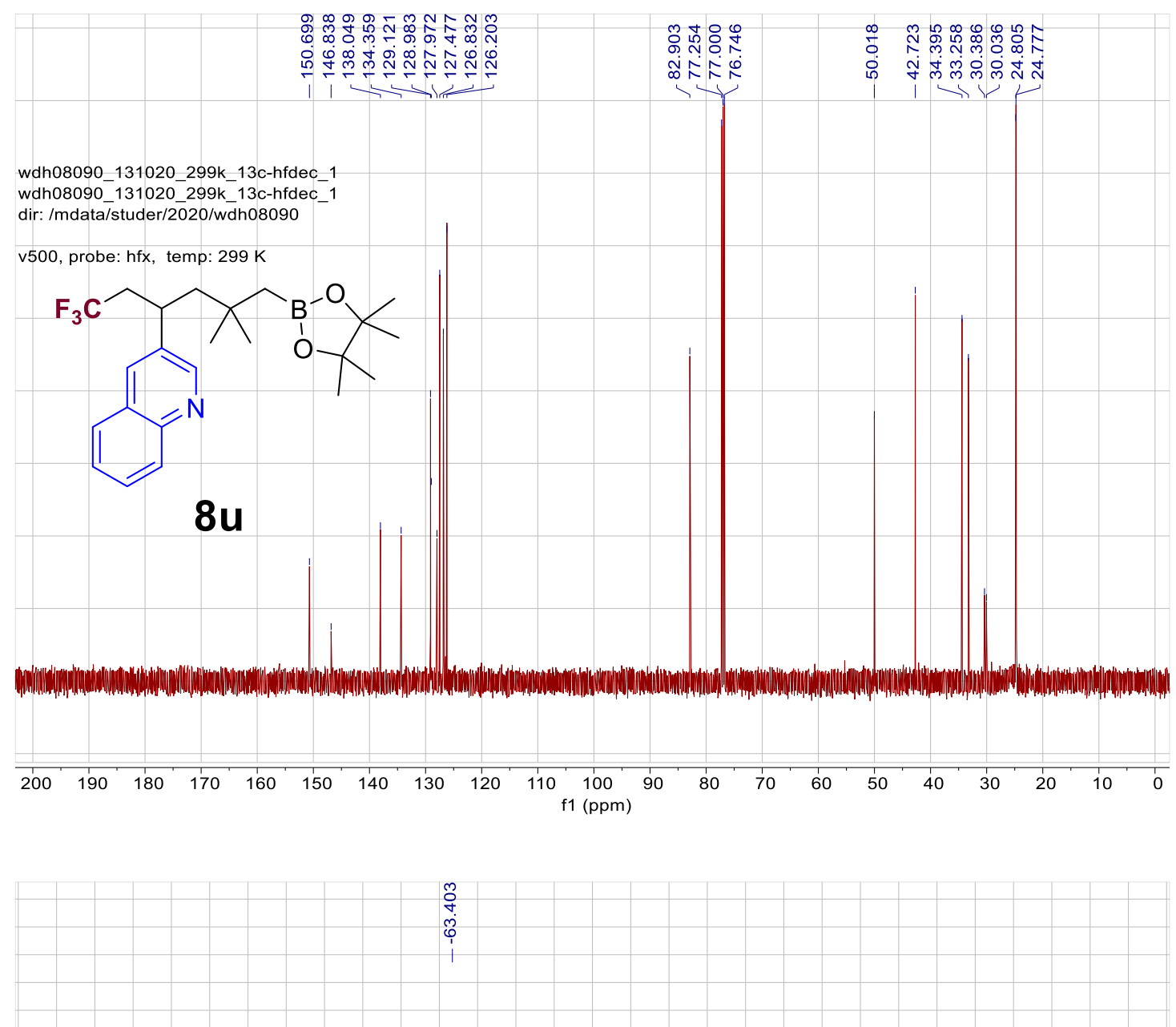

\section{Oct12-2020.743 fid}

stu wdh-08-090-p

f19cpd CDCl3 /opt/topspin av1 1

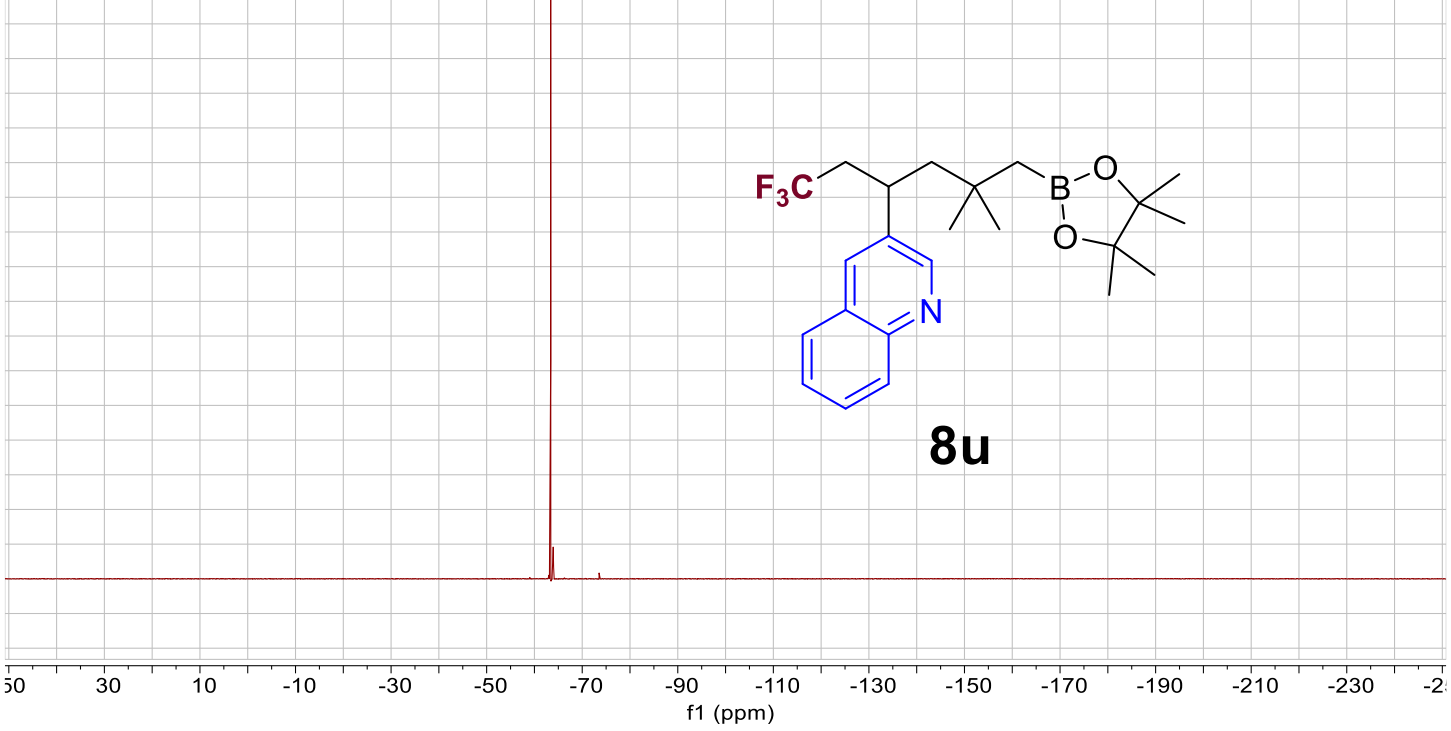



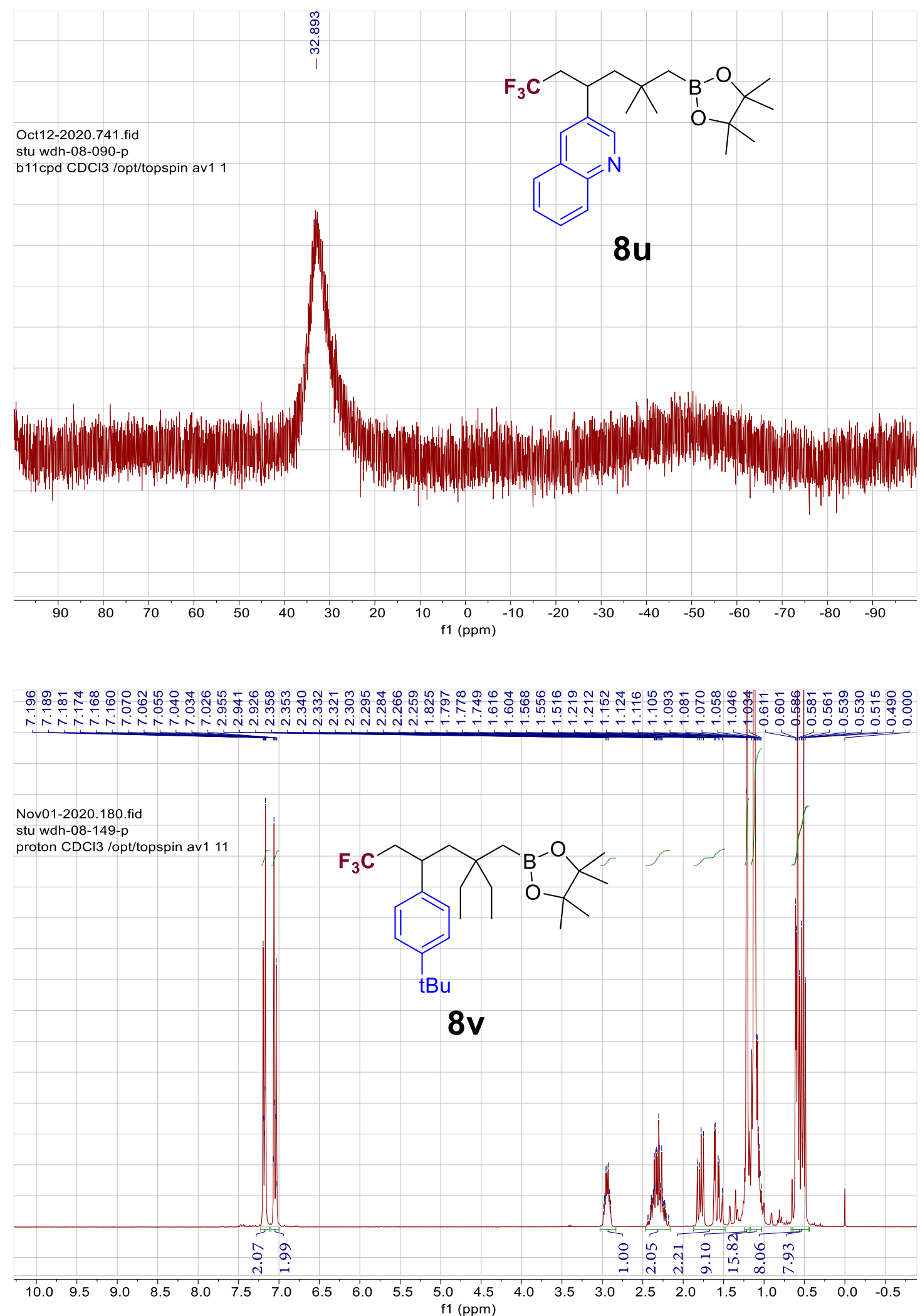

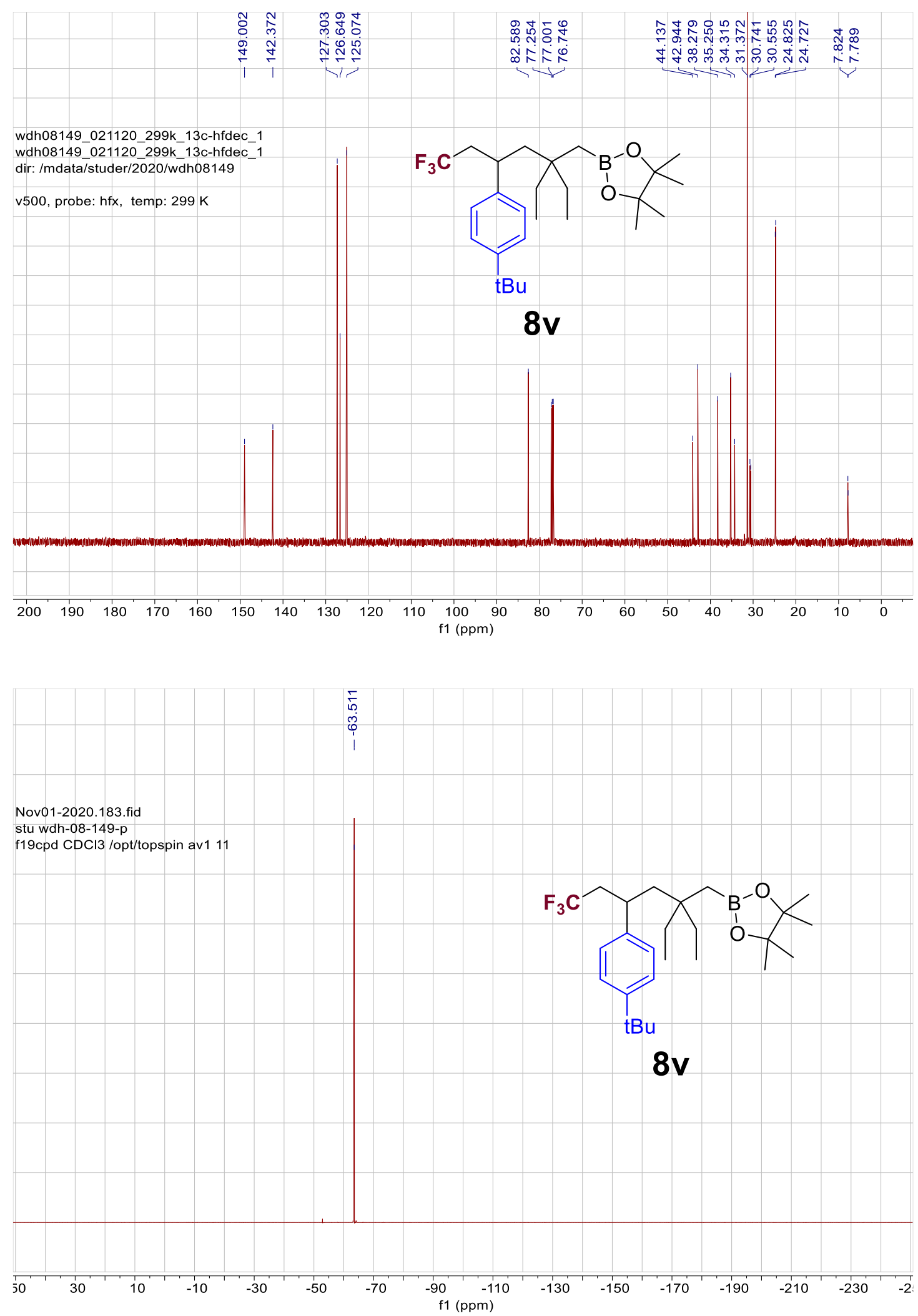

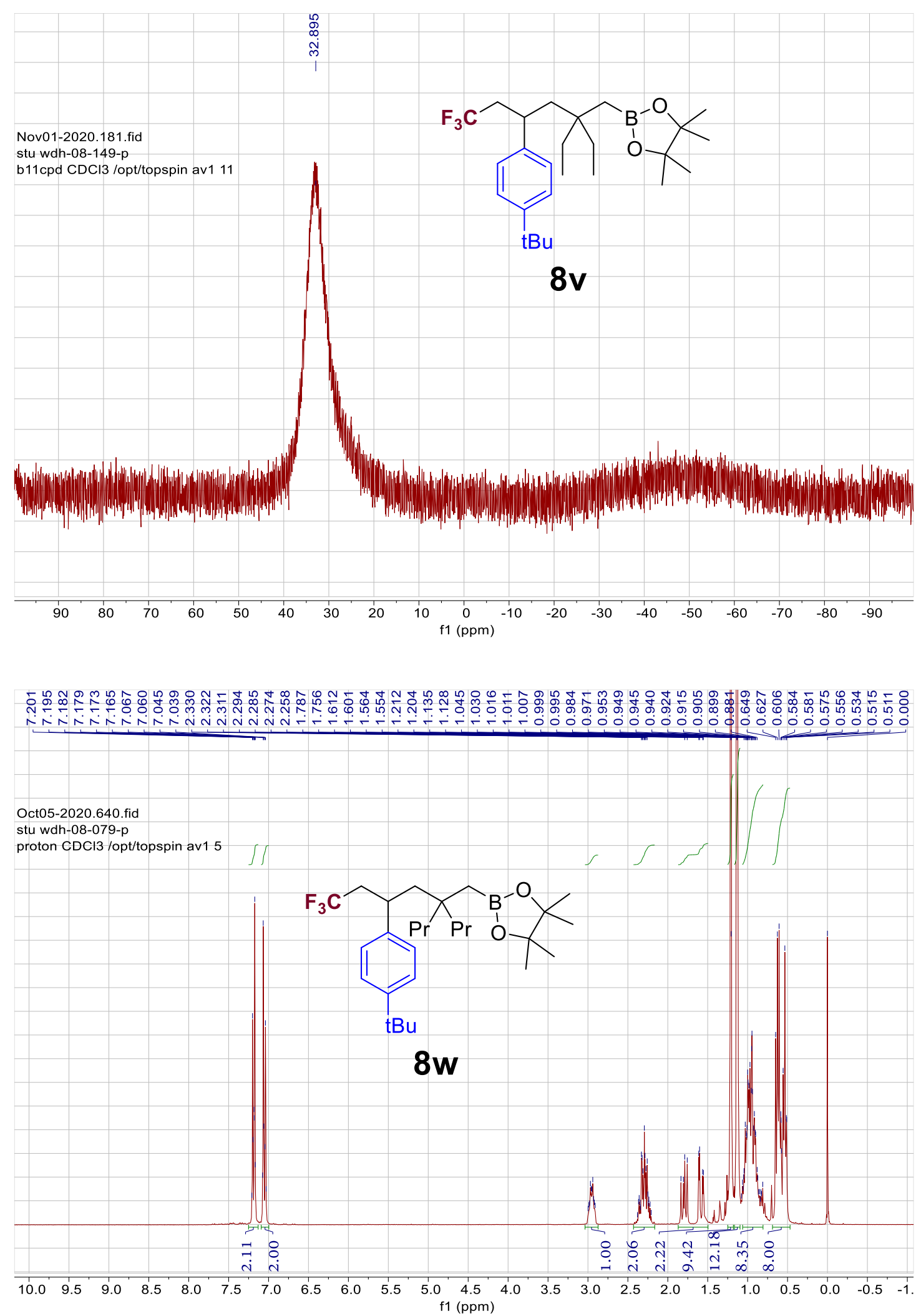

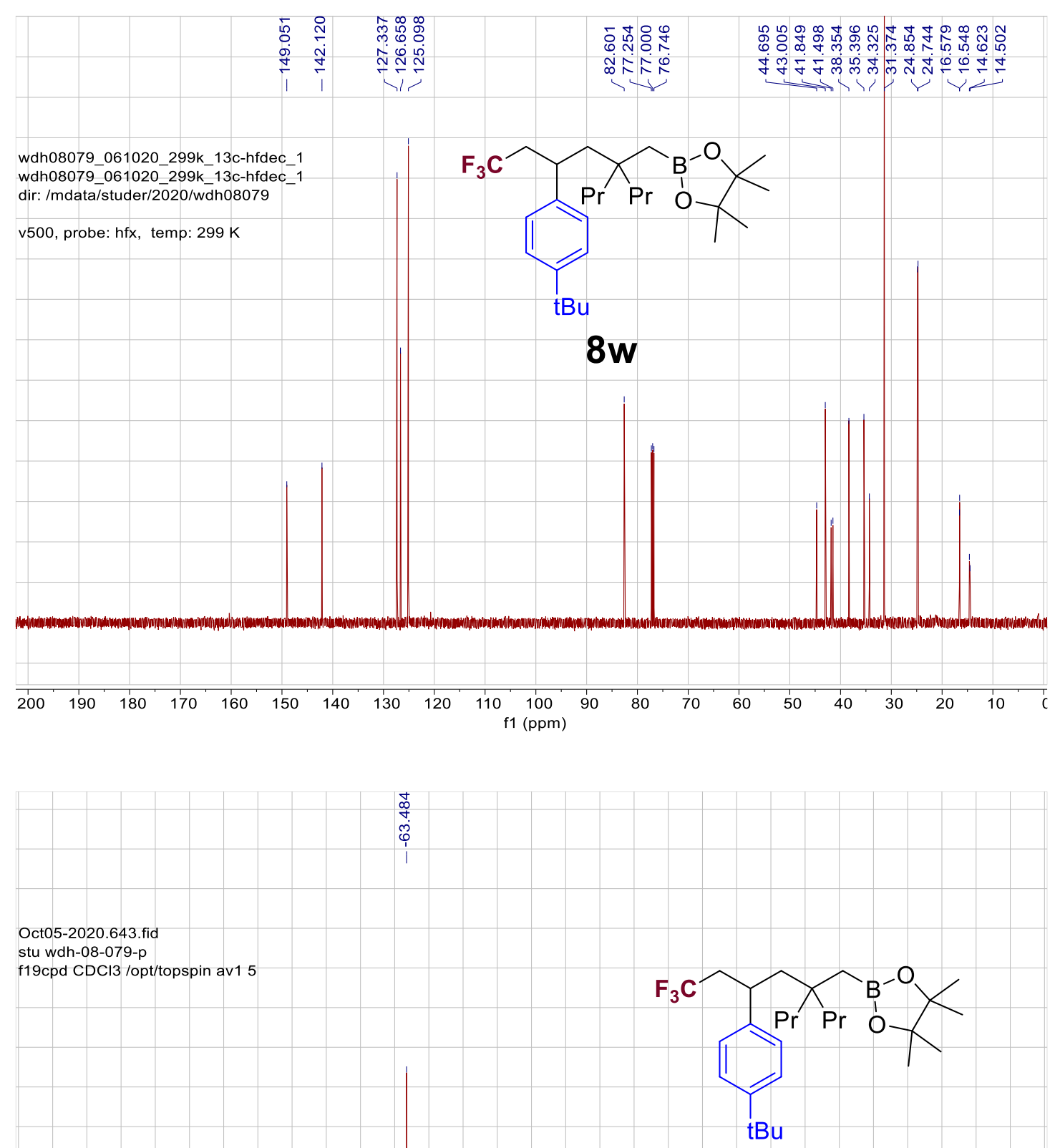

$8 w$ 

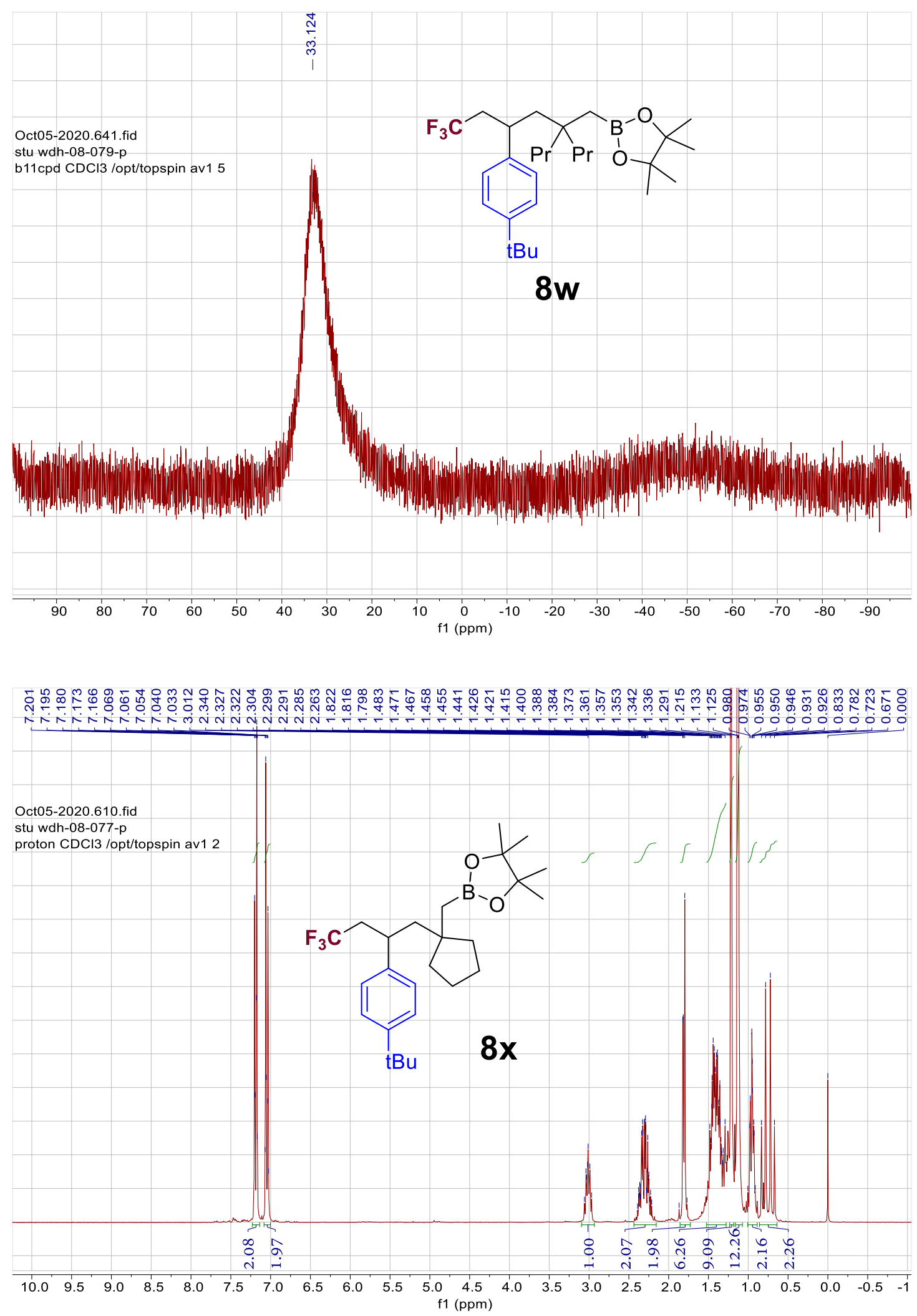

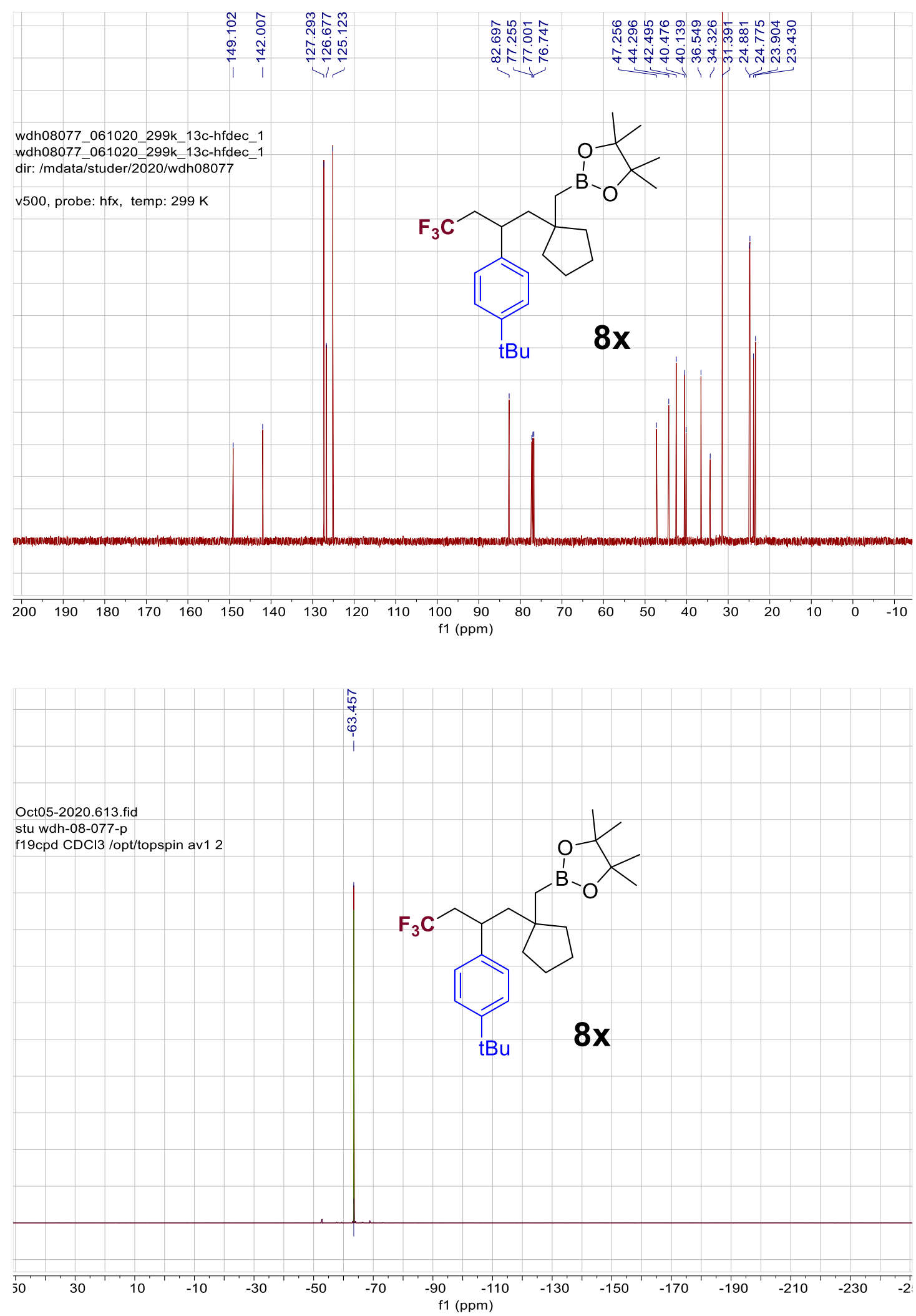

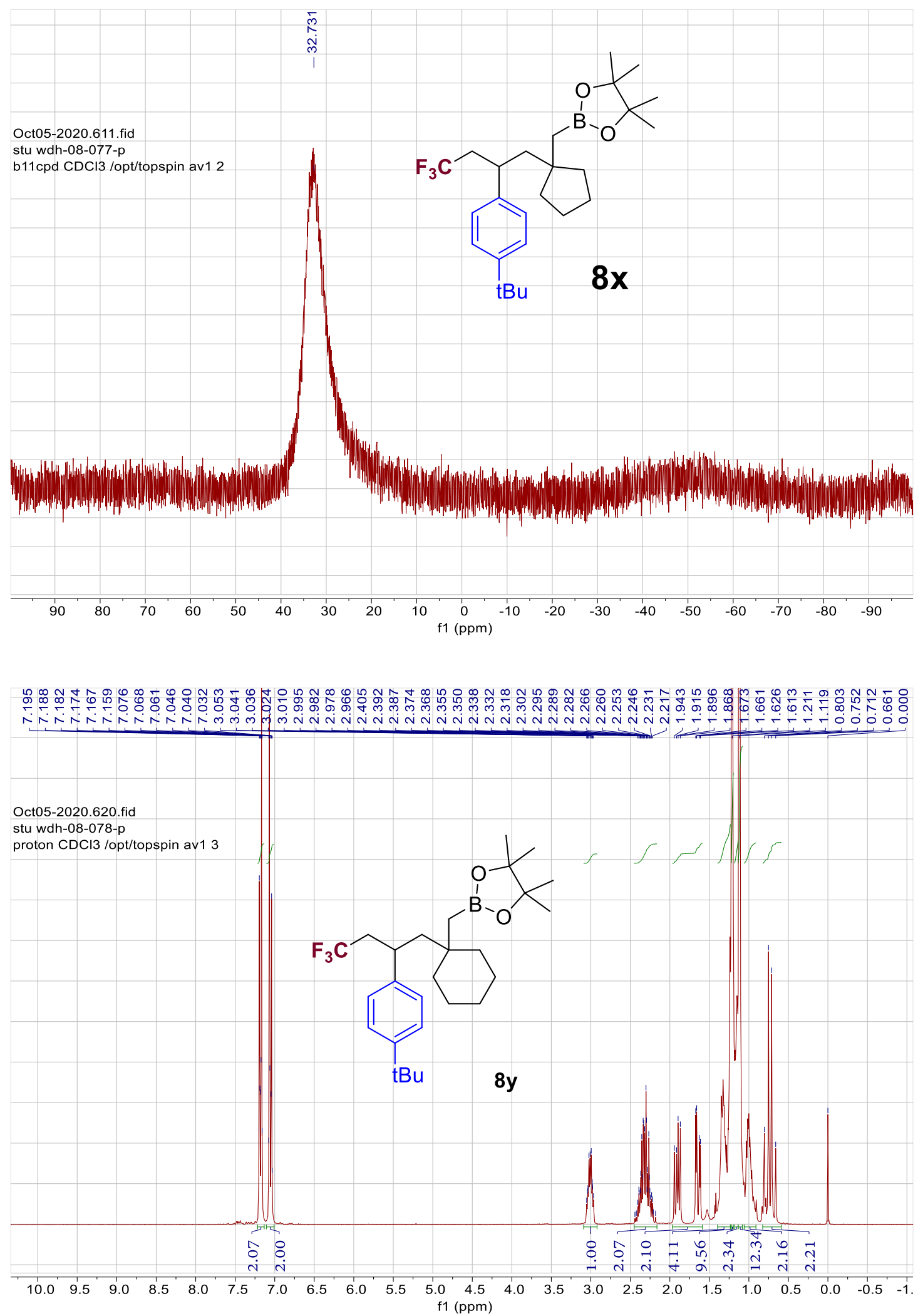

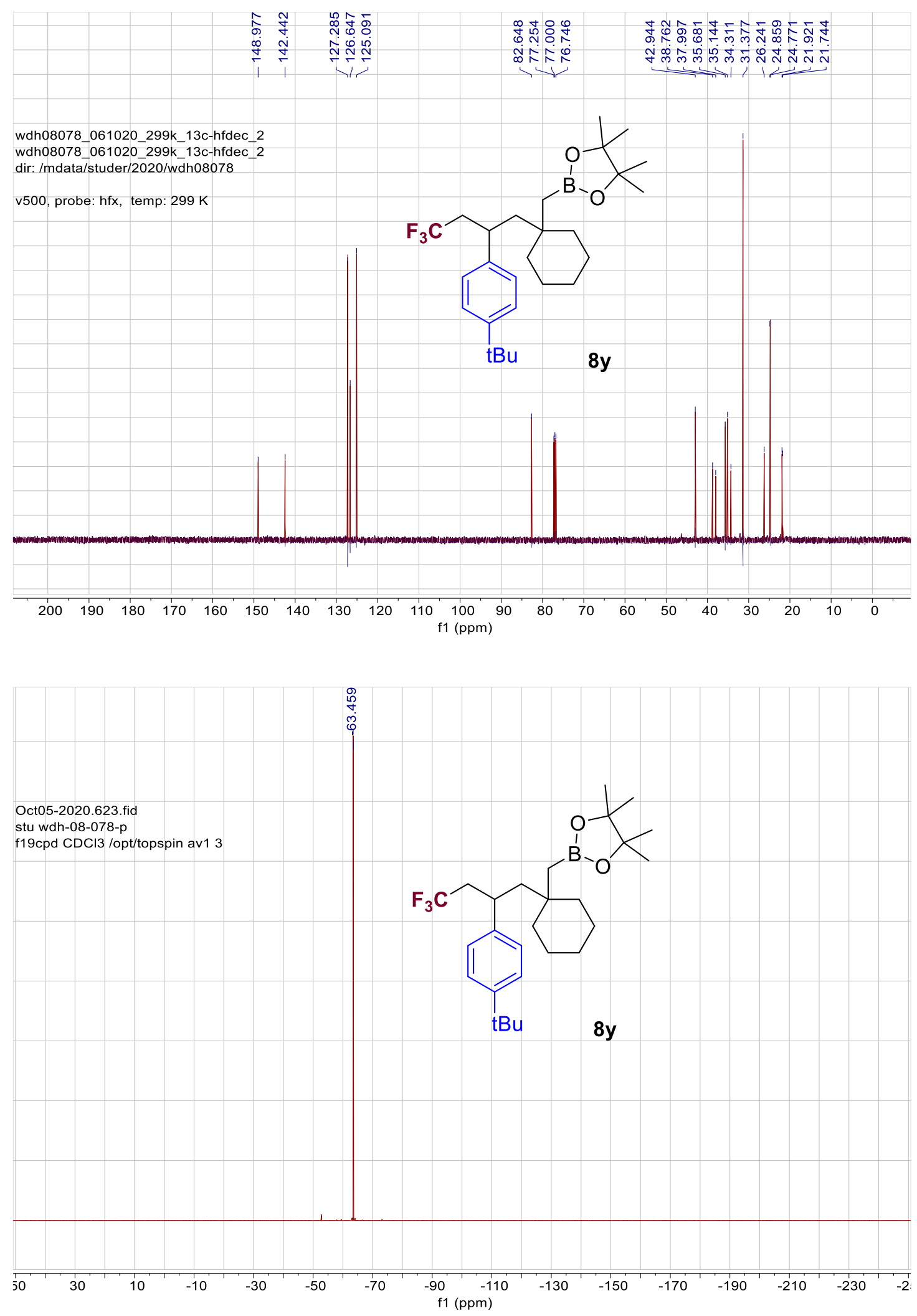

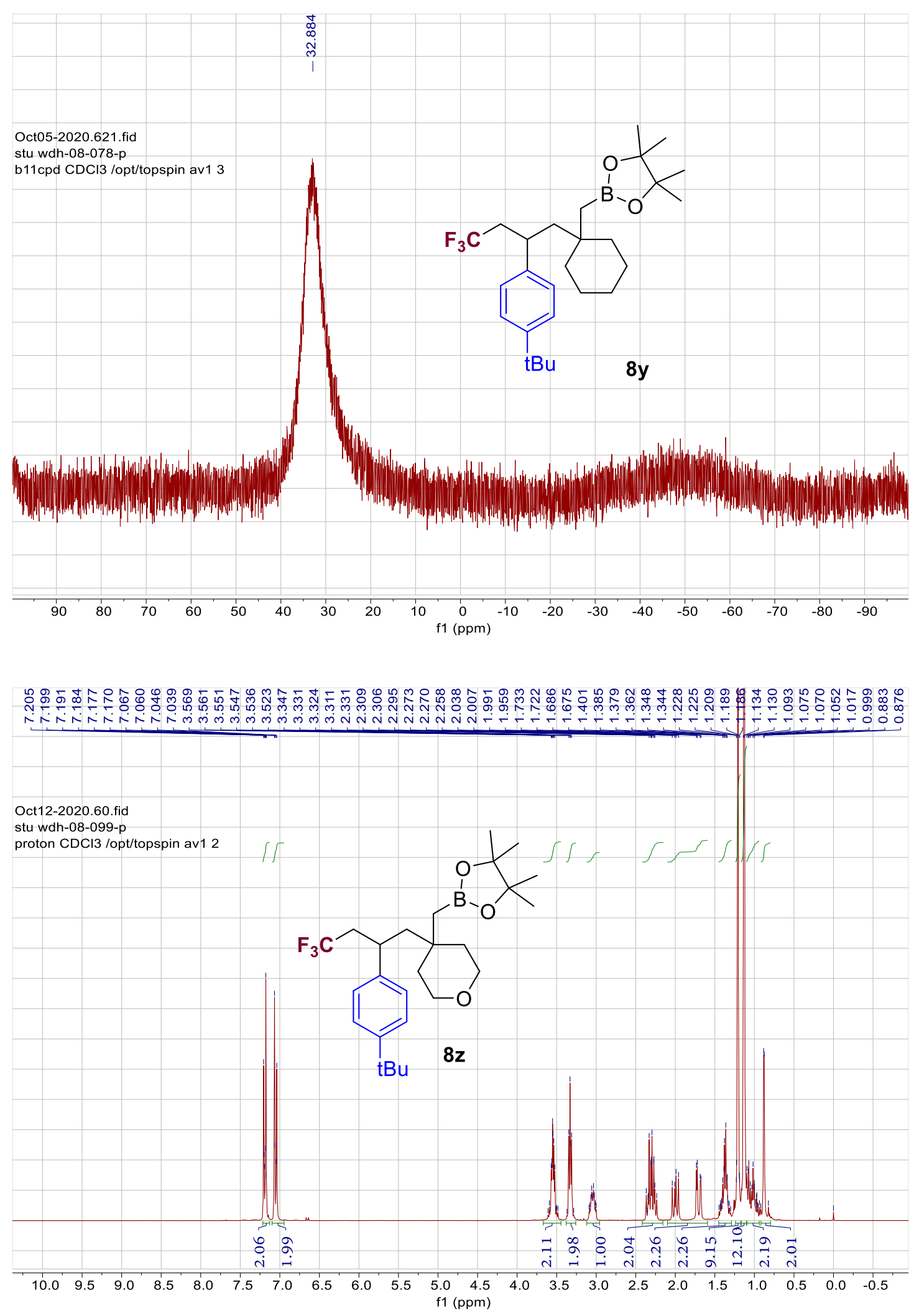

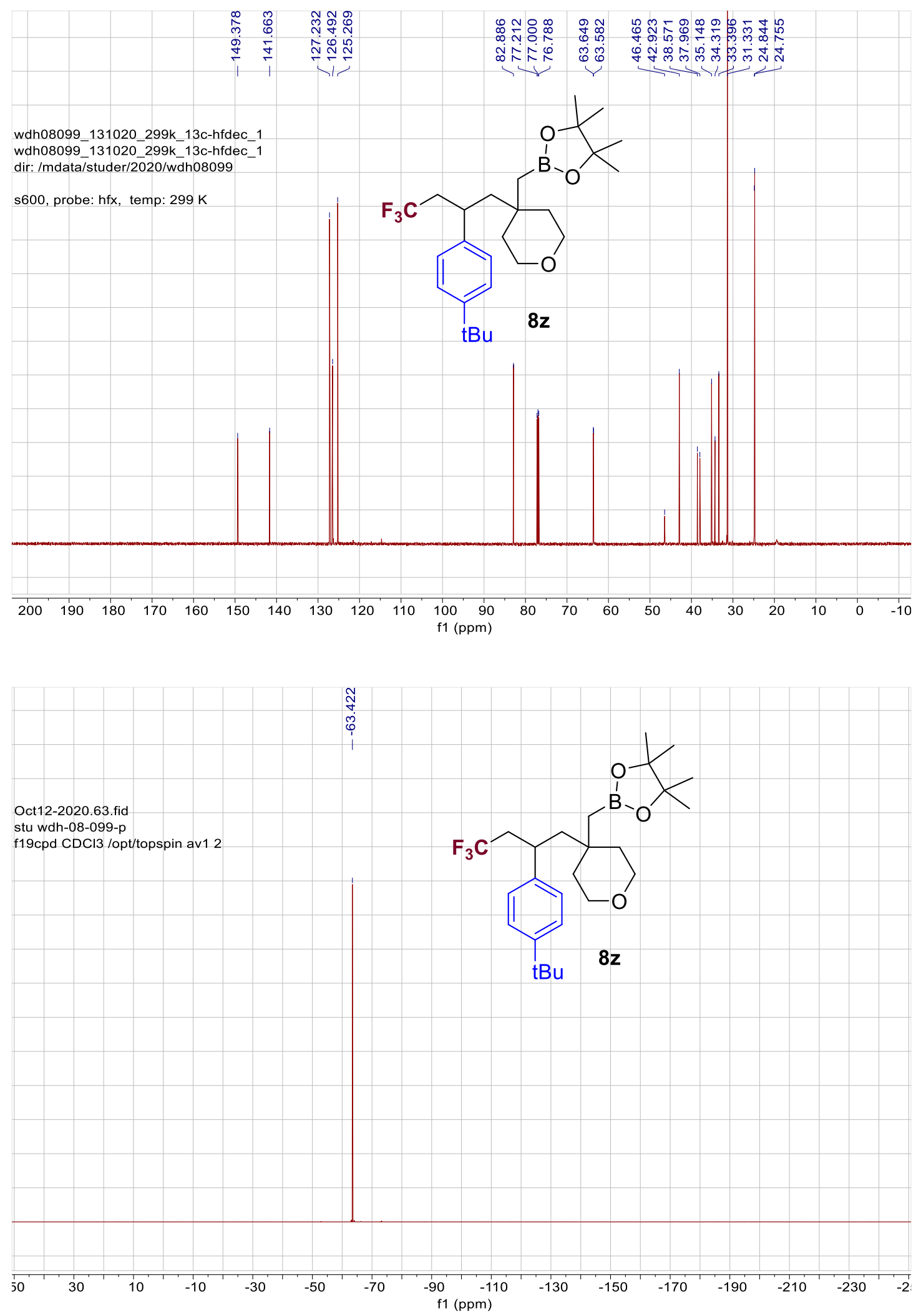


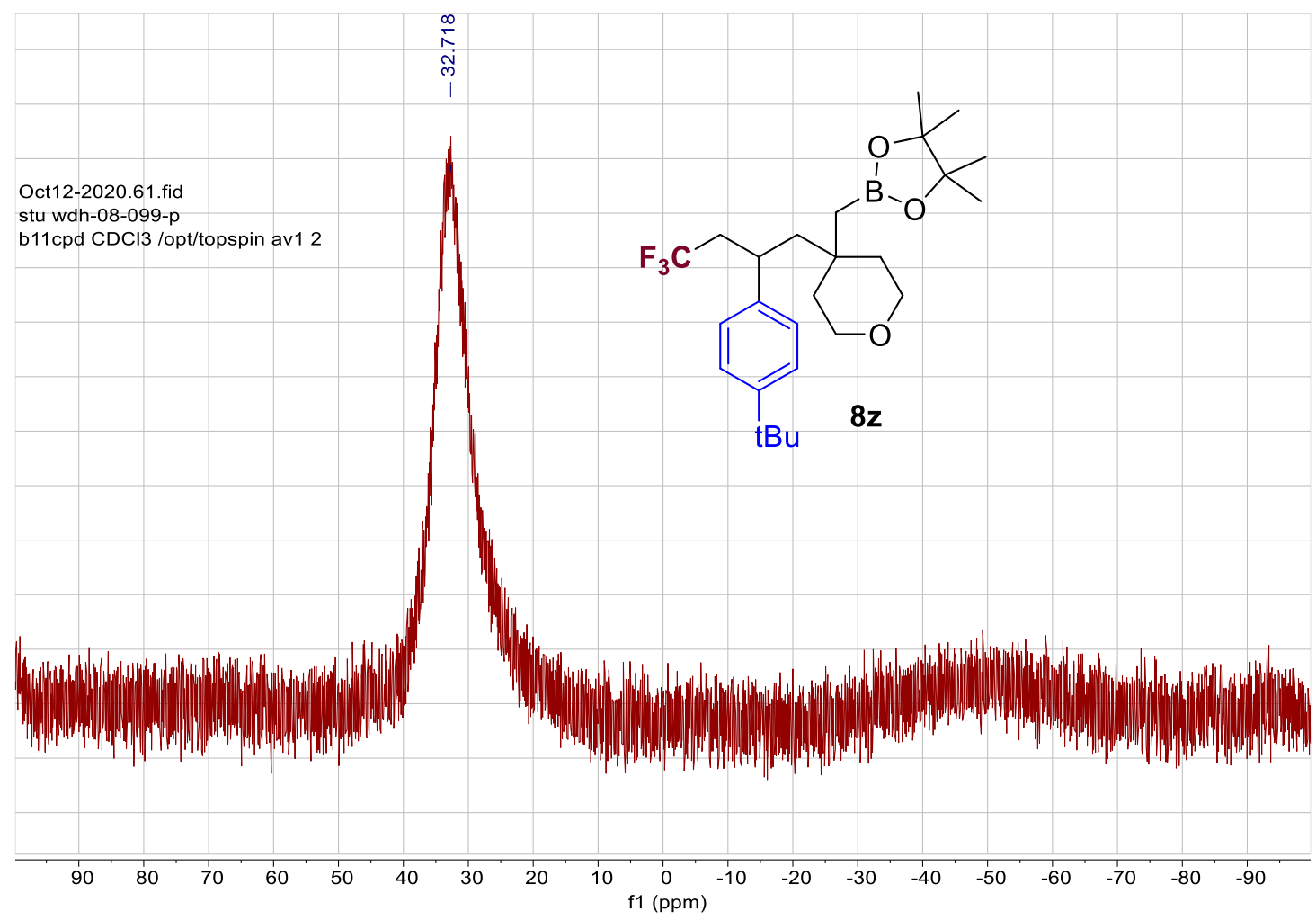

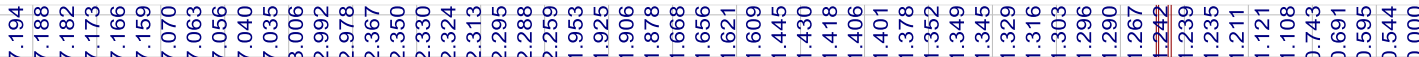

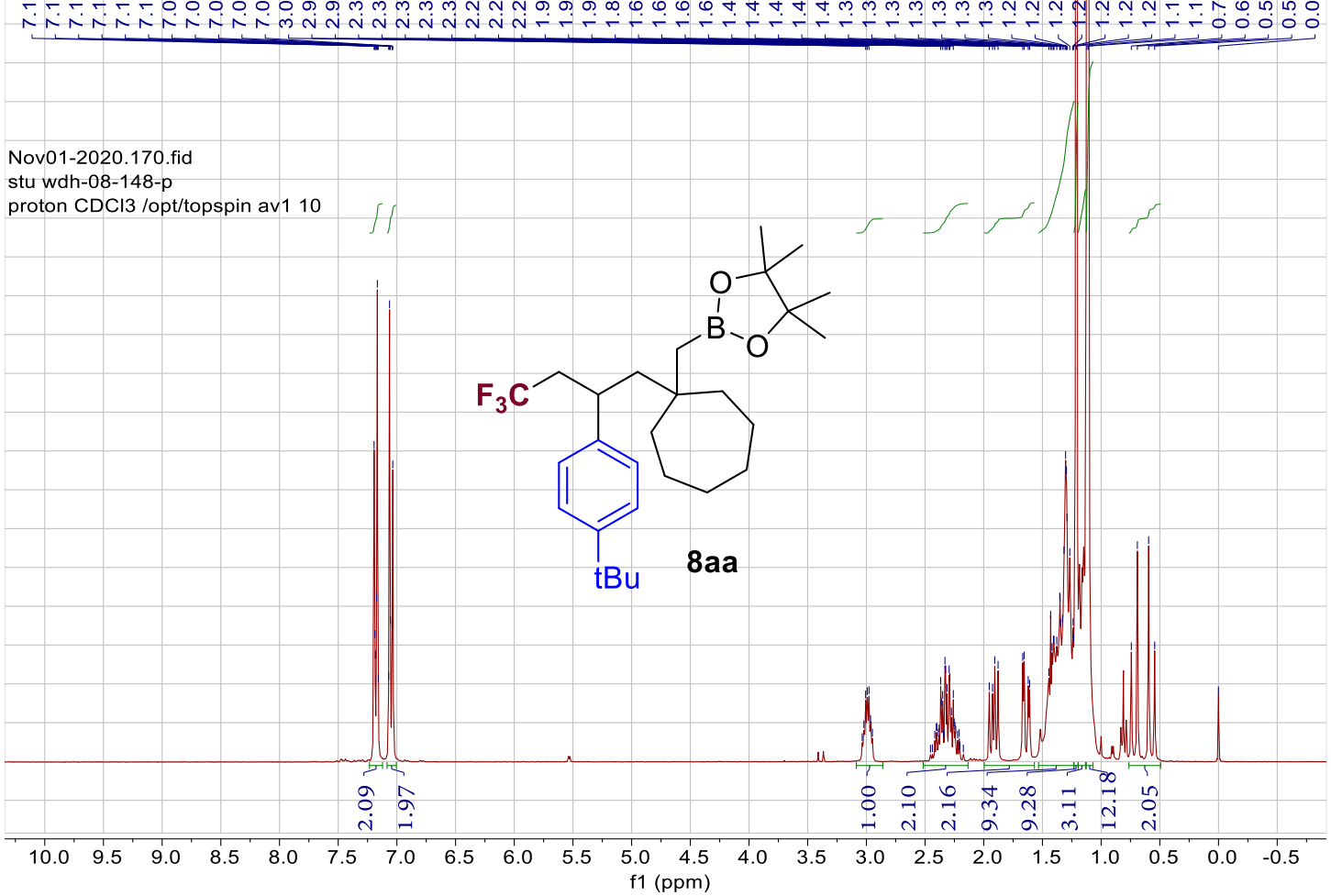



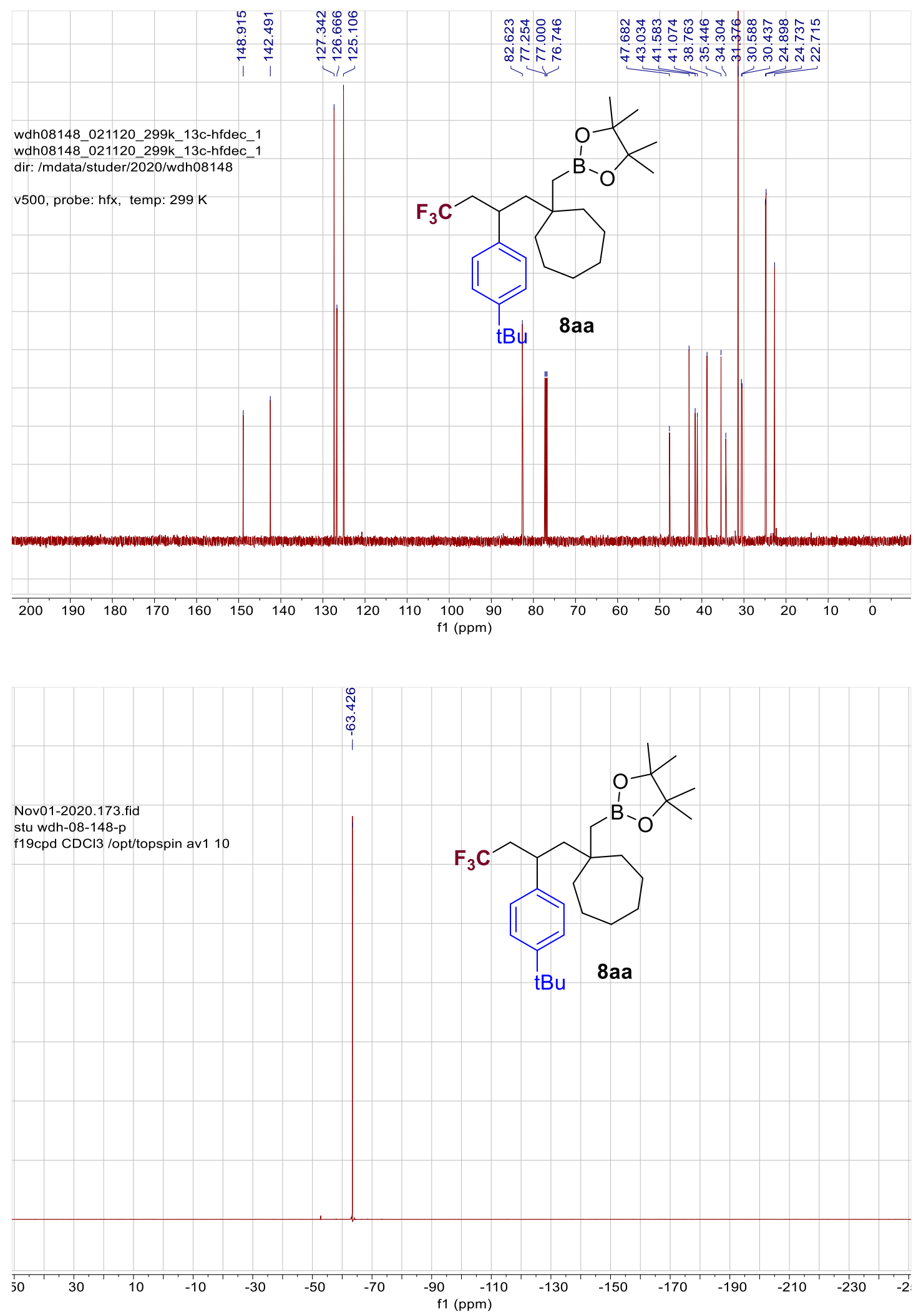

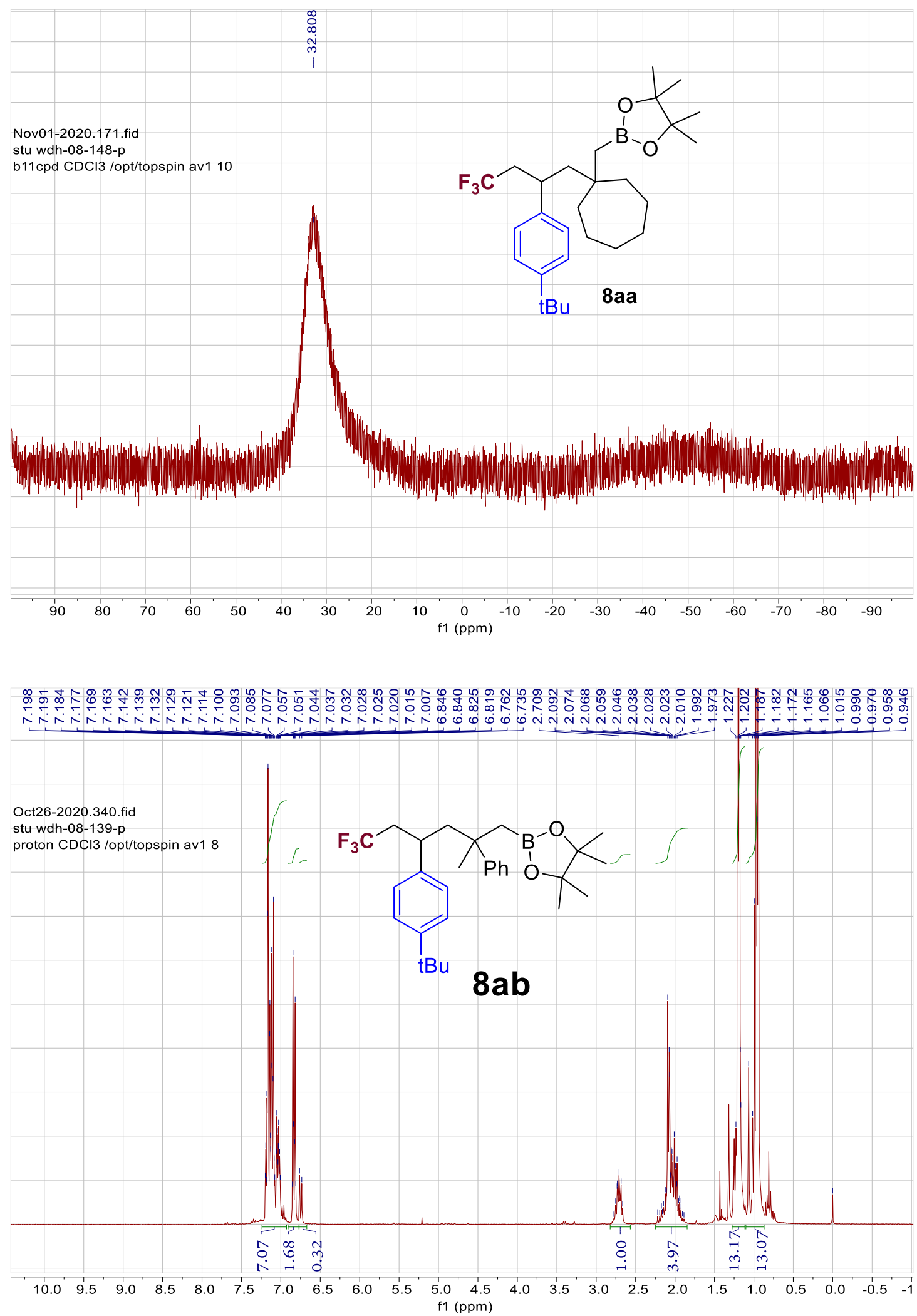

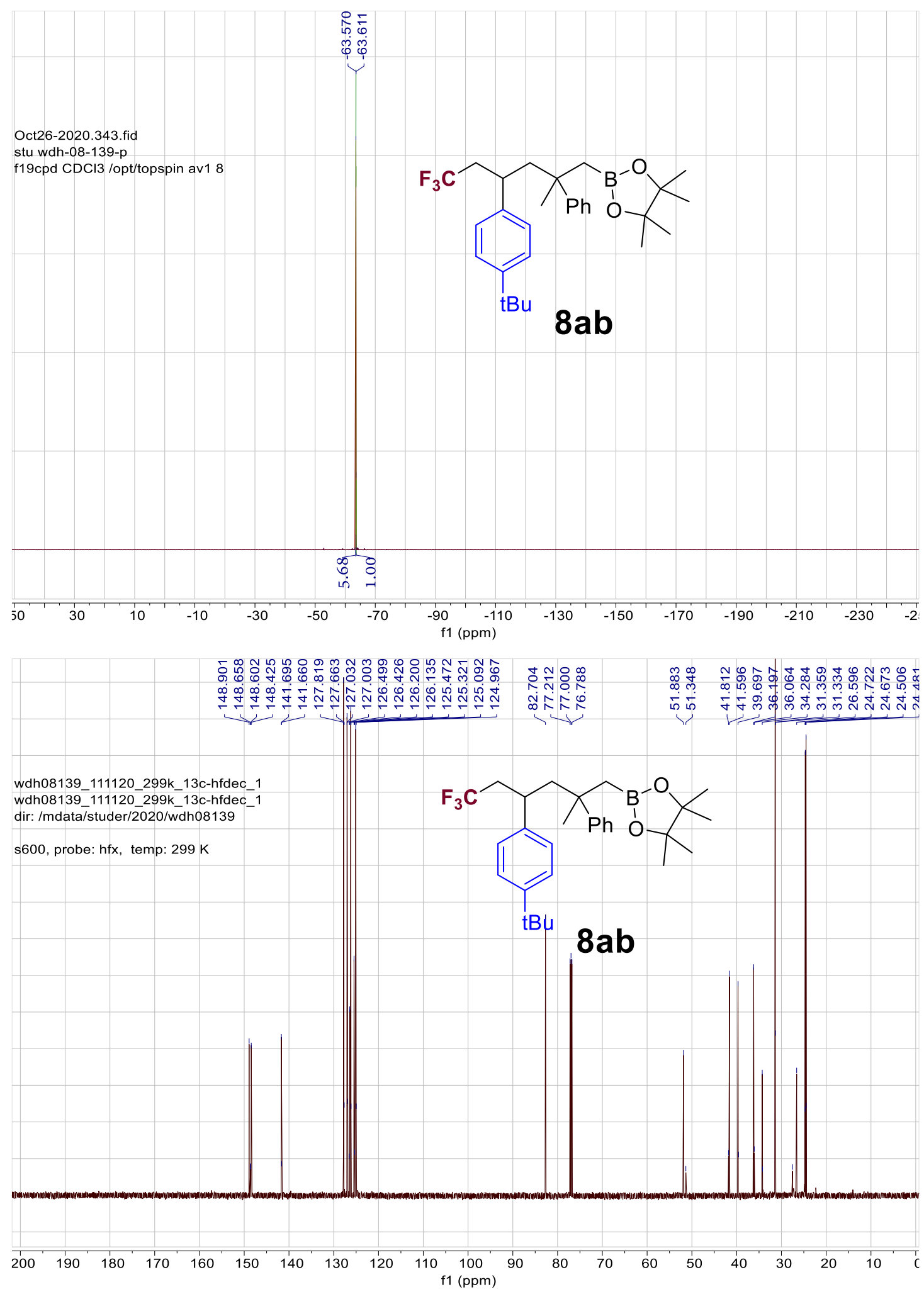

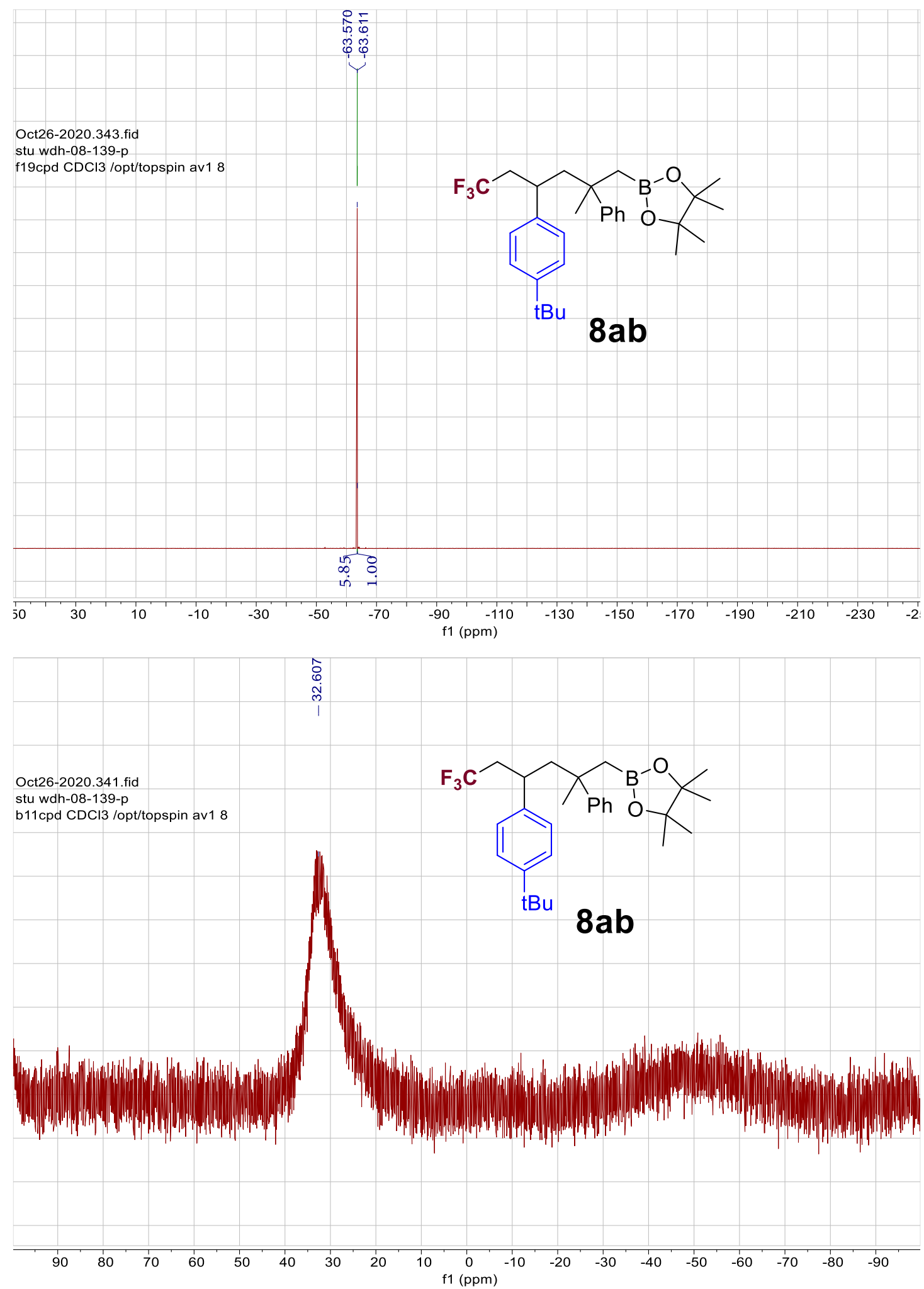

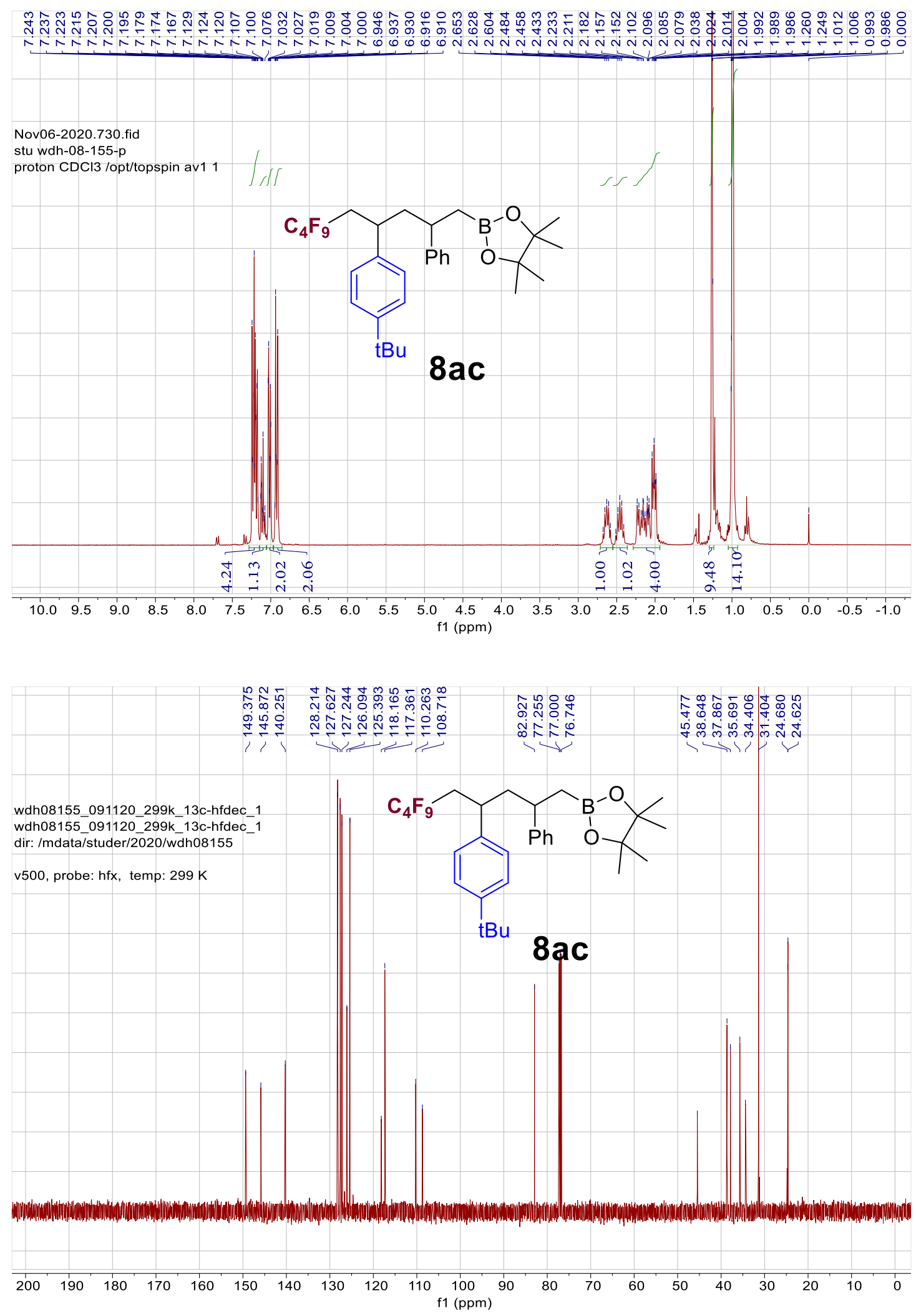

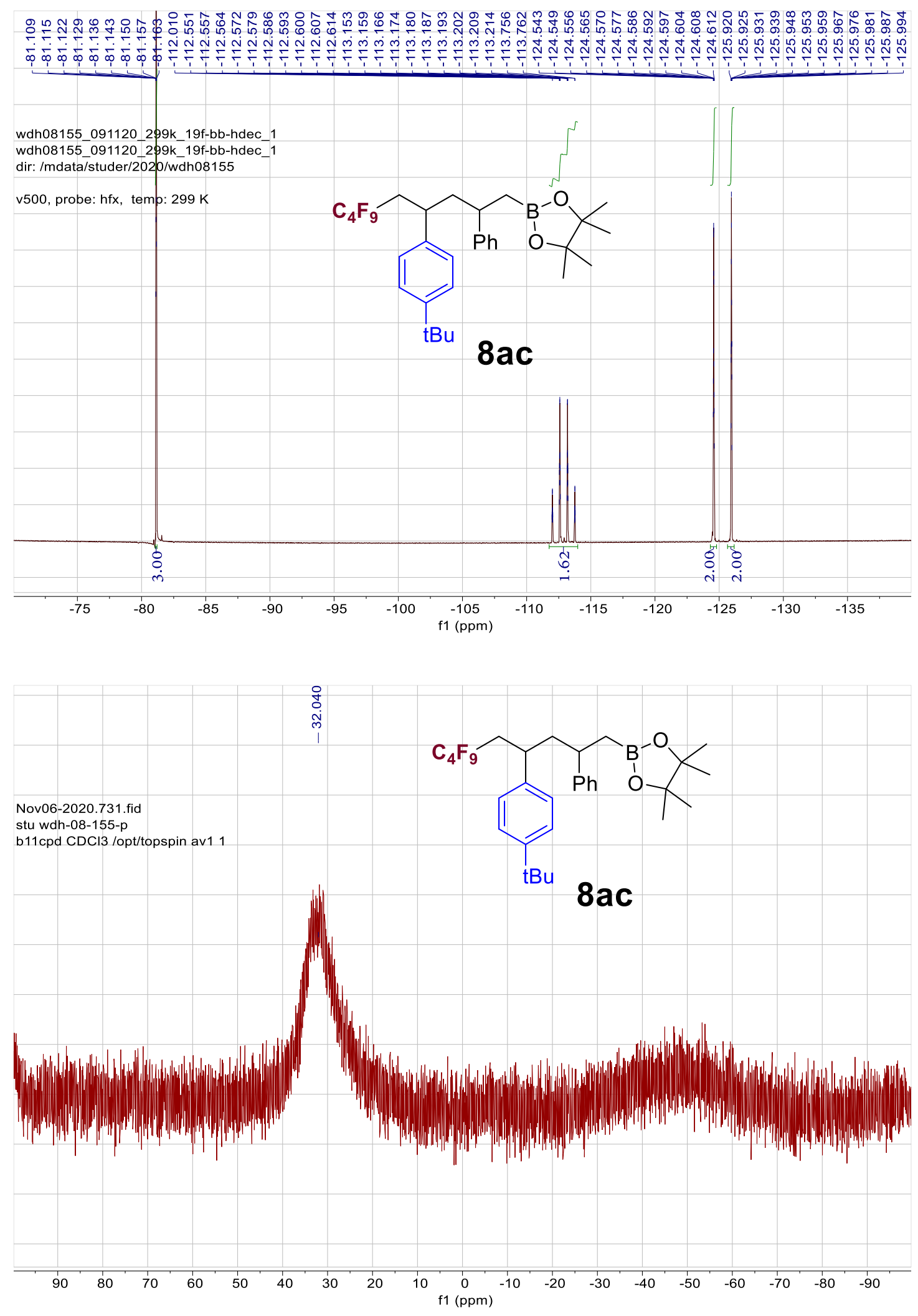

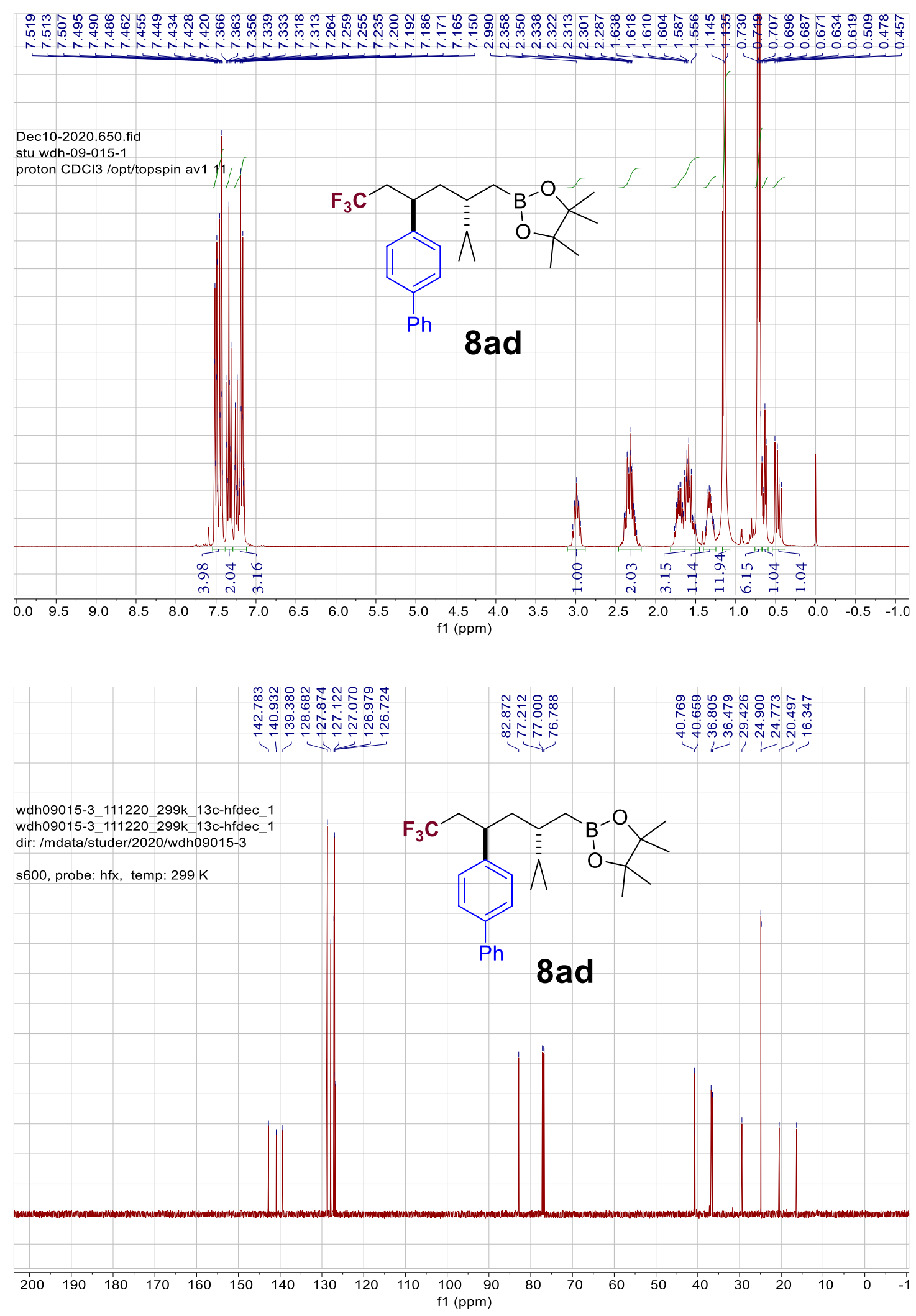

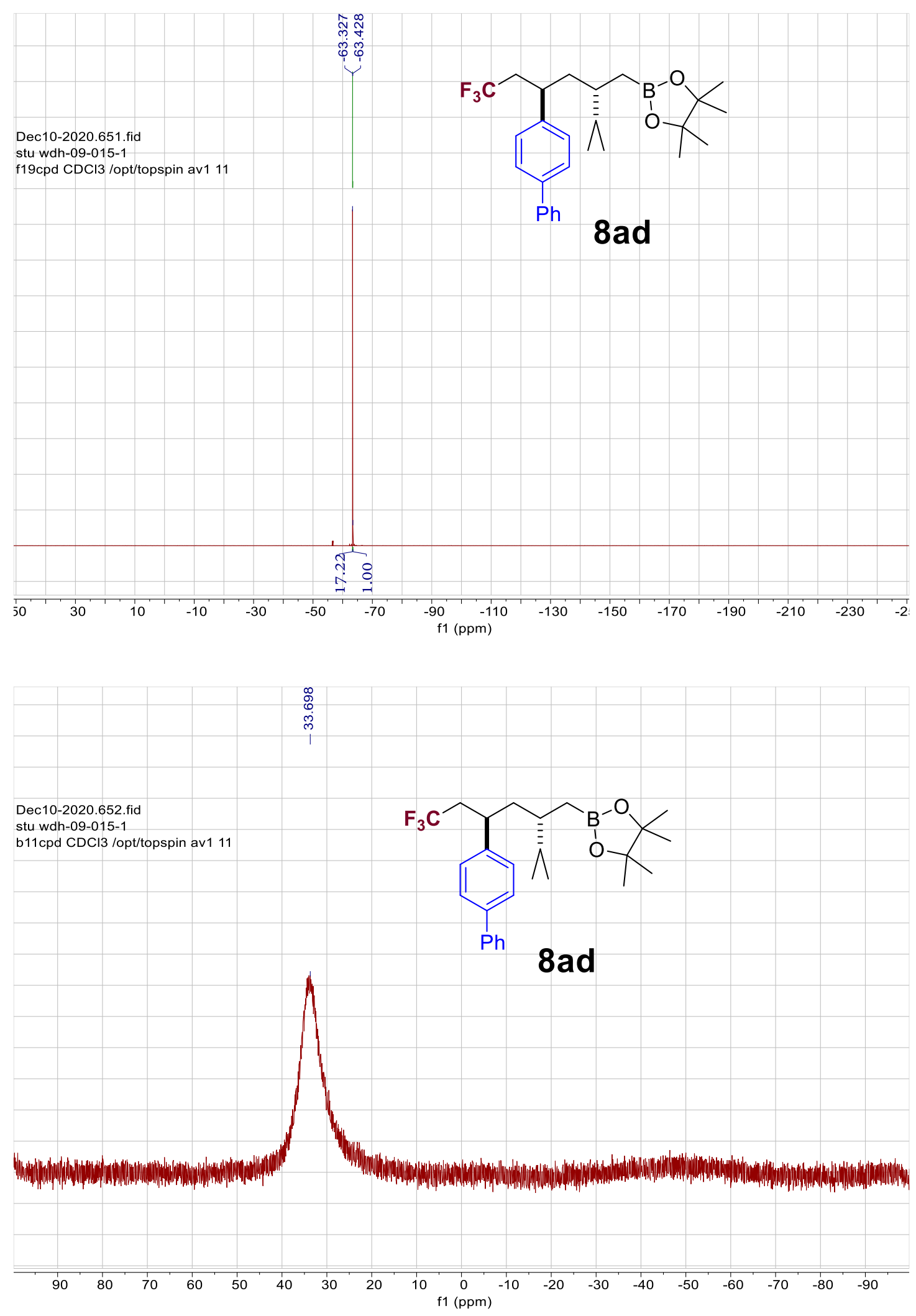

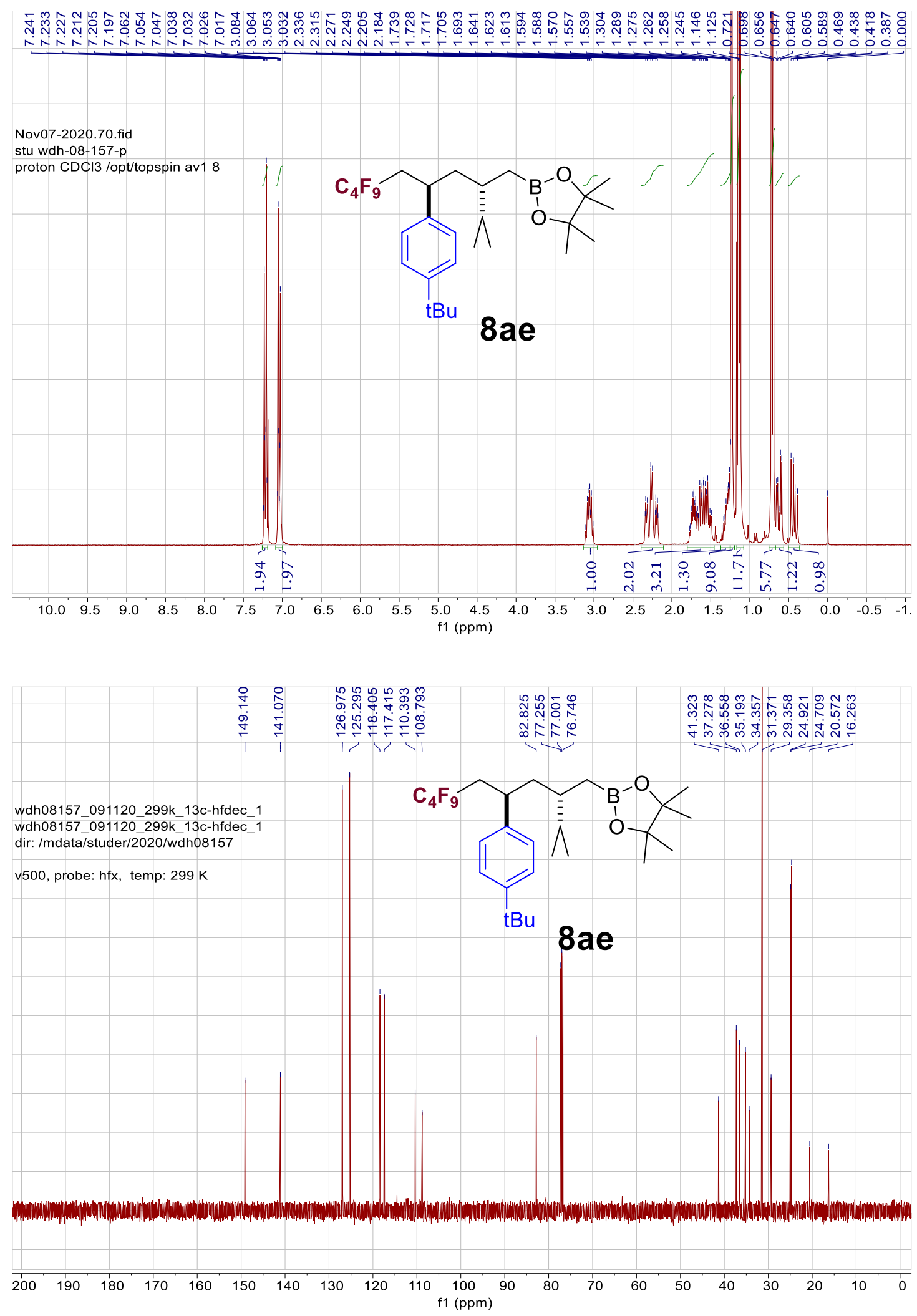


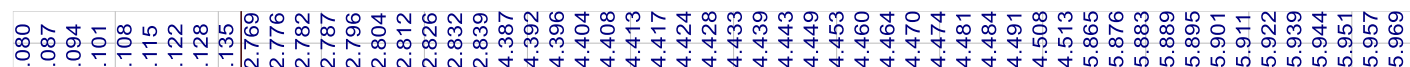

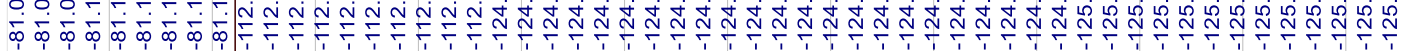
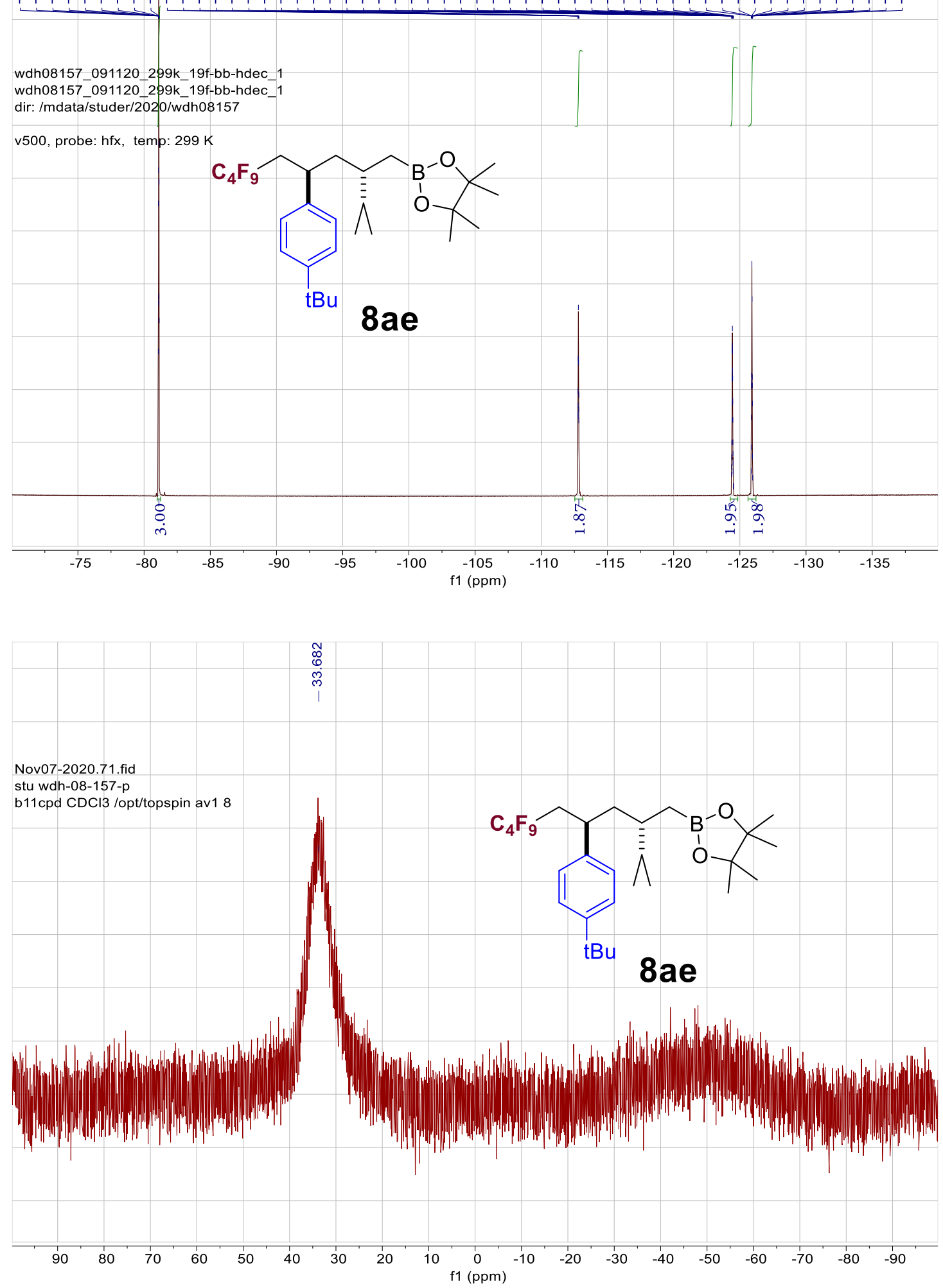

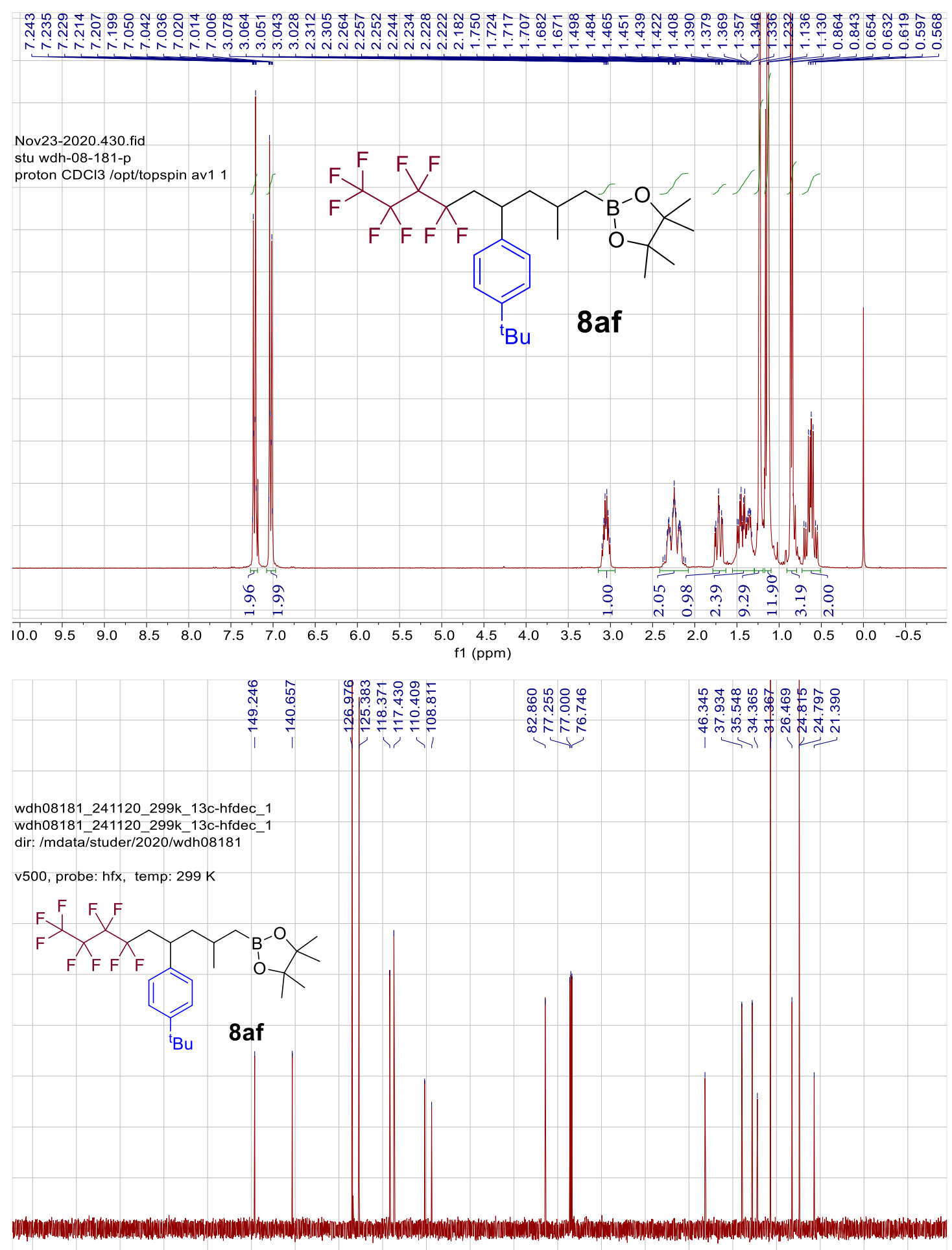

$\begin{array}{llllllllllllllllllll}1 & 190 & 180 & 170 & 160 & 150 & 140 & 130 & 120 & 110 & \begin{array}{c}100 \\ \mathrm{f} 1(\mathrm{ppm})\end{array} & 90 & 70 & 60 & 50 & 40 & 30 & 20 & 10 & 0\end{array}$ 

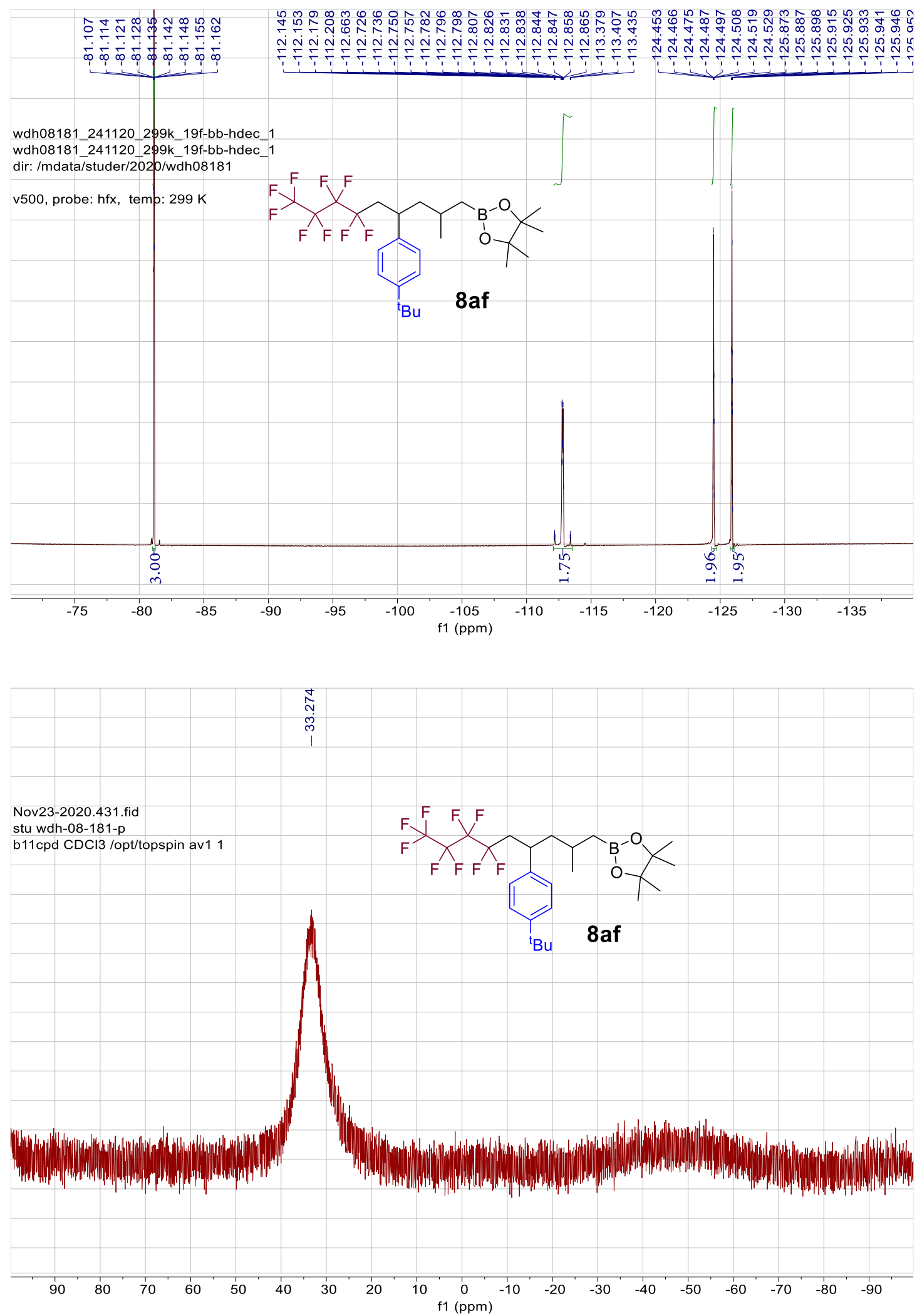

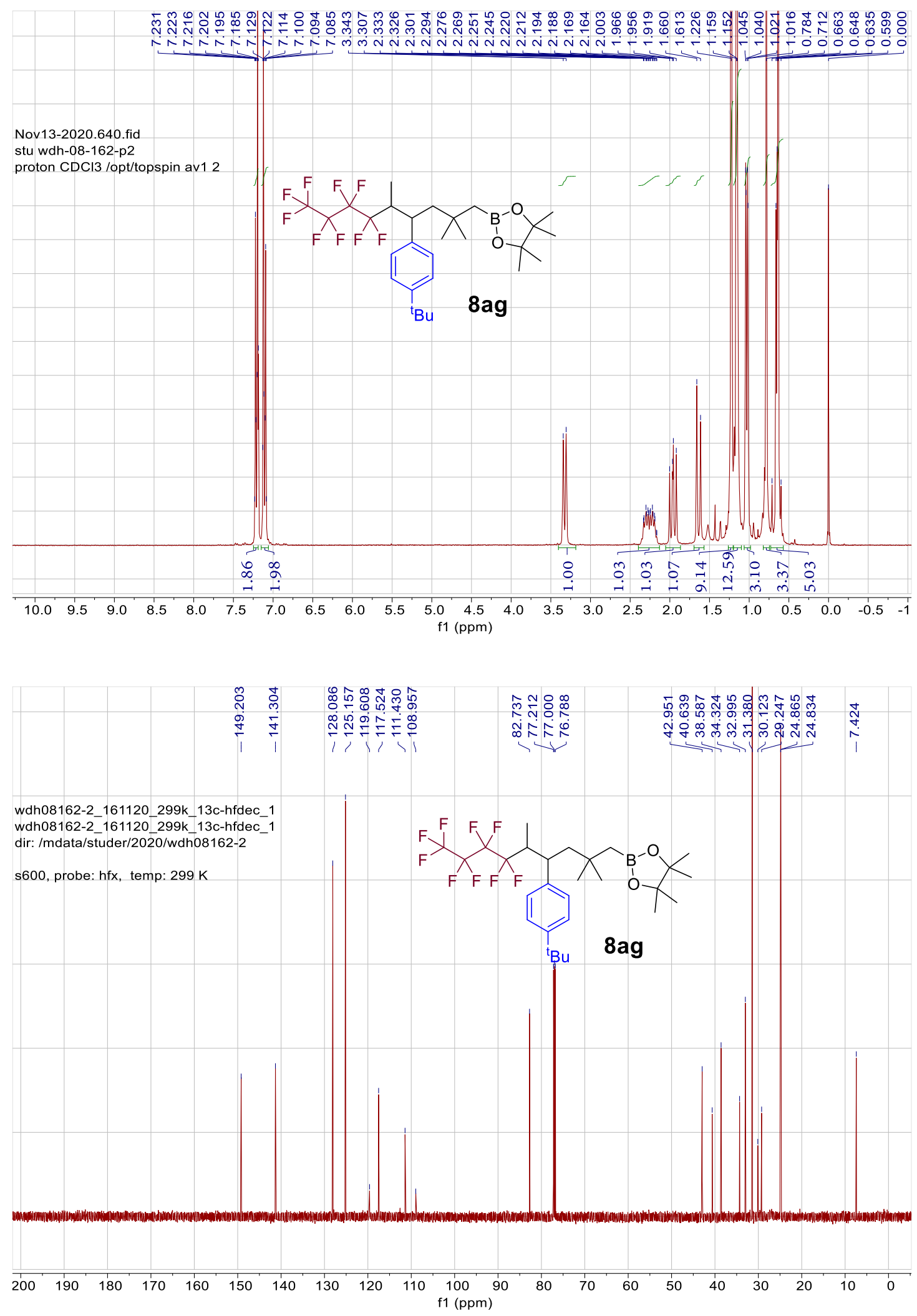
wdh08162-2 161120 299k 19f-bb-hdec

wdh08162-2 161120 299k 19f-bb-hdec

dir:/mdata/studer/2020/wdh08162-2

s600, probe: $\mathrm{hfx}$, temp: $299 \mathrm{~K}$
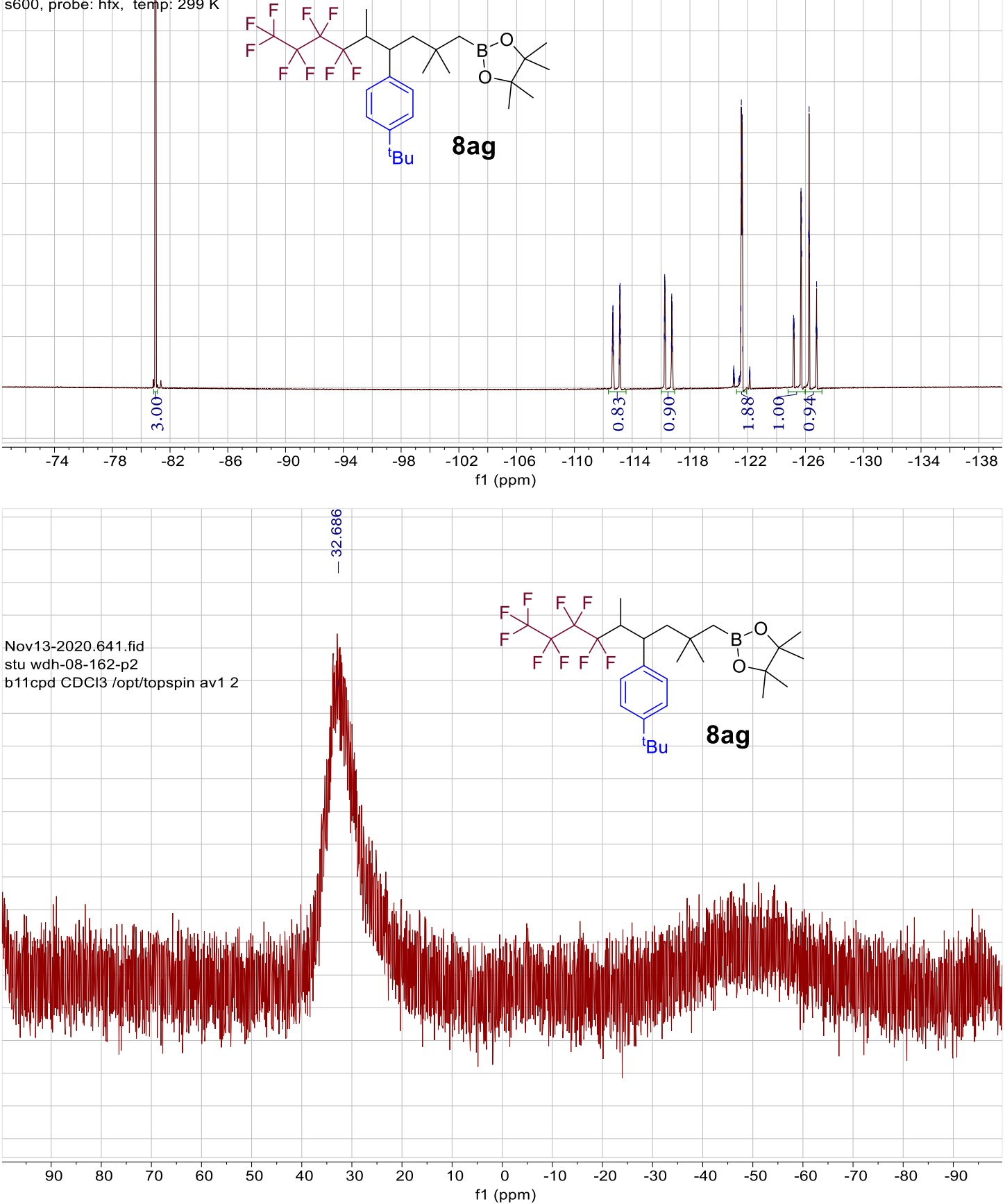

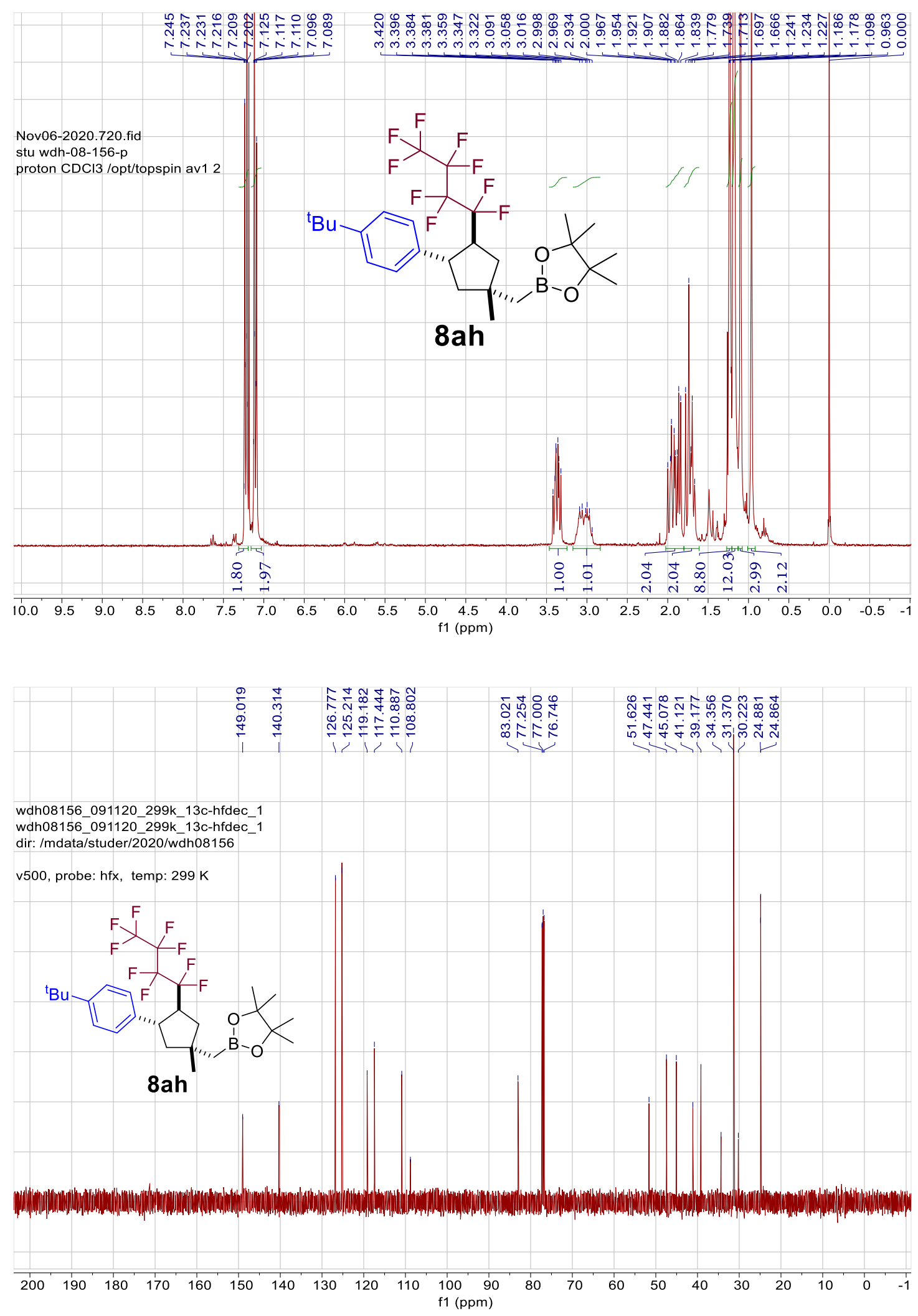

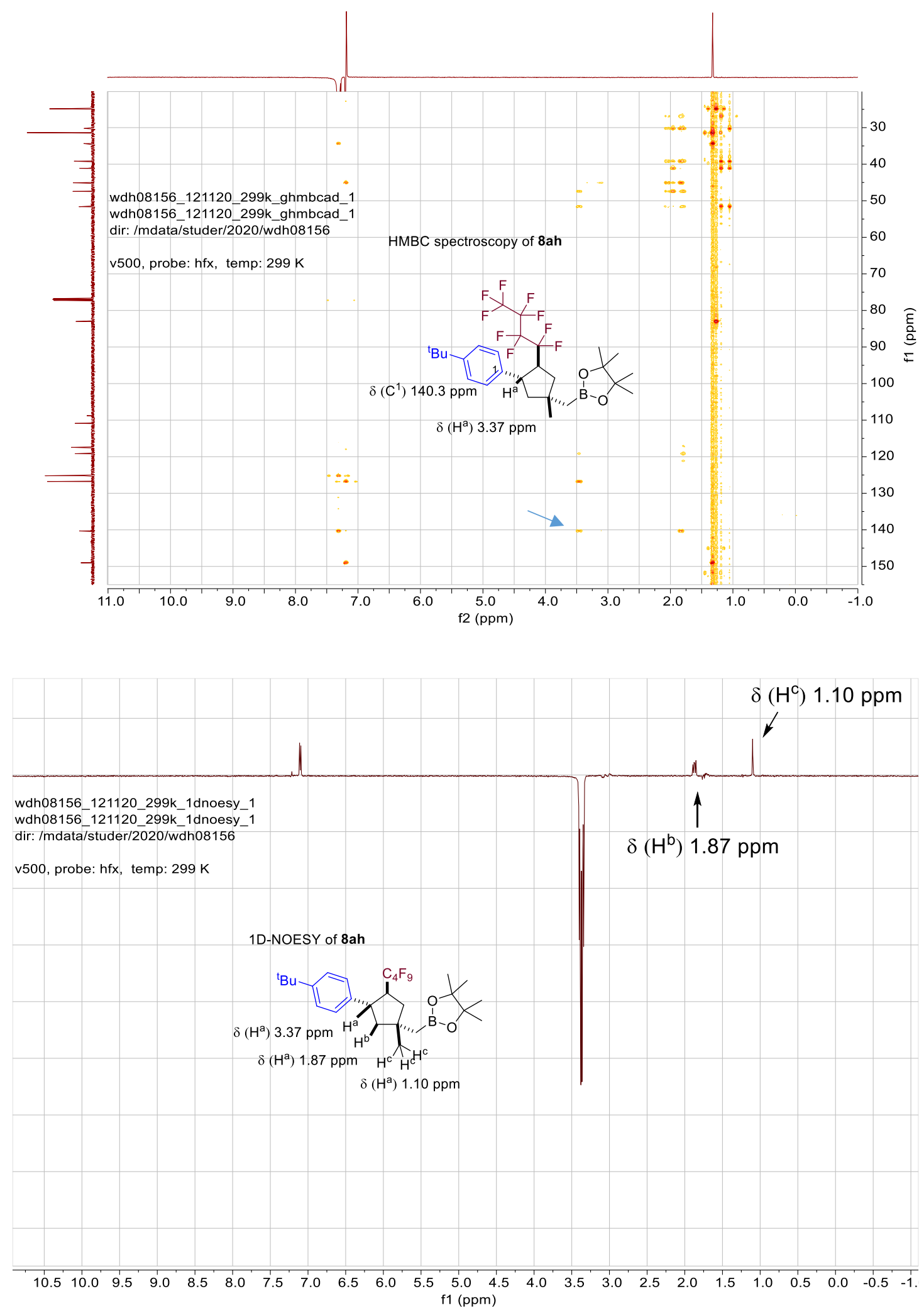


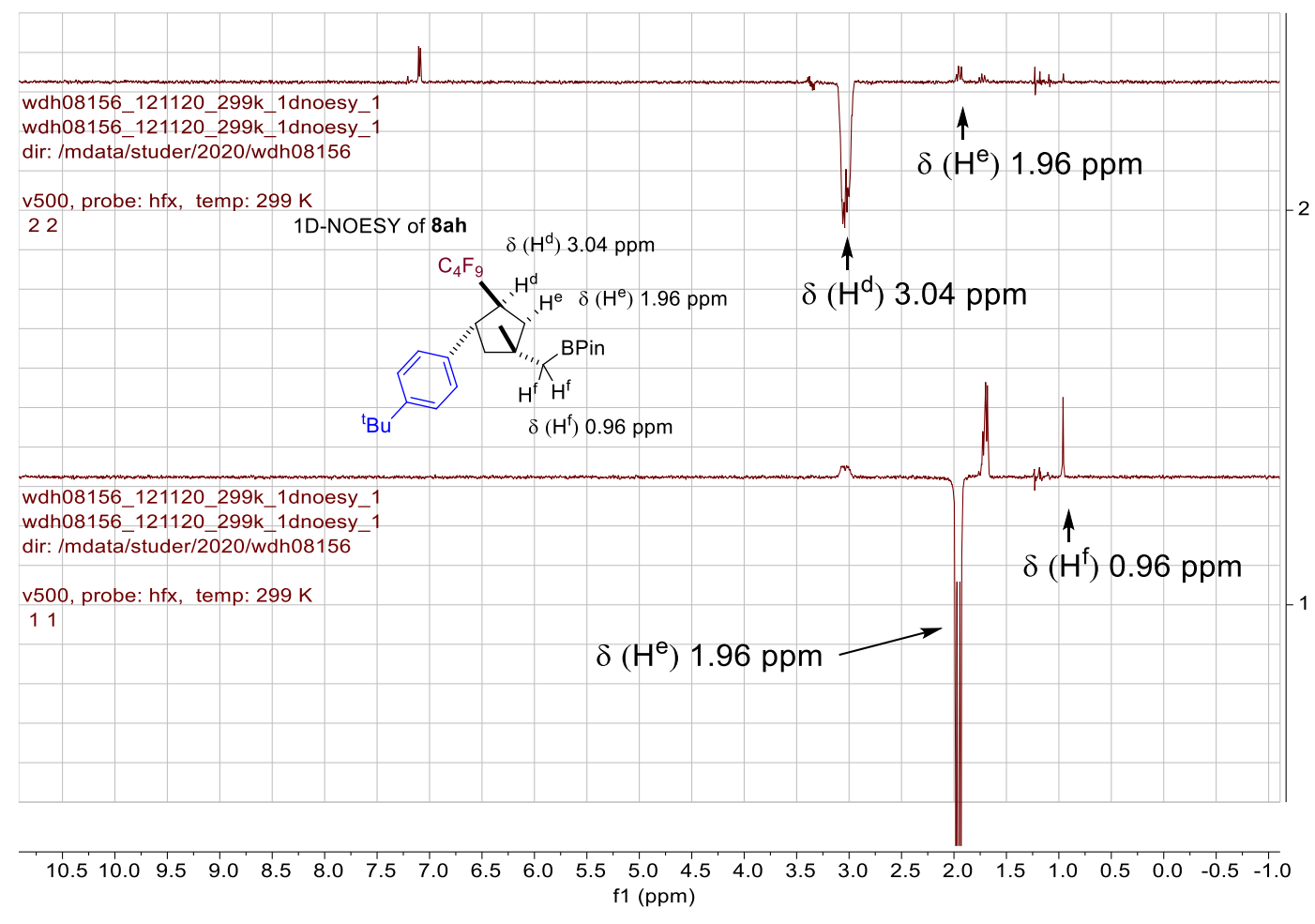

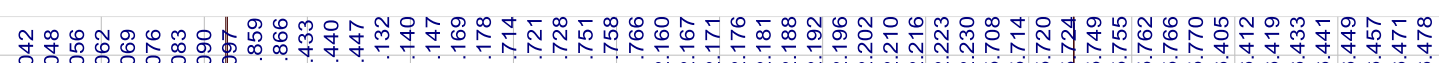

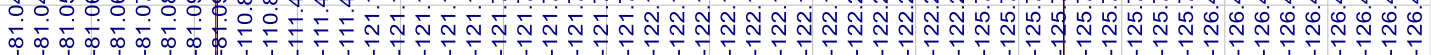

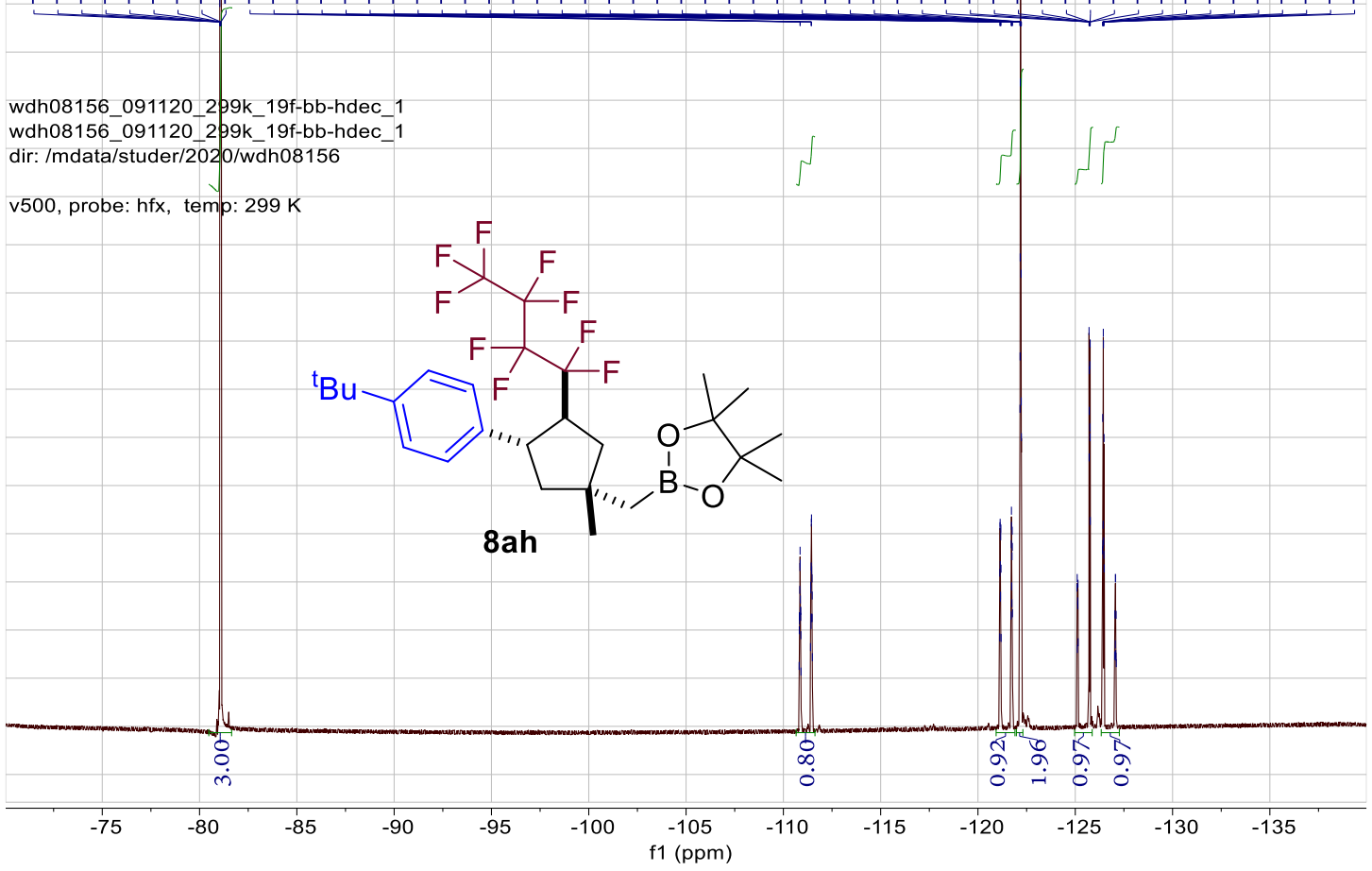



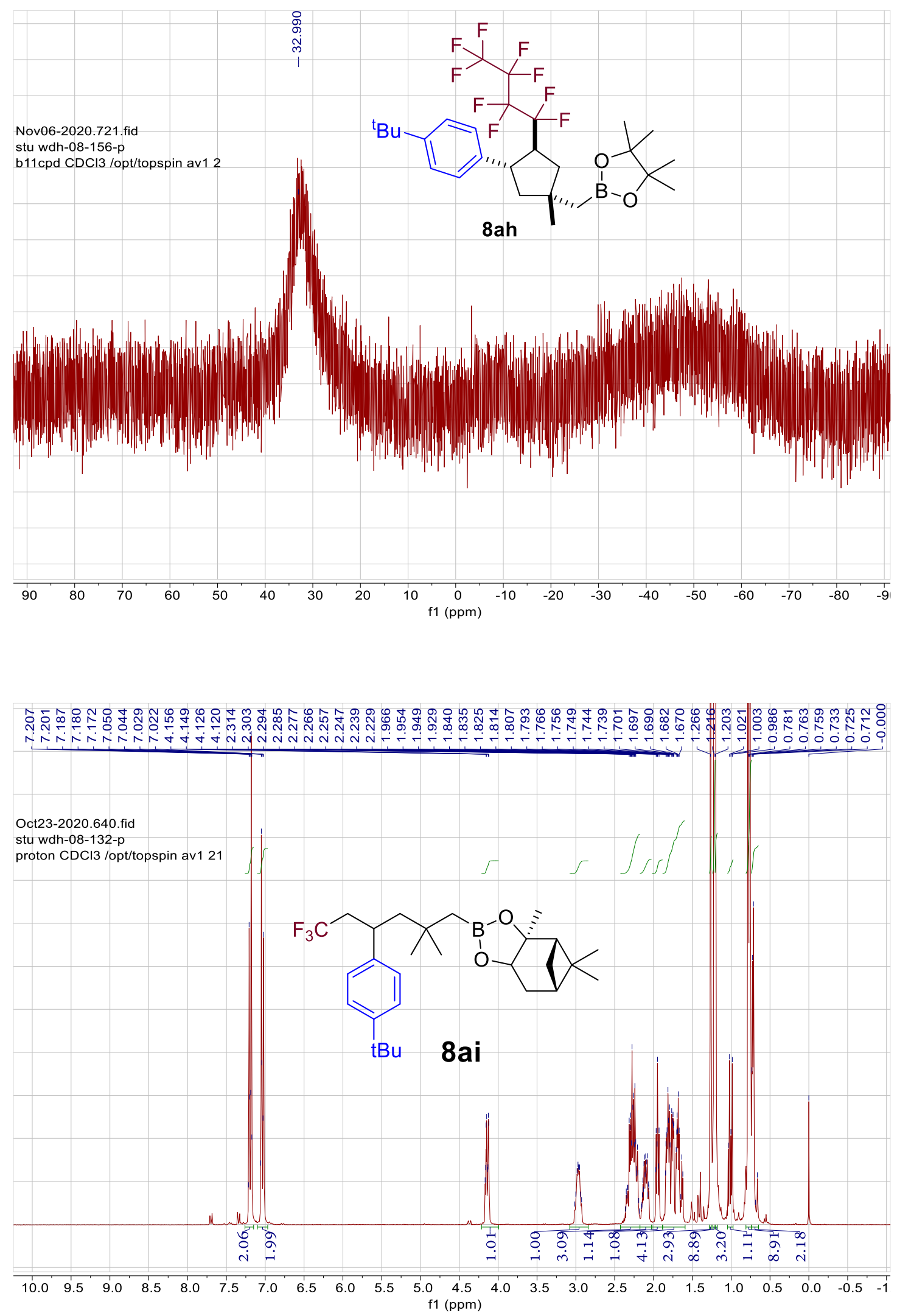

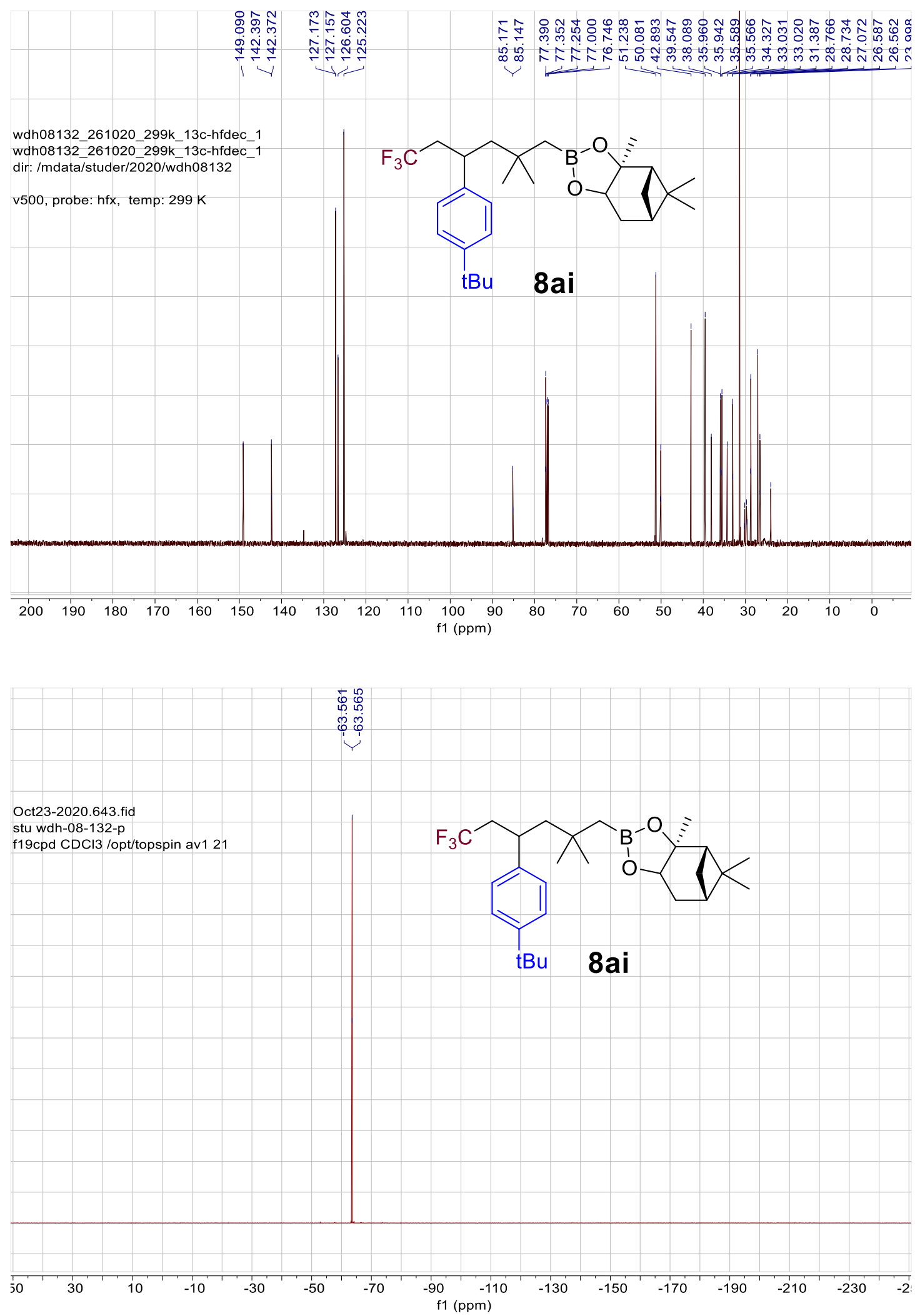

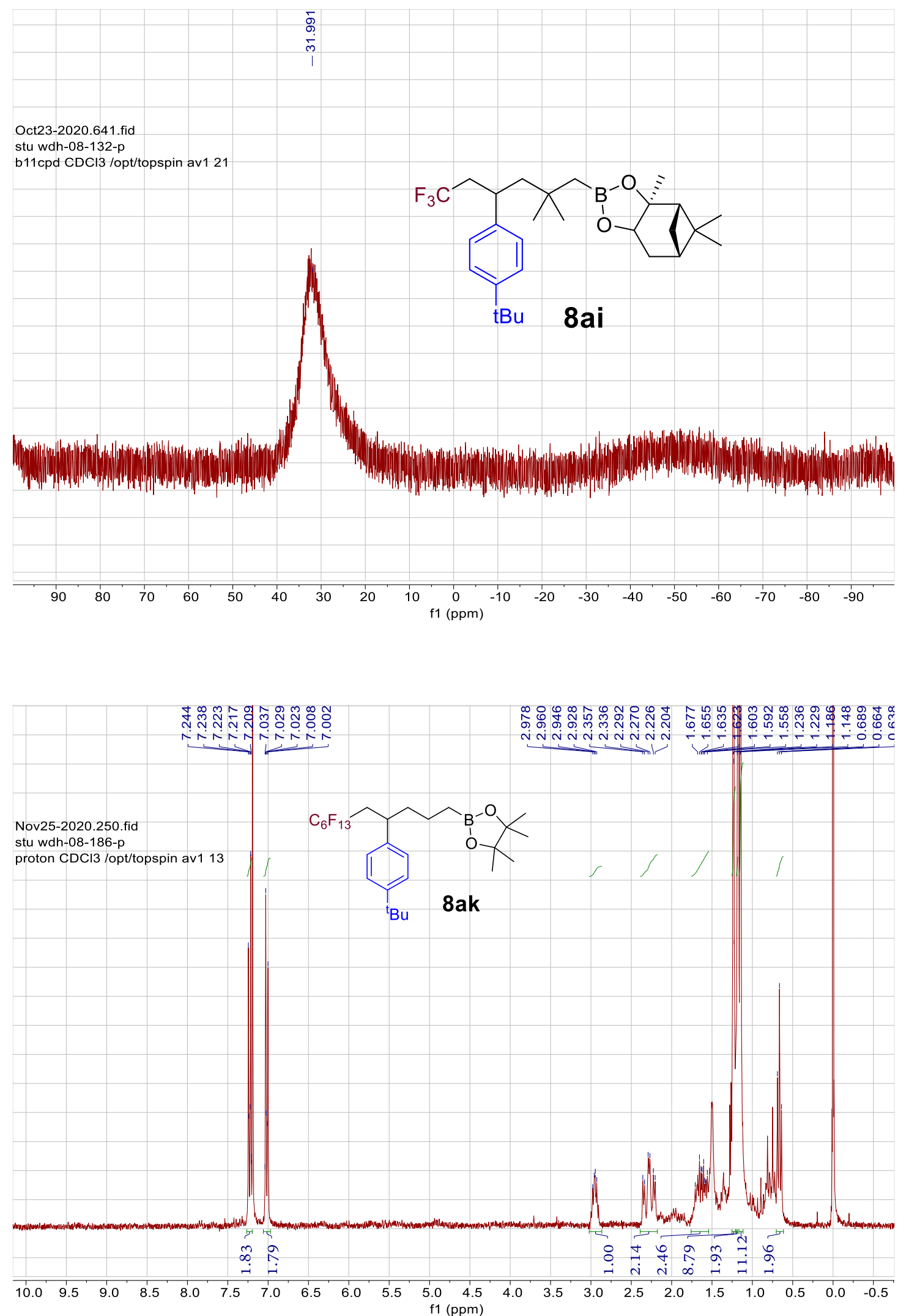

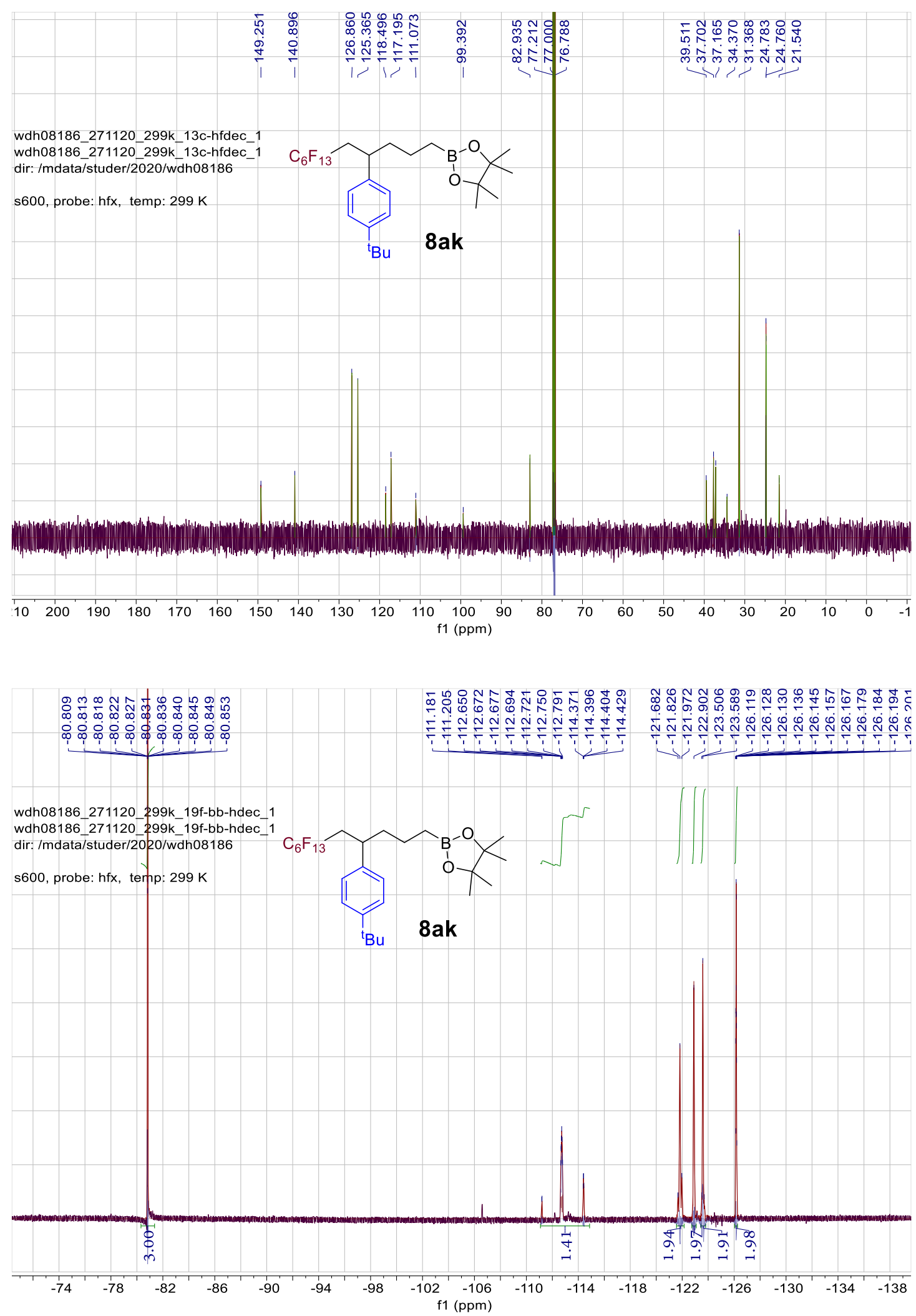
wdh08186_271120_299k_11b_1
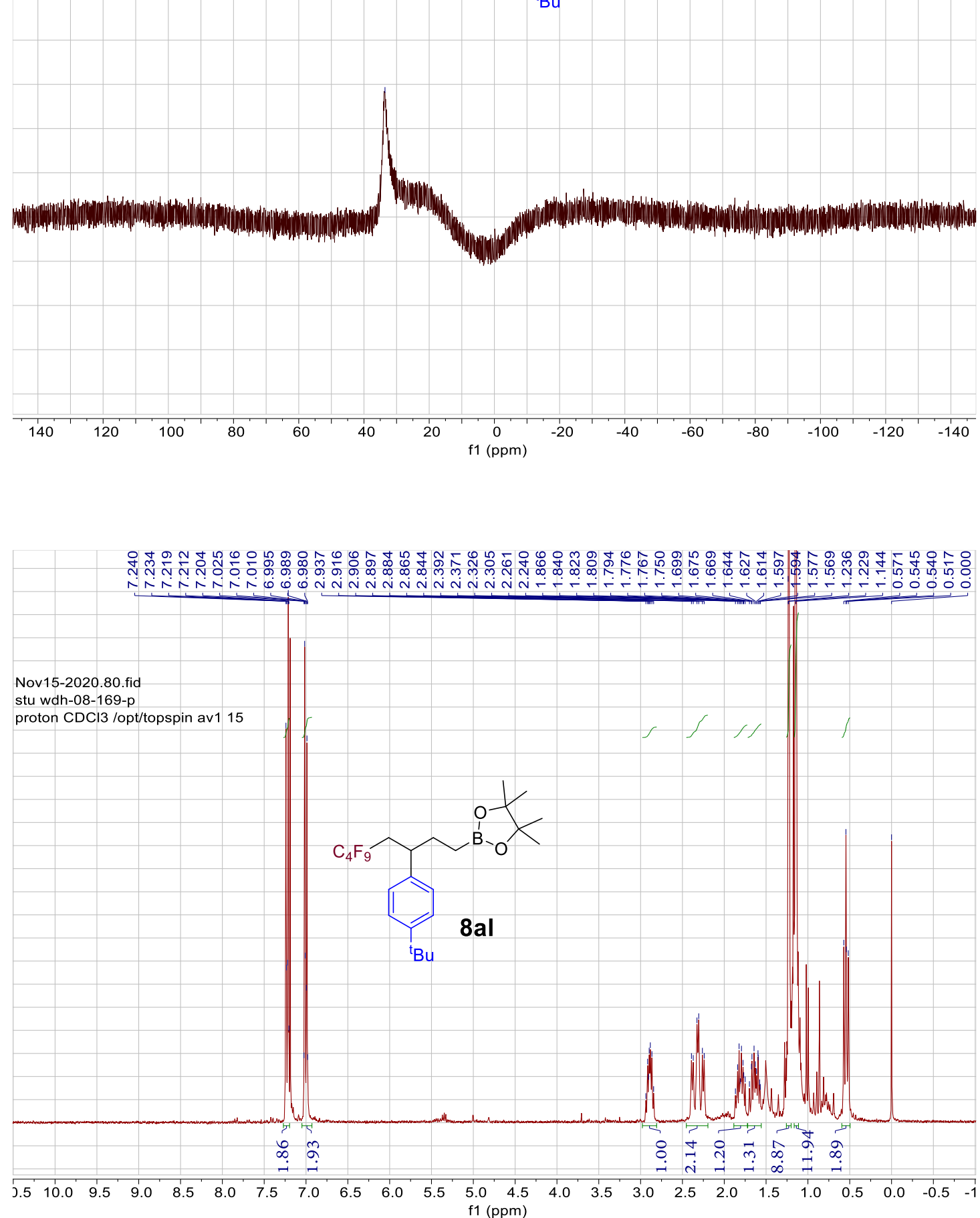

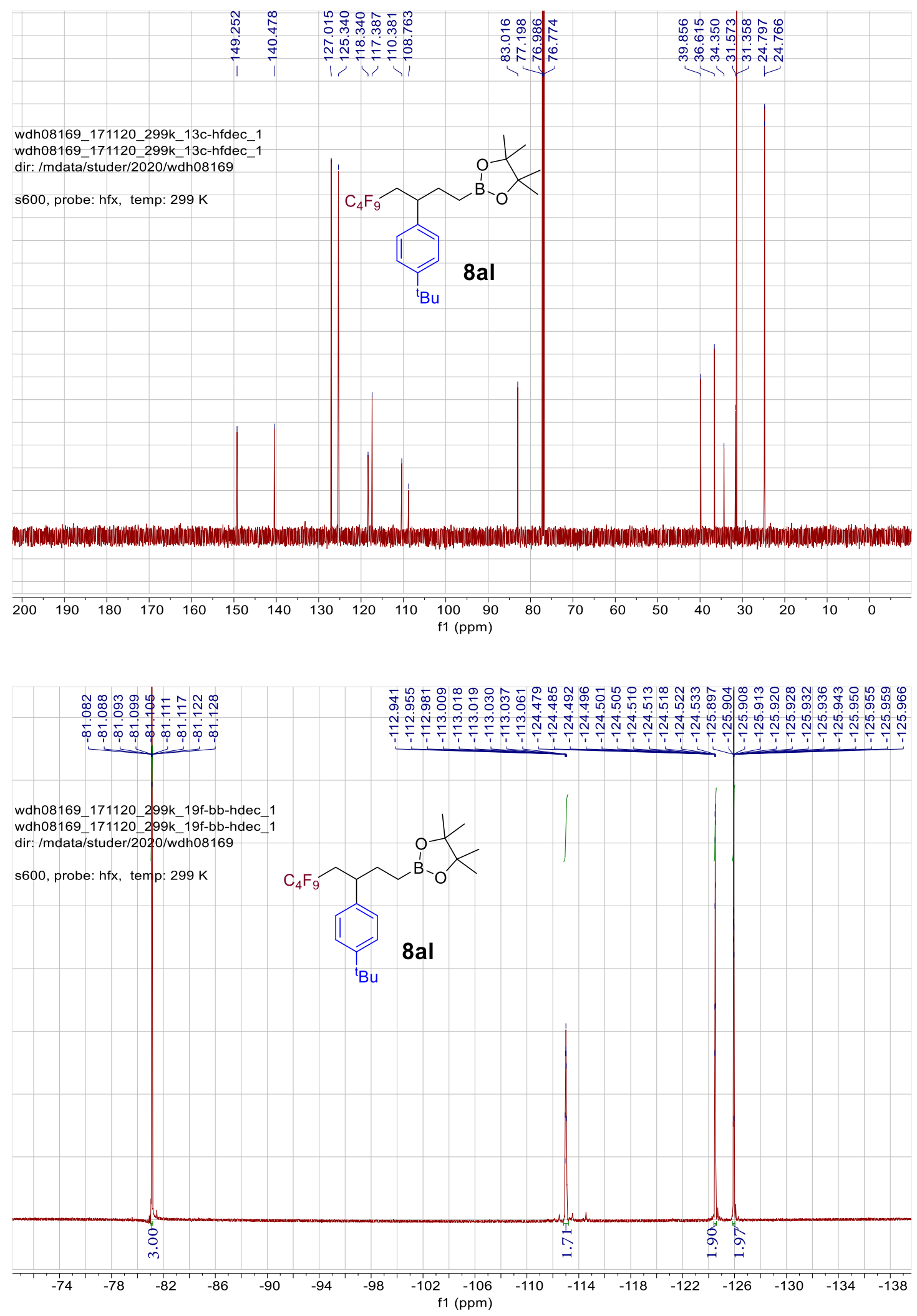

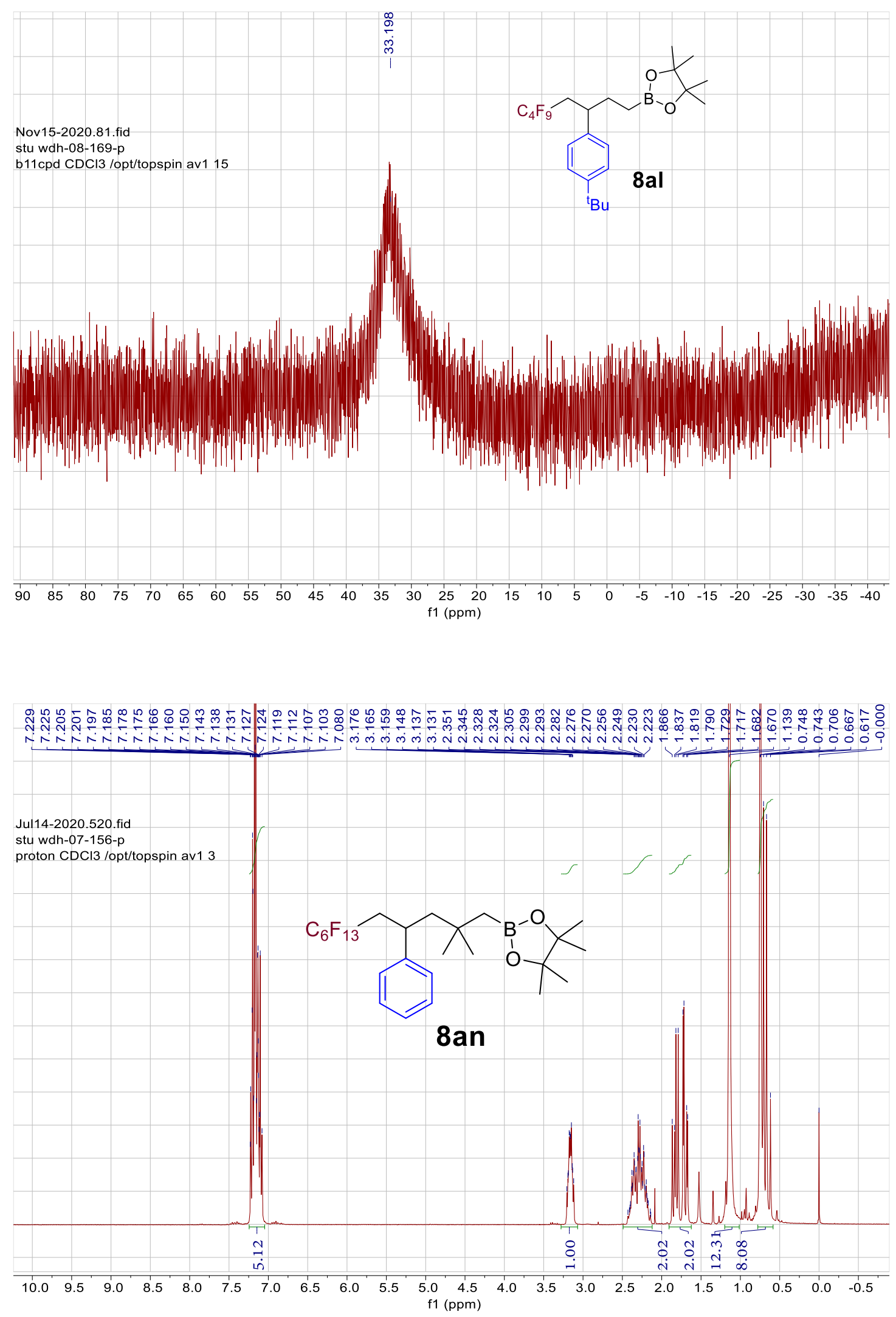

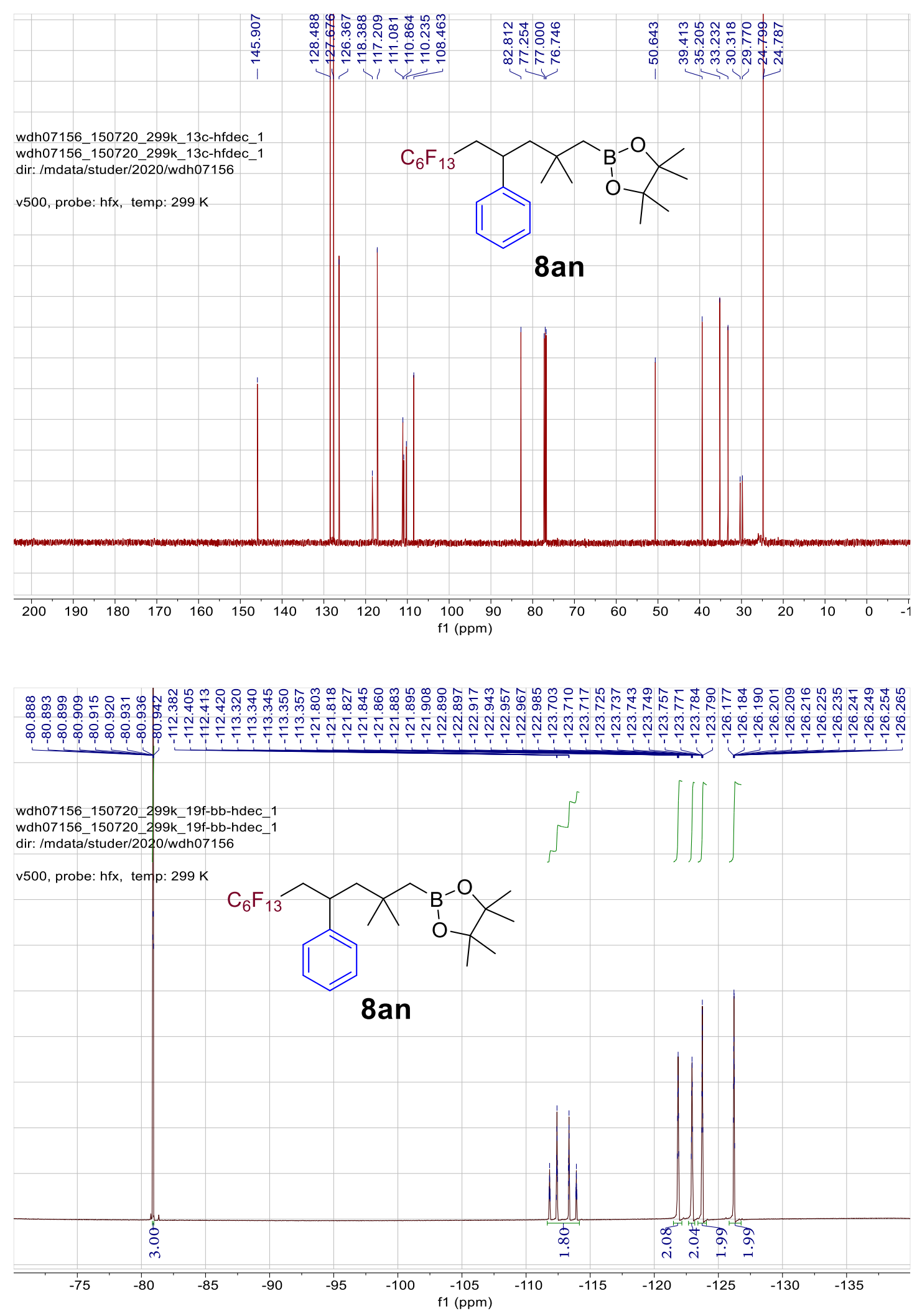

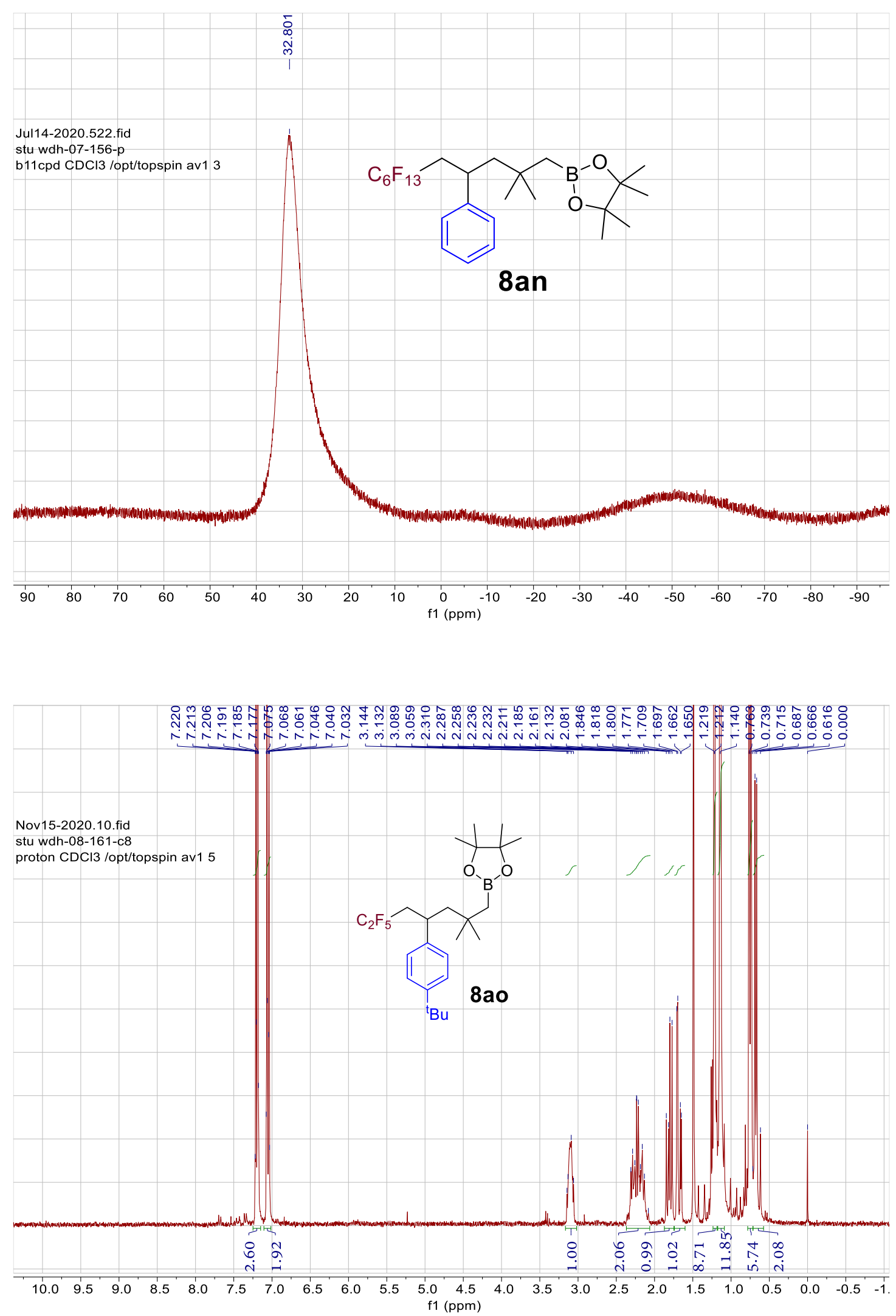

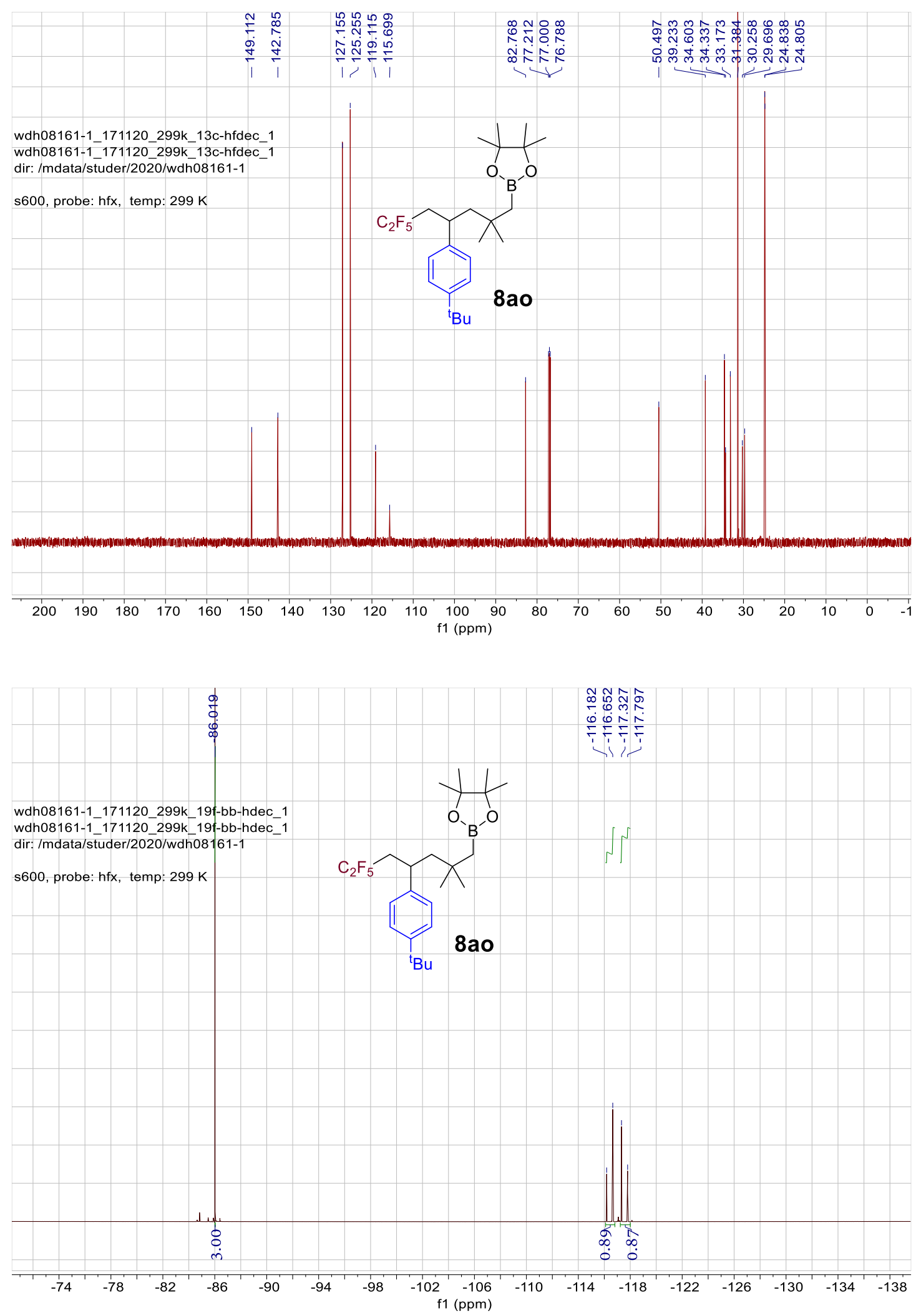


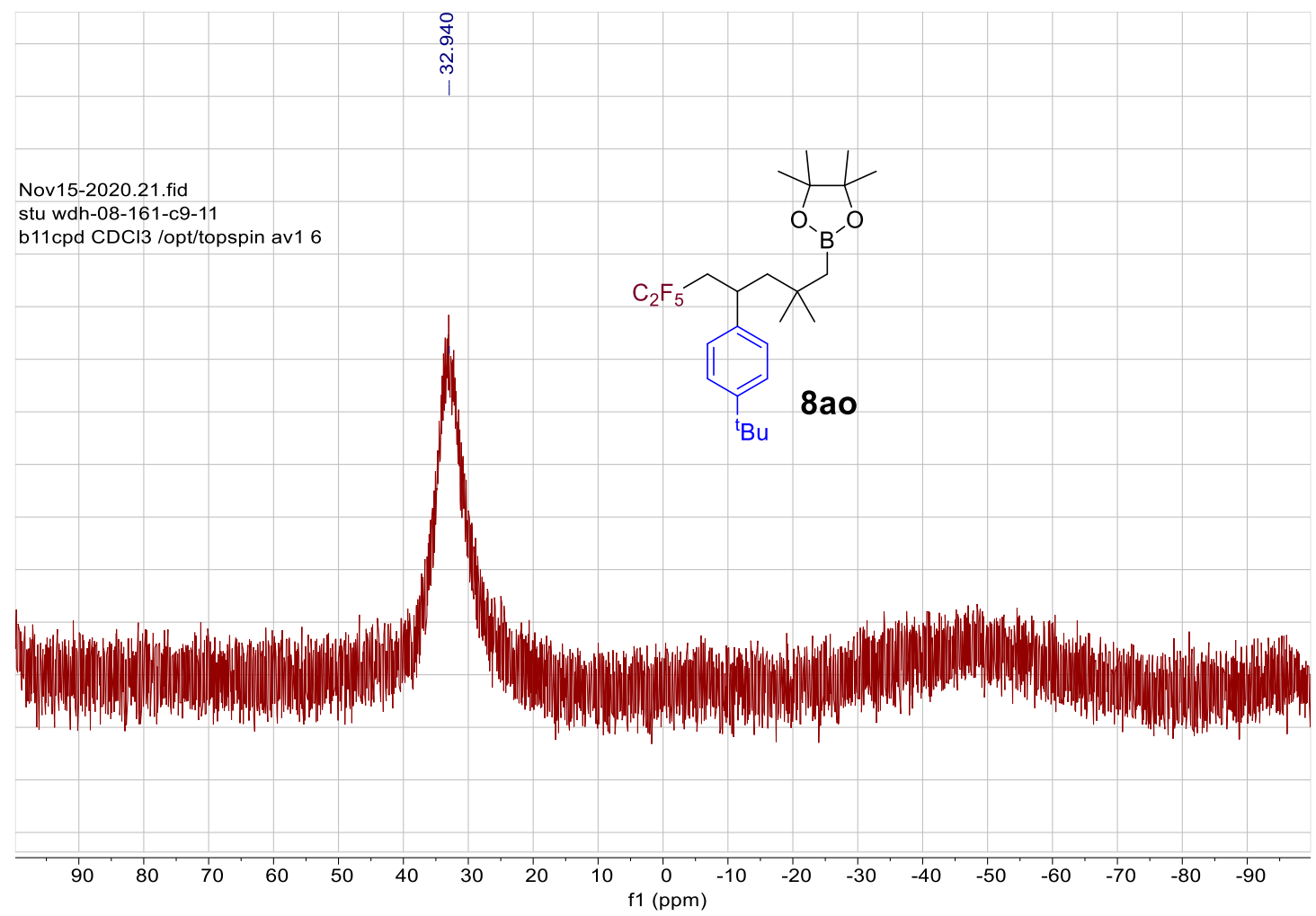

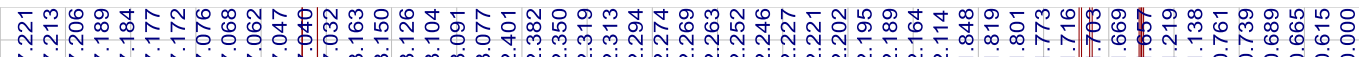

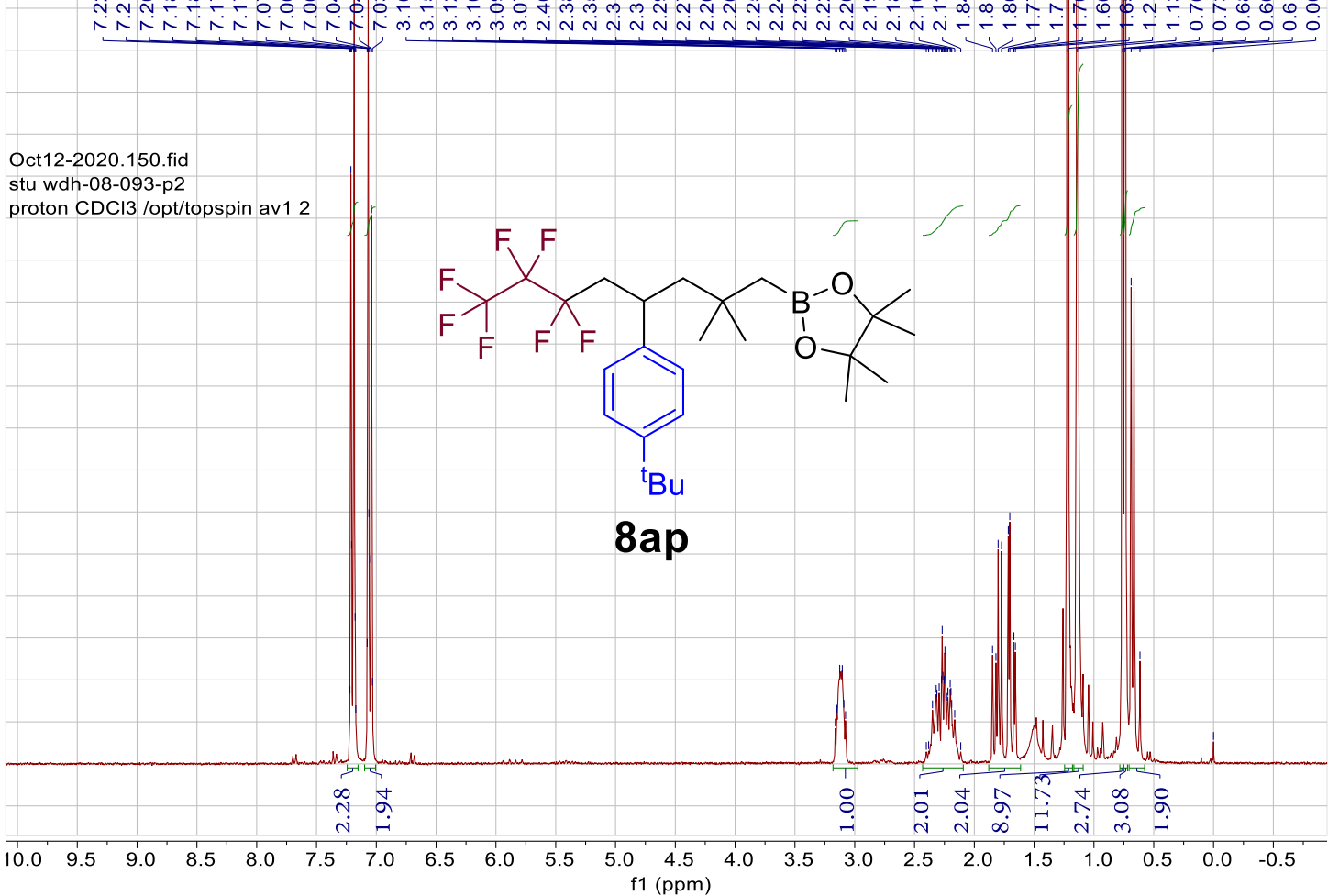



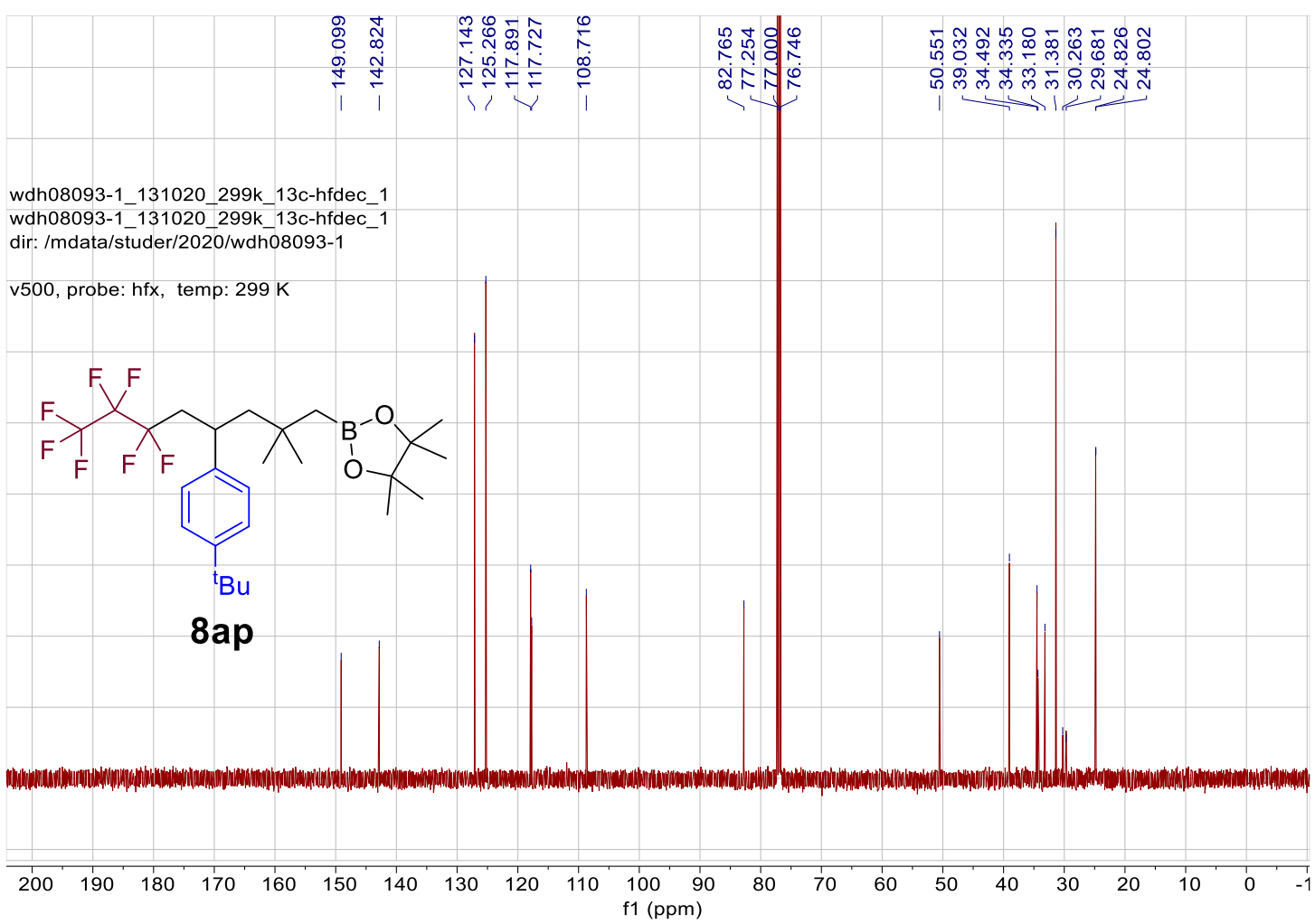

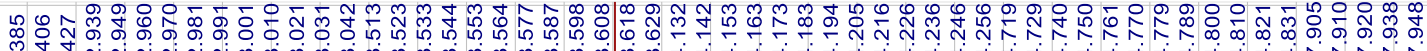

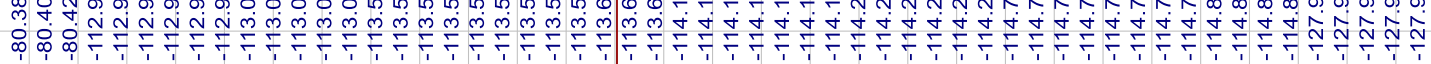

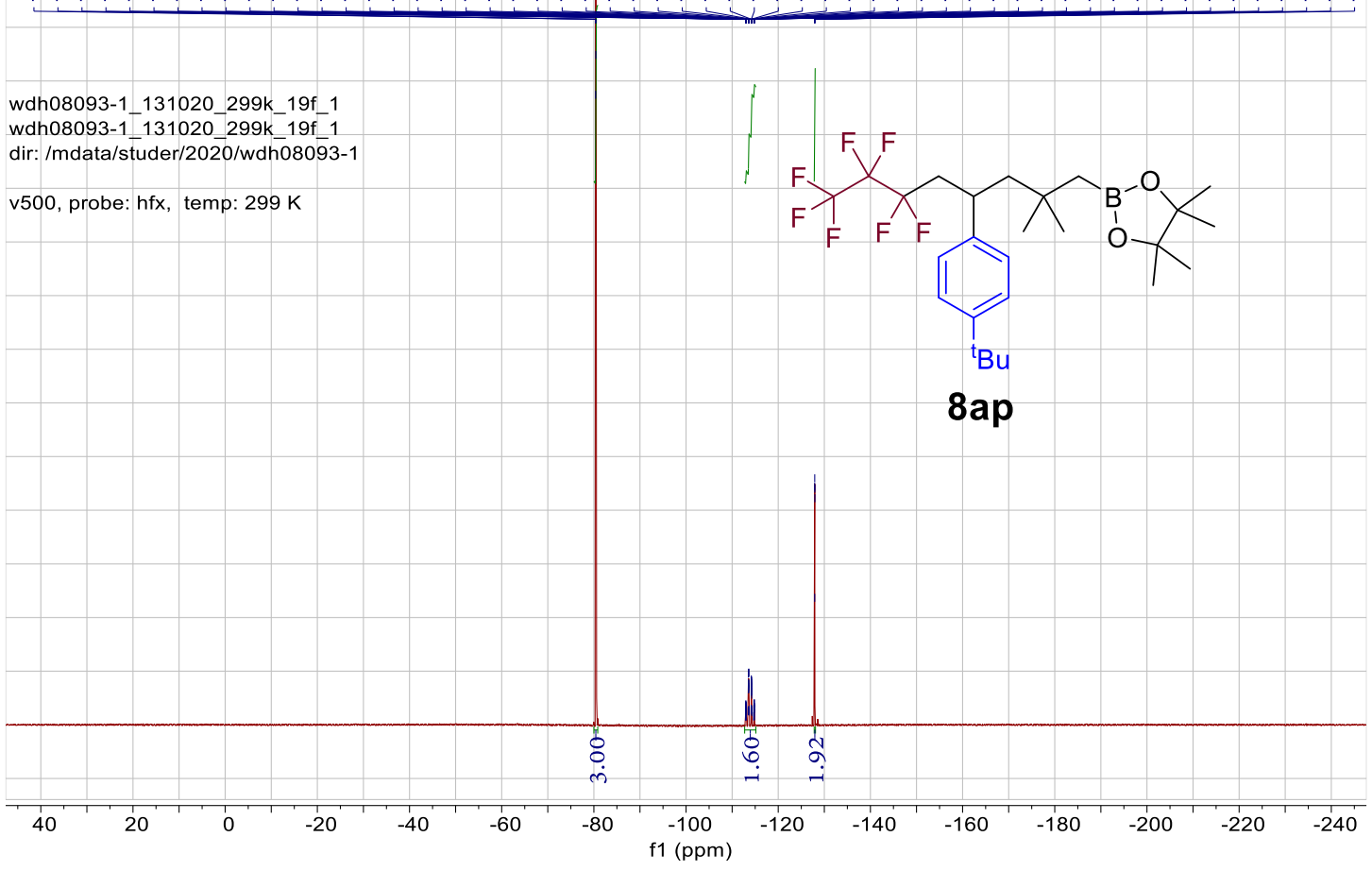



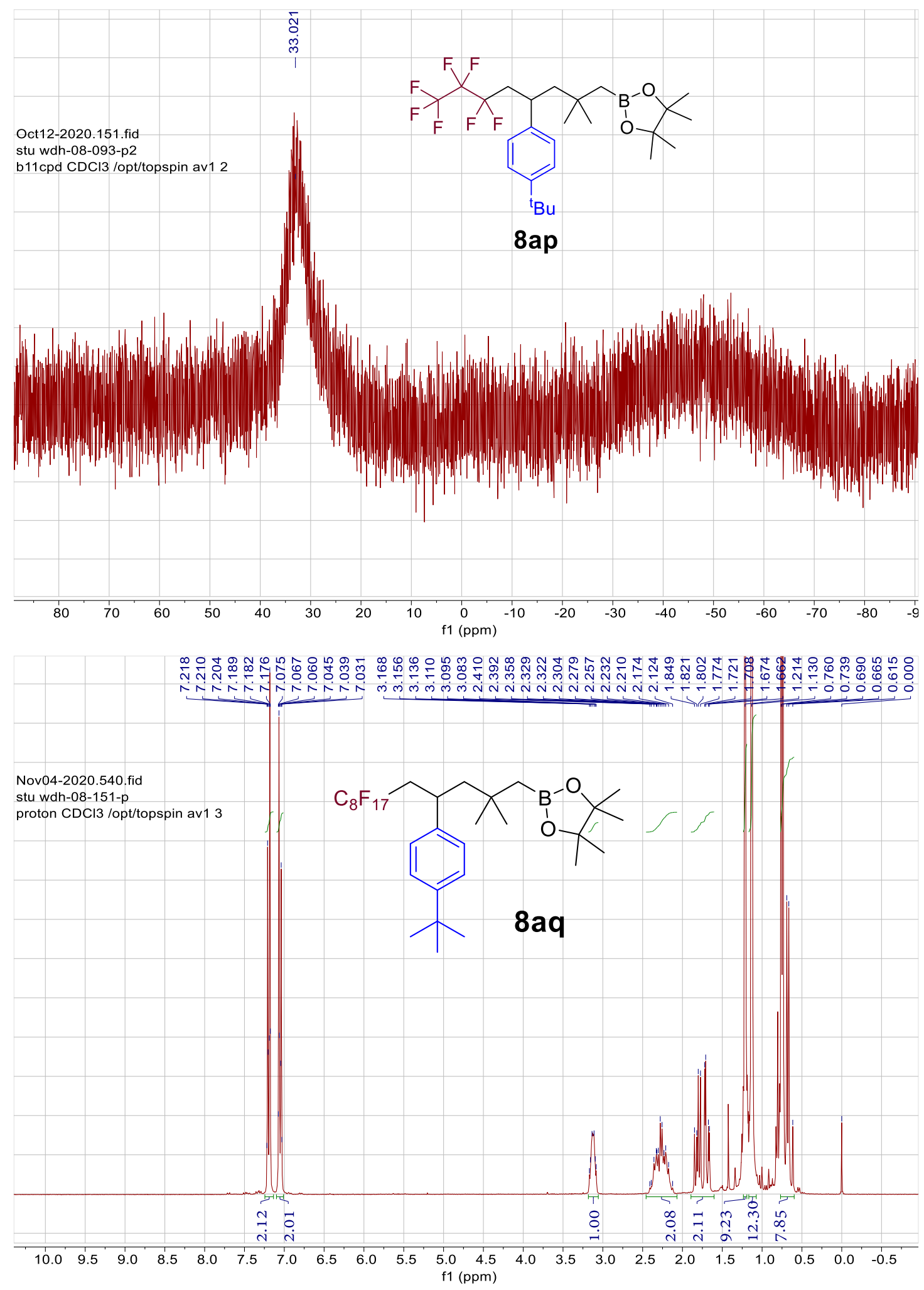

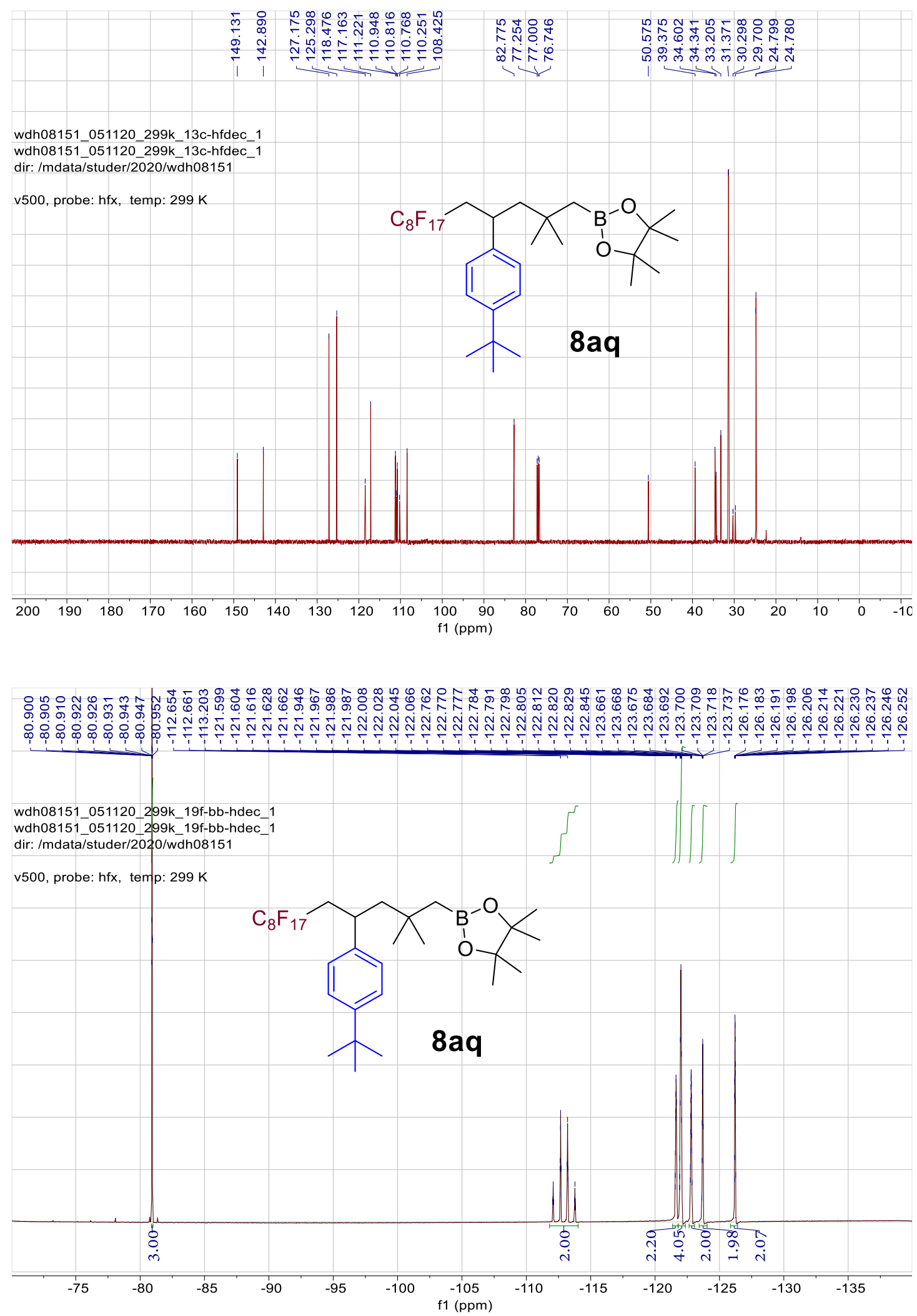

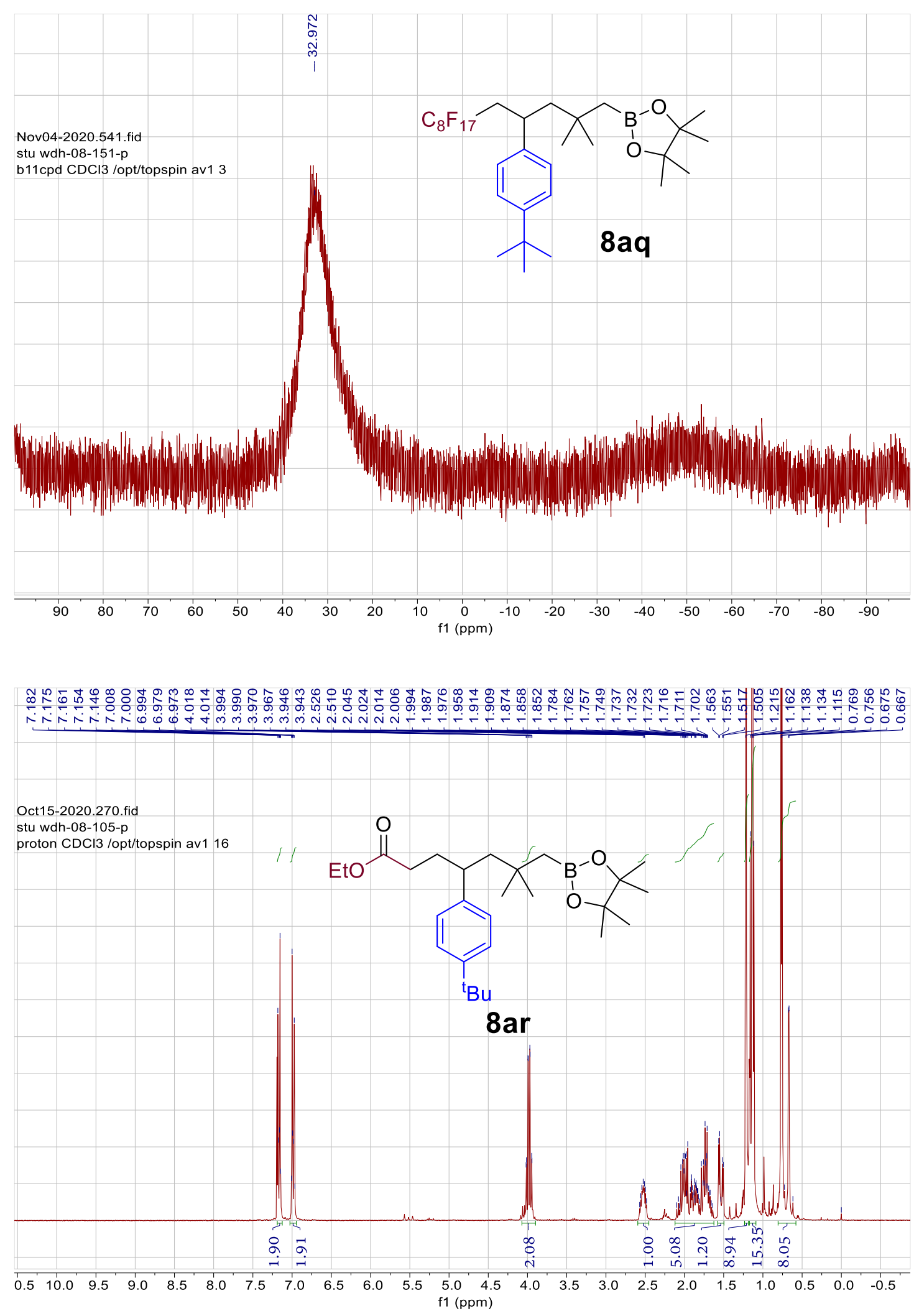

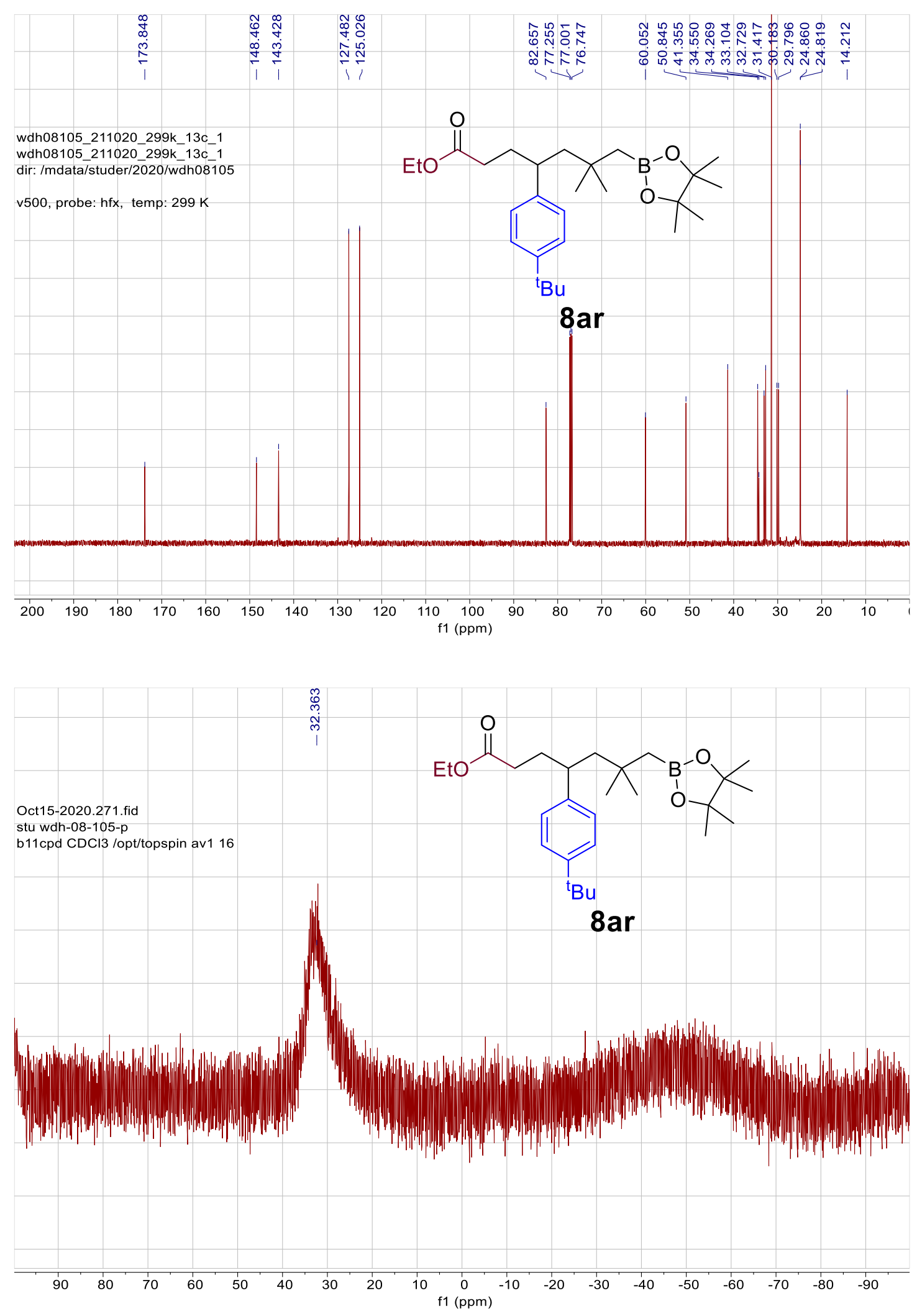

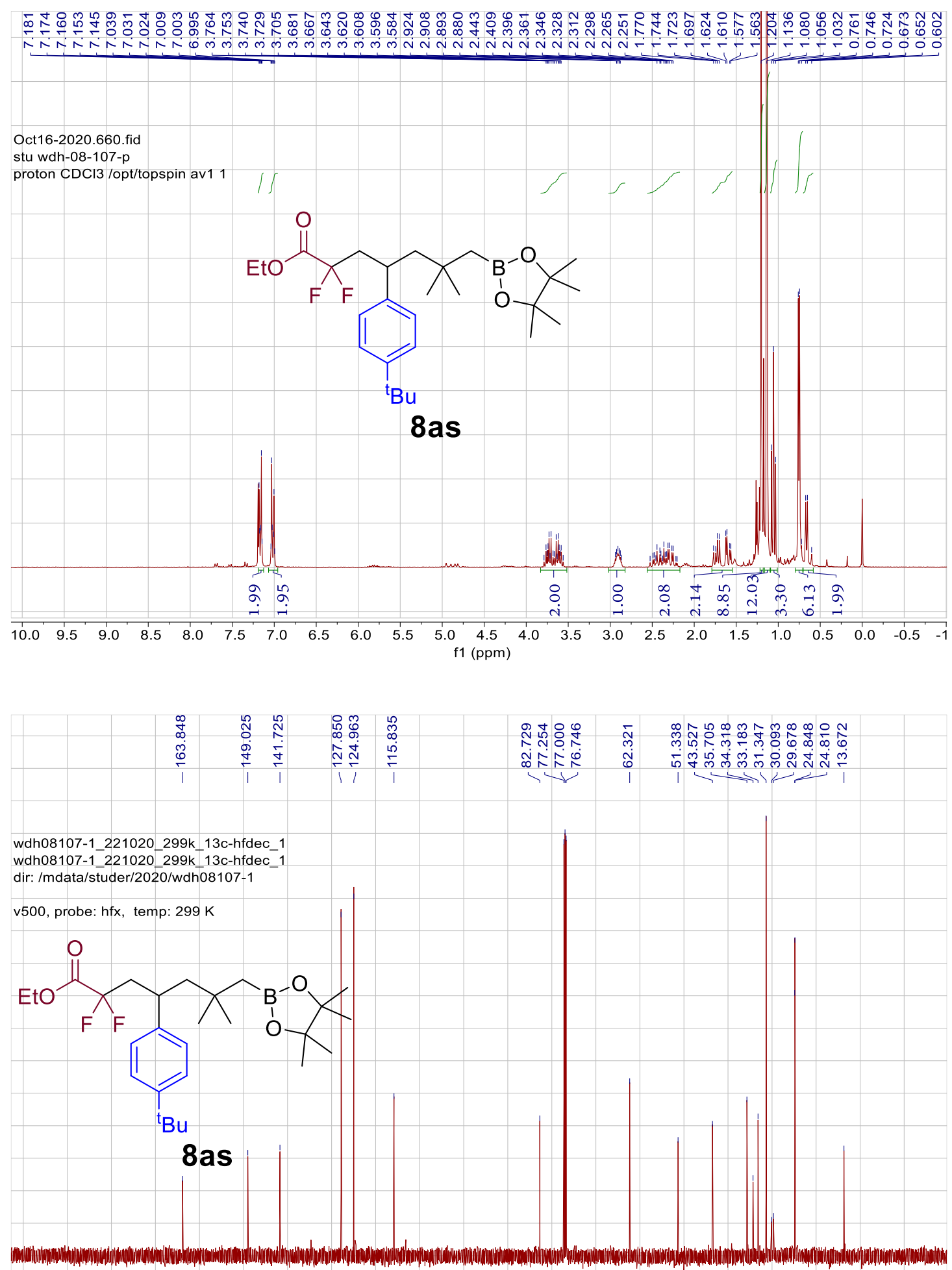

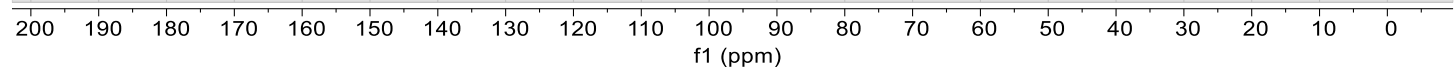



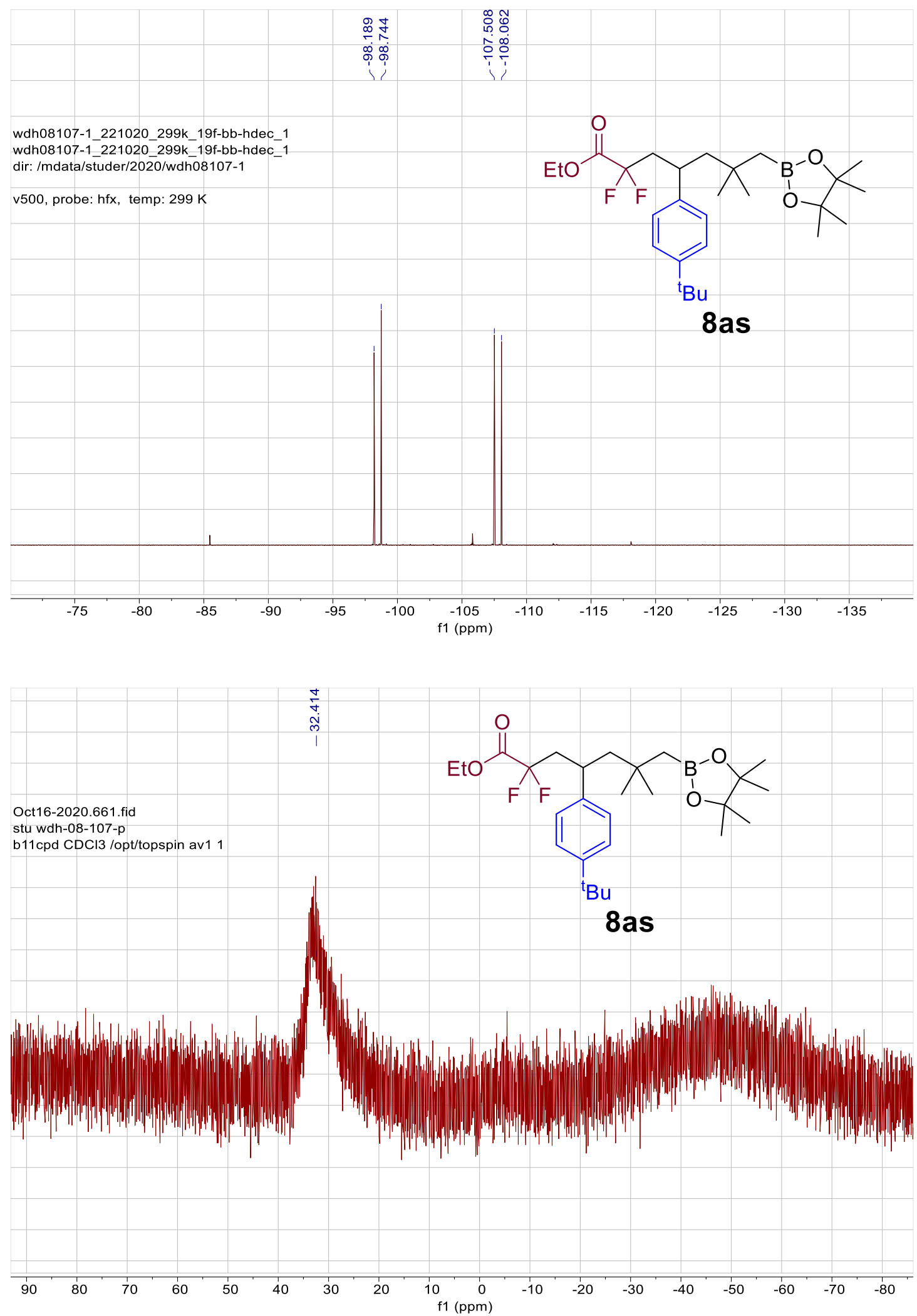

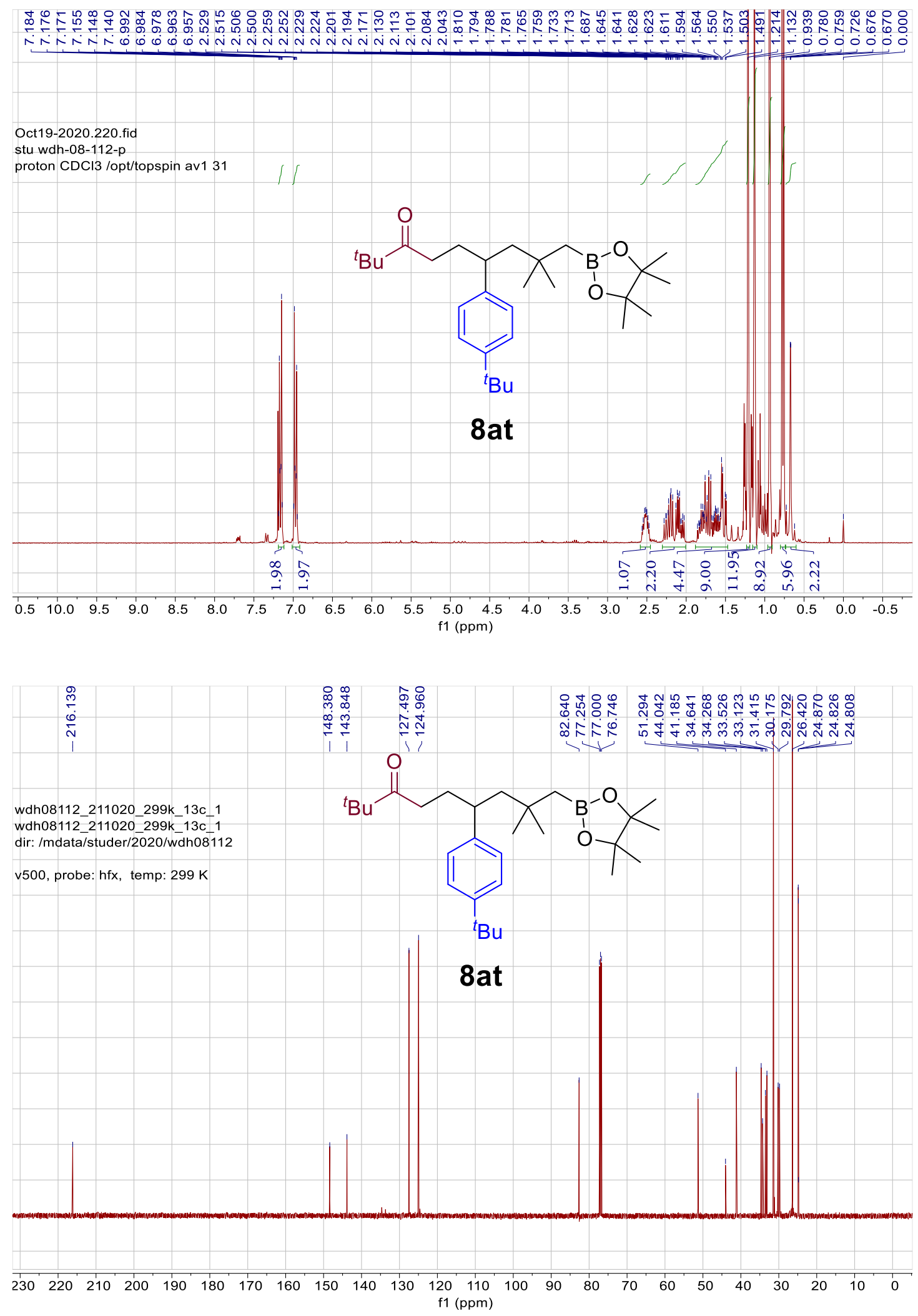

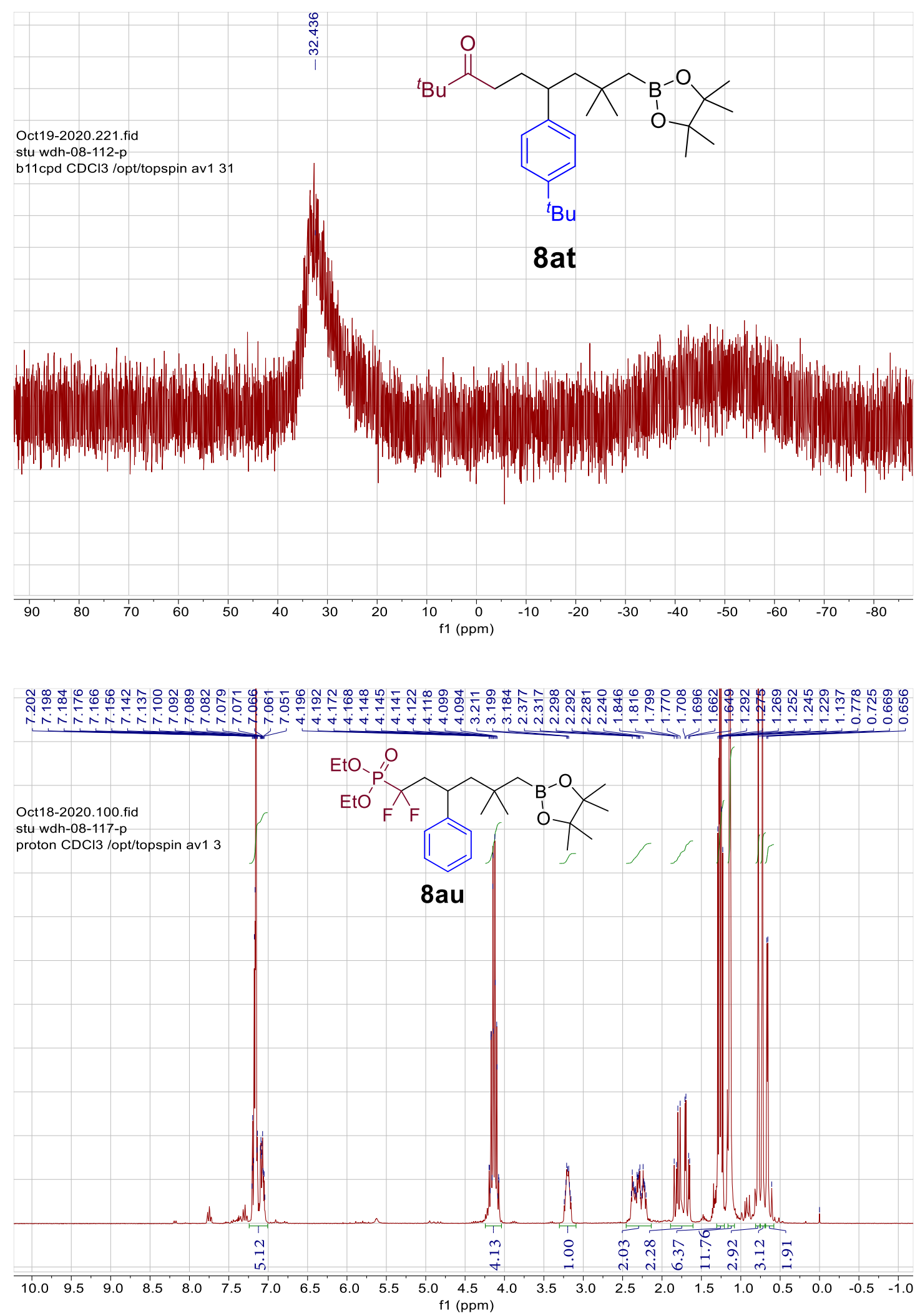

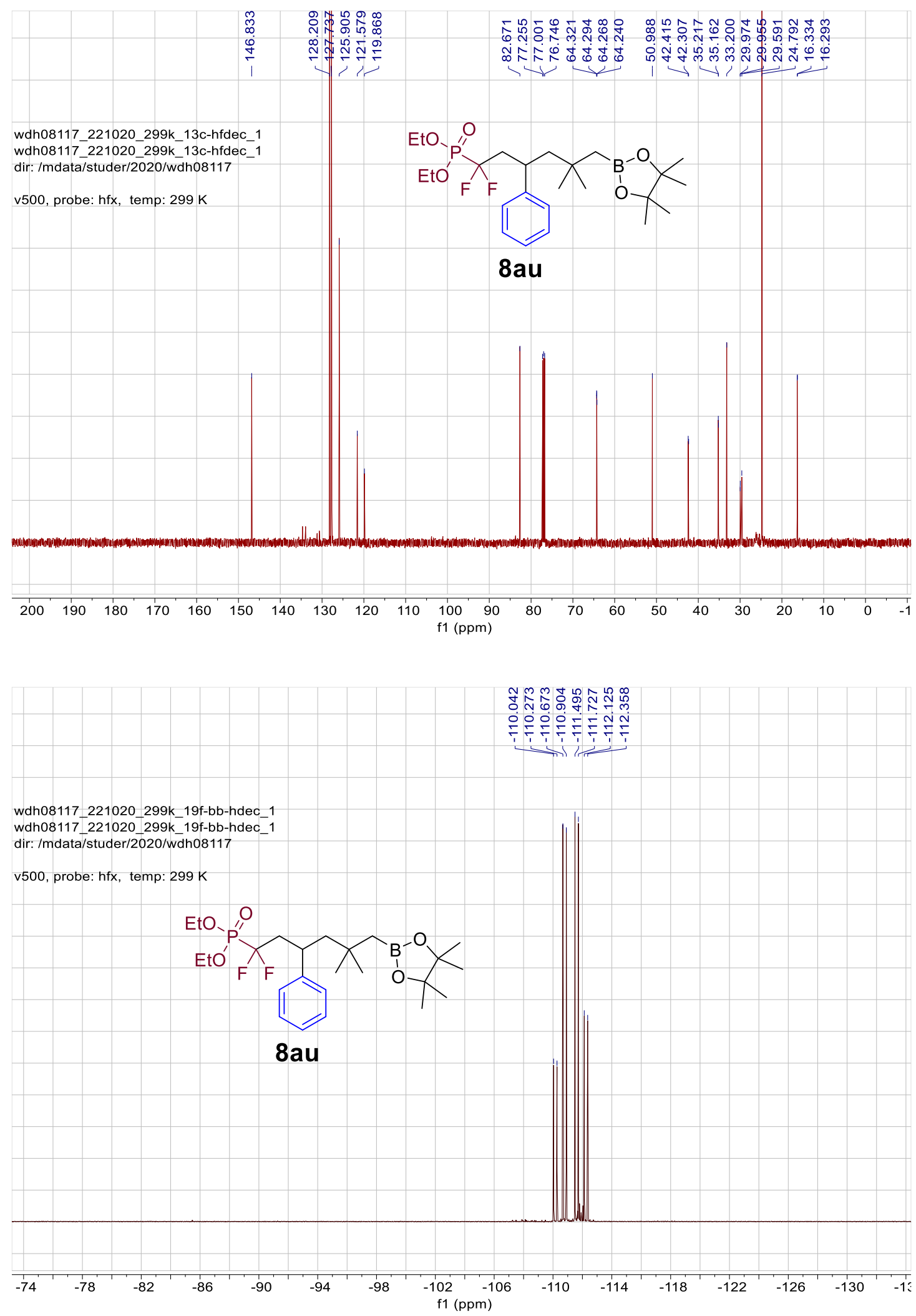

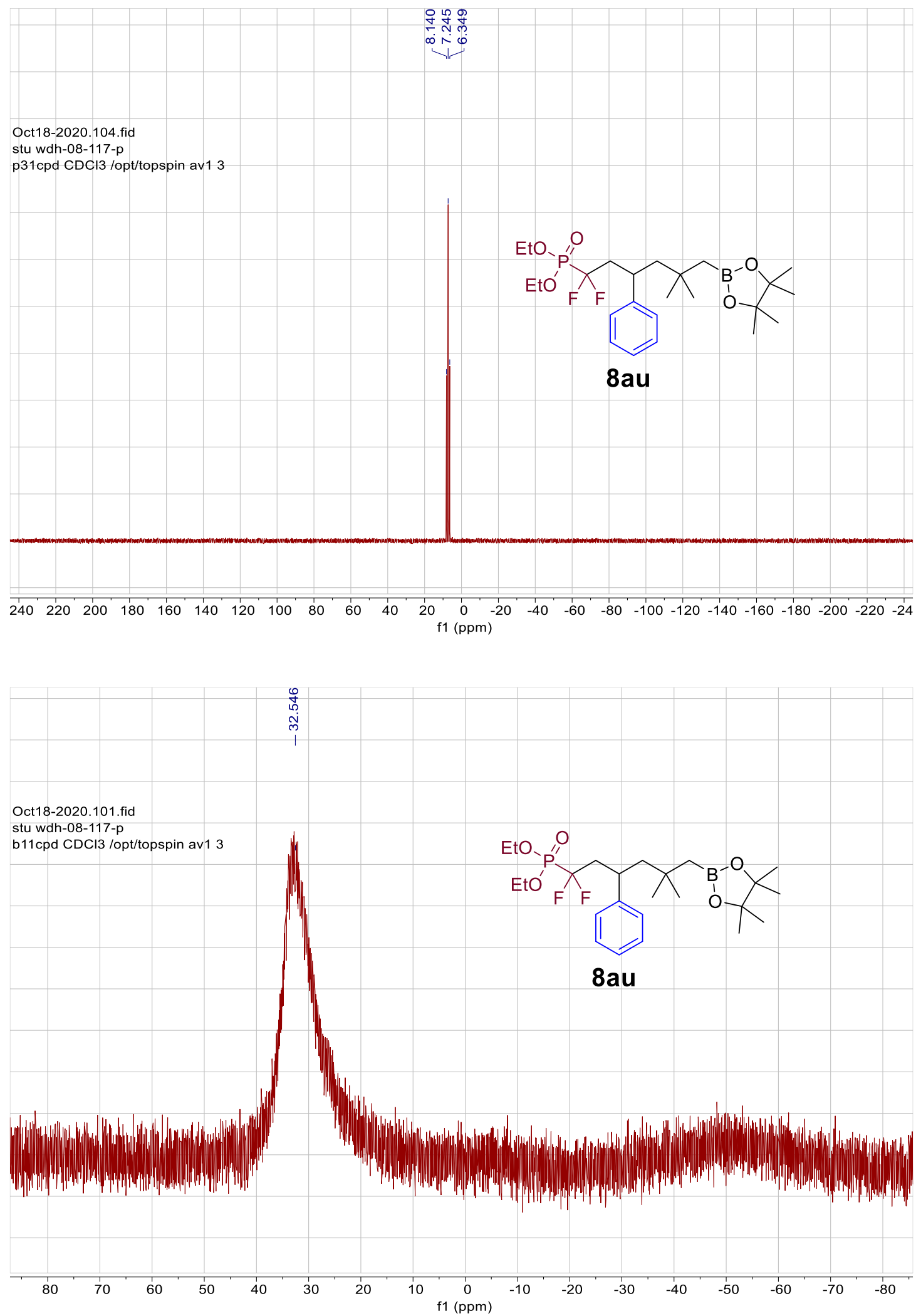

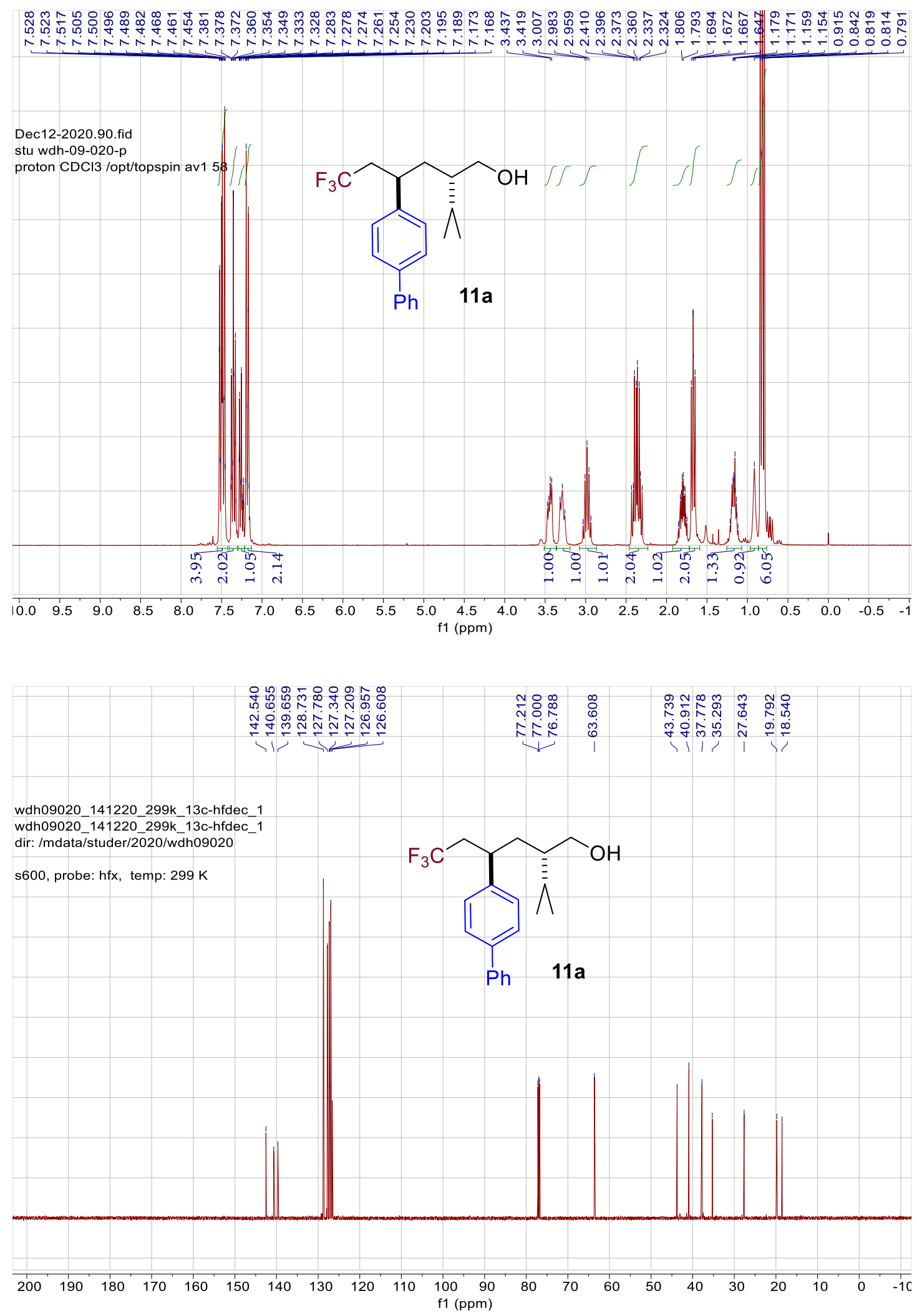

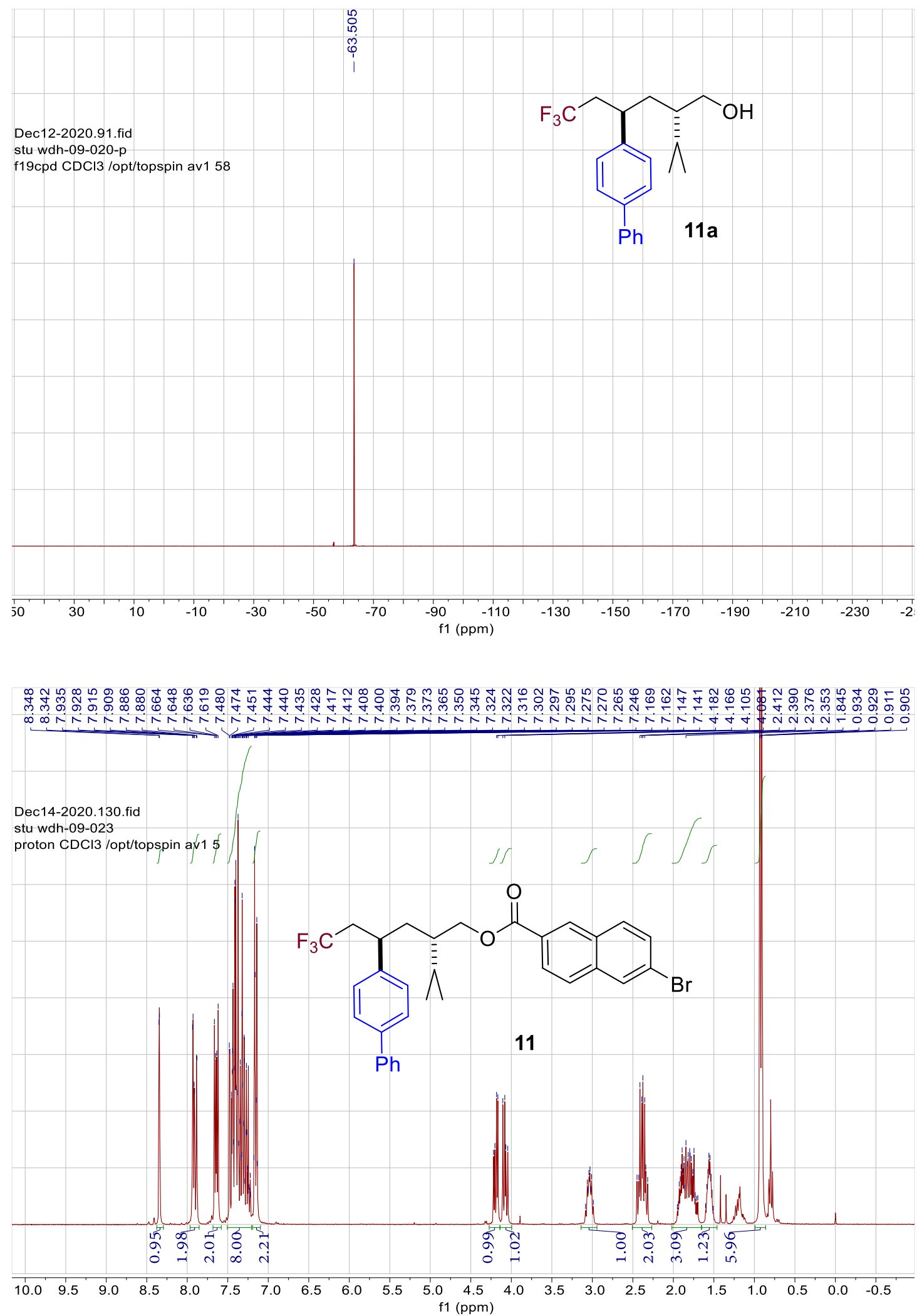

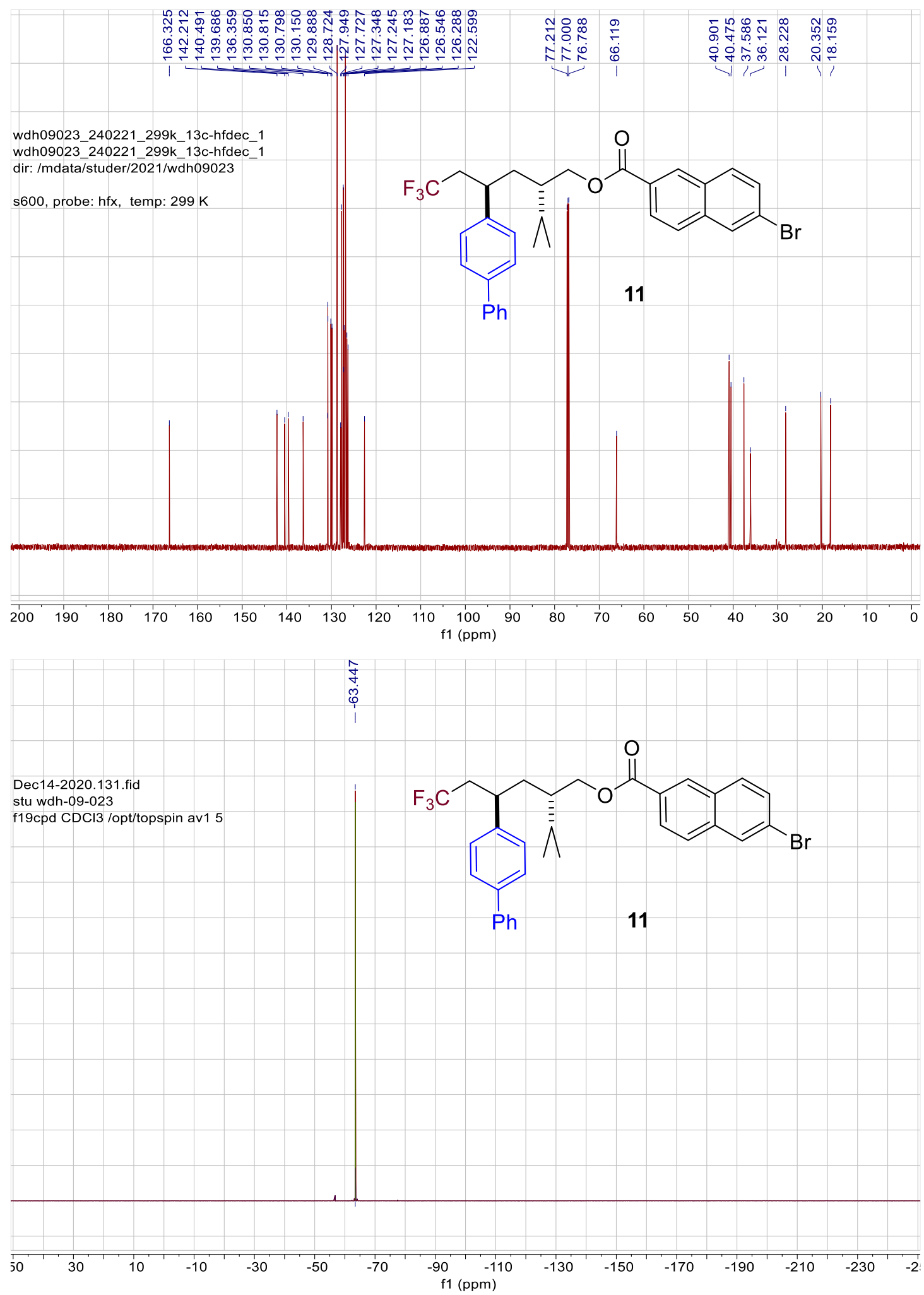

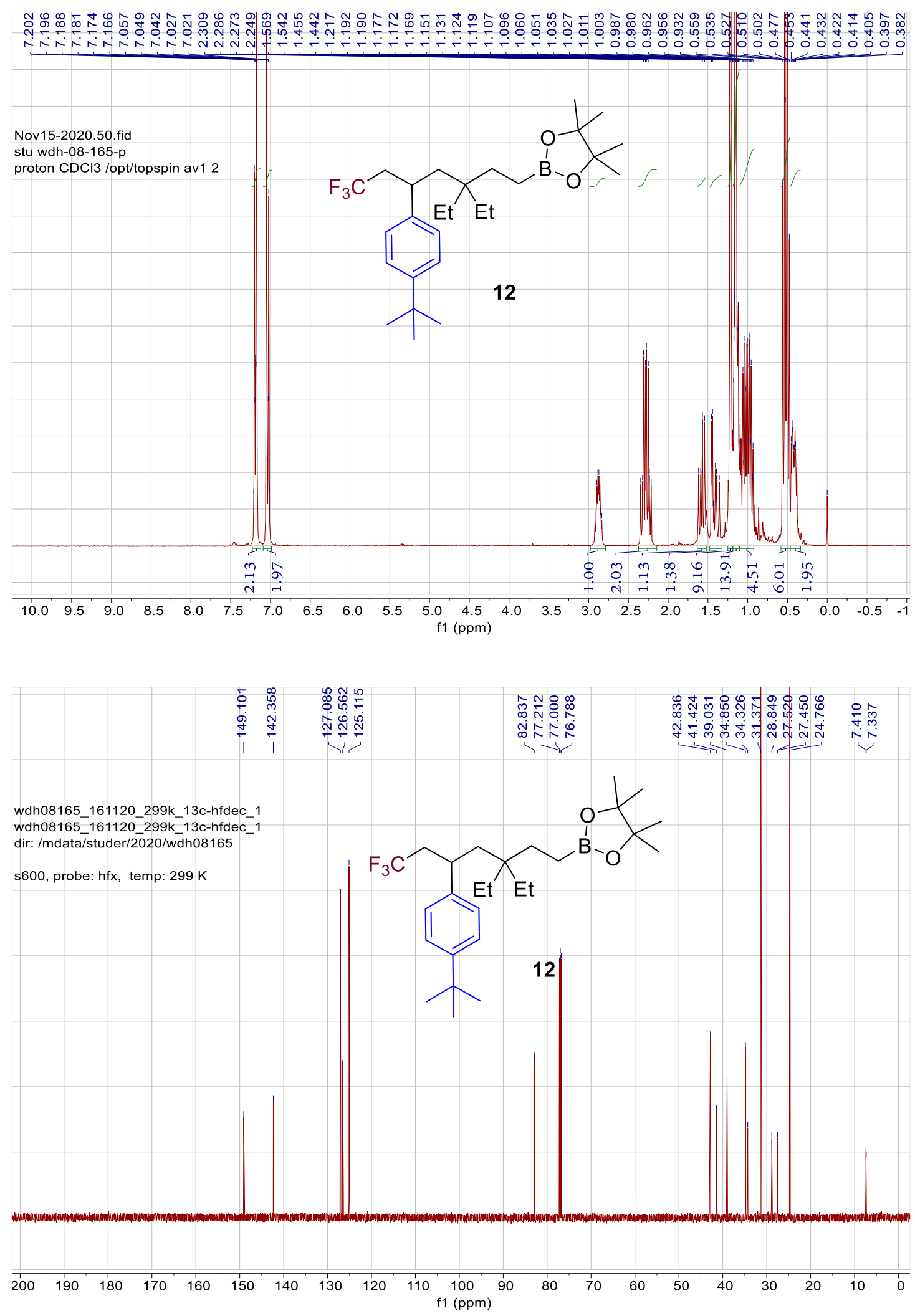

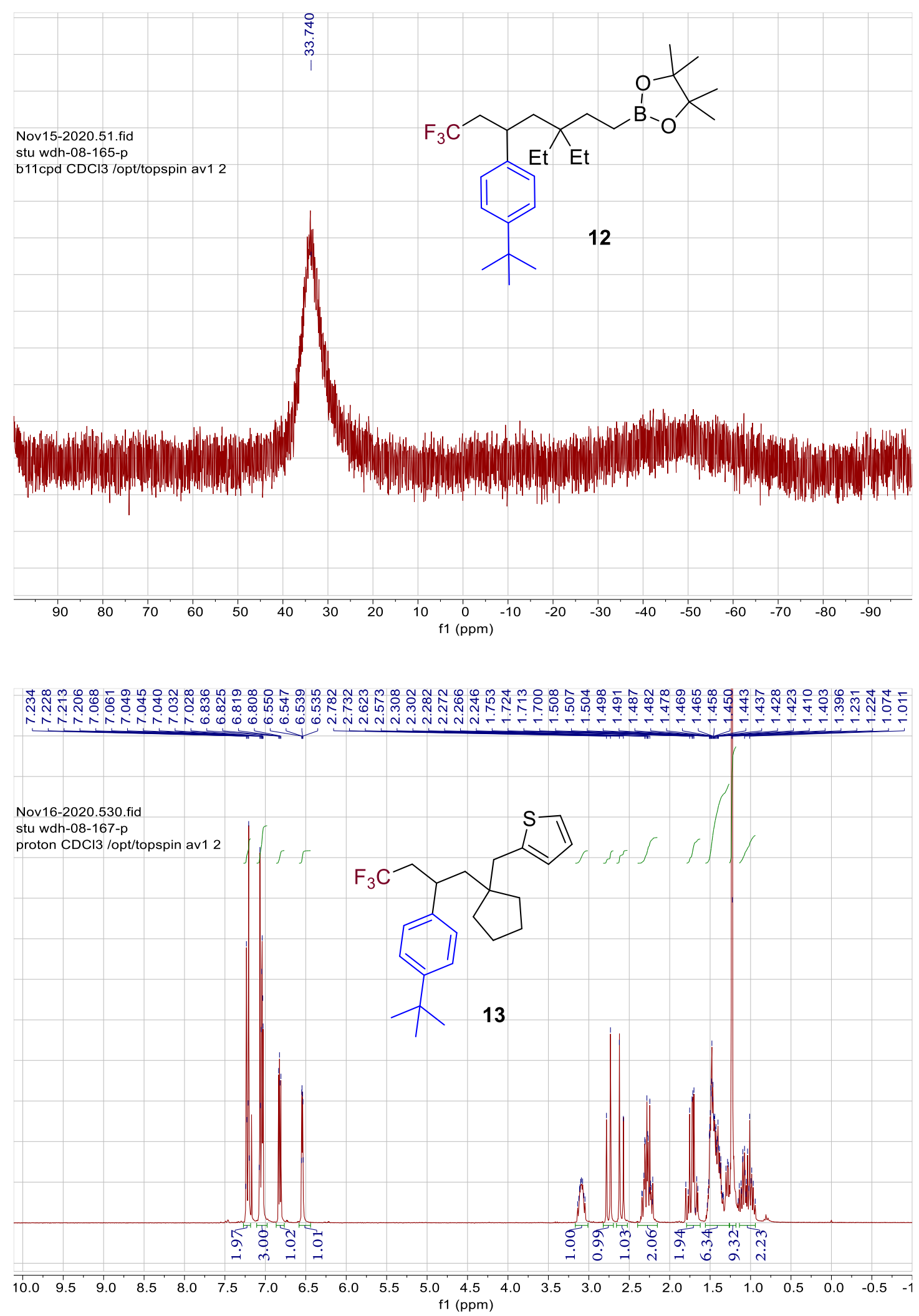

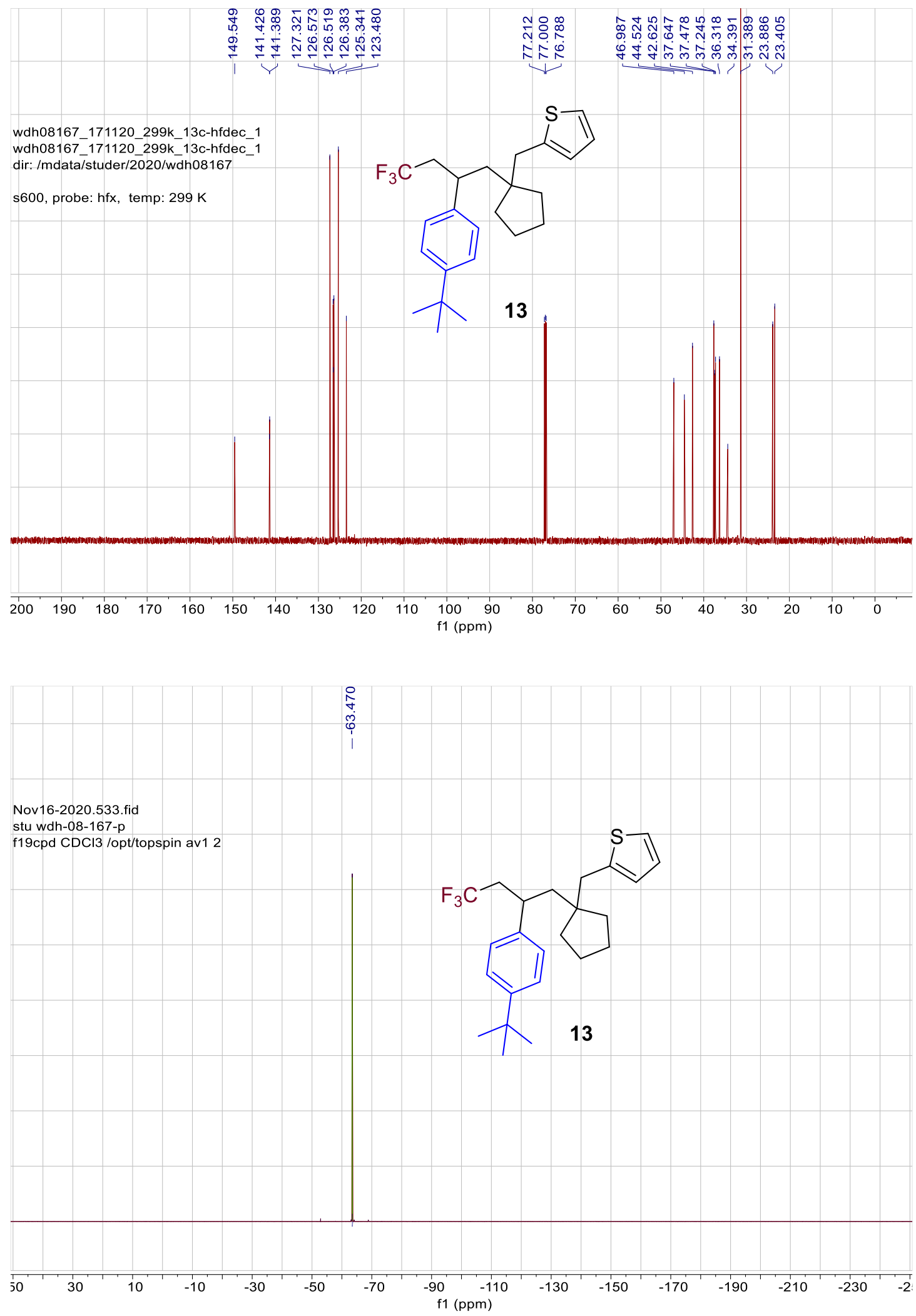

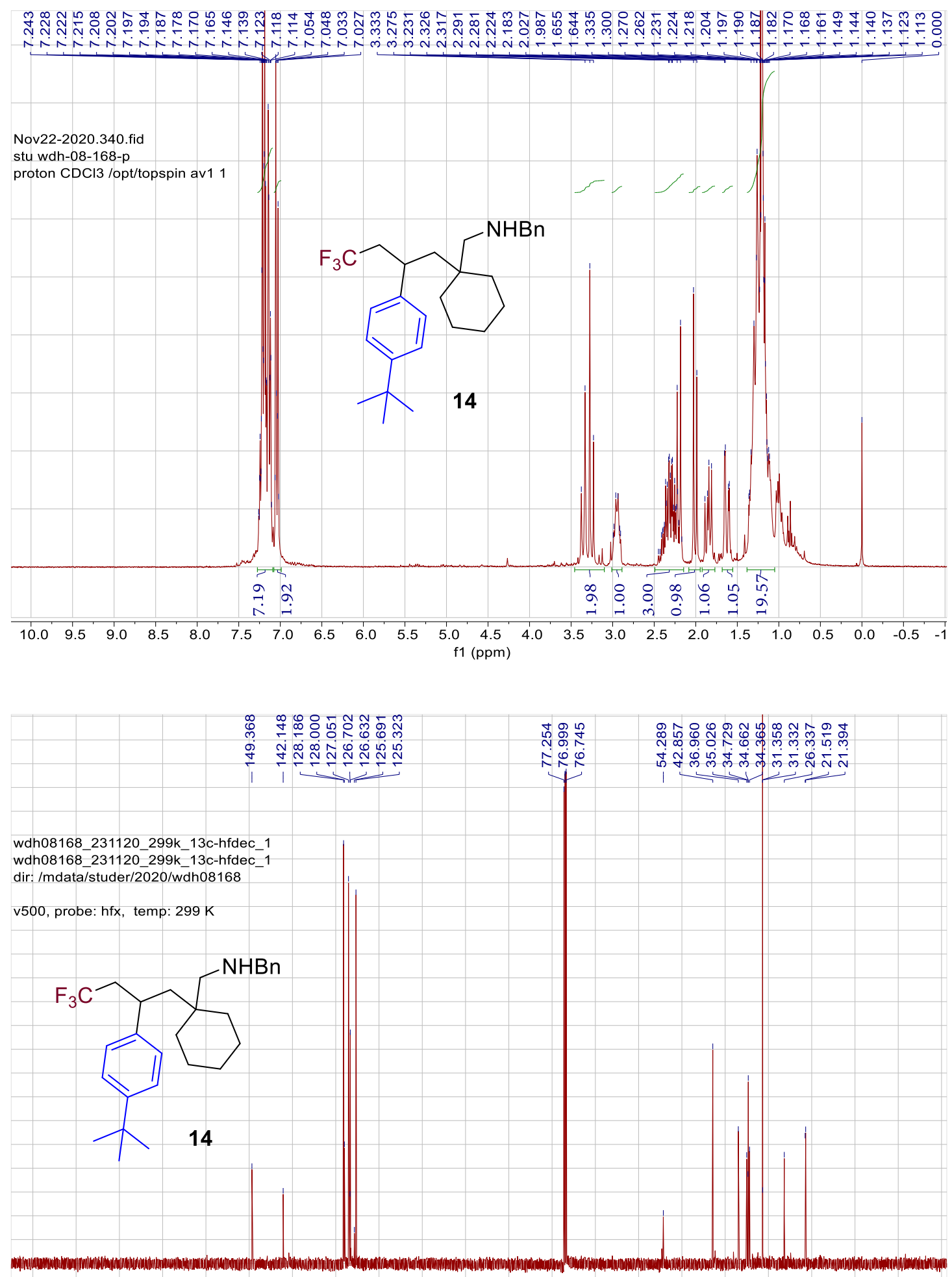

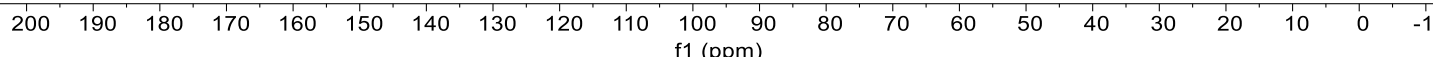



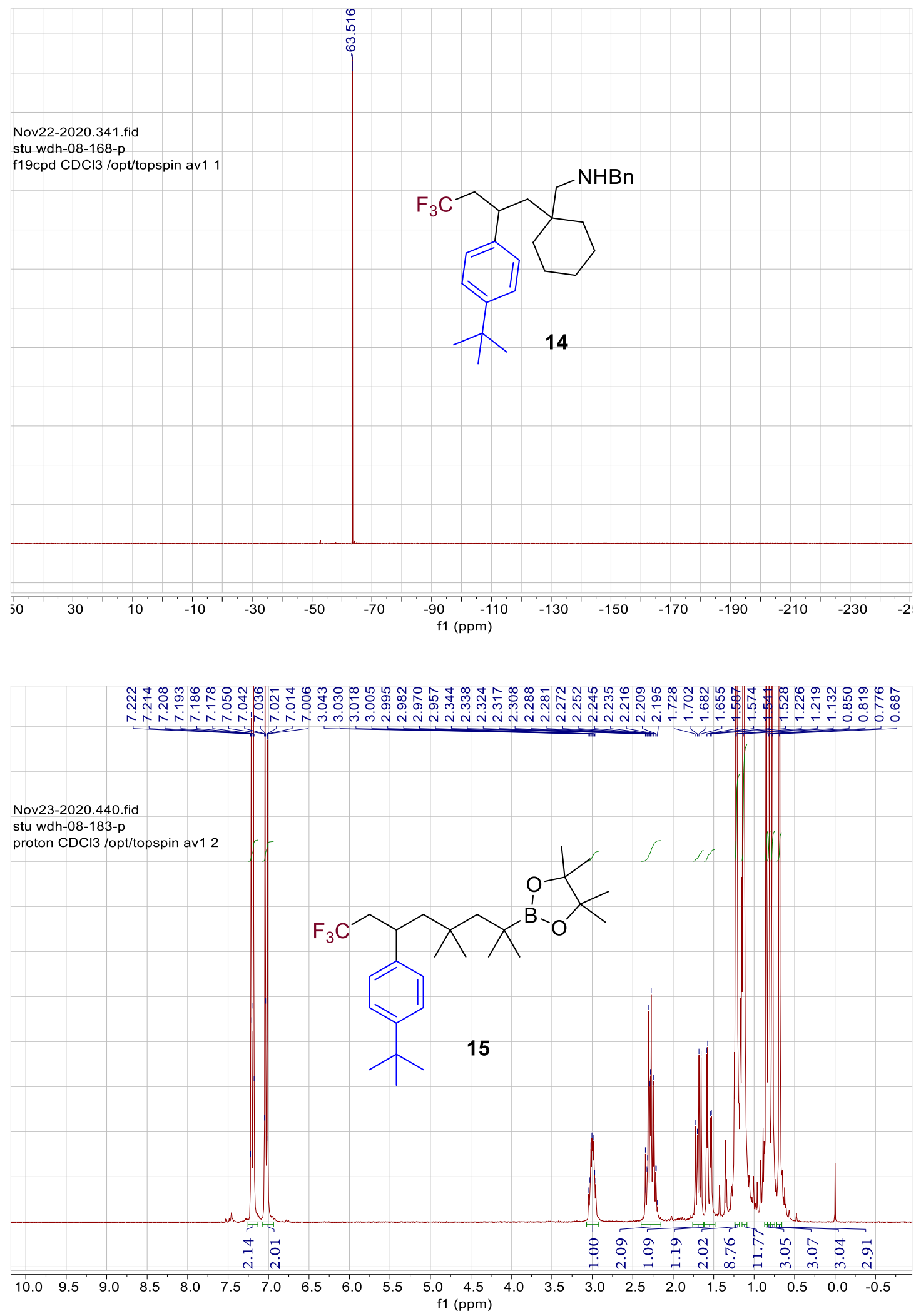

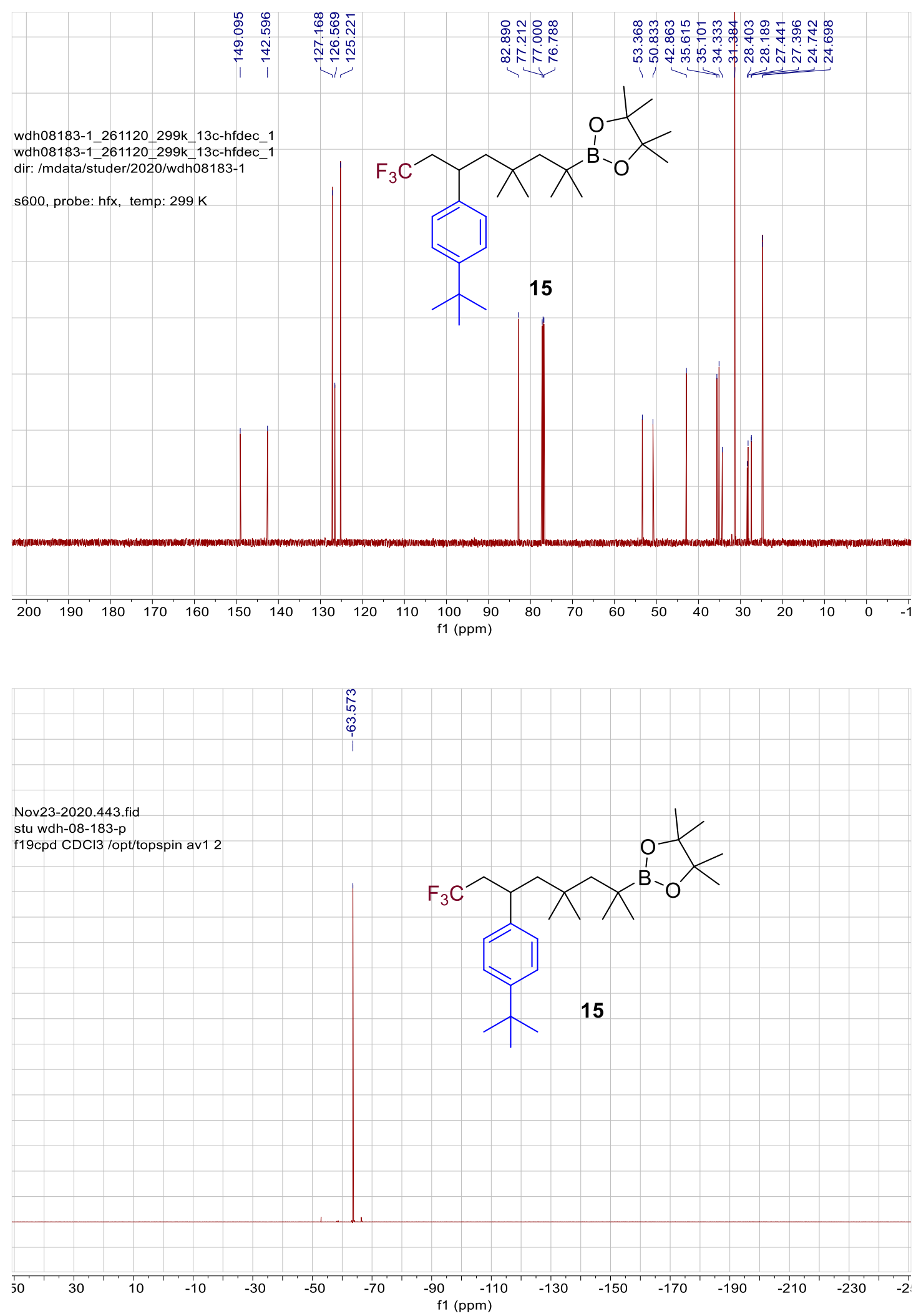


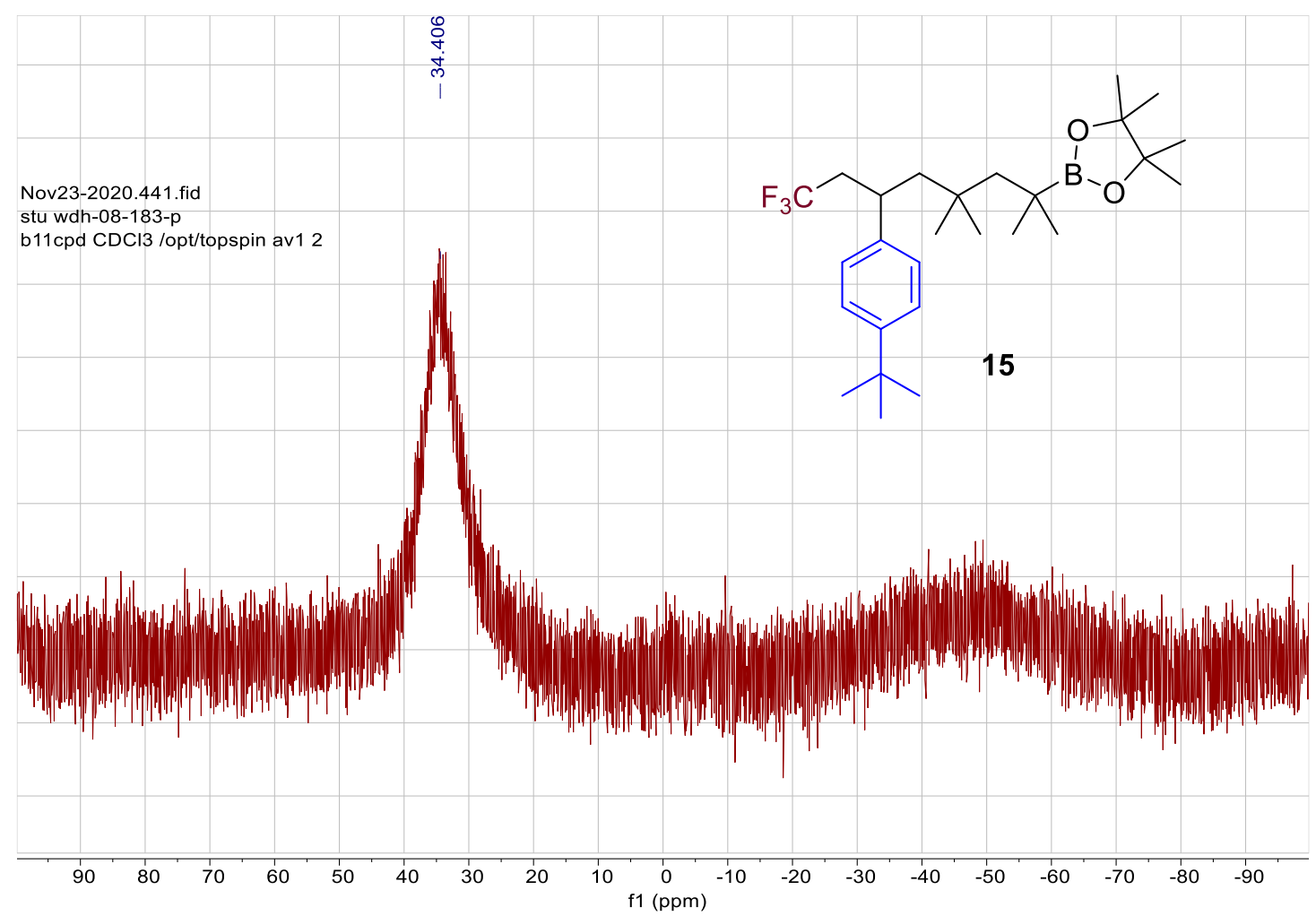

